\title{
L'eterno ritorno del Droit des gens di Emer de Vattel (secc. XVIII-XIX)
}

L'impatto sulla cultura giuridica in prospettiva globale 

GLOBAL PERSPECTIVES
ON LEGAL HISTORY 


\title{
Global Perspectives on Legal History
}

\author{
A Max Planck Institute for European Legal History \\ Open Access Publication
}

http://global.rg.mpg.de

Series Editors: Thomas Duve, Stefan Vogenauer

Volume 8

Global Perspectives on Legal History is a book series edited and published by the Max Planck Institute for European Legal History, Frankfurt am Main, Germany.

As its title suggests, the series is designed to advance the scholarly research of legal historians worldwide who seek to transcend the established boundaries of national legal scholarship that typically sets the focus on a single, dominant modus of normativity and law. The series aims to privilege studies dedicated to reconstructing the historical evolution of normativity from a global perspective.

It includes monographs, editions of sources, and collaborative works. All titles in the series are available both as premium print-on-demand and in the open-access format. 
ELISABETTA FIOCCHI MALASPINA

\section{L'eterno ritorno del}

Droit des gens di Emer de Vattel (secc. XVIII-XIX)

L'impatto sulla cultura giuridica in prospettiva globale

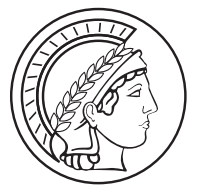

MAX PLANCK INSTITUTE

FOR EUROPEAN LEGAL HISTORY

2017 
ISBN 978-3-944773-07-0

eISBN 978-3-944773-17-9

ISSN 2196-9752

First published in 2017

Published by Max Planck Institute for European Legal History, Frankfurt am Main

Printed in Germany by epubli, Prinzessinnenstraße 20, 10969 Berlin

http://www.epubli.de

Max Planck Institute for European Legal History Open Access Publication

http://global.rg.mpg.de

Published under Creative Commons CC BY-NC-ND 3.0 DE

http://creativecommons.org/licenses/by-nc-nd/3.0/de

The Deutsche Nationalbibliothek lists this publication in the Deutsche Nationalbibliographie; detailed bibliographic data are available on the Internet at http://dnb.d-nb.de

Cover illustration:

Paul Klee, polyphon gefasstes Weiss, 1930

(C) akg-images, Berlin

Cover design by Elmar Lixenfeld, Frankfurt am Main

Recommended citation:

Fiocchi Malaspina, Elisabetta (2017), L'eterno ritorno del Droit des gens di Emer de Vattel (secc. XVIII-XIX). L'impatto sulla cultura giuridica in prospettiva globale, Global Perspectives on Legal History, Max Planck Institute for European Legal History Open Access Publication, Frankfurt am Main, http://dx.doi.org/10.12946/gplh8 
A Gianna,

al suo infinito ed esemplare amore per la vita 



\section{Indice}

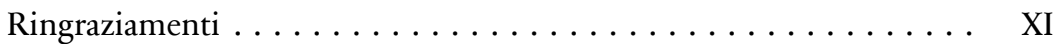

Il contrappunto di Emer de Vattel: note introduttive . . . . . . . 1

1. La regolamentazione statale: il successo del Droit des gens.... . 3

2. Pluralità di riflessioni nelle ricerche sul Droit des gens. . . . . . 5

3. Itinerari e problemi metodologici globali . . . . . . . . 10

4. Emer de Vattel in un'ottica interdisciplinare . . . . . . . . 14

Cenni biografici su Vattel: una vita tra il Principato di Neuchâtel, la Prussia e la Sassonia . . . . . . . . . . . . . . . 20

Parte prima

Dalle traduzioni al discorso politico del Droit des gens . . . . . . . 27

Capitolo I

Divulgare e ricreare un nuovo diritto delle genti . . . . . . . . . . 29

1. L'emergere di una strategia divulgativa: la «Scuola romanda del diritto naturale» . . . . . . . . . . . . . . . 29

2. Il Droit des gens e il garbuglio editoriale del $1758 \ldots \ldots \ldots . .37$

3. Meccanismi giuridici nelle traduzioni settecentesche

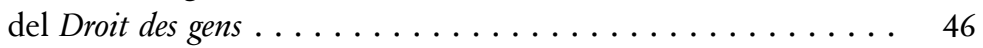

4. La Scuola romanda nella penisola italiana del Settecento: il « comune desiderio $» \ldots \ldots \ldots \ldots \ldots \ldots \ldots \ldots$

5. Il Droit des gens edito da Charles-Guillaume Frédéric Dumas

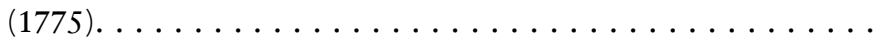

Capitolo II

La «governabilità » dello Stato e delle relazioni internazionali . . . . 75

1. Verso la regolamentazione giuridica delle forme sociali . . . . 75 
2. Innovazione all'interno della tradizione: l'obiettivo del

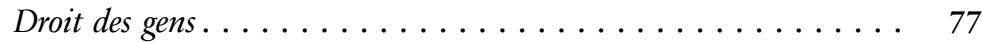

3. Il «piano» della Nazione: la costituzione . . . . . . . . . 90

4. L'arte di governare: l'organizzazione dello spazio sociale . . . . 101

5. «Stato », « Nazione » e « Potenza »: la ieraticità dei trattati internazionali e della loro interpretazione . . . . . . 111

Parte seconda

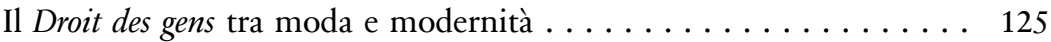

Capitolo III

Il ritorno del Droit des gens tra la fine del Settecento e l'Ottocento . . 129

1. Intrecci tematici intorno al Droit des gens . . . . . . . . . . . 129

2. Teorie vatteliane in Ferdinando Galiani e Giovanni Maria Lampredi . . . . . . . . . . . . . . . 131

3. Il punto di vista del neocastellano Jean Pierre Chambrier d'Oleyres . . . . . . . . . . . . . . . . . . . 137

4. Giuristi e politici inglesi: «Vattel esprime il senso del tempo in cui viviamo" . . . . . . . . . . . . . . . 143

5. «Vattel » nella corrispondenza tra i cardinali Ercole Consalvi e Bartolomeo Pacca . . . . . . . . . . . . . . . . . . . . 152

6. Controversie giudiziali in Nord-America: il richiamo al Droit des gens . . . . . . . . . . . . . . . . . . . 157

7. Il Droit des gens nel panorama delle controversie internazionali 161

Capitolo IV

Commentare e annotare il Droit des gens nel XIX secolo:

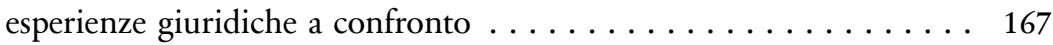

1. Commentare il diritto e commentare il diritto internazionale. . 167

2. Il Droit des gens nel XIX secolo in Francia tra diritto naturale

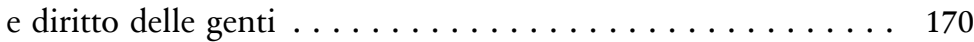

2.1 Il Droit des gens riletto dal portoghese Silvestre Pinheiro Ferreira: le Notes del 1838 . . . . . . . . . . . 175

2.2 Un altro Droit des gens nelle note di Paul Pradier-Fodéré

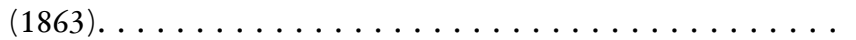


3. Diritto costituzionale nelle note di Manuel Pascual Hernández al Derecho de Gentes (1820 e 1834) . . . . . . . . . . 188

4. Il Droit des gens manuale didattico-politico in America Latina. . 193

5. L'utilizzo casistico del Law of Nations: le note di Joseph Chitty e di Edward Ingraham alle edizioni inglesi e nord americane $(1834$ e 1852$) \ldots \ldots \ldots \ldots \ldots \ldots$

6. L'autonomia del diritto internazionale e il Droit des gens nei paesi di lingua tedesca . . . . . . . . . . . . . 214

7. «La relazione diretta con i bisogni e gli affari presenti della nostra patria »: il Droit des gens in Grecia (1825 e 1831) . 220

8. Diritto pubblico e diritto costituzionale: il Droit des gens

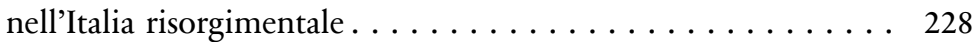

8.1 Nel Regno delle Due Sicilie: Terenzio Sacchi rilegge il Diritto delle genti (1854) . . . . . . . . . 234

Epilogo

1. Il fenomeno del Droit des gens in prospettiva globale:

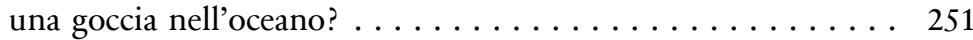

2. Il Droit des gens nella sua polifonia . . . . . . . . . . 257

Prospetto delle edizioni consultate del Droit des gens di Emer de Vattel pubblicate nei secoli XVIII e XIX . . . . . . . . . . 261

Bibliografia . . . . . . . . . . . . . . . . 273

About the Author . . . . . . . . . . . . . . . . . . 349 



\section{Ringraziamenti}

Questo libro racconta la storia di un viaggio: un lungo viaggio tra Sette e Ottocento, realizzatosi tramite le numerose edizioni e traduzioni del Droit des gens di Emer de Vattel, a partire dalla sua prima pubblicazione nel 1758. Le idee viaggiano, migrano, assumono forme nuove e si sviluppano nei diversi contesti, tuttavia il loro percorso sarebbe decisamente più arduo senza i libri e i testi che le veicolano. Le sezioni e i capitoli che compongono questa monografia si presentano come un insieme di molteplici itinerari giuridici diversificati e disomogenei in cui è stato studiato l'impatto che ha riscosso il trattato di Vattel.

Ma Emer deVattel e il suo Droit des gens rappresentano anche il mio viaggio personale iniziato nel 2008 con la tesi di laurea discussa con la professoressa Maria Gigliola di Renzo Villata presso l'Università degli Studi di Milano, proseguito tra il 2009 e il 2012 all'Università degli Studi di Genova con la tesi di dottorato, il cui tutor è stato il professore Rodolfo Savelli.

$\mathrm{Ci}$ sono state molte tappe significative in cui ho incontrato colleghi ma soprattutto amici che hanno arricchito e caratterizzato il mio percorso. La gioia per me più grande si concretizza nell'esprimere la mia infinita gratitudine a tutti coloro che mi hanno sempre sostenuto. Un grazie destinato ad andare oltre le parole è per il Max-Planck-Institut für europäische Rechtsgeschichte di Francoforte sul Meno, che sin dal primo soggiorno di studio, avvenuto nel 2011, ho sentito come la mia seconda casa. Il professore Thomas Duve ha seguito dall'inizio lo sviluppo della ricerca, incoraggiandomi ad investigare il fenomeno vatteliano nella sua più ampia complessità, dandomi l'opportunità di confrontarmi, discutere, elaborare e presentare il mio lavoro in un contesto scientifico unico e molto speciale. Sono veramente grata per questa collocazione editoriale perché la maggior parte delle mie ricerche sono state condotte presso il Max-Planck-Institut, nella sua meravigliosa biblioteca e ora qui cristallizzate.

Il dottore Heinz Mohnhaupt ha saputo consigliarmi ed ascoltarmi con tanta passione ed energia, indicandomi sempre linee di ricerca alternative per ricostruire in modo critico la «fortuna » del Droit des gens. 
Ho avuto l'opportunità di partecipare alle attività del progetto diretto dal professore Miloš Vec, intolato «Das Völkerrecht und seine Wissenschaft, 1789-1914 » che ringrazio unitamente al suo gruppo composto dagli amici Lea Heimbeck, Nina Keller, Stephan Kroll, Friederike Kuntz e Kristina Lovric-Pernak. Come ringrazio la dottoressa Lena Foljanty per avermi coinvolta nelle splendide iniziative organizzate per lo studio della traduzione giuridica nel senso più ampio.

Nicole Pasakarnis mi ha sempre accolto con estrema dolcezza sostenendomi soprattutto nell'ultima e delicata fase del libro, insieme alla professionalità e attenzione del dottore Karl-Heinz Lingens e alla generosa pazienza di Karin Reichstein, Beate Jaeger, Nadine Göbel, del dottore James Thompson, nonché di tutto lo staff editoriale, verso cui sono riconoscente.

Ringrazio altresì Carola Schurzmann, quale responsabile dell'amministrazione del Max-Planck-Institut e Anna Heym e Anne-Lotte Zimmermann per la loro gentilissima disponibilità.

A Helsinki, presso l'Erik Castrén Institute for International Law and Human Rights, il professore Martti Koskenniemi mi ha insegnato la bellezza della libertà delle idee, unita alla complicità della loro criticità: quei mesi sono stati intensi e produttivi e ne conservo un ricordo vivissimo. Sono veramente grata per quanto ho potuto apprendere e sviluppare scientificamente, per l'accoglienza ricevuta dai professori Lauri Hannikainen, Jan Klabbers e Jarna Petman, per tutte le amicizie che si sono formate e rafforzate, ricordo in particolare Paolo Amorosa, Mónica García-Salmones Rovira, Manuel Jiménez-Fonseca, Liisi Keedus, Walter Rech, anche María José Belmonte Sánchez, Luca Bonadiman, Sanna Villikka, e per l'amorevole ospitalità delle Suore Orsoline che ringrazio nella persona di suor Renata Glucha.

La mia ricerca si è completata con il soggiorno di ricerca a Oxford, dove ho approfittato della splendida Bodleian Library, ringrazio professore Paul Yowell per l'attenzione e il tempo che mi ha dedicato.

Il professore Santiago Legarre durante l'intero iter "vatteliano » mi ha sempre esortato a credere nei miei risultati e a continuare, tenendo ben presente il valore e il sacrificio di ogni investigazione.

Vi sono progetti scientifici che partendo dallo studio del Droit des gens stanno prendendo sempre più forma, indirizzandomi verso un aperto e costruttivo dialogo anche interdisciplinare: ringrazio il dottore Frank Grunert, i professori Knud Haakonssen, Béla Kapossy, Gabriella Silvestrini, 
Simone Zurbuchen, così come i professori Luigi Nuzzo e Antonio Trampus per il fruttuoso confronto su tematiche comuni e i dottori Raphaël Cahen e Frederik Dhondt.

Un sentito ringraziamento per il periodo dottorale «genovese » ̀̀ per il professore Vito Piergiovanni per tutti i suoi preziosi e saggi consigli ed inoltre per i professori Roberta Braccia, Riccardo Ferrante, Maura Fortunati, Rodolfo Savelli, Lorenzo Sinisi, Daniela Tarantino, e per Valter Montallegro. Ho avuto l'opportunità di collaborare con l'Università degli Studi di Trieste anche con esercitazioni seminariali e per questo sono grata al dottore Davide Rossi, ai professori Romana Pacia, Fabio Padovini, e alle interessanti discussioni con i professori Stefano Amadeo, Mario Fiorentini e Roberto Scarciglia.

Vorrei anche ringraziare i professori Catherine McCauliff, Giuseppina De Giudici, Lorenzo Gagliardi, Valerio Gigliotti, Gian Savino Pene Vidari, Roberto Sacchi e in particolare Chiara Tenella Sillani per avermi sostenuta umanamente.

Amicizie sincere e vere hanno reso speciale ogni tappa del mio viaggio, aiutandomi con tanto affetto a superare anche i momenti più difficili, esprimo il mio più caro pensiero ai dottori: Benedetta Albani, Lorena Atzeri, Manuela Bragagnolo, Cristina Ciancio, al professore Giuseppe Conti, Francesco di Chiara, Julietta Marotta, Jessika Nowak, Osvaldo Rodolfo Moutin, Agustin Parise, Belinda Rodriguez Arrocha, João Henrique Roriz, nonché a Mariana Armond Dias Paes, Pamela Cacciavillani e Audrey Dauchy.

Ringrazio coloro che hanno realizzato il referaggio per le puntuali osservazioni, ed infine i direttori del Max-Planck-Institut für europäische Rechtsgeschichte i professori Thomas Duve e Stefan Vogenauer per aver accolto la mia ricerca in questa prestigiosa collana, di cui mi sento estremamente onorata.

Sono lieta di condividere l'esito della mia analisi sul Droit des gens con la Rechtswissenschaftliche Fakultät der Universität Zürich, ad inaugurazione di una nuova esperienza.

Zurigo, 27 Marzo 2017 



\section{Il contrappunto di Emer de Vattel: note introduttive}

Una parte essenziale dell'attività storica, quale componente integrante del riconoscimento del valore di un autore, è lo studio della sua fortuna, o meglio ancora, dell'eco che la sua opera « ha avuto ai suoi tempi o più tardi, l'influsso che ha esercitato sui contemporanei e sui posteri, la forma con la quale ha inciso sulla tradizione, modificandola e improntandola della propria potente personalità ${ }^{\mathbf{1}}{ }^{\mathbf{1}}$

La circolazione del Droit des gens ou Principes de la loi naturelle, appliqués à la conduite et aux affaires des Nations et des Souverains di Emer de Vattel, iniziata immediatamente dopo la sua pubblicazione tramite le varie edizioni e le prime traduzioni settecentesche, ha permesso la diffusione del pensiero vatteliano in un'ottica giuridico-politica che supera il tempo e lo spazio.

L'impatto che riscosse il Droit des gens fu estremamente versatile per la disomogeneità con cui venne accolto nelle diverse realtà politiche, nei contesti storico-giuridici e nei tentativi di attualizzazione nella pratica e nella dottrina: infatti moltissimi giuristi e anche politici tra Sette e Ottocento avevano come punto di riferimento il testo di Vattel, a dimostrazione della sua particolare autorità.

Nasce così spontanea la domanda se i continui richiami possano essere inseriti nel novero delle tipiche citazioni di stile, in cui semplicemente veniva riportato il pensiero di Vattel, oppure siano un evidente segnale di un significato più profondo, che affonda le radici proprio nelle peculiarità insite nel Droit des gens e nella sua capacità di organizzare e regolare l'entità statale nei suoi rapporti interni e nelle relazioni internazionali.

La diffusione del Droit des gens, verrà ricostruita non tanto sotto l'aspetto esegetico, già ampiamente e ben sviscerato, quanto piuttosto attraverso una panoramica complessiva delle dinamiche che concretamente gestivano l'utilizzo del trattato: dall'influenza sul potere politico negli affari interni e in quelli esterni, all'essere un vademecum per diplomatici e consoli e anche un manuale per uso didattico.

1 Petronio (1986) 36. 
La compresenza di più livelli normativi, politici e sociali che percorrono proprie direttive, pur coesistendo all'interno di un unico quadro, caratterizzano il fenomeno vatteliano. Si è scelto il termine «contrappunto" sia nel senso della critica letteraria, inteso come il modo di condurre una narrazione o altra opera letteraria su temi e motivi, giocando su effetti e toni a contrasto, seppur complementari, sia nel senso musicale. Il contrappunto indica il tipo di composizione che si realizza quando numerose voci si imitano l'una con l'altra; è l'arte di combinare con una data melodia (che viene definita canto dato) una o più melodie contemporanee, vocali o strumentali, più o meno autonome (che si dicono anch'esse contrappunti rispetto al canto dato). ${ }^{2}$ Nella sua accezione traslata è stato utilizzato anche per riferirsi alle diverse forme culturali, sostenendo la necessità di comparare esperienze differenti nella loro costante coesistenza e interazione reciproca. ${ }^{3}$ L'obiettivo si snoda nella dialettica tra due poli: nello studio di un canto dato, la prima edizione del Droit des gens di Emer de Vattel del 1758 nel suo costante dialogo con i cosiddetti contrappunti cioè le successive edizioni, traduzioni, le versioni commentate dai giuristi del XIX secolo, il suo accoglimento e le sue criticità nei più complessi contesti.

Il contrappunto al Droit des gens, le sue più svariate interpretazioni e applicazioni hanno prodotto di volta in volta una opera continuamente nuova e leggibile in un determinato spazio-tempo, ma al tempo stesso sempre identica: Vattel incarna l'emblema del personaggio "passato", ma sempre " attuale »: che ha detto ed è in grado di dire (ancora). Il successo del trattato verrà evidenziato in questa ricerca con una alternanza di tematiche, meccanismi e aspetti che si relazionano nella loro continuità storica e presente. Con molteplici prospettive, tipiche della polifonia contrappuntistica, è possibile mantenere uno sguardo storico-giuridico ampio: accanto al profilo della storia editoriale del Droit des gens ci sarà il profondo interesse, per lo studio del trattato, che è avvento anche in tempi abbastanza recenti, in occasione degli anniversari della sua pubblicazione e della nascita di Vattel. Inoltre

2 Basso (coord.) (1995), Contrappunto, 244-245.

3 Così scriveva Said: "Abbiamo a che fare con la formazione di identità culturali intese non come essenze date (nonostante parte del loro perduto fascino è che esse sembrino e siano considerate tali), ma come insiemi contrappuntistici, poiché si dà il caso che nessuna identità potrà mai esistere per se stessa e senza una serie di opposti, negazioni e opposizioni »: SAID (1998) 77. Partendo da queste riflessioni e sull'uso del contrappunto sempre in senso traslato, tra i molti studi, cfr.: Baratta (2007). 
l'aspetto metodologico, fortemente legato ad una esigenza di scrivere una storia del diritto globale, sarà sostenuto da una apertura all'interdisciplinarietà, in cui la traduzione, nel senso più ampio del termine, avrà un ruolo chiave. I concetti di moda e modernità saranno investigati all'interno di intrecci giuridici pratici e dottrinali tra Sette e Ottocento, grazie alla voce di autorevoli giuristi e politici che si servivano del Droit des gens, e, traducendolo o ripubblicandolo con annotazioni, favorivano una assimilazione, molto spesso mediata, del pensiero vatteliano.

\section{La regolamentazione statale: il successo del Droit des gens}

Molti autori sostengono che l'accoglimento dell'opera sia dovuto principalmente a tre fattori:

The first was his readability which was the vehicle whereby the Law the Nations gained a popular significance it had never entertained before and [...] left the narrow circle of the doctrinaire to enter the wide and more influential circle of the man of letters. The second factor was the relevance of his work to the political facts of the day, especially state sovereignty, and the third factor was the system borrowed almost in toto from Wolff, whereby Vattel's system was given coherence as well as grace and relevance. $^{4}$

La « leggibilità », la chiarezza espositiva e l'apparente linearità si rispecchiano nell'elaborazione di fondamentali concetti che riguardano tanto lo Stato, come l'esigenza di avere una costituzione, quanto la comunità internazionale, ben rappresentata dal principio dell'equilibrio del potere e dalla guerre en forme. ${ }^{5}$

Uno degli aspetti più singolari del trattato risiede nella straordinaria capacità di regolare lo Stato: il potere politico, soprattutto nel primo libro dedicato alla Nazione, è il risultato di una serie di meccanismi giuridici di "governabilità », che si realizzano nella relazione tra lo Stato e i cittadini a livello nazionale e tra gli Stati a livello internazionale, per evolversi nella «bio-competenza » foucaultiana. ${ }^{6}$ Vattel organizza lo spazio sociale della Nazione mettendo al primo posto la ricerca del buon governo, della propria perfezione e felicità, che coincidono con il mantenimento della sicurezza e

4 Ruddy (1975) 309-310; Ruddy (1972) 177-196. Cfr. anche: Fenwick (1913) 395-410; Fenwick (1914) 375-392; Guggenheim (1956) 23; Thévenaz (1957) 9-16.

5 Chetail (2014) 295.

6 Jounnnet (2006) 58. Si rimanda specialmente a: Foucault (2005). 
del benessere dei propri membri. ${ }^{7} \mathrm{Si}$ tratta di porre «norme che producano vita » e non reprimano; tale principio applicato a livello internazionale è fondato sulle regole da rispettarsi in tempo di pace e in tempo di guerra, che sono contemporaneamente funzionali e vitali alla sopravvivenza di ogni singola Nazione.

Diritto costituzionale, diritto interno e internazionale sono tenuti insieme dall'esigenza di scrivere per «la pratica », esclusivamente per i sovrani e per coloro che, come diplomatici e pratici, si trovavano quotidianamente ad affrontare questioni legate al diritto delle genti:

Mais il importerait peu d'en instruire seulement des particuliers, qui ne sont point appelés au Conseil des Nations, et qui n'en déterminent point les démarches. Si les Conducteurs des Peuples, si tous ceux qui sont employés dans les affaires publiques daignoient faire une étude sérieuse d'une Science, qui devrait être leur Loi et leur boussole, quels fruits ne pourrait-on attendre d'un bon Traité du Droit des Gens? ${ }^{8}$

Il Droit des gens, come dice Vattel, è la loro bussola e, poiché i principi di uno stato altro non sono che il presupposto per le successive regole nelle relazioni internazionali, non si può non cogliere, nello studiare le teorie vatteliane, l'importanza manifestata per il diritto costituzionale e per il diritto interno, da cui come conseguenza inevitabile discende il diritto internazionale.

Non è infatti casuale che durante il XIX secolo le edizioni del trattato manifestino la dicotomia insita nel testo e, alcuni contesti europei, come la Spagna, accentuino la lettura in chiave costituzionalista, altri come la Francia o l'Inghilterra invece risaltino una lettura più internazionalista.

L'utilizzo della comparazione è d'aiuto per ricostruire il successo di una opera: nel caso specifico si tratta di un approccio comparativo sincronico (con una dovuta analisi anche delle edizioni settecentesche), che permette di individuare le differenze e le analogie della circolazione del Droit de gens e, al tempo stesso, di cogliere alcune caratteristiche del fenomeno Vattel, andando però oltre l'indagine sulle semplici influenze e sulle interrelazioni tra culture e contesti tra loro diversi.

Sotto questo profilo si constata la presenza di due costanti nell'Ottocento: la prima è la ripubblicazione di testi sul diritto delle genti di autori fondamentali quali Ugo Grozio, Jean Barbeyrac, Jean-Jacques Burlamaqui e Georg Friedrich von Martens; la seconda è che questi ultimi, esattamente come accadde per il trattato di Vattel, risultano tutti commentati, con lo scopo

7 Jouannet (2006) 58.

8 Vattel (1758a) Préface, XXIII. 
di fornire soluzioni ai problemi dell'allora nuova comunità internazionale e/ o a quelli di politica interna.

Le numerose edizioni vatteliane pubblicate nell'Europa occidentale, in paesi come la Francia, la Spagna, l'Inghilterra, il Nord America conservano tutte caratteristiche pressoché identiche: sono commentate e sono edite per lo più nell'arco di tempo che intercorre tra il 1830 e il 1870. L'operazione compiuta da giuristi come Silvestre Pinheiro Ferreira, Manuel Pascual Hernández, Joseph Chitty e Paul Luis Pradier-Fodéré, come verrà illustrato nelle successive pagine, è la testimonianza che l'interesse nasceva in primo luogo dalla lettura testuale del Droit des gens, da cui derivava una particolare inclinazione ad interpretare le teorie vatteliane alla luce dei progressi della storia del diritto delle genti e ad aggiornare il testo con i casi pratici inerenti al diritto costituzionale e poi al diritto internazionale.

\section{Pluralità di riflessioni nelle ricerche sul Droit des gens}

Nel 2008, in occasione dei duecentocinquanta anni dalla pubblicazione del Droit des gens (1758-2008), si è assistito ad una vera e propria « fioritura » di monografie, saggi e convegni sulla sua figura, la sua opera e il suo pensiero. ${ }^{9}$ Senza contare le iniziative internazionali che sono state organizzate in Svizzera e in altri contesti europei per i trecento anni dalla nascita di Vattel $(1714-2014) .^{10}$

9 Tra i numerosi contributi: Chetail/Haggenmacher (eds.) (2011); Good (2011); Toyoda (2011) in particolare 161-190; SANDOz (éd.) (2010); cfr. anche il numero 31 del 2010 della rivista Grotiana curato da Béla Kapossy dedicata a Vattel. Nel 2008 Richard Whatmore e Béla Kapossy ripubblicarono l'edizione inglese del trattato di Vattel del 1797, fornendo una densa nota introduttiva in: Vattel (2008a) IX-XX; nello stesso anno compare una edizione in lingua portoghese, con un saggio introduttivo redatto da Francesco Mancuso in: VATtel (2008b) 17-67; nel 2011 viene riedita la versione inglese del trattato curata nel 1834 da Chitty: Vattel (2011).

10 Diversi sono stati i convegni e seminari, basti pensare all'incontro di Neuchâtel del 30 aprile 2014 intitolato La genèse et l'impact du Droit des Gens. A l'occasion du 300 ème anniversaire de la naissance d'Emer de Vattel (relatori André Bandelier e Yves Sandoz); cfr. per l'occasione anche gli articoli comparsi sui quotidiani: SEnger (22. April 2014) Seite 22, Teil 01; Senger (Vendredì, 25 avril 2014) Dèbats, 12. Anche l'Italia, insieme alla Svizzera e a Neuchâtel, ha partecipato attivamente all'importante anniversario: a Venezia si è tenuto il workshop organizzato da Antonio Trampus e dalla School of International Relations deil'Università Ca' Foscari, dal titolo Vattel's International Law System: Politics, Institutions and Diplomacy 1714-2014, gli interventi e i risultati del colloquio sono in corso di stampa. 
Gli internazionalisti, gli storici del diritto e delle relazioni internazionali hanno ricostruito il contesto storico e politico in cui Vattel scrisse, hanno fornito in modo critico una rilettura del Droit des gens, facendo emergere le caratteristiche sue per la decisiva formazione del diritto internazionale durante i secoli XVIII e XIX.

In effetti negli ultimi dieci anni l'interesse è incredibilmente aumentato, $\mathrm{e}$ le pubblicazioni, soprattutto in forma di articoli, hanno avuto una crescita esponenziale su tutto il territorio mondiale. Il risultato è una straordinaria e vivace costellazione di studi intorno al pensiero vatteliano: non sono mancate, solo a titolo di esempio, approfondite ricerche sulle diverse letture del trattato, ${ }^{11}$ sul concetto di persona morale dello Stato, ${ }^{12}$ sulla ragione di stato, ${ }^{13}$ sul buon governo, ${ }^{14}$ sul sistema del Droit des gens, ${ }^{15}$ sulla guerra, ${ }^{16}$ sul nemico, ${ }^{17}$ sul diritto di resistenza, ${ }^{18}$ sul commercio, ${ }^{19}$ sull'intervento, ${ }^{20}$ sul colonialismo, ${ }^{21}$ sulla ricezione negli Stati Uniti d'America, ${ }^{22}$ sui trattati internazionali, ${ }^{23}$ sul ruolo delle traduzioni del trattato ${ }^{24}$ e sulla comparazione delle sue teorie con importanti figure settecentesche. ${ }^{25}$

11 Kapossy (2010) 5-21.

12 Holland (2011) 438-445.

13 Koskenniemi (2010) 297-339 anche Devetak (2011) 105-128.

14 Legarre (2012) 431-445.

15 KosKenniemi (2011a) 51-75.

16 Silvestrini (2008) 381-400; Silvestrini (2010a) 44-68. Anche: Zurbuchen (2009) 408-417. Gli scritti di Hunter (2011) 87-104; Hunter (2010a) 108-140.

17 Mancuso (2009) 1277-1310, e anche i più recenti contributi: Mancuso (2015a) 65-81; Mancuso (2015b) 29-51 e Senellart (2004).

18 Carrera (2012) 81-109.

19 Nakhimovsky (2007) 157-173; Porras (2014) 641-660.

20 Zurbuchen (2010) 69-84; Pitts (2013) 132-153.

21 Cavallar (2008) 181-209; Anghie (2014) 81-99; Anghie (2011) 237-253.

22 Richardson (2012) 547-571, in particolare 548-550; Hunter (2012) 173-206 e Chetail (2014) 251-300.

23 Dupuy (2011) 151-166; Lesaffer (2011a) 353-384.

24 Sulla diffusione del Droit des gens nella penisola italiana del Settecento e sulla prima edizione italiana del 1781 cfr.: Trampus (2011a) 81-108, qui 89; Trampus (2011b) 217-232; Trampus (2013) 153-174. Cfr. i seguenti contributi sul sistema internazionale di Vattel: Trampus (2016); su Vattel e le costituzioni italiane prima di Cadice: Trampus (2015) e il Droit des gens nel panorama europeo del Settecento: Stapelbroek/ Trampus (2014) 181-206.

25 Dhondt (2015) 367-394; Dhondt (2016a) 247-250. Vi è poi l'articolato contributo di Hunter (2013a) 477-502 e la successiva risposta di Nardin (2014) 122-134. Su Rousseau e Vattel anche: Christov (2013) 167-187 e Silvestrini (2011) 101-129. 
Contestualmente importanti monografie si sono soffermate su delicatissimi temi facendo risaltare: l'apporto di Vattel rispetto ai suoi predecessori, il suo contributo per il diritto internazionale pubblico moderno e il dualismo presente nel Droit des gens; ${ }^{26}$ l'attenzione al diritto umanitario; ${ }^{27}$ l'aspetto rivoluzionario di Vattel nel tentativo di razionalizzare le relazioni internazionali; ${ }^{28}$ le dinamiche legate al diritto delle genti che si sono protratte fino al Novecento; ${ }^{29}$ nonché il complesso e molto attuale concetto di nemico dell'umanità. ${ }^{30}$

La presente ricerca traccia una storia in chiave giuridico-politica dell'opera vatteliana, attraverso l'analisi della sua diffusione e circolazione. Nel reperire le fonti, inquadrare l'argomento e raggiungere gli obiettivi stabiliti si è prestata anche attenzione alle critiche ricevute e alla loro fondatezza e non solo all'accoglimento del Droit des gens. Celebri sono le posizioni del diplomatico Jean-Pierre Chambrier d'Oleyres durante le guerre rivoluzionarie francesi, che si opponeva al sistema elaborato nel trattato di Vattel. ${ }^{31}$ Immanuel Kant ironizzava su Grozio, Pufendorf e Vattel, mutuando una espressione tratta dal Libro di Giobbe definendoli «leidige Tröster ». ${ }^{32}$ Per Van Vollenhoven, Vattel era paragonato a «satan $» .{ }^{33}$ Hersch Lauterpacht nella prima metà del XX secolo diresse forti critiche alla teoria interpretativa dei trattati internazionali, contenuta nel Droit des gens nel libro II, al capitolo XVII, constatando come essa « a toujours été beaucoup plus remarquable par son manque de pertinence et son excès d'artifice que par sa réelle utilité ». ${ }^{34}$

E' opportuno precisare, però, che questa tensione di accettazione e contrasto al pensiero vatteliano, si potrebbe dire di «amore e odio", si percepisce soprattutto nell'Ottocento, un secolo che è stato determinante per l'evoluzione del diritto internazionale. Come è stato sottolineato da Luigi

26 JouAnnet (1998); si segnalano della stessa i successivi e sempre illuminanti contributi: Jouannet (2011) 133-150; Jouannet (2012a); JouAnNEt (2012b) 1118-1121.

27 Good (2011); anche Good (2013) 117-128; Good (2012) 15-33.

28 Mancuso (2002).

29 LAZZARich (2012).

30 ReCH (2013).

31 Chambrier d’Oleyres (1795); Chambrier d’Oleyres (1793) 436-459; Chambrier D'Oleyres (1796), per la sua posizione nei riguardi del Droit des gens cfr. NAKHIMOvsKY (2010) 141-164.

32 KANT (1795) 33.

33 Vollenhoven (1919) 93.

34 LAUTerpacht (1934) 713. 
Nuzzo, nel XIX secolo si assiste al processo di specializzazione e professionalizzazione del diritto internazionale, che si realizza nella sua trasformazione in un sapere scientifico del tutto svincolato dalla prassi diplomatica e dal diritto naturale. ${ }^{35}$

I protagonisti di questo cambiamento sono stati i giuristi ed in particolare gli internazionalisti, che hanno colto pienamente l'iter iniziato con le Rivoluzioni Americana e Francese alla fine del XVIII secolo, la presa al potere di Napoleone e il suo successivo crollo, il Congresso di Vienna e l'inizio di un nuovo ordine nelle relazioni tra gli Stati, che porterà progressivamente al Trattato di Parigi del 1856, in cui si sancì ufficialmente l'ingresso della Turchia «ai vantaggi del diritto pubblico e del concerto europeo ». ${ }^{\mathbf{3 6}}$

Essi erano chiamati a ricoprire un difficilissimo ruolo perché dovevano bilanciare differenti interessi e al contempo diffondere le teorie della disciplina, promuovendo la « coscienza delle nazioni civili », e

mediate between universalism and nationalism, humanitarian aspirations and colonial impulses, technical, economic and financial challenges, nations and states, recognized states as subjects of knowledge; yet they also thought it necessary to spread a new legal science over the world - with regard to that they incorporated a deep supranational dimension into their general principles. International law became the product of a historical reflection by an elite of intellectuals that, thought an organic relationship with the conscience of civilized nations, translated value into a scientific system. ${ }^{37}$

Infatti alcuni dei più importanti internazionalisti, tra cui gli italiani Pasquale Stanislao Mancini e il genero Augusto Pierantoni, unirono le loro forze per la creazione a Gand, l'8 Settembre 1873, dell'Institut de droit international; la finalità era di favorire il progresso del diritto internazionale, formulando principi generali, diffondendo la conoscenza e dando il suo concorso ad ogni serio tentativo di codificazione graduale e progressiva del diritto internazionale. ${ }^{38}$

E' proprio nell'Ottocento che si assiste ad un capovolgimento dell'uso dei trattati internazionali che assumono il valore di veri e propri strumenti di codificazione del diritto internazionale, al fine di creare disposizioni e prin-

35 Nuzzo (2012a) 4.

36 Storti (2012) 53. Anche: Vec (2012) 654-678; Steiger (2009) 513-551. Per i rapporti tra Impero Ottomano ed Europa: Augusti (2013).

37 Nuzzo/Vec (2012) XII.

38 Nuzzo (2012a) 133; Nuzzo (2009a) 1311-1381. 
cipi comuni a tutti gli Stati. ${ }^{39}$ Al centro dell'attenzione sia pratica sia dottrinale vi sono i trattati di pace e l'emergere di un «new enforcement means", che si sarebbe protratto anche per tutto il secolo successivo; ${ }^{40}$ la regolamentazione del diritto bellico, ${ }^{\mathbf{4 1}}$ unitamente alle convenzioni di diritto internazionale umanitario e alla fondazione nel 1863 a Ginevra della Croce Rossa. ${ }^{42}$ Risoluzioni che, nella realtà, dovevano sempre scontare i paradossi delle dinamiche del diritto internazionale, soggetto a logiche di potere e politiche che inevitabilmente poco si conciliavano con gli spiriti di pace. $^{43}$

Inoltre, come argomentato da Gustavo Gozzi, nell'Ottocento prese forma «il diritto internazionale della società internazionale globale », soprattutto con l'ingresso della Cina, del Giappone nel diritto europeo e la gestione dei rapporti anche giuridici con le colonie d'Africa, e di conseguenza si assistette all'esportazione globale delle teorie sul diritto internazionale elaborate in Europa e nel Nord America. ${ }^{44}$ Un chiaro esempio sono le prime traduzioni in lingua cinese e giapponese dell'opera dell'americano Henry Wheaton, Elements of International Law, edito nel 1836, e contemporanea la circolazione su scala mondiale di trattati del «passato" come il Droit des gens di Vattel e il De iure belli ac pacis di Ugo Grozio. ${ }^{45}$

Nell'Ottocento, altresì, l'idea di nazione si rafforzò e raggiunse la sua acme: lo spirito liberale continuava a soffiare sia in Europa sia oltreoceano, traducendosi e plasmandosi in molteplici realtà politiche, giuridiche, sociali, economiche e scientifiche. La costruzione dell'identità nazionale veniva segnata dai moti rivoluzionari che si estesero «a macchia d'olio ", disegnando una nuova mappa geopolitica del mondo, con confini diversi e nuovi Stati, che a loro volta si identificarono nella loro carta costituzionale.

39 Augusti (2015) 37-61, anche Mannoni (2014).

40 Lesaffer (2012) 84.

41 Passero (2009) 1479-1504; Pietropaoli (2009) 1169-1213.

42 Lovric-Pernak (2013); O'Connell (2013) 101-151, in particolare 105-125; Focarelli (2013a); Focarelli (2013b) 103-128.

43 Hippler/Vec (eds.) (2015).

44 Gozzi (2010) 157. Cfr.: Kroll (2012); Stolleis/Yanagihara (Hrsg.) (2004).

45 Solo sinteticamente per la circolazione del Droit des gens nei territori asiatici, che verrà ripresa nel corso del volume: WeIfang (2008) 75-78; Toyoda (2010) 59-74; Svarverud (2001) 128. Per quanto riguarda la diffusione dell'opera di Wheaton: Armitage (2013) 28 ss; Liu (2004) 108-139 e Liu (1999) 127-164. 
In un contesto di grandi cambiamenti globali possono le edizioni, riedizioni, traduzioni di un autore come Vattel essere state determinanti per la formazione del diritto e del diritto internazionale nell'Ottocento?

\section{Itinerari e problemi metodologici globali}

Per rispondere all'interrogativo è necessario soffermarsi sia sulle più recenti tendenze storiografiche e metodologiche, perché accanto alla parola storia si affianca sempre più spesso l'aggettivo "globale ", sia sul profondo dibattito che nei diversi contesti socio-culturali è stato proposto sulle tematiche legate alla globalizzazione e all'integrazione europea con una partecipazione sostanziale anche delle scienze storiche.

Negli anni Sessanta del secolo scorso è stato messo in discussione, infatti, il concetto di cultura, utilizzando strumenti nuovi tratti dalla semiotica, dallo strutturalismo e dallo studio dei linguaggi. E' diventato perciò imprescindibile confrontarsi con le teorie di Walter Benjamin, Claude LéviStrauss, Roland Barthes, Michel Foucault e Jacques Derrida per una ricerca approfondita e applicata e per la formulazione di nuovi modelli culturali.

In questa trasformazione culturale sono state coinvolte più discipline, basti pensare all'antropologia, alla sociologia e alla storiografia. La critica avanzata si basa sul presupposto che la maggior parte di esse abbiano continuato a concentrarsi su valori e categorie, quali i concetti di orientale-occidentale, di identità-diversità, per lungo tempo condizionati e vincolati al modello eurocentrico, considerato l'unico e il migliore. ${ }^{46}$ Pertanto da una situazione omogenea e compatta della visione del mondo e della ricerca si sono fatti spazio, con molto coraggio, le culture postcoloniali, che da quelle parti «marginali » del pianeta sostengono con forza una battaglia contro il sentirsi « periferia ${ }^{\mathbf{4 7}}$

46 Luminari sotto questo profilo sono state le riflessioni di Edward Said nel celebre Orientalismo, pubblicato nel 1978, a cui l'autore, nel 1994, ha aggiunto una postfazione. Il saggio, come è noto, propone un nuovo modo di pensare la teoria e la pratica del colonialismo attraverso una critica radicale alla rappresentazione che gli studiosi occidentali hanno costruito dell'Oriente, generalmente basata su una concezione eurocentrica e su una serie di false alternative, ideologicamente motivate e spesso storiograficamente errate, tra occidente e oriente, europeo ed extraeuropeo: SAID (2002) e SAID (1998).

47 Per quanto riguarda la bibliografia italiana si rimanda a: Lombardi-Diop/Romeo (eds.) (2012); Bassi/Sirotti (a cura di) (2010); di Piazza (2004) 417-424. 
All'interno degli studi postcoloniali le riflessioni si spostano verso atteggiamenti che privilegiano il metissaggio, la (re-)invenzione creativa e politica della diversità, i fenomeni ibridi e tutto ciò che avviene nello scambio culturale non inteso in senso statico e univoco ma, viceversa, come un processo di trasformazione congiunta.

Viene così crearsi un "terzo spazio ", liminale e di transito, che produce necessariamente qualcosa di nuovo coinvolgendo più livelli: sociale, politico, linguistico, antropologico e giuridico, partendo dal presupposto che l'ibridità generatasi abbia una sua precisa rilevanza ontologica. ${ }^{\mathbf{4 8}}$

Gli storici hanno sentito fortemente l'esigenza di partecipare al cambiamento " globale ", concentrando le loro ricerche nell'individuazione di strumenti più adeguati per concettualizzare e per analizzare le nuove tematiche: sotto questo profilo fondamentale sono stati gli studi di storia comparata, ${ }^{49}$ della world history o della global history, ${ }^{50}$ dell'histoire croisée ${ }^{51}$ e della storia trasnazionale. $^{52}$

La storia letta in prospettiva globale ha il mondo come orizzonte e deve essere arricchita grazie una narrazione che dialoghi con il passato, superando le rigide categorie tipicamente eurocentriche e molto spesso nazionalistiche. Come è stato sostenuto da Tommaso Detti « le nostre usuali periodizzazioni epocali devono essere passate ad un consapevole vaglio critico, rivedute e in ogni caso considerate non come frontiere invalicabili entro le quali racchiudersi, ma come confini da attraversare ${ }^{53}$

48 E' Homi K. Bhabha ad introdurre il concetto di «terzo spazio »: «Teoricamente innovativo, e politicamente essenziale, è il bisogno di pensare al di là delle tradizionali narrazioni relative a soggettività originarie e aurorali, focalizzandosi invece su quei momenti o processi che si producono negli interstizi, nell'articolarsi delle differenze culturali. Questi spazi "inter-medi" costituiscono il terreno per l'elaborazione di strategie del sé - come singoli o come gruppo - che danno il via a nuovi segni di identità e luoghi innovativi in cui sviluppare la collaborazione e la contestazione nell'atto stesso in cui si definisce l'idea di società. E' negli interstizi - emersi dal sovrapporsi e dal succedersi delle differenze che vengono negoziate le esperienze intersoggettive e collettive di appartenenza ad una nazione, di interesse delle comunità e di valore culturale »: ВнавнA (2001) 12.

49 Haupt / Коска (Нg.) (1996); Наupt/Коска (2009) 12-30; e anche Коска (2003) 39-44; KAelble / Schriewer (Hg.) (2003) e Rossi (1990). Anche: IbBetson (2013) 1-11; Iвbetson (2012) 131-145.

50 Douki / Minard (2007) 7-21; Iggers / Wang/Mukherjee (2008) e Meriggi (2015) 1-10.

51 Werner/ZimmermanN (2002) 607-636; Werner/ZimmermanN (2003) 7-36.

52 Abbattista (2011) 773-780. Cfr.: Randeria (1999) 87-96; Haupt/Kocka (2009) e Schulze (2013).

53 DetTI (2005) 5. 
Anche la storia del diritto ne è rimasta coinvolta, basti pensare alle recenti pubblicazioni che hanno incoraggiato a studiare nelle pieghe di un fenomeno giuridico le realtà e le dinamiche locali, dove produzione giuridica e normativa si trovano e si «intrecciano » con cambiamenti sociali e processi di traduzione culturale. Il criterio auspicato è infatti: "Good 'Global history' is by no means total history but the combination of local histories, open for global perspectives $»{ }^{54}$

L'interesse profondo verte su quali possano essere le tecniche e le teorie che gli storici del diritto utilizzano nel ricostruire il processi di interazione tra differenti ordini normativi, sui motivi della scelta di alcuni concetti e non di altri, e soprattutto sugli strumenti metodologici a disposizione degli studiosi.

Se la disciplina è stata anche concepita e costruita sul modello ottocentesco del binomio Stato-nazione, cercando energicamente la costruzione dell'identità nazionale al fine di dare rilievo alle particolarità e uniformità delle tradizioni proprie, oggi occorre, invece, prendere coscienza di orizzonti diversi in cui scoprire molto attraverso spazi che sono sempre stati considerati come marginali.

Non possiamo accontentarci, come scrive Thomas Duve, della mappa tracciata nel XIX secolo in cui lo spazio è dipinto come omogeneo e semplificato. "Necesitamos una réflexión más profunda sobre los procesos de reproducción de un orden simbólico, come lo es el derecho, fuera de su lugar de origen: un proceso en el cual intervienen actores, artefactos, e ideas, entre otros aspectos $\gg .55$

Un approccio storico di questo tipo pone in primo piano le profonde, strutturali, irreversibili dinamiche dello scontro tra culture e, di conseguenza, costringe lo studioso, a investigare sui processi di influenza reciproca, di attrazione, ma anche di repulsione. Rileggere la storia del diritto in prospettiva globale significa cogliere che tutte le entità in relazione tra loro sono il risultato del loro intrecciarsi. Tutte le dipendenze e interferenze sono il punto di partenza di un vero e proprio quadro storico transnazionale, frutto di commistioni sociali, culturali e giuridiche con ripercussioni tanto nella storia del diritto in prospettiva globale quanto nella storia della globalizzazione del diritto. ${ }^{56}$

54 Duve (2014a) 61.

55 Duve (2014b) 2; anche Duve (2014c) 16-48.

56 Duve (2016) 2-6. Per riflessioni sulla congiunzione della dimensione spazio-temporale nella storia del diritto: Meccarelli/Solla Sastre (eds.) (2016). 
La storia del diritto internazionale per la sua peculiare inclinazione alla dimensione globale è al centro di questo cambiamento. È stato Martti Koskenniemi, in un articolo apparso nel 2004, ad auspicare che le future ricerche si orientassero verso tre differenti prospettive: una storia intellettuale attenta ai contesti, alle influenze sociali, culturali e politiche; una storia che analizzasse quanto "gli altri » o le cosiddette "periferie » avessero condizionato lo sviluppo del diritto internazionale occidentale; infine, una storia sociale che tenesse conto delle diverse tipologie di società internazionali studiate in connessione con sistemi normativi ad essi collegati. ${ }^{57}$

Sono passati oltre dieci anni da quelle riflessioni, si è assistito ad un vero e propria "svolta storica " 58 ma soprattutto gli studi si sono sempre più orientati a superare la visione tipicamente eurocentrica, proponendo un approccio "globale». Si pensi all'imponente pubblicazione e raccolta di saggi curata da Bardo Fassbender e Anne Peters, ${ }^{59}$ alla ricostruzione fornita da Mamadou Hébié ${ }^{\mathbf{6 0}}$ e alle ricerche di Arnulf Becker Lorca, il quale racconta una storia intellettuale, in cui i protagonisti sono gli Stati e i giuristi non europei, riscritta attraverso la prospettiva di coloro che si trovavano fuori dall'Europa e, dalle remote periferie o semiperiferie del globo, aspiravano a farne parte. ${ }^{61}$

L'obiettivo è quello di mettere in luce l'ibrido della disciplina e dei meccanismi giuridici. In effetti, se pensiamo al diritto internazionale l'ibridità è una delle sue condizioni essenziali e costitutive: oltre all'incontro e allo scontro tra Stati europei e Stati non appartenenti al sistema europeo esso stesso è un ibrido. Ibrido nella sua evoluzione da diritto naturale a diritto

57 Koskenniemi (2004a) 61-66 e Koskenniemi (2012a) 943-971. Anche: Nuzzo (2013) 681-699; Hueck (2001) 194-217.

58 Bandeira Galindo (2005) 539-559, Kemmerer (2008) 71; Skouteris (2012) 99-122; Craven (2016). Sulle ragioni dell'allontanamento degli storici del diritto dal diritto internazionale: LESAFFER (2007) 27-42; sulla necessità invece di un maggior incentivo (o meglio ancora ritorno), come afferma Nuzzo, negli sudi della storia del diritto internazionale in Italia: Nuzzo (2009b) 75-99. Per un bilancio delle più recenti ricerche: Dhondt (2016b) 313-334.

59 Fassbender/Peters (ed.) (2012).

60 HéBIÉ (2015).

61 Becker Lorca (2014), Becker Lorca (2010) 475-552 e Becker Lorca (2012) 1034-1057. Sui recenti sviluppi della storia del diritto internazionale globale si veda anche: KeMmerer (2015) 1-14. 
delle genti e poi infine a diritto internazionale, nell'utilizzo di concetti e categorie appartenenti a differenti fonti normative, ma anche nella struttura e nel contenuto della gran parte dei testi pubblicati tra il XVI e il XVIII secolo.

Tuttavia la « visione » globale porta inevitabilmente ad interrogarsi sulla sua metodologia, i cui dibattiti sono vivissimi. Occorre solo menzionare gli scritti di Anne Orford e le sue riflessioni sull'anacronismo effettuate nella convinzione che una rigida cronologica compartimentazione sia inadeguata per la storia del diritto, sostenendo, invece, come si possa «estendere » il concetto di contesto al di là del passato e del presente; ${ }^{62}$ la difesa di Ian Hunter, viceversa, per la necessaria contestualizzazione, perfettamente in linea con il pensiero degli storici intellettuali; ${ }^{63}$ la posizione di Koskenniemi, il quale mira ad una investigazione storica che si interroghi su come $\mathrm{i}$ «contexts were formed and to what extent they have persisted to make the world into what it has become today ${ }^{\mathbf{6 4}}$

Alla luce di queste prime considerazioni si può comprendere quanto il diritto internazionale sia plurale. Ciò permette dal punto di vista metodologico di valutare differenti prospettive di narrazione, dando spazio ad un intreccio di più ampio respiro e offrendo un punto di vista dinamico, diversificato e poliedrico. La storia del diritto internazionale globale prende forma proprio attraverso la localizzazione, la storicizzazione e la contestualizzazione delle storie stesse, che pluralmente creano la disciplina e simultaneamente la arricchiscono di contorni e sfumature sempre più dettagliati. Ma con quali strumenti si localizza, storicizza e contestualizza la storia giuridica in prospettiva globale?

\section{Emer de Vattel in un'ottica interdisciplinare}

Un approccio interdisciplinare e non più squisitamente settoriale contribuisce a porre nella giusta dimensione il complesso meccanismo che regola le dinamiche di un determinato fenomeno giuridico, fornendo delle risposte

Orford (2014) 369-385; Orford (2013a) 97-118; Orford (2013b) 166-197.

63 Hunter (2010b) 11-29. Cfr. anche Hunter (2013a) 477-502; Hunter (2013b) 289-307 e le riflessioni pubblicate nell'articolo: Hunter (1982) 80-91. Sulla storia intellettuale si rimanda a: Whatmore (2015) e Whatmore/Young (eds.) (2016).

64 Koskenniemi (2013) 238; Koskenniemi (2014) 119-138; Koskenniemi (2016) 104-112. 
all'interrogativo formulato. In European Legal History - Global perspectives, Thomas Duve ha lanciato una sfida suggerendo una apertura alla interdisciplinarità nello studio della storia del diritto, che tendesse verso una epistemologia del diritto nel processo globale di traduzione culturale: « to do so, we have to open the field of observation, and obviously, seek a wellbalanced interdisciplinary approach that does not consider 'law' as something categorically different from other fields of cultural production, but as one modus of normativity ${ }^{65}$

Considerare il diritto come un «modo della normatività » significa analizzarlo nel suo insieme, senza svincolarlo da procedimenti sociali e politici, tanto nel contesto di produzione quanto nel contesto di arrivo attraverso la sua diffusione e circolazione.

La sua contestualizzazione con l'ausilio di altre discipline contribuisce a spiegare particolari eventi che si verificano, ad esempio, durante l'utilizzo di modelli giuridici o la circolazione di un trattato in differenti situazioni spazio-temporali. Questi eventi non possono essere ricondotti alla semplice "ricezione ", il cui significato anche nella storia del diritto è messo giustamente in discussione, perchè il risultato finale è sempre caratterizzato dall'originalità e non da una passiva assimilazione delle idee e del diritto. ${ }^{66} \mathrm{Si}$ privilegiano così le fratture, le zone d'ombra mediante le quali un trattato, come il Droit des gens, ha subito le trasformazioni e gli adattamenti che erano completamente estranei a Vattel mentre si accingeva a pubblicare l'opera. La storia del diritto delle genti può essere letta come un continuto processo di assimilazione e di produzione nell'attualizzazione di testi giuridici ad opera del giurista-lettore che li conosce, del giurista-scrittore che si trasforma a sua volta in produttore e del giurista critico (in questo caso lo storico) che ragiona su di essi.

Inoltre come aveva sostenuto Bruno Paradisi, l'indagine storica del diritto internazionale, nella sua dimensione giuridica non può svincolarsi da uno studio che tenda verso l'unità del diritto attraverso nel contesto storico nel quale esso viene creato. ${ }^{67}$ E' pertanto importante investigare anche sugli

65 Duve (2013) 18.

66 Duve (2012) 52-53.

67 Bruno Paradisi, nell'esporre i motivi che lo avevano avvicinato alla storia del diritto internazionale e alla pubblicazione della sua celebre Storia del diritto internazionale nel Medio Evo, affermava che: "Quella Storia del diritto internazionale era un tentativo, per quanto imperfetto, di risolvere la dogmatica nella storia giuridica senza peraltro trascurare lo 
intrecci, sulle interazioni che, durante il corso dell'Ottocento, l'evoluzione del diritto internazionale ha avuto con le altre discipline giuridiche, nonchè sulle dinamiche che regolavano e determinavano la fruibilità di una determinata opera giuridico-politica.

Illuminanti, sotto questo profilo, sono le riflessioni sulla globalità di Paolo Grossi: «Proprio perché lo storico del diritto è storico e giurista, direi che anche il diritto gli appare nella sua globalità. [...] Perché storia è sempre storia di una complessità di vita, di quel complesso di forze spirituali, intellettuali, strutturali, che noi chiamiamo civiltà ${ }^{68}$

Partendo da queste posizioni Vattel viene così analizzato come un vero e proprio «caso » sotto il profilo metodologico, interdisciplinare, e storiografico. Egli, infatti, è l'esempio dell'intellettuale calato nel suo tempo, inserito negli ambienti illuministi di Neuchâtel, della Svizzera e di altri Stati europei, come la Francia e la Prussia. La rete di persone nella duplice veste di amici e conoscenti creatasi attorno a Vattel è una fonte inesauribile di studio: come l'amicizia con Jean-Henri Samuel Formey, con cui Vattel costantemente si confronta durante la stesura della sua opera, l'amico fidato a cui spedisce una parte del manoscritto che va direttamente nelle mani di Elie Luzac, editore dei lavori di Formey ed artefice dell'edizione contraffatta del Droit des gens nel 1758. Intorno a Vattel ruota anche l'affascinante e operosa Societé Typographique, al cui vertice vi era Samuel Ostervald, anch'egli amico stretto e curatore di una edizione del Droit des gens, successiva alla morte di Vattel.

Lo studio del trattato ha risvolti diversi: nella storia, nella storia dell'editoria e nella storia del diritto, e soprattutto le molteplici edizioni e traduzioni influiscono sui modelli di circolazione giuridica e delle idee.

Nel momento in cui si verifica una sorprendente diffusione di un trattato grazie alle riedizioni e alle traduzioni nelle più diverse circostanze spaziotemporali, la ricerca necessita l'utilizzo di specifiche competenze che possano arrivare «nell'intimità del dinamismo del fenomeno giuridico ». ${ }^{69}$

strumento filologico ed erudito di affermare come fosse possibile e desiderabile che la storia del diritto esprimesse in una sola proposizione un'analisi ed una sintesi nelle quali l'esame della natura e della struttura degli istituti di una parte del diritto fosse una cosa sola con la storia complessiva del diritto, considerato nel quadro della storia tutta intera »: PARAdisi (1973) 193.

68 Questa citazione è tratta dall'intervista realizzata da Meccarelli / Solimano (2007).

69 Checchini (1950) 392.

16 Note introduttive 
Nell'aprirsi alla interdisciplinarità si è scelto di concentrare l'attenzione alle teorie offerte dagli studi sulla traduzione. Le prime teorie sulla traduzione si sono sviluppate all'inizio dell'Ottocento nel momento in cui l'ermeneutica ha assorbito la traduzione, per poi riemergere successivamente nei primi anni del Novecento grazie i determinanti saggi di Walter Benjamin, Über Sprache überhaupt und über die Sprache des Menschen del 1916 e Die Aufgabe des Übersetzers del 1921. Per Benjamin tra il testo tradotto e il testo originale si instaura un vero e proprio rapporto necessario: quest'ultimo è la sorgente da cui deriva la traduzione, la quale però non deve mirare a diventare una mera copia dell'originale: «la vera traduzione è trasparente, non copre l'originale, non gli fa ombra, ma lascia cadere tanto più interamente sull'originale, come rafforzata dal suo proprio mezzo, la luce della pura lingua ${ }^{70}{ }^{70}$ Le riflessioni di Benjamin sono confluite all'interno dei cosiddetti Translation Studies, che sono emersi intorno agli anni Settanta del Novecento, soffermandosi in particolare sul processo traduttivo, discostandosi dalla proposizione di meri modelli e analizzando una prospettiva più generale della traduzione. Sono state affrontate le questioni centrali della tecnica di traduzione (come il problema dell'equivalenza, il concetto e di traducibilità e intraducibilità), dimostrando quanto l'opera tradotta non possa essere considerata secondaria rispetto all'originale, ma una creazione assolutamente autonoma. $^{71}$

Gli studi d'impostazione post-strutturalista, influenzati dal decostruzionismo (basti pensare alle riflessioni di Jacques Derrida) arricchirono quelli traduttivi di nuovi stimoli, permettendo di ricercare una relazione tra la traduzione e gli studi culturali. Susan Bassnett e André Lefevere si fecero portavoci della cultural turn o svolta culturale, soffermandosi sulle connessioni tra la traduzione e i diversi campi di studio, collaborando al fine di individuare le relazioni interdisciplinari. ${ }^{72}$

70 Benjamin (1976) 47.

71 « 11 traduttore individua la diversità tra la cultura di partenza e quella di arrivo e valuta come procedere nel suo lavoro, in modo da decidere quali adattare e come presentare al pubblico destinatario gli elementi peculiari della cultura originaria. Il traduttore costituisce l'anello di collegamento che determina l'interazione di culture diverse »: ULRYCH (1997) XII. Per maggiori approfondimenti, si segnalano tra i più recenti: Millán/ Bartrina (eds.) (2013); Munday (2013). Anche: Snell-Hornby (2006); Shuttleworth / Cowie (1997); Bassnett (1980); Lefevere (1978) 234-235.

72 Bassnett/Lefevere (1998) e Bassnett/Lefevere (1990). Anche Lefevere (1992a); Lefevere (1992b). Sul Cultural Turn sinteticamente: Bachmann-Medick (2006). 
Ogni traduzione, di conseguenza, non è mai un fenomeno passivo ma impone una partecipazione totale di chi effettua la traduzione vera e propria e di chi poi sarà il fruitore stesso del lavoro, ${ }^{73}$ anche nel caso di una traduzione rigorosamente letterale. ${ }^{74}$

Essa non può essere definita soltanto come equivalenza linguistica e lessicale, in cui ciò che conta è l'attenzione sfrenata all'originale, mentre la copia è solo "the final component of a static dichotomy " ${ }^{75} \mathrm{ma}$, viceversa, è il risultato di una vera e propria mediazione culturale. ${ }^{76}$

Susan Bassnett afferma che la traduzione non avviene mai in un vacuum, bensì in un continuum; non è un atto isolato, ma parte di un processo dinamico di transfer interculturale. Inoltre è un'attività altamente manipolativa, mai innocente, che coinvolge ogni tipo di livello in quel processo di passaggio tra confini linguistici e culturali. ${ }^{77}$

Lena Foljanty, con riflessioni inerenti alla storia del diritto, argomenta quanto

the concept of translation draws our attention to the nuanced dynamics and processuality of transfer processes as well as the constants shifts as well as to the constants shifts in the forms of legal thinking, acting, and perceiving that take place during these processes. It enables us to design investigative frameworks that critically reflect on the dichotomy of giving and receiving. ${ }^{78}$

73 Dimitru (2006).

74 Il celebre teorico della traduzione Lawrence Venuti sostiene che: "Translation changes the receiving cultural situation by bringing into existence something new and different, a text that is neither the source text nor an original composition in the translating language, and in the process it changes the values, beliefs, and representations that are housed in institutions »: VeNUTi (2013) 21.

75 Lianeri (2002) 4.

76 Per Bassnett "What is generally understood as translation involves the rendering of a source language ( $\mathrm{sl}$ ) text into a target language $(\mathrm{tl})$ so as to ensure that (1) the surface meaning of the two will be approximately similar and (2) the structure of the sl will be preserved as closely as possible but not so closely that the tl structure will be seriously distorted. [...] What is analyzed in such studies is the product only, the end result of the translation process and not the process itself »: BASSNETT (2014) 14-15. Allo stesso modo Hans Josef Vermeer: "eine Translation ist nicht die Transkodierung von Wörtern oder Sätzen aus einer Sprache in eine andere, sondern eine komplexe Handlung, in der jemand unter neuen funktionalen und kulturellen und sprachlichen Bedingungen in einer neuen Situation über einen Text (Ausgangssachverhalt) berichtet, in dem er ihn auch formal möglichst nachahmt »: Vermeer (1986) 33.

77 Bassnett / Trivedi (1999) 2.

78 Foljanty (2015) 7. 
Il Droit des gens non fu di certo il primo trattato nella storia del diritto delle genti ad essere stato tradotto: è sufficiente solo citare la prima edizione francese del De iure belli ac pacis di Ugo Grozio, tradotta da Antoine de Courtin, edita nel $1687 .{ }^{79}$ L'aspetto traduttivo giocò un ruolo chiave nella fruibilità dei testi sul diritto delle genti: è d'uopo nel prossimo capitolo soffermarsi sul ruolo di Barbeyrac per la diffusione del diritto naturale, grazie alle sue traduzioni delle opere di Grozio e Pufendorf.

Per la storia del diritto internazionale l'incredibile fortuna del Droit des gens di Vattel, suscita numerose riflessioni partendo proprio dall'aspetto traduttivo. $^{80}$

Il legal transfer inteso come un "processo di traduzione culturale " ${ }^{\mathbf{8 1}}$ può essere agevolato proprio dai contenuti e dalla finalità di una opera, che ben riesce ad adattarsi, piegarsi o meglio ancora essere riletta a seconda delle diverse esigenze storiche e geografiche. Emer de Vattel con il suo Droit des gens pone al centro lo Stato da cui fa derivare la legittimazione alla formazione di una costituzione, intesa come regolamento fondamentale, scelta e non imposta da ingerenze esterne, la necessità che si persegua il bene comune per i cittadini e, una volta esaurite le caratteristiche essenziali di uno stato-nazione, allarga l'indagine allo Stato come uno soggetto in grado di relazionarsi nella sfera internazionale. ${ }^{82}$ Il trattato di Vattel « rappresenta [...] un caso tipico di riproduzione di un modello culturale al di fuori dell'originario contesto storico e geografico e adattato sempre più spesso, subendo l'interferenza di una cultura terza, in nuovi ambiti politici e sociali ». ${ }^{83}$

79 Nel suo Avertissement du traducteur, Courtin si sofferma a lungo sulle diverse modalità di traduzione, sottolineando che « La traduction est la même chose que l'enluminure. Il ne suffit pas, pour bien enluminer, de suivre l'estampe et de rendre espèce pour espèce, c'està-dire de ne pas faire un homme pour un arbre; il ne suffit pas de démêler les choses qui se perdent les unes dans les autres ; [...] il faut encore, ce qui est l'art, suivre la pensée du Graveur, garder par tout son œconomie [...] afin que l'œil voyant beauté pour beauté, croye voir au naturel les choses mêmes qu'il ne voit que sur le papier [...]». E ancora: "C'est $[\ldots]$ pour cette raison, que l'on a voulu se tenir prez de l'Auteur, et comme le suivre pas à pas, autant que l'a pû permettre le génie de nôtre langue [...] On a voulu disje, conserver autant qu'il se pourrait le caractère de l'Auteur, ce qui est même de l'essence de la traduction ; afin que ceux qui la liraient eussent toûjours devant eux l'Auteur même et non pas le Traducteur »: Courtin (1687) II-IV.

80 Si rimanda a: Fiocchi Malaspina/Keller-Kemmerer (2014) 214-227.

81 Foljanty (2015) passim.

82 Trampus (2013b) 153.

83 Trampus (2013b) 153-154. 


\section{Cenni biografici su Vattel: una vita tra il Principato di Neuchâtel, la Prussia e la Sassonia}

Emer de Vattel nacque a Couvet, nella provincia di Neuchâtel, il 25 aprile del $1714,{ }^{84}$ da David de Vattel, un pastore della chiesa protestante e da Marie de Montmollin, figlia di Jean, più volte receveur à Valangin ${ }^{85}$ e sorella di Emer, uno dei più zelanti sostenitori del governo prussiano di cui divenne consigliere e cancelliere ${ }^{\mathbf{8 6}}$ Iniziò a seguire le orme paterne studiando teologia a Basilea e nonostante fosse riuscito a superare brillantemente gli esami di ammissione al corso rifiutò questa opportunità, decidendo di iscriversi all'accademia di Ginevra, per dedicarsi al diritto.

Non si conoscono i motivi della rinuncia, si suppone che il rifiuto fosse legato all'eccessiva durata degli studi teologici, o forse, più probabilmente, la morte prematura del padre, avvenuta nell'aprile del 1730, gli fece cambiare idea. ${ }^{87}$ Si trasferì così a Ginevra, dove approfondì, presso l'Accademia, discipline giuridico-filosofiche Barbeyrac attraverso le opere di Leibniz, di Wolff e di Barbeyrac, ma soprattutto ebbe come maestro Jean-Jacques Burlamaqui, che lo influenzò moltissimo ad avvicinarsi al diritto naturale e al diritto delle

84 Il testo più importante sulla vita di Vattel è il contributo di Béguelin (1929) 35-176. Tra i molti scritti che si sono soffermati sulla vita di Vattel, senza fini esaustivi, si rimanda: Guggenheim (1959) XV-XXXII; Manz (1971) 9-54; Jouannet (1998) 13-14; Jouannet (2012b) 118-1121. Anche Beaulac (2003) 237-292 in particolare 242-247; Beaulac (2004); Kapossy/Whatmore (2008a) IX-XX. Sul contesto di Neuchâtel e il Principato: Toyoda (2009) 103-124.

85 Il padre Jean de Montmollin fu anche consigliere di Stato nel 1685 e nel 1694; morì a Neuchâtel il 5 febbraio 1696. Di lui si trova scritto: «très versé dans le droit public et dans les affaires de l'État »: Journal Helvétique (août 1755) 212.

86 Emer de Montmollin (1664-1714) fu maitre bourgeois (1702) e avvocato (1706) di Neuchâtel. Partecipò inoltre in missione diplomatica alle diete di Langenthal (1707) e di Aarau (1708) e alle conferenze che condussero alla pace di Utrecht. Si distinse perché sostenne molto energicamente la candidatura del re di Prussia per il governo del Principato di Neuchâtel al posto di quella francese (tale vicenda sarà approfondita nel II capitolo del presente lavoro): cfr. Toyoda (2011) 166 ss.

87 BÉGUeLIN (1929) 40.

$20 \quad$ Note introduttive 
genti. ${ }^{88}$ Terminati gli studi, nel 1741, pubblicò il suo primo saggio filosofico, La défense du système leibnizien, in cui sostenne fermamente Leibniz e il suo pensiero. ${ }^{89}$ Il testo era dedicato a Federico II, forse con la speranza forse di avere un appoggio per la carriera diplomatica, ma, non poté per il momento percorrere questa strada. Béguelin racconta che arrivò a Berlino nel marzo 1742 «sur l'invitation de l'ambassadeur de France, et dans l'espoir d'y trouver quelque emploi » e fu ospitato da Jean Henri Formey, l'amico, come si vedrà nel prossimo capitolo, con cui avrebbe mantenuto rapporti epistolari per tutta la vita. ${ }^{90}$

Tuttavia, non avendo trovato un impiego redditizio a Berlino, Vattel cambiò radicalmente prospettiva e da suddito prussiano si trasferì a Dresda presso la dimora del conte Heinrich von Brühl, il primo ministro dell'elettorato di Sassonia, dove ottenne anche se non immediatamente un'occupazione temporanea e fu insignito del titolo di conseiller d'ambassade. ${ }^{91}$

88 Ibidem; Kapossy/Whatmore (2008a) X.

89 Sul saggio cfr.: Zurbuchen (1998) 91-113; Zurbuchen (2004) 189-211.

90 BÉGueLIN (1929) 44.

91 Toyoda (2011) 169. Il passaggio dalla Prussia alla Sassonia è per Vattel decisivo. Nel 1747, infatti, circa dieci anni prima della pubblicazione del Droit des gens presentò a Brühl una Mémoire et autres pièces concernant la création et l'objet d'une raprésentation diplomatique de la Cour de Dresde à Berne, in cui propose il trasferimento del Principato di Neuchâtel dalla Prussia alla Sassonia. Secondo Vattel l'operazione era possibile poiché il sovrano prussiano aveva perso ogni interesse verso il Principato di Neuchâtel e si era posto come unico obiettivo la conquista della Slesia, che effettivamente ottenne a seguito della pace di Aquisgrana nel 1748. A tal fine avanzava tre motivi per cui la Sassonia avrebbe dovuto acquisire Neuchâtel: « $1^{\circ}$ Le Comté de Neuchâtel est une Principauté absolument souveraine, ne relevant que de Dieu seul: Son Prince peut créer des nobles, reconnus comme tels dans toute l'Europe. $2^{\circ}$ Sa Majesté pourroit en tirer de bons Officiers et de bons soldats et y lever même d'autres Suisses du voisinage. $3^{\circ}$ Le Roi, comme Prince de Neuchâtel se trouveroit Allié des Quatre Cantons de Berne, Lucerne, Fribourg et Soleure; car deux des Cantons Catholiques qui n'ont pas voulu renouveller les anciennes alliances avec la Maison de Brandebourg, les renouvelleroient très volentiers avec un Prince de leur religion ", e così concludeva: "Si mon idée est goûtée, je m'estimerai très heureux de l'avoir conçûe. Mais quoiqu'il en soit, j'éspére qu'on daignera la regarder comme un effet de mon zèle pour le service du Roi, et de la certitude où je suis qu'il ne pourroit arriver à ma Patrie de plus grand bonheur que celui de passer sous la domination de Sa Majesté ». A questa Mémoire seguì nello stesso anno un Memorandum inviato al sovrano della Sassonia in cui presentò ufficialmente la sua idea; Vattel si sentiva in grado di fare una simile proposta perché sapeva che poteva contare sull'appoggio dei cugini Montmollin che, dalla morte dello zio di Vattel, erano sempre stati membri del Conseil d'État del Principato di Neuchâtel. Tuttavia il loro aiuto non fu così determinante, il Principato rimase nelle mani 
Nel 1747 venne inviato in missione diplomatica a Berna, in qualità di ministre accrédité du Grand Electeur de Saxe. ${ }^{92}$ Sfortunatamente questo incarico non gli portò nessun profitto economico e di conseguenza fu costretto a ritornare a Neuchâtel..$^{93}$

Per Vattel il periodo vissuto tra Berlino, Dresda e Neuchâtel fu, come lo ha dipinto Béguelin, il più triste: il lavoro era precario, lo stipendio misero e saltuario, ${ }^{94}$ anche se altre fonti affermano che in quegli anni Vattel conducesse una vita agiata, ${ }^{95} \mathrm{ma}$ al di là delle condizioni di vita, non smise di scrivere testi di carattere più squisitamente letterario. ${ }^{\mathbf{9 6}}$

Nel 1758, però, dopo la pubblicazione del Droit des gens il successo fu considerevole con sorpresa dell'autore stesso: venne chiamato nel 1759 a Dresda ad occupare l'incarico diplomatico che da molto tempo aspirava e fu nominato da Augusto III, re di Sassonia, suo consigliere privato per gli affari esteri. ${ }^{97}$

Fu questa l'unica e la tanto attesa occasione per Vattel di dimostrare le sue capacità anche in ambito diplomatico: fortemente preso dal nuovo lavoro

della Prussia, ma, come si vedrà, dopo la pubblicazione del Droit des gens, venne nominato consigliere privato di Augusto III, re di Sassonia. Per la trascrizione di entrambe le memorie: BÉGUelin (1929) 164-167. Sulla vicenda cfr. ToYoda (2011) 171 ss. e Roulet (1987) 115.

92 Toyoda (2011) 169.

93 La missione a Berna durò pochissimo: attraverso le lettere inviate sia a Formey che a Brühl si calcola che non superò i quattro mesi: BÉGUELIN (1929) 77.

94 BÉGUeLIN (1929) 49.

95 Cfr. Godet (1893) 221-222; anche Manz (1971) 16 nota 15.

96 Occorre precisare inoltre che tra il 1746 e il 1761 pubblicò diversi saggi letterari: Loisir philosophique, ou piéces diverses de Philosophie de Morale et d'Amusemens nel 1747 (a Ginevra, ma in realtà Dresda) e nel 1757 Poliergie au mélange de littérature et de poesie (ad Amsterdam). Il Journal Helvétique, con una recensione, elogiò Poliergie in cui « toutes ces diférentes Piéces portent le caractère de l'esprit judicieux et aimable. Elles anoncent le Philosophe éclairé, l'Home de goût et le Citoïen. L'Auteur veut instruire et plaire. Il réussit également à l'un et l'autre de ces égards". E terminava: "Cet ouvrage de Mr. de Vatel fait autant l'éloge de son cœur que de son Esprit, ceux qui ont l'avantage de le conoitre savent l'emploi qu'il fait de ses talents, pour l'avancement des Sciences et le bonheur des homes »: Journal Helvétique (avril 1757) 471-481, qui 472 e 481. Vattel in quel periodo scrisse anche: Mélanges de littérature, de morale, et de politique, edito nel 1760, che ebbe una ulteriore ristampa nel 1765, con il titolo Amusemens de littérature, de morale, et de politique: Kapossy/Whatmore (2008b) 77-103.

97 In questo contesto si può collocare l'incontro tra Vattel e il giurista italiano Bartolomeo Valdrighi: TAVILla (2009) 85-108, in particolare 89-90.

$22 \quad$ Note introduttive 
pubblicò nel 1762 un saggio sul diritto naturale intitolato Questions de Droit Naturel. ${ }^{98}$ L'opera, in realtà, fu terminata ancora prima della stesura del Droit des gens ed esattamente nel marzo 1753. Il ritardo della pubblicazione, secondo alcune fonti, sembra sia dovuto a causa della morte di Wolff, avvenuta l'anno successivo, e della contestuale difficoltà che ebbe Vattel nel trovare un editore. $^{99}$

Il saggio si presenta non tanto come uno scritto polemico, quanto invece una critica costruttiva rivolta allo Jus naturae del filosofo tedesco, anzi " comme un commentaire, destiné à rendre [le] Traité [de M. Wolff] plus utile ». ${ }^{\mathbf{1 0 0}}$ Tutte le riflessioni e osservazioni sulla dottrina di Wolff che il giurista di Neuchâtel elaborò nel corso degli anni sono raccolte in queste

98 Vattel (1762) 439. Il Journal Encyclopédique, il 15 giugno 1763, si espresse sulla nuova pubblicazione: "Quel autre que M. de Vattel pouvoit développer les sentimens de M. Wolf, commenter ses Écrits, expliquer ses principes et les combattre quelquefois? A quel autre qu'à cet Auteur célèbre étoit-il reservé d'ajoûter de nouvelles lumières aux excellens préceptes de M. Wolf, et d'applanir les obstacles qui rendent si difficile l'étude et la connoissance du Droit naturel? Ce n'étoit pas assez pour lui que d'avoir fondé les profondeurs du Droit des gens et d'en avoir donné aux Peuples et aux Rois de si sages leçons: il a cru que l'immortel ouvrage qu'il a publié sur cette matière seroit imparfait jusqu'à ce qu'il auroit examiné les règles, et parcouru tous les rameaux de la plus importante de toutes les notions de la Morale, celle du Droit de la Nature ; car quoique ce Droit soit gravé dans tous cœurs, il est cependant encore bien loin de ce dégré d'évidence que le génie et l'industrie ont donné à presque toutes les connoissances humaines. En effet, quelques efforts qu'eut fait l'illustre M. Wolf pour écarter les doutes que la différence des mœurs, les préjugés et les erreurs ont répandu sur cette intéressante matiere, la plûpart des grandes questions du Droit de la Nature subsistoient encore dans leur ancienne incertitude; et pour les décider, il ne falloit pas moins que les lumières réunies de ces deux Ecrivains célèbres »: Journal Encyclopédique (15 juin 1763) 3-4. Successivamente le riflessioni comparvero anche in latino in Jus naturae methodo scientifica pertractatum, pars prima [-octava], et nunc primum cum viri cl. de Vattel animadversionibus, editio novissima emendatior, et auctior che fu pubblicato a Francoforte e Lipsia tra il 1764 e il 1766, presso aere Societatis Venetae. In realtà il luogo di edizione è falso come rilevano BravetTi/Granzotto (a cura di) (2008) n. 571, 206.

99 Bandelier (2012) 52-53. Il titolo iniziale dell'opera era Observation sur le Droit Naturel de M. Wolff, in una lettera inviata a Formey il 21 ottobre 1753 si trova scritto: « Mes Observations sont à Lausanne [...], où je crois qu'elles s'impriment; croiriez-vous que le grand nom de Wolf est précisément ce qui fait trouver de la difficulté à placer ce manus[crit] les libraires de Hollande et de Suisse me répondent que Wolf ni ses critiques ne sont plus de mode; et pour ceux de France, il ne faut pas seulement leur en parler »: BÉGuelin (1929) 136, nota 173 .

100 Vattel (1762) Avertissement, VIII. 
pagine: «à mesure que j'avançois, et que je les voyois s'étendre sur des matieres intéressantes, je commençai à penser, qu'il ne seroit peut-être pas inutile de les donner au public ». ${ }^{101}$ Lo scopo di Vattel è quello di cercare di perfezionare e chiarire la disciplina del diritto naturale sulla base delle definizioni fornite da Wolff, ${ }^{102}$ e di conseguenza per raggiungere tale scopo « une petite remarque peut prévenir ce mal ». ${ }^{103}$ Esso è dedicato ad un pubblico giovane e Vattel pone in esso un intento squisitamente didascalico, utilizzando molti esempi, ragionamenti logici netti e precisi, ${ }^{\mathbf{1 0 4}}$ ma non ebbe la risonanza del Droit des gens.

Nel 1764, ormai in età matura, sposò Marie-Anne de Chêne, una giovane donna discendente da una nobile famiglia francese trasferitasi a Dresda e dalla loro unione, il 30 gennaio, 1765 nacque a Dresda Charles Adolphe Maurice de Vattel. ${ }^{105}$

Probabilmente furono gli sforzi o la concentrazione per l'impegnativo incarico da consigliere di Augusto III le cause del peggioramento delle sue condizioni di salute, tanto che scrisse a Formey: "l'air de Dresde ne me convient pas [...], et je ne suis point content de ma santé. Depuis huit ou neuf mois, des incommodités me tracassent et commencent à m'affaiblir; cela joint à mes occupation de devoir me laisse à peine le temps de jetter quelquefois les yeux sur Homère et Cicéron ». ${ }^{\mathbf{1 0 6}}$

Rientrò nella sua città natale, quando forse già intuiva che le forze gli stavano venendo a meno e, il 28 dicembre 1767 a soli 53 anni, morì. ${ }^{107}$

Il decesso fu annunciato qualche mese più tardi nel marzo 1768 dal Journal Encyclopédique con queste parole:

101 Ivi, III. Cfr. anche: Beaulac (2003) 246.

102 Vattel (1762) Avertissement, IV-V: «l'ouvrage [de Wolff] traite d'une matière dans laquelle aucune erreur n'est indifférente; s'il est échappé à un Auteur d'un si grand poids quelque décision peu juste, son autorité entraînera plus d'un lecteur dans des erreurs dangereuses pour la pratique ».

103 Ibidem.

104 Affermava infatti che: "si j’y ai réussi, et si ces jeunes gens veulent se former, par ces exemples, dans l'art de saisir l'état d'une Question, d'en démêler les vrais principes de faire de ces principes une juste application, et d'en suivre avec sagacité les vêritables conséquences ; je me flatte que par cela seul je leur aurai été très utile, et je me féliciterai d'un si heureux succès »: Ivi, III.

105 BÉGUELIN (1929) 62-63.

106 BÉGUELIN (1929) 64.

107 BÉGuelin (1929) 65, anche 140 nota 191.

$24 \quad$ Note introduttive 
M. Emer de Vattel, Conseiller privé de la Cour de Saxe, au Département des Affaires étrangères, est mort à Neuchâtel, sa patrie où il était allé de Dresde dans l'espérance que l'air natal lui rendroit la santé. [...] Tous ceux qui ont lu les ouvrages de ce sçavant seront sensibles à la perte que nous venons de faire, et dont ceux qui étaient liés avec lui se consoleront très difficilement. ${ }^{\mathbf{1 0 8}}$

La morte del giurista di Neuchâtel non cancellò di certo la sua fama che invece continuò sempre più a crescere, con caratteristiche e peculiarità del tutto singolari.

108 Journal Encyclopédique (I ${ }^{\mathrm{er}}$ mars 1768) 149. 



\section{Parte prima}

\section{Dalle traduzioni al discorso politico del Droit des gens}

« Divulgazione » e " traduzione » sono le parole chiave che ricorreranno all'interno delle prossime pagine, intese come strategie specifiche per la circolazione delle teorie del diritto naturale e del diritto delle genti, i cui effetti si riscontrano tanto a livello giuridico quanto a livello politico.

Nel primo capitolo saranno analizzati diversi profili che, intersecati offrono una visione peculiare del Droit des gens. Verranno illustrate le interazioni storiche e dottrinali nella quali Vattel si è formato come giurista, i riflessi, all'interno del suo pensiero, di autori come Jean Barbeyrac e Jean-Jacques Burlamaqui, che hanno dato un incisivo contributo alla diffusione delle teorie del diritto delle genti anche fuori dal territorio svizzero. E' stato proprio Barbeyrac a realizzare l'acquisizione del diritto naturale moderno all'interno della cultura politica, giuridica ed etica in ambiente sia protestante che cattolico; ${ }^{1}$ ed è stato Burlamaqui, insegnando il diritto naturale e delle genti e con la pubblicazione delle sue opere, ad influenzare anche Vattel.

L'interessante coincidenza di diverse e molteplici edizioni del Droit des gens nel 1758, proprio in contemporanea alla prima, può essere un ottimo terreno per riflettere sulle ragioni non solo di stampa ma anche politiche, dal momento che il trattato ha visto la luce durante la Guerra dei Sette Anni. Le iniziative editoriali, avvenute nel 1758 , sono, infatti, significative: la contraffazione olandese aggiunse al Droit des gens il sottotitolo Ouvrage qui conduit à developper les veritables Interêts des puissances e le edizioni « ridotte " del Droit des gens, intitolate Droit de la guerre e Mémoires politiques, consistono nella pubblicazione del III e IV libro del Droit des gens, rispettivamente dedicati alla guerra e alla pace.

1 Bazzoli (1986) 191. 
Le immediate traduzioni (in lingua inglese, tedesca, spagnola e italiana) sono la prova di come il processo traduttivo sia un complicato, ma necessario meccanismo di appropriazione di un testo, delle teorie e del linguaggio giuridico in esso contenuto. La versione francese del 1775 edita da Charles-Guillaume Dumas sarà approfondita come sorta di case-study per indagare sulle relazioni tra editoria, politica e diritto, soprattutto per quanto concerne il contesto della Rivoluzione americana.

L'accoglimento delle teorie vatteliane risiede nelle elaborazioni dei concetti di sovranità, indipendenza, uguaglianza degli stati e di equilibrio del potere, che anche se non state in toto "create » dal giurista di Neuchâtel, sono state senza dubbio precisate nei contenuti e maggiormente divulgate attraverso il Droit des gens. Il secondo capitolo dimostrerà, infatti, come alcune dinamiche giuridiche siano state mutuate in gran parte delle fonti dai predecessori, ma il giurista di Neuchâtel ne ha trasformato l'utilizzo e le finalità.

Le molteplici forme «sociali » attraverso cui il potere sovrano si relaziona giuridicamente con i soggetti, cittadini o sudditi sono i punti cardine su cui si snoda il pensiero di Vattel. Il fine della società civile è la realizzazione della felicità dei cittadini e l'ottenimento della giustizia attraverso la sicurezza: si ha pertanto il passaggio da uno stato che impone ad uno stato che regola e si determina sulla vita, prendendo forma e concretezza nella Nazione, la quale aspira ad esercitare non una funzione repressiva, bensì " regolativa ».

Nel leggere il trattato e le sue diverse edizioni si colgono importanti differenze semantiche che hanno anticipato molte teorie ottocentesche: "Stato », « Nazione» e « Potenza ». In primis lo Stato viene visto come l'entità giuridica e istituzionale, quasi fosse una sorta di contenitore astratto e generale; quindi "la Nazione ", intesa come «contenuto", è formata dai cittadini e acquista sostanza attraverso la promulgazione della costituzione che rappresenta il "piano » della Nazione e non più un "patto »; infine la «potenza», relativa all'esercizio del potere politico si identifica con la « ragion di stato» ed è la manifestazione delle dinamiche e delle esigenze nazionali e inter-nazionali. 


\section{Divulgare e ricreare un nuovo diritto delle genti}

\section{L'emergere di una strategia divulgativa: la « Scuola romanda del diritto naturale"}

La connotazione quasi sempre riduttiva che assume la parola «divulgazione " tende ad identificare il suo processo come una semplice modalità di trasmissione del sapere, che consente la condivisione della cultura con altri soggetti diversi dai produttori della stessa; ma, in realtà, l'iter divulgativo è caratterizzato da precise scelte e dalla necessità di acquisizione di determinate conoscenze per rispondere ad una utilità pratica, politica e anche dottrinale.

Al tempo stesso si è inclini a ritenere che la storia della sopravvivenza di un particolare autore si realizzi prevalentemente nell'esposizione delle tesi critiche sui suoi scritti, rilegando quasi in second'ordine l'analisi dei concreti meccanismi che portano alla loro espansione e fruibilità e hanno permesso nel corso del tempo e dello spazio di trarne insegnamento diverso. Esiste, invece, una precisa dicotomia tra la destinazione intenzionale dell'autore e la sua diffusione «due fatti che possono benissimo non combaciare, perché le vicende storico-culturali possono far giungere un'opera, almeno in alcune età e per alcune sue parti, a un pubblico tutto diverso da quello a cui essa era diretta, nelle intenzioni dell'autore ». ${ }^{1}$

Emer de Vattel, il «divulgatore di Wolff», ha risposto ad una esigenza del suo tempo, articolando il linguaggio giuridico e tecnico ad una comunità che chiedeva certezza in ambito "internazionale ", attraverso un sistematico trattato sul diritto delle genti. Egli aveva intuito, nonostante le esitazioni del suo Droit des gens, e ignaro della sua successiva fortuna, l'esigenza di una divulgazione indirizzata ai sovrani e a coloro che detenevano ruoli di governo e politici.

Questo tipo di approccio è stata la conseguenza diretta della sua appartenenza ad un particolare ambiente scientifico: la cosiddetta « Scuola Romanda

1 Petronio (1981) 15-16. 
del diritto naturale». L'esperienza giusnaturalistica settecentesca avvenuta nell'attuale Svizzera francofona, mediante i differenti esponenti, è divenuta sempre più raffinata, sino a giungere a una più completa sistematizzazione nello studio e nella trattazione del diritto delle genti. Si è infatti assistito ad un graduale indirizzamento del sapere giuridico da un contesto più squisitamente dottrinale e letterario ad un utilizzo politico, accademico e formativo, che è culminato con il Droit des gens di Vattel. Se è vero che i progressi di una scienza sono il frutto del lavoro collettivo di un certo numero di autori, ognuno dei quali apporta, migliora, precisa e amplia i risultati di chi lo ha preceduto, non sarà affatto scontato né di poco conto l'elaborato finale, che viceversa apparirà più incisivo e decisivo e meglio potrà rispondere $\mathrm{a}$ determinati contesti spazio-temporali.

L'École Romande du Droit Naturel, come afferma Alfred Dufour, fu una sorta di mediazione tra la cultura tedesca, rappresentata tra i molti da Pufendorf, Thomasius e Wolff, e la cultura francese che si riconosceva, ad esempio, nelle opere di Montesquieu, Rousseau e Voltaire. ${ }^{2}$ Ginevra, Losanna, Neuchâtel e Yverdon erano principali poli di diffusione in cui circolavano idee e trattati di diritto naturale e delle genti, che riscossero un grande successo nell'Europa del Sette e Ottocento. Tra gli esponenti vi erano illustri giuristi e filosofi, basti pensare a Jean Barbeyrac (1674-1744), professore di diritto e storia all'Accademia di Losanna e poi di diritto pubblico a Groninga; ${ }^{3}$ all'archeologo e naturalista Louis Bourguet (1678-1742); ${ }^{4}$ a Jean-Jacques Burlamaqui (1694-1748), professore di diritto naturale a Ginevra; ${ }^{5}$ ad Emer de

2 Dufour (1976) 2; Dufour (1979) 133-143, Dufour (1999) 35-74. Anche: Orestano (1982) 169-178 e Keller (2006) 387-409; ZuRbuchen (2004) 189-211.

3 Su Barbeyrac storico è il contributo di Meylan (1937). Senza pretesa di esaustività si rimanda ad alcuni tra i più recenti contributi che riguardano il lavoro di Barbeyrac traduttore, il suo ruolo di professore e le sue riflessioni sul diritto naturale e delle genti e su alcuni specifici temi: Zurbuchen (1991); Zurbuchen (2003) 49-70; Hochstrasser (1993) 289-308; Goyard-Fabre (1996) 11-74; Korkman (2002) 109-122; Korkman (2003) 195-225; Hunter (2004) 670-699; Korkman (2006) 257-282; Pott (2002); Labriola (2003); Poudret (2006) 105-110; Tillet (2007) 373-396; Di Donato (2007) 409-418; Silvestrini (2007) 103-116; Tappy (2010) 2-8; Palladini (2011).

4 Per la vita e le opere di Bourguet: HaAg (1847) 484-486; JeAnneret/Bonhôte (1863a) 59 ss.; Perrochon (1951); Bork (1974) 49-77; Carozzi (1986) 25-51; Rieppel (1987) 125-132; Schaer (1996) 16-22; SLOAN (2006) vol. 2, 903-938.

5 Per la figura di Burlamaqui: Harvey (1937); Borgeaud (1938) 73-82; Gagnebin (1944); Habscheid (1967) 5-24; Simonin (2007) 89-101. Anche: Johnston (2005) 331-374; 
Vattel (1714-1767); infine a Fortunato Bartolomeo de Felice (1723-1789), promotore dell'imponente Encyclopédie d'Yverdon. ${ }^{\mathbf{6}}$

Pur considerando che all'interno del giusnaturalismo non si possa parlare di una vera e propria scuola, ${ }^{7}$ la dicitura école è stata attribuita dagli storici per indicare e riunire caratteristiche comuni di autori che, durante la loro carriera di professori ed editori, hanno avuto un ruolo determinante per la diffusione di idee giusnaturalistiche, con una marcata apertura però alle teorie più illuministiche nell'ambito geografico specifico della Svizzera francofona. $^{8}$

L'interesse per «il sapere" e per la sua massima divulgazione è una costante nelle opere di Barbeyrac. A Losanna nel 1714, nel contesto accademico, pronunciava il celebre Discours sur l'utilité des lettres et des sciences par rapport au bien de l'Etat, all'interno del quale non vi era soltanto l'esaltazione della cultura in se stessa, bensì del suo potere pubblico, della sua utilità e necessità in relazione alla società civile e politica, al «bien publique ». 9

Tuttavia Barbeyrac non si limitò a riflessioni sul valore della conoscenza, ma agevolò la complessa diffusione delle teorie del diritto naturale con le sue traduzioni in francese alle opere di Ugo Grozio e Samuel Pufendorf: l'aspet-

Korkman (2006) 257-282; Gomez (2007/2008) 41-56; Douglass (2011) 209-230; DevetaK (2012) 105-128; Broussois (2015).

6 Su de Felice e la sua opera enciclopedica: Maccabez (1903); Perret (1945) 155-178; Cernuschi (1993) 85-109; Donato (1993) 53-86; Donato (1997) 374-396; Donato (2002) 1-26; Cernuschi (2005) 470-476; Ferrari (2008); Ferrari (2010) 87-106.

7 Padoa Schioppa (2007) 329.

8 Dufour (1976) 2: “Si l'on s'accorde pour qualifier d'Ecole un mouvement intellectuel attaché à défendre autour d'un ou de plusieurs maîtres un certain nombre de principes et les propageant par la parole ou par l'écrit, il nous paraît difficile, au vu de l'impressionnante continuité de l'enseignement du Droit naturel à Lausanne et à Genève comme de la production littéraire qui s'y rapporte du début à la fin du XVIIIe siècle, de contester l'existence à cette époque d'une Ecole Romande du Droit naturel, vouée à la diffusion en terre de culture française des principes de Grotius, de Pufendorf, de Thomasius et de Wolff relatifs aux fondements rationnelles du Droit positif, aux règles méthodologiques de leur détermination et à celles de leur formulation ".

9 «Je dis donc, que les Arts Libéraux et les belles Connaissances sont un des meilleurs remparts de la Liberté [...] L'ignorance abbaisse l'Esprit, elle étousse les sentimens d'honneur et de magnanimité, elle dispose à subir le joug sans peine pour quelque vil intérêt, elle ôte le courage d'entreprendre de belles actions pour le bien de la Patrie». E ancora sosteneva che: «Aussi voions- nous qu'entre les moiens dont les Tyrans se sont avisez pour affirmir leur domination, un de ceux qu'ils ont cru les plus efficaces, ç'a été d'empêcher, autant qu'ils ont pû, que les Citoiens ne s'attachassent aux Lettres»: Barbeyrac (1714) 16. 
to traduttivo giocò, dunque, una funzione chiave nella fruibilità dei testi sul diritto delle genti, ma soprattutto fu il vero e proprio punto di forza dell'École. Barbeyrac, infatti, tradusse in francese nel 1706 il De iure naturae et gentium di Pufendorf, ${ }^{\mathbf{1 0}}$ successivamente, nel 1724, il De iure belli ac pacis di Grotius. ${ }^{11}$ Egli incarna concretamente la tensione verso l'accessibilità della cultura o, meglio ancora, per usare le parole di Labriola, il suo lavoro ebbe come fine « la divulgazione massima delle teorie del diritto naturale, oltre il pubblico ristretto e tradizionale degli eruditi $»,{ }^{\mathbf{1 2}}$ e le sue traduzioni erano rivolte alle « jeunes gens» e alle " gens sans lettres ». ${ }^{\mathbf{1 3}}$

Illuminante è leggere anche il metodo e l'approccio utilizzato da Barbeyrac per le sue traduzioni:

je ne parle pas des transitions, qu'il a fallu souvent suppléer [...] mais il y avait quelque fois des pensées mal rangées que je pouvais transposer sans aucun inconvénient [...] Aussi ai-je trouvé des transpositions à faire dans des endroits où les choses étoient mal rangées; [...] Il y en a même quelques-uns de ceux-ci [...] dans lesquels j’ai été obligé ou de transposer certaines pensées d'un paragraphe à l'autre, ou de transposer des paragraphes entiers: au lieu que partout ailleurs, les périodes dont l'ordre a été changé, se trouvent toutes dans un même paragraphe. ${ }^{14}$

Il procedimento adottato da Barbeyrac, l'inserzione di note esplicative, la correzione e semplificazione del testo groziano hanno creato qualcosa di radicalmente nuovo con ripercussioni tanto a livello locale, quanto su scala «internazionale », al punto tale che le versioni di Barbeyrac, a loro volta, sono state tradotte e riedite molto di più che gli originali stessi. ${ }^{15}$

10 Pufendorf (1706).

11 Grotius (1724).

12 Labriola (2003) 17. Anche: Baldwin (2007) 101-124. Saunders (2003) 473-490, in particolare 483-484.

13 Cfr. sul punto Pärvärinne (2012) 33-47 e anche Gordley (2013) 130.

14 Barbeyrac, Préface du traducteur, in: Grotius (1724) XXIV.

15 Nel 1867, nel criticare la versione francese del giurista di Losanna, Louis Pradier-Fodéré, sosteneva che «bien que la lecture de l'original latin soit d'un grand attrait, l'usage de lire et de citer Grotius dans la paraphrase que Barbeyrac en a faite a prévalu généralement, et il en est résulté que le traité du Droit de la Guerre et de la Paix a été le livre le plus cité, et le moins connu »: Pradier-Fodéré, Avant-Propos, in: Grotius (1867) III-XII, qui III. Era proprio Pradier Fodéré, professore di diritto pubblico e fondatore della facoltà di scienze politiche a Lima, a proporre con forza, benchè fosse trascorso oltre un secolo dalla versione di Barbeyrac, una traduzione francese del trattato di Grozio, che fosse però "parola per parola ». Egli concepiva, come una pura e aderente trasposizione lessicale, la traduzione delle opere sul diritto delle genti, e nel caso specifico del De iure belli ac pacis, per portare il trattato alla sua originalità, era disposto a sacrificare la sua lingua materna, e a 
Oltre alle traduzioni di Barbeyrac deve essere aggiunta, sempre a fini divulgativi, la produzione di testi sul diritto delle genti. Jean-Jacques Burlamaqui dal 1723 docente di diritto a Ginevra, pubblicò nel 1747 i Principes $d u$ droit naturel e nel 1751, apparvero postumi i Principes du droit politique, entrambi gli scritti ottennero una notevole influenza non solo nei cantoni della Svizzera francese ma anche nella vicina Francia e in altri contesti europei. ${ }^{16}$ Importante fu l'insegnamento di Burlamaqui nella formazione di Vattel: durante gli studi giuridici ginevrini rimase particolarmente colpito e influenzato dalle sue lezioni sul diritto naturale e delle genti.

Per comprendere, altresì, il clima respirato dagli esponenti della Scuola Romanda è opportuno rilevare, che il territorio svizzero del XVIII secolo, parafrasando Voltaire, era una delle regioni europee più fiorenti dal punto di vista dell'istruzione e dell'arte: ${ }^{17}$ l'immagine della vivacità, della ricchezza della vita culturale e intellettuale svizzera era rappresentata dalla proliferazione dell'editoria e delle riviste, sulle quali venivano discusse in primo piano questioni legate al diritto naturale e al diritto delle genti.

Significativa è l'esperienza della Bibliothèque Italique, vera e propria fucina di diffusione della cultura e del pensiero italiano, che venne pubblicata a Ginevra dal 1728 al $1734:^{18}$ questa rivista quadrimestrale nacque grazie

riordinare le note, apponendo ad esse la sigla di paternità: Grozio, Barbeyrac, oppure proprio Pradier-Fodéré. Ancora in un altro passaggio affermava: «Pénétré de l'idée que le devoir du traducteur est de faire abstraction de lui-même, et de faire connaître l'auteur tel qu'il est, non tel qu'on aurait désiré qu'il fût, je me suis attaché à suivre de très-près le texte ; préférant toujours l'exactitude à l'élégance du style, et ne craignant jamais de répéter un mot, lorsque ce mot se trouvait répété dans l'original. [...] Lorsque le style de Grotius a résisté pas sa concision au tout de la phrase français, je me suis efforcé de ne pas abandonner pour cela le texte ; j'ai sacrifié volontairement les lois de ma langue maternelle »: Pradier-Fodéré, Avant-Propos, in: Grotius (1867) VII-VIII. L'aggiunta di note esplicative, anche con precisi riferimenti alla storia del diritto delle genti dell'Ottocento, non era nuova a Pradier-Fodéré perché, come si vedrà nei prossimi capitoli, qualche anno prima, nel 1863, aveva curato una delle migliori edizioni del Droit des gens di Emer de Vattel, che era completata da un fitto apparato di note con spiegazioni dottrinali e pratiche dell'utilizzo del pensiero del giurista e diplomatico di Neuchâtel.

16 Goyard-Fabre (1996) 11-74.

17 Voltaire (1994) 34. Anche: Biucchi (1983) 67 ss.

18 PAPa (1993) 106. Per approfondimenti sulla Bibliothèque Italique si rimanda: CRUCITtIUllrich (1974); Rosset (1996) 193-223. La diffusione della opere di Giannone, Muratori, Maffei e Vallisneri (o Vallisnieri) fu al centro dell'iniziativa: Crucitti-Ullrich (1991) n. $165,191$. 
all'iniziativa di un gruppo di intellettuali ugonotti rifugiati in Svizzera, tra cui vi era anche un ulteriore esponente della Scuola Romanda, Louis Bourguet, amico di Jean Barbeyrac e successivamente di Emer de Vattel, originario di Nîmes, ma residente a Neuchâtel. La Bibliothèque Italique fu fondata grazie ad un progetto di Gabriel Seigneux de Correvon, ma l'esecuzione fu resa da Bourguet, il quale già dal 1725 era stato incaricato di ottenere il budget per l'iniziativa. ${ }^{19}$

Lo storico del diritto Philippe Meylan descrive Bourguet come un uomo dal sapere universale, dalla «nature franche et généreuse », al punto tale che «Genève, Lausanne, Neuchâtel se disputaient cet homme extraordinaire, trop inventif à la fois, trop modeste et trop pauvre pour songer à répandre par le livre de productions de son esprit ${ }^{\mathbf{2 0}}$

Nel novembre 1716, dopo che Bourguet aveva informato Jean Barbeyrac sulla decisione di stabilirsi a Neuchâtel, quest'ultimo così gli rispose:

J'aurais bien souhaité que vous vous fussiez déterminé pour Lausanne: mais à vous dire le vrai, quoique vous voulussiez bien témoigner y avoir quelque inclination, lorsque nous eûmes le bonheur de vous voir ici, je ne me suis jamais flatté que vous prissiez ce parti $[\ldots]$ Indépendamment des raison de famille, les personnes qui vous y attirent méritent bien cette préférence. A votre place, j’en aurais fait autant que vous. $^{21}$

Bourguet è ricordato non solo per essere sostenitore della filosofia di Leibniz ma anche per aver fondato nel 1732, a Neuchâtel, il foglio più diffuso in tutta la regione svizzera il Mercure Suisse o Recueil de nouvelles historiques, politiques, littéraires et curieuses, il quale vide tra i collaboratori Emer de Vattel. ${ }^{22}$ A partire dal gennaio 1738, alla suddetta rivista venne unita mensilmente la pubblicazione del Journal Helvétique. ${ }^{23} \mathrm{Fu}$ proprio il Journal Helvétique nell'aprile del 1758 ad annunciare la prima edizione del Droit des gens, con queste parole:

Le style est clair, net et coulant, d'une noble et élégante simplicité, tel que doit être le langage de la vérité, surtout quand elle parle aux Rois et aux Ministres des Rois: Il

19 Ibidem.

20 Meylan (1937) 110.

21 Così riportato in: Meylan (1937) 110.

22 Candaux (1988) 49-57, qui 49-50; anche Candaux (1991a) n. 950, 884-885; Candaux (1973) 126-171. Anche Huguenin/Léchot (2012) 55-71; Huguenin/LÉchot (dir.) (2016).

23 Papa (1993) 117. Anche: Schlup (1988) 59-71; Candaux (1991b) n. 743, 682-683; Huguenin (2012) 315-327. 
est d'ailleurs vif et animé dans l'ocasion, tel que le langage d'un Home qui veut des Vérités importantes, et qui les voit bien dans ce qu'elles ont d'intèressant pour le bonheur et la gloire des Nations et de leurs Conducteurs. ${ }^{24}$

La pubblicazione nel contesto svizzero di tutte queste iniziative editoriali avveniva ad opera di Daniel Wavre e Abraham Droz e successivamente del figlio Abraham II Droz, al quale si deve anche la prima edizione dell'opera di Vattel. ${ }^{25}$ A partire dal 1769 il Mercure Suisse fu affidato alla celebre Société typographique de Neuchâtel che, in assenza di una specifica e uniformata disciplina sul diritto d'autore, si specializzò nella riproduzione illegale e nella ristampa di opere di successo, quali ad esempio l'Encyclopédie di Diderot, rivestendo un ruolo fondamentale per la diffusione dei Lumi. ${ }^{26}$ Chiusa nel 1789 , è considerata dagli storici una delle più importanti imprese editoriali del Settecento e vanta la pubblicazione, in soli vent'anni, di oltre cinquecento opere. ${ }^{27}$ Tra i fondatori vi era Frédéric Samuel Ostervald, amico intimo di Vattel nonché curatore di un'edizione del Droit des gens, pubblicata dalla Société nel $1773{ }^{28}$

Per ragioni di chiarezza editoriale è opportuno precisare che la direzione del Mercure Suisse, prima di essere affidata alla Société typographique, per un tempo intermedio, seppur breve, tra il 1767 e il 1769, fu assunta dal romano Fortunato Bartolomeo de Felice, che aveva sposato nel 1759 Suzanne-Catherine Wavre, figlia di uno dei fondatori della rivista svizzera. ${ }^{29}$

24 Journal Helvétique (avril 1758) 444 ss.

25 Quartier-la-Tente (1903) 245. Anche: Schlup/Tissot/Donzé et al. (éd.) (1983) 36; Donato (1992a) 74-111.

26 Darnton (1982); Darnton (1984a) 342-359; Darnton (1984b) 477-492; Machet (1986) 158-185; Pasta (1990a) 281-320; Pasta (1997a); Via (1995); Darnton/Schlup (éd.) (2005); VALERI (2006).

27 VAleri (2006) 23.

28 Frédéric Samuel Ostervald (1713-1795) apparteneva all'alta borghesia di Neuchâtel, fece parte del Gran Consiglio di Neuchâtel nel 1746 e nel 1751 del Piccolo Consiglio. Venne definito un oratore affascinante. Appunto insieme Jean Elie Bertrand, Samuel Fauche e Jonas-Pierre Berthoud fondò la Société typographique de Neuchâtel, di cui fu direttore dal 1769 al 1789: JeAnneret/Bonhôte (1863b) 145-148. Sul contributo di Ostervald alla Société: Valeri (2006) 13; Darnton (2009) 39. Sulla Société e la distribuzione dei libri si veda: Schlup (éd.) (2010); Seaward (2014) 439-457.

29 «Par ses deux premières femmes, Suzanne-Catherine Wavre e Louis-Marie Perrelet, Félice, bourgeois de Thièle, n'était pas étranger au pays de Neuchâtel, si proche d'ailleurs d'Yverdon »: Perret (1945) 123. 
De Felice fu, senza dubbio nel contesto delle iniziative divulgative uno dei personaggi più singolari del Settecento; nel 1762 a Yverdon aprì una stamperia che divenne in poco tempo famosa in tutta Europa: "nei libri che scrisse e in quelli che pubblicò, egli portò un fermento intellettuale basato sulla cultura eclettica ma aggiornata ed entusiasta, non comune fra gli editori del $700 »^{30}$

Fu il promotore della celebre Encyclopédie d'Yverdon, dedicata a Haller, stampata tra il 1770 e il 1780 e composta da cinquantotto volumi. Essa aveva come punto di riferimento l'opera di Diderot e d'Alembert, ma De Felice volle discostarsi del prestigioso modello, «riplasmando » e "aggiornando » le voci e tenendo conto del progresso delle conoscenze: infatti la principale differenza tra l'Encyclopédie d'Yverdon e l'Encyclopédie parigina risiede proprio nello spirito meno filo francese e meno antireligioso, che le valse il nome di « enciclopedia protestante » con una più ampia diffusione nell'Europa centro settentrionale. $^{31}$

Benché la maggior parte delle voci fossero state redatte dallo stesso De Felice, egli si avvalse della collaborazione di moltissimi scrittori di fama europea, tra i quali anche il celebre illuminista (nonché amico di Vattel) Jean Henri Samuel Formey, sostenitore della pubblicazione dell'Encyclopédie e insieme a De Felice ideatore dell'iniziativa. ${ }^{32}$

De Felice si distinse anche per un'interessantissima edizione " considérablement augmenté » del trattato di Burlamaqui, Principes du droit de la nature et des gens, che pubblicò tra 1766 e il 1768 a Yverdon e recante la letteradedica a Formey, nella quale illustrò il pensiero giusnaturalista di Burlamaqui e tra le fonti, di cui si era servito per annotare e aumentare l'opera del giurista ginevrino, compare anche il Droit des gens di Vattel.

30 Pejrone (1987) 682-686, in particolare 684.

31 Donato (1992a) 99 ss.; Donato (1992b) 243-247.

32 Jean Henri Formey (1711-1797), docente di retorica e filosofia all'Università di Berlino. Membro dell'Accademia delle scienze e, a partire dal 1748, segretario a vita dell'università di Berlino. Diresse riviste molto importanti, quali la Bibliothèque Impartiale e la Nouvelle Bibliothèque Germanique. Sostenitore, come Vattel, di Leibniz e di Wolff, ma anche di Locke e di Hume, criticò fortemente Diderot ne Le système du vrai bonheur (1750) e alcune posizioni di Rousseau nell'Anti-Émile (1763) e nell' Émile Chrétien (1764). Sulla biografia di Formey si rimanda sinteticamente a: Marcu (1953) 296-305; Donato (1996) 87-98; Häseler (2005) 121-140; cfr. anche Bandelier (2012) VII-XVIII, qui VIII. 
Il vivo panorama della Svizzera settecentesca era così caratterizzato da una pluralità di elementi culturali, sociali e politici all'interno dei quali prendevano forma le iniziative editoriali, le traduzioni e l'insegnamento, favorendo una dinamica circolazione della dottrina giuridica proveniente anche dagli altri Stati europei e influendo in modo decisivo sulla formazione di Emer de Vattel e del suo Droit des gens. Era lo stesso Vattel ad elogiare la sua terra, dimostrandosi fiero di esservi nato: "Je suis né dans un pays, dont la Liberté est l'âme, le trésor et la Loi fondamentale: je puis être encore, par ma naissance, l'ami de toutes les Nations $»{ }^{33}$

\section{Il Droit des gens e il garbuglio editoriale del $\mathbf{1 7 5 8}$}

La genesi del Droit des gens è iniziata nell'anno 1747, undici anni prima della reale pubblicazione: tutti i passaggi, le esitazioni e le difficoltà sono racchiusi nel carteggio, oggi interamente a stampa, tra Vattel e Jean Henri Samuel Formey, ${ }^{34}$ anch'egli impegnato a scrivere un trattato sul diritto naturale e delle genti. ${ }^{35}$ Entrambi, Formey e Vattel, sostenevano la necessità che il celebre filosofo e giurista di Halle, Christian Wolff, venisse apprezzato anche nei paesi francofoni e si ponevano come scopo la massima fruibilità del suo pensiero. Nel giugno 1749, Vattel proponeva, sulla scia di Barbeyrac, di tradurre e adattare in francese lo Ius gentium methodo scientifica pertractatum di Wolff appena pubblicato, trasformandolo « dans un ouvrage françois d'un stile moins rebutant ", proprio al fine di rendere accessibile ad un pubblico più vasto le teorie di colui che considerava il suo maestro scientifico. ${ }^{\mathbf{3 6}}$

Tuttavia il progetto inaspettatamente fu accantonato. Il modello di traduzione, tanto «manipolativa » quanto più « visibile » che circolava nella Scuola Romanda, a Vattel non sembrava più consono ai suoi obiettivi. Egli voleva un suo diritto delle genti. ${ }^{37}$

33 Vattel (1758a) Préface, XXVI. Guggenheim (1956) 24: «Fut Emer de Vattel le premier auteur suisse de droit international à être conscient de sa nationalité. Son sens de mesure, sa prudence, mais aussi son amour de l'humanité ont été le précieux héritage qu'il a légué à ceux qui sont venus après lui ».

34 Bandelier (2012) e anche Bandelier (1996).

35 Formey (1758).

36 Bandelier (2012) XII. Cfr. la lettera di Vattel a Formey, 27 giugno 1749, trascritta in: Bandelier (2012) 103-105.

37 Per la ricorrenza del possessivo « moi », per indicare la propria opera sul diritto delle genti cfr. BANDELIER (2012) XIII. 
Il 25 marzo 1753 informava Formey che si stava dedicando esclusivamente alla redazione di una «sua » opera sul diritto delle genti, avendo come riferimento gli scritti di Wolff; questo suo nuovo lavoro cominciava a prendere forma, confidava in una fortunata pubblicazione e anche in un riscontro economico: « je ne veux plus travailler uniquemente pour la gloire, j'ai besoin de songer à un profit plus réel, et je veux qu'un libraire me paie mes ouvrages $» .{ }^{\mathbf{3 8}}$

Dopo aver ricevuto la premessa dei Principes du droit de la nature et des gens di Formey, Vattel, il 14 novembre 1754, rispondeva che il suo Droit des gens procedeva lentamente perché Wolff gli risultava carente nella trattazione di alcuni temi legati al diritto delle genti e pertanto doveva ricorrere alla consultazione e allo studio degli scritti di Grozio e di Pufendorf. ${ }^{39}$

L'8 novembre 1755 Vattel sottoponeva al giudizio dell'amico il piano della sua opera e chiedeva consiglio per la scelta dell'editore. ${ }^{40} \mathrm{Fu}$ così che Formey, molto entusiasta del lavoro, lo mise subito in contatto con l'editore e scrittore di Leida, Elie Luzac, che all'epoca curava le sue pubblicazioni, inviandogli sia il programma sia « une partie du manuscrit » del giurista di Neuchâtel. ${ }^{41}$

38 Lettera di Vattel a Formey, 25 marzo 1753, in: Bandelier (2012) 143-146, qui 144.

39 Ed aggiungeva: «Au reste, je ne charge point mon ouvrages [sic] d'autorités; je l'orne seulement d'exemples et je dresse les décisions sur ce qui me paroit découler des principes »: Lettera di Vattel inviata a Formey, 14 novembre 1754, in: Bandelier (2012) 154-156, qui 155 .

40 Lettera di Vattel inviata a Formey, 8 novembre 1755, in: BANDELIER (2012) 157-164, qui 157.

41 Lettera di Vattel inviata a Formey, 17 febbraio 1757, in: Bandelier (2012) 179-180. Elie Luzac (1721-1796), figlio del rifugiato ugonotto Elie e di Anne-Marie Cabrolle, studiò filosofia a Leida e, come lo zio, Jean Luzac (1702-1783), dedicò tutta la sua vita all'editoria, divenendo uno dei più famosi e quotati editori olandesi. Scrisse anche saggi a sostegno della libertà di stampa come l'Essai sur la liberté de produire ses sentimens nel 1749. Per una più completa biografia si rimanda a: Kossmann (1966) 60 ss.; Velema (1985) 427-444; Velema (1987) 143-156; Velema (1993) 34 ss. Per quanto riguarda il programma del Droit des gens di Vattel lo si trova pubblicato sia sul Journal Helvétique (septembre 1757) 376, che sul Mercure Suisse (septembre 1757) 80-89. Sul punto è interessante anche confrontare i commenti a tale programma che furono apportati da uno dei più cari amici di Formey, Nicolas Trublet, il quale gli scrisse: "J'ai envoyé à M. de Boissy pour le Mercure programme du Droit des gens. Il est très bien fait, et j'achèterai l'ouvrage, quand il paraîtra »: lettera di Trublet edita all'interno del volume che raccoglie la corrispondenza di Trublet e Briasson a Formey: Briasson / Trublet (1996) 226, anche 216, 255-256. 
Luzac, il 17 maggio 1756, riscontrata l'avvenuta ricezione del testo, si rendeva subito disponibile alla pubblicazione:

Je lui promets $[\ldots]$ bonne et belle impression, correcte, prompte, mais je n'entrerai pas en négociation à moins que l'auteur ne s'engage de ne [pas] donner le même livre, corrections, augmentations etc. à quelque autre. Il faut que j'en aye seul la possession

e terminava la sua lettera aggiungendo:

voici le sommaire des chapitres qui doivent composer l'ouvrage de M. Vattel. Je vous le renvoie Monsieur, et j’y ajoute les remerciements pour la préférence que vous m'avez donnée en me parlant de ce livre. Je vous remets le soin de répondre à $\mathrm{M}$. Vattel auquel vous me ferez plaisir avec lequel je serais charmé d'être en liaison. ${ }^{42}$

Il repentino e inaspettato accoglimento del testo da parte della casa di Leida, creò non pochi problemi sui diritti di stampa e tale questione si fece molto complicata e ambigua: Vattel, non intenzionato a concedere l'esclusiva della pubblicazione del trattato a Luzac, si discostò dalla proposta accentratrice dall'editore dell'amico, esprimendo invece, il desiderio di vedere curata « sotto i suoi stessi occhi » la stampa della sua opera.

Nella lettera del 17 febbraio 1757 sottolineava all'amico che Luzac: «n'a pas voulu démordre de son droit de copie, et cette condition désagréable me fait prendre le parti d'imprimer ici sous mes yeux. Je verrai moi-même les épreuves. L'ouvrage sera fort bien imprimé, et en beau papier ${ }^{43}$ Egli riteneva ormai di poter vantare sul manoscritto di Vattel un diritto di copia (che verrà esercitato durante il XVIII secolo anche da altri editori olandesi) e, anche senza aver avuto l'autorizzazione di Vattel, pubblicò nel 1758 una edizione del Droit des gens (a Leida), che non sarà mai riconosciuta dal diplomatico di Neuchâtel e verrà da lui stesso dichiarata contraffatta. ${ }^{\mathbf{4 4}}$

Dalla corrispondenza intercorsa tra i due amici, nella fase antecedente la pubblicazione emerge non solo un vero e sincero legame affettivo, ma anche una complicità culturale, infatti, senza timore, Vattel confidava all'amico l'obiettivo principale della sua opera, e cioè la stesura di un testo sistematico

42 Lettera di Luzac inviata a Formey il 17 maggio 1756, in: Bots/Schillings (2001) 290-291. La corrispondenza tra Luzac e Formey è composta da più di duecento lettere spedite tra il 1748 e il 1770, si rimanda anche al contributo di: Marx (1968) 779-786, in particolare 785 .

43 Lettera di Emer de Vattel a Formey, 17 febbraio 1757, in: Bandelier (2012) 179-181, qui 179.

44 Cfr. Bandelier (2012) 179, nota 2; Fiocchi Malaspina (2014) 733-754. 
sul diritto delle genti che fosse semplice da leggere, piacesse « alla gente del mondo ", fosse ornato di esempi riguardanti la storia dell'epoca: un libro aggiungeva - diverso dall'opera di Wolff. ${ }^{45}$

L'11 aprile 1757 scriveva a Formey che il trattato avrebbe riportato come luogo di edizione Londres, ma che personalmente stava curando la stampa a Neuchâtel con l'editore del Mercure Suisse (e del Journal Helvétique) Abraham II Droz: «Elle sera belle et très-correcte, en grand papier et en caractéres $S^{t}$ Augustin; l'ouvrage formera près de cent feuilles, ou 800 . p. in $4^{\circ}$ et on pourra le diviser en deux parties $» .{ }^{\mathbf{4 6}}$

Il 17 dicembre 1757 il manoscritto, ormai terminato, veniva inviato a Formey: «Voici enfin mon Droit des gens. Je le fais mettre dans un ballot pour Francfort, d'où vous le recevrez, mon cher ami, par le chariôt de poste $» .{ }^{47}$ In questa circostanza Vattel pregava l'amico di annunciare la pubblicazione del Droit des gens sulle riviste che dirigeva e con cui collaborava assiduamente, come la Bibliothèque Impartiale e la Nouvelle Bibliothèque Germanique, al fine di poter avere un ampio successo e di poter vendere molte copie. ${ }^{48}$ Formey prontamente sostenne la pubblicazione dell'opera, secondo le direttive espresse da Vattel: ${ }^{49}$ "Je vous prie d'en parler dans votre Journal le plustôt possible, en insistant particulièrement sur ce qui distingue mon ouvrage de tous ceux qui ont été écrits sur ces matières ${ }^{50}$ Ma soprattutto insisteva sull'aspetto tipografico: «Je crois que vous pouvez la louer en

45 Lettera di Emer de Vattel a Formey, 17 febbraio 1757, in: Bandelier (2012) 179-181, qui 179.

46 Lettera di Vattel a Formey, 11 aprile 1757, in: Bandelier (2012) 184-186, qui 185, in una altra lettera del 6 ottobre 1757, Vattel scriveva a Formey: «Mon Droit des gens formera 2 vol[umes] in $4^{\circ}$ faisant ensemble 122. feuilles, sur le modèle du Burlamaqui; mais mon édition est plus belle »: Bandelier (2012) 189-192, qui 191. Cfr. anche Lapradelle, Introduction, in: VatTel (1916) XXVI; Olivart (1905) 1092-1093; ThÉVENAZ (1958) 129-135; Jelmini (2010) 496.

47 Lettera di Vattel a Formey, 17 dicembre 1757, in: Bandelier (2012) 192-194, qui 192.

48 Negli anni in cui scrisse il Droit des gens Vattel, come si è visto, si trovava in precarie condizioni economiche e sperava di migliorare la sua posizione ambendo ad un importante incarico diplomatico, come poi di fatto avvenne, a seguito della pubblicazione del suo trattato. Sulla Bibliothèque Impartiale cfr. Marx (1991) vol. I, n. 164, 189-191; sulla Nouvelle Bibliothèque Germanique cfr. Kämmerer (1991) vol. I, n. 163, 188-189.

49 Bibliothèque Impartiale (1758) 187-189.

50 Lettera di Vattel a Formey, 17 dicembre 1757, in: Bandelien (2012) 192-194, qui 192193. 
conscience. Cela est nécessaire, pour qu'on ne s'avise pas d'attendre la contrefaction annoncée en Hollande ». ${ }^{\mathbf{1}}$

La preoccupazione più grande di Vattel rimaneva sempre l'edizione del Droit des gens, che era stata prodotta in Olanda da più editori « aux dépens de la compagnie » e pubblicamente dichiarata contraffatta nell'aprile del 1758 , in quanto era un "mauvais tour de Luzac.${ }^{52}$ Vattel temeva che la contraffazione avesse modificato la sua opera e il suo pensiero e a Formey chiedeva incessantemente di diffondere al più presto la notizia di questo «mauvais tour $» .53$

Di conseguenza nell'articolo di Formey, pubblicato sulla Bibliothèque Impartiale, sono state illustrate con precisione le differenze tra l'edizione

51 Bandelier (2012) 193.

52 Bandelier (2012) 55. Per quanto riguarda la formazione della compagnia " aux dépens de la Compagnie », Luzac il $1^{\circ}$ aprile scrisse a Rey che era stata composta da lui e da altri editori olandesi: Verbeek, Wetstein, Eyk e Haack: Bots/Schillings (2001) 314, nota 1. Della contraffazione del Droit des gens e dell'impossessamento dei diritti di autore da parte degli olandesi vi è traccia anche nella corrispondenza tra Pierre Alexandre Du Peyrou, l'esecutore testamentario di Rousseau, nonché il curatore delle sue opere complete pubblicate a Ginevra tra il 1780 e il 1790, e Marc Michel Rey, editore di origini ginevrine ma residente ad Amsterdam. Du Peyrou, qualche anno dopo la pubblicazione, il 18 ottobre 1766, faceva infatti sapere che riteneva "singulier que la société de Leyde ayant contrefait son Droit des Gens, ait acquis par là, le droit exclusif de l'imprimer en Hollande »: Lettera da Neuchâtel di Pierre Alexandre Du Peyrou a Marc Michel Rey del 18 ottobre 1766, trascritta nell'imponente opera che contiene la corrispondenza e molti altri documenti di Voltaire (1974) D13616, 30.

53 Il disconoscimento pubblico della versione contraffatta avverà nell'aprile 1758 sul Journal Helvétique, in cui Vattel invitava il suo pubblico ad acquistare la sola edizione che portava la scritta Londres « et pour laquelle il a obtenu l'entrée exclusive en France, on peut se la procurer, non seulement à la Haye et à Neuchâtel, mais aussi à Paris chez Mrs Guerin \& De la Tour, à Francfort, chez Mrs les Frères van-Duren et à Genève chez Mrs les Frères Philibert »: Journal Helvétique (avril 1758) 440; è interessante riportare anche quanto il Journal Encyclopédique, il 15 giugno, scriveva: "Quel droit peut avoir un Libraire de frustrer un Ecrivant des fruits de son travail, en contrefaisant son ouvrage ? Cette coutume qui, depuis longtemps, s'est introduite dans tous les pays où il y a des presses ne sauroit légitimer cet abus, Outre cet inconvénient, il est toujours à craindre que dans la contrefaction d'un ouvrage de la nature de celui de Mr Vattel, où l'on traite plusieurs matières délicates, il ne se glisse des altérations et des interpolations. Ce sont ces deux motifs qui ont déterminé à ne permettre d'entrée en France qu'à l'édition originale faite sous les yeux de cet estimable auteur »: Journal Encyclopédique (15 juin 1758) 43. 
olandese e quella originale, differenze che sono esclusivamente stilistiche e non attengono affatto al contenuto, alle teorie e al pensiero di Vattel. ${ }^{54}$

L'edizione olandese fu pubblicata qualche mese dopo quella originale e le diversità tra le due consistono nella riduzione del numero delle pagine attraverso l'utilizzo di spazi e caratteri di stampa differenti e l'aggiunta del sottotitolo Ouvrage qui conduit à développer les véritables intérêts des puissances, modifica che permetterà di riconoscere l'edizione contraffatta anche nelle rispettive traduzioni americane e inglesi del XIX secolo. ${ }^{55}$

Questa disputa editoriale mette in luce il grande interessamento degli editori per la novità che suscitava il trattato, infatti la straordinaria diffusione si manifesta nelle molteplici versioni contemporanee: anche l'editore Benjamin Gibert de L'Aia distribuiva l'edizione di Londra (in realtà) Neuchâtel, modificando solo il frontespizio e la prima pagina della Préface dell'opera.

Sempre nel $1758 \mathrm{fu}$ data alle stampe un'ulteriore edizione del Droit des gens, questa volta in tre volumi in $12^{\circ}$ e recante la scritta Londres: Ostervald all'interno del suo Abrégé de la vie de Vattel, edito all'interno del Droit des gens del 1773, scrisse che il trattato fu pubblicato « en suite à Paris, en Hollande et ailleurs, sans la participation de l'auteur ${ }^{56}$

I numerosi articoli apparsi sul Journal Helvétique e sul Journal Encyclopédique dissuasero il pubblico dall'acquisto della versione contraffatta di Leida e in qualche modo contribuirono al successo immediato del Droit des gens, che già veniva considerato un capolavoro e paragonato all'opera di Grozio. ${ }^{57}$

Sull'Année Littéraire del 1759, il polemista francese Élie Fréron, uno dei più accesi oppositori dei philosophes, scriveva di avere letto il Droit des gens «con gusto e piacere » e che:

54 Bibliothèque Impartiale (Janvier et Février 1758) 138 ss. Cfr. le numerosissime lettere indirizzate a Formey e successivamente edite da BÉGuelin (1929), in cui Vattel, ad esempio, così affermava: " je vous prie de faire mettre dans le premier volume de votre Bibliothèque Germanique, l'Annonce ou l'Avertissement que je vous transcrirai ci-dessous » BéGUelin (1929) 127, nota 146. Anche sulla contraffazione il contributo di: Bandelier (2012) 45-46. Dalla corrispondenza tra Formey e Luzac risulta che fosse proprio quest'ultimo a pubblicare l'opera: Krauss (1963) 207-216.

55 VATTEL (1758b).

56 Ostervald (1773) 6.

57 Journal Helvétique (avril 1758) 443 ss.; Journal Encyclopédique (1 mars 1758) 41: «Ce Traité réunit l'importance des matières à la solidité de leur exposition; il a été travaillé avec tout le soin possible [...] et trouvera désormais sa place dans toutes les bibliothèques à côté de Grotius, de Pufendorf et de Burlamaqui ». 
On ne peut s'empêcher d'en ressentir, en voyant défendre les droits de l'humanité et poser ces principes respectables qui doivent, dans tout les tems et dans tout les cas, diriger la conduite des maîtres qui nous gouvernent. [...] C'est un des meilleures ouvrages que nous ayons sur tout ce qui concerne la science politique du Droit de gens. $^{58}$

Analogamente l'opera di Vattel incuriosì moltissimo anche i Philosophes: il 21 giugno 1766 il diplomatico francese Pierre-Michel Hennin, dopo aver personalmente incontrato Vattel, scrisse all'amico Voltaire una lettera nella quale lo dipinse come l'autore di un'eccellente trattato sul diritto delle genti, ma ancora più apprezzabile era il candore del suo animo e la saggezza del suo spirito. $^{59}$

Anche il contesto storico bellicoso ebbe una valenza decisiva nella stesura: l'opera, come ha ben illustrato Tetsuya Toyoda, venne scritta durante la disastrosa Guerra dei Sette Anni, che si svolse tra il 1756 e il 1763 e coinvolse le principali potenze europee dell'epoca, fra cui la Gran Bretagna, la Prussia, la Francia, l'Austria e la Russia. ${ }^{60}$ Il 28 febbraio 1757, Vattel inviava all'Avoyer e al Conseil di Berna una lettera aperta di protesta contro l'invasione della Sassonia da parte della Prussia; ${ }^{61}$ successivamente al conte Heinrich von

58 Fréron (1759) 48-63. Su Élie Fréron (1718-1776) Balcou/Barthélémy / Cariou (dir.) 2001; Vier (1969) 475-483.

59 BÉguelin (1929) 64. Su Pierre-Michel Hennin (1728-1807) si rimanda a: Brandli (2006); Brandli (2014) 65-93 e Brandli (2012a); Brandli (2012b) 15-26.

60 ToyodA (2011) 178-179.

61 «C'est un principe reconnu de toute la Terre et sur lequel repose la sûreté et la tranquillité des Nations, que quand un Souverain croit avoir quelque sujet de plainte contre un autre, il doit proposer ses griefs, faire ses demandes, avant que de courrir aux armes, et c'est seulement après qu'on lui a refusé une juste satisfaction, ou lorsqu'il ne peut raisonnablement l'espérer, que nâit pour lui le droit de faire la guerre: Ou si le cas pressant l'oblige de pourvoir sans délai à sa sûreté, au moins doit-il être toujours prêt à accepter les conditions équitables qui lui seront offertes. La Saxe avoit désarmé, bien loin de faire des préparatifs menaçans. Le Roi de Prusse ne se plaignait de rien; il faisoit des protestations d'amitié et de bon voisinage au moment qui a précédé son invasion dans ce païs. Il a requis même le passage »: lettera trascritta in BéGUeLIN (1929) 172-173, ora anche in BAndelier (2012) 181-184. Per quanto riguarda l'organizzazione del governo di Berna nel XVIII secolo è opportuno ricordare che il consiglio esercitava il potere sovrano ed era formato da duecentonovantanove membri; il potere esecutivo era invece affidato ad un senato, mentre tra i magistrati solo due erano nominati avoyers. Tale carica era a vita e uno di essi rivestiva un ruolo molto importante e decisorio all'interno del consiglio: « uno degli avoyer occupa un seggio particolare nella sala de gran consiglio, più elevato degli altri, e coperto da un baldacchino: il sigillo della repubblica è posto su di una tavola che 
Brühl, primo ministro dei principi elettori di Sassonia, scriveva che nel suo trattato c'era un preciso passaggio in cui illustrava l'obbligo di tutte le potenze di riunirsi per fermare chiunque volesse introdurre delle "prassi [internazionali] così funeste ${ }^{62}{ }^{62}$ ed è proprio attraverso l'esplicita posizione d'appoggiare la Sassonia che si riesce a comprendere perché un anno dopo la pubblicazione del suo trattato, nel 1759 , fu nominato consigliere privato di Augusto III e nel 1763 fu trasferito al dipartimento degli affari esteri a Dresda. ${ }^{63}$

La Guerra dei Sette Anni e le sue ripercussioni politiche si intrecciano con la storia del Droit des gens dal punto di vista editoriale: infatti l'editore della versione contraffatta "Aux dépens de la compagnie », sempre nel 1758, diede alle stampe Le droit de la guerre, ou principes de la loi naturelle appliqués à la conduite et aux affaires des nations et des souverains (Amsterdam e Leida). Nello stesso anno, vennero modificati sia il luogo di edizione (Francoforte e Lipsia) sia in parte il titolo in Mémoires politiques concernant la guerre ou principes de la loi naturelle appliqués à la conduite et aux affaires des nations et des souverains. L'autore delle due opere è Vattel: il Droit de la guerre e le Mémoires politiques non sono altro che la ripubblicazione del terzo e quarto libro di cui è composto il Droit des gens, dedicati rispettivamente alla guerra e alla pace.

Nell'Avis di entrambe le edizioni è manifestata la necessità di una trattazione separata sulla guerra:

En un mot la matière y est traitée d'une façon nouvelle : Tout est lié : nulle ambigüité de termes. Les principes y sont établis avec toute la solidité possible :

gli sta davanti. Egli non dice mai il suo parere se non quando glielo chieggono, e non dà il suo voto se non quando i suffragi sono eguali. Il termine avoyer, corrisponde allo schlteiss, advocatus o praetor dei latini »: Ferrario (1833) 94-95.

62 «Il se trouve justement dans mon Droit des Gens, un passage où je fais voir que toutes le Puissances doivent se réunir pour châtier celle qui veut introduire des coutumes si funestes »: BÉGuelin (1929) 57. Il passaggio a cui Vattel allude è contenuto nel libro III al capitolo IX, all'interno del quale Vattel affronta il complesso argomento della guerra con riferimento a "quelles choses » che appartengono al nemico e che sono sul suo territorio: VatTel (1758a) L. III, chap. IX, $\mathbb{\$} 168,139$.

63 Il Conte Brühl il 15 settembre 1759 scriveva a Vattel: «Sa Majesté agrée de vous employer doresnavant à la Chancellerie du Conseil privé pour des expéditions françoises »: Lettera trascritta in: BÉGuelin (1929) 131-132 nota 156. Vattel così gli rispose l'8 ottobre 1759: «Je ne trouve point de termes, Monseigneur, pour vous bien exprimer toute l'étendue de ma reconnoissance. [...] Daignez, Monseigneur, assurer Sa Majesté de toute ma fidélité et du zèle qui me feroit exposer mille fois ma vie avec joie, pour son service »: Lettera trascritta in: BÉGuelin (1929) 132, nota 156; cfr. anche BANDELIER (2012) IX. 
les conséquences tirées avec une justesse d'esprit administrable; et les loix qui en résultent sont éclaircies par des faits, qui ont rapport à l'Histoire de notre temps. De sort que cet Ouvrage pourra servir de clef à l'interprétation des Événements présents. ${ }^{64}$

Non vi è alcuna testimonianza di queste particolari edizioni (ridotte) nella corrispondenza di Vattel, né con Formey né con il conte Brühl, e sembrerebbe una iniziativa nata esclusivamente in ambiente olandese, dove già era stata stampata la copia contraffatta del trattato, l'editore di entrambi i volumi si firma "Aux dépens de la compagnie ». La pubblicazione di una sola parte del Droit des gens è la prova concreta che il pensiero vatteliano potesse essere uno strumento utile per rispondere alla delicata e difficile situazione europea durante la Guerra dei sette anni e al desiderio comune di una pace di cui si faceva portavoce.

«Aux dépens de la compagnie » pubblicò anche altri scritti che hanno fatto sorgere dubbi sull'identificazione della loro paternità a Emer de Vattel: si tratta di alcuni volumi di argomento storico-politico dati alle stampe a Francoforte e a Lipsia tra 1757 e il 1763, e siglati «par L'Observateur Hollandois, Rédigez et Augmentez par M.D.V. ». ${ }^{65}$ Secondo alcune ricostruzioni storiche potrebbe essere uno pseudonimo il primo e un acronimo il secondo, si ritiene, infatti, che L'Observateur Hollandois sia Jacob-Nicolas Moreau (poi-

64 "Cet ouvrage [...] est véritablement neuf et tout différent de ceux que l'on avoit jusquesici sur la même matière. Le Droit de la Guerre y est traité nettement, et non pas noḯ dans une longue déduction du Droit Naturel. [...] L'auteur y a répandu de l'agrément, du feu et de l'intérêt, dans tous les endroits qui en étoient susceptibles. Il a écrit pour les personnes qui joignent la politesse du grand Monde aux lumières de Cabinet; et la clarté de son style le met à la portée de tous. [...] L'Auteur a joint fort souvent aux Maximes, des Exemples capables de les confirmer, de les éclaircir, ou de délasser agréablement et utilement le Lecteur; et pour ne point répéter ceux qui se trouvent dans Grotius et ses commentateurs, il a pris la plus grande partie des siens dans l'Histoire Moderne »: Avis, in: VATTEL (1758c) 2-3.

65 Si riportano: le Mémoires pour servir à l'Histoire de notre temps (1757); Mémoires pour servir à l'Histoire de notre temps, par rapport à la Guerre Angli-Gallicane (1758); Mémoires pour servir à l'Histoire de notre temps, ou l'on déduit historiquement le Droit et le Fait de la Guerre Sanglante qui trouble actuellement toute l'Europe (1758); Mémoires pour servir à l'Histoire de notre temps, contenants les réflexions politiques sur la guerre présente (1758). A queste si aggiunsero ulteriori Memoires edite da "Aux dépens de la compagnie » con l'acronimo M.D.V. oppure Mr.D.V., edite tra il 1759 e il 1763. Per la Mémoire sur les affaires de Courlande, pubblicata poi in tedesco nel 1763, con il titolo Memoire über die Curländische Affairen, cfr.: Good (2011) 15. 
ché era l'editore dell'omonima rivista pubblicata tra il 1755 e il 1759), e "M.D.V. » sia Monsieur de Vattel. ${ }^{66}$ In realtà l'attribuzione al giurista di Neuchâtel è molto discutibile perché l'editore, appunto, "Aux dépens de la compagnie », come si è visto per la contraffazione del Droit des gens, era lo stesso Luzac e anche questa iniziativa non compare né nei carteggi di Vattel né nei cataloghi delle opere pubblicate all'interno delle edizioni successive. ${ }^{67}$

La dinamicità e varietà di divulgazione del pensiero vatteliano costituiscono la sua unicità e peculiarità nella storia del diritto: la diversificazione in modo capillare della circolazione del Droit des gens, nelle molteplici edizioni, segna il decisivo passo in avanti di un giurista, quale Vattel, che, nato in seno alla Scuola Romanda, è riuscito ad elaborare un sistema giuridico vincente, rendendo il suo trattato una chiave di lettura e di interpretazione della realtà contingente.

\section{Meccanismi giuridici nelle traduzioni settecentesche del Droit des gens}

Le molteplici traduzioni del Droit des gens sono la prova della più ampia utilizzazione del trattato. Il corollario fondante di tutto il Settecento è senza dubbio la concezione della circolazione come fruibilità di idee attraverso il loro continuo mutamento, grazie ai meccanismi di scambio, di importazione e di esportazione messi in atto dalle traduzioni.

Sebbene sia stato scritto che «se sert de la langue française, c'est-à-dire du meilleur filtre qui ait jamais su clarifier l'abstraction allemande ${ }^{68}{ }^{68}$ la ragione del successo di Vattel non risiede nell'aver utilizzato la lingua francese, tipica dell'ambiente culturale e intellettuale settecentesco, e conforme a

66 Beaulac (2003) 244-245: Jacob-Nicolas Moreau (1717-1803), celebre avvocato francese, acceso sostenitore dell'Ancien Régime e nemico dei philosophes. A partire dal 1755 lavorò al servizio del Ministero degli Affari Esteri, primo consigliere di Luigi XVI, bibliotecario di Maria Antonietta, storiografo di Francia (1774), fu appunto il redattore del periodico L'Observateur Hollandois: Hervouet (2009); Gembicki (1979). È opportuno ribadire che l'attribuzione delle suddette opere sia a Vattel che allo stesso Moreau anche in passato ha sollevato numerosi dubbi, per Quérard è Moreau l'autore delle Mémoires: Quérard (1834) 289; il dibattito è ampiamente illustrato da Jouannet (1998) 430-431.

67 Basti solo ricordare l'edizione del 1773 edita dalla Société Typographique di Neuchâtel, Ostervald nel catalogo delle opere di Vattel non menziona le Memoires: Ostervald, Abrégé de la vie de Vattel, in: Vattel (1773) V-VI.

68 Lapradelle, Introduction, in: VatTel (1916) XXIII. 
moltissimi trattati dell'epoca, come afferma Chetail, egli: «écrit dans la langue internationale de son temps simplement pour mieux s'addresser à son public, comme d'autres l'ont fait avant lui et le feront après lui ».69

Il Droit des gens vide la luce esattamente quattro anni prima de Le contrat sociale di Rousseau e dieci anni dopo l'Esprit des Lois di Montesquieu. Nel 1746 Mably aveva altresì pubblicato il suo Droit public de l'Europe, a cui seguì nel 1757 Principes des négociations: l'obiettivo era di mettere in risalto quale fosse l'emergenza del diritto pubblico europeo, di fare conoscere, mediante la lingua diplomatica (divenuta tale con Utrecht), la politica dell'Europa dalla pace di Westfalia sino ad allora, non limitandosi ad un elenco di trattati, bensì esponendo i principi e gli interessi che li hanno determinati, ed illustrando la metodologia per interpretare e leggere il sistema giuridicopolitico europeo. ${ }^{70} \mathrm{Nel} 1747$ Burlamaqui, pubblicava i suoi Principes $d u$ droit naturel, che conobbero un forte successo ed ebbero più di una edizione.

A sostegno di questa tesi, cioè che di per sé la stesura in lingua francese non possa essere sufficiente a giustificare il successo dell'opera, vi sono appunto le numerose traduzioni del trattato di Vattel: nel 1759, soltanto un anno dopo la prima edizione francese, fu pubblicata la traduzione in inglese del Droit des gens. ${ }^{71}$

69 Chetail (2011) 395. Circa trent'anni dopo la pubblicazione del Droit des gens nel 1788 Georg Friedrich von Martens pubblicava il suo Précis du Droit des gens moderne de l'Europe fondé sur les traités et l'usage, opera che ebbe un grande successo non solo in ambiente tedesco. Martens scrisse l'opera in francese perché «il était assez naturel de parler des droits des nations dans la langue qui depuis longtemps est devenue presque universelle en Europe, surtout pour les affaires étrangères », ma nel commentare questa scelta aggiungeva anche che «la langue française semble offrir des difficultés particulières pour ce genre d'écrits. Il est aisé de devenir obscur en voulant éviter d'être prolixe. Plusieurs écrivains français, d'ailleurs estimés, semblent en avoir fait l'éprouve »: Martens (1788) Préface, XIV; Chetail (2011) 395-396.

70 Bazzoli (2001) 44-45. Su Mably: Baker (1990); Wright (1997); Deleplace (2002); Mazzanti Pepe (2010); Belissa (2013) 111-128; Belissa (2015) 523-558, in particolare 527 ss.; Friedemann (2014); Bosica (2015). Sul dibattito settecentesco francese sul diritto pubblico europeo: Koskenniemi (2012b) 43-73.

71 Reeves nei primi anni del XX secolo affermerà che: "Il est probable qu'une traduction anglaise de Vattel fut la première œuvre de droit international imprimée hors d'Europe »: Reeves (1924) 37. Nel XVIII secolo furono pubblicate diverse edizioni inglesi, oltre alla prima del 1759, si ricordano quelle edite a Dublino nel 1787 e nel 1792 dall'editore Luke White. Un'altra nel 1793 da Robinson e l'ultima, per quanto riguarda il XVIII secolo, di cui si parlerà ampiamente, nel 1797. 
E' lo stesso Vattel a scrivere, nel settembre 1765, al suo amico Formey: «Mon Droit des gens a fait grande fortune en Angleterre, où on l'a traduit; à Vienne, il est sur les bureaux d'expédition, et on le cite dans les écrits comme livre classique ${ }^{72}{ }^{72}$

La versione inglese intitolata The Law of Nations; or Principles of the law of nature applied to the conduct and affairs of nations and sovereigns. A work tending to display the true interests of powers, è stata pubblicata a Londra in due volumi tra il 1759 e il 1760 dagli editori Coote e J. Newbery. Come si legge dal sottotitolo, essa è la traduzione del Droit des gens pubblicato a Leida che Vattel dichiarò contraffatto. A questa prima versione inglese non è stato apportato nessun commento, né note né premesse o introduzioni aggiuntive.

Dalle ricerche effettuate emerge in modo molto curioso, ma estremamente significativo, la presenza di riferimenti al trattato di Vattel all'interno dei quotidiani e delle riviste inglesi del Settecento. Occorre distinguere dalle recensioni, tra l'altro numerosissime, edite in occasione delle diverse pubblicazioni dell'opera in inglese, dall'inserimento sui giornali, come articoli, di interi e specifici passaggi del Droit des gens, con grande apprezzamento del suo pensiero: ad esempio: il Lloyd's Evening Post, l'8 luglio 1765 scriveva che: « M. Vattel of Geneva, a most amiable Philosopher, strongly declares for Grotius's opinion ». ${ }^{73}$

72 BÉGUELIN (1929) 137, nota 179.

73 Lloyd's Evening Post, issue 1248 (July 8, 1765-July 10, 1765). L'annuncio della pubblicazione in lingua francese si trova in: Public Advertiser, issue 7509 (Wednesday, 22 November 1758). Molteplici sono le segnalazione della prima versione inglese del trattato: London Evening Post, issue 5091 (19 July 1760); London Chronicle, issue 548 (June 28, 1760-July 1, 1760). Il Public Ledger or The Daily Register of Commerce and Intelligence, issue 162 (Friday, 18 July 1760), riporta i titoli dei vari capitoli del Droit des gens. Viene anche annunciata la pubblicazione delle Questions de droit naturel di Vattel: St. James Chronicle or British Evening Post, issue 334 (April 26, 1763-April 28 1763). Per quanto riguarda il richiamo all'autorità di Vattel ad esempio: Morning Chronicle and London Advertiser, issue 4258 (Thursday, 9 January 1783), in cui nel discorrere sulla relazione tra individui e la Nazione, l'obbligo di conservazione di quest'ultima e l'ipotesi che possa essere smembrata si faceva riferimento a Vattel "whose authority will not disputed 》 $\mathrm{e}$ venivano citati parte delle riflessioni contenute al libro I, capitolo XVII, $\mathbb{1} 16$ del Droit des gens: « if the individuals that compose a nation should unanimously agree to break the link that binds them, it would be lawful for them to do so, and thus to destroy the state or nation; but they would doubtless incur a degree of guilt, if they took this step without just and weighty reasons; for civil societies are approved by the Law of Nature, which 
Sembrerebbe che l'influenza di Vattel in Inghilterra, e anche in America, non fosse limitata alla sfera giuridico-politica, ma ricomprendesse una vasta categoria di lettori, poiché era visto come un " autore alla moda » e leggibile da chiunque.

Inoltre, come verrà approfondito nel prossimo capitolo, dal punto di vista più squisitamente concettuale, le elaborazioni di Vattel sulla costituzione intesa come il « regolamento fondamentale » dello Stato, contenute nel primo libro del Droit des gens al capitolo III, richiamano le vicende storicopolitiche inglesi e anticipano quelle nordamericane. La British Constitution, infatti, nella sua accezione al singolare era stata proposta già dal 1689 con la Glorious Revolution; mentre i coloni americani, intendendo Colonial Charters come costituzioni, ad esse si riferirono durante la rivoluzione per la redazione della carta fondamentale americana, configurandone « il concetto dal punto di vista contenutistico mediante le caratteristiche della scrittura, della paternità del popolo, dell'indisponibilità dei governanti e della garanzia dei diritti umani ". ${ }^{74}$

Nel contesto delle terre di lingua tedesca il Droit des gens venne tradotto da Johann Philip Schulin. L'opera fu pubblicata nel 1760 a FrancoforteLipsia con il titolo Völkerrecht, oder gründliche Anweisung wie die Grundsäze des natürlichen Rechts auf das Betragen und auf die Angelegenheiten der Nationen und Souveräne angewendet werden müsen. Ein Werk welches Anleitung giebt, das wahre Interesse souveräner Mächte zu entdecken. Egli si servì per la traduzione dell'edizione francese pubblicata a Leida senza inserire alcuna nota o saggio introduttivo. ${ }^{75}$

Poco si sa sulla vita di Schulin, ma la sua opera di traduttore fu particolarmente attiva: pubblicò tra il 1762 e il 1767 la versione tedesca della Science

recommends them to mankind, as the true means of supplying all their wants, and of effectually advancing towards their own perfection. Moreover civil society is so useful, nay so necessary to all citizens, that it may well be considered as morally impossible for them to consent unanimously to break it without necessity ». Molto frequenti sono i richiami anche sul The Times: Vattel, ad esempio, è la fonte principale per un approfondimento circa la naturalizzazione di un individuo proveniente da un altro Stato, in questo caso si trattava di cittadini inglesi da lungo tempo trasferitisi in territorio nordamericano: cfr. The Times, issue 2796 (Thursday, 3 October 1793) 4.

74 Hofmann (2009a) 9; Hofmann (2005) 228, nota 4. Anche: Grimm (2002) 103 ss.; WinterHOFF (2007) 24 ss.

75 Meusel (1815) 231-232. 
du gouvernement di Gaspard Real de Curban. ${ }^{76}$ Quella di Schulin fu l'unica edizione del trattato vatteliano in tedesco e nel XIX secolo non vide né una nuova pubblicazione né una ristampa. In effetti nel contesto tedesco la diffusione del pensiero vatteliano ebbe un particolare percorso, poiché fu inserito e inglobato nel già vivo panorama di autori di testi di diritto delle genti e di diritto naturale. Il maestro scientifico di Vattel, Christian Wolff nel 1749, all'età di settant'anni, scrisse Ius gentium methodo scientifico pertractatum, in quo jus gentium naturale ab eo quod voluntarii, pactitii et consuetudinarii est accurate distinguitur, in cui, discostandosi profondamente da Grozio sostenne che gli elementi fondamentali del diritto delle genti fossero: una legge universale di natura, un diritto volontario universale, un diritto convenzionale particolare ed infine un diritto consuetudinario particolare, derivati e riconducibili alla natura dell'uomo. ${ }^{77}$ Tali distinzioni furono ribadite da Wolff nella sua ultima opera composta soltanto un anno dopo, nella quale riassunse gran parte delle sue teorie: Institutiones iuris naturae et gentium.

L'interesse costante e attento al diritto naturale e al diritto delle genti costituiva una sorta di piattaforma teorica per l'evoluzione del discorso giuridico e mise le premesse per il contributo degli autori di lingua tedesca tanto per il diritto pubblico quanto per il diritto internazionale tra il Sette e l'Ottocento: basti solo citare Johann Jacob Moser, contemporaneo di Vattel, autore di numerosi trattati di diritto delle genti, ${ }^{78}$ tra $\mathrm{i}$ quali spicca la monumentale opera in dieci volumi intitolata Versuch des neuesten Europäischen Völkerrechts in Friedens- und Kriegszeiten edita tra il 1777 e il 1780 e considerata come « un monolito nel panorama pubblicistico del XVIII secolo ». ${ }^{79}$ Vi furono anche ulteriori autori come Ludwig Julius Friedrich Höpfner che nel 1780 pubblicò Naturrecht der einzelnen Menschen, der Gesellschaften und der Völker; Gottlob August Tittel diede alle stampe il suo Natur und Völkerrecht nel 1786 e un anno dopo apparve il Europäische Völkerrecht in Friedenszeiten nach Vernunft di Karl Gottlob Günther.

76 Per Schulin si rimanda a: WeIDlich (1783) 290-291. La Science du gouvernement, in otto volumi, fu pubblicata per la prima volta a Parigi nel 1754 a cura del nipote dell'autore, l'abate De Burle. Su Real de Curban e Vattel cfr.: Dhondt (2015a) 367-394 e Dhondt (2015b) 500 ss.

77 Stolleis (2008) 369.

78 Stolleis (2008) 322 ss. Si ricordano: Moser (1750) e Moser (1752).

79 Stolleis (2008) 325. 
In una così vasta e ben rappresentata letteratura tedesca, le teorie di Vattel circolarono ed ebbero una influenza molto particolare; significativo è il Précis $d u$ droit des gens di Martens, all'interno del quale il Droit des gens è stato annoverato tra le fonti principali del trattato. ${ }^{\mathbf{8 0}}$ Heinrich Gottfried Scheidemantel nel suo Das allgemeine Staatsrecht überhaupt und nach der Regierungsform indicava il Droit des gens per lo studio del diritto delle genti e per le sue teorie sul diritto interno. ${ }^{\mathbf{8 1}} \mathrm{Nel}$ variegato panorama tedesco meritano alcune precisazioni sul termine « costituzione ». Mentre nelle lingue neolatine esso è entrato nel linguaggio giuridico ed è stato sostenuto che si fosse diffuso e in qualche modo "rafforzato " proprio dal Droit des gens di Vattel, alla stregua della terminologia inglese di constitution; nei paesi di lingua tedesca, come è stato rilevato da Antonio Trampus, constitutio si riferiva per lo più ad atti legislativi, ed era circoscritto alla legge imperiale, la cosiddetta Kaisergesetz: ${ }^{\mathbf{8 2}}$ il vero e proprio passaggio linguistico-costituzionale si ebbe verso la fine del Settecento quando Konstitution fu assimilato alla legge fondamentale dello Stato. ${ }^{83}$

In Spagna, invece, la censura colpì l'opera di Vattel tanto da essere stato definito da Gutierrez Vega una « larva retracta ». ${ }^{84}$ Con l'editto del 20 giugno 1779 il Droit des gens fu ufficialmente proibito e messo all'indice ${ }^{85}$ dalla « negra censura inquisitorial ». ${ }^{86}$ L'opera era ugualmente conosciuta, benché

80 Martens (1788) 20, 92, 97, 115, 132, 143, 178, 258, 271, 347, 390, 402, 407, 445-446, 465, 467, 489, 494, 500, 527, 585. Cfr.: D’Aspremont (2011) 64-65.

81 Scheidemantel (1775) 5, 260-266. È altresì autore di altre due opere: Scheidemantel (1770-1773); SCHEIDEMANTEL (1778).

82 Trampus (2004) 302. Mohnhaupt (1990) 858-870; Mohnhaupt (2003) 969-982.

83 Trampus (2002) 37.

84 Tale termine sta proprio a significare la pressione della censura per uno studio completo e «libero » del Droit des gens: Gutierrez Vega (2010) 119-125.

85 Indice ultimo de los libros prohibidos (1790) 273; sul punto si rimanda anche a Tomás Y VAliente (1964) 417-462.

86 «En el estudio del Derecho Natural, de Gentes y Público que va a emprender iquántas dificulatades no le amenazan desde el primer paso! ¿Dónde, me dice vm., encontraré libros para hacer este estudio? No lo sé. Veo notados con negra censura los nombres de los más ilustres maestros y no me atrevo a proponerlos ni a desecharlos, Grocio, Puffendorf, Burlamaqui, Wolf, Wattel ", autori che, in ogni caso, "convendría depurar de la heces que obligaron a proscribirlos ": Reflexiones sobre la Constitución, la leyes, usos y costumbres de Castilla così riportato in CoRONAS GonzÂles (1998) 93-100, in particolare 95; Coronas GonzÂles (2008) 97 ss. È necessario sottolineare che simile destino fu riservato anche alla cattedra di diritto naturale: Rus Rufino (1993) traccia un profilo completo 
non vi fossero traduzioni del Droit des gens fino al 1820, influenzando notevolmente la cultura giuridica dell'epoca e tracciandone addirittura la strada per il secolo successivo. ${ }^{\mathbf{8 7}}$

L'assimilazione delle teorie vatteliane risulta infatti mediata dal lavoro di « adattamento » da parte dei giuristi spagnoli, per mezzo della pubblicazione di trattati sul diritto delle genti che prendevano come riferimento il Droit des gens, incentivando una vera e propria circolazione «indiretta ». José de Olmeda y León (1740-1805) fu, come affermò de Olivart oltre un secolo dopo, il primo giurista spagnolo a scrivere un'opera sistematica sul diritto delle genti. ${ }^{88} \mathrm{Nel} 1771$ pubblicò Elementos del derecho público de la paz y de la guerra, ilustrados con noticias, históricas, leyes y doctrinas del derecho espanõl. ${ }^{\mathbf{9 9}}$ Nella Introducción indirizzò il suo trattato alla pratica giuridica al fine di poter essere utile allo Stato, riconoscendo quanto la disciplina del diritto delle genti in Spagna non fosse oggetto di studio approfondito. ${ }^{\mathbf{9 0}}$

La caratteristica più importante di questa opera consiste nell'avere ampiamente attinto da Vattel, non solo per quanto riguarda le tematiche affrontate, ma anche nell'organizzazione del trattato. ${ }^{91}$ Secondo Gutierrez, la somiglianza tra i due testi raggiunge addirittura il $95 \%,{ }^{92}$ per Martinez l'Elementos è un vero e proprio plagio del Droit des gens. ${ }^{93}$ Olmeda si limitò a eliminare quei passaggi del trattato di Vattel che potevano essere censurati e

della travagliata storia della cattedra di diritto della natura e delle genti. Cfr. anche Aguilar Piñal (1969); Peset (1974) 178-179; Enciso Recio (1985) 191-239; Alvarez de Morales (1979); Azcárate (2002) in particolare VIII-XVIII; Alvarez de Morales (2003) 141-158. Nel 1742 fu interamente proibita l'opera di Pufendorf e anche la traduzione francese di Barbeyrac e la decisione fu ribadita successivamente nel 1787 « en todas las lenguas, con o sin las notas de M. Barbeyrac »: Defourneaux (1973) 155.

87 Alvarez de Morales (1998) 49-60, in particolare 51-53. In questo periodo in Spagna si segnala anche la pubblicazione di opere sul diritto delle genti, marittimo e raccolte di trattati: Valiente (1744); Bertodano (1740); Bertodano (1746); Pecquet (1747).

88 Olivart (1891) 13.

89 Olmeda y Leon (1771). Molto interessante è anche la successiva edizione ottocentesca del trattato in cui vi sono due saggi uno biografico sull'autore e l'altro critico sulla sua dottrina: Olmeda y Leon (1891). Cfr. anche: Herrero Rubio (1947); Herrero Rubio (1952) 309-450; Herrero Rubio (1953).

90 Olmeda y Leon (1771) 7.

91 Herrero Rubio (1954) 132-133.

92 Gutierrez Vega (2010) 120.

93 Martínez Martínez (2013) 1-118, qui 39, nota 59.

52 Capitolo I 
in particolare le sezioni dedicate alla religione in cui il giurista di Neuchâtel manifestava le sue posizioni anticuriali. ${ }^{94}$

Il trattato di Olmeda si inserisce in una precisa corrente spagnola del tempo che progessivamente agevolò l'ingresso anche delle teorie del diritto delle genti; come sostiene Deforuneaux « la Inquisición no ha abogado la libertad del espíritu; no ha impedido la penetración de ideas nuevas en españa ${ }^{95}$

Negli stessi anni, a seguito della riforma universitaria spagnola, venne istituita la prima cattedra di diritto naturale e delle genti presso gli Estudios de San Isidro di Madrid e venne affidata a Joaquín Marín y Mendoza, ${ }^{\mathbf{9 6}}$ che considerava l'opera di Vattel come « la obra mejor que ha salido del Derecho de Gentes ».97 I docenti universitari spagnoli riuscirono così ad introdurre un insegnamento che fu una sorta di mediazione tra la ricezione delle dottrine sul diritto naturale provenienti dall'Europa e la politica censoria, contribuendo, in questo modo alla realizzazione di un diritto delle genti: « una tonalidad cristiana original que les permitirá ser aceptada sin inconvenientes en un país catolico y con la Inquisicíon como era España, y que la aleja de la Escuela de Derecho natural francés que se desarrolla en este país en el último tercio del siglo XVIII y que es de claro matiz anticristiano $»{ }^{98}$

Curiosa è un'opera dal tono satirico pubblicata a Madrid nel 1772 e che prendeva le mosse dagli studi sul diritto della natura e delle genti, intitolata: Los eruditos a la violeta ó courso completo de todas las ciencias dividido en siete lecciones para los siete dias de la semana con el Suplemento de este compuesto por don Joseph Vazquez, quien lo publica en obsequio de los que pretenden saber mucho, estudiando poco. Alla sezione Jueves, quarta leccion. Derecho natural, y

94 Herrero Rubio (1954) 132-133; anche Ortiz de la Torre (1970) 122-132, in particolare 128.

95 Defourneaux (1973) 210.

96 JohQuín Marín y Mendoza (1727-1782), celebre professore di diritto e avvocato dei Reales Consejos, oltre a pubblicare nel 1776 a Madrid una edizione del manuale di Eineccio: Elementa Juris Naturae et Gentium, castigationibus ex Catholicorum doctrina, nello stesso anno fu l'autore della Historia del derecho natural y de gentes et iuris historia aucta, in cui dopo aver circoscritto il tema, fornito le definizioni e il campo di applicazione del diritto delle genti, offre una panoramica dei più importanti autori che hanno trattato il tema e vi inserisce Vattel. Si rimanda a: Alvarez de Morales (2008) 271-281; Rus Rufino (2000) 257-282; Rus Rufino (1998) 163-198.

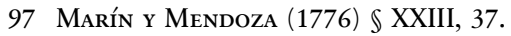

98 Alvarez de Morales (1988) 134. 
de las Gentes, l'autore Joseph Vazquez, lo pseudonimo di José Cadalso, affermava: « No hay cadete, estudiante de primer año ni mancebo de mercader que no hable de $[\ldots]$ Grocio, Wolfio, Puffendorf, Vatel [...] ».99

Un tentativo di tradurre il Droit des gens fu effettuato nel 1774 dal bibliotecario e dottore in legge José Ortiz de la Peña, ${ }^{\mathbf{1 0 0}}$ intitolato Derecho de Gentes o principio de la Ley natural [...], traducido del francés al castellano por el B. D. Josef Ortiz. Tale opera, mai pubblicata e consistente solo nella traduzione del primo libro (interamente) e dei capitoli I e II del secondo libro, è tutt'oggi conservata presso la Biblioteca dell'Università di Salamanca. ${ }^{\mathbf{1 0 1}}$

Per la prima ufficiale edizione in lingua spagnola è necessario attendere il XIX secolo, quando l'avvocato Hernandez nel 1820 pubblicò il Derecho de gentes di Vattel.

Anche il fermento divulgativo in una Spagna censurata dimostra che non fu la lingua francese la ragione del successo dell'interessamento al trattato vatteliano, quanto piuttosto il bisogno di un cambiamento radicale per la trattazione del diritto delle genti e Vattel incarnava la nuova prospettiva concreta e pertinente alla realtà del tempo, anche nel contesto spagnolo.

99 Cadalso (1772) 36 ss.; Muela (1945) 103-131; Andreu (1977).

100 Bibliotecario della Università di Salamanca, autore di due diversi cataloghi in questa biblioteca, il primo del 1776 è per autori, Index librorum qui in amplissima Salmanticensis Academiae Bibliotheca publico studentium usui custodiuntur alphabetico ordine digestus labore et studio e nel 1777 scrisse il secondo indice, questa volta per materia: Bibliotheca Classica Salmantina, seu Index Librorum omnium, qui in publica Salmaticensis Academiae Bibliotheca reponuntur per classes et materia dispositus in usum iuvenentutis studiosae: BeCEDAs GonzáLEZ (2004) 311-312 e Becedas Gonzáles/Lilao Franca (2006) 880-953, in particolare 935-936; Astigarraga (2008) in particolare nota 15.

101 Biblioteca universitaria di Salamanca, Ms. 93: Lilao/Castrillo Gonzáles (1997) 94; Becedas Gonzáles (2005) 181-207; sul punto anche: Robledo (2003) 49-80, in particolare 67-68, 80, 117. Come scrive Defourneaux " un cualificator del Santo Oficio emprendió después la tarea de preparar una edición sexpurgada de la obra, cuyo texto fue sometido en 1785 a la censura del P. Muñoz, para que la examine con reflexión; y si contiene algo de los pasajes o expresiones que dieron motivo a la prohibición, o por lo respectivo al dogma o al sistema público de gobierno de España > »: Defourneaux (1973) 193. 


\section{La Scuola romanda nella penisola italiana del Settecento: il « comune desiderio »}

I testi della scuola romanda del diritto naturale nella penisola italiana del Settecento ebbero un iter peculiare: i piccoli Stati che componevano l'Italia dell'epoca predispongono ad una ricerca incline a riflessioni comparate e capillari sui trattati del diritto delle genti, a partire dalle traduzioni delle opere di Barbeyrac, di Burlamaqui ed infine di Vattel.

Importanti e recenti studi hanno infatti illustrato le vicende delle versioni italiane dei testi di Pufendorf, tra le quali spiccano le traduzioni effettuate sul modello di Barbeyrac. Tra il 1757 e il 1759, esattamente quando Emer de Vattel terminava il suo trattato e lo consegnava alle stampe, veniva pubblicata la traduzione di Pufendorf ad opera di Giovambattista Almici, Il Diritto della Natura e delle Genti o sia Sistema generale De' Principii li più importanti di Morale, Giurisprudenza, e Politica, edita a Venezia dall'editore Pietro Valvasense. ${ }^{102}$

Il lavoro di Almici fu condotto proprio sul testo francese annotato da Barbeyrac, con l'obiettivo di rivedere l'impostazione del professore di Losanna. Il motivo di questa traduzione italiana rettificata e illustrata si basava sulla necessità che venisse diffusa anche in Italia un'opera che presentasse il diritto naturale nella sua più profonda e attuale scientificità, senza che venissero però messi in discussioni i dettami della religione cattolica. ${ }^{103}$ Almici, come ben argomentato da Bazzoli, tuttavia si è confrontato puntualmente con i commenti e le note di Barbeyrac: nonostante nella Prefazione vi sia un

102 Panizza (1969) 483-528; Bazzoli (1979) 3-100; Bazzoli (2005) 41-60; Quaglioni (1999) 23-250; Stoffella (2000) 137-175; Stoffella (2001) 173-199; Stoffella (2013) 45.

103 Bazzoli (1979) 18. Nella Prefazione si trova scritto: "Era ben di dovere, che un'Opera di tanto merito, e di tanta importanza, qual è il sistema del diritto della natura e delle genti del Barone di Puffendorf, a giudizio di tutti i dotti, vedesse anche la Repubblica Letteraria d'Italia, che in nulla cede all'altre d'Europa, in Italiano ridotta. [...] Tanto appunto mi sono ingegnato d'eseguire in questa mia, anzi che traduzione, rettificazione, e illustrazione che vogliasi dire. Mentre infatti ho procurato di combatterlo, e di confonderlo in ogni luogo, dove hollo ritrovato dissentiente dalla Cattoliche Romane massime, istabilendovi all'incontro le solide, e sane sentenze della Chiesa nostra sostenute e difese: come potrà con facilità rilevare chi legge, in proposito della bugia, dell'usura, del matrimonio, della poligamia, dei voti, dei patti, dell'omicidio in sè, dei duelli, dei tradimenti, e delle convenzioni di guerra; e in varj altri capi, ne' quali tutti ho impugnato l'Autore, e corretto, e a buoni, e retti sentimenti ridotto »: Almici, Prefazione, in: Pufendorf (1757) I. 
riferimento limitato al lavoro compiuto da Barbeyrac, Almici « lo segue più di quanto non appaia, o per prudenza, non ammetta ». ${ }^{104}$

Le note di Almici sono numerosissime all'interno del testo, e contengono soprattutto nel volume IV edito proprio nel 1759 moltissimi riferimenti al Droit des gens di Vattel, a sua volta dato alle stampe l'anno precedente. Nell'annotatore c'è un grandissimo apprezzamento per il pensiero del giurista di Neuchâtel, dallo stesso chiamato "Vettel », e in più di una occasione non esita a sostenerlo energicamente. Si prenda come esempio la celebre definizione di «caractère représentatif » degli ambasciatori: Vattel nel libro IV al capitolo VI intitolato Des divers ordres de ministres publics, du caractère représentatif, et des honneurs qui sont dus aux ministres affermava che «ce qu'on appelle le caractère représentatif par excellence, est la faculté qu'a le ministre de représenter son maître, quant à sa personne même et à sa dignité », ${ }^{105}$ continuando che «le caractère représentatif, ainsi dit par excellence, ou en opposition avec les autres sortes de représentations, constitue le ministre du premier ordre, l'ambassadeur ${ }^{\mathbf{1 0 6}}$

Vattel distingueva così tra gli ambasciatori ordinari e straordinari, come una dicotomia dettata per ragioni inerenti alla missione da essi ricevuta; ${ }^{\mathbf{1 0 7}}$ ad un livello inferiore collocava gli Envoyés, sprovvisti del carattere rappresentativo propriamente detto e come tali ministri di second'ordine; ${ }^{\mathbf{1 0 8}}$ ad un terzo livello i Résidents, che rappresentavano la persona del sovrano non nella sua dignità ma solo nei suoi affari. ${ }^{109}$ Secondo Vattel, a causa della complessità del cerimoniale, si era creata una altra figura, che non aveva nessun tipo di determinazione particolare riguardo al carattere, era il ministre, incaricato di rappresentare il sovrano in modo indeterminato. ${ }^{\mathbf{1 1 0}} \mathrm{Il}$ ministro plenipotenziario, infine, pur non fruendo di «aucune attribution particuliére de rang et de caractère", aveva acquisito nella pratica un ruolo immediatamente successivo a quello dell'ambasciatore. ${ }^{\mathbf{1 1 1}}$

Questa ripartizione e distinzione di ruoli è stata accolta in toto da Almici, che nel commentare il capitolo X di Pufendorf dedicato agli ambasciatori,

104 Bazzoli (1979) 22.

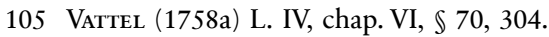

106 Vattel (1758a) L. IV, chap. VI, $\mathbb{\$} 71,305$.

107 Ibidem.

108 Vattel (1758a) L. IV, chap. VI, $\mathbb{~ 7 2 , ~} 306$.

109 Vattel (1758a) L. IV, chap. VI, $\mathbb{\$} 73,306$.

110 VATtel (1758a) L. IV, chap. VI, $\mathbb{\$} 74,307$.

111 Ibidem. 
parafrasa nelle sue note, in moltissimi passaggi, il pensiero vatteliano: « $\mathrm{Al}$ giorno d'oggi si danno vari nomi, e titoli a questi pubblici Ministri, cioè Inviati, Residenti, Ambasciatori. Li primi due non hanno il carattere di rappresentazione personale propriamente del Principe loro; ma lo presentano solo per quei tai affari, che trattare inviati sono; al contrario che gli Ambasciatori hanno il carattere rappresentativo del proprio Principe per eccellenza, personale, onde di maggior dignità sono ». ${ }^{\mathbf{1 1 2}}$

Quello che si realizza con la traduzione di Almici è un metodo scientifico elaborato, ma anche un raffinato strumento di rapida diffusione del pensiero: Almici si serve della versione di Pufendorf tradotta in francese da Barbeyrac, e al tempo stesso annota lui stesso il testo utilizzando il Droit des gens di Emer de Vattel, appena pubblicato.

Un ulteriore testo di Pufendorf, anch'esso tradotto in francese da Barbeyrac (Amsterdam, 1708), ebbe una singolare vicenda nella penisola italiana: si tratta De officio hominis et civis, che vide due versioni, una veneziana ad opera di Michele Grandi e un'altra ad opera di Domenico Amati edita da Raymondi a Napoli nel 1780 e poi nel 1785 .

La traduzione di Grandi si compone di tre volumi in quarto: i primi due vennero pubblicati nel 1761 e il terzo nel 1767 da Francesco Pitteri. ${ }^{113}$ Ad indurre Grandi a cimentarsi nell'impresa fu proprio il « vedere pubblicata in italiano la suddetta Opera Grande di Pufendorf dal sig. Giovanni Battista Almici ». Egli respinse pubblicamente il metodo adottato da Almici di intervenire direttamente sul testo, interpolandolo e manomettendolo, riconoscendo invece il diritto, cioè il «comune desiderio ", dei lettori di «avere sott'occhio le opinioni di questi celebri Scrittori esposte in quella stessa maniera con cui essi le pubblicarono $»{ }^{\mathbf{1 1 4}}$

Nella sua strategia traduttiva, completamente differente da quella di Almici, appare molto evidente la propensione intellettuale di Grandi che pur ancorato alle preoccupazioni più moralistiche e di ortodossia religiosa contribuisce alla circolazione delle idee illuministiche. Come sostenuto da

112 Così nella nota di Almici in: Pufendorf (1759) vol. IV, L. VIII, cap. X, $\mathbb{\$} 1$, 469, nota 1 , cfr. anche 472, 474, 479 ss., nelle quali Almici sviluppa i concetti del carattere della rappresentatività degli ambasciatori prendendo come punto di riferimento Vattel. Per una disamina sugli ambasciatori all'interno delle teorie sul diritto delle genti: Vec (2015) 487-522.

113 Per riferimenti biografici su Grandi: Morelli di Schönfeld (1855) 124-129.

114 Grandi, Avvertimento, in: Pufendorf (1761) t. I, XI-XII. 
Bazzoli, Grandi, servendosi della notorietà di Pufendorf, ha voluto intenzionalmente diffondere e far conoscere ai lettori italiani l'interpretazione di Barbeyrac. Egli difendeva con fermezza il professore di Losanna dalle critiche formulate da Almici e, quasi a voler divulgare ulteriori suoi scritti, pose in appendice altri due saggi di Barbeyrac: il Discours sur la permission des Loix del 1715, e il Discours sur le Bénéfice des Loix del 1716. ${ }^{115}$

A Napoli, qualche anno più tardi, invece venne pubblicata un'altra versione del De Officio ad opera del giureconsulto napoletano Domenico Amato.

Il trattato di Pufendorf, sia in latino sia nella traduzione francese di Barbeyrac, rappresenta una sorta di lettura obbligata per le generazioni napoletane durante l'età delle riforme, come lo era la versione italiana del De Iure Belli ac Pacis di Grozio edita a Napoli nel 1777 a cura di Antonio Porpora, con l'editore De Dominicis. ${ }^{116}$

Nel Granducato di Toscana, prendeva avvio, invece, la traduzione delle teorie di Burlamaqui. Come ben illustrato da Sandro Landi, la versione italiana dei Principes du droit de la nature et des gens, corredata dalla note di Bartolomeo Fortunato de Felice (1767-1768), fu iniziata in Toscana nel 1772 da parte dell'editore Bindi, ma fu subito interrotta dalla censura. ${ }^{117}$ Il responsabile della stampa di Siena, Stefano Bertolini, era convinto che il testo di Burlamaqui e le note di De Felice potessero essere pericolose perché elaboravano una originale teoria sul fondamento del potere sovrano, sui suoi limiti e sulle prerogative dei principi nei confronti della religione. Bertolini, infatti, giustificava la scelta di interrompere la stampa affermando che vi erano dei «punti troppo interessanti al diritto regio ». ${ }^{\mathbf{1 1 8}}$ Due erano in particolare i punti «scottanti »: il primo erano le teorie contrattualistiche sviluppate da Burlamaqui e approfondite da De Felice, e il secondo punto era la posizione favorevole nei confronti di una monarchia « limitata ", sulla base del modello inglese. ${ }^{119}$ In ogni caso l'interruzione fu solo momentanea perché Bindi, tra il 1780 e il 1782, pubblicò l'opera; il repentino cambio di vedute fu molto probabilmente dettato dalla maggiore apertura alla libertà di stampa e ai limiti più ridotti adottati dal governo. ${ }^{\mathbf{1 2 0}}$

115 BAzzoli (1979) 46.

116 Conti (a cura di) (2002).

117 Landi (2000) 254; anche Trampus (2013) 172.

118 LANDi (2000) 255.

119 Ibidem.

120 Burlamaqui (1780-1782). 
In un altro contesto della penisola italiana, nel 1780, l'editore Giovanni Gatti, pubblicava a Venezia la traduzione ai Principes $d u$ droit naturel di Burlamaqui, introducendo l'opera con queste parole: «E' abbastanza noto, con quanto ardore fiensi rivolte le più colte Nazioni d'Europa allo studio della giurisprudenza Naturale, da che Grozio lo ridusse a sistema [...] Tra queste si è pure distinta non poco l'Italia, non solo colle ristampe, traduzioni, confutazioni [del] Grozio, dell'Obbes, del Pufendorf e del Wolff, ma eziandio colle opere originali dei suoi più dotti, e più profondi scrittori ». ${ }^{\mathbf{2 1}}$

Il traduttore era il conte Benedetto Crispi che si firmò con l'acronimo C.B.C., e dopo aver elencato tutte le diverse versioni europee del trattato di Burlamaqui, in Inghilterra, Olanda e Danimarca, precisò di avere scelto per la sua traduzione la prima edizione dei Principii di diritto naturale (appunto del 1747) al posto di quella successiva annotata da De Felice, poiché quest'ultima oscurava il pensiero del professore di Ginevra. ${ }^{\mathbf{1 2 2}}$

Crispi tradusse anche i Principes du droit politique (1751) di Burlamaqui, che furono pubblicati nello stesso anno sempre dall'editore Gatti. Il Conte Benedetto Crispi era un importante aristrocratico, si distinse per essere un ottimo traduttore dal tedesco, dall'inglese, ma soprattutto dal francese: oltre ai lavori di Burlamaqui si annovera nel 1784 la versione italiana (edita con Gatti) dell'opera dell'abate Mably, Il diritto pubblico dell'Europa. Il Conte era amico e protettore del gruppo di gesuiti che, espulsi dalla Spagna nel 1767, si erano rifugiati in quella zona della penisola italiana e non esitava a coinvolgerli nelle iniziative culturali ed editoriali da lui promosse. ${ }^{123}$

121 Crispi, Il traduttore a chi legge, in: Burlamaqui (1780) III-IV.

122 La traduzione del 1780 ebbe una particolare fortuna in Italia soprattutto nel contesto accademico: ancora nel 1812, Pietro Antonio Magalotti, professore di diritto naturale a Perugia, utilizzava moltissimo il pensiero di Burlamaqui perché era « un modello di sintesi che non separava il giusnaturalismo groziano dall'utilitarismo pufendorfiano e consentiva al Magalotti di percorrere quella via di mezzo che i tempi suggerivano ", senza contare che nella prima parte del suo corso seguiva l'ordine e il titolo dei capitoli e paragrafi di Burlamaqui, trascrivendo letteralmente le sue parole quasi come se lo avesse adottato come un libro di testo, per poi ampliare le fonti e arrivare a discostarsi nella seconda parte, entrando in polemica con Burlamaqui sul delicato problema se la ragione fosse un fondamento sufficiente dell'obbligazione morale, indipendentemente dalla volontà di Dio: Comparato (2014) 221-242.

123 Guasti (2006a) 282 e nota 75. Guasti precisa che su Benedetto Crispi è disponibile un'ampia documentazione archivistica ed epistolare. Il Conte era una figura molto interessante per la condivisione di orientamenti e interessi culturali con quella parte dell'emigrazione 
Le traduzioni dei trattati di Burlamaqui sono completate dalle note di un ex gesuita, il catalano Luciano Galissà y Costa, specialista in lingue orientali e prefetto della libreria di Ferrara dal 1782. E' stato giustamente rilevato come lo scopo di Gallissà e di Crispi non si riduceva a prendere possesso di questi testi di diritto naturale (di provenienza protestante), attraverso un approccio che non offendesse i dettami della Chiesa Cattolica, ma di contribuire alla circolazione e alla diffusione delle teorie di Burlamaqui. ${ }^{\mathbf{1 2 4}}$

L'editore Giovanni Gatti, nell'Avvertimento dei Principes de droit naturel di Burlamaqui, annunciava la pubblicazione della versione italiana al testo di Vattel entro il maggio del 1781. La traduzione effettivamente avvenne ad opera di Lodovico Antonio Loschi, ${ }^{125}$ con il titolo Il diritto delle genti, ovvero principii del diritto naturale applicati alla condotta e agli affari delle nazioni e de' sovrani, in tre volumi in ottavo, ripubblicata poi nel $1804 .{ }^{\mathbf{1 2 6}}$ Essa ebbe come punto di riferimento l'edizione pubblicata a Neuchâtel nel 1773, curata da Ostervald. ${ }^{127}$

Le particolarità dell'edizione italiana, come sottolinea Antonio Trampus, sono sinteticamente due: il rispetto dell'intento dell'autore e l'arricchimento

gesuitica in Italia, che era portatrice di idee riformatrici della ilustración spagnola: Cfr: Guasti (2006b) 15-52; anche Trampus (2011a) 95. Per la traduzione al testo di Mably: Roggerone (1991) 179.

124 Berti (2010) 330.

125 Lodovico Antonio Loschi, letterato e traduttore modenese, vissuto tra il 1744 e il 1811: « istruito assai bene nelle lingue inglese e francese, divenne un instancabile traduttore, appigionando per cosí dire la vendereccia sua penna », fu altresì docente universitario di etica e morale ed ebbe un ruolo, anche se non primario, nella vita politica nel Triennio Repubblicano e nel napoleonico Regno d'Italia: Cagnoli (1837) 335 ss.; Armani (1979) 352-354; Venturi (1990) 163 e 224. Si rimanda interamente al già citato contributo di: Trampus (2011a) 81-108.

126 Vattel (1781-1783). Trampus illustra le tappe della pubblicazione dell'opera segnalando, in particolare, che dalle licenze ottenute per la stampa dell'opera, sembrerebbe essere stata realizzata in un tempo breve, cioè tra il 1780 e il 1781 . Inoltre il manoscritto del primo volume ottenne l'autorizzazione alla stampa il 22 marzo 1781, con falso luogo di Lione, concessa al tipografo Giovanni Gatti, in base al parere del revisore Gasparo Gozzi; mentre il secondo volume, poi successivamente suddiviso in due tomi, ottenne l'autorizzazione il 22 settembre 1781, sulla base del parere favorevole del revisore Cosimo Mei: Cfr. Trampus (2011a) 92. Nel 1804 e 1805 questa edizione vide una ulteriore ripubblicazione in tre tomi, in $8^{\circ}$, senza che vi fossero apportate aggiunte o modifiche rispetto alla precedente e con altresì in appendice la voce Droit Naturel redatta da Boucher d'Argis.

127 Questa edizione sarà analizzata nel prossimo paragrafo. 
di note da parte del traduttore. ${ }^{128}$ Infatti Loschi non si limitò ad una semplice divulgazione italiana del testo, anzi intervenne apponendo note esplicative soprattutto nel primo volume. ${ }^{129}$ È Appiano Buonafede a scrivere che Loschi «pubblicò una traduzione accompagnata di giusti avvertimenti ». ${ }^{\mathbf{3 0}}$

Occorre precisare che le note apportate da Loschi risultano comunque riconoscibili e tali da non alterare il testo originale. Nell'Avvertimento è il traduttore a specificare: " una traduzione esser non può bella ed elegante che a misura del suo originale. Basta bene che l'opera sia riconosciuta eccellente pel metodo e per la sostanza delle cose ». ${ }^{\mathbf{1 3 1}}$

In appendice all'edizione italiana venne inclusa la voce Diritto naturale scritta da Antoine-Gaspard Boucher d'Argis nel 1755 per l'Encyclopédie di Denis Diderot. La traduzione era necessaria per Loschi perché fosse palese « in quale stato [si] ritrovasse questa nobilissima ed utilissima scienza, quando prese a trattarla il nostro autore ${ }^{\mathbf{1 3 2}}$

Nella sua voce Boucher d'Argis aveva argomentato che il diritto naturale fosse « una giurisprudenza » stabilita dalla ragione su certe materie fra tutti gli uomini e allo stesso modo dovesse essere osservata da tutte le nazioni. Le riflessioni dell'illuminista francese si «limitavano " ad un breve excursus storico, in cui partendo dall'epoca romana si soffermava sulle opere dei più famosi giusnaturalisti, quali il De iure belli ac pacis di Grozio e illustrava in forma riassuntiva il contenuto dei diversi libri che compongono il trattato.

128 Trampus (2011a) 89.

129 Le note di Loschi, evidenzia Trampus, riguardano essenzialmente commenti o critiche alle posizioni più palesemente anticuriali di Vattel, quando questi, ad esempio, rivendicava la libertà di religione e la libertà di coscienza come diritti inviolabili dell'uomo. Inoltre il traduttore fece diverse note anche sulle libertà personali. Ad esempio nel momento in cui Vattel esponeva i doveri in capo al popolo e alla Nazione nell'obbligo di concorrere alla costruzione e alla manutenzione delle opere pubbliche, Loschi, nelle sue note, sottolineava invece che "debbono i Sovrani astenersi dal comandar lavori pubblici per servire soltanto alla loro vanità, e le cui immense spese sieno di gran lunga sproporzionate al profitto che ne può mai trarre uno stato »: cfr. Trampus (2011a) 100-101.

130 Buonafede (1792) cap. XLV, 213, nota 1.

131 Loschi, Avvertimento del volgarizzatore, in: VaTtel (1781) VI.

132 Ibidem. Boucher d'Argis (1708-1791) illustre giurista e avvocato francese, dedicò tutta la sua vita allo studio del diritto francese, diritto romano e diritto canonico. Pubblicò anche diverse opere specifiche su alcuni isituti del diritto privato, soprattutto sul diritto di famiglia, tra cui si ricordano: Traité des gains nuptiaux e de survie, qui sont en usage dans les païs de droit écrit (Lyon, 1738) e il Traité de la criée des meubles au dessus de leur prisée (Paris, 1741). Per riferimenti biografici: Gaven (2007) 113-115. 
Un paragrafo sintetico era altresì dedicato a Pufendorf e a Burlamaqui e all'ordine in cui sono impostate le loro opere, senza però entrare in riflessioni nè filosofiche e nè tantomeno giuridiche.

In effetti il contenuto e la struttura della voce enciclopedica ricevettero moltissime critiche e il dibattito sui motivi che spinsero Diderot a redigere personalmente un'altra voce Droit naturel (Morale) e di pubblicarla nell'Encyclopédie proprio dopo quella di Boucher d'Argis, è ancora aperto. ${ }^{133}$

Questa disamina del lavoro di Boucher d'Argis, seppur concisa, è necessaria per comprendere quale fosse il clima in cui si è inserita la traduzione italiana: Loschi si sentiva quasi obbligato a prendere le mosse da una voce enciclopedica, si potrebbe dire «incompleta », sul diritto naturale, fornendo in tutta risposta l'ampio e ben strutturato trattato di Vattel.

Per quanto riguarda le sorti della versione di Loschi, essa ebbe un infelice destino a Napoli durante la rivoluzione francese in quanto fu messa all'indice. All'epoca l'attività di revisione era svolta da Francesco Conforti, professore di storia dei concili all'Università di Napoli e teologo presso la corte napoletana, incaricato del controllo sull'ammissione dei libri esteri. ${ }^{\mathbf{1 3 4}}$

Conforti censurava Rousseau, definendo il suo Émile "pernicioso alla religione » e giudicava non solo Vattel e il suo Droit des gens un'opera « sediziosa, perchè riconosce nel Popolo il diritto di riformare la costituzione e di cambiare il governo », ma soprattutto inveiva contro Loschi poichè nella sua traduzione «si sono conservate le stesse massime, che si dettano nell'originale ». ${ }^{135}$

Quella di Loschi fu l'unica edizione italiana stampata nel XVIII secolo, ma il trattato, che già circolava sin dalla prima versione francese, destava certamente un grandissimo interesse nei più svariati contesti della penisola

133 LUPORINI (1987) 679-697.

134 « Di grave responsabilità in quegli anni in cui si voleva ad ogni costo impedire che penetrassero nel Regno i principi della rivoluzione francese e addirittura le notizie degli avvenimenti di Francia. Il Conforti doveva rivedere ed esaminare con ogni diligenza [...] tutte le casse, i colli, e gli involti che per la Dogana e per lo Procaccio s'immetevano in Napoli. Doveva visitare le librerie e doveva curare che non si introducessero libri malvagi, e sediziosi, sotto un titolo e un frontespizio innocente, e di autore cognito e provato. Doveva inoltre informare il governo dei libri ritrovati che egli ritenesse dannosi alla sovranità e alla religione e che dovevano essere sequestrati e banditi »: VILLANI (1962) 240; sul punto si rimanda anche a RAO (1990) 469-520, in particolare 478.

135 Villani (1962) 249.

$62 \mid$ Capitolo I 
italiana: dalla dottrina del diritto, alla diplomazia, alla giurisprudenza, all'accademia. Autori come Cesare Beccaria, Pietro Verri e Gaetano Filangeri furono influenzati notevolmente dall'opera di Vattel: frequentissimi, ad esempio, sono i richiami diretti e indiretti al Droit des gens in Dei delitti e delle pene che "assieme a quella di Burlamaqui sembra costituire una delle fonti principali per l'elaborazione del saggio ». ${ }^{\mathbf{1 3 6}}$

Significativo è un passaggio di Alessandro Verri in cui nel Caffe, discorrendo Di alcuni sistemi di diritto pubblico, scriveva di Vattel come l'unico che «abbia colta la verità [del diritto pubblico] e sia quello che abbia spogliata questa scienza dalle chimere e dagli equivoci riducendola a sistema di idee, non di parole ${ }^{137}$

L'illustre giurista Alberto de Simoni, altresì, fu affascinato dalle teorie della scuola romanda, cercò di tradurre Burlamaqui e considerava Vattel «l'umanissimo». Frequenti sono i richiami alle teorie vatteliane nel suo Del furto e sua pena del 1776 , che in più di una occasione non esitava a definire "giustissime ${ }^{\mathbf{1 3 8}}$ e della stessa idea era anche Paolo Risi. ${ }^{139}$

In un contesto geografico, politico e sociale completamente differente, il Droit des gens è stato ampiamente consultato per la difesa del vescovo di Mileto, Giuseppe Maria Carafa, intitolata Difesa del vescovo di Mileto e del Collegio dei Greci in Roma contro un'istanza fiscale ed una scrittura stampata col

136 Trampus (2011a) 83. Gianni Francioni scrive che Burlamaqui e Vattel « hanno un posto fisso sul tavolo di composizione dei Delitti »: Francioni (1984) 334; inoltre nelle annotazioni al celebre testo di Beccaria, Francioni esplicita in moltissime occasioni la provenienza vattelliana delle idee del giurista milanese: Beccaria (1984) 20, 27, 29, 31-32, 34, 39-40, 49, 52, 73, 79, 83, 85, 87-88, 97, 105, 112, 119. Anche: PASTa (1990b) 515.

137 Il Discorso IX, Di alcuni sistemi del diritto pubblico, si trova insieme agli altri scritti di Alessandro Verri pubblicati sul Caffe e raccolti in: VerRI (1818) 370.

138 De Simoni (1776) 13. De Simoni si riferisce al capitolo XIII $₫ 171$ del libro I del Droit des gens, in cui Vattel discorre della «mesure des peines » ed afferma: "Quand on réfléchit sur la pratique criminelle des anciens Romains, quand on se rappelle leur attention scrupuleuse à épargner le sang des citoyens, on ne peut manquer d'être frappé de la facilité avec laquelle il se verse aujourd'hui dans la plupart des Etats. La république Romaine étoit-elle donc mal policée ? Voyons-nous plus d'ordre, plus de sûreté parmi nous? C'est moins l'atrocité des peines, que l'exactitude à les exiger, qui retient tout le monde dans le devoir. Et si l'on punit de mort le simple vol, que réservera-t-on pour mettre la vie des citoyens en sûreté ». Anche PASta (1997b) 119-148, qui 132; Birocchi (2013) 718-720.

139 Osserva Solimano che Risi si servì delle teorie di Vattel per dimostrare che i romani facevano ricorso raramente alla pena di morte: Solimano (2001) 419-519, qui 440, nota 59. Su Risi: Solimano (2013) 1694-1696. 
titolo di Dimostrazione del padronato della Real Corona, sulla chiesa e badia della Trinità di Mileto (Napoli 1769), in cui rivendicava la proprietà e diritti sulla badia della Trinità di Mileto vantati invece dalla corte napoletana. La questione era annosa e molto complicata, ma la particolarità della sua tesi centrale, poi non accolta in giudizio, era la corretta interpretazione del Concordato del 2 giugno 1741 tra il Re di Napoli Carlo Borbone e il Papa Benedetto XIV, che doveva essere letto alla stregua delle regole vatteliane, le quali venivano richiamate in toto dal vescovo per l'attribuzione della proprietà della badia alla Chiesa. ${ }^{\mathbf{1 4 0}}$

In ambiente accademico tracce consistenti della scuola romanda e in particolare del testo di Vattel si possono riscontrare in alcuni atenei, dopo l'istituzione, attraverso riforme attuate in tempi differenti, della cattedra di diritto naturale: ad esempio è stato dimostrato quanto le opere di Burlamaqui e di Vattel fossero considerate validissime per lo studio del diritto naturale nell'Università di Pavia, basti solo ricordare le citazioni nelle Institutiones iuris naturalis et Institutiones iuris publici universalis di Jean Baptiste Noël de Saint Clair, professore di diritto naturale e pubblico universale, ${ }^{141} \mathrm{o}$ i richiami ai modelli giusnaturalistici elaborati da Barbeyrac e Vattel all'interno delle lezioni e degli scritti dell'abate Pietro Tamburini. ${ }^{\mathbf{1 4 2}}$

Al tempo stesso Vattel affascinava perché godeva di riflesso dell'apprezzamento che il pensiero di Wolff aveva nel contesto accademico: a Padova nel 1773 l'abate Matteo Franzoja insegnava diritto naturale, adottando come libro di testo le Institutiones juris naturae et gentium; a Pisa, Giovanni Maria Lampredi, nel redigere i Theoremata, a sua volta, come si vedrà nel terzo capitolo, fu fortemente influenzato da Vattel. ${ }^{143}$

All'interno di questo variopinto panorama si coglie che nella penisola italiana settecentesca non era certo mancato il "comune desiderio » di (ri)leggere il Droit des gens nei diversi e più svariati contesti.

140 Carafa (1769) XXXV-XL.

141 Di Renzo Villata (2008) 583-612. L'interesse verso Vattel nel contesto giuridico milanese è riscontrato anche nelle citazioni all'interno delle Allegationes: Di Renzo Villata (2004) 64 ss.

142 Stella (1993) 193. Su Tamburini vedasi anche: Mantese (1942). Anche: Mazzetti (1948); Comini (1992); Verzella (1998); Vismara (2012) 95-114; Van Kley (2014) 109-162, e Carrera (2015).

143 Di Simone (2005) 221-268, qui 254; Di Simone (2006) 135-158, qui 139. Anche Zordan (1999) 5-76. 


\section{Il Droit des gens edito da Charles-Guillaume Frédéric Dumas (1775)}

Nel 1773 la Société typographique di Neuchâtel pubblicò una nouvelle édition augmentée del Droit des gens in due volumi. Tra i fondatori e i membri della Société c'era anche un caro amico di Vattel, Frédéric Samuel Ostervald, il quale riuscì ad intessere legami con società analoghe come la Typographische Gesellschaft Bern e la Société typographique de Lausanne, ma soprattutto costruì una rete continentale di corrispondenti in grado di offrire tutti gli apporti necessari per una oculata pianificazione della produzione editoriale, la cosidetta « ricerca di mercato».

Ostervald chiedeva direttamente ai librai un parere determinante per la pubblicazione o meno di una opera. Per questo, nel 1770, scrisse una lettera a Pierre Gosse, titolare con Daniel Pinet di una importante libreria distributrice in tutta Europa e con sede all'Aia, per chiedergli una opinione sulla ristampa del Droit des gens di Vattel. Gosse, pur sapendo che il trattato fosse eccellente, rifiutò la collaborazione richiamando alla memoria la contraffazione di Ledia edita, come si è visto, da "Aux depens de la compagnie »: gli editori olandesi vantavano un diritto di copia sul trattato « comme les libraires de Leyde ont acquis par l'impression de la première édition ce que nous appelons ici le droit de copie et non le privilège, que nous respectons saintement entre nous dans les libraires d'Hollande ce droit de copie, nous n'en pouvons point faire l'enterprise en ce pays ». ${ }^{\mathbf{1 4 4}}$

Gosse in questo contesto affrontò lucidamente il problema della politica editoriale, terreno di scontro tra intellettuali e uomini di affari, che si dividevano tra la ricerca della qualità culturale e il primato delle vendite. Egli infatti scriveva: «il est très bien nécessaire, Messieurs, aujourd'hui de suivre le goût du siècle, le goût de public, dans l'entreprises. MM. les savants s'y trompent très souvent. Un libraire, qui a du débit, juge plus sûrement du goût du public qu'un savant puisse le faire ». ${ }^{\mathbf{1 4 5}}$

Nonostante queste considerazioni Ostervald, valutò opportuno, anche per ragioni di mercato, di stampare a Neuchâtel nel 1773 una nuova edizione del Droit des gens, con diverse aggiunte tra le quali l'inserimento di una biografia dell'autore curata da lui, intitolata Abrégé de la vie de M. de Vattel, ${ }^{\mathbf{1 4 6}}$ alcune

144 Lettera così riportata in: DARNTON (2008) 277-292, in particolare 281.

145 Darnton (2008) 283.

146 Tale Abrégé si trova nel II volume del Droit de gens del 1773. Ostervald terminava il suo saggio biografico con una nota malinconica della prematura scomparsa dell'amico nonché 
note che Vattel aveva posto a margine del suo esemplare, riguardanti precisazioni di carattere bibliografico e approfondimenti storici a sostegno delle argomentazioni delle sue tesi che di volta in volta esponeva. ${ }^{\mathbf{1 4 7}}$

Nel 1775, per motivi sia editoriali che filologici, Charles-Guillaume Frédéric Dumas curò e pubblicò, ad Amsterdam presso van Harrevelt, un nuovo Droit des gens, per correggere gli errori, a suo dire, presenti nella versione di Ostervald del $1773 .{ }^{\mathbf{1 4 8}}$

Dumas, servendosi dell'espediente letterario intitolato Lettre de l'éditeur à Monsieur ***, presentava il lavoro svolto per la pubblicazione, affermava di aver aggiunto delle note riconoscibili e sottolineava la non precisione dell'edizione della Société typographique del $1773 ;{ }^{149}$ in quest'ultima gli errori erano molto grossolani e di stampa, Vattel, ad esempio, nell'Abrégé è citato Madame e non Monsieur ${ }^{\mathbf{1 5 0}}$ ed è per questo che Dumas si sentiva quasi obbligato ad una nuova e corretta versione. ${ }^{151}$

grande giurista ed elogiandolo con le seguenti parole: « Nous ne nous arrêterons pas à la faire ici l'éloge de feu M. de Vattel. Quelque qu'il pût être pour nous de répandre quelques fleurs sur la tombe d'une publiciste si digne de notre admiration et de notre estime. Tout le monde sait qu'il réunissait à un rare degré les qualités de l'esprit et celles réunissant du coeur; qu'il joignait à la justesse, à l'étendue du géniel, les vertus les plus essentielles, la candeur, la droiture, la générosité, les sentiments nobles et élevés. Invariable dans ses principes, il fut toujours bon citoyen, ami fidèle, empressé à faire le bien. Ses ouvrages, d'ailleurs, suffisent pour le faire connaitre. Il s'y est peint lui-même par des traits qui caractérisent la plus belle âme. Ce que nous y ajouterions ne pourrait qu'affaiblir un tableau qui, en nous rappelant tout ce qu'il fu, honore et sa patrie et l'humanité »: Ostervald, Abrégé de la vie de Vattel, in: VatTel (1773) V.

147 Una nota importantissima è sicuramente legata alla primordiale concezione di blocco totale, di cui si parlarà nel capitolo successivo. Vattel infatti sentì il bisogno di confortare questa ardita decisione con documenti che trasse per lo più dagli Annales et Histoires des Troubles des Pays-Bas di Ugo Grozio, cfr. VAtTel (1758a) L. III, chap. VII, $\mathbb{\$} 112$.

148 Nato nel 1721 da genitori immigrati francesi a Kloster Heilbronn nel principato tedesco di Ansbach, risiedette per gran parte della sua vita Olanda, dove morì nel 1796. Su Charles-Guillaume-Frédéric Dumas: Schaeper (1995) 53.

149 Dumas, Lettre de l'éditeur à Monsieur ***, in: Vattel (1775) I-II: «L'édition de Neuchâtel a été fait après la mort de l'Auteur, sur un exemplaire où il avoit mis quelques additions en marge. Mais de toutes ces augmentations, il paroît qu'aucune n'a rien changé au texte, lequel j'ai trouvé tout à fait conforme à celui de Leide; avec cette seule différence, que l'impression de Leide est correcte, et que celle de Neuchâtel est cruellement maltraitée par la négligence de l'Imprimeur. On est tenté, en lisant, de croire que celui-ci a voulu épargner les fraix de la correction, et qu'il a mis ses formes sous presse à la mesure que ses ouvriers se hâtoient de les composer ».

150 Ostervald, Abrégé de la vie de Vattel, in: Vattel (1773) II.

151 Dumas, Lettre de l'éditeur à Monsieur ***; in: Vatrel (1775) I : «sollicité par le libraire, qui réimprime ce Livre à Amsterdam, de le revoir et d'y ajouter des remarques, je me suis 
Inoltre nella Lettre l'editore olandese annunciava di voler distinguere le note della prima edizione di Vattel (1758) da quelle che Vattel aveva aggiunto successivamente al suo manoscritto (pubblicate da Ostervald) e di contrassegnare le sue stesse osservazioni con numeri arabi e l'apposizione dell'iniziale « D. » al termine di ogni nuova annotazione.

L'intento di Dumas, altamente filologico, permette di risalire alla paternità della nota, conservando il lavoro originale e evidenziandone le interpolazioni successive.

Per quanto riguarda le note redatte da Vattel (nelle versioni del 1758 e del 1773) si tratta per la maggior parte di precisazioni bibliografiche e di riferimenti, ad esempio, ad autori come Cicerone o Grozio. Dumas identifica le note presenti nella prima edizione con l'apposizione delle lettere dell'alfabeto a), b) ecc. e invece le successive (pubblicate postume nel 1773) con gli asterischi; è pertanto molto semplice orientarsi nel testo e riconoscere immediatamente non solo la paternità delle note, ma anche la datazione.

Le aggiunte sono di carattere esegetico e con diversi riferimenti dottrinali, che sembrerebbero colmare alcuni passaggi del Droit des gens in cui Vattel, a detta del curatore, è stato più generico. Per utilizzare le parole di Dumas tali note: " ont passées sous vos yeux, Monsieur, ces Remarques; vous les avez presque toutes approuvées, et vous avez si bien caractérisé l'esprit dans lequel je les ai composées, que je ne saurais me refuser à la satisfication de transcrire ici vos propres paroles: J'ai lu - dites vous - vos réflexions avec tout l'attention possible; et je trouve qu'elles servent presque toutes à bien éclaircir le texte, ou (ce qui est à mon avis plus intéressant) à donner au Lecteur d'autres vues encore que celles de l'Auteur, afin qu'il compare, qu'il pense, et qu'il pèse ${ }^{\mathbf{1 5 2}}$

Il lavoro minuzioso di Dumas, è indirizzato a fornire al lettore spunti di riflessione su particolari temi ed è completato dall'esposizione di teorie di giuristi diversi da Vattel per meglio chiarire determinate riflessioni presenti nel testo originale: le annotazioni contrassegnate dalla « D. » maiuscola sono

prêté à ses désir, parce que il m'a paru que mon travail pourra être utile au grand nombre de ceux pour qui cet Ouvrage est fait, je veux dire aux jeunes gens, et à tant d'autres Lecteurs qui, par état, ne sont point à même de consulter et juger ceux d'où Mr. de Vattel a tiré le sien. Sans y avoir dit du nouveau, on ne saurait lui contester le mérite d'avoir mis à la portée d'un beaucoup plus grand nombre de Lecteurs les travaux des grands hommes qui ont ouvert cette importante carriere. Je n'en dirai rien de plus, par la raison que, depuis qu'il a paru, les Journaux et le Public lui ont suffisamment rendu justice ».

Dumas, Lettre de l'éditeur à Monsieur ${ }^{* * *}$, in: Vattel (1775) III. 
relativamente poche, se si tiene conto della vastità dei temi trattati nel Droit des gens, e si concentrano soprattutto nel primo libro, quello dedicato al diritto interno di uno Stato.

Un esempio interessante del procedimento metodologico di Dumas si può riscontrare nelle argomentazioni di Vattel contenute al libro I, capitolo I, $\mathbb{S}$, in cui si soffermava sulle forme di governo e, sebbene non avanzasse nessuna preferenza, in nota riconosceva nel governo monarchico la tipologia preferita, purché il potere fosse esercitato in modo limitato e non assoluto. Per definire il termine « limitato » Vattel rievocava la vicenda del re di Sparta, Teopompo, elogiato anche in parte da Aristotele, che, per rendere duraturo il suo regno, aveva limitato i suoi poteri attraverso l'istituzione dell'eforato con il compito di governare la polis e munito di ampi potere anche sanzionatori. ${ }^{153}$ L'esempio tratto dalla storia greca serviva per esplicitare una situazione politica contemporanea: il caso della politica interna svedese, in cui il sovrano Adolfo Federico (1710-1771) aveva ristretto molto i suoi poteri. Ma proprio sulla storia svedese Dumas aggiunse la sua nota storica e dottrinale, aggiornando la situazione svedese con le dichiarazioni del successore Gustavo III (1746-1792), il quale, il 19 agosto 1772, progettò un colpo di Stato con la contestuale approvazione di una costituzione che aumentasse i poteri regi a discapito del Parlamento. ${ }^{154}$ Dumas terminava la sua riflessione riferendosi all'Inghilterra e citando Montesquieu nel suo tagliente frammento dell'Esprit des Lois: "On peut appliquer aux Suédois ce que Montesquieu dit des Anglais du siècle passé, qu'ils cherchaient la liberté, et ne la trouveraient nulle part, et qu'enfin il a fallu se reposer dans le Gouvernement même qu'on avait proscrit. Aussi leur nouvelle forme de gouvernement paroît-elle plusieurs égards assez semblable à celle qui fait le bonheur et la prospérité de l'Angleterre. Je comparerois volontiers l'ancien Sénat à une cinquième roue, dont le mouvement rompoit l'unité des quatre autres et du conducteur ». ${ }^{\mathbf{1 5 5}}$

Un secondo esempio del metodo adottato da Dumas si può ricondurre alle vicende per la spartizione della Polonia nel 1772 ad opera dell'Impero Russo, di quello austriaco e del regno di Prussia. Nel momento in cui Vattel al libro I, capitolo II, $\mathbb{S} 24$, affrontava la tematica relativa al raggiungimento

153 Vattel (1775) L. I, chap. I, $\mathbb{S} 3,11-12$, nota *. Sulle scelte politiche di Teopompo si rimanda molto sinteticamente a: Occhipinti (2011) 291-307.

154 Dumas in: Vattel (1775) L. I, chap. I, $\mathbb{~ 3 , ~ 1 2 , ~ n o t a ~} 2$.

155 Ibidem. 
della perfezione e della conservazione dello Stato, attraverso la costituzione, richiamava illustri esempi: elogiava l'Inghilterra, definendola la nazione con una "mirabile costituzione", e al tempo stesso spostava l'attenzione verso un'altra, che non menzionava ma dipingeva come una nazione altrettanto forte e valorosa, sebbene troppo restia a non voler cambiare e migliorare la sua costituzione, « excessivement jalouse de sa liberté, cette nation a pris des précautions qui mettent sans doute le roi hors d'état de rien entreprendre contre la liberté publique. Mais ne voit-on pas que ces mesures passent le but, qu'elles lient les mains du prince le plus juste et le plus sage, et lui ôtent les moyens d'assurer cette même liberté contre les entreprises des puissances étrangères, et de rendre la nation riche et heureuse? ». ${ }^{156}$ E' Dumas a precisare con una chiara e dettagliata nota e con informazioni supplementari la triste sorte della Polonia, realizzatasi nel 1772 e già anticipata quasi profeticamente da Vattel nel testo.

Dumas, dopo aver citato la posizione critica di Voltaire sulla Polonia contenuta nel suo Essay sur l'Histoire générale, si poneva in aperta condanna al sistema di governo polacco, basato su quella che è stata definita libertà dorata o democrazia nobiliare (aurea libertas in latino e in polacco Ztota Wolność), più ricordata come l'« anarchia nobiliare ", in cui l'intera nobiltà godeva di ampie libertà, aveva gli stessi diritti e obblighi e aveva condotto progressivamente all'indebolimento del governo centrale, favorendo così la conquista ad opera di altre potenze europee. ${ }^{157}$

Pertanto all'interno dell'edizione del 1775 si intercalano le note di Dumas, a volte come le precedenti più storiche, altre, invece sono di taglio più dottrinale, ad esempio, nel primo libro, quando Dumas si preoccupava di fornire una definizione più completa delle "leggi criminali", esposte dal giurista di Neuchâtel nel capitolo XIII al paragrafo 170, in cui veniva demandata ad ogni singolo Stato la scelta delle leggi che più convenissero alla sua situazione interna. ${ }^{158}$ Apponendo la sua «D. », Dumas, invece, illustrava l'intenso dibattito settecentesco sulla necessità e proporzionalità della pena, utilizzando come fonti ad esempio Rousseau, proprio al fine presen-

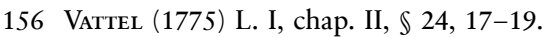

157 Dumas in: Vattel (1775) L. I, chap. II, $\mathbb{2} 24,18-19$, nota 3. Sulla «Libertà dorata »: Madonia (2013) 37 ss.

158 Vattel (1775) L. I, chap. XIII, $\$ 170$, 94-96. 
tare al lettore molteplici riflessioni su una tematica così importante e all'epoca attuale. ${ }^{159}$

Con Dumas inizia l'approccio più esegetico al testo di Vattel, che si svilupperà durante tutto il XIX secolo e vedrà coinvolti diversi internazionalisti ma anche politici, intenti nel cercare nel Droit des gens una risposta ai diversi problemi di politica sia interna sia esterna. Sotto questo profilo, come noto, il trattato di Vattel rientra tra i maggiori testi europei consultati durante la guerra di indipendenza americana, per la costruzione di una nuova nazione che guarda, ammira e prende spunto dai più importanti giuristi europei dell'epoca, nella convinzione di trovare in essi delle basi solide per il diritto pubblico e per quello diritto internazionale. ${ }^{\mathbf{1 6 0}}$

Dumas, inoltre rafforza la strategia di una lettura di uno specifico testo giuridico in base ad una determinata necessità storico-contingente: l'edizione del 1775 , che poteva sembrare una semplice pubblicazione per esigenza di mercato in realtà, assume un ruolo rilevante e di primo piano nelle vicende politiche europee e nordamericane; la decisione di Dumas di stampare il testo di Vattel è ben documentata all'interno dell'importante carteggio con uno dei padri fondatori degli Stati Uniti di America, Benjamin Franklin, che nel 1766 lo incontrò in Olanda. Tra il 1768 e il 1786 Dumas ebbe una corrispondenza molto consistente con Franklin, al quale spediva diverse copie di libri di impronta politico-giuridica, lo informava anche delle pubblicazioni europee e della sua intenzione di curare una nuova edizione del Droit des gens. Da L'Aia, il 17 maggio 1775, l'editore olandese così informava l'amico americano:

J'ai donné depuis peu une nouvelle Edition du Droit des Gens de Vattel in 4o avec des notes de ma façon, et une Lettre aussi de moi à la tête, où je parle de vos Provinces avec un intérêt qui part du fond du cœur. J'en ai deux Exemplaires en

159 Dumas in: Vattel (1775) 95-96, nota 14.

160 Reeves (1909) 549. Anche: Balch (1915) 113-140; Wilson, (1936) 14-26. Armitage, (2002) 39-64; Morley (2002) 126-128. Sui riferimenti di Vattel a livello giurisprudenziale nel contesto nordamericano si rimanda a: Oosterveld (2015) 23 ss.; Chetail (2014) 251ss; Chetail (2011) 400 ss. e anche a Richardson (2012) 547-571 in particolare 548-550. Cfr. sul punto: Sylvester (1999) 67, afferma che nelle sentenze Americane, « in all, in the 1780 s and 1790 s, there were nine citations to Pufendorf, sixteen to Grotius, twenty-five to Bynkershoek, and a staggering ninety-two to Vattel ». Sui riferimenti al diritto delle genti europei: Janis (2004); Bellia / BradFord, (2011) 445-552, in particolare 471. Da ultimo anche: Keller (2003) 84 e ss.; Janis (2010) 24 ss. 
réserve pour vous et pour la Bibliothèque de Philadelphie; j’y ajouterai quelques autres petites nouveautés que je croirai pouvoir vous faire plaisir, et ferai partir le paquet par le premier Vaisseau qui fera voile de Rotterdam pour Philadelphie. ${ }^{\mathbf{1 6 1}}$

Successivamente Dumas, il 30 giugno 1775, informava Franklin di avere terminato la stampa del trattato di Vattel inviadogli tre copie, ${ }^{162}$ e con queste parole, il 9 dicembre dello stesso anno, l'illustre politico americano lo ringraziò: «I am much obliged by the kind present you have made us of your new edition of Vattel. It came to us in good season, when the circumstances of a rising State make it necessary frequently to consult the Law of Nations. According that copy [...] has been continually in the hand of our Congress now sitting ", aggiungendo che, come da accordi, la terza copia l'avrebbe consegnata al college di Massachusetts Bay affinchè fosse continuamente: " in the hands of the members of our congress, who are much pleased with your notes and preface and have entertained a high and just esteem for their author ». 163

161 Lettera di Dumas inviata a Franklin, 17 maggio 1775, in: Willcox (ed.) (1982) 46-49.

162 Lettera di Dumas inviata a Franklin, 30 giugno 1775, in: Willcox (ed.) (1982) 74-78. Dumas nella stessa continuava dicendo: «On a fini d'imprimer l'hiver passé une nouvelle édition du Droit des gens de Vattel. Je n'ai pu refuser aux sollicitations de certaines gens d'en être l'Editeur. Ce qu'il y a de curieux, c'est qu'à la réserve de mes idées touchant les peines, que ces gens connoissent et approuvent, toutes les autres theses que je soutiens, tant dans mes notes que dans une Lettre que j'ai mise à la tête de l'édition, sont justement l'opposé de ce qu'on vouloit de moi; et mon histoire à cet égard ressemble fort au conte de Balaam: on s'attendoit que je maudirois des tyrans beaucoup moins odieux que ceux que l'on bénit; et j'ai fait le rebours: je doute qu'on me le pardonne; et je m'en console ». La lettera si trova parzialmente trascritta anche in CHETAIL: (2011) 397.

163 Lettera di Franklin inviata a Dumas, 9 dicembre 1775, in: Willcox (ed.) (1982) 287-291. È anche interamente trascritta nella raccolta di corrispondenza diplomatica edita da Wharton (ed.) (1889) 64-66 e in: The European Magazine and London Review (1804) 347-349. Si trova parzialmente anche in Reeves (1909) 552; Reeves (1937) 697-702. Thayer segnala che presso la biblioteca dell'Università di Harvard è presente proprio questa edizione di Vattel (Amsterdam, 1775) registrata come dono personale di «B. Franklin »: Thayer (1895) 951; questa ricostruzione trova conferma anche dalla lettera che Benjamin aveva inviato all'amico nonchè celebre politico James Bowdoin il 24 marzo 1776: "I have put into Mr Adam's Hands directed for you, the new Edition of Vattel When you have perus'd it, please to place it in your College Library » (intesa come la biblioteca di Harvard), trascritta nella raccolta di lettere dei delegati del Congresso Continentale edita da Smith (ed.) (1976) 435. Cfr. anche Lapradelle, Introduction, in: Vattel (1916) XXX; SEARs (1919) 379-399, in particolare 379-380; Barton Korty (1965) 1-83, in particolare 20,39 e 65 . 
Questo celebre carteggio dimostra, da un lato, la circolazione dell'opera nell'ambiente americano, dall'altro la valenza politica del Droit des gens. Dumas seguì con attenzione le vicende americane, e, attraverso le lettere scritte a Franklin è stato possibile ricostruire alcuni passaggi cruciali della guerra di indipendenza americana facendo emergere soprattutto quale tipologia di letture europee erano predilette dai rivoluzionari. ${ }^{164}$ Dumas fece cenno di ciò che stava accadendo in nord America anche nella sua Lettre (posta come premessa alla nuova edizione di Vattel): «Dans les Résolutions vigoureuses de ces braves colons, vous reconnoîtrez la voix de la vrai Liberté aux prises avec l'oppression. Vous frémirez, vous vous révolterez contre la morgue et la dureté inconcevable de ceux, qui, jaloux à l'extrême de leur propre liberté, pensent de pouvoir devenir plus puissants, de pouvoir rester libres eux-mêmes en asservissant leurs frères ». ${ }^{165}$

Dumas fu particolarmente attivo come traduttore, oltre ad inviare le copie del nuovo Droit des gens di Vattel, a Franklin faceva pervenire anche la traduzione in francese dell'estratto degli atti del primo Congresso continentale americano, che era in circolazione gia in Europa. ${ }^{166}$ Tradusse inoltre testi americani e incentivò la loro lettura nel vecchio continente, come il trattato di William Smith's An Historical Account of the Expedition Against the Ohio Indians, in the Year 1764, Under the Command of Henry Bouquet (1769).

Egli non fu un semplice "osservatore», infatti, nella stessa lettera del 9 dicembre 1775 con cui Franklin, non solo lo ringraziava per l'invio del Droit des gens, ma lo nominava agente del Committee of Secret Correspondence, poiché «as you are situated at the Hague, where Ambassadors from all the Courts reside, you would make use of the opportunity that situation affords you, of discovering, if possible, the disposition of the several Courts with

164 SAdosky (2009) 78-79. Cfr.: Wright JR. (1926) 524-547; Bourguignon (1977) 270-295; Widmer (1988) 359-389; Moynihan (1990) 17; Rabkin (1997) 305-306; Golove/ Hulsebosch (2010) 932-1066; Hunter (2012) 173-206; Hunter (2013c) 12-22.

165 Dumas, Lettre de l'éditeur à Monsieur *:*;, Vattel (1775) III-IV.

166 Lettera di Dumas inviata a Franklin, 30 giugno 1775, in: Willcox (ed.) (1982) 74-78: "J'ajoute au paquet une couple d'exemplaires d'une traduction hâtée que j'ai faite de l'Extrait des Actes de votre Congrès général dans le temps où ces pièces parurent chez nous dans leur primeur en Anglois. Il me seroit difficile de vous dire, Monsieur, combien les Pieces qu'il contient ont été goûtées et admirées généralement, et l'horreur avec laquelle on voit Saturne dévorer ses enfans ». 
respect to such assistance or alliance, if we should apply or the one, or porpose the other ». ${ }^{\mathbf{1 6 7}}$ Dumas accettò e mantenne la carica dal 1775 al 1796.

Il singolare «caso Dumas " permette di interrogarsi in modo più ampio sulle dinamiche politiche che possono nascere da uno scambio epistolare: come editore, Dumas, ha selezionato determinate tipologie di libri giuridici da far pervenire oltre oceano, contribuendo anche al progressivo indirizzamento del discorso giuridico nel pensiero di alcune delle più illustri figure della rivoluzione americana come Franklin. ${ }^{168}$ Non si tratta «banalmente » di una diffusione, ma di una precisa scelta che, partendo da una sfera editoriale sposta il campo di indagine nel diritto e nella politica. Nel frenetico clima culturale settecentesco europeo Dumas diciassette anni dopo la prima edizione francese del Droit des gens fece pervenire una copia della "sua » nuova versione oltreoceano, inaugurando la circolazione "mondiale» del trattato e favorendo in altri contesti l'acquisizione delle teorie vatteliane, che hanno assunto rilevanza per l'evoluzione del diritto costituzionale e internationale.

167 Così continuava Franklin nell'identificare il lavoro e la prudenza che Dumas doveva tenere: «As it may possibly be necessary, in particular instances, that you should, for this purpose, confer directly with some great Ministers, and show them this letter as your credential, we only recommend it to your discretion, that you proceed therein with such caution, as to keep the fame from the know ledge of the English Ambassador, and prevent any public appearance, at present, of your being employed in any such business, as thereby, we imagine, many inconveniencies may be avoided, and your means of rendering us service increased »: Lettera di Franklin inviata a Dumas, 9 dicembre 1775, in: WiLlcox (ed.) (1982) 287-291. Sulla partecipazione di Dumas agli eventi americani: Morice (1961) $17-28$.

168 Sul possesso del Droit des gens da parte del primo presidente degli Stati Uniti curioso è inoltre un aneddoto che risale a maggio 2010: la New York Society Library, una delle biblioteche americane più antiche dopo più di duecento anni è riuscita a recuperare la copia di The law of nations di Vattel che aveva prestato a George Washington il 5 ottobre 1789 e, ironicamente, il direttore della biblioteca Mark Bartlett, scherzò sulla multa dovuta al ritardo che ammonterebbe a più di trecentomila dollari. La copia presa in prestito da Washington è l'edizione inglese (London 1779). La notizia ha riscosso un notevole successo soprattutto attraverso internet, cfr. l'articolo su Reuters di Goldsmith (2010) e si trova riportata anche in: Chetall (2011) 397, nota 45. 



\section{La « governabilità » dello Stato e delle relazioni internazionali}

\section{Verso la regolamentazione giuridica delle forme sociali}

Le riflessioni argomentate nel primo capitolo, attorno alle edizioni del Droit des gens del 1758 e alle successive traduzioni, hanno avuto lo scopo di ricostruire la poliedrica figura di Emer de Vattel. Agli occhi del lettore la «vicenda Vattel » appare un groviglio di implicazioni semplicemente editoriali, invece genera problematiche e interrogativi sul discorso politico-giuridico contenuto nel Droit des gens e sulle motivazioni che hanno reso il trattato un vero e proprio best-seller durante il Settecento e la prima metà dell'Ottocento.

Se è vero che la strategia traduttiva e le contestuali decisioni editoriali sono sempre il frutto di una ponderata valutazione in termini di impatto e di utilizzazione del testo, allora si possono considerare le operazioni di divulgazione del diritto delle genti, compiute con il trattato di Vattel, come concrete risposte per le esigenze politiche e giuridiche degli Stati, tanto sul piano delle relazioni interne quanto su quelle esterne.

L'accoglimento maggiore del Droit des gens si articola su due aspetti distinti, ma profondamente intersecati tra di loro, che assicurano un incredibile interesse temporale e spaziale: da un lato vi sono le regole contenute nel primo libro, poste a fondamento di una Nazione, dall'altro vi è tutto ciò che concerne le relazioni internazionali, la guerra e la diplomazia, tematiche elaborate rispettivamente nel secondo, nel terzo e nel quarto libro.

La creazione, infatti, di un ordine giuridico interstatale con determinati principi presuppone una profonda e analitica circoscrizione di tutto quello che appartiene ad uno Stato e ad una Nazione: Vattel costruisce un concetto di Nazione, al cui interno attribuisce precisi dettami per regolare la vita dello Stato stesso e dei suoi cittadini. L'attenzione è talmente capillare che anche l'individuo assume una connotazione marcatamente politica, portandolo a contribuire al proprio bene insieme a quello comune e raggiungendo di 
conseguenza una analogia tra la sfera privata e quella pubblica, esattamente come lo Stato si realizza internamente ed esternamente. ${ }^{\mathbf{1}}$

Così si esprime Jouannet:

en 1758 , émerge réellement le droit des gens classique au sens d'un ensemble de règles individualisées et autonomisées, destinées à régir une société internationale non hiérarchisée dont le fondement est la notion de souveraineté étatique et dont la finalité est d'assurer le respect d'un certain nombre de droits et devoirs parfaits des États. $^{2}$

Egli utilizza parte delle teorie sul diritto delle genti elaborate dai suoi predecessori, le «fa proprie » e instaura, con il Droit des gens, un dialogo giuridico con il potere politico. Ci sono alcune parole chiave nel trattato di Vattel che anticipano quelli che saranno i temi centrali di tutto l'Ottocento: è sufficiente pensare alla distinzione tra Stato e Nazione, alla costituzione, nella sua accezione al singolare, come "regolamento fondamentale» e ai diritti e doveri di uno Stato nei confronti dei suoi cittadini. Paradossalmente si compie una innovazione partendo da quella che è la tradizione; tradizione contrassegnata dai topoi giusnaturalistici e dell'illuminismo, ma che con Vattel assumono una valenza completamente diversa, perché sono distinti da una connotazione più marcatamente politico-giuridica.

Luminare è in questo senso la posizione di Norberto Bobbio che caratterizza la diffusione delle teorie del diritto naturale del Settecento, grazie all'uscita di esse dalla sfera strettamente dottrinale: «la dottrina del diritto naturale, chiusa ormai nelle Università, diventata togata ed accademica, relegata in voluminosi trattati o in manuali istituzionali ad uso delle scuole, lontana dai problemi sociali e politici da cui da cui era pure sorta (si pensi a Grozio e ad Hobbes), era una cultura morta. Montesquieu, Voltaire, Rousseau, l'Enciclopedia rappresentavano la cultura viva. Anche se gli strumenti che essi adoperavano erano gran parte gli stessi, lo spirito era cambiato ». $^{3}$ Vattel rientra all'interno di questa "cultura viva ». La sua notorietà è soprattutto giustificata grazie all'elaborazione dei concetti di sovranità, indipendenza, uguaglianza degli Stati e di equilibrio del potere; sebbene tutte le definizioni presenti chiaramente non siano state in toto "create», molte di esse sono state senza dubbio da lui precisate nei contenuti e maggiormente divulgate attraverso il Droit des gens.

1 Cfr. Piro (1999) 441-443; Piro (2000) 65-94; Birocchi (2002) 219, nota 342.

2 Jounnnet (1998) 419.

3 Bоввіо (2009) 265. 
Specularmente alle pagine precedenti nelle quali si è illustrato il ruolo assunto dalla divulgazione attraverso il fenomeno traduttivo, lo scopo di questa sezione è invece di analizzare l'obiettivo prefissato nel trattato. La maggiore accessibilità alle teorie giuridiche, in questo caso alle teorie del diritto naturale e delle genti, non è sinonimo di «semplicità » e "diffusione ", bensì di attenzione ad un cambiamento, anche giuridico, che si realizza in un determinato contesto storico e sociale e si ripercuote tanto a livello europeo quanto a livello globale.

Non si tratta di aver delineato tout court le caratteristiche politico-giuridiche, ma di aver dato l'avvio ad una esigenza concreta che ha iniziato a prendere forma nel XVIII secolo proprio con il Droit des gens. Vattel scrive per coloro che sono chiamati ad esercitare un ruolo di potere politico: è una presa di coscienza importante, il messaggio contenuto è destinato a chi governa, e di conseguenza il linguaggio è strutturato in modo incisivo.

Non si intende illustrare il pensiero di Vattel, né affrontare il Droit des gens con un intento esegetico, né esporre teorie già ampliamente analizzate nella storia del pensiero politico e nella storia del diritto internazionale, ma soffermarsi su alcuni meccanismi giuridici, definibili di " governabilità », che sono Stati elaborati dal giurista di Neuchâtel, mutuando gran parte delle fonti dai predecessori, trasformandone l'utilizzo e le finalità. Si tratta di svolgere in questo senso una concreta ricerca delle molteplici forme sociali attraverso cui il potere sovrano si relaziona giuridicamente con i soggetti, cittadini o sudditi, al fine di comprendere gli elementi specifici identificati da Vattel sui quali esso si realizza.

\section{Innovazione all'interno della tradizione: l'obiettivo del Droit des gens}

Ad introdurre i quattro libri del Droit des gens vi è una lunga préface, nella quale sono ampiamente presentati i principi fondamentali della dottrina e le fonti, attraverso un breve excursus sulle diverse teorie del diritto delle genti formulate dai predecessori. ${ }^{4}$ Centrale e illuminante è la definizione che Vattel dà al diritto delle genti, inteso come " une science particulière, laquelle consiste dans une application juste et raisonnée de la Loi Naturelle aux

4 Si veda l'analisi della Préface presentata da: Mancuso (2002) 248 ss. 
affaires et à la conduite des Nations ou des Souverains ${ }^{5}{ }^{5}$ Il diritto delle genti per essere definito tale è esclusivamente quello che regola gli «affari » e la condotta delle Nazioni e dei Sovrani, prima ancora che a livello internazionale all'interno dello Stato stesso.

Per Vattel il tema, tuttavia, non è Stato affrontato con la cura necessaria ed è Stato rilegato ad una nozione vaga e imprecisa, precisando che in tutti i trattati a lui precedenti e contemporanei, il diritto delle genti si trova «mescolato e confuso » con il diritto naturale e pertanto (codesti trattati) non risultano sufficienti a delinearlo giuridicamente. ${ }^{\mathbf{6}}$

Grozio, secondo il giurista di Neuchâtel, nel De iure belli ac pacis, sembra ridurre il diritto delle genti semplicemente agli usi delle nazioni, individuando così un diritto delle genti volontario dipendente dal consensus gentium, ${ }^{7}$ "conoscibile con un metodo empirico analizzante la storia e l'agire dei popoli e ben distinto dal diritto naturale ${ }^{8}{ }^{8}$ È necessario sottolineare che $\mathrm{i}$ presupposti della teoria di Grozio sono anticipati già nei Prolegomena, all'interno dei quali il giurista olandese contesta il pensiero di Carneade di Cirene, l'impersonificazione di coloro che negano una legge universalmente obbligatoria. Ciascun individuo sarebbe mosso solo dai propri interessi, pertanto le leggi umane hanno origine da considerazioni di opportunità e non sono fondate né si riferiscono ad una legge naturale, poiché questa non esiste. ${ }^{9}$

Grozio risponde a questa obiezione con un'accurata dissertazione sull'uomo che in quanto tale è definito un animale di specie superiore, con alcuni tratti caratteristici, tra i quali il desiderio di tendere all'impostazione di una struttura sociale pacifica e organizzata conformemente alla sua intelligenza. È per questo, che, contrariamente a Carneade, non si può accettare come verità universale l'asserzione secondo cui ogni animale è spinto dalla natura a cercare solo il suo bene; vi è un ordine sociale intimamente ricercato dall'uomo in quanto uomo, per il cui perseguimento e mantenimento occorre la legge. Quest'uomo sociale ha la capacità di valutare cosa sia van-

5 Vattel (1758a) Préface, VI.

6 Ivi, III.

7 Ivi, VIII.

8 Mancuso (2002) 251: si rimanda alle bellissime pagine di Mancuso per una disamina più approfondita dei concetti elaborati da Vattel e delle relazioni con i suoi predecessori, nonché alla già citata monografia di JouANnet (1998) passim.

9 Grotius (1625) Prolegomena. 
taggioso o dannoso per il perpetuarsi, presente e futuro, della società civile: qualunque cosa sia chiaramente in contrasto con il vivere sociale è pensata come contraria anche alla legge di natura, cioè, alla natura dell'uomo. ${ }^{\mathbf{1 0}}$

Tutto ciò che proviene dalla ragione umana è il diritto naturale, inteso quale dettame della retta ragione che vuole dimostrare un atto come moralmente turpe o moralmente necessario secondo la conformità della stessa natura razionale e a far vedere che tale atto è in conseguenza di ciò vietato o comandato da Dio in quanto autore della natura. ${ }^{\mathbf{1 1}}$

Mettendo dunque al centro la retta ragione e non la volontà divina, Grozio pone «i principi del diritto di natura stabilendo la conformità $o$ difformità, rispetto alla natura umana, delle azioni umane le quali, in conseguenza di questa decisione della ragione umana, sono vietate e prescritte da Dio ». ${ }^{12}$ Al tempo stesso il diritto naturale è complementare il diritto positivo, che sorge dall'obbligazione consensuale, " consensu obligatio, quae cum ex naturali iure vim suam habeat $" ;^{13}$ altresì vi è il diritto dei popoli o delle genti, che si basa esclusivamente sul consenso degli Stati e dal quale si sono originate alcune norme giuridiche che regolano i rapporti tra le nazioni ed è ben distinto dal diritto naturale. ${ }^{14}$

Alle stregua delle argomentazioni groziane, Vattel, servendosi De iure belli ac pacis tradotto da Barbeyrac, sostiene come egli "a entrevû la vérité », ma « comme il défrichoit, pour ainsi dire, une matière importante, fort négligée avant lui, il n'est pas surprenant que, l'esprit chargé d'une immense quantité d'objets et de citations qui entroient dans son plan, il n'ait pû parvenir toûjours aux idées distinctes, si nécessaires cependant dans les Sciences ». ${ }^{\mathbf{1 5}}$

Vattel contesta a Grozio che «les Sociétés Politiques ou les Nations vivent entr'elles dans une indépendance réciproque, dans l'état de Nature, et qu'elles sont soumises, dans leur qualité de Corps Politiques, à la Loi Naturelle » e pertanto, restringendo il diritto delle genti alle sole massime stabilite dal consenso dei popoli «semble donner à entendre, que les Souverains ne peuvent presser entr'eux que l'observation de ces dernières maximes, réser-

10 Ibidem.

11 Grotius (1625), L. I, cap. I, X, 6.

12 GozzI (2010) 61.

13 Ibidem.

14 Ibidem.

15 Vattel (1758a) Préface, VIII-IX. 
vant le Droit interne pour la direction de leur Conscience ». ${ }^{\mathbf{1 6}}$ Se Grozio, viceversa, avesse considerato le caratteristiche e le peculiarità delle nazioni avrebbe di certo riconosciuto il diritto delle genti naturale come una scienza particolare, la quale produce per le nazioni sia una obbligazione interna sia una esterna, indipendentemente dalla loro volontà e avrebbe inoltre intuito che il consenso dei popoli è soltanto la base e la fonte di un particolare diritto delle genti chiamato diritto delle genti arbitrario. ${ }^{17}$

Thomas Hobbes, secondo Vattel, è l'unico dei giusnaturalisti ad aver fornito un'idea distinta, ma ancora incompleta del diritto delle genti. E' interessante osservare che Vattel illustra le tesi di Hobbes, come specifica in una nota avvalendosi dell'opera di Pufendorf, nella traduzione francese realizzata da Barbeyrac; questo conferma che il diplomatico di Neuchâtel si serviva delle versioni « divulgative » francesi della scuola romanda, di cui lo stesso fa parte. $^{\mathbf{1 8}}$

Hobbes identifica una uguaglianza tra la società naturale degli uomini e quella degli Stati, negando sostanzialmente l'esistenza di un diritto delle genti in tempo di pace e di guerra: entrambe le società sono prive di legge e di un «terzo » in grado di dirimere i conflitti. In effetti egli è convinto che gli Stati siano nella titolarità di agire e operare all'interno dei limiti dettati unicamente dalle regole della propria autoconservazione, ${ }^{19}$ ed afferma l'esistenza di due tipi di legge quella naturale e quella umana, assimilando nel diritto naturale il diritto delle genti, e puntualizzando categoricamente che non vi è alcuna distinzione tra lo ius gentium e il diritto naturale. ${ }^{20}$ Vattel

16 Ibidem.

17 Vattel (1758a) Préface, X. Sul punto cfr. Mancuso (2002) 250-251.

18 Vattel (1758a) Préface, X, nota (g).

19 Gozzi (2010) 75-76. Come è Stato argomentato da Gustavo Gozzi, in questo Stato di natura dominato dalle guerre che potevano essere combattute e quelle realmente combattute, « inevitabilmente vengono meno alcuni capisaldi della precedente concezione dello ius gentium, in particolare la dottrina della guerra giusta [sostenuta ad esempio da autori come de Vitoria e dallo stesso Grozio]: vige solo la logica dello Stato improntata alla propria autoconservazione [...] ed è per questi motivi che la dottrina internazionalistica di Hobbes vien definita realistica ".

20 Новвеs $(1760)$ cap. XIV, $\mathbb{S}$ IV, 320: «Rursus naturalis dividi potest in naturalem hominum, quæ sola obtinuit dici lex naturae, et naturalem civitatum, quae dici potest lex Gentium, vulgo autem jus Gentium appellatur. Præcepta utriusque eadem sunt: sed quia civitates semel institutæ induunt propriaetates hominum personales, lex quam loquentes de hominum singulorum officio, naturalem dicimus applicata totis civitatibus, nationibus, sive 
obietta ad Hobbes di essersi illuso proprio sul punto in cui ritiene che il diritto naturale non subisca modifiche nella sua applicazione da parte degli Stati, stabilendo pertanto l'uguaglianza sostanziale e formale tra diritto naturale e diritto delle genti. ${ }^{21}$

Vattel dedica pochissime righe a Pufendorf contestandogli, sulla scia delle riflessioni avanzate nei confronti di Hobbes, l'uguaglianza e la contestuale confusione tra diritto naturale e diritto delle genti. ${ }^{22}$

Né Grozio, né Hobbes e nemmeno Pufendorf hanno convinto pienamente con le loro teorie sul diritto naturale e delle genti Vattel, il quale, invece, dimostra sincera riconoscenza e stima nei riguardi di Barbeyrac, perché nel suo intenso lavoro di autore e traduttore ha ben compreso e chiarito che

il y des Loix communes à tous les Peuples, ou des choses que tous les Peuples doivent observer les uns envers les autres : Et si l'on veut appeler cela Droit des gens, on le peut très bien. Mais, outre que le consentement des Peuples n'est pas le fondement de l'obligation où l'on est d'observer ces Loix, et ne saurait même avoir lieu ici en aucune sorte, les principes et les loix d'un tel droit sont au fond les mêmes que celles du Droit Naturel proprement ainsi nommé : Toute la différence qu'il y a, consiste dans l'application, qui peut se faire un peu autrement, à cause de la différence qu'il y a quelquefois dans la maniére dont les Sociétés vuident les affaires qu'elles ont les unes avec les autres. ${ }^{23}$

Barbeyrac, in moltissimi punti della sua traduzione francese al De iure belli ac pacis, aveva criticato la teoria sul diritto naturale e delle genti elaborata da Grozio, affermando che qualora il diritto delle genti nascesse dal semplice consenso, questo pregiudicherebbe l'intero sistema. Per il professore di Losanna il diritto delle genti trova il fondamento nel diritto naturale e si perfeziona in un insieme di regole comuni a tutti i popoli; egli respinge l'idea che possa esservi un criterio di fondazione del diritto che sia diverso dall'obbligo di rispettare una legge naturale e che sia interamente fondato sull'elemento volontaristico del consenso espresso dai popoli: «ce droit des gens, positif et distinct du droit naturel, c'est un pure chimére ». ${ }^{24}$

gentibus, vocatur jus Gentium. Et quæ Legis et juris naturalis Elementa hactenus tradita sunt, translata ad civitates et gentes integras, pro legum juris Gentium Elementis sumi possunt ».

21 Vattel (1758a) Préface, X.

$22 \mathrm{Ivi}, \mathrm{X}$.

23 Ivi, XI. Cfr. la nota di Barbeyrac in Grotius (1724) L. I, chap. I, $\mathbb{S}$ XIV, nota 3, 56 ss.

24 Grotius (1724) L. I, chap. I, $\mathbb{X}$ XIV, nota 3, 56 ss. 
Dunque Barbeyrac, asserendo che il diritto delle genti debba necessariamente essere ricondotto ad una legge naturale, ha sostenuto che le regole contenute nel diritto naturale non possano essere esclusivamente applicate alle nazioni, necessitando una "modellazione » alla luce della diversa natura alle quali sono destinate.

Nonostante questa perspicace riflessione, secondo Vattel «il ne paroît pas qu'il ait vû toute l'étenduë de cette idée, puisqu'il semble ne pas approuver que l'on traite le Droit des Gens séparément du Droit Naturel des particuliers ", ${ }^{25}$ e a tal riguardo si riscontra una citazione indiretta ad una altra opera, gli Elementa philosophiae practicae di Budde del 1697: Vattel in nota precisa che non ha potuto consultarla ma che Barbeyrac, nel richiamarla, si è limitato a lodarla, senza però andare oltre e comprendere la sua portata. $^{26}$

La fonte principale del Droit des gens, su cui si articolano le forme del diritto delle genti è la dottrina di Wolff. Vattel, infatti, sottolinea che mai avrebbe pensato di redigere un simile trattato se non avesse avuto l'onore di studiare l'opera di Wolff, che gli ha chiarito la portata del fondamento del diritto delle genti e il concetto di diritto naturale. ${ }^{27}$

Così Vattel nella sua Préface, esplicitando quanto già aveva confidato all'amico Formey, scrive il Droit des gens sulla falsariga dell'opera di Wolff, prendendo come corollari le sue definizioni e i suoi principi generali, ma al tempo stesso però sottolinea, che sin dal momento in cui ha deciso di

25 Vattel (1758a) Préface, XI-XII.

26 Scrive Vattel: «Il loüe seulement la méthode de Buddeus, disant, que cet Auteur a eû raison de marquer (dans les Elementa Philos, pract.) après chaque matière du Droit Naturel, l'application qu'on en peut faire aux Peuples les uns par rapport aux autres; autant du moins que la chose le permettoit ou l'exigeoit ", in nota precisa di non essere riuscito a procurarsi il testo di Buddeus utilizzato da Barbeyrac: VatTel (1758a) Préface, XII. Il passaggio a cui Vattel allude è: Buddeus (1697) 206-207.

27 Vattel (1758a) Préface, XII-XIII: «La gloire en étoit réservée à M. le Baron de Wolff. Ce grand Philosophe a vû que l'application du Droit naturel aux Nations en Corps, ou aux États, modifiée par la nature des Sujets, ne peut se faire avec précision, netteté et avec solidité, qu'à l'aide des Principes généraux et des notions directrices, qui doivent la règler; que c'est par le moyen de ces Principes seuls que l'on peut montrer évidemment, comment, en vertu du Droit Naturel même, les décisions de ce Droit à l'égard des particuliers doivent être changées et modifiées, quand on les applique aux États, ou Société Politiques, et former ainsi un Droit des Gens naturel et nécessaire: D'où il a conclu qu'il étoit convenable de faire un systême particulier de ce Droit des Gens; et il l'a exécuté heureusement ». 
scrivere il suo trattato, si è discoStato dal pensiero di Wolff, evitando quindi di fare dell'opera del giurista tedesco una semplice versione francese. ${ }^{28}$

Alla base del pensiero del giurista tedesco vi è il classico parallelismo tra la società naturale degli individui e quella degli Stati: « natura civitates diversae inter se spectantur tanquam personae liberae ${ }^{29}$ e che "ad eadem officia tum erga se ipsas, tum erga gentes alias obligantur, qua singuli singulis tenentur ${ }^{30} \mathrm{Di}$ conseguenza anche « la grande società di Stati » pone al centro il diritto naturale, che è per definizione immutabile e perfetto. Tuttavia, come accade tra gli uomini, che sono al contrario mutevoli e imperfetti, un diritto con le caratteristiche intrinseche dell'immutabilità e della perfezione non è di per sé sufficiente per regolare tutti i rapporti umani, così, allo stesso modo, tra gli Stati è necessario un diritto che sia "meno rigoroso" dello ius naturae e che pertanto meglio si possa adattare alla complessità delle relazioni internazionali. Tale diritto ha una origine ben diversa dal « consenso degli Stati » elaborato da Grozio: per Wolff il diritto delle genti non deriva né da un patto né da una consuetudine ma dal fatto che i popoli si trovano in una sorta di civitas gentium maxima, la quale impone loro determinate norme di condotta. ${ }^{31}$

Questa società per il professore di Halle deriva dall'esigenza « di radunare le forze » al fine di raggiungere la propria perfettibilità: « cum gentes conjunctis viribus se statumque suum perficere obligentur; ipsa natura societatem

28 Vattel (1758a) Préface, XV-XVI: «Je me fuis donc borné prendre dans l'Ouvrage de M. Wolf ce que j'y ai trouvé de meilleur, fur- tout les Définitions et les Principes généraux; mais j’ai puisé avec chóix dans dans cette source, et j'ai accommodé à mon plan les matériaux que j'en tirois. Ceux qui auront lû les Traités du Droit Naturel du Droit des Gens de M. Wolf verront combien j'en ai profitté. Si j’eusse voulu marquer par-tout ce que j'en empruntois, mes pages se trouveroient charges de citations également inutiles désagréables au Lecteur. II vaut mieux reconnoître ici une fois pour toutes les obligations que j'ai ce grand Maître. Quoique mon Ouvrage comme le verront ceux qui voudront se donner la peine d'en faire la comparaison soit très-différent du sien j'avoüe que je n'aurois jamais eû l'assurance d'entrer dans une si vaste carrière si le célèbre Philosophe de Hall n'eût marché devant moi ne m'eût éclairé ».

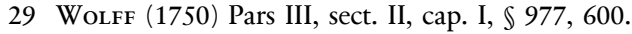

30 Wolff (1750) Pars IV, cap. I, $\mathbb{\$} 1088,679$.

31 Wolff (1750) Pars IV, cap. I, $\mathbb{\$} 1090,680-681$. Sul concetto di Civitas Maxima, si rimanda a: Brierly (1967) 38-40; Onuf (1994) 280-303; Onuf (1998) 59 ss.; Mancuso (2002) 259 ss.; Hunter (2007) 146-147; Brandt (2007) 113-132; Hofmann (2009b) 116; KosKenNiemi (2011) 23. 
quandam inter gentes instituit, in quam ob obligationis naturalis indispensabilem necessitatem consentire tenetur, ut quasi pacto contracta videatur $\gg .{ }^{32}$

Come argomentato da Scipione Gemma, « il cosiddetto diritto volontario è tale soltanto di nome, mentre in sostanza ha del diritto di natura quasi tutti i caratteri e principalissimi quelli della necessità e dell'assolutezza ${ }^{33} \mathrm{Al}$ contrario non necessario e al tempo stesso non assoluto, è il diritto che si basa sul consenso, che a sua volta può essere esplicito e allora costituisce il diritto delle genti pattizio «ius quod ex pactis oritur inter gentes diversas initis cum obligationibus respondentibus vel adhaerentibus », ${ }^{34}$ o tacito che forma così il diritto delle genti consuetudinario «quod longo usu inter quasdam gentes introductum et instar juris observatum ». ${ }^{35}$

Il pensiero giuridico wolffiano è un corollario di deduzioni scientificamente fondate, questa volta di carattere non più antropologico, ma sociopolitico, che deve rapportarsi con una impostazione rigorosamente geometrica e poco accessibile. ${ }^{36}$ Jouannet parla di "fixisme méthodologique», poiché a suo avviso tutta la dottrina wolffiana può essere riassunta come una successione ininterrotta di diritti e obbligazioni all'interno delle quali il diritto civile e delle genti restano ancorati al diritto naturale; ${ }^{37}$ osserva inoltre valorizzando Wolff che il diritto delle genti non è un diritto che regola le relazioni tra gli Stati, bensì è il diritto degli Stati considerati prima come singoli e poi di conseguenza anche nelle loro dinamiche esterne. ${ }^{38}$

In effetti sarà proprio Vattel a continuare il pensiero wolffiano ponendo al centro il diritto delle genti come legge vigente tra gli Stati e a collocarlo in una dimensione tanto nazionale quanto internazionale, acquisendo così

32 Wolff (1750) P. IV, cap. I, $\mathbb{S} 1090,680-681$.

33 Gemma (1902) 16.

34 WolfF (1750) P. IV, cap. I, $\mathbb{S} 1091,682$.

35 WolfF (1750) P. IV, cap. I, $\mathbb{S} 1092,683$.

36 Bobbio (2009) 140.

37 JOUANNET (1998) 129-130.

38 JouAnNet (1998) 400-401: continua Jouannet sottolineando come «Le droit des gens wolffien possède un champ d'application déjà beaucoup plus étendu que notre droit international public contemporain car même s'il est vrai que le domaine réservé des États varie en fonction de leurs engagements internationaux, une distinction de principe n'en demeure pas moins établie entre ce domaine et celui du droit international. [...] Le droit des gens devient réellement avec Wolff, fondateur à ce titre des grands principes de la vision classique du droit international, un droit autonome, destiné à régir la conduite de ceux qui seront désormais les sujets traditionnels du nouveau droit international, à savoir les Etats souverains». 
definitivamente il suo significato classico. ${ }^{39}$ Nella Préface egli contesta Wolff soprattutto nell'ambito della classificazione delle diverse forme di diritto delle genti, precisa che il diritto delle genti volontario di Wolff sarebbe una sorta di diritto civile, soffermandosi così sul concetto di Civitas maxima, la quale permette di individuare nelle norme del diritto delle genti (il cui contenuto è costituito dal diritto naturale), l'equivalente del diritto civile vigente all'interno delle singole nazioni. ${ }^{40}$ Vattel, non condividendo affatto questa idea, vede invece le nazioni quali soggetti qualitativamente differenti dagli individui in ragione della loro perfetta indipendenza e quindi della loro sovranità. ${ }^{\mathbf{4 1}}$

Secondo Carl Schmitt nell'opera vatteliana avviene il decisivo rafforzamento della consapevolezza e coscienza degli Stati moderni, il cui nucleo è la sovranità statale. ${ }^{42}$ Il diritto delle genti, benché ancora ancorato al diritto naturale, è la scienza da applicare esclusivamente alle relazioni tra le nazioni ed è con questa affermazione che Vattel indirettamente ammette (come per tutte le scienze) una serie di caratteristiche: la sua dinamicità, la possibilità di apportare « progressi » e la sua conformazione alla contingente realtà storica, prevedendo ovviamente anche il suo superamento per il raggiungimento della sua perfettibilità.

Tale posizione di scientificità del diritto permette di innescare una serie di meccanismi giuridici che portano il diritto delle genti alla sua massima espressione, attraverso la costruzione sostanziale della Nazione, la quale si basa sul concetto di costituzione, come presupposto imprescindibile per l'ammissione della stessa all'interno della comunità internazionale.

Da un lato vi è il diritto delle genti cosiddetto necessario, che deriva dalla natura ed è un diritto interno e relativo alla coscienza, ${ }^{43}$ dall'altro il diritto

39 Ivi, 401.

40 Vattel (1758a) Préface, XVII.

41 Mancuso (2002) 262. Nippold (1934) XLIV: «Gerade so wie im Staate die bürgerlichen Gesetze sich auf die natürlichen zurückführen, und wie dort das Naturgesetz selbst vorschreibt, auf welche Weise dies zu geschehen habe, so müssen auch in der Civitas Maxima, in der Staatengesellschaft, aus den natürlichen Gesetzen die bürgerlichen Gesetze abgeleitet werden, auf dieselbe Weise, wie im einzelnen Staate das Naturgesetz dies vorschreibt». Cfr. anche: Brownlie (1984) 357-369, in particolare 358; KoskenNiemi (2005) 76 ss., anche Koskenniemi (2011a) 70 ss.; Whelan (1999) 403-434 in particolare 404; Christov (2005) 561-584, in particolare 571 ss.

42 Sснмiтt (1991) 132.

43 Vattel (1758a) Préface, XXI. 
delle genti volontario, subordinato al primo, che ne raccomanda l'osservan$\mathrm{za}$, «en considération de l'état où les Nations se trouvent les unes avec les autres, et pour le bien de leurs affaires $»{ }^{\mathbf{4 4}}$

All'interno del diritto delle genti volontario si identifica il diritto delle genti arbitrario, cioè quello costituito dal diritto pattizio e dal diritto consuetudinario, contribuendo in modo determinante, per usare le parole di Francesco Mancuso, al «consolidamento di alcuni concetti politico-giuridici fondamentali dell'età contemporanea, sia per quanto riguarda il diritto pubblico interno, sia per quanto concerne il diritto internazionale ». ${ }^{45}$

Koselleck illustra che la divisione realizzata da Vattel tra diritto delle genti necessario e diritto delle genti volontario è stata la base per la razionalizzazione dello Stato e della guerra consacrando "nel primato della politica la chance che anche le esigenze morali $[. .$.$] avrebbero trovato il loro adempi-$ mento ». ${ }^{46}$

In questa prospettiva vengono tracciati i doveri e i diritti delle nazioni, precisando che le nazioni sono corpi politici, società di uomini tenuti insieme al fine di procurare, con tale riunione di forze, la loro salvezza e il loro vantaggio. ${ }^{47}$

E' sempre Jouannet ad indagare sulla tensione duale contenuta nel Droit des gens nel suo rapporto tra diritto naturale e diritto positivo e sull'elaborazione spinosa del diritto delle genti volontario, ${ }^{48} \mathrm{ma}$ al tempo stesso individua ulteriori dualismi: una prima dicotomia contrappone il dovere di conservazione con il dovere di perfezione; una seconda i doveri inter gentes e

44 Ivi, XXI.

45 Ibidem. Vattel, infatti, come afferma Mancuso, rende autonomo il diritto volontario «attraverso una concezione che mira ad illustrare da un lato il contenuto minimo del diritto naturale positivizzabile per le Nazioni e dall'altro a identificare i principi generali di un diritto, come quello arbitrario, che è indipendente dalle nazioni, ma che stabilisce un quadro di ordine minimale tra queste ultime »: Mancuso (1998) 265. Sul punto si rimanda anche a: Reibstein (1958) 607-636; Linklater (1982) 84-95; Hochstrasser (2000) 176-183; NAKHIMOVSKY (2007) 158.

46 Koselleck (1972) 46.

47 Vattel (1758a) L. I, chap. I, $\mathbb{1} 1$ 17. Rafael Domingo evidenzia come la struttura del Droit des gens possa essere letta in prospettiva comparata con le Istituzioni di Gaio, creando un paradigma alternativo alla tripartipartizione gaiana con una portata internazionale. Esso è composto dallo Stato, dalle relazioni tra gli Stati e dalla guerra: Domingo (2011) 627-647, in particolare 635 .

48 Jounnnet (1998) 85 ss.

86 Capitolo II 
intra gentes; una terza i diritti di uno Stato verso se stesso e verso gli altri; ed infine una ultima i diritti e doveri perfetti e imperfetti (interni ed esterni). ${ }^{49}$

La negazione della Civitas Maxima è un aspetto importante della divergenza dal pensiero di Wolff, ma è bene sottolineare che il binomio Wolff/Vattel deve essere approfondito anche riguardo alla somiglianza tra i due trattati. Nel 1785 Dietrich Ludwig von Ompteda diede alle stampe il suo Literatur des gesammten sowobl natürlichen als positiven Völkerrechts, che verrà poi continuato e completato da Karl Albert Kamptz ed edito nel 1817. L'autore dedica diverse pagine all'opera di Vattel e si concentra soprattutto nel comparare il Droit des gens al lavoro di Wolff. ${ }^{50}$

Ompteda riporta interamente l'indice del trattato del giurista di Neuchâtel e successivamente in una tabella pone a confronto la sistemazione dei capitoli nell'opera di Wolff e in quella vatteliana, evidenziando i molti punti in cui quest'ultimo ha ridotto e semplificato l'opera del giurista di Halle, ordinando o raggruppando i capitoli e rendendo così più agevole la lettura. ${ }^{51}$

Ompteda illustra come i nove capitoli dello Ius gentium, corrispondano ai quattro libri del Droit des gens: il primo libro coincide con il capitolo primo; il secondo con i capitoli terzo, quarto e quinto; il terzo con i capitoli sesto e settimo ed infine il quarto con i restanti capitoli del trattato Wolff. ${ }^{52}$

In effetti è Vattel stesso, come si è visto, ad ammettere di avere scritto il trattato sulla falsariga dell'opera del giurista di Halle: egli non esita a dire che preferisce esplicitare sin dalle prime pagine il suo grande debito alle teorie wolffiane, da cui ha ampiamente attinto:

Ceux qui auront les Traités du droit naturel et du droit des gens de M. Wolff, verront combien j'en ai profité. Si j'eusse voulu marquer par-tout ce que l'en empruntois, mes pages se trouveroient chargées de citations également inutiles et désagréables au Lecteur. Il vaut mieux reconnoître ici, une fois pour toutes, les obligations que j'ai à ce grand Maître. ${ }^{53}$

Vattel scrisse il trattato per gli uomini di governo e il suo intento era quello di creare un testo che fosse facile da consultare e al cui interno fossero contenute tutte le materie che potessero servire a tale fine; infatti, pur attin-

49 JOUANNEt (2011) 135-136.

50 OMPTEDA (1785) 339-345.

51 OMpteda (1785) 345; la tabella si trova anche riportata nel celebre testo di WheAton (1845) 185.

52 OMPTEDA (1785) 345.

53 Vattel (1758a) Préface, XVII. 
gendo anche nei contenuti al lavoro di Wolff ha fatto il significativo passo in avanti rispetto ai predecessori e al suo maestro. L'adattamento delle teorie wolffiane ad una dimensione più reale e concreta, permette a Vattel di creare un sistema di regole da applicarsi sia allo Stato sia alle relazioni tra gli altri Stati e di conseguenza di staccarsi inevitabilmente dal pensiero del maestro.

Inoltre occorre ricordare che vi sono numerosi altri passaggi del Droit des gens mutuati anche dalle opere di altri giusnaturalisti. E' sufficiente riscontrare che la famosissima citazione per cui molto spesso Vattel è ricordato «Un nain est aussi bien un homme, qu'un géant une petite République n'est pas moins un Etat souverain que le plus puissant Royaume " ${ }^{54}$ è la traduzione latina del passaggio contenuto nello Ius Gentium di Wolff ${ }^{55}$ e si trova in forma simile nei Principes du politique di Burlamaqui. ${ }^{56}$ Tuttavia era Stato Jean Bodin, molto tempo prima, nel 1576 ad affermare che « un petit Roy est autant souverain que le plus grand monarque de la terre $»{ }^{57}$

Celebre è l'elaborazione vatteliana del principio di equilibrio e l'analisi della situazione europea durante la prima metà del Settecento. L'idea che gli Stati creino una società e debbano intrattenere tra loro un certo numero di relazioni, oggetto specifico del diritto delle genti sono Stati meglio elaborati nel Droit des gens alla luce della realtà storica e politica. L'Europa rappresenta un esempio di sistema di Stati indipendenti, posti tra loro in una condizione di equilibrio politico. Alla base di queste teorie vi è una ragionata posizione di Vattel, che prendendo coscienza della realtà politica internazionale dei suoi tempi, sviluppa, sebbene in forma quasi utopica, il principio dell'equilibrio di potenza tra le nazioni, concepito come alleanze che si creano appositamente per le loro esigenze politiche. ${ }^{58}$

54 Vattel (1758a) Préliminaires, $\mathbb{S} 18,11$.

55 Wolff (1764) Prolegomena, $\mathbb{S} 16$, 6: "Quemadmodum itaque homo procerissimus non magis homo est, quam nanus ; itaque quoque Gens, quantumvis parva non minus Gens est, quam Gens maxima ».

56 Burlamaqui $(1751)$ t. $2,4^{\text {ème }}$ partie, chap I, $\mathbb{S} 5,3$ : «Toutes les Nations doivent se regarder comme naturellement égales et indépendantes les unes des autres ».

57 È stata consultata l'edizione di Bodin (1583) L. I, chap. II, 13.

58 Vattel (1758a) L. III, chap. III, $\ 47,39-40$ : «L'Europe fait un système politique, un corps où tout est lié par les relations et les divers intérêts des Nations qui habitent cette partie du monde. Ce n'est plus, comme autrefois, un amas confus de pièces isolées, dont chacune se croyant peu intéressée au sort des autres, et se mettait rarement en peine de ce qui ne la touchait pas immédiatement. L'attention continuelle des souverains à tout ce qui se passe, les ministres toujours résidents, les negociations perpétuelles, font de l'Euro- 
Il sistema è dunque incentrato sull'attività dei sovrani, costruito su una trama di negoziazioni continue, e forma una specie di repubblica, i cui membri sono indipendenti ma, al tempo stesso, legati dall'interesse comune per la conservazione dell'ordine e della pace. È su questi principi che si forma l'equilibrio di potere: esso rappresenta una disposizione delle cose, per mezzo delle quali nessuna potenza si trova nella condizione di predominare in modo assoluto ed esclusivo rispetto alle altre. ${ }^{59}$

Vattel è collocato al termine e al tempo stesso all'inizio di una nuova concezione giuridica tanto dottrinale quanto pratica. E' Stato rilevato che mentre Wolff è il più grande epigone della corrente leibniziana, Burlamaqui lo è per quanto riguarda la corrente pufendorfiana, tramandata in Svizzera attraverso l'attività di traduzione e di interpretazione di Barbeyrac. Bobbio, con acume, sostiene che la filosofia eclettica di Burlamaqui sia la manifestazione della fine della corrente giusnaturalistica. Tuttavia dal tramonto delle teorie giusnaturalistiche ebbe origine il «moto di formazione spirituale sfociato nella rivoluzione francese, di cui la scuola del diritto naturale del '600 e della prima metà del '700 era stata una preparazione, per così dire negativa, che aveva sbarazzato il terreno dai rami vecchi e aveva spianato gli ostacoli che impedivano di vedere più lontano ${ }^{\mathbf{6 0}}$ ed è in questo ambiente così complesso e vivace che Vattel realizza un " ponte » vincente, dialogando con il potere politico attraverso una costruzione teorico-giuridica.

Questa analisi permette di circoscrivere la portata del pensiero vatteliano all'interno di una sostenuta e corposa schiera di giuristi, che lui stesso considerava e stimava validi maestri e suoi predecessori e dai quali ha attinto per

pe moderne une espèce de république, dont les membres indépendent, mais liés par l'intérêts commun, se réunissent pour y maintenir l'ordre et la liberté. C'est ce qui a donné naissance à cette fameuse idée de la balance politique, ou de l'équilibre du pouvoir. On entend par-là une disposition des choses, au moyen de laquelle aucune puissance ne se trouve en état de prédominer absolument, et de faire la loix aux autres $\%$. La stessa citazione si trova anche nell'edizione curata da De Felice: Burlamaqui (1768) t. VI, 345. Occorre precisare che Michel Foucault esprimendosi sull'equilibrio di potere lo definiva come una situazione pratica «storicamente falsa " ma tale idea, in un certo senso, oltrepassava la realtà divenendo una sorta di principio cardine: Foucault (2005) 221. Per una completa disamina dei rapporti tra bilanciamento dei poteri e diritto nel Settecento si rimanda all'interessantissima monografia di DHоNDt (2015b) e DHоNDt (2016c) 329349; Dноndt (2016d) 67-83.

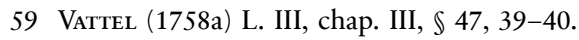

60 Воввіо (2009) 161. 
il suo Droit des gens, ma non si è identificato completamente. La grande differenza è insita nel fine che contraddistingue il trattato. In effetti nel momento in cui viene deliberatamente scelto di scrivere una opera per coloro che esercitano un ruolo di potere si esplicita una strategia politica e giuridica (contenuta soprattutto nel primo libro del trattato) che mira alla sociabilità e al raggiungimento della felicità della Nazione, obiettivo che $\mathrm{i}$ trattati precedenti giusnaturalisti non avevano chiarito così palesemente.

Per il contenuto del trattato altamente politico e giuridico è necessaria una precisazione lessicale e terminologica che ha ripercussioni sulla ricostruzione del pensiero vatteliano. Nel Droit des gens, infatti, si possono identificare $\mathrm{i}$ tre termini semantici a cui corrispondono le tre diverse categorie concettuali di «Stato », « Nazione» e « Potenza ». Tali termini dalle connotazioni iterative e ieratiche permettono di cogliere le differenze, le specificità e le reversibilità dei rapporti giuridici e politici che tendono ad intersecarsi, talvolta ad assumere apparentemente lo stesso significato, altre volte ancora a contraddirsi.

\section{Il « piano » della Nazione: la costituzione}

All'interno del capitolo I del I libro del Droit des gens viene circoscritto il problema della sovranità statale: le nazioni hanno volontà propria e il diritto delle genti stabilisce i loro diritti e i loro obblighi. Ogni Nazione che si governa da sé medesima, sotto qualsiasi forma, repubblicana o monarchica, senza essere dipendente da qualsiasi altro Stato, si definisce Stato sovrano e i suoi diritti sono naturalmente gli stessi di qualsiasi altra Nazione. ${ }^{\mathbf{6 1}}$ Soltanto una Nazione sovrana ed indipendente, cioè che si governi con la propria autorità e le sue leggi, può entrare e far parte della grande società delle nazioni. ${ }^{62}$

Gli unici Stati che non sono soggetti del diritto delle genti sono quelli che "sont passés sous la Domination d'un autre », ${ }^{63}$ discostandosi ancora una volta dal pensiero wolffiano, il quale invece, aveva ricondotto il concetto di

61 Vattel (1758a) L. I, chap. I, $\mathbb{S} 4,18$.

62 Ibidem: "Pour qu'une Nation ait droit de figurer immédiatement dans cette grande Société, il suffit qu'elle soit véritablement souveraine et indépendante, c'est-à-dire qu'elle se gouverne elle-même, par sa propre autorité et par ses Loix ».

63 Vattel (1758a) L. I, chap. I, $\mathbb{\$} 11,21$.

$90 \quad$ Capitolo II 
dipendenza, in funzione del «summum imperium » e al « rector civitatis », con particolare riguardo ai trattati ineguali e alle unioni federative. ${ }^{\mathbf{6 4}}$

Se per Vattel dunque gli Stati sono composti da uomini naturalmente liberi ed indipendenti, allo stesso modo anche le nazioni devono essere considerate libere ed indipendenti le une dalle altre, ed è mediante questa concezione che critica la posizione di Wolff sul fondamento dei cosiddetti «Stati patrimoniali ». Il professore di Halle, accogliendo le posizioni di autori precedenti e contemporanei, dedicò moltissimo spazio all'esistenza degli Stati o regni patrimoniali, senza rigettare o correggere, per usare le stesse parole di Vattel, una teoria così umiliante per l'umanità, ${ }^{65}$ e di cui quest'ultimo non ammette neppure la denominazione che trova impropria, offensiva e pericolosa nei suoi effetti. ${ }^{66}$

Egli afferma che lo Stato non può assolutamente essere considerato come un patrimonio, perché ogni sovranità ha insita la caratteristica della inalienabilità; quando un principe elegge il suo successore o quando cede ad un altro la corona, non fa altro che nominare in forza del potere che gli è Stato conferito o sotto forma espressa o tacitamente, colui che governerà al suo posto; ${ }^{67}$ ne consegue che lo Stato non è un oggetto bensì un soggetto e non può in nessun caso essere qualificato come un patrimonio nelle mani di un sovrano. ${ }^{68}$ Questa visione dello Stato-soggetto, unita all'affermazione dello Stato-collettività (e non più civitas maxima) permetterà al giurista di Neuchâtel di rivestire durante tutto il Settecento, ma anche l'Ottocento, un posto centrale sia nel campo del diritto internazionale sia nell'elaborazioni delle costituzioni moderne.

Vattel, per rafforzare la sua tesi, presenta una serie di esempi concreti attraverso cui la sovranità possa essere esercitata e a chi debba spettare il giudizio sulla contestazione di una successione. Il più interessante è senza dubbio il caso del 1707 e che riguarda proprio il Principato di Neuchâtel. Dopo la morte di Marie de Nemours (appartenente alla dinastia francese d'Orléans-Longueville) avvenuta nel 1707, il Tribunale sovrano dei Tre Stati,

64 Sul punto si rimanda a: Bachmann (1977) 158 ss.

65 Vattel (1758a) Préface, XVI.

66 Vattel (1758a) Préface, XVI. Per una disamina della critica agli Stati patrimoniali cfr. MANCuSO (2002) 205 ss.

67 Vattel (1758a) L. I, chap. V, $\$ 69,69-70$.

68 Jouannet (2000) 161. 
che rappresentava l'organo giudiziario supremo del principato (che nel 1694 aveva posto Marie al governo di Neuchâtel) aveva il compito di indicare il suo successore. Durante l'estate del 1707, diciannove presunti eredi sostennero energicamente la loro causa davanti ai dodici giudici di Neuchâtel che costituivano il tribunale. Solo nove pretendenti, che giustificavano la loro legittimità, invocando diritti testamentari o ereditari nella successione dei d'Orléans-Longueville o in quella del casato de Châlon, vennero infine ammessi al processo. I giudici manifestarono la loro opposizione ad ogni influenza francese e il 3 novembre 1707 designarono come principe di Neuchâtel Federico I, re di Prussia. Fu proprio in questa vicenda che lo zio di Vattel, Emer de Montmollin, appoggiò la politica di Federico I e venne nominato cancelliere di Stato prussiano. ${ }^{69}$

Con queste parole orgogliose Vattel riporta la coraggiosa scelta del Principato di Neuchâtel, chiamata a decidere chi fosse il successore nel suo governo: «Les Etats de la principauté de Neuchâtel ont souvent prononcé, en forme de Sentence juridique, sur la succession à la souveraineté. En l'année 1707, ils jugérent entre un grand nombre de Prétendants ; et leur Jugement, rendu en faveur du roi de Prusse, a été reconnu de toute l'Europe dans le Traité d'Utrecht ${ }^{\mathbf{7 0}}{ }^{\mathbf{0}}$

Egli delinea inoltre i principi generali dei doveri di una Nazione verso se stessa, che confluiscono nel binomio «conservazione e perfezione» di uno Stato. ${ }^{71}$ Il primo consiste nella durata dell'associazione politica che costituisce la Nazione e qualora essa volga al termine, secondo Vattel, esistono solo gli individui che la componevano; mentre la perfezione di una Nazione si trova in tutto ciò che permette ad essa di raggiungere il suo fine « et l'état d'une

69 Grandpierre (1829) 15 ss.; Chambrier (1840) 494-509; Roulet (1987) 99-151; ScheuReR (1987) 54 ss.; ThÉvenaz (1948) 52 ss.; Bachmann (1993) 193 ss.; Stribrny (1998) 21. Cfr. anche Toyoda (2011) 165 ss.

70 VATtel (1758a) L. I, chap. V, $\$ 66,66$.

71 Vattel (1758a) L. I, chap. II, $\mathbb{S} 13$, 22: «Une nation est un être déterminé par ses attributs essentiels, qui a sa nature propre, et qui peut agir convenablement à cette nature. Il est donc des Actions d'une Nation, comme telle, qui la concernent dans sa qualité de Nation, et qui sont convenables ou opposées à ce qui la constituë telle, ensorte qu'il n'est point indifférent qu'elle commette quelques-unes de ces actions et qu'elle en omette d'autres. La Loi Naturelle lui prescrit des Devoirs à cet égard». 
Nation est parfait, lorsqu'il n'y manque rien de tout ce qui lui est nécessaire pour arriver a cette fin ${ }^{72}$

Viene illustrato quale è il fine della società civile, inteso come il procurare ai cittadini tutte le « cose » di cui essi hanno bisogno per le loro necessità, per la convenienza e per le comodità della vita, che concorrono in modo più generale alla felicità $e$, ancora più importante, occorre che ciascuno possa godere del suo, ottenere giustizia con sicurezza e difendersi contro ogni violenza esterna. ${ }^{73}$

Identificare il fine della società civile nella realizzazione della felicità dei cittadini e nell'ottenimento della giustizia attraverso la sicurezza, sono principi estremamente importanti e contribuiscono al passaggio da una concezione di Stato che impone ad uno Stato che regola e si determina sulla vita dei suoi cittadini. Ciò significa l'attuazione di una serie di meccanismi che sono Stati progettati nel XVIII, nell'era definita da Foucault della "governabilità », la quale si manifesta sul piano interno ed esterno, sul piano pubblico e privato e permette allo Stato, come entità astratta, di determinarsi e di prendere sostanza nella Nazione. ${ }^{74}$

Il pensiero vatteliano si innesta e prende forza all'interno di questo movimento settecentesco: nel Droit des gens sono tracciate le caratteristiche essenziali della costituzione di uno Stato, in cui si ribadisce la massima che ogni società deve stabilire un'autorità pubblica che ordini gli affari comuni e prescriva ad ognuno la propria condotta tenendo ben presente il bene pubblico; un'autorità che appartiene al corpo della società, sebbene possa essere esercitata in modi diversi. La costituzione è la carta superiore e fondamentale di uno Stato, la quale risulta da un atto di sovranità della Nazione, che determina il modo attraverso cui l'autorità pubblica deve essere esercitata: «En elle se voit la forme sous laquelle la Nation agit en qualité de Corps

72 VAttel (1758a) L. I, chap. II, $\mathbb{\$} 14,23:$ «On sait que la perfection d'une chose consiste en général, dans un parfait accord de tout ce qui constitue cette chose-là, pour tendre à la même fin. [...] Une Nation étant une multitude d'hommes unis ensemble en Société Civile; si dans cette multitude tout conspire à obtenir la fin que l'on se propose, en formant une Société Civile, la Nation est parfaite: Et elle le sera plus ou moins parfait, selon qu'elle approchera plus ou moins de ce parfait accord. De même, son état externe sera plus ou moins parfait, selon qu'il concourra avec la perfection intrinsèque de la $\mathrm{Na}$ tion ".

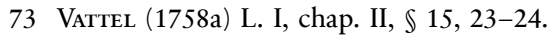

74 Foucault (2005) 89; Jouannet (2006) 58. 
Politique; comment et par qui le Peuple doit être gouverné, quels sont les droits et les devoirs de ceux qui gouvernent. Cette Constitution n'est dans le fond autre chose que l'établissement de l'ordre dans lequel une Nation se propose de travailler en commun à obtenir les avantages en vue desquels la Société Politiques s'est établie ». ${ }^{75}$

La costituzione dello Stato decide della sua perfezione e della sua attitudine al raggiungimento del fine della società, aggiungendo, che nel momento in cui viene emanata, vengono posti i fondamenti per la conservazione statale, per la sua sicurezza e per la sua felicità. ${ }^{76}$ Per la prima volta viene attribuito al concetto di costituzione "una definizione autonoma, indipendente da altri contesti, ed una nuova dimensione contenutistica, anche se questa in parte si ricollega agli elementi tradizionali quali la forma di Stato, il bene pubblico, il corpo statale, le leggi fondamentali e l'efficacia vincolante $»^{77}$

L'autorità pubblica stabilisce le leggi, alcune delle quali regolano le relazioni tra privati e perciò vengono chiamate leggi civili, mentre ve ne sono altre direttamente orientate al bene pubblico « et dans cette classe, celles qui concernent le Corps même et l'essence de la Société, la forme du Gouvernement, la manière dont l'Autorité Publique doit être exercée, celles, en un mot, dont la concours forme la Constitution de l'État, sont les Loix Fondamentales $\gg .^{78}$ Come ben indicato da Heinz Mohnhaupt, il giurista di Neuchâtel (esattamente come aveva argomentato Montesquieu) nega l'esistenza di una costituzione che possa essere valida per tutti i popoli, in quanto l'adattamento alle condizioni particolari e alle singole circostanze è un requisito indispensabile e necessario. ${ }^{79}$

Vattel precisa, ancora una volta, che la costituzione dello Stato e le sue leggi sono la base della pubblica tranquillità, « il più fermo sostegno dell'autorità politica e il pegno della libertà dei cittadini », tuttavia essa è destinata a rimanere "lettera morta », un vano simulacro, se non viene rigorosamente osservata. La Nazione deve vegliare incessantemente su di essa e deve garantire il suo rispetto tanto da parte di coloro che governano, quanto da parte

75 VATtel (1758a) L. I, chap. III, $\mathbb{S} 27,31$.

76 Vattel (1758a) L. I, chap. III, $\mathbb{2} 28,31-32$.

77 Mohnhaupt (2008) 103.

78 VATtel (1758a) L. I, chap. III, $\mathbb{S} 29,32$.

79 Mohnhaupt (2008) 104. 
del popolo. Attaccare la costituzione, violare le sue leggi è un «delitto capitale» contro la società e i soggetti che hanno compiuto un tale crimine devono essere repressi. ${ }^{\mathbf{8 0}}$

Partendo dalle considerazioni di Vattel si intuisce il passaggio dalla legge fondamentale, intesa come astratta, alla costituzione di uno Stato come legge positiva, cioè come "réglement fondamental ». ${ }^{\mathbf{8 1}}$

Mentre per Grozio la costituzione era l'insieme delle regole essenziali per lo Stato, ed era chiamato «Lex », ${ }^{\mathbf{8 2}}$ per Pufendorf era definita «Lex regni », cioè l'insieme di leggi che formano un piccolo gruppo di regole essenziali riguardanti l'esercizio del potere, ${ }^{\mathbf{8 3}}$ tradotta poi con Barbeyrac in "Loi fondamentale $;^{84}$ per Burlamaqui le leggi fondamentali «proprement ainsi nommés ne sont que des précautions plus particulières que prennent les peuples, pour obliger plus fortement les souverains à user de leur autorité, conformément à la règle générale du bien public ${ }^{85}$ Anche Wolff si poneva in linea con tali definizioni tradizionali, approfondendo peró le leggi fondamentali nella loro vasta pluralità. ${ }^{86}$

80 Vattel (1758a) L. I, chap. III, $\mathbb{3} 30,33-34$.

81 Tomás y Valiente (2003) 34-35.

82 Grotius (1625) L. I, cap. III, $\mathbb{S}$ XVIII, 83 ss.

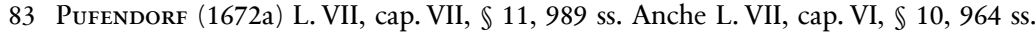

84 Riflessioni argomentate da Barbeyrac all'interno della sua traduzione a Pufendorf (1712) L. VII, chap. VII, $\mathbb{S} 11,319$.

85 Burlamaqui (1751), t. II, premier partie, chap. VII, $\mathbb{S}$ XLI, 57.

86 WolfF (1750) P. III, cap. I, $\mathbb{9} 984,603-604$ : «Leges, ad quas exercitium imperii adstringitur, fundamentales dicuntur. Quamobrem lex quoque fundamentalis est, ut Rector civitatis in certis negotiis requirat consensum vel populi universi, vel certorum quorundam, et qua disponitur de modo trasmittendi, vel deferendi imperium. Patet praetera, Rectorem civitatis obligari ad legum fundamentalium observantiam, nec eas pro lubito suo mutare posse. Cum vero leges fundametales fiant consensu populi; eodem quoque tolli et mutari possunt, modo ius Rectori civitatis, aut eius successoribus inde quaesitum non diminuatur. Enimvero quia leges fundamentales a populo fiunt pro lubitu imperium in Rectorem civitatis trasferende; $s i$ Rector civitatis, qui absolutum imperium habet, ipse certas leges constituit, iuxta quas imperium exercere velit, vel etiam concilium, sine cuius approbatione quid facere nolit, eadem leges fundamentales non sunt, ac per consequens Rector civitatis non semper necessario easdem observare tenetur, immo si ita visum fuerit, eas prorsus tollere potest. Quamobrem nec ea de causa imperium esse definit absolutum atque summum. Idem valet, si populus saltem exprimit voluntatem suam de nonnullis, qua Rector civitatis facere, aut non facere debeat, non vero expresse declarat, se eius iudicio stare molle, nec concilium constituit, sine cuius consensu nibil eorum decernere valet; cum nullum sibi ius in actus Rectoris reservet populus ». 
$\mathrm{Al}$ contrario, invece, Vattel si colloca nella prospettiva " de lege ferenda ${ }^{87}$ e soprattutto identifica esplicitamente che cosa sono la costituzione e le leggi fondamentali: un "piano" «sur lequelle la Nation est résolu de travailler à son bonheur ». ${ }^{88}$

Identificando la costituzione come un "piano", egli si discosta dalla concezione della costituzione come patto dei consociati: il patto, per gran parte dei giusnaturalisti, era lo strumento attraverso cui si realizzava e prendeva forma il patto sociale, mentre per Vattel è lo strumento con cui la società civile si determina politicamente cerca il suo vantaggio, la sua fortuna e la sua felicità nella sua socializzazione. ${ }^{89}$ Questa è la versione concretamente moderna della precedente concezione dello Stato di natura e dell'associazione politica che ora si realizza attraverso il contratto sociale e come una autorità « esente da calcoli utilitaristici ».90

Tale definizione ha ricadute anche sul processo legislativo, perché la costituzione viene cristallizzata rispetto al normale corso della legislazione statale e non subisce alcuna modifica né per il passato né per il futuro. Il giurista di Neuchâtel non ammette la modifica della costituzione da parte degli organi legislativi qualora la Nazione non abbia espressamente conferito loro il potere della sua modifica e in forza di questa disposizione che la costituzione contravviene al principio lex posterior derogat legi priori, restando «immunizzata contro la legislazione successiva ». ${ }^{91}$ Questi principi vengono elaborati per la Nazione e Vattel specifica che è esclusivamente ad essa che si rivolge, abbracciando così una chiara e netta distinzione tra lo Stato e la Nazione. ${ }^{92}$ Spetta, dunque, a chi governa e detiene il potere rispettare e mantenere le leggi fondamentali di uno Stato, come se si trattasse di una osservanza religiosa, e pertanto esse devono essere considerate come regole sacre e inviolabili. ${ }^{93}$

Significativo è il richiamo alle teorie vatteliane all'interno della Représentations des citoyens et bourgeois de Genève au Premier Syndic de cette République avec les Réponses du Conseil à ces Représentations dell'8 agosto 1763, quando

87 Tomás y Valiente (2003) 34-35.

88 Vattel (1758a) L. I, chap. IV, $\mathbb{4} 46,44$.

89 Hofmann (2005) 227-238, qui 231.

90 Hofmann (2005) 228.

91 Vattel (1758a) L. I, chap. III, $\int \mathbb{S} 33-34,35-37$. Hofmann (2005) 229.

92 VAttel $(1758 \mathrm{a})$ L. I, chap. III, $\mathbb{S} 32,35$.

93 Vattel (1758a) L. I, chap. IV, $\$ 46,44$.

96 Capitolo II 
un centinaio di cittadini ginevrini difesero pubblicamente Rousseau. ${ }^{94} \mathrm{Si}$ aprì un vero e proprio caso di diritto costituzionale in merito ai poteri che esercitò le Petit Conseil, sotto l'impulso del procuratore generale JeanRobert Tronchin, nel censurare gli scritti di Rousseau. Come è noto il 9 giugno 1762, l'Emile venne condannato dal Parlamento di Parigi, Rousseau colpito dal mandato di cattura, prevenne l'arresto lasciando Montmorency e recandosi in Svizzera. Il 14 giugno arrivò a Yverdon, nel territorio bernese, ma nello stesso periodo il Contrat Social e l'Emile vennero vietati e respinti anche a Ginevra. Espulso anche dal territorio di Berna, nel luglio dello stesso anno si rifugiò a Môtiers-Travers, nella contea di Neuchâtel, e nel frattempo anche i Paesi Bassi e il Consiglio Scolastico di Berna condannarono l'Emile. Il 28 agosto fu pubblicato il Mandement contro l'Emile scritto e pubblicato dall'arcivescovo di Parigi Christophe de Beaumont du Repaire, che lo definì come un uomo "plein du langage de la philosophie, sans être véritablement philosophe ».95 Rousseau rispose alle accuse nel marzo 1763, descrivendosi a sua volta come un pensatore "plus ardent qu'éclairé », ${ }^{\mathbf{9 6}}$ ma il Petit Conseil il 29 aprile proibì la sua ristampa. ${ }^{97} \mathrm{Fu}$ così che con una lettera indirizzata al Premier Syndic, il 12 maggio, Rousseau rinunciò al diritto di cittadinanza nella repubblica ginevrina e come ha scritto Jean-Daniel Candaux «les citoyens et Bourgeois en furent vivement impressionnés et ils en conçurent même un complexe de culpabilité qui les porta à l'action : $^{98}$ i cittadini reagirono attraverso le cosiddette Représentations.

Uno dei punti della Représentation dell'8 agosto 1763 si basava "sur la nature de nôtre Constitution et sur la liberté publique établie dans nôtre Ville a l'egard des ouvrages de Droit et de Politique »: a supporto delle rimostranze dei cittadini ginevrini viene espliciatamente citato il trattato di Vattel e il passaggio in cui afferma che «S'il s'eleve dans l'Etat des contestations sur les Loix fondamentales, sur l'administration publique, sur les droits des differentes Puissances qui y ont part; il appartient uniquement à la Nation d'en juger et de les determiner conformement à la Constitution

94 Représentations des citoyens et bourgeois de Genève au Premier Syndic de cette République avec les réponses du Conseil à ces représentations (1763) 76 e anche 233.

95 Beaumont du Repaire (2012) 1128.

96 Rousseau (2012a) 1173.

97 Gatti (2005) 5-40; Gatti / Chiodi (a cura di) (2014).

98 Gouhier (1983) 206-207. 
politique ».99 A tale iniziativa, che rimase inascoltata, qualche settimana, dopo il 20 agosto, se ne aggiunse un'altra, questa volta sostenuta da quattrocentottanta cittadini. Essi domandavano che la questione venisse sottoposta al Conseil Général, considerato, in definitiva, come il solo interprete delle leggi. La risposta fu resa il 31 agosto da Tronchin, il quale legittimava le prerogative del Petit Conseil alla stregua delle leggi della Repubblica. Il 29 settembre i cittadini energicamente rifiutarono le argomentazioni del procuratore generale attraverso una nuova Représentation, ma la pubblicazione delle Lettres écrites de la campagne di Tronchin diede una nuova dimensione alla discussione, a cui seguì la risposta di Rousseau edita nel dicembre del 1764 attraverso le Lettres écrites de la montagne. ${ }^{\mathbf{1 0 0}}$

Oltre all'esempio all'interno della vicenda di Rousseau, l'applicazione del concetto di costituzione elaborato da Vattel lo si può altresì riscontrare tanto nella divulgazione scientifica quanto sul versante storico-politico del tardo Settecento. Si è assistito ad una vera e propria diffusione del suo pensiero ad opera di Fortunato Bartolomeo de Felice: la voce Constitution d'État pubblicata nella sua Encyclopédie ou dictionnaire universel raisonée des connaissances humaines, è scritta sulla falsariga del pensiero del giurista di Neuchâtel, da cui ha attinto tanto nell'impostazione quanto nei contenuti. ${ }^{101}$

Si comprendono i motivi dell'accoglimento vatteliano anche nelle rivoluzioni americana e francese e in molte costituzioni moderne. La ricapitolazione sistematica delle norme fondamentali viene raggiunta politicamente nelle colonie del Nord America, dove le proposizioni di Vattel erano sempre citate e molto hanno influito su uomini come James Otis e Benjamin Franklin. ${ }^{\mathbf{1 0 2}}$ Allo stesso modo con la Rivoluzione francese la costituzione viene

99 Vattel (1758a) L. I, chap. III, $\mathbb{3} 36,38$.

100 Rousseau (2012b) 159-540. Cfr. Gouhier (1983) 206-207. Anche: Bernardi/Guérard/ Silvestrini (sous la direction de) (2005); Whatmore (2006) 385-413, qui in particolare 399 ss.; Silvestrini (2010b) 69-87; Silvestrini (2010c).

101 De Felice (1772) 189 e ss. Sul punto: Baker (1990) 256; Kates (1998) 70.

102 Ossipow e Gerber hanno recentemente pubblicato un saggio concernente l'influenza del Droit des gens negli Stati Uniti, distinguendo in particolare tra ricezione politica e giuridica; sotto il primo profilo si riscontra l'utilizzo di Vattel in molti dibattiti politici, soprattutto all'interno del Primo e del Secondo Congresso Continentale (1774, 1775-1781), del Comitato degli Affari Esteri (1777-1781), del Congresso della Confederazione svoltosi tra il 1781 e il 1789; mentre per quanto riguarda la ricezione giuridica, essa inizia con la Dichiarazione d'Indipendenza e culmina nel secolo XIX con l'utilizzo di Vattel in ambito 
definita in base alle sue finalità, come organizzazione del potere statuale necessario a favorire la libertà e la garanzia della sicurezza nazionale, raggiungendo la sua cristallizzazione nell'articolo 16 della Déclaration secondo cui: «Toute société dans laquelle la garantie des droits n'est pas assurée, ni la séparation des pouvoirs déterminée n'a point de constitution ». ${ }^{103}$

Significativa è la stretta somiglianza con le teorie costituzionali di uno dei più celebri politici rivoluzionari, Emmanuel Sieyès, secondo Paolo Colombo, « le omogeneità dottrinali tra de Vattel e quello che viene universalmente riconosciuto come il padre francese delle teoria del potere costituente sono assai più che marcate e quasi sicuramente esulano dal campo delle semplici coincidenze, quantomeno perché hanno probabili precedenti comuni alle spalle ». ${ }^{104}$

Le argomentazioni vatteliane si ritrovano in quelle di Sièyes: la Nazione è il soggetto del potere costituente; viene esplicitata l'accettazione del principio maggioritario per il processo della legislazione ordinaria; l'individuazione dello strumento istituzionale attraverso il quale la Nazione può intervenire sulla costituzione negli organi legislativi ordinari, investiti però di un preciso mandato costituente; e l'importantissima differenza, che influenza il contrattualismo moderno, tra potere costituente e potere costituito. I poteri ordinari, come si è visto, per Vattel non possono intervenire sulla costituzione perché un soggetto delegato non può intervenire sull'atto della delega: questo rappresenta la divisione, ripresa in Sièyes, tra potere costituente e potere costituito che sarà alla base della Rivoluzione Francese, ${ }^{105}$ e di molti altri contesti europei e non europei ottocenteschi. ${ }^{106}$

Anche in una altra occasione della Rivoluzione Francese, Vattel ha assunto un ruolo singolare. L'abate Henri Grégoire (1750-1831) propose per ben due volte alla Convenzione (la prima il 18 giugno 1793 e la seconda il 23

giurisprudenziale: Ossipow/Gerber (2010) 79-118. Cfr. anche il già citato: ChetaiL (2014) passim .

103 Hofmann (2005) 229.

104 Соцомво (2014) 117-128, qui 119.

105 Cоцомво (2014) 119-120.

106 Cfr. anche: Jouannet (1998) 339. Anche Manz (1971) 89; Nussbaum (1954) 158. Mallarmé scriveva: «si l'on veut rattacher Vattel à la Révolution française il ne faut le considérer que comme un précurser, sans doute hardi, mais ancore éloigné »: Mallarmé (1904) 598. Cfr. Nys (1896) 318-406; Géraud-LlorCa, (1993) 211-225; Edelstein (2008) 229262; Edelstein (2009). 
aprile 1795), una Déclaration du droit des gens. ${ }^{107}$ Essa, composta da ventuno articoli, sancisce numerosi principi generali e assoluti, soprattutto in tema di sovranità, di indipendenza, della libertà di scelta della migliore forma governativa, del potere esclusivo della Nazione al cambiamento di essa, dei trattati internazionali e del loro rispetto. ${ }^{\mathbf{1 0 8}} \mathrm{Si}$ tratta di una vera e

107 Egli è una delle figure più significative del Settecento, propugnatore dell'abolizione dei privilegi e della schiavitù e sostenitore del suffragio universale. Reso celebre dal punto di vista pittorico dall'immortalazione che David ne fece nel Serment du Jeu de Paume, si battè per tutta la vita per gli Ebrei residenti in Francia, che vennero difesi da Grégoire nell'altrettanto celebre Essai sur la régénération physique, morale et politique des Juifs del 1788. L'abate Grégoire pubblicò nel 1808 De la littérature des nègres, ou Recherches sur leurs facultés intellectuelles, leurs qualités morales et leur littérature. Quest'opera intende dimostrare l'uguaglianza fondamentale di tutte le società e di tutti gli uomini, sostenendo che tutti possono raggiungere lo stadio di evoluzione civile presente nelle più alte élite culturali europee. Nello specifico Grégoire ha ricostruito il profilo biografico di artisti e scrittori neri e meticci, illustrandone i meriti e le caratteristiche dei loro lavori. De la littérature riscosse un immediato successo e fu tradotta sia in inglese che in tedesco ed influenzò il movimento antischiavista dei secoli XIX e XX. Cfr. Lüsebrink (1997) 168-178; HermonBelot (2000); Popkin / Popkin (ed.) (2000), anche Grewe (2001) 416. Scrive Losada: "A diferencia de los utopistas de "Las Luces", muy pronto econtramos a Henri-Baptiste Grégoire movilizado por las grandes causas de la humanidad: la libertad de los judíos, la emancipación de los negros (especialmente los de Haíti), la suerte de los pobres y los humildes, en una grandiosa visión de una confederación de sabios, filósofos y teólogos de toda Europa que trabajarían para exaltar los beneficios aportados por el cristianesimo a la humanidad, desde sus orígines, y para reunir las iglesias separadas »: Losada (1989) 71.

108 Belissa (octobre 2010). Anche Belissa (1998). Questo è il testo: " $1^{\circ}$ Les peuples sont entre eux dans l'état de nature; ils ont pour lien la morale universelle; $2^{\circ}$ Les peuples sont respectivement indépendants et souverains quel que soit le nombre d'individus qui les composent et l'étendue du territoire qu'ils occupent. Cette souveraineté est inaliénable; $3^{\circ}$ Un peuple doit agir à l'égard des autres comme il désire qu'on agisse à son égard; ce qu'un homme doit à un homme, un peuple le doit aux autres; $4^{\circ}$ Les peuples doivent, en paix, se faire le plus de bien, et, en guerre, le moins de mal possible; $5^{\circ}$ L'intérêt particulier d'un peuple est subordonné à l'intérêt général de la famille humaine; $6^{\circ}$ Chaque peuple a le droit d'organiser et de changer les formes de son gouvernement; $7^{\circ} \mathrm{Un}$ peuple n'a pas le droit de s'immiscer dans le gouvernement des autres; $8^{\circ}$ II n'y a de gouvernement conforme aux droits des peuples que ceux qui sont fondés sur l'égalité et la liberté; $9^{\circ} \mathrm{Ce}$ qui est d'un usage inépuisable ou innocent, comme la mer, appartient à tous, et ne peut être la propriété d'aucun peuple; $10^{\circ}$ Chaque peuple est maître de son territoire; $11^{\circ}$ La possession immémoriale établit le droit de prescription entre les peuples; $12^{\circ} \mathrm{Un}$ peuple a droit de refuser l'entrée de son territoire et de renvoyer les étrangers, quand sa sûreté l'exige; $13^{\circ}$ Les étrangers sont soumis aux lois du pays et punissables par elles; $14^{\circ} \mathrm{Le}$ bannissement pour crime est une violation indirecte du territoire étranger; $15^{\circ}$ Les entreprises contre la liberté d'un peuple sont un attentat contre tous les autres; $16^{\circ}$ Les ligues 
propria «sintesi dei dibattiti politici dell'illuminismo sulle relazioni tra $\mathrm{i}$ popoli e sugli Stati. La prospettiva cosmopolita è riassunta in un modo che non ha uguali ». ${ }^{109}$ Egli si inspira palesemente alle idee di Vattel, che viene citato più volte all'interno del suo discorso dell'aprile 1795. Chevalley sostiene che sia sufficiente solo leggere il titolo della Dichiarazione di Grégoire per comprendere quanto racchiuda in sé i principi vatteliani: egli infatti «suit son guide, et le suit pas à pas ». ${ }^{\mathbf{1 1 0}}$

Secondo Théodore Ruyssen questa dichiarazione, benchè non fu mai sottoposta a votazioni nè venne mai accolta, ${ }^{111}$ fu la " première énonciation précise des principes du droit international qui ait été soumise à une assemblée politique » ed è veramente indicativo che il pensiero di Vattel sia ripreso in toto perché sembra così confermare l'esigenza della proceduralizzazione del diritto interno costituzionale con ricadute su quello internazionale. ${ }^{\mathbf{1 1 2}}$

\section{L'arte di governare: l'organizzazione dello spazio sociale}

Si rileva una costante nella stesura del Droit des gens: è la straordinaria capacità sia di regolare e organizzare lo Stato che prende forma e concretezza

qui ont pour objet une guerre offensive, les traités ou alliances qui peuvent nuire à l'intérêt d'un peuple, sont un attentat contre la famille humaine; $17^{\circ} \mathrm{Un}$ peuple peut entreprendre la guerre pour défendre sa souveraineté, sa liberté, sa propriété; $18^{\circ}$ Les peuples qui sont en guerre doivent laisser un libre cours aux négociations propres à amener la paix; $19^{\circ}$ Les agents publics que les peuples s'envoient sont indépendants de $s$ lois du pays où ils sont envoyés, dans tout ce qui concerne l'objet de leur mission; $20^{\circ}$ II n'y a pas de préséance entre les agents publics des nations; $21^{\circ}$ Les traités entre les peuples sont sacrés et inviolables »: Interamente riportato in: Le Fur/ChkLaver (éd.)(1934) 68-69. E' Stato inoltre scritto: «Parce que l'abbé Grégoire n'était pas juriste de formation, il est fort probable qu'il s'est servi dans la rédaction de son projet de Déclaration de l'ouvrage de Vattel. On peut trouver chez eux les mêmes formulations en langue française. Néanmoins il avait complètement ignoré la doctrine vattelienne sur les devoirs d'une nation envers elle-même, sur le droit de nécessité, sur les droits parfaits et imparfaits, etc. Etant donné que Vattel était largement compilateur des idées de Christian Wolff, l'abbé Grégoire est, paradoxalement, successeur de la solidarité wolffienne à travers Vattel. Il est cependant moins probable, mais point exclu, qu'il ait eu accès aux ouvrages de Wolff, publiés en latin »: Degan (1989) 110, nota 12.

109 Belissa (octobre 2010).

110 Chevalley (1912) 91-92; Pisanó (2002) 300 ss.; Sepinwall (2005) 81 ss.

111 Keitner (2007) 94. Cfr. anche Baker (1994) 177 ss.

112 RuYsSen (1961) 57. 
nella Nazione, sia di aspirare alla creazione di esso con una funzione non repressiva, bensì « regolativa ». Le leggi per Vattel altro non sono che delle regole stabilite dall'autorità pubblica per essere osservate dalla società e indirizzate al bene dello Stato e dei cittadini. ${ }^{113}$

La regolazione e l'organizzazione della società in termini spaziali e sociali è una priorità per il giurista di Neuchâtel e solo quando uno Stato ha realizzato al suo interno i suoi doveri, può dirsi Nazione e dialogare con le altre, articolando le «sue » relazioni internazionali. Lo Stato rappresenta l'entità astratta, il cosiddetto contenitore, il cui contenuto è la Nazione stessa, che si determina attraverso la promulgazione della costituzione, il buon governo, inteso come il consequenziale raggiungimento della felicità e del benessere dei cittadini.

Una volta delineato il cosiddetto sistema interno di uno Stato, stabilendo la sua sovranità e il concetto di costituzione, Vattel, infatti, illustra i tre principali «oggetti» di un buon governo: il primo consiste nel provvedere ai bisogni di una Nazione, nel quale fanno parte il dovere di incoraggiare tanto il lavoro quanto l'industrializzazione, la circolazione della moneta, la coltivazione delle terre (definita come un obbligo imposto dalla natura), la libertà di commerciare e anche la libertà di poter rifiutare il commercio estero. ${ }^{114}$

Ancora una volta viene ribadito che la Nazione si deve attivare affinchè ci sia nel suo territorio "une heureuse abondance de toutes les choses nécessaires à la vie » ma ancora più significativo, quasi a rafforzare il legame tra la «fabbricazione e aspirazione alle vita » e il governo, le "même des modités et des agréments innocents et louables ». E specifica che «Outre qu'une vie aisée, sans mollesse, contribue au bonheur des hommes, elle les met en état de travailler avec plus de soin et de succès à leur propre perfection; ce qui est leur grand et principale devoir ». ${ }^{\mathbf{1 1 5}}$

Il secondo oggetto è procurare la vera felicità alla Nazione attraverso l'istruzione, l'educazione, l'amore per la patria, definita (quasi anticipando il significato ottocentesco) come: « lo Stato dove si è membri ». ${ }^{116}$ E' oppor-

113 VAttel (1758a) L. I, chap. III, $\ 29,32$.

114 Sull'analisi del rapporto tra commercio, proprietà e bene comune della Nazione: Porras (2014) 655 ss.

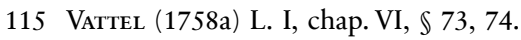

116 VATtel (1758a) L. I, chap. XI, $\$ 122,112-113$. 
tuno precisare che il termine «felicità » compare numerose volte nel Droit des gens, più di cinquanta volte all'interno dei quattro libri. Tale ricorrrente e volontaria ripetizione denota quanto essa (ri)comprenda anche quello di sicurezza e di benessere e sia un punto focale per l'organizzazione dello Stato: tutto ciò che può produrre ad un uomo la vera felicità, deve essere l'aspirazione di un buon governo e merita dunque la più seria attenzione da parte dei governanti. La felicità è il centro, a cui tendono tutti i doveri di un uomo e di un popolo ed è il più alto fine della legge naturale. Il desiderio di essere felice è quella forza vigorosa che fa muovere gli uomini e spetta dunque a chi governa dedicarsi e attivarsi per la realizzazione di essa, "promuovendola attraverso l'esercizio del loro potere $»{ }^{\mathbf{1 1 7}}$

Sempre in questa sezione si sofferma lungamente anche sulla pietà e sulla religione: «la Piété et la Religion influent essentiellement sur le bonheur d'une Nation. [...] Rien n'est si propre que la Piété à fortifier la Vertu, et à lui donner toute l'entenduë qu'elle doit avoir. [...] Une nation doit donc être pieuse. Que les Supérieurs chargés des affaires publiques, se proposent constamment de mériter l'approbation de leur divin Maître : Tout ce qu'ils sont au nom de l'État doit être réglé sur cette grande vuë. Le soin de former tout le peuple à la piété, sera toujours l'un des principaux objets de leur vigilance, et l'État en recevra de très grands avantages ». ${ }^{\mathbf{1 1 8}}$

La delicata riflessione riguarda anche « l'établissement public de la Religion: devoirs et droits de la nation » e in particolare si concentra sulla tolleranza che deve essere presente in uno Stato. Il mezzo più sicuro e più equo per evitare che all'interno di uno Stato possano verificarsi rivolte e scontri per la differenza di religione, pericolosi non tanto per i costumi quanto per lo Stato, è «l'universale tolleranza di tutte le professioni ». Vattel richiama la situazione politica dell'epoca a lui contemporanea: l'Olanda e il Regno di Prussia ne sono la prova, poiché convivono cattolici, luterani, ebrei, e tutti sono protetti dal sovrano per il raggiungimento della pace interna. ${ }^{119}$

Inoltre tra i mezzi per il raggiungimento della felicità vi sono anche la justice e la police, che si articolano nella necessità per uno Stato di avere giuste leggi, di istituire tribunali garanti del loro rispetto; viene così affrontato sinteticamente e, senza contribuire in modo incisivo, il problema delle pene

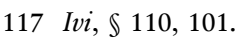

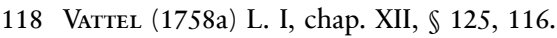

119 Ivi, chap. XII, $\mathbb{1} 135,125-126$. 
e della loro esecuzione per poi soffermarsi sulla police (tradotta « buon governo » nell'edizione italiana di Vattel ad opera del Loschi), che consiste nell'attenzione del sovrano e dei magistrati per il mantenimento dell'ordine. I regolamenti devono prescrivere tutto ciò che aspiri alla sicurezza, all'utilità e alla comodità pubblica; «Le souverain, par une sage police, accoûtume les peuples à l'ordre et à l'obéissance ; il conserve la tranquillité, la paix et la concorde parmi les Citoyens ${ }^{\mathbf{1 2 0}}$

Da questa definizione derivano una serie di circostanze in cui si manifesta il buon governo quali: i motivi che conducono alla proibizione del duello; le norme che ad esempio il sovrano, come un buon padre, deve emanare al fine di impedire tra i sudditi prevaricazioni economiche; i limiti della proprietà privata e il rispetto delle regole, soprattutto in questioni economiche; la repressione dei monopoli commerciali e di tutte le operazioni che tendono a rincarare i viveri e i beni di prima necessità. ${ }^{\mathbf{1 2 1}}$

Santiago Legarre ha giustamente osservato come Vattel, ponendo l'attenzione all'obbligo del sovrano a proposito del mantenimento dell'ordine interno, abbia contribuito in modo incisivo al passaggio tra "police into police power - what today is a settled legal category of the Constitutional Law of the Western states", elevandolo a " one of the great exponents of the idea of police - a domestic concept $»{ }^{\mathbf{1 2 2}}$

Proseguendo nell'illustrazione del contenuto del primo libro di Vattel, il terzo ed ultimo gruppo di oggetti essenziali al « bon gouvernement » è composto dalla necessità per uno Stato di «se fortifier contre les attaques du déhors », perché "si la Société n'est pas en état de repousser un agresseur, elle est très-imparfaite, elle manque à sa principale destination», e ancora «la

120 VATtel (1758a) L. I, chap. XIII, $\ 174,165$.

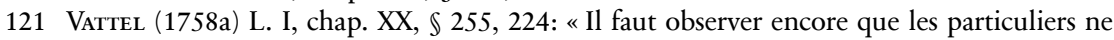
sont pas tellement libres dans l'économie ou le gouvernement de leurs biens, qu'ils ne demeurent sujets aux Loix et aux règlements de police faits par le Souverain. Par exemple, si les vignes se multiplient trop dans un pays, et qu'on y manque de bleds, le Souverain peut défendre de planter de la vigne dans les champs propres au labourage; car le bien public et le salut de l'État y sont intéressés. Lorsqu'une raison de cette importance le demande, le Souverain ou le Magistrat peut contraindre un particulier à vendre ses denrées, dont il n'a pas besoin pour sa subsistance, et en fixer le prix. L'Autorité publique peut et doit empêcher les monopoles, réprimer toutes les manœuvres tendantes à faire enchérir les vivres".

122 Legarre (2012) 437-438; Legarre (2007) 753 ss. 
Nation doit se mettre en état de repousser et de dompter un injuste Ennemi; c'est un devoir important, que le soin de sa perfection, de sa conservation même, lui impose, et à son Conducteur ». ${ }^{123}$ In tale categoria rientra tutto ciò che compete all'esercizio del potere statale che si articola nel "nombre des citoyens, leurs vertus militaires et les richesses ", comprendenti a loro volta « les forteresses, l'artillerie, les armes, les chevaux, les munitions ». ${ }^{\mathbf{1 2 4}}$

Al centro di questa sezione vi è tutto ciò che deve essere tutelato al fine di garantire la miglior sicurezza all'interno della Nazione, tra cui i rapporti tra una Nazione e lo status di cittadini, di emigranti, di esiliati. Successivamente si concentra sui beni pubblici, sui beni demaniali con particolare riguardo alla loro alienazione e sulle imposte, per poi concludere con una dettagliata trattazione sul diritto dei fiumi, dei laghi, del mare, e dei limiti spaziali dello Stato costiero sui mari. ${ }^{\mathbf{1 2 5}}$

Molto interessante è, senza dubbio, la disamina inerente l'occupazione di una Nazione presso un altro paese, tema che immediatamente evoca la conquista: ${ }^{126}$ Vattel assume una posizione « tipica » dell'epoca, giustificando la conquista inglese nel Nord-America, esaltando la figura di William Penn, esattamente come altri suoi contemporanei. E' sufficiente richiamare l'attenzione sull'opera pubblicata dall'abate Guillaume-Thomas François Raynal nel 1772, intitolata Histoire philosophique et politique des établissements et du commerce des Européens dans les deux Indes. ${ }^{\mathbf{1 2 7}}$ Essa è considerata una vera e propria enciclopedia dell'anticolonialismo del XVIII secolo e si pone all'interno dei cosiddetti "voyages philosophiques", molto diffusi durante l'epoca illuministica, e rappresenta sia un pretesto per la riflessione sul diritto natu-

123 Vattel (1758a) L. I, chap. XIV, $\mathbb{S} 177,171$.

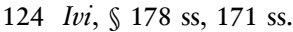

125 VAttel (1758a) L. I, chap. XXIII, $\mathbb{2} 279,243$.

126 «Le Droit des Gens ne reconnoîtra donc la propriété et la Souveraineté d'une Nation que sur les pays vuides qu'elle aura occupés réellement et de fait, dans lesquels elle aura formé un Etablissement, ou dont elle tirera un usage actuel »: Vattel (1758a) L. I, chap. XVIII, \208, 194.

127 In realtà l'opera fu pubblicata anonima nel 1770 , e venne vietata nel 1772 . Raynal allora la ripubblicò anonima nel 1774 e nuovamente venne messa all'indice. Ripubblicata con il suo ritratto nel 1780, l'Histoire venne fatta bruciare pubblicamente dal Parlamento di Parigi ed egli fu costretto a fuggire in esilio. Nel 1781 scappò in Svizzera, poi cercò rifugio presso la corte prussiana, successivamente in Russia da Caterina II, fino al ritorno in Francia nel 1784 ma con il divieto di soggiornare a Parigi. 
rale sia una manifesta denuncia contro il dispotismo, il clericalismo, la tratta degli schiavi e il colonialismo europeo. ${ }^{\mathbf{1 2 8}}$

Per la realizzazione di questa imponente opera Raynal si è servito dell'ausilio di una figura centrale dell'illuminismo francese, Denis Diderot. Come affermato da Lucio Villari si assiste alla fusione tra ideale e scienza: Raynal affronta il problema delle relazioni internazionali ed economiche fra l'Europa e le Indie orientali e occidentali, mentre Denis Diderot si sofferma sulla critica al colonialismo. ${ }^{129}$

Denis Diderot e Guillaume-Thomas Raynal, nelle edizioni del 1774 e del 1780 dell'Histoire des deux Indes, ai termini «civilisation » e « civilisé » associano una ampia riflessione sulla storia dell'espansione europea nelle Indie, dai primi viaggi dei navigatori portoghesi del secolo XV sino alla Rivoluzione Americana. Ma l'Europa pioniera della «civilizzazione » è anche culla di contraddizioni, basti pensare alla legittimazione della tratta dei neri, alla guerra giusta soprattutto per questioni inerenti al commercio. ${ }^{130}$ Questioni squisitamente giuridiche che affondano le radici nel diritto delle genti.

Tenendo ben in considerazione le diverse riflessioni e finalità che presentano le opere di Vattel e di Raynal è, in ogni caso, anche possibile stabilire un parallelo tra il loro pensiero e il contesto storico in cui sono vissuti. Entrambi

128 Il capolavoro monumentale di Raynal è diventato un vero e proprio best-seller durante tutto il XVIII secolo. Nella prima parte Raynal ha voluto chiarire il suo lavoro storicopolitico, mentre nella seconda parte ha scritto la storia delle due parti del pianeta, Est e Ovest, raggruppando le due sfere della colonizzazione europea. Al centro dell'indagine vi è l'Europa e il ruolo che essa ha rivestito nella storia: una Europa paladina della civilizzazione che viene "ridimensionata " da Raynal, il quale pone a confronto la civiltà europea con le altre civiltà e viene discussa in relazione al concetto di «diversità »: VILLARI (1996) 29. Come ha sottolineato Bancarel all'interno della recente riedizione della Histoire "lire l'Histoire au XXIe siècle ce n'est pas seulement ouvrir les yeux sur le siècle des Lumières, c'est appréhender un univers qui commence avec la découverte du Nouveau-Monde et nous conduit jusqu'à l'actualité la plus contemporaine. C'est aussi se souvenir d'événements parfois oubliés qui ponctuent l'histoire du monde et dont les conséquences trop souvent ignorées ont des répercussion jusqu'à nos jours: 1598- l'édit de Nantes, 1776- la déclaration de l'Indépendance des États Unis, 1789- La Révolution française, 1794- l'abolition de l'esclavage, 1948- la déclaration universelles des Droits de l'homme »: Bancarel (2006) 9-10; Bancarel (2004) passim; Bancarel (2011) 23-34; Benot (2000) 165-171; Fioravanti (2012); cfr. anche Tuccillo (2013) 243 ss.; Courtney/Mander (ed.) (2015).

129 Villari (1996) 31.

130 LÜSEBRINK (1997) 168-178. 
sono stati testimoni della Guerra dei Sette Anni, che esplose in Europa, si diffuse nelle colonie e fu combattuta nei quattro continenti. Proprio in quegli anni Vattel scriveva il suo Droit des gens, mentre Raynal scriveva la sua Histoire al termine del conflitto, come una sorta di denuncia ai disastri coloniali francesi che seguirono la Guerra dei Sette Anni.

In linea con le posizioni illuministiche della loro epoca entrambi si soffermarono sulla "conquista » delle terre da parte degli europei, e sulla legittimità della stessa. Raynal, esattamente come Vattel nel libro I, capitolo XVIII sostiene le modalità di occupazione territoriale messe in atto da Quaccheri. ${ }^{131}$ Raynal nel IX tomo, libro XVIII, intitolato Colonies Anglaises fondées dans la Pennsylvanie, dans le Maryland, dans la Virginie, dans la Caroline, dans la Georgie et dans la Floride. Considérations générales sur tous ces établissements, si occupa del buon e del cattivo governo, ${ }^{132}$ elogiando la personalità Penn. ${ }^{133}$

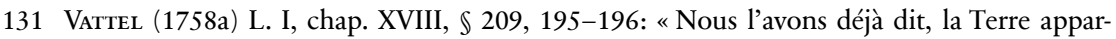
tient au Genre- humain pour sa subsistance: Si chaque Nation eût voulu dès le commencement s'attribuer un vaste pays, pour n'y vivre que de chasse, de pêche et de fruits sauvages; notre globe ne suffiroit pas à la dixième partie des hommes qui l'habitent aujourd'hui. On ne s'écarte donc point des vuës de la Nature, en resserrant les Sauvages dans des bornes plus étroites. Cependant on ne peut que louer la modération des Puritains Anglois, qui les premiers s'établirent dans la Nouvelle-Angleterre. Quoique munis d'une Charte de leur Souverain, ils achetèrent des Sauvages le terrein qu'ils voulaient occuper. Ce loüable exemple fut suivi par Guillaume Penn et la Colonie de Quackers qu'il conduisit dans la Pensilvanie".

132 «Ces deux sortes de gouvernement sont également inconnues dans les annales du monde. Elles ne nous offrent que des ébauches imparfaites, plus ou moins rapprochées de l'atroce sublimité, plus ou moins éloignées de la beauté touchante de l'un et de l'autre de ces grands tableaux. Les nations qui ont joué le rôle le plus éclatant sur le théâtre de l'univers, entraînées par une ambition dévorante, présentèrent de traits de conformité avec le premier [un mauvais gouvernement]. D'autres, plus sages dans leurs constitutions, plus simples dans leurs mœurs, plus limité dans leurs vues, enveloppées d'un bonheur secret, s'il est permis de parler ainsi, paroissent ressembler davantage au second [bon gouvernement] »: RaYnal (1781) L. XVIII, I, 2-3.

133 «Son arrivée au Nouveau-Monde fut signalée par un acte d'équité, qui fit aimer sa personne et chérir ses principes. Peu satissant du droit que lui donnoit sur son établissement la cession du ministère Britannique, il résolut d'acheter des naturels du pays, le vaste territoire qu'il se proposait de peupler. On ne fait point le prix qu'y mirent les sauvages: mais quoiqu'on les accuse de stupidité pour avoir vendu ce qu'ils ne devaient jamais aliéner, Penn n'en eut pas moins la gloire d'avoir donné en Amérique un exemple de justice et de modération, que les Européens n'avoient pas même imaginé jusqu'alors. [...] »: Raynal (1781) L. XVIII, IV, 10. 
Il «buon governo » e la conquista sono temi molto cari anche a Vattel, il quale condivide con Raynal la soluzione in Nord America effettuata da Penn e, ad esempio, per quanto riguarda la conquista spagnola in molti passaggi del Droit des gens, non esita ad affermare che: "Quand l'Espagnol attaquoit les Américains, sous prétexte que ces peuples refusoient de commercer avec lui, il couvroit d'une vaine couleur son insatiable cupidité ${ }^{134}$ e a condannare la distruzione degli Imperi del Perù e del Messico. ${ }^{\mathbf{1 3 5}}$

Da un lato in questa ultima sezione del I libro, Vattel affronta non solo il discorso inerente alla legittimità dell'espansione territoriale, dall'altro si preoccupa anche di individuare quali siano le minacce alla sicurezza di uno Stato, soffermandosi sul concetto di nemico interno, esterno e dell'umanità, che è Stato oggetto di una recente analisi. ${ }^{\mathbf{1 3 6}}$

Nel paragrafo 232 Vattel illustra i limiti della giurisdizione territoriale nei confronti di coloro che sono rifugiati o esiliati nella patria di origine: «Si un exilé ou un banni a été chassé de sa patrie pour quelque crime, il n'appartient point à la Nation chez laquelle il se réfugie de le punir pour cette faute commise dans un pays étranger. Car la nature ne donne aux hommes et aux Nations le droit de punir que pour leur défense et leur sûreté; d'où il suit que l'on ne peut punir que ceux qui on a été lésé ». ${ }^{137}$

134 VATtel (1758a) L. II, chap. II, $\mathbb{\$} 25,277$.

135 Vattel (1758a) L. II, chap. I, $\mathbb{S} 7,262$ : « Mais si une Nation est obligée de contribuer de son mieux à la perfection des autres, elle n'a aucun droit de les contraindre à recevoir ce qu'elle veut faire dans cette vuë. L'entreprendre, ce serait violer leur Liberté naturelle. Pour contraindre quelqu'un à recevoir un bienfait, il faut avoir autorité sur lui; et les Nations sont absolument libres et indépendantes. Ces ambitieux Européens, qui attaquoient les Nations américaines, et les soumettoient à leur avide domination, pour les civiliser, disoient-ils, et pour les faire instruire dans la véritable Religion; ces usurpateurs, dis-je, se fondoient sur un prétexte injuste et ridicule». Si rimanda anche a: Cavallar (2008) 181-209; Anghie (2011) 237-253; Anghie (2014) 81-99.

136 RECH (2013). Rech analizza concetto di nemico dell'umanità, alternando riflessioni filosofico-politiche a ricostruzioni sulla storia delle idee e utilizzando come fonte il Droit des gens di Vattel per delineare le teorie sulle figure di nemici dell'umanità «fuori » e « dentro » l'Europa. I nemici risultano essere coloro che hanno violato gravemente il diritto delle genti e la necessità che vengano eliminati nasce direttamente da una esigenza statale che si estende sul fronte internazionale: « for Vattel [...] the ultimate essence of law was to preserve the basic conditions without which human society would be impossible, or unthinkable. This utilitarian conception of law- classical, modern and anti-medieval-lay at the basis of Vattel's theory of international law enforcement »: RecH (2013) 228.

137 Vattel (1758a) L. I, chap. XIX, \$232, 211-212. 
Il giurista di Neuchâtel sostiene che il diritto di punire si fondi unicamente sul diritto alla sicurezza e il mantenimento di quest'ultimo altro non è che un compito delegato dai cittadini a chi governa: lo Stato infatti, come persona morale deve mantenere la sicurezza, punire chi offende, perseguendo tutti i reati pubblici. ${ }^{\mathbf{1 3 8}}$

Tuttavia il principio di territorialità non si applica in un solo caso, quello del perseguimento di coloro che sono identificati come i nemici dell'umanità: «Les empoisonneurs, les assassins, les incendiaires de profession, peuvent être exterminés partout où les saisit; car ils attaquent et outragent toutes les Nations, en foulant aux pieds les fondements de leur sûreté commune ${ }^{139}$

Come sostenuto da Walter Rech, in Vattel è presente l'analogia tra il nemico dello Stato e il nemico dell'intera comunità internazionale, la quale è legittimata ed è chiamata ad agire nella repressione di coloro che sono nemici dell'umanità, al fine di mantenere un livello di sicurezza e tranquillità tanto a livello nazionale che internazionale, anticipando così la delicata tematica di una giurisdizione universale. ${ }^{\mathbf{1 4 0}}$

138 Vattel (1758a) L. I, chap. XIII, $\sqrt{ }$ 169: «quand les hommes s'unissent en Société, comme la Société est désormais chargée de pourvoir à la sûreté de ses membres, tous se dépouillent en sa faveur de leur droit de punir. C'est donc à elle de venger les injures particulières, en protégeant les Citoyens. Et comme elle est une personne morale, à qui on peut aussi faire injure; elle est en droit de maintenir la sûreté, en punissant ceux qui l'offensent, c'est-à-dire, qu'elle a le droit de punir les délits publics ». Sul concetto di persona morale come aspetto basilare per l'ordine internazionale: Holland (2011) 438-445.

139 Vattel (1758a) L. I, chap. XIX, $\mathbb{2} 233,212$ : «C'est ainsi que les Pirates sont envoyés à la potence par les premiers entre les mains de qui ils tombent. Si le Souverain du pays, où des crimes de cette nature ont été commis, en réclame les auteurs, pour en faire la punition; on doit les lui rendre, comme à celui qui est principalement intéressé à les punir exemplairement. Et comme il est convenable de convaincre les coupables et de leur faire leur procès dans toutes les formes, c'est une séconde raison pourquoi on livre ordinairement les malfaiteurs de cet ordre aux États qui ont été le théâtre de leurs crimes ».

140 ReCH (2013) 28-29. La concezione di Vattel sul nemico dell'umanità richiama immediatamente la situazione politica e internazionale dei nostri giorni: mai come nell'ultimo decennio si è assistito ad un elevatissimo uso della dicitura "nemico dell'umanità » per identificare, soprattutto nel contesto occidentale, la guerra contro il terrorismo, in tutte le sue manifestazioni. E' stato argomentato da Rech come: «Vattel's advocacy for the repression of the heinous international crimes has, directly or indirectly through the medium of later publicists familiar with it, contributed to the shaping of a modern doctrine of $j u s$ congens, war crimes and crimes against humanity and to the doctrine of military intervention for humanitarian purposes »: RECH (2013) 221. 
E' interessante notare che Vattel termina il I libro riassumendo le finalità per cui ha descritto esclusivamente la Nazione « in se stessa »:

"Voilà tout ce que nous avions à dire dans ce premier Livre. Un plus grand détail sur les Devoirs et les Droits d'une Nation considérée en elle-même, nous mènerait trop loin : Il faut [...] le chercher dans les Traités particuliers de Droit Public et de Politique. Nous sommes fort éloignés de nous flatter que nous n'avons omis aucun article important. C'est ici une légère Esquisse d'un immense Tableau. Mais un Lecteur intelligent suppléera sans peine à toutes nos omissions, en faisant usage des Principes généraux. Nous avons donné tous nos soins à établir solidement ces principes, et à les développer avec précision et netteté ». ${ }^{\mathbf{1 4 1}}$

Esaurita l'analisi sulla Nazione che è stata sviscerata in ventitré capitoli, viene affrontato il tema delle relazioni tra Stati, e dei diritti e obblighi che tra essi derivano. Il primo obbligo che vige tra le nazioni è il dovere di mutua assistenza a cui Vattel fornisce una spiegazione insieme al dovere essenziale di libertà ed indipendenza delle nazioni, distinguendo pertanto tra obbligo interno «tant qu'elle lie la Conscience, qu'elle est prise de règles de notre devoir » ${ }^{\mathbf{1 4 2}}$ e obbligo esterno, "en tant qu'on la considère relativement aux autre hommes, et qu'elle produit quelque droit entre eux ", che a sua volta può essere perfetta o imperfetta. ${ }^{\mathbf{1 4 3}}$

Anche la libertà e l'indipendenza delle nazioni è modellata sulla libertà originaria degli uomini ma: "Une nation est donc maîtresse de ses actions, tant qu'elle n'intéresse pas les droits propres et parfait d'une autre, tant qu'elle n'est liée que d'une obligation interne, sans aucune obligation externe parfaite $»{ }^{\mathbf{1 4 4}}$

Tale affermazione comporta una serie di corollari: si sancisce una perfetta uguaglianza di diritto tra le Nazioni, la reciprocità delle obbligazioni nascenti tra esse, che non possono essere attivate se non in presenza di una «lesione » all'autonomia e all'indipendenza delle nazioni ed infine si giunge all'elaborazione del principio di autoconservazione degli Stati, che mutuato

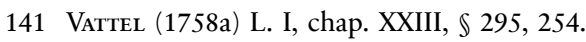

142 Vattel (1758a) L. I, Préliminaires, $\mathbb{1} 17,10$.

143 Vattel (1758a) L. I, Préliminaires, $\mathbb{S} 17,10$ : «Le droit parfait est celui auquel se trouve joint le droit de contraindre ceux qui ne veulent pas satisfaire à l'obligation qui y répond; et le droit imparfait est celui qui n'est pas accompagné de ce droit de contrainte. L'obligation parfaite est celle qui produit le droit de contrainte; l'imparfaite ne donne à autrui que le droit de demander ».

144 Vattel (1758a) L. I, Préliminaires, $\mathbb{2}$ 20, 11. 
dalla sfera dei singoli, è sostanzialmente inteso da Vattel come "une faculté morale d'agir, c'est-à-dire de faire ce qui est moralement, ce qui est bien et conforme à nos devoirs $»{ }^{\mathbf{1 4 5}}$

Dunque le Nazioni hanno quattro caratteristiche: la libertà naturale, l'indipendenza, l'uguaglianza e la sovranità che sostanzialmente funge da comune denominatore.

\section{5. «Stato ", « Nazione » e « Potenza »: la ieraticità dei trattati internazionali e della loro interpretazione}

Tre sono i termini che emergono in modo solenne all'interno del Droit des gens: lo «Stato » viene visto come l'entità giuridica e istituzionale, quasi fosse una sorta di contenitore astratto e generale; la " Nazione " è il contenuto, che è formata dai cittadini e acquista sostanza attraverso il perseguimento del piano costituzionale e dei tre principali oggetti di un buon governo. Basta, infatti, semplicemente leggere il titolo del trattato per comprendere pienamente il messaggio di Vattel che indirizza l'opera esplicitamente e concretamente ai sovrani e alle nazioni e non agli Stati: Droit des gens ou Principes de la loi naturelle appliqués à la conduite et aux affaires des Nations et des Souverains. ${ }^{146}$

L'edizione contraffatta prodotta da Elie Luzac nel 1758, permette di completare la triade semantica con "Potenza ", che, relativa all'esercizio del potere politico, conduce progressivamente alla terminologia di ragion di Stato ed è più vicina alle esigenze della comunità internazionale, tanto in tempo di pace quanto in tempo di guerra. Luzac, come si è visto nel precedente capitolo ha aggiunto un sottotitolo eloquente e molto chiarificatore per i posteri: Ouvrage qui conduit à développer les véritables intérêts des puissances.

Lo spazio sociale delineato da Vattel si articola nella regolamentazione a livello interno di una nazione e successivamente si estende, con il principio di bilanciamento dei poteri, a livello internazionale: le relazioni internazionali devono garantire tra le nazioni ciò che la singola deve perseguire a livello nazionale.

145 Vattel (1758a) L. II, chap. IV, $\mathbb{S} 49,295$.

146 Devetak (2011) 105-128. 
Gli strumenti con cui «dialogano » le potenze e le nazioni, titolari della loro sovranità, sono per eccellenza i trattati, ai quali Vattel dedica molti capitoli del secondo, terzo e quarto libro. Egli afferma che tale materia è, senza alcun dubbio, la più importante per le relazioni tra gli Stati e definisce il trattato come il patto stipulato in virtù del bene pubblico da potenze sovrane. ${ }^{\mathbf{1 4 7}}$ Essi « mettent en règle les prétentions des Souverains; ils doivent faire reconnoître les Droits des Nations, assurer leurs intérêts les plus précieux. Entre des Corps Politiques, des Souverains, qui ne reconnoissent aucun Supérieur sur la terre, les Traités sont l'unique moyen d'ajuster les prétentions diverses, de se mettre en règle, de savoir sur quoi compter et à quoi s'en tenir ». ${ }^{\mathbf{1 4 8}}$

Nell'elaborare le sue teorie sul diritto dei trattati Vattel viene influenzato dalle posizioni dei suoi predecessori, e in particolare da Grozio, inspirandosi alla sua disamina e struttura; all'interno del De iure belli ac pacis, Grozio dedica interamente il capitolo XV del libro II alle convenzioni pubbliche e partendo dai giuristi romani, utilizza come fonte principale per la sua trattazione Ulpiano. ${ }^{149}$ Per il giurista $i$ trattati sono stipulati in forza di una autorità pubblica, o suprema e questa è la caratteristica che li distingue non solo dai contratti tra privati ma anche da quelli stipulati dai sovrani nei loro affari privati.

Grozio illustra anche un'ulteriore tripartizione delle convenzioni: il foe$d u s$, inteso come trattato concluso per ordine del sommo potere e in cui il popolo è soggetto alla vendetta divina se non viene rispettato; poi vi è la sponsio, quando le persone pubbliche hanno promesso ma senza ordine della potestà sovrana, ed infine altri trattati che non possono rientrare in nessuna delle due precedenti categorie. ${ }^{\mathbf{1 5 0}}$

I trattati sono anche divisi in base al loro rapporto con la legge naturale e Grozio anche in questa separazione sembra seguire i giuristi romani, evidenziando che nella prima categoria fanno parte le convenzioni stipulate sulla base dei criteri direttamente desunti e esplicitamente riscontrabili nel diritto naturale, mentre nella seconda categoria ci sono quei trattati che «iure naturae aliquid adijciunt ${ }^{151}$

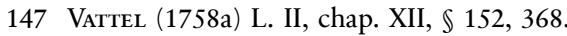

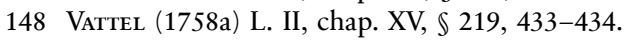

149 Grotius (1625) L. II, cap. XV, $\mathbb{I}$ I, 322 ss.

150 Ibidem.

151 Ibidem. Cfr. Vitanyi (1980) 546; Del Vecchio (1964) 68. 
I trattati che appartengono al primo gruppo sono per Grozio in realtà delle manifestazioni della teoria del diritto naturale: diritti come quello all'ospitalità, al commercio sono dunque sempre esistiti poiché figli del diritto naturale.

Le convenzioni, invece, che "aggiungono» qualcosa al diritto naturale sono a loro volta divise in due parti: « aut aequales sunt, aut inaequales ». ${ }^{\mathbf{1 5 2}}$ Nelle convenzioni uguali vi è eguaglianza riguardo al rapporto che le parti hanno nello stipulare il trattato: ciò significa una parità totale, in tutti i settori e in tutti i campi. ${ }^{153}$ Mentre i trattati ineguali sono caratterizzati da una posizione di superiorità di una delle parti, sia nel corso delle trattative che nella conclusione e negli effetti: «Aequales sunt quae utrinque eodem modo se habent », ${ }^{\mathbf{1 5 4}}$ e ancora Grozio afferma che: «ex aequalibus qualia sunt inaequalia facile est intellegi. Promittuntur autem inaequalia aut ex parte digniori aut ex minus digna $[. .$.$] et haec ipsa aut cum imminutione$ sunt imperii aut sine eius imminutione ${ }^{155}$

Secondo Grozio, la legge naturale resta il principio cardine di tutto il diritto umano; non può esserci un diritto che vada contro i principi della natura. I diritti di sovranità sono subordinati alle norme del diritto naturale come se dipendessero da un ordine giuridico superiore, "ius naturale, cui summa imperia subiacent $\gg .{ }^{\mathbf{1 5 6}}$

Il diritto positivo non può prescrivere ciò che il diritto naturale proibisce, ma può porre dei limiti alla libertà naturale: lo ius naturale pertanto, studiato nel suo complesso, non ha una validità assoluta e generale; il diritto delle genti infatti si distingue tra $\mathrm{i}$ popoli in base alle regole permissive nei confronti dell'originario diritto naturale. ${ }^{157}$

Le tesi di Grozio sui trattati sono state in parte rielaborate da Pufendorf, che fondava la libera vita degli Stati unicamente sul diritto naturale, attraverso una rappresentazione realistica dello Stato e della sua potenza, sviluppando così, in opposizione a Grozio, la dottrina degli interessi degli Stati.

152 Grotius (1625) L. II, cap. XV, $\mathbb{S}$ VI, 325.

153 Ibidem.

154 Ibidem.

155 Ivi, $\mathbb{S}$ VII, 326.

156 Grotius (1625) L. II, cap. IV, $\mathbb{X}$ XIII, 173.

157 Gozzi (2010) 61 ss. 
Pufendorf critica altresì la posizione del giurista olandese sul rispetto delle convenzioni stipulate con un nemico. Egli afferma che tale rispetto è vero solo per quanto riguarda i trattati che mirano alla pace, mentre non è così per quelli che lasciano inalterata una condizione di guerra. Nel caso di una situazione di pace si impone la fides verso i patti stipulati; al contrario in una situazione di guerra, una condizione di ostilità consente la licenza senza limiti di recar danno agli altri. ${ }^{158}$

Pufendorf si sofferma ampiamente anche sulla consuetudine che è individuata come «tacito quodam consensu usurpari solita ", ${ }^{159}$ ma se una consuetudine deriva dal diritto naturale vuol dire che non assume molta importanza l'accordo in sé, bensì ciò da cui deriva tale patto, ovvero dallo ius naturale stesso: per il giurista non occorre creare un'autonoma categoria di diritto per identificare le consuetudini perché queste trovano il loro posto proprio all'interno del diritto naturale. ${ }^{\mathbf{1 6 0}}$

Il diritto delle genti è di per sé considerato come immutabile e duraturo e la molteplicità dei trattati conclusi in diverse circostanze temporali e ambientali è inconciliabile con la possibilità di identificare tali convenzioni all'interno del suddetto diritto. Le tesi di Pufendorf sul diritto delle convenzioni, secondo Gozzi, mirano a porre al centro della sua analisi lo Stato: il diritto deriva non più da Dio, bensì dallo Stato e ciò comporta che « la recta ratio della dottrina del diritto naturale si muti nella ratio civitatis, ossia nella ragion di Stato ». ${ }^{\mathbf{1 6 1}}$

Tuttavia è Wolff che riconosce alla legge naturale solo una funzione indiretta; nasce proprio con lui una vera e propria concezione diversa del diritto naturale, da cui si deduce che lo ius gentium non coincide con il diritto naturale: «atque ita Ius Gentium non per omnia idem manet cum iure naturae, quatenus singulorum actus regit $» .{ }^{\mathbf{1 6 2}}$

Il diritto delle genti che deriva direttamente dal diritto naturale non è sufficiente di per sé stesso a regolare i rapporti tra gli Stati, i quali hanno il dovere di completare o modificare le regole dello ius naturae a tal punto che la legge creata può discostarsi totalmente da quella naturale e può non seguire le prescrizioni di essa. ${ }^{163}$ Fondamentale diventa il consenso espresso,

158 Pufendorf (1672a) L. VIII, cap. VII, $\mathbb{S} 2$, 999; anche Gozzi (2010) 79.

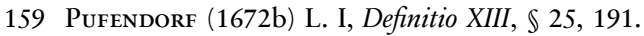

160 Ibidem.

161 Gozzi (2010) 79.

162 WolfF (1764) Praefactio, II.

163 Ibidem. 
che può scaturire solo attraverso un trattato. Uno Stato dunque non può essere obbligato a concludere un trattato senza il suo consenso e ancora di meno contro la sua volontà e una Nazione non acquisterà mai un diritto da un'altra senza una espressa rinuncia da parte di quest'ultima. ${ }^{164}$

I trattati, sostiene Wolff, hanno effetto solo per gli Stati partecipanti e di conseguenza il diritto pattizio non sarà mai universale bensì particolare: « ius gentium pactitium est, quod ex pactis oritur inter diversas Gentes initis. [...] pacta quoque non obligant nisi gentes inter quas inita, consequenter ius gentium quod ex pactis oritur, seu pactitium universale non est, sed particulare ». ${ }^{\mathbf{1 6 5}}$

Nella stesura dei capitoli sui trattati, Vattel si rifa principalmente alla struttura groziana, ma approfondisce molto di più la materia fornendo definizioni circoscritte e precise: « un traité [...] est un pacte fait en vue du bien public par les princes supérieurs », ${ }^{\mathbf{1 6 6}} \mathrm{e}$ ancora, i trattati pubblici non possono nascere se non in nome della sovranità propria di ciascuno Stato: "Ainsi les Conventions que les Souverains sont entre eux, pour leurs affaires particulières, et celles d'un Souverain avec un particulier ne sont pas des traités publics ». ${ }^{\mathbf{1 6 7}}$

Il principio, ben noto in Grozio " pacta sunt servanda ", assume un ruolo fondamentale anche per Vattel: dinanzi ad una promessa fatta secondo diritto e priva di vizi sorge non solo un obbligo in capo colui che ha promesso, ma un diritto anche a colui che attende la realizzazione obbligatoria, e pertanto non vi sarà più sicurezza neanche nel commercio fra gli uomini se essi non si credono obbligati a serbare fede e tener parola al contratto stipulato. Quest'obbligazione è dunque necessaria per mantenere l'ordine e la pace nelle relazioni tra i vari Stati. ${ }^{168}$

Se pertanto gli impegni di un trattato impongono un'obbligazione perfetta producono necessariamente un diritto perfetto: violare un trattato significa dunque violare il diritto perfetto di colui con il quale si è stipulato e pertanto « est une injure ». ${ }^{169}$

164 Mancuso (2002) 279-283.

165 WolfF (1764) Prolegomena, $\$ 23,8$.

166 VATtel (1758a) L. II, chap. XII, $\ 152,368$.

167 Ivi, $\$ 154,368$.

168 Ibidem. Cfr. anche Genta (2004) 120-121.

169 Vattel (1758a) L. II, chap. XII, $\mathbb{1} 164,375$. 
Addirittura Vattel individua in essi qualcosa di sacro, esattamente come sacra è la legge fondamentale della Nazione: i trattati sono «des choses sacrées entre les Nations ». ${ }^{\mathbf{1 7 0}}$ La sola legittima eccezione ammessa è quella derivante dalla necessità di rispettare « les devoirs d'une Nation envers ellemême » che corrispondono cioè all'autoconservazione della stessa. ${ }^{171}$ Mancuso sotto questo profilo ha rilevato quanto "nella mutevolezza dei fenomeni giuridici e politici, la stella polare di una "giusta » politica deve essere la ricerca dell'autoconservazione ", mostrando in queste posizioni quanto non solo Wolff abbia caratterizzato il suo pensiero ma anche Hobbes, con la differenza però che Vattel "cercherà di individuare un tessuto di norme volontarie $[\ldots]$ destinate a ridurre lo Stato di natura interstatuale ». ${ }^{\mathbf{1 7 2}}$

Seguendo la divisione dei trattati in due classi già formulata da Grozio, afferma che le convenzioni contenti le prescrizioni del diritto naturale "servent à se procurer un droit parfait à des choses auxquelles n'avait qu'un droit imparfait, en sorte qu'on peut exiger désormais ce qu'auparavant on étoit seulement fondé à demander comme un office d'humanité ». ${ }^{173}$

In questa prima classe rientrano tutti i trattati di pace e amicizia stipulati tra i vari Stati e Vattel porta come esempi i trattati che contengono la non offensività reciproca. In essi uno Stato si impegna ad astenersi dall'effettuare lesioni al proprio alleato. Anche se non producono concretamente un nuovo diritto, tuttavia diventano utilissimi e accidentalmente necessari qualora vi siano Stati che si credono «en droit de tout oser contre les étrangers ». ${ }^{\mathbf{1 7 4}}$

Per quanto riguarda invece la seconda categoria « que s'engage à quelque chose de plus », gli Stati si impegnano a rispettare le obbligazioni non contenute nella legge naturale, stipulando di volta in volta trattati eguali o ineguali. ${ }^{\mathbf{1 7 5}}$

Tra i trattati eguali Vattel elenca ad esempio un'alleanza difensiva, in cui gli Stati stipulano il soccorso reciproco, viceversa in quella offensiva le parti possono promettersi che ciascuno degli alleati fornirà lo stesso numero di vascelli o di armi. ${ }^{176}$ Mentre i trattati ineguali sono quelli in cui le parti non

170 Vattel (1758a) L. II, chap. XIV, $\$ \int ~ 218-219,433$.

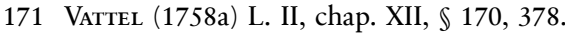

172 Mancuso (2002) 282-283.

173 VAttel (1758a) L. II, chap. XII, $\mathbb{S} 169,377$.

174 Ivi, $\mathbb{1 7 1 ,} 379$.

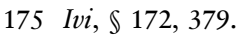

176 Ibidem. 
si promettono obblighi reciproci uguali. ${ }^{177}$ A loro volta questi si dividono in due specie: la prima è costituita dai trattati « où l'inégalité se trouve du côté de la Puissance la plus considérable et se manifeste seulement dans une supériorité d'honneurs ", in cui senza attribuire al più potente alcun diritto sul più debole viene concesso soltanto una «superiorità di onori e di considerazione ». A tale proposito Vattel porta come esempio la politica di un sovrano interessato a uno Stato più debole, che promette a quest'ultimo soccorsi in caso di necessità, scambi vantaggiosi, ma al tempo stesso esige dal suo alleato atti di rispetto. ${ }^{\mathbf{1 7 8}}$

I trattati in cui invece "l'inègalité se trouve du côté de la puissance inférieure » sono quelli che impongono al più debole obbligazioni più ampie, carichi più gravosi. Queste condizioni sono imposte dal vincitore o dettate dalla necessità che obbliga uno Stato debole a ricercare la protezione o l'assistenza ad uno Stato più potente e Vattel afferma che nella maggior parte dei casi il più debole non solo si impegna a non fare guerra senza il consenso del più forte, ma anche ad avere i suoi stessi nemici e amici e a non concludere accordi con determinati Stati. ${ }^{179}$ Le alleanze dove " l'inègalité se trouve du côté de la Puissance inférieure "si dividono a loro volta in due tipi, a seconda che offendano in qualche parte la sovranità di ciascuno Stato o non la offendano. ${ }^{\mathbf{1 8 0}}$

Per Vattel la sovranità trova la sua ragione d'essere nell'integrità, cioè quando i diritti fondamentali di uno Stato sono propri e non sono delegati a nessun'altra Nazione, e tale integrità rimane lesa se viene ceduto uno dei suoi diritti che la compongono. Ad esempio un trattato non "offende la sovranità » quando uno Stato debole promette di non attaccare una certa Nazione senza il consenso del suo alleato. Con ciò, infatti, non si spoglia del suo diritto, ma semplicemente opera una restrizione nei confronti di questo a favore del suo alleato: ${ }^{181}$ è proprio in questa circostanza che Vattel sottolinea che vi sono dei doveri scambievoli delle nazioni rispetto alle alleanze disuguali. ${ }^{182}$ Occorre convenire che non è lecito obbligare gli Stati deboli,

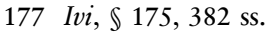

178 Ivi, $\mathbb{S} 175,383$.

179 Ibidem.

180 Ivi, $\mathbb{1} 175,386$.

181 Ibidem.

182 VATtel (1758a) L. II, chap. XII, $\mathbb{1} 178,387-388$. 
senza che vi siano giustificati motivi, a compromettere la loro dignità: « rien n'affermit plus sûrement la puissance d'un grand Monarque, que ses égards pour tous les Souverains ${ }^{183}$

L'agire dello Stato forte, qualora contragga convenzioni con uno più debole, è guidato dalla legge naturale e deve essere data un'assistenza generosa senza esigere contraccambio né tanto meno l'equivalente. In questo senso il giurista di Neuchâtel è molto preciso nell'illustrare il rapporto tra i cosiddetti trattati ineguali e la legge naturale. ${ }^{\mathbf{1 8 4}}$

Nel continuare ad esporre le teorie sul diritto dei trattati si sofferma anche sulla forma di governo scelta da ogni singolo Stato e, in relazione alle convenzioni, opera una distinzione a seconda che gli Stati siano monarchie o repubbliche: vi sono pertanto alleanze personali e alleanze reali. Le prime sono quelle che si riferiscono alla persona dei contraenti, mentre quelle reali sono rivolte unicamente agli argomenti dei quali si tratta, prescindendo da ogni dipendenza della persona dei contraenti. L'alleanza personale si scioglie nel momento in cui muore colui che l'ha contratta; quella reale invece fa parte del corpo stesso dello Stato e sussiste proprio quanto lo Stato. ${ }^{\mathbf{1 8 5}}$

Ogni alleanza fatta da una repubblica è reale di sua natura: «Quand un peuple libre, un État populaire, ou une république aristocratique, fait un traité, c'est l'État même que contracte; ses engagements ne dépendent point de la vie de ceux qui n'en ont été que les instruments: Les membres du peuple, ou de la Régence, changent et se succèdent; l'État est toujours le même ». ${ }^{186}$

Benché i rappresentanti del popolo o del governo cambino, dice Vattel, lo Stato è sempre lo stesso. Ugualmente anche i trattati conclusi da un re o da qualsiasi monarca sono trattati di Stato: il re rappresenta solo al momento

183 Ivi, $\mathbb{1 7 8 ,} 388$.

184 Ibidem: "Quoiqu'en dise une Politique intéressée, il faut ou soustraire absolument les Souverains à l'autorité de la Loi Naturelle, ou convenir qu'il ne leur est pas permis d'obliger, sans de justes raisons, les États plus foibles à compromettre leur dignité, moins encore leur liberté, dans une Alliance inégale. Les Nations se doivent les mêmes secours, les mêmes égards, la même Amitié que les particuliers vivant dans l'état de Nature. Loin de chercher à avilir les foibles, à les dépouiller de leurs avantages les plus précieux; elles respecteront, elles maintiendront leur dignité et leur liberté, si la vertu les inspire plûtôt que l'orgueil, si elles sont plus touchées de l'honnêteté que d'un grossier intérêt: que disje? Si elles sont assez éclairées pour connaître leurs véritables intérêts ».

185 Ivi, L. II, chap. XII, $\mathbb{S} 183,191$.

186 Ivi, $\mathbb{1} 185,192$. 
della stipula i suoi sudditi i quali saranno tenuti a mantener fede agli obblighi contenuti nella convenzione. ${ }^{\mathbf{1 8 7}}$

Uno dei capitoli del Droit de gens che ebbe numerosi accoglimenti e altrettante critiche durante i secoli XVIII e XIX, è senza dubbio quello in cui Vattel affronta il problema dell'interpretazione dei trattati e nel quale spiega perché occorre stabilire delle regole per interpretare. ${ }^{\mathbf{1 8 8}}$

L'interpretazione è per Vattel un procedimento esplicativo nel caso in cui i trattati siano oscuri. Se infatti le idee degli uomini fossero sempre distinte e perfettamente determinate se, nel momento in cui vengono pronunciate tutte le espressioni fossero chiare, precise, «suscettibles d'un sens unique", non ci sarebbero problemi a comprendere la volontà contenuta nelle parole e non sussisterebbe, dunque, nemmeno il problema interpretativo. ${ }^{\mathbf{1 8 9}}$

Le regole interpretative nascono dalla ragione e sono riconosciute tramite il diritto naturale, ${ }^{190}$ sono "capables de répandre la lumière sur ce qui est obscur », di definire un atto certo o incerto, di deludere le aspettative di una parte che può contrarre in mala fede, e infine "vont particulièrement à réprimer la fraude, à prévenir l'effet de ses artifices ». ${ }^{191}$

Esse sono necessarie perchè oltre ad avere il compito essenziale di guidare l'interprete, le une con le altre si devono « combiner ensemble et l'Interprétation se faire de manière qu'elle s'accommode à toutes, selon qu'elles sont applicables au cas » e nel caso in cui tali regole sembrerebbero «se croiser, elles se balancent et se limitent réciproquement, suivant leur force et leur importance, et selon qu'elles appartiennent plus particulièrement au cas dont il est question ». ${ }^{\mathbf{9 2}}$

La regola principale ricordata come l'aforisma e considerata « comme la pierre angulaire de toute l'interprétation juridique », ${ }^{193}$ statuisce che: «n'est pas permise d'interpréter ce qui n'a pas besoin d'interprétation ». ${ }^{194}$ Quando

187 Ibidem.

188 Per l'analisi delle regole interpretative elaborate da Vattel cfr.: Orakhelashvili (2011) 94-96. Cfr. anche: Neri (1958) 91; Vitanyi (1980) 535 ss.; Viola (2001) 30 ss.; DupuY (2011) 151-166; Chetail (2014) 293 ss.; DjefFal (2016) 50.

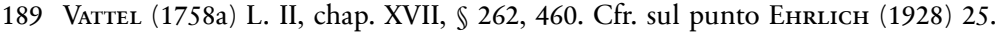

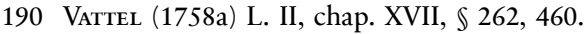

191 Ibidem.

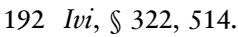

193 Vitanyi (1980) 558.

194 Vattel (1758a) L. II, chap. XVII, $\mathbb{S} 263$, 461. Sulla famosa massima: Sснотт (2001), 155-190, in particolare 156. 
un atto è concepito in termini chiari e precisi, quando soprattutto il senso è talmente manifesto « on n'a aucune raison de se refuser au sens que cet Acte présente naturellement ». L'andar oltre il testo cercando di interpretarlo, per restringerlo o ampliarlo, è un voler eluderlo. Vattel esorta quindi a redigere atti in forma più chiara possibile: «tout cela vous sera inutile, s'il est permis de chercher des raisons étrangères, pour soutenir qu'on ne peut le prendre dans le sens qu'il présente naturellement $»{ }^{\mathbf{1 9 5}}$

Nell'elaborazione delle regole interpretative è Vattel a compiere il passo in avanti, quello decisivo, riguardo la preminenza dell'elemento logico-letterale; egli rappresenta un vero e proprio distacco, non solo nei confronti del pensiero groziano, ma anche del suo maestro Wolff. Per il giurista di Neuchâtel la forza e l'obbligazione di ogni contratto derivano da una promessa perfetta e, poiché chi promette non può essersi impegnato oltre la sua volontà che ha sufficientemente dichiarato, per conoscere il vero senso del contratto "il faut principalement faire attention aux paroles de celui qui promet ${ }^{196}$ L'interpretazione di ogni atto e trattato si deve fare secondo regole certe, atte a determinare il senso attraverso cui dovessero naturalmente intenderlo le parti interessate nel momento in cui l'atto fu redatto e poi accettato. ${ }^{197}$

Vattel stabilisce una massima generale nella quale sembrerebbe porre al centro la reale volontà delle parti, dando quindi maggiore preminenza all'interpretazione soggettiva. Egli statuisce che "l'interpretazione legittima di un atto deve ricercare soltanto il pensiero dell'autore o degli autori stessi »: ${ }^{\mathbf{1 9 8}}$ «i cavillosi, i quali contestano il senso di una disposizione chiara e precisa, hanno l'abitudine di ricercare i loro vani pretesti nell'intenzione, nelle finalità, che essi attribuiscono all'autore di quella disposizione ", ${ }^{199}$ ma per Vattel deve essere interpretato ciò che per chiarezza di significato, non richiede alcuna interpretazione. ${ }^{200}$

Sergio Neri sottolinea l'importanza della presa di posizione vatteliana, ben più netta e consapevole di quanto lo fosse per Grozio e Wolff, circa

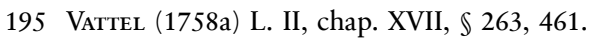

196 Ivi, $\mathbb{2} 263,461$.

197 Ivi, $\mathbb{2} 268,464$.

$198 \mathrm{Ivi}, \mathbb{2} 270,465$.

199 Ivi, $\mathbb{2} 264,462$.

$200 \mathrm{Ivi}, \mathbb{S} 263,461$.

120 Capitolo II 
la possibilità che la ricerca interpretativa diventi un'attività giuridicamente malsicura. ${ }^{201}$ Questi ultimi sostengono anch'essi qualcosa di analogo parlando della lettera « chiara » del trattato e della necessità, in certi casi, di applicarla, ma non altrettanto incisivi quanto Vattel: resta per loro soltanto un'affermazione generica, fine a se stessa e svincolata da ogni applicazione concreta. ${ }^{\mathbf{2 0 2}}$

Entrambi si limitano a sostenere l'obbligo di attenersi all'interpretazione logico-letterale del trattato, allorché niente faccia presumere una differenza di contenuto tra quest'ultimo e la comune intenzione dei contraenti, e pongono in aggiunta, come condizione all'applicazione diretta del significato obiettivo del testo giuridico, la piena coincidenza tra questo significato e la reale volontà delle parti. ${ }^{203}$

Per Vattel invece è tutto diverso, egli sottopone l'applicazione diretta al testo giuridico alla sola condizione che si tratti di un testo chiaro di per sé stesso; tale, cioè, che la sua chiarezza non risulti dal confronto effettivo con la reale volontà delle parti, ma venga piuttosto constatata come un dato obiettivo. $^{204}$

Il compito dell'interprete è la ricerca della volontà comune dei contraenti, la quale, quando non sia afferrabile nella chiarezza logico-letterale del testo, sarà da ricercarsi in tutti gli elementi ad essa relativi ed estrinseci al contenuto oggettivo dell'accordo.

Egli afferma che "puisque l'interprétation légitime d'un acte ne doit tendre qu'à découvrir la pensée de l'auteur, ou des auctores de cet acte; dès qu'on y rencontre quelqu'obscurité, il faut chercher quelle a été vraisemblablement la pensée de ceux qui l'ont dressé, et interpréter en conséquence ${ }^{205}$

Le teorie interpretative permettono a Vattel di rivestire un ruolo particolare tra l'Otto e il Novecento, in cui sono, come si è già anticipato molto apprezzate (tanto da trovare addirittura una loro collocazione nella Conven-

201 NERI (1958) 94.

202 Ibidem.

203 Ibidem.

204 Afferma Neri: "Quel perfetto sincronismo tra l'espressione formale del testo giuridico e la reale intenzione dei contraenti, che, nel pensiero del Grozio e del Wolff, si presenta ancora come un dato accertabile a posteriori acquista, nel Vattel, i caratteri di una presunzione assoluta basata sulla chiarezza intrinseca del testo giuridico »: NeRI (1958) 95.

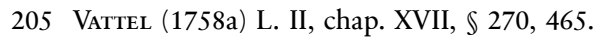


zione di Vienna sul diritto dei trattati del 1969), ${ }^{206}$ oppure fortemente criticate, come nel caso ad esempio di Hersch Lauterpacht. ${ }^{207}$

Sia le regole interpretative sia il rispetto dei trattati, devono essere letti in funzione della convivenza pacifica tra le nazioni, perchè per usare le parole di Vattel

toute la tranquillité, le bonheur et la sûreté du Genre humain reposent sur la Justice et sur l'obligation de respecter les droits d'autrui. [...] Plus de sûreté, plus de commerce entre les hommes, s'ils ne se croient point obligés de garder la foi, de tenir leur paroles. Cette obligation est donc aussi nécessaire, qu'elle est naturelle et indubitable, entre les Nations qui vivent ensemble dans l'état de nature, et qui ne connaissent point de Supérieur sur la terre, pour maintenir l'ordre et la paix dans leur Société. ${ }^{208}$

Tra le righe del trattato vatteliano sono molteplici le riflessioni che emergono e qui ci si limita a richiamarne alcune: l'importanza delle dinamiche presenti nel Droit des gens possono dare molte risposte a livello teorico e pratico per quanto riguarda i concetti di costituzione e di relazioni internazionali. La solennità e la forza con cui i termini «Stato ", "Nazione » e "Potenza » vengono perceptiti, sono una chiave di lettura della realtà politico-giuridica dell'epoca e permettono di cogliere le contraddizioni e le tensioni che si realizzeranno nel diritto internazionale lungo il corso dell'Ottocento. Infine Vattel pone al centro la Nazione e il raggiungimento della sua perfezione tanto a livello nazionale quanto a livello internazionale, attraverso una sistematica attenzione al contesto a lui contemporaneo.

Come sostiene Mancuso «nel Droit des gens, infatti, è sempre ben presente la distanza che intercorre tra la giustizia teorica e l'effettiva pratica del potere: Vattel ben comprende l'inefficacia del solo diritto naturale e la risolve nell'individuazione di norme, a base volontaristica, organizzanti la preservazione di un ordine minimale interstatuale ». ${ }^{209}$

I meccanismi sociali introdotti al fine di mantenere « un ordine minimale interstatuale » che garantiscano alla singola Nazione il raggiungimento della felicità dei suoi cittadini sono un topos che compare in tutto il Droit des gens. I trattati internazionali, il loro rispetto e la loro interpretazione non sono che

206 Viola (2001) 60. Cfr. anche Dörr (2012) 477-604, qui 529.

207 Lauterpacht (1934) 713-819.

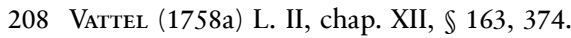

209 Mancuso (2002) 239 ss.

122 Capitolo II 
un modello di tali meccanismi: essi rappresentano il fil rouge delle teorie vatteliane e si trovano in tutte le relazioni riguardanti lo Stato verso se stesso e verso gli altri, all'interno delle regole per la condotta della guerra e per la diplomazia, che verranno affrontate nelle pagine successive prendendo come esempio la vera applicazione di esse nella realtà politica, giuridica e diplomatica.

Il Droit des gens tiene insieme il diritto internazionale e il diritto interno attraverso lo strumento della regolamentazione: sotto questo punto di vista può essere indagato nelle numerose fonti giuridiche dell'Ottocento tanto pratiche quanto dottrinali nella sua duplice veste di trattato moderno, come anticipatore di fondamentali temi, e trattato alla moda, come sinonimo non solo di successo editoriale, ma anche nell'identificazione di concetti di natura giuridico-politica. 



\section{Parte seconda}

\section{Il Droit des gens tra moda e modernità}

Una lettura del Droit des gens può essere ricercata nella straordinaria capacità di regolamentazione in esso contenuto, soprattutto per quanto riguarda la creazione di una nazione, che realizza di fatto i presupposti per l'evoluzione del diritto internazionale.

L'indagine presentata in questa sezione procede attraverso una ricognizione di nessi tematici che ruotano attorno alla figura del giurista di Neuchâtel nel corso del Settecento e dell'Ottocento, e contribuendo a delineare l'immagine complessiva di un fenomeno squisitamente giuridico. Si tratta della ripubblicazione di testi commentati di diritto delle genti del Sei e Settecento.

È stato posto giustamente in discussione il ruolo dei testi dottrinali, collocandoli nella gerarchia delle fonti del diritto internazionale come « fonti persuasive », «text-books» 0 «books of authority ${ }^{1}{ }^{1}$ tuttavia tali testi rappresentano un leitmotiv nella storia del diritto e nella storia del diritto internazionale e sono un fertile terreno di investigazione, perché permettono di comprendere le complessa circolazione e articolazione del diritto nelle sue molteplici e svariate realtà sociali, culturali e politiche.

Il richiamo costante alle teorie vatteliane in diversi e numerosi contesti ottocenteschi, conduce ad una analisi dei meccanismi che, anche nella storia del diritto delle genti, hanno favorito l'affioramento del Droit des gens, il quale oscilla tra due ambiti intimamente legati ed attratti, ma al tempo stesso distinti: quello di un trattato «di moda» e quello di un trattato « moderno ".

I concetti di « moda » e «modernità » sembrano dipingere e connotare il Droit des gens dalla sua pubblicazione nel 1758 sino al corso di tutta la prima metà dell'Ottocento, individuando due campi di ricerca, dai quali si giunge a conclusioni divergenti.

1 Genta (2004) 5. 
Prima di entrare nel merito della «vicenda Vattel », è opportuno illustrare, seppur brevemente, i richiami concettuali ai termini moda e modernità. La moda è, in primo luogo, uno dei dispositivi più validi attraverso cui leggere i molteplici percorsi di identità, sia questa personale, nazionale o trasnazionale; percorsi che coinvolgono temi sociali, economici ma anche giuridici e, nel caso specifico, di diritto costituzionale e internazionale.

In secondo luogo la moda è un processo che viene dettato dal mercato, regolato dal desiderio e della domanda del consumatore: questo è stato ben visibile nel Settecento, grazie all'esponenziale domanda di edizioni del Droit des gens sia in lingua francese sia nelle diverse traduzioni. Tale fenomeno viene incrementato anche nel corso dell'Ottocento con peculiari caratteristiche: quello dell'aggiunta dei commenti al Droit des gens di Vattel da parte giuristi specializzati nel diritto internazionale.

Le dinamiche della moda, quindi, non sono legate, come è consuetudine pensare, soltanto all'abbigliamento, agli stili, al linguaggio, all'arte, ai gusti, all'estetica ma anche più ampiamente a tutte le aree della cultura, che comprende l'inclinazione a leggere determinati testi e trattati giuridici con un fine più squisitamente formativo. Le mode condizionano anche le forme più alte della cultura generando concetti e figure del pensiero, influenzando il linguaggio e la realità e mettendo in evidenza le possibili connessioni. Esse «sembrano non poter prescindere dal riferimento a determinati topoi figurali e che finiscono per diventare veri e propri concetti passe-partout, parole d'ordine atte a convalidare la veridicità del discorso con l'autorità imposta dalla loro stessa presenza $»^{2}$

In terzo luogo, la moda dipende da due movimenti simultanei, che coesistono e realizzano di per sé il concetto stesso di «moda»: imitazione e distinzione. Con l'imitazione gli individui cercano di partecipare ad un modello universale, ad una comunità immaginaria, ad un segno generale della cultura oggettiva di quel momento, ad un gruppo sociale meritevole di prestigio e considerazione. Ma al tempo stesso i soggetti, come affermato da Georg Simmel, sentono il bisogno di distinguersi, di trovare segni attraverso cui differenziarsi da ciò che è troppo comune, banale, scontato. ${ }^{3}$

Rileggere il Droit des gens di Vattel attraverso la lente della «moda» significa soffermarsi su determinati dispositivi di pensiero, di storia delle

2 Corio (2007) 1.

3 Così Simmel (1998) 62. 
idee e di diffusione delle stesse che coinvolgono più livelli e permettono la rappresentazione di realtà, quale quella nazionale e internazionale, che sono il frutto di intrecci costanti, di letture giuridiche, di imprese editoriali di grandissimo impatto sulla circolazione della cultura giuridica dell'Ottocento.

La moda in sé propone una immagine dialettica tra ciò che è vecchio e ciò che è nuovo e si identifica nell'immagine dell'eterno ritorno, o per usare le parole di Walter Benjamin «la moda è l'eterno ritorno del nuovo ». ${ }^{4} \mathrm{Il}$ ritorno ai vecchi contenuti rielaborati in forma nuova, porta alla creazione di una vera e propria spirale senza fine in cui passato e il mutamento convivono in una apparente alternanza: "la moda è sempre nuova e sempre vecchia al tempo stesso, nella misura in cui l'imitazione è mimesi di qualcosa che già esiste e la sua proposizione ne mette in luce un nuovo aspetto ${ }^{5}$

Nel concetto di modernità risiede, invece, la straordinaria capacità di sperimentare l'innovazione attraverso un preciso atto volontario che è frutto del presente vissuto. Questo atteggiamento focalizzato al presente, come si è visto nel primo capitolo, è stato percepito da Vattel ed è stato concretizzato e teorizzato nel Droit des gens con la regolamentazione dei rapporti e delle istanze politiche e giuridiche di uno Stato nella sua capacità di relazionarsi con i cittadini e con gli altri Stati. Regolamentazione che ha influenzato gran parte delle teorie della prima metà del XIX secolo, proprio perché Vattel è riuscito a cogliere le contraddizioni e le tensioni del suo presente, anticipando alcune dei più importanti dibattiti sette e ottocenteschi. ${ }^{\mathbf{6}}$

Michel Foucault, nel commentare le posizioni di Charles Beaudelaire in De l'héroïme de la vie moderne sul concetto di moderno, ha sostenuto che " per l'atteggiamento moderno, il grande valore del presente è indissociabile dall'accanimento con cui lo si immagina diversamente da com'è e lo si trasforma, non per distruggerlo, ma per captarlo in quello che è ${ }^{7}$ Come si è detto precedentemente nel caso di Vattel, in coincidenza con la Guerra dei Sette Anni, vi era l'esigenza di scrivere per coloro che si occupavano di politica, divenendo così un vero e proprio vademecum per politici e diplomatici.

4 Benjamin (2006) $\$ 29,197$.

5 Pedroni (2013) 113.

6 Foucault (2012) 33.

7 Ivi, 36. Cfr. le riflessioni sul concetto di moderno di Gumbrecht (1997) 499 ss.; anche Gumbrecht (1978) 93-131. 
Vattel chiaramente non è stato completamente un innovatore ma nel suo atteggiamento ci sono tutti i presupposti per una visione moderna sia del diritto costituzionale e sia del diritto internazionale. Ciò permette di investigare, in una dimensione completamente diversa, l'influenza del Droit des gens sull'elaborazione del pensiero giuridico moderno, partendo dalla « ripresa » delle teorie vatteliane in forma di critica o di accoglimento attorno a precise tematiche e in circoscritti contesti storici e geografici, proseguendo con l'analisi del ruolo svolto dalle edizioni commentate del Droit des gens nel contesto europeo, per poi soffermarsi sulla versatilità del trattato che traspare dalla lettura dei giuristi.

Il legame tra la moda e la modernità si snoda sulla straordinaria concomitanza di interessi che lega l'una all'altra; entrambe proiettate verso il futuro, si rivolgono al passato, solo se questo può essere reinterpretato con gli occhi dell'attualità. ${ }^{8}$ Ma quale rapporto intercorre tra moda e moderno nel caso specifico di Emer de Vattel? Vattel è contemporaneamente alla moda e moderno? 


\section{Il ritorno del Droit des gens tra la fine del Settecento e l'Ottocento}

\section{Intrecci tematici intorno al Droit des gens}

Tra la fine del Settecento e i primi anni dell'Ottocento si registra un duplice fenomeno: da un lato la maggior parte delle nuove edizioni del trattato di Vattel non contengono particolari caratteristiche, ma sono per lo più ristampe delle precedenti; dall'altro però si assiste, in diversi contesti europei, ad una presa di posizione critica nei confronti del Droit des gens, che non verrà tralasciata dagli internazionalisti dell'Ottocento. Le riflessioni contenute nel Questions de Droit des Gens di Chambrier d'Oleyres o nel Discourse on the Study of the Law of Nature and Nations di James Mackintosh, ad esempio, saranno inserite in alcune delle più importanti edizioni francesi commentate del XIX secolo.

La fase di transizione, storica, politica e giuridica coinvolge fortemente il diritto delle genti nel suo processo di trasformazione in diritto internazionale: le guerre rivoluzionarie, la presa al potere da parte di Napoleone Bonaparte e i lavori del Congresso di Vienna, i moti rivoluzionari, hanno condizionato i giuristi e il loro ruolo all'interno del dialogo tra gli Stati. Come è noto l'espressione diritto internazionale nacque alla fine del Settecento da un'acuta riflessione di Jeremy Bentham, il quale sosteneva che la parola «internazionale» dovesse essere riconosciuta e utilizzata come un termine nuovo, come "branch of law » capace di racchiudere in sé tutte quelle caratteristiche proprie del «law of nations ${ }^{1}$

1 Jeremy Bentham utilizza il termine diritto internazionale e sottolinea che: «there remains then the mutual transaction between sovereigns, as such, for the subject of that branch of jurisprudence which may be properly and exclusively termed international ». Colui che, secondo Bentham, più si è avvicinato al termine "internazionale » è stato D'Aguesseau; egli raccomandava a suo figlio che la disciplina del diritto delle genti aveva un altro senso cioè il diritto tra le nazioni: Bentham (1789) chap. XVII, n. XXV, 236. Bentham si riferisce 
Tali peculiarità si riscontrano anche nel diverso utilizzo del trattato vatteliano: la sua dinamicità è presentata in quei contesti spazio-temporali in cui esso è stato richiamato per la sua «autorità » 0 , viceversa messo in discussione. Vengono così affrontati alcuni nessi tematici che ruotano attorno al Droit des gens e prendono forma grazie all'applicazione, alla critica e all'approvazione delle elaborazioni vatteliane da parte di celebri giuristi, i quali si sono cimentati nel diritto delle genti vatteliano. Gli esempi riguardano la neutralità nel dialogo scientifico tra Galiani e Lampredi; le regole per la condotta della guerra nei dibattiti parlamentari inglesi; le immunità dei consoli nella corrispondenza tra i cardinali Pacca e Consalvi; il diritto delle genti nelle sue molteplici articolazioni nel pensiero del diplomatico di Neuchâtel Chambrier d'Oleyres; e infine il valore del rispetto dei trattati internazionali e la loro interpretazione in alcuni casi americani ed inglesi e nelle controversie internazionali.

Il Droit des gens, come è stato rilevato da Koskenniemi, era un "'realistic' book, especially useful for diplomats and practitioners, not least because it seemed to offer such compelling rhetorics for the justification of most varied kinds of State action ». $^{2}$

Al fine di cogliere il legame tra moda e modernità e l'ambivalenza dell'uso del trattato, la struttura del capitolo si presenta, volutamente in senso figurato, come se fosse un polittico a più pannelli interconnessi tra di loro, con un sistema ad intreccio al cui centro ci sono le molteplici teorie vatteliane e la loro fortuna.

alla Première Instruction, contenant un Plan général d'Etudes, et en particulier celle de la Religion etcelle du Droit che aveva indirizzato al figlio il 27 settembre 1716. Queste sono le parole del cancelliere: "Je pourrois ici en ajouter une troisième, qui appartient en quelque manière au droit public; c'est ce qu'on appelle le droit des gens; ou, pour parler encore plus correctement (parce que le nom de droit des gens a un autre sens que vous apprendrez dans l'étude du droit romain), le droit entre les nations, jus inter gentes, qui comprend les règles que les nations doivent observer entr'elles, soit dans la guerre, soit dans la paix. Mais, comme cette espèce de droit n'a point d'autre force pour être exécuté, que celle que les idées de justice et d'équité naturelle peuvent lui donner; et qu'il n'y a aucune autorité supérieure qui puisse en affermir l'observation entre des princes ou des nations qui ne dépendent point l'une de l'autre, on ne peut lui donner le nom de droit que dans un sens général, et non pas dans l'exacte précision; parce que, comme vous le verrez ailleurs, la notion exacte du nom de droit renferme toujours l'idée d'une puissance suprême qui puisse contraindre les hommes à s'y soumettre ": così nella raccolta delle sue opere in una edizione successiva: D'Aguesseau (1819) 13.

2 Koskenniemi (2005) 112. 


\section{Teorie vatteliane in Ferdinando Galiani e Giovanni Maria Lampredi}

Il primo pannello vede come protagonisti l'abate Ferdinando Galiani e Giovanni Lampredi, per aver dato un notevole contributo al diritto delle genti e alle spinosissime tematiche della neutralità e delle relazioni commerciali tra le comunità neutrali e quelle belligeranti. ${ }^{3}$ Il giurista di Neuchâtel rappresenta l'anello di congiungimento tra i due. Alla base della celebre controversia scientifica tra Galiani e Lampredi sui limiti del commercio neutrale, sorta tra il 1782 e il 1788 , vi è la distinzione tra commercio neutrale passivo e attivo, che non solo era stata esplicitata da Vattel, ma questi ha fornito una precisa definizione della diversità dei regimi giuridici in un caso e nell'altro. ${ }^{4}$

Nella redazione del Droit des gens, infatti, Vattel non si è sottratto dal presentare alcune riflessioni su uno dei più complessi argomenti del diritto delle genti, quale è il concetto di neutralità all'interno della cornice giuridica, dando un contributo sostanziale alla dottrina. ${ }^{5}$ Egli ha dedicato alla neutralità l'intero capitolo, VII del suo terzo libro (sulla guerra), intitolato De la neutralité, et des troupes en pays neutre.

I popoli neutrali, scrive:

sont ceux qui n'y prennent aucune part, demeurant Amis communs des deux partis, et ne favorisant point les armes de l'un, au préjudice de l'autre. [...] Pour bien saisir cette question, il faut éviter de confondre ce qui est permis à une Nation libre de tout engagement, avec ce qu'elle peut faire, si elle prétend être traitée comme parfaitement neutre, dans une Guerre. Tant qu'un Peuple neutre veut jouïr sûrement de cet état, il doit montrer en toutes choses une exacte impartialité entre ceux qui se sont la guerre. ${ }^{6}$

3 La neutralità è sempre stata al centro dei dibattiti europei tanto della dottrina del diritto delle genti quanto nella pratica, sulle dinamiche tra Sette e Ottocento, senza pretesa di esaustività: cfr. Schnakenbourg (2013), Alimento (ed.) (2011). Per una ricostruzione del periodo storico nel contesto europeo e nord americano di fine Settecento: Grewe (2000) 384-393. Per quanto riguarda la sua evoluzione nel diritto delle genti: Helfman (2005) 549-586; Neff (2005) 153 ss.; Boye (1938) 157-231; Royen (1938) 18.

4 Miglio (2009) 51; anche il contributo Spagnesi (2011) 233-246. Un anno dopo la pubblicazione del Droit des gens le riflessioni di Hübner per il commercio degli Stati neutrali iniziavano a diffondersi in tutta Europa: HüBner (1759). Cfr. su Hübner: Naguib LeERberg (2015) e il contributo di SchNakenbourg (2011) 203-216.

5 Miglio (2009) 22. Sulle teorie relative alla neutralità con particolare attenzione a Vattel e al suo importante contributo Grewe (2000) 372-377. Su Vattel e la neutralità anche: Oeter (2011) 335-352; Oeter (1988) 470 ss.

6 Vattel (1758a) L. III, chap. VII, $\$ \$ 103-104,79$. 
L'imparzialità quale caratteristica essenziale dello Stato neutrale soggiace alle regole del diritto delle genti naturale, «résultent d'une juste combinaison des Droits de la Guerre, avec la Liberté, le salut, les avantages, le Commerce et les autres Droits de Nations neutres ». ${ }^{7}$ Difende energicamente la legittimità delle leve di truppe neutrali e dei prestiti di denaro a fronte di stati belligeranti. Il fine è la conservazione della nazione neutra, come principio generale, su cui si basa l'intero Droit des gens, e che rafforza il suo intento di « regolare » lo Stato fornendo tutto quello che serve ed è indispensabile alla vita dei propri abitanti. Non può infatti essere considerato contrario ai principi neutrali tutto ciò che una nazione realizza nell'esercitare i suoi diritti, mirando unicamente al proprio bene comune, in modo imparziale e senza favorire una delle due parti in guerra. Diversamente è incompatibile con le regole della neutralità quando si realizza facendo un torto ad uno dei belligeranti. In tutte le guerre che agitano l'Europa, gli abitanti della Svizzera, precisa Vattel, si mantengono neutrali e consentono ad ognuno indistintamente di andare a comprare viveri, cavalli e munizioni. ${ }^{\mathbf{8}}$

Vattel diversifica il commercio neutrale passivo, cioè quello svolto sul territorio dello stesso Stato neutrale, dal commercio neutrale attivo, cioè che si realizza con le vendita per trasporto da parte dello Stato neutrale, sottoponendo a regimi diversi l'attività del neutro. Nel caso del commercio passivo, la posizione del neutro si giustifica in base alle regole generali della libertà assoluta del commercio e dell'imparzialità: pertanto questi sarà legittimato a vendere, anche ai belligeranti che si trovano sul suo territorio ogni genere di merci, dalle vettovaglie ai cavalli, al legno da costruzione, dalle navi alle armi, alle munizioni da guerra, in virtù del principio della libertà degli scambi economici. ${ }^{9}$ L'eccezione alla regola, che impedisce al neutro di commerciare le armi, è quando questa attività si perfeziona con il trasporto a destinazione delle merci stesse; mentre il commercio effettuato sul proprio territorio può essere limitato in presenza di un trattato che impegni lo Stato neutro ad essere imparziale con entrambi.

Il commercio neutrale attivo è trattato in modo molto peculiare da Vattel, in quanto sostiene che lo Stato neutro, anche nel caso della vendita per trasporto, goda del pieno diritto di rifornire i belligeranti di qualunque tipo di merce, a condizione che si comporti in maniera uguale con ambedue le

7 Ivi, $\$ \int 110,83$.

$8 \mathrm{Ivi}, \mathbb{S S} 118,93$.

9 Miglio (2009) 52.

132 Capitolo III 
parti in guerra: il neutro pertanto « esercita un diritto - un diritto alla libera attività commerciale - che non è obbligato per nessuna ragione a sacrificare in favore del belligerante ». ${ }^{\mathbf{1 0}}$

Gianfranco Miglio ritiene che la classificazione minuziosa delle merci oggetto del commercio di uno Stato neutro sia « un punto essenziale del sistema vatteliano ». ${ }^{\mathbf{1 1}}$ Il giurista di Neuchâtel, a differenza dell'illustre predecessore Ugo Grozio, distingue due categorie: la prima sono le merci che non hanno alcun rapporto con la guerra e con le sue necessità, che di conseguenza possono essere sempre liberamente fornite e trasportate agli stati belligeranti:

les puissances en guerre n'ont aucune raison de le leur refuser, d'empêcher le transport de pareilles marchandises chez l'ennemi : Le soin de leur sûreté, la nécessité de se défendre, ne les y autorise point, puisque ces choses ne rendront pas l'Ennemi plus formidable. Entreprendre d'en interrompre, d'en interdire le commerce, ce seroit violer les droits des nations neutres et leur faire injure; la nécessité, comme nous venons de le dire, étant la seule raison qui autorise à gêner leur Commerce et leur navigation dans les ports de l'Ennemi. ${ }^{\mathbf{1 2}}$

La seconda tipologia di merci è legata alla guerra e costituisce la mercanzia di contrabbando: il trasporto di esse ad uno degli Stati in conflitto può legittimamente essere ostacolato. Pertanto solo se il trasporto e la fornitura della merce è a nome dello Stato neutrale si realizza la violazione dei doveri di neutralità, viceversa se avviene per iniziativa dei privati non c'è alcuna violazione. ${ }^{13}$ Vattel elenca nel dettaglio le merci che appartengono all'una e all'altra categoria: « Les choses qui sont d'un usage particulier pour la Guerre, et dont on empêche le transport chez l'Ennemi, s'appellent marchandises de contrebande. Telles sont les Armes les Munitions de Guerre, les bois et tout ce qui sert à la construction et à l'armement des Vaisseaux de Guerre, les Chevaux ». ${ }^{14}$

Anche « les vivres», quando si « espere de réduire l'ennemi par la faim », ${ }^{15}$ possono rientrare in questa categoria, anticipando, in forma primordiale, la moderna e attuale nozione di blocco totale. ${ }^{16}$

10 Miglio (2009) 53.

11 IVI, 54.

12 Vattel (1758a) L. III, chap. VII, $\mathbb{1} 112,87-88$.

13 Miglio (2009) 55.

14 Vattel (1758a) L. III, chap. VII, $\mathbb{\$} 112,88$.

15 Ibidem.

16 Miglio (2009) 55. 
L'abate Ferdinando Galiani non condivise, in particolare, la libertà di commercio del neutro che precede la bipartizione tra commercio attivo e passivo. Egli pubblicò nel 1782 il suo De' doveri e de' principi neutrali verso $i$ guerreggianti e di questi verso $i$ principi neutrali, opera che ebbe particolare apprezzamento in tutta Europa e «ben potrebbe essere il coronamento della sua carriera di scrittore politico $» .{ }^{\mathbf{1 7}}$

Galiani definì la neutralità come " un bene universale della misera spezie umana ${ }^{\mathbf{1 8}}$ ma, al tempo stesso, sottolineava "che così bella materia di studi, e di meditazioni sia stata poco men, che dell'intutto trascurata! ». ${ }^{19}$ Dopo un breve excursus storico commentava l'opera di Grozio, il quale «col favore della sua immensa lettura e prodigiosa memoria, avendo d'un ricamo ricchissimo d'erudizione abbellita la vecchia stoffa del Diritto Pubblico, acquistò fama di fondatore d'una scienza, cha alla vaghezza, ed al lustrore di quell'insolito ornamento parve tutta nuova, appena destinò uno, o due capitoli del suo libro De Jure Belli ac Pacis a parlare de' dritti, e dei doveri della neutralità ", ${ }^{20}$ per poi rivolgersi a coloro che avevano seguito le orme di Grozio, cioè Hobbes, Pufendorf, Wolff e Barbeyrac, all'interno delle cui opere « invano si cercheranno profonde discussioni sulla materia della neutralità ", ma solo nozioni elementari «e spesso poco correttamente pronunziate, $\mathrm{o}$ anche in tutto false vi s'incontrarono $»^{\mathbf{2 1}}$

Galiani, pur riconoscendo che Vattel fosse stato l'unico ad essersi addentrato in tale materia, avanzò non poche critiche concernenti la sua metodologia. Secondo l'Abate «E' rimarchevole [...], che spesso unisce, e mischia i dettami della giustizia con i consigli della prudenza politica; il che [...] genera confusione, e turba la concatenazione degli argomenti, e delle pruove, dalla quale sola ha da dipendere la verità delle dottrine del Gius ». ${ }^{22}$

17 Stapelbroek (2011) 77. In un altro saggio Stapelbroek contestualizza Galiani ponendo in relazione la sua prospettiva con la storia del commercio nel Mediterraneo: Stapelbroek (2008a) 63-89; per una ricostruzione dell'ambiente napoletano e della penisola italiana: Stapelbroek (2008b) 127 ss.; Stapelbroek (2006a) 361-367; Stapelbroek (2006b) 406429; Stapelbroek (2001) 137-169. Per approfondimenti su Galiani anche: Diaz (1968) 854-909; Deloche (1988) 1143-1157; de Majo (1998) 456-465.

18 Galiani (1782) L. I, Introduzione, 1.

19 Ivi, 2.

20 Ibidem.

21 Ivi, 4-5.

22 Ivi, 5, nota 1. 
Si scagliava poi sulla mancanza di spiegazioni fornite dal giurista di Neuchâtel: "Argomenti o ragioni da stabilire il suo sentimento non ne dà, $\mathrm{e}$ forse non ne aveva nel suo capo. L'esempio della nazione Svizzera e di altre in Europa formano tutta la sua prova ${ }^{23}$ egli definì la dottrina di Vattel «bizzarra » e con la quale questi «se n'applaudisce egli stesso come d'un insegnamento più umano, e capace di diminuire le calamità dell'Europa, coll'estinguere le scintille di nuove guerre $[\ldots] »{ }^{24}$

Galiani continuava nella sua invettiva: «Egli avea nel suo frontespizio annunziato un libro del Diritto delle genti e non una opera della Dissimulazione Politica, o della Pazienza Cristiana ${ }^{25}$

Le critiche si fanno sempre più serrate al capo IX, quando Galiani trattò del commercio tra i neutrali e i belligeranti: Vattel «tra tutti si contradistingue per la facilità d'accozzare opinioni contradittorie », e dicendo altresì: «Se insegnando il dritto publico tra così palpabili, e grossolane contradizioni si renda servizio, o disservigio al genere umano lo giudichi ogni lettore. Io non ho riferito questi luoghi del Vattel con altro disegno, fuorché di dimostrare il caos, l'intrigo, l'incertezza, in cui rimane il dritto di vietare ai neutrali il commercio con talun de'guerreggianti ». ${ }^{\mathbf{2 6}}$

Lampredi, invece, ebbe una posizione molto diversa, diametralmente opposta, e il suo tono non fu così polemico, anzi molto spesso pur non citandolo tra le sue fonti, si coglie la sua influenza; il Droit des gens, come argomentato da Paolo Comanducci, «è una delle opere più utilizzate dal

23 Galiani (1782) 120, e in nota continua che «questo rimprovero di non addurre ragioni, frequente a potersi fare al Vattel, ed a non pochi altri, è grave quando si scrive sul Diritto delle Genti. Nelle decisioni di diritto basterebbe citar la legge; ma le nazioni tra loro non hanno altre leggi scritte, che i trattati volontariamente stipulati. Quando questi tacciono, altro non rimane fuorché quell'eterno, e general lume di natural filosofia comune a tutti gli uomini, e tanto impresso in noi, che fa parte della nostra stessa non viziata natura. Con quest'arme alla mano dovea il Vattel ragionare, e non coll'esempio degli usi attuali d'Europa, esempio fallace, e che a ben rifletterlo neppure s'adatta alle sue dottrine ». Ivi, 120-121, nota 2. Per una panoramica del contesto e del dibattito dell'epoca sulle questioni relative alla neutralità: GREwE (2000) 372-376.

24 Galiani (1782) 123.

25 Ivi, 124. Nel cercare di trovare una spiegazione alla teoria di Vattel, Galiani afferma che compose il suo Droit des gens « al fine di far piacere alla generosa Corte di Sassonia e ai Cantoni Svizzeri. Ma appunto perché tra gli Svizzeri egli dimorava è meno scusabile di non essersi avveduto, che l'antico uso loro di dare truppe ai Sovrani d'Europa nulla ha che fare con la sua questione »: Ivi, 124, nota 2.

Ivi, $178-179$, nota 1. 
giurista toscano in tema di diritto delle genti ». ${ }^{27}$ Già nello Iuris publici universalis, sive Iuris naturae et gentium theoremata, iniziato nel 1767 , ci sono moltissimi rinvii diretti e indiretti alle teorie elaborate da Vattel. ${ }^{28}$ La terza parte dei Theoremata, è interamente dedicata allo ius gentium, in cui Lampredi affrontava e cercava di sviscerare la complicata relazione tra diritto naturale e diritto delle genti, prendendo come riferimento i più importanti esponenti del giusnaturalismo: così se da un lato veniva respinta la civitas maxima di Wolff, dall'altro si accoglievano le posizioni, ad esempio, di Barbeyrac e di Heinecke. ${ }^{29}$

Qualche anno più tardi nel 1788 diede alle stampe Del commercio dei popoli neutrali in tempo di guerra, ${ }^{30}$ dove sostenne energicamente la libertà di commercio degli Stati neutrali, fondata sul diritto delle genti e su quello pattizio in risposta alle tesi di Galiani. ${ }^{31}$ L'introduzione si presenta come una articolata disamina dei principi che regolano il commercio tra gli Stati neutrali e delle più significative posizioni dottrinali che si sono susseguite nel tempo; per Lampredi, senza dubbio, il più rappresentativo fu Alberico Gentili, che tentò di creare « un sistema di giustizia anche tra lo strepito dell'ar$\mathrm{mi} »,{ }^{32}$ le cui posizioni segnarono « in certo modo la strada al celebre » Ugo Grozio, «il quale scrivendo con maggiore eleganza, ed ornando il suo Trattato di forbita e pellegrina erudizione fece quasi scordare il suo Maestro, di cui però seguitò quasi totalmente il disegno ». ${ }^{33}$ Per Lampredi, Gentili si soffermava sul problema del commercio dei popoli neutrali « per incidenza, e dice quanto basta per concludere che [...] è difficile da sciogliersi sembrando, dic'egli, che tanto il Belligerante, che impedisce e vieta il commercio dei Neutrali, quanto ai Neutrali, che si chiamano offesi da questo impedimento siano fondati in ragione ${ }^{34}$ Tale questione era, come scriveva il professore di Pisa, purtroppo ancora pendente e mai risolta, nonostante

27 Comanducci (1981) 269.

28 Ivi, 267.

29 Ivi, 262-264.

30 Su Lampredi per una ricostruzione del pensiero, del contesto e delle influenze dottrinali si rimanda a: Comanducci (1981); VAnNini (2004) 259-262. Su Lampredi professore titolare della cattedra di diritto pubblico a Pisa: Marini (2000) 635-661.

31 Comanducci (2013) 1138-1141.

32 LAMPREDI (1788) 4.

33 Ivi, 6.

34 Ivi, 5.

136 Capitolo III 
fossero trascorsi quasi duecento anni dalla pubblicazione del De iure belli libri tres di Gentili, e per questo motivo era intenzionato ad affrontarla, tenendo in grande considerazione, la realtà in cui viveva, gli usi delle nazioni e i motivi da cui scaturivano confusioni nella trattazione del commercio.

L'attenzione alla pratica e alla situazione storico-politica caratterizzano anche l'opera di Vattel, che viene richiamato nel corso della trattazione da Lampredi. Egli parlò di «Wattel », come di un autore che riuscì sempre a dare risalto al diritto delle genti illustrato da Wolff: « Ma più chiaramente di lui ha parlato il suo redattore Wattel, che traducendolo in lingua francese gli ha tolto la disgustevole aria scolastica, e l'ha ornato di vesti assai più leggiadre ${ }^{35}$ Lampredi accolse, riprese in toto e ampliò maggiormente le teorie vatteliane avversate da Galiani, argomentando il tema del commercio effettuato da Stati neutrali e la contestuale distinzione tra la vendita di merci utilizzabili da uno solo dei contendenti (da qualificarsi illecita), e la vendita delle stesse merci ad entrambi (lecita). Resta senza dubbio un dato certo: le posizioni di Vattel e Lampredi furono conformi al contesto storico, politico e giuridico nel quale vivevano e rappresentano uno specchio delle tensioni interne ed esterne dei rispettivi Stati di origine. Il giurista di Neuchâtel, infatti, assegnò un regime privilegiato al commercio neutrale passivo, osservando e valutando le scelte economiche della Svizzera, esattamente come Lampredi aveva ben presente la medesima prassi adottata e difesa dal Granducato di Toscana. ${ }^{36}$

\section{Il punto di vista del neocastellano Jean-Pierre Chambrier d'Oleyres}

Alla fine del XVIII secolo e durante tutta l'epoca successiva non sono mancate monografie e saggi che, partendo da precise situazioni storiche-politiche, hanno criticato fortemente il Droit des gens, delineando quanto la sua teoria fosse inefficace specialmente sul piano delle relazioni internazionali. Jean-Pierre Chambrier d'Oleyres appartiene a queste correnti saggistiche che tendono ad offuscare $\mathrm{o}$, più precisamente $\mathrm{a}$ " ridimensionare » l'operato della dottrina vatteliana.

35 Ivi, 65.

36 Miglio (2009) 57. 
Chambrier condivide con Vattel il luogo di nascita, Neuchâtel, lo stesso amore per la sua terra, la stessa passione per gli studi umanistici, ma, di estrazione sociale molto elevata, la sua esistenza di vita, fu opposta a quella molto modesta e faticosa di Vattel.

Appartenente ad una importante famiglia nobile, Chambrier fu impegnato sin da giovanissimo in importanti missioni diplomatiche. ${ }^{37} \mathrm{Nel}$ febbraio 1780 Federico II lo aveva nominato proprio ambasciatore presso la corte di Torino. ${ }^{38}$ L'incarico, rinnovato il 20 agosto 1786, fu mantenuto sino al fatidico dicembre 1798, e con l'occupazione di Torino da parte delle truppe francesi Chambrier abbandonò la città sabauda per rientrare a Neuchâtel nel castello ereditato dal padre presso Comondrèche. ${ }^{39}$ Egli realizzò la delicata operazione politica e diplomatica del passaggio di consegne del Principato di Neuchâtel dalla Prussia alla Francia napoleonica. ${ }^{40}$ Quando nel marzo del 1814 il Principato di Neuchâtel fu restituito alla Prussia, Chambrier fu allora investito della carica di governatore e luogotenente generale, che mantenne sino alla morte avvenuta a Neuchâtel il 22 dicembre 1822 , come cittadino ormai svizzero. ${ }^{41}$

37 Egli era nato il 4 ottobre 1753 da Charles-Louis de Chambrier (1690-1770) e da Madelain Brun d'Oleyres (1711-1786). Figlio unico e celibe, nel 1797 adottò un lontano cugino, Frédéric de Chambrier per impedire l'estizione del casato, che è ancora oggi esistente.

38 Basso (2004) 943-962, qui 948. Su Chambrier d'Oleyres: NaKhimovsky (2010) 152; Weber (2012) 142-157. E' attualmente in corso un progetto sulla figura del diplomatico presso l'università di Berna, curato da Christian Widler con la collaborazione di Nadja Ackermann, intitolato: « ¿Sattelzeit > de la diplomatie? Sémantiques politiques et pratiques de négociation durant la carrière diplomatique de Jean-Pierre de Chambrier d'Oleyres (1753-1822) »: http://www.hist.unibe.ch/forschung/forschungsprojekte/sattelzeit_der_ diplomatie/.

39 Basso (2004) 948-949. Chambrier fu particolarmente attento e attivo alla storia locale durante questo periodo, nel 1789, pubblicò le sue Notices Préliminaires sur des recherches historiques relatives à l'Etat de Neuchâtel et Vallengin.

40 Chambrier aveva ricoperto la carica di ambasciatore prussiano, perché ancora in quegli anni Neuchâtel, come si è visto nel capitolo precedente, era sede di un autonomo principato, e dal 1707, era passato sotto il regno di Prussia. Successivamente fu annesso da Napoleone alla Francia nel marzo del 1806, e consegnato nelle mani del maresciallo Louis-Alexandre Berthier, capo di stato maggiore dell'imperatore: il passaggio delle consegne fu appunto svolto proprio da Chambrier.

41 Basso (2004) 948-949. Infatti, nel 1815, il Principato entrò a far parte della Confederazione Elvetica, Chambrier di fatto fu cittadino «svizzero » solamente negli ultimi sei anni di vita, pur continuando ad essere un possedimento del re di Prussia e tale rimanendo sino al 1857, quando Federico Guglielmo IV, rinunciò definitivamente alla sovranità e si limitò a conservare il titolo di principe di Neuchâtel. 
Conosciuto come un uomo di straordinaria cultura che spaziava dalla storia alla musica, ${ }^{42}$ nel 1792 Chambrier divenne membro dell'Accademia Reale Prussiana di Scienze e Belle Arti di Berlino, dove pubblicò vari studi nei campi della storia e della letteratura, tra cui le Questions de Droit des Gens et Observations sur le Traité du Droit des Gens de M. de Vattel, ${ }^{\mathbf{4 3}}$ che verranno raccolte e pubblicate nel 1795 a Parma sotto il titolo l'Essay sur le Droit des Gens. ${ }^{44}$

Le scettiche posizioni di Chambrier nei confronti dell'opera vatteliana divennero il punto di partenza per le riflessioni contenute nelle edizioni del Droit des gens del XIX secolo, come rilevano, ad esempio, le note che Pradier-Fodéré appose nel 1863.

Il saggio di Chambrier è stato oggetto di un attento studio da parte di Isaac Nakhimovsky e si colloca all'interno del dibattito, che vedeva in prima linea schierato anche Kant con la pubblicazione nello stesso anno della sua Per la Pace perpetua. ${ }^{45}$ L'Europa dell'epoca viveva l'impeto delle guerre rivo-

42 Chambrier fu definito da Giovanni Maria Rutini un uomo «amabilissimo come bravissimo nella musica ». Come illustrato da Alberto Basso, durante il suo soggiorno a Torino, fu allievo del celebre violinista e compositore torinese Gaetano Pugnani e che lo fece diventare membro dell'Accademia Filarmonica di Bologna: BAsso (2004) 949, anche 948, nota 18; Schnoebelen (1979) 131-132. E' opportuno precisare, per comprendere la caratteristica poliedrica di Chambrier, che con Isabella Agneta de Charrière (nata Tuyll van Serooskerken), famosa scrittrice olandese naturalizzata svizzera, ebbe una intensa corrispondenza con importanti risvolti non solo musicali, ma anche politici e rappresenta una vera e propria testimonianza di come veniva percepita e vissuta la Rivoluzione fuori dalla Francia. I due si incontrarono all'Aja probabilmente nell'agosto 1772 ed ebbero scambi all'inizio saltuari, prima di avviare una fitta corrispondenza nel dicembre 1784. La scrittrice aveva fatto avere al diplomatico una copia del suo romanzo epistolare Lettres neuchâteloises da lei pubblicato ad Amsterdam all'inizio dello stesso anno. Negli anni della Rivoluzione Isabelle entrò in contatto anche con Benjamin Costant. Nel settembre 1789 la stessa scriveva a Chambrier: « on a la tête si pleine de politique en ce moment que l'on ne peut s'empecher de considerer tous les objet sour leur point de vue politique », e si esprimeva in un primo momento contro poi, dopo il 1793 a favore degli immigranti francesi che scappavano dalla loro terra, frammento di lettera riportata in LetzTer (1998) 55. Dal punto di vista musicale, oltre alla proposta di Chambrier di mettere in contatto Isabelle con Domenico Cimarosa (che denota quanto il diplomatico fosse inserito nell'ambiente musicale), occorre solo ricordare che il 5 novembre 1788 Isabella Agneta de Charrière, gli comunicava di aver spedito la sua tragedia lirica Les Pheniciennes direttamente a Mozart: EISEN (1991) 1788; BASSO (2004) 943-962.

43 NaKhimovsky (2010) 143.

44 Chambrier d'Oleyres (1795).

45 NaKhimovsкy (2010) 141-164. 
luzionarie, che si succedettero a seguito delle ostilità tra il governo rivoluzionario francese e la maggior parte delle potenze europee dal 1792 fino al 1802, quando venne firmato il trattato di Amiens; benché l'Inghilterra fosse costantemente in guerra con la Francia, esse si divisero tra prima coalizione (1792-1797) e seconda coalizione (1798-1802), a cui seguirono i successivi scontri all'interno delle guerre napoleoniche.

Nel 1795, a seguito dell'occupazione francese dei Paesi Bassi e alla creazione di uno Stato satellite, la Repubblica di Batavia, la Prussia si poneva il problema se restare in guerra con la coalizione contro la Francia o negoziare con essa la pace. La pace fu firmata il 5 aprile 1795 con il trattato di Basilea alla presenza per la Francia di de Barthélemy e per la Prussia dell'ambasciatore von Hardenberg e con la quale si riconobbe alla Francia il possesso della riva sinistra del Reno, la Renania. ${ }^{46}$

In questo conflittuale contesto storico si trovano le opposte posizioni di Kant e di Chambrier, come rilevato da Nakhimovsky. ${ }^{47}$

In Kant, traspare in modo evidente il suo entusiasmo per la Rivoluzione francese in atto, sostenendo che "se infatti la fortuna volesse che un popolo potente e illuminato possa costituirsi in repubblica (la quale per la sua natura deve tendere ad una pace perpetua), allora si avrebbe in ciò un nucleo dell'unione federativa per gli altri Stati, che sarebbero indotti ad associarsi ad essa, a garantire così lo stato di pace fra gli Stati in conformità all'idea del diritto internazionale e ad estendersi sempre più mediante ulteriori unioni della stessa specie ${ }^{\mathbf{4 8}}$

Al contrario Chambrier giustificava il ricorso alla guerra contro la Francia rivoluzionaria argomentando la necessità di fermare e di coalizzarsi contro chi violasse in modo così manifesto le leggi che sono alla base della società $\mathrm{e}$ mettono a rischio gli Stati stessi e la loro sicurezza interna:

si une nation étoit parvenue, par le délire des chefs d'un parti prédominant chez elle, à inspirer une défiance manifestement fondée à toute l'Europe, et que chaque Souverain fût ouvertement menacé de voir ses sujets se révolter contre lui par les

46 Per una ricostruzione storica: Fremont-Barnes (2001); Blanning (1986); Blanning (1996). Per le ripercussioni delle guerre rivoluzionarie sul diritto delle genti: Kubben (2011) passim. Si rimanda anche all'intero volume edito da Jacobs/Kubben/Lesaffer (eds.) (2008); Grewe (2001) 416-424.

47 NaKhimovsky (2010) 141-164.

48 Kant (2004) 65. Sulla ricezione di tale passaggio: Ruiz (1980) 47-193; Azouvi / BureL (1991). 
manœuvres des émissaires envoyés dans son pays, lorsque ceux-ci ne feroient pas formellement désavoués; si elle se permetroit même de consacrer par ses délibérations des principes de conduite à l'egard des autres qui lésassent leur sureté commune en autorisant l'insurrection des pays voisins, et leur coalition avec elle: les autres seroient fondées à se réunir contre celle qui violeroit aussi manifestement les loix générales sur lesquelles repose leur société. ${ }^{49}$

Dall'analisi dei rispettivi saggi emerge che il Droit des gens risulta in un certo qual modo, anche in questo caso, il loro anello di congiungimento. Kant non si era concentrato come Chambrier su una critica precisa ed esclusiva del pensiero e dell'opera di Vattel, ma, in un famoso passaggio della sua Per la pace perpetua, si dimostrò fortemente in disaccordo con Grozio, Pufendorf e anche con il giurista di Neuchâtel, in quanto nei loro scritti avevano prestato sempre più attenzione alla guerra (a cui come ben è noto Vattel dedicò interamente il terzo libro), ritenendola quasi come parte integrante della vita e giudicandola evidentemente ineliminabile, piuttosto che concentrarsi ed impegnarsi per porre condizioni per stabilire una pace vera. ${ }^{50}$

Dal punto di vista dello ius gentium, così concepito da Vattel e dai suoi predecessori la pace sarebbe stata impossibile: dunque, secondo Kant non si possono considerare giuristi, giacché per poterlo essere avrebbero dovuto ammettere una autorità superiore che facesse rispettare il diritto, la cui esistenza era però incompatibile con l'essenza stessa della sovranità statale. ${ }^{51}$

Chambrier, invece, sviscerò molti passaggi del Droit des gens e delle Questions de droit naturel, elaborando le sue considerazioni, facendo riferimento alla realtà storica della sua epoca e inserendo anche le posizioni di altri autori a lui contemporanei. Il suo principale intento era però quello di far risaltare le teorie di Wolff a discapito del suo "concittandino ». Si schierò contro Vattel soprattutto per quanto concerne le caratteristiche del diritto delle genti volontario, sottolineando che la dicotomia diritto delle genti necessario e diritto delle genti volontario apparteneva a Wolff, del quale Vattel era solo un semplice populizzatore. Ad esempio, contro le critiche di Vattel alla civitas maxima, Chambrier sosteneva che:

Vattel ne trouve pas cette idée satisfainte; il ne reconnoit point d'autre Société naturelle entre les nations que celle que la nature a établie entre tous les hommes, et il se promet de faire voir que toutes les modifications, les restrictions et tous les

49 Chambrier d’Oleyres (1795) 49.

50 Kant (2004) 61-62. Cfr. Koskenniemi (2009) 395-422.

51 Kant (2004) 61-62. 
changemens en un mot qu'il faut apporter dans les affaires des nations à la rigueur du droit naturel, et dont se forme el droit volontaire, se déduisent de la liberté naturelle des nations, des intérêts de leurs devoirs réciproques, et des distinctions de droit interne et externe, parfait. ${ }^{52}$

La disapprovazione è decisa e puntuale, perché le posizioni di Vattel, rifiutata l'idea wolffiana, avrebbero compromesso le intere basi normative per giudicare e punire le azioni commesse dagli Stati contro il diritto delle genti. Chambrier all'interno del suo saggio procedeva inoltre a sistematiche osservazioni che coinvolgevano tutta l'opera vatteliana. Ad esempio argomentava che Vattel nel dare la sua definizione di diritto delle genti, aveva creato una sorta di incertezza, in una scienza dove i principi «sont si lumineux par euxmêmes, a été corrigée de nos jours par des écrivains philosophes qui ont développé ces principes dans leur ordre naturel, en les appliquant aux cas les plus controversés ».53

Chambrier, altresì, sosteneva che il principio dell'indipendenza assoluta delle nazioni come teorizzato da Vattel, creasse una sorta di libero arbitrio da parte dei singoli Stati e l'unico modo per arginare la situazione di incertezza ed evitare sopraffazioni consistesse nel riconoscere che gli Stati non fossero assolutamente indipendenti ma facessero parte di una "société générale des nations $» .54$

Tra le fonti utilizzate per scagliare le invettive contro Vattel oltre alle teorie di Sulzer, una, in particolare, gli è consona: si tratta del pensiero di Ferdinando Galiani.

Chambrier era concorde con Galiani sulla "confusione » che aleggiava nel Droit des gens e, sulla materia della neutralità e del contestuale commercio con gli Stati neutri, affermava che l'economista di Napoli aveva elaborato un sistema sicuramente più solido e più articolato. ${ }^{55}$ Aveva ripercorso quei

52 Chambrier d'Oleyres (1795) 9-10.

53 Ivi, 6.

54 Ivi, 11 ss. A questo punto Chambrier citava il saggio di Johann Georg Sulzer intitolato Recherches sur un principe fixe, qui serve à distinguer les devoirs de la morale de ceux du droit naturel e pubblicato lo stesso anno del Droit des gens. Sulzer (1720-1779), esponente di una forma moderata di estetica razionalista di stampo wolffiano si interroga sui criteri distintivi tra i doveri della morale e quelli del diritto naturale e delle genti. Sulzer, come è noto, fu allievo di Johann Jacob Bodmer e docente all'Accademia di Berlino: NAKHIMOvSKY (2010) 156. E' interessante notare che anche le riflessioni di Sulzer verranno riprese e inserite nelle edizioni francesi ottocentesche del trattato di Vattel.

55 Chambrier d'Oleyres (1795) 40 ss. 
passaggi vatteliani oggetto di critica da parte di Galiani alla luce della situazione politica europea, proponendo una visione distante da quella del Droit des gens e delle Questions, in quanto i principi del diritto delle genti sono analizzati nei suoi progressi e nelle dinamiche esistenti con il diritto naturale, nella più ampia cornice delle relazioni internazionali e diplomatiche dell'epoca.

Stupisce l'attenzione che il brillante ed eclettico Chambrier, il diplomatico della realpolitik, ha avuto nei confronti di Vattel e delle sue teorie; certamente aveva percepito che l'opera, per la sua caratteristica non più solo filosofico-concettuale, ma anche pratica e concreta, potesse entrare nelle più svariate situazioni storiche, politiche e sociali e apportare il proprio contributo al diritto delle genti, entrando di fatto nel novero dei « famosi ».

\section{Giuristi e politici inglesi: «Vattel esprime il senso del tempo in cui viviamo"}

L'illustre politico inglese Edmund Burke, sostenitore dell'intervento contro la Francia rivoluzionaria, fu, in un primo momento, un profondo ammiratore di Vattel: non solo lo considerava una autorità nel diritto delle genti, ma veniva identificato come colui che meglio rappresentava « il senso del tempo » a lui contemporaneo. ${ }^{56}$

Tra il 1792 e il 1793 approfondì gli studi annotando il Droit des gens. Le parti che attirarono l'attenzione di Burke nel suo Extracts from Vattel's Law of Nations riguardavano le teorie in merito al diritto di asilo (L. I, cap. XIX), ai casi stabiliti da Vattel in cui era legittimo l'intervento di un Stato nel ter-

56 Burke proferiva queste parole all'interno dello Speech on the Seizure and Confiscation of Private Property in St. Eustatius, discorso edito in: Burke (1816) 259. Occorre precisare che tale testo è pervenuto grazie ad una sintesi effettuata da una terza persona, e non è quello redatto di proprio pugno da Burke. Sulla vicenda di St. Eustatius oggetto dello Speech nella politica inglese e per la posizione assunta da Burke, sulle citazioni al Droit des gens, ma al tempo stesso sul suo superamento e l'utilizzo di altre fonti dottrinali, si rimanda a: Авватtista (2008) 1-33. Sul rapporto tra Burke e Vattel cfr. Welsh (1995) 32-35; diverse considerazioni sull'influenza vatteliana in: STANLIS (1953), 397-413, 403, nota 19. Sulle posizioni di Burke contro la Francia rivoluzionaria: Armitage (2000) 617-634, in particolare 627-631; Hampsher-Monk (2005) 65-100; Dreyer (1979) 6-23 in particolare 7-8; Calamo Specchia (2015) 71-88. Sul confronto tra Vattel e Burke e i rapporti tra l'Inghilterra e la Francia: Whatmore (2010) 85-107, in particolare 104 ss. 
ritorio di un altro (L. II, cap. IV), ai casi di alleanza (L. II, cap. XII), al sistema europeo (L. III, cap. III), ai beni che appartengono ai nemici e al loro trattamento in guerra (L. III, cap. IX) e nel contesto diplomatico, al diritto di ricevere e di inviare i ministri degli affari esteri (L. IV, cap. V). ${ }^{57}$

Burke si era addentrato con scrupolo nel Droit des gens, proprio per cercare una convincente giustificazione nel portare avanti il suo disegno contro la Francia, ma scoprì che per il giurista di Neuchâtel non c'è un universale diritto di punire perché «le droit de punir dérive uniquement pour les hommes, du droit de sûreté, par conséquent il ne leur appartient que contre ceux qui les ont offensés ». ${ }^{\mathbf{8}}$

Diventava difficile per Burke giustificare un intervento dell'Inghilterra attingendo esclusivamente dalla dottrina vatteliana. Già dal 1793, come argomentato da Nakhimovsky, la stima e devozione di Burke cominciavano a diminuire e quando scrisse le sue Letters on a regicide peace aveva già messo da parte Vattel, descrivendo l'Europa come una singola comunità. ${ }^{59}$

Le posizioni prese nei confronti del Droit des gens a fine Settecento provengono da uomini di un certo peso politico e si può argomentare che il diritto internazionale fosse ormai in cerca di punti fermi e ben definiti in una Europa di grandi sconvolgimenti: se, infatti, Chambrier sin dall'inizio contestava il diritto delle genti vatteliano, in Burke la critica è più lenta e si sviluppa nei corso degli anni, fino alla decisione definitiva di accantonare il trattato.

Iniziavano altresì ad essere pubblicati saggi con l'intento di ricostruire la storia del diritto delle genti. Nel 1795, un altro famoso giurista inglese Robert Ward pubblicò in due volumi, un'opera intitolata An Enquiry into the Foundation and History of the Law of Nations in Europe since the Time of the Greeks and Romans to the Age of Grotius. ${ }^{\mathbf{6 0}}$

Ward, dopo aver esposto il pensiero di Grozio e successivamente di Pufendorf, in merito alla distinzione tra diritto delle genti e diritto naturale, introduceva la figura di Vattel elogiando il Droit des gens per aver reso acces-

57 Nakнimovsky (2010) 159; Nakhimovsky (2011a) 92. Cfr. anche Nakhimovsky (2011b) 212-228.

58 VAttel (1758a) L. II, chap. I, $\$ 7,263$.

59 NaKhimovsky (2010) 159.

60 Ward (1795). Su Robert Ward cfr: Phipps (1850); Patmore (1854) 1-202; Panizza (1997). Anche: LeSAFFer (2011b) 149-184. 
sibile il diritto delle genti, ${ }^{61}$ e per l'utilizzo di un metodo eccellente, ${ }^{\mathbf{6}}$ ma avanzava dubbi circa la genericità della trattazione « and often too flight, in his reasoning, and attends too little to its particular application ", che poteva anche essere «insoddisfacente » e addirittura "pericolosa ${ }^{63}$

Le posizioni critiche nei confronti del trattato furono ogetto di studio, qualche anno dopo, nel 1799, da James Mackintosh che pubblicò il celebre Discourse on the study of the law of nature. James Mackintosh (1765-1832) fu un deputato del Parlamento inglese; dopo la laurea in medicina e alcuni anni di professione, abbandonò tale carriera per dedicarsi al diritto. ${ }^{64}$ Estremamente attivo, con una spiccata intelligenza e sensibilità, al punto che il professore di Harvard, George Ticknor, scrisse di non aver mai incontrato una persona "whose conversation was more richly nourished with knowledge, at once elegant and profound [...] What is best in modern letters and

61 «the model of which is more light and elegant than that of those heavy though magnificent structures which we have surveyed; for he has thoroughly cleared them from the cumbersome ornaments which were supposed to adorn them, and had rendered the way into the interior less difficult and obscure »: WARD (1795) 626.

62 Ibidem: "he marshals, in the outset, a series of preliminary principles, on which he professes to found all his future reasoning, and to which, in the course of it, he regularly refers to. Whether his preliminaries, however, will always bear out his conclusions, does not come within our intention to examine ".

63 Ivi, 626-627. Secondo Ward la conseguenza di questo « is to avoid the opposite fault of his [...] predecessors, that his work, though stored with excellent argument, is not sufficiently supported by the authorities of cases, without which even the reasoning upon international law will want much useful elucidation, but which forms the very essence and base of all the concerns, what he calls the positive law of nations ». Il trattato di Vattel "does not appear by any means to preclude the necessity of studying the works of his masters. Whoever, indeed, would understand his subject throughout, and become acquainted with the Law of Nations in all its nicety and extent, let what will be his own stores of knowledge, or the depth of his thought, can hardly arrive at the end of the proposes, without giving all his mind to the Treaties of this wonderful man »: Ivi, 627.

64 Mackintosh era considerato « eminent as a jurist, a statesman, and a writer, equally distinguished for his extensive learning, his large views, and his liberal principles in law, politics and philosophy»: Godwin (1866) 605. Cfr. Cunningham (1839) 208-220. A livello più generale si rimanda anche al testo pubblicato dal figlio di Mackintosh, Robert James: Mackintosh (1835). È stato inoltre scritto che « he was one of the most distinguished Members of the House of Commons; and his speeches upon all great questions which were agitated in his time were remarkable, not merely for their eloquence, but the large and comprehensive views of national policy, which were supplied by his almost unrivalled knowledge of history and political philosophy »: The London and Edinburgh philosophical Magazine and Journal of Science (1833) 139. 
culture seems to have passed through his mind and given a peculiar raciness to what he says ${ }^{65}$

Dopo un viaggio in Francia, diede alle stampe nel 1791 in risposta alla posizione di Burke (in merito alla Rivoluzione), Vindiciae Gallicae, più conosciuto solo come Defence of the French Revolution. Tra i due politici i rapporti non erano affatto sereni ma con il tempo, a seguito dei drammatici eventi che segnarono la Rivoluzione francese, si dovettero ricredere, e da un incontro chiesto da Mackintosh a Burke nacque molto di più di una reciproca stima intellettuale. ${ }^{\mathbf{6 6}}$

Mackintosh acquistò notorietà quando, ammesso all'esercizio della professione forense e incaricato di insegnare diritto delle genti presso la Lincoln's Inn, il 13 febbraio 1799 presentò il suo corso con una Introductory Lecture, che pubblicò nello stesso anno con il titolo Discourse on the Study on the Law of Nature and Nations. ${ }^{67}$ Il saggio oltre ad avere un enorme successo, fu utilizzato come premessa per moltissime edizioni francesi del Droit des gens di Vattel nel corso del XIX secolo. ${ }^{68}$ Sin dalle prime righe del suo discorso emerge l'intento didascalico, illustrando la complessa materia del diritto delle genti, con un excursus storico, in cui venivano citati Cicerone, Bacone, e poi Grozio, il quale, secondo Mackintosh, aveva dato una sistemazione coerente al diritto delle genti. ${ }^{69}$

65 O'Leary (1989) 148.

66 Nel 1796 Mackintosh chiese di essere ricevuto da Burke. Aveva infatti letto Two Letters on a Regicide Peace, scrivendo una recensione favorevole sul Monthly Review, di cui era diventato collaboratore nel luglio del 1795. I due si incontrarono dopo il Natale del 1796: entrambi rimasero profondamente segnati dalle loro conversazioni che cambiarono idea l'uno dell'altro. Fu proprio Mackintosh nel 1799, a seguito della morte di Burke, a definirlo «the most eloquent of men, the perpetual force and vigour of his arguments being hid from vulgar observation by the dazzling glories in which they were enshrined »: O'Brien (1992) 414 e Ayling (1988) 266-267.

67 Mackintosh (1846) 27-43.

68 Armitage afferma che rispetto al testo di Ward, il saggio di Mackintosh: "was the preface to the enormously successful-but now, unfortunately, lost, set of lectures, eclectically derived from German histories and philosophy, the jurisprudence of Montesquieu and the canon of international law from Grotius to Vattel »: Armitage (2013) 150. Sul discorso di Mackintosh anche: Murphy (1985) 56-58 e Storti (2012) 79 ss.

69 Mackintosh (1846) 30-31: " he produced a work which we now, indeed, justly deem imperfect, but which is perhaps the most complete that the world has yet owned, at so early a stage in the progress of any science to the genius and learning of one man ». 
Dopo brevi riflessioni sulle opere di Pufendorf e Wolff così affrontava il trattato vatteliano:

«the abridger [of Wolfius], deserves considerable praise. He is a very ingenious, clear, elegant, and useful writer, but he only considers one part of this extensive subject, namely, the law of nations, strictly so called; and I cannot help thinking, that, even in this department of the science, he has adopted some doubtful and dangerous principles, not to mention his constant deficiency in that fullness of example and illustration, which so much embellishes and strengthens reason. I was unwilling to have expressed more strongly or confidently my disapprobation of some parts of Vattel: though I might have justified more decisive censure by the authority of one of the greatest lawyers of the present age. ${ }^{{ }^{70}}$

Ma «l'abbreviatore » di Wolff è da ritenersi il punto di partenza delle indagini del saggio di Mackintosh, pur avendo riscontrato le lacune e gli errori nel Droit des gens. ${ }^{71}$ Il giurista inglese intendeva pertanto arricchire il suo Discourse di esempi pratici, in cui potesse illustrare i progressi del diritto delle genti nell'Europa alla fine del XVIII secolo, in quanto «So much of these treaties has been embodied in the general law of Europe, that no man can be master of it who is not acquainted with them. The knowledge of them is necessary to negotiators and statesmen; it may sometimes be important to private men in various situations in which they may be placed; it is useful to all men who wish either to be acquainted with modern history, or to form sound judgement on political matters ${ }^{72}$

Mentre la dottrina giuridica appoggiava non incondizionatamente il pensiero vatteliano, la stampa e, in particolare il The Times, utilizzava sempre più frequentemente brani e interi passaggi del Droit des gens come fonti per i propri articoli. ${ }^{73}$ Inoltre nei discorsi parlamentari della fine del Settecento e

70 Mackintosh (1846) 33.

71 Ibidem: "His politics are fundamentally erroneous; his declamations are often insipid and impertinent; and he has fallen into great mistakes in important practical discussions of public law».

72 Ivi, 35.

73 Come si è visto in uno dei giornali detentore del primato di citazioni, riferimenti e interi articoli dedicati a Droit des gens è The Times, che in moltissime occasioni, durante il corso del XIX secolo e il primo ventennio del XX, riportava interi passaggi del trattato di Vattel e a seconda delle circostanze storiche, alternava paragrafi sulla neutralità, sulla guerra, sulla diplomazia elaborati dal giurista di Neuchâtel, in parte si trattava della pubblicazione delle sedute parlamentari, ma anche dell'invio di lettere all'editore. A titolo esemplificativo: sui conflittuali rapporti tra Spagna e Inghilterra poche settimane dopo la battaglia di 
dei primi vent'anni del secolo successivo si riscontravano ampie e corpose citazioni del Droit des gens, anche da parte del Mackintosh nella sua veste di politico. ${ }^{74}$

Si registra soprattutto il progressivo richiamo alle teorie vatteliane nelle controversie internazionali da parte di alcuni membri del Parlamento inglese. Così ad esempio, il 17 febbraio 1783, durante la discussione degli articoli preliminari per il trattato di pace di Parigi, che verrà stipulato il 3 settembre dello stesso anno, con cui terminò la guerra d'indipendenza degli Stati Uniti d'America, il conte di Carlisle, Frederik Howard, invocava Vattel, e la sua autorità affinché i territori americani venissero alienati dal re solo a seguito del consenso del parlamento: «The Kings of England have the power of concluding treaties of peace and of making alliances; but they cannot alienate any of the dominions without the consent of the Parliament $»^{75}$

Durante le guerre napoleoniche, e precisamente mentre l'Inghilterra affrontava l'intervento della Spagna nel conflitto a favore della Francia, conclusosi nell'ottobre 1805 con la battaglia di Trafalgar, l'avvocato Jonathan Raine, nell'adunanza del 12 febbraio 1805, si schierava contro tale decisione, argomentando che essa non solo fosse ingiustificabile ma che fosse contraria ai principi del diritto delle genti e quanto statuito da Vattel. ${ }^{76}$

Durante il congresso di Vienna, a seguito della decisione di annettere la Liguria al Regno di Sardegna, avvenuta il 12 novembre 1814, dalla House of Commons si levò forte la voce dello stesso James Mackintosh che vedeva in questo atto una vera e propria ingiustizia: «I say injustice, because I contend that we have been guilty of injustice towards the independent and innocent people of Genoa, even if we had not been pledged by any declarations, or bound by any promises. In charging this as a substantive crime, independent

Capo di Santa Maria del 5 ottobre 1804 e sul tema della dichiarazione di guerra vi è un espresso richiamo alle teorie di Vattel: The Times, issue 6159 (Tuesday, 23 October 1804) 2; cfr. una lettera all'editore che aveva ad oggetto il trattamento dei prigionieri di guerra: The Times, issue 8507 (Wednesday, 12 August 1812) 2; una altra lettera questa volta riguardante la situazione europea e le vicende di Napoleone Bonaparte in: The Times, issue 9086 (Wednesday, 8 December 1813) 3; su questioni inerenti ad uno scambio di beni militari: The Times, issue 10636 (Thursday, 1 April 1819) 3.

74 Mestad (2015), 48-64.

75 Howard (17 February 1783) 36.

76 Raine (12 February 1805) 214-220. 
of all engagements, I conceive that I am supported by the highest authorities in public law ». ${ }^{77}$ Tra queste "altissime " autorità spiccava chiaramente il Droit des gens, di cui veniva citato gran parte del paragrafo 203, capitolo XIII, del libro III, dedicato all'annessione dei territori a seguito di guerra o di conquista, e in cui veniva affrontato il tema dell'ottenimento della libertà da parte di un popolo che fosse stato ingiustamente conquistato dal nemico. Per Vattel, infatti «It has been observed that we may be obliged, if not externally, yet in conscience, and by the laws of equity, to restore to a third party the booty we have recovered out of the hands of an enemy who had taken it from him in an unjust war ${ }^{78}$ Un popolo che è stato spogliato della sua libertà non rinuncia mai alla speranza e al desiderio di riaverla: «If they have not voluntarily incorporated themselves with the state by which they have been subdued, - if they have not freely aided her in the war against us, we certainly ought so to use our victory, as not merely to give them a new master, but to break their chainse ${ }^{79}$

Secondo Mackintosh questo passaggio di Vattel sembrava essere stato scritto proprio per i genovesi e inveiva contro l'annessione dicendo che: «the Genoese were our friends, if not our allies; they were not voluntarily incorporated into the conquering state, they did not freely contribute their aid against us. We have used our victory to change their master, not to break their chains; every part of our conduct is condemned by the impartial expositor of the sense of mankind on the morality between nations. How will the condemnation be evaded? ». ${ }^{\mathbf{8}}$

Numerosissime sono i richiami a Vattel anche nella pratica politicoamministrativa inglese: per legittimare i cosiddetti Navigation Acts vennero parafrasati interi passaggi del Droit des gens, sostenendo che essi fossero «a legitimate judgement by a sovereign nation of the means necessary for the preservation of its subject and were therefore strictly comfortable to the principles of the laws of nature and of nations ${ }^{81}$ Il trattato di Vattel fu molto consultato da parte dell'amministrazione delle colonie inglesi, come

77 Mackintosh (21 February 1815) 933-936.

78 Vattel (1797) L. III, chap. XIII, \$ 203, 391-392.

79 Ivi, $\$ 203,392$.

80 Mackintosh (21 February 1815) 933-936.

81 Brooke Clarke (1803) 1-3. Sull'uso di Vattel nei Navigation Acts cfr: Nakhimovsky (2010) 160 ss. 
avvenne per la Colonia del Capo o per il Nuovo Galles del Sud, essendo una valida fonte per "giustificare e spiegare la dominazione europea ». ${ }^{\mathbf{2}}$

Di rilevanza sono le citazioni vatteliane in occasione della decisione dell'esilio di Napoleone all'isola di Sant'Elena. È ben noto che il 15 luglio 1815 Napoleone si arrese agli inglesi salendo a bordo della nave Bellerophon a Plymouth Sound e vi rimase a bordo quindici giorni. La condizione della sua consegna era la deportazione in Inghilterra o negli Stati Uniti e l'attribuzione dello status di privato cittadino.

Ma Napoleone fu trasferito sulla nave Northumberland e fu portato a Sant'Elena. La questione dello status dell'ex imperatore generò un acceso dibattito tra $\mathrm{i}$ giuristi e i politici inglesi «some saying that he has to be regarded as a prisoner of war; others as a pirate, or hostis humani generis, carrying about with him caput lupinum $\gg .{ }^{\mathbf{8 3}}$

Gli inglesi lessero attentamente il Droit des gens per cercarvi una valida giustificazione su come dovesse essere riconosciuto giuridicamente Napoleone Bonaparte e, benchè il Droit des gens non contemplasse una simile ipotesi, «the law of self-preservation would justify the keeping of Napoleon under restraint in some distant region, where he should be treated with all the indulgence compatible with a due regard for the peace of making ». ${ }^{\mathbf{8 4}}$

Se da un lato il panorama giuridico pratico e dottrinale vedeva un vivace utilizzo delle teorie vatteliane, dall'altro le edizioni erano per lo più ristampe delle versioni francesi. Esse furono pubblicate in Inghilterra e in Irlanda (a Dublino) tra il 1787 e il 1797, con il titolo The law of nations, or principles of the law of nature, applied to the conduct and affairs of nations and sovereigns from the French Monsieur De Vattel.

In particolare quella del 1797 pubblicata a Londra da J. Robinson, Paternoster-Row è considerata come la più importante edizione inglese del trattato di Vattel. ${ }^{85}$ Nell'Advertisement l'editore sottolineava la necessità nel tradurre il testo di Vattel di eliminare tutti gli errori che erano contenuti nell'edizione francese del 1773, e continuava precisando che nel momento in cui aveva deciso di apportare delle note queste erano diventate sempre più

82 Benton (2002) 207. Anche Benton / Ford (2016).

83 Prendergast (1855) 233.

84 Forsyth (1853) 20; GoldwORTh (1904).

85 Hurd (1858) 345, nota 1. Cfr. Starke (1972) 164. Su questa edizione anche Kapossy/ WHATMORE (2008a) XXI-XXIII. 
numerose ma « whether they will be acknowledged as amendments, it must rest with the reader to determine. Even if this decision should be more favourable than I have any reason to expect, I lay no claim to praise for my humble efforts, but shall esteem myself very fortunate if I escape the severity of censure for presenting this work to the public in a state still so far short of perfection ${ }^{\mathbf{8 6}}$

Dal punto di vista generale l'apparato di note è costituito da correzioni per lo più di carattere storico, con il preciso intento di fornire al lettore una maggiore completezza riguardo alle citazioni o esempi che Vattel fornì durante la sua stesura e seguendo anche la versione francese edita da Dumas nel $1775 .^{87}$ Inoltre nel 1811 e nel 1812 furono pubblicate a Londra dall'editore Clarke altre due edizioni di Vattel: esse furono semplici ristampe della precedente del $1797,{ }^{88}$ che non mancarono però di essere annunciate, come nei casi precedenti, sui giornali inglesi a cui seguì, tra Sette e Ottocento la pubblicazione di interi brani tratti del Droit des gens, con relativi commenti alla situazione contingente. ${ }^{\mathbf{9}}$

86 "add the author's valuable notes from the posthumous edition printed at Neuchâtel in 1773 , - to correct some errors I had observed in the former version, - and occasionally to amend the language where doubtful or obscure, -where the utmost limits of my original plan », così gli editori nell'Advertisement in: VATTEL (1797).

87 Prendendo come esempio i Préliminaires (che nella versione inglese diventano Idea and general principles of the law of nations) nel momento in cui Vattel, specifica che cosa intende per diritto delle genti, il commentatore annota che lo studio di questa scienza presuppone una conoscenza approfondita del diritto naturale: «Nature herself, therefore, has established that society, whose great end is the common advantage of all its members: and the means of attaining that end constitute the rules that each individual is bound to observe in his whole conduct. Such are the natural laws of human society. Having thus given a general idea of them, which is sufficient for any intelligent reader, and is developed at large in several valuable works, let us return to the particular object of this treatise »: Ivi, XVI-XVII.

88 Cfr. The Edinburgh Annual register, (1811) CXI; Catalogue of the library or congress, (1830) 112; A catalogue of the printed books and manuscripts in the library of the Inner Temple (1833) 104; A catalogue of the Library of the London Institution (1813) 700. Ancora più interessante è il catalogo dell'editore di Vattel di questi anni: Clarke's Bibliotheca legum. Complete catalogue of the common and statute law-books of the United Kingdom with an account of their dates and prices, arranged in a new manner, interspersed with observations, from the Authorities, on the principal works (1819) sect. vi. (civil, feudal, ecclesiastic and universal law) 97.

89 In particolare per l'annuncio della pubblicazione delle nuove edizioni del Droit des gens: London Chronique, issue 5673 (Thursday, 27 December 1792); Morning Post, issue 6142 


\section{5. «Vattel » nella corrispondenza tra i cardinali Ercole Consalvi e Bartolomeo Pacca}

In altri contesti geo-politici, sempre durante l'epoca napoleonica, non mancarono i riferimenti al Droit des gens. Un esempio è quello dell'utilizzo di Vattel nelle relazioni diplomatiche della Santa Sede: i cardinali Ercole Consalvi e Bartolomeo Pacca hanno in comune la carica di segretario e prosegretario ed entrambi hanno vissuto gli anni travagliati del papato tra Sette e Ottocento e, coinvolti nelle tristi situazioni a difesa della Chiesa e dei suoi diritti, non hanno esitato, quando lo ritenevano opportuno, a rifarsi al testo del protestante Vattel.

Pacca è considerato uno dei fautori della resistenza del Pontefice nei confronti della politica napoleonica e delle iniziative delle autorità francesi a Roma. ${ }^{90}$ Le letture di Pacca spaziavano dai padri della Chiesa alla pubblicistica contemporanea, anche tedesca e francese e tra esse spiccava un grande interesse per il Droit des gens. La sua vita all'interno della Chiesa non fu facile, rivestendo un ruolo rilevante nelle conflittuali relazioni tra il papato e Napoleone. Il 19 giugno 1808 Pio VII lo nominò prosegretario di Stato in seguito all'arresto del cardinale Giulio Gabrielli, che, a sua volta sostituiva il segretario Consalvi, costretto alle dimissioni. Pacca fu arrestato a sua volta il 6 settembre dello stesso anno e solo per l'intervento di Pio VII evitò l'allontanamento da Roma per Benevento. Rimase pertanto confinato negli appartamenti pontifici, dove redasse, con Michele Di Pietro, la bolla Quam memorandum (10 giugno 1809), che in risposta all'annessione degli Stati romani all'Impero francese, scomunicava di fatto Napoleone. Nella notte fra il 5 e il 6 luglio 1809, quando il generale Étienne Radet occupò il Quirinale, Pacca fu prelevato insieme al Papa, esiliato a Grenoble e poi internato, su ordine di Napoleone, nella fortezza di Fenestrelle in Piemonte. L'isolamento, durato dal 1809 al 1813, non gli impedì di dettare le sue memorie, che contengono dei rimandi espliciti all'opera di Vattel. ${ }^{91}$

Egli esortava che sarebbe stato "lecito di permettere al popolo di liberarsi da quegl'ingiusti aggressori » poiché « tutte le leggi naturali, divine ed

(Saturday 29 December 1792); Star, issue 1456 (Tuesday, 8 January 1793); St. James's Chronicle or the British Evening Post, issue 6536 (November 7, 1799-November 9, 1799).

90 Sulla figura del cardinale Pacca si rimanda: Armando (2014) 39-45; Curzi (2010) 207-215.

91 Armando (2014) 39.

152 Capitolo III 
umane danno il diritto agli oppressi di respingere la forza con la forza, e di scuotere il giogo che senz'alcuna ragione fu loro imposto ${ }^{92}$ e a sostegno di ciò riportava interamente il passo di Vattel contenuto nel libro IV al capitolo IV, $\mathbb{3} 3$, che così recita:

Qu'un avide et injuste conquérant subjugue une Nation, qu'il la force à accepter des conditions dures, honteuses, insupportables, la nécessité le contrainte à se soumettre: mais ce repos apparent n'est une paix; c'est une oppression que l'on souffre tandis qu'on manque de moyens pour s'en délivrer, et contre laquelle des gens de cœur se soulèvent à la première occasion favorable. ${ }^{93}$

In questo paragrafo Vattel citava la drammatica fine dell'impero Azteca e di Montezuma ad opera degli spagnoli guidati da Hernán Cortés, affermando che

Toutes ses maximes vont au plus grand bien de l'humanité. [...] Celui, qui rompt lui-même tous les liens de la société humaine, pourra-t-il les réclamer s'il arrive, qu'un peuple abuse de cette maxime pour se soulever injustement, et recommencer la guerre? Il vaut mieux l'exposer à cet inconvénient, que de donner aux usurpateurs un moyen aisé d'éterniser leurs injustices, et d'asseoir leur usurpation sur un fondement solide. ${ }^{94}$

Il cardinale Pacca, nel richiamare la situazione romana, suggeriva di sostituire Hernán Cortés con il generale Sextius-Alexandre-François Miollis, e Montezuma con Pio VII, affinché fosse « ben provato ciò, che poteva lecitamente fare l'ottimo Pontefice in quelle triste, e dolorose circostanze, giacché al citato passo di Vattel nulla v'è da opporre quando si tratti di restituire la libertà, e il dominio al legittimo Sovrano ${ }^{95}$

Il 24 maggio 1814 Papa Pio VII faceva trionfalmente ritorno a Roma insediandosi nuovamente al Quirinale e ripristinando l'antico Stato della Chiesa. Il cardinale Ercole Consalvi, nuovamente segretario di Stato, fu il

92 PACCA (1843) parte I, cap. IV, 179; tale testo si trova anche riportato in: Cantù (1864) 255, nota 2.

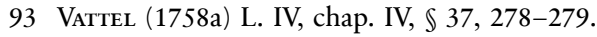

94 Ivi, $\mathbb{3} 37,279$.

95 PACCA (1843) 178-179. Per quanto riguarda il generale Sextius-Alexandre-François Miollis dal 1807 al 1814 fu governatore di Roma e degli Stati del Papa, dando prova di «tatto » diplomatico nelle dure vertenze sorte tra Pio VII e Napoleone; ma non poté sottrarsi all'ordine categorico d'impadronirsi della persona del Pontefice e di farlo prigioniero a Savona (1809): sulla vicenda Nardi (2005); Vandiver Nicassio (2009) 178 ss. 
vero protagonista di questa fase postnapoleonica e inviato come negoziatore al Congresso di Vienna, non esitò a citare il Droit des gens. ${ }^{\mathbf{9 6}}$

L'esempio più famoso di quanto l'autorità di Vattel sia stata un valido sostegno alle richieste di Consalvi è avvenuto il 23 giugno 1814, quando il Cardinale inviava la sua nota diplomatica ufficiale ai ministri delle principali potenze d'Europa, all'interno della quale chiedeva che il Pontefice fosse rimesso nel possesso delle Legazioni, delle Marche, di Benevento, di Pontecorvo, di Avignone e del Venosino e inoltre riaffermava i diritti del Pontefice sul ducato di Parma e di Piacenza. La Santa Sede, sosteneva veemente Consalvi, non riconosceva valido il Trattato di Tolentino concluso, il 19 febbraio 1797, con la Repubblica francese, rappresentata da Napoleone Bonaparte, e con Pio VI. ${ }^{97}$ Come è noto tale trattato imponeva forti limitazioni al Papa che, oltre a pagare trenta milioni di franchi alla Francia, si impegnava a rinunciare ai suoi diritti su Avignone, sul contado Venassino e sulle legazioni di Bologna, Ferrara e Ravenna, accettava l'occupazione di Ancona fino alla conclusione della pace generale e consegnava molti importanti manoscritti e opere d'arte. ${ }^{98}$ Secondo la nota del segretario Consalvi esso era da ritenersi nullo, perché era il risultato di una ingiusta e illegittima aggressione contro uno Stato da sempre dichiaratosi neutrale.

96 Per la partecipazione di Consalvi al Congresso di Vienna: Ruck (1917); Cassi (1931); Regoli (2006).

97 Consalvi (1834) 36-40, qui in particolare 36-37.

98 Scovazzi (2012) 9-19, qui in particolare 13. Scovazzi, all'interno del suo studio, riporta la posizione di Antoine-Chrysostome Quatremère de Quincy (1755-1849), il quale si scontrava contro la spoliazione delle opere d'arte nella penisola italiana. L'archeologo e critico d'arte, infatti, aveva pubblicato nel 1796 le sue Lettres sur le préjudice qu'occasionneroient aux Arts et à la Science, le déplacement des monumens de l'art de l'Italie, le démembrement de ses Ecoles, et la spoliation de ses Collections, Galeries, Musées, [...], che erano state riedite a Roma nel 1803 e nel 1815. In queste lettere Quatremère de Quincy sosteneva l'idea di un interesse collettivo, condiviso da tutti gli esseri umani per la tutela del patrimonio culturale e la sua salvaguardia nel contesto in cui è stato creato. Questa riflessione è perfettamente in linea con Vattel (1758a) L. III, chap. III, $\mathbb{\$} 168$, 140: «Pour quelque sujet que l'on ravage un pays, on doit épargner les édifices qui font honneur à l'humanité, et qui ne contribuent point à rendre l'Ennemi plus puissant; les Temples, les Tombaux, les Bâtiments Publics, tous les Ouvrages respectables par leur beauté. Que gagne-t-on à les détruire? C'est se déclarer l'ennemi du Genre humain, que de le priver de gaieté de Cœur, de ces Monuments de l'Art, de ces Modèles du Goût ": E' vero che non c'è nessun riferimento a Vattel nello scritto di Quatremère, ma nella biblioteca personale di quest'ultimo era presente una copia del trattato di Vattel: Bibliothèque de M. Quatremère de Quincy [...] collection d'ouvrages relatifs aux beaux arts et à l'archéologie (1850) 137, n. 789. 
A sostegno della sua argomentazione Vattel, anzi Wattel, veniva citato, insieme Grozio. Scriveva Consalvi che: « Grozio asserisce, che gli articoli di un trattato hanno forza di condizione, LA DI CUI MANCANZA LO RENDE NULLO. Wattel, parlando dell'assioma, che i trattati contengono delle promesse perfette e reciproche, stabilisce, che l'alleato oltraggiato, o leso in ciò, che costituisce l'oggetto del trattato, può scegliere, o di costringere il mancatore all'adempimento de' suoi impegni, o di dichiarare il contratto risoluto per il pregiudizio arrecatogli: ed altrove dice, quando il trattato di pace è violato da uno de' contraenti, l'altro è in facoltà di dichiarare il trattato risoluto ». ${ }^{99}$ Consalvi riprendeva le posizioni di Vattel sul diritto dei trattati, contenute, come si è visto, nel secondo libro del Droit des gens $\mathrm{e}$ in modo ancora più incisivo incalzava: "Questi principi hanno una forza ancor più grande, allorchè la violazione del' trattato da una delle parti contraenti è stata spinta fino alla distruzione dell'altra. In un caso simile la parte distrutta non conserva alcuna obbligazione verso il suo distruttore; come questi non conserva alcun diritto sopra di essa. Wattel afferma: Quando uno Stato è distrutto, o soggiogato da un conquistatore, mai i suoi trattati PERISCONO con la potenza publica, che li aveva contratti ». ${ }^{\mathbf{1 0 0}}$

Mentre Consalvi era impegnato al congresso a Vienna, Pacca da Roma, come prosegretario in sua sostituzione temporanea, lo teneva informato periodicamente sulle dinamiche della Santa Sede e sui rapporti con i consoli degli altri Stati. Ulteriori riferimenti a Vattel si possono riscontrare proprio nella corrispondenza tra i due, in merito ad alcune questioni che riguardavano i consoli esteri a Roma. ${ }^{101}$ Occorre precisare che i rapporti tra il governo pontificio e i consoli esteri hanno spesso generato numerosi contrasti in merito alla definizione delle regole cui dovevano attenersi. In particolare in una vicenda fu coinvolto in prima persona Pacca in lite con il console a Roma del Regno di Napoli, il cavalier Zuccari. Pacca, il 18 febbraio 1815, in una lettera che aveva principalmente ad oggetto i preparativi di guerra da parte di Napoleone e di Murat, illustrava anche a Consalvi il suo problema: Ludwig von Lebzeltern, inviato straordinario e ministro plenipotenziario d'Austria presso la Santa Sede dall'aprile 1814 caldeggiava la causa napoletana e le pretese di Zuccari, in merito alla immunità consolari.

99 Consalvi (1834) 37.

100 Ibidem. Anche: Robinson (1987) 102 ss.

101 Hochstrasser (2004) 182-183. 
Il Console era infatti stato accusato di essere l'organizzatore di una rete occulta di spionaggio; di diffondere una stampa tendenziosa; e di aver messo in atto una serie di tentativi tesi ad influenzare l'opinione dei sudditi pontifici in senso contrario al governo. La questione spinosa da risolvere riguardava l'immunità personale dei consoli, che veniva invocata rimandando quanto stabilito da Vattel. Lebzeltern si faceva portavoce che «i riguardi particolari » spettanti ai consoli andassero «proporzionati con quelli che merita il sovrano a cui appartiene il console da quello presso il quale risiede ». ${ }^{102}$ Il leitmotiv delle immunità verso i consoli ritorna ancora nella lettera di Consalvi, che da Vienna il 4 aprile 1815, dava suggerimenti a Pacca in merito agli atteggiamenti da tenere nei confronti dei Napoletani per quanto riguarda i privilegi e $\mathrm{i}$ « riguardi particolari ». Vattel, ancora una volta, è citato dal Cardinale come uno degli autori più accreditati e più recenti e si dice " espressamente favorevole alle immunità personali ", ma nonostante ciò, la posizione di Zuccari doveva essere vagliata. ${ }^{103}$ Consalvi precisava quale sarebbe stata la soluzione migliore per chiudere la vicenda: far comprendere al Console « in poche parole ", che qualora avesse violato le leggi o compromesso la sicurezza e la quiete dello Stato, «l'intenzione del Santo Padre è di fargli in tal caso significare la necessità di una immediata partenza dai suoi Stati, non senza aggiungere che se in seguito di questa risposta crede di voler i suoi passaporti, gli saranno rimessi ». ${ }^{104}$ E proprio sulla remissione dei passaporti Pacca, il 29 aprile 1815, aggiornava Consalvi sulle richieste di Zuccari, a cui il Pacca rispose sempre "secondo i principi di gius pubblico ». ${ }^{105}$ La disputa finì «colla cessione in punto teoretico dei Napolitani; e all'ultima istanza, avanzata da essi, per sapere se voleva accordarsi al console di Napoli in Roma quella stessa immunità personale, che si asserisce godere in Napoli il console pontificio, restò senza risposta ». ${ }^{\mathbf{1 0 6}}$

102 Lettera n. 261 di Bartolomeo Pacca al Cardinale Consalvi, Roma 18 febbraio 1815, in: Roveri/Fatica/Cantù (a cura di) (1973) 136-139, qui 138.

103 Lettera n. 297 del Cardinale Consalvi a Bartolomeo Pacca, Vienna 25 marzo 1815, in: Roveri / Fatica/Cantù (a cura di) (1973) 332-339, qui 333.

104 Ivi, 333-334.

105 Lettera n. 322 di Bartolomeo Pacca al Cardinale Consalvi, Genova 29 aprile 1815, in: Roveri / Fatica/Cantù (a cura di) (1973) 446-456, 447.

106 Ibidem. 
Le citazioni vatteliane presenti nelle memorie di Pacca, nella nota diplomatica ufficiale di Consalvi e nel carteggio tra i due Cardinali permettono di cogliere quanto il dinamismo del Droit des gens fosse presente nei primi anni dell'Ottocentro e permeato nella cultura giuridica. Esse sono agli occhi dello storico una ulteriore sfaccettatura di un prisma, al cui interno sono contenute le molteplici critiche e accettazioni che il Droit des gens ebbe in questo periodo, coinvolgendo traduzioni, edizioni del trattato, saggi critici e pratica diplomatico-consolare e politica. La conoscenza del testo e l'uso delle teorie vatteliane nello Stato della Chiesa per questioni temporali ed ecclesiastiche dimostrano quanto il Droit des gens fosse visto esclusivamente come opera giuridica neutra e legittimata a leggersi in ambienti anche clericali.

\section{Controversie giudiziali in Nord America: il richiamo al Droit des gens}

Alcune ricerche dimostrano che il Droit des gens ebbe la sua massima fioritura a livello politico, pratico, diplomatico e dottrinale durante la prima metà del XIX secolo anche in Nordamerica: «Vattel's treaty on the law of nations was quoted by judicial tribunals, in speeches before legislative assemblies, and the decrees and correspondence of executive officials ». ${ }^{107}$

Il Droit des gens fu utilizzato come manuale per gli studenti e fu punto di riferimento sia per gli uomini di governo sia per i teorici delle dottrine politico-internazionali. ${ }^{108}$ Uno studio condotto da Dickinson illustra quanto negli Stati Uniti Vattel tra il 1789 e il 1820 fosse citato nei «findings of American cases ", cioè nelle decisioni delle corti, nelle difese degli avvocati, rispetto ai suoi predecessori quali Pufendorf e Bynkershoek. ${ }^{109}$ Altresì Ossi-

107 Fenwick (1913) 395.

108 Ibidem.

109 Dickinson (1932) 259, nota 132. Dickinson illustra attraverso una tabella il numero di citazioni mettendo a confronto Grozio, Pufendorf, Bynkershoek ed infine Vattel. Tale tabella è riportata in diversi testi di storia del diritto delle genti, tra cui in particolare in Nussbaum (1954) 162; Guggenheim (1959) XIX; Lengel (1999) 117-132. Lachs (1987) 159-160: «Grotius seems to have been in great demand throughout the $18^{\text {th }}$ century. As records show, in the period between 1701 and 1800 De iure belli ac pacis was published in 34 editions: 20 in the original Latin, 2 in Dutch, 6 in French (amongst these the famous edition by Jean-Pierre Barbeyrac); translations into other languages followed in the $19^{\text {th }}$ century and present century: 2 in English and 4 in German. However Grotius was surpassed by Vattel, who enjoyed great popularity from the middle of the $18^{\text {th }}$ century ». 
pow e Gerber all'interno del già citato saggio, hanno evidenziato che tra il 1762 e il 1820 il nome Vattel compare duecentoventiquattro volte in centodiciotto fonti differenti (sia politiche che giuridiche). ${ }^{\mathbf{1 1 0}}$

Nemmeno Grozio ebbe a quell'epoca un simile successo: « a century ago not even the name of Grotius was more potent in its influence upon the questions relating to international law than Vattel ». ${ }^{\mathbf{1 1}}$

Il testo di Vattel e le teorie sul diritto internazionale contenute in esso, quali l'identificazione dei diritti fondamentali degli Stati, la loro sovranità, l'indipendenza e l'uguaglianza, divennero nozioni elementari di diritto internazionale al punto tale che in moltissimi trattati del XIX secolo vi era più di un riferimento al Droit des gens. ${ }^{\mathbf{1 1 2}}$

Negli Stati Uniti contemporaneamente il trattato di Vattel continuò ad acquistare sempre più un ruolo essenziale in famosi giornali: è, ad esempio, il National Intelligencer a pubblicare interi paragrafi del Law of Nations con particolari riflessioni sulla situazione internazionale dell'epoca. ${ }^{\mathbf{1 1 3}}$

In particolare Hinsley afferma che opere come quella di Vattel e di Montesquieu nelle prima metà del XIX secolo ebbero un notevole impatto poi-

110 Ossipow / Gerber (2010) 93.

111 Dickinson (1932) 259, nota 132.

112 «Et comme l'avenir fut à Vattel, plus qu'à tout autre, favorable, les auteurs qui se sont succédés, philosophes ou juristes qui l'ont étudié, se sont, de cela, montrés surpris et ont été, pour lui, sévères. Vattel est sans cesse cité: pas une controverse où il n'apparaisse; mais dans une histoire des doctrines du Droit des gens, sa place est loin d'être aussi grande que dans la jurisprudence, soit interne, soit internationale, est grande son autorité ». LAPRADELLE, Introduction, in: VATTEL (1916) XLIII.

113 Solo a titolo di esempio: nel 1807 in occasione della battaglia di Copenaghen degli inglesi contro i danesi, effettuata senza una preventiva dichiarazione di guerra, comparivano diverse posizioni nei giornali dall'epoca che prendevano posizione sull'accaduto, citando anche Vattel: "The attack on Copenhagen, by order of the British government, is allowed to be the most desperate and unprincipled event of the present war » in: The National Intelligencer and Washington Advertiser, issue 1105 (Friday, 13 November 1807). Compaiono interi brani del Droit des gens, ad esempio, sulla materia della immunità degli ambasciatori e dei loro diritti (L. IV, chap. VI, $\mathbb{S} 80 \mathrm{ss}$.): The National Intelligencer and Washington advertiser, issue 1421 (Friday, 17 November 1809). In tema di neutralità dei vascelli: National Intelligencer, issue 1663 (Saturday, 8 June 1811) e anche Daily National Intelligencer, issue 2240 (Friday, 17 March 1820); in tema di guerra: Daily National Intelligencer, issue 116 (Saturday, 15 May 1813); sulle ragioni per dichiarare una guerra: The Mississipi State Gazette, issue 14 (Saturday, 4 April 1818); The Mississipi State Gazette, issue 15 (Saturday, 11 April 1818); The Mississipi State Gazette, issue 17 (Saturday, 25 April 1818). 
ché: «these had emphasised that Europe was divided into independent states. They had also assumed that the relations between those states were moderated by the recognition that they were members of a common civilisation, no less than by expediency and the wise policy of maintaining among themselves $[\ldots]$ an equal balance of power ${ }^{\mathbf{1 1 4}}$

Rispetto al numero di citazioni e frequenza di esse all'interno della lista dei case laws americani, nonostante i precisi studi sopracitati, è necessario però procedere con le dovute cautele e differenziare $\mathrm{i}$ casi in cui si tratti di citazioni definibili di stile, molto spesso ricorrenti, nelle quali il riferimento a Vattel è seguito dall'indicazione ad esempio di Grozio, di Pufendorf, e di Burlamaqui e quindi svolge una funzione si potrebbe dire « rafforzativa» di una tesi, da, invece, citazioni di sostanza, in cui le posizioni di Vattel, emergevano ed erano la fonte principale delle arringhe degli avvocati e delle decisioni dei giudici.

Per riflettere sulle modalità di richiamo all'opera vatteliana e sull'entità di esse potrebbe essere interessante citare come esempio un case law americano discusso presso la Corte Suprema: Armitz Brown contro gli Stati Uniti. Il fatto storico che fa da cornice è la guerra anglo-americana, che, come è noto, fu dichiarata dagli Stati Uniti all'Inghilterra il 18 giugno 1812 e si concluse con la pace di Gand (Londra, 24 dicembre 1814 e Washington, 17 febbraio 1815). Essa fu chiamata anche «la guerra del 1812 », ed è stata un riflesso lontano della lotta tra l'Inghilterra e la Francia rivoluzionaria e napoleonica (1793-1815), lotta in cui ambedue i combattenti si permettevano, ora per un verso ora per un altro, di sorvegliare il ricco commercio marittimo, neutrale, degli Stati Uniti. ${ }^{\mathbf{1 1 5}}$

Il caso preso in considerazione riguarda proprio il momento dello scoppio della guerra e di un carico di legname di proprietà dell'inglese Armitz Brown che era stato trasportato sulla nave americana Emulous, scaricato sul territorio americano nell'aprile 1812, e confiscato un anno dopo nell'aprile del 1813, come bene appartenente al nemico. Il ragionamento dell'avvocato del signor Brown, si fondava proprio sul costante richiamo al Droit des gens. Per l'avvocato la causa non poteva che essere decisa alla stregua del libro III, paragrafo 63, nel quale si statuiva la condotta che doveva essere mantenuta dagli Stati all'atto della dichiarazione di guerra, nel momento in cui vi erano

114 Hinsley (1963) 187.

115 Per riferimenti: Stagg (1983); Perkins (1970). 
beni di proprietà del nemico. Secondo la teoria vatteliana, riportando la versione inglese del trattato:

«The sovereign declaring war can neither detain the persons nor the property of those subjects of the enemy who are within his dominions at the time of the declaration. They came into his country under the public faith. By permitting them to enter and reside in his territories, he tacitly promised them full liberty and security for their return. He is therefore bound to allow them a reasonable time for withdrawing with their effects; and if they stay beyond the term prescribed, he has a right to treat them as enemies, as unarmed enemies, however. But if they are detained by an insurmountable impediment, as by sickness, he must necessarily, and for the same reasons, grant them a sufficient extension of the term ». ${ }^{\mathbf{1 1 6}}$

L'autorità di Vattel fu accolta dalla Suprema Corte degli Stati Uniti la quale, nel febbraio 1814, statuì che il legname inglese non potesse essere confiscato come bene appartenente al nemico senza un preciso atto legislativo, poiché la dichiarazione di guerra del 1812 non doveva valutarsi come tale al fine del fermo del carico stesso, ma era indispensabile un atto finalizzato alla confisca dei beni del nemico. ${ }^{\mathbf{1 1 7}}$

Molto recentemente Chetail si è occupato dello studio dell'importanza del Droit des gens all'interno della giurisprudenza della Corte Suprema degli Stati Uniti, evidenziando le ragione di tale influenza, articolandole su tre distinti aspetti: «First his systematic coverage of international law has proven particularly useful for domestic tribunals, that are unfamiliar with international law. [...] Second, the endorsement of Vattel by the Founding Fathers and the diplomatic community made him a natural source of reference for domestic tribunals. Third, the common law tradition of judicial precedent has steadily reinforced his enduring influence in the case law ${ }^{\mathbf{1 1 8}}$

L'utilizzo di Vattel e della sua autorità ha di fatto creato i presupposti per un continuo ed ininterrotto riferimento al suo pensiero soprattutto in un contesto come quello americano in cui vige un sistema di Common Law, all'interno del quale il precedente giudiziario vincola sostanzialmente le successive decisioni. ${ }^{119}$

116 VATtel (1797) L. III, chap. IV, $\$ 63,318$.

117 Brown v. United States, in: Report of Decisions in the Supreme Court of the United States (1855) 46-73.

118 Chetail (2014) 266.

119 Molte sono le memorie degli avvocati e le decisioni giudiziarie nelle quali l'autorità di Vattel fu significativa per la causa stessa: Report of a Case, decided on Saturday, the 16th of November, 1793, in the General Court of Virginia (1794) 17, 45; Proceedings on the Impeach- 


\section{Il Droit des gens nel panorama delle controversie internazionali}

Prima di entrare nel merito del fenomeno Vattel all'interno delle controversie internazionali, è opportuno riflettere su alcuni radicali cambiamenti che hanno coinvolto la disciplina nel corso dell'Ottocento. In questo secolo, nella concretezza, i veri protagonisti di questa disciplina sono i trattati, il cui ruolo muta profondamente rispetto all'epoca precedente, tanto da assumere sempre più importanza al fine di garantire, in diversi ruoli, una sorta di stabilità tra gli Stati ed essere:

instrumental in the judicial organisation of political constellations, which were in no way compared with one another, either in content or in structure. They were used for bringing an end to classical European wars; for the ordination of general political and judicial relationships in Europe as well as in other parts of the world [...] for a comprehensive regulation of the fundamentals of economic cohesion, the position of the citizens and also regulating general problems in international law regarding war and peace, etc. ${ }^{\mathbf{1 2 0}}$

Tutto il corso dell'Ottocento è caratterizzato dalla tensione e dall'attenzione a riconoscere che il diritto internazionale sia un diritto non in balia del potere politico e della diplomazia statale, ma abbia un preciso fondamento giuridico, il quale si realizza attraverso i trattati e le convenzioni, come scrive Mannoni, una «vera probatio diabolica! ». ${ }^{\mathbf{2 1}}$

Inoltre l'aumento dei traffici, il conseguente moltiplicarsi di relazioni politiche e commerciali e lo sviluppo industriale contribuirono ad apportare un contenuto radicalmente nuovo all'interno dei rapporti umani rispetto ai secoli precedenti e concorsero in modo decisivo all'evolvere di una disciplina, che gradualmente risentì di una straordinaria attenzione per quanto riguarda il diritto pubblico e il diritto privato internazionale.

ment of William Blount, a Senator of the United States from the State of Tennessee, for High Crimes and Misdemeanors (1799) 76; The Claim and Answer with the Subsequent Proceedings, in the Case of the Right Reverend Charles Inglis, against the United States, (1799) 11, 18, 88, 95; BACHE, Richard (reported by) (1813), the Case of Alien Enemies, considered and decided upon a writ of Habeas Corpus, allowed on the petition of Charles Lockington, an Alien Enemy (1813) 14, 17; Opinions of the Judges of the Supreme Court of the United States, in the Case of the Proceedings of Charles River Bridge vs. the Proprietors of Warren Bridge and Others. Delivered at the January Term of the Court, at Washington (1837) 107-108. Per ulteriori controversie giudiziali cfr. ChetaIL (2014) 265 , nota 70 .

120 STEIger (2004) 59.

121 Mannoni (2003) 485-498, qui 492. 
I primi fermenti critici contro l'immobilismo dottrinale nacquero nella scuola tedesca nella seconda metà dell'800: Bergbohm, Jellinek e Triepel partendo dall'osservazione diretta della realtà internazionale, misero in luce l'unilateralità di vedute proprie della dottrina contrattuale e cercarono di dare al trattato una definizione giuridica che fosse capace di risolvere l'enorme complessità di forme, che quest'ultimo andava assumendo. ${ }^{\mathbf{1 2 2}}$

Nell'Ottocento si colloca l'inizio vero e proprio di una nuova era nelle relazioni internazionali, sancito poi ufficialmente con la Dichiarazione di Parigi sul diritto marittimo del 1856. Se, infatti, fino al XVIII secolo erano stati rari i casi in cui le relazioni internazionali tra gli Stati uscirono dal quadro di uno scambio reciproco di prestazioni a carattere principalmente bilaterale, a partire soprattutto, dalla seconda metà del secolo seguente si sentì la necessità di creare una serie di regole che avessero una disciplina unitaria e comune a tutti gli Stati. ${ }^{123}$ Non si trattava più soltanto di regolare le diverse esigenze statali, ma di stabilire, di comune accordo, i mezzi e i modi giuridici con cui tutelare, o raggiungere, un identico interesse, di cui si facevano portatori numerosi membri della Comunità Internazionale.

Con la fondazione dell'Institut de Droit International del 1873 venne inaugurato il diritto internazionale come specifico " progetto condiviso da professionisti del diritto europei, pur provenendo da diversi retroterra nazionali- dal Belgio, dalla Gran Bretagna, dalla Germania, dalla Svizzera, dall'Italia naturalmente-, avessero idee ampiamente coincidenti sul significato e lo scopo dei loro sforzi ». ${ }^{\mathbf{1 2 4}}$ Il fine ultimo del diritto internazionale era perciò la promozione in Europa di riforme liberali e al tempo stesso la civilizzazione di tutte le realtà coloniali. ${ }^{125}$

122 Ibidem. Vedasi anche: Mannoni (2014).

123 Westfalia e Utrecht, come argomentato da Nussbaum, sono trattati differenti da quelli successivi del XIX secolo per due caratteristiche fondamentali. La prima è che questi ultimi sono raramente ristretti ad un numero limitato di partecipanti, e tendono invece a lasciare ad ogni Stato la possibilità di aderirvi o meno; mentre le Convenzioni precedenti erano sempre chiuse. La seconda caratteristica è che i trattati multilaterali anteriori a quelli del XIX secolo avevano per oggetto la distribuzione dei territori, la delimitazione di frontiere o il regolamento di altre questioni interessanti esclusivamente gli Stati partecipanti; laddove le Convenzioni successive rivelano invece la tendenza sempre più spiccata a stabilire norme generali di condotta degli Stati: Nussbaum (1954) 197.

124 Koskenniemi (2012c), Prefazione all'edizione italiana del Gentle Civilizer of Nations, XXIII.

125 Ibidem. 
In Europa nel contempo erano nate le prime cattedre specializzate nella disciplina: la prima a Torino nel 1851 fondata da Pasquale Stanislao Mancini, a Oxford nel 1859 a Cambridge nel 1866, in Olanda nel 1876, mentre nel $1884 \mathrm{fu}$ istituita in Belgio, dove a Bruxelles insegnava Alphonse Rivier. ${ }^{\mathbf{1 2 6}}$

In una simile realtà, così profondamente in cambiamento, stupisce che si sentisse fortemente l'esigenza di utilizzare autori passati, come Vattel, per la creazione di un diritto delle genti (poi internazionale), e tenesse conto però della ormai nuova comunità internazionale dell'Ottocento.

Il XIX secolo è per eccellenza il periodo dell'utilizzo degli arbitrati, quali strumenti per la risoluzione delle controversie internazionali. Tra il 1851 e il 1875 i casi di arbitraggio furono un centinaio e quasi il doppio, centonovantanove, tra il 1876 e il 1900, per poi assestarsi sul numero di centoquarantanove tra il 1901 e il 1914. Solo nella seconda metà dell'Ottocento prenderà corpo un'accelerazione degli arbitrati, soprattutto su alcune questioni specifiche di conflitto tra Stati. ${ }^{127}$ Questa fase di passaggio, irrobustitasi a cominciare dagli anni settanta del secolo, si concretizzò anche nella partecipazione alla redazione degli arbitrati internazionali di alcuni networks transnazionali di esperti. Nonostante aumentassero le richieste, in sedi istituzionali rilevanti, di costituzione di una corte internazionale, gli Stati occidentali preferivano, per ovvie opportunità politiche, agire all'interno di un quadro di risoluzione dei conflitti vincolato allo strumento del trattato gestito da un arbitrato internazionale. ${ }^{\mathbf{1 2 8}}$

L'influenza del Droit des gens si riscontra anche nel numero di riferimenti nelle controversie in ambito internazionale, distinguendo però tra le citazioni avvenute all'interno di un documento diplomatico oppure nei richiami presenti nelle note dottrinali. Entrambi i casi sono una testimonianza di quanto il giurista venisse chiamato come una sorta di deus ex machina per la risoluzione delle liti tra Stati.

La Recueil des arbitrages internationaux curata da Lapradelle e Politis, edita nei primi anni del Novecento, ricca di documentazione diplomatica e politica, è divisa in tre volumi, il primo dal 1798-1855, il secondo dal 1856 al 1872 infine il terzo dal 1872 al 1875 . Jouannet rileva quanto vengano citati Vattel e il suo Droit des gens all'interno di alcune controversie internazionali.

126 De la Rasilla del Moral (2013) 48-65, qui 48.

127 LANGHORNe (1996), 43.

128 Lovric-Pernak (2015) 62-74. 
Questo atteggiamento, che risulta costante nel tempo, sembra confermare e ad attribuire al giurista di Neuchâtel una sorta di vera e propria autorità. I temi per i quali si richiama Vattel sono molteplici e molto differenti: vi sono, infatti, citazioni per quanto riguarda il diritto interno, il diritto dei trattati internazionali, la loro interpretazione, la neutralità nonché il problema della guerra. ${ }^{129}$ Occorre tuttavia una precisazione in merito, poiché la raccolta di Lapradelle e Politis contiene i documenti diplomatici, le posizione delle commissioni incaricate, le sentenze ma anche le note dottrinali, che «tient compte de l'état du droit international au moment où le litige étant jugé et son état actuelle, de sorte qu'on a ainsi une très utile contribution à l'histoire interne du droit international ». ${ }^{\mathbf{1 3 0}}$

Così ad esempio la nota dottrinale alla spinosa questione relativa all'interpretazione dell'articolo I del trattato di Gand che ha posto fine alla guerra americana, è fondata sulle regole interpretative elaborate da Vattel al libro II del Droit des gens. Il 24 dicembre 1814 l'Inghilterra e gli Stati Uniti avevano firmato a Gand le condizioni della pace. Il Trattato, ristabiliva lo status quo territoriale preesistente al conflitto, risolveva i problemi inerenti alla linea di frontiera nordorientale tra il Canada e gli Stati Uniti, alla navigazione del Mississippi e si soffermava sulla restituzione da parte degli inglesi degli schiavi catturati durante il conflitto. Proprio su questo punto all'articolo 1 era statuito che:

«All hostilities both by sea and land shall cease as soon as this Treaty shall have been ratified by both parties as hereinafter mentioned. All territory, places, and possessions whatsoever taken by either party from the other during the war, or which may be taken after the signing of this Treaty, excepting only the Islands hereinafter mentioned, shall be restored without delay and without causing any destruction or carrying away any of the Artillery or other public property originally captured in the said forts or places, and which shall remain therein upon the Exchange of the Ratifications of this Treaty, or any Slaves or other private property ».

129 Jounnnet (1998) 15, nota 22. Per il numero di citazioni riguardanti Vattel, mi limito così a richiamare tra i molti solo: Lapradelle / Politis (1905) per i riferimenti a Vattel: sul domicilio del nemico, 75-76, 83, 172; indipendenza degli Stati, 453; interpretazione dei trattati, 366 ss.; in materia di fiumi, 321-324. Lapradelle / Politis (1923) per i riferimenti a Vattel: diniego di giustizia, 9, 19, 103, 156; interpretazione dei trattati, 92, 113, 156-157, 455, 468, 516; Lapradelle / Politis (1954) per i riferimenti a Vattel: amministrazione della giustizia, 134-135, 732, proprietà privata in tempo di guerra, 227, prestando però sempre attenzione che gran parte di queste citazioni non riguardano il documento stesso ma il loro richiamo all'interno delle note dottrinali.

130 Renault, Préface, in: Lapradelle / Politis (1905) XII. 
Ma gli inglesi si rifiutarono di restituire gli schiavi, sollevando la presunta cavillosità dell'articolo in tema di interpretazione, la controversia fu oggetto della sentenza arbitrale resa dall'imperatore russo Alessandro I, il 22 aprile 1822 , il quale si pronunciò sul senso letterale del trattato e sulla sua interpretazione, statuendo nel caso specifico che gli americani avessero tutte le legittime ragioni per pretendere un indennizzo per la mancata restituzione degli schiavi e fu così che la Gran Bretagna pagò duecentocinquantamila sterline. ${ }^{131}$ L'autore della nota dottrinale partendo da questa controversia, valutava il trattato di Gand, e in particolare l'articolo I, alla luce delle più importanti teorie internazionali dell'epoca, quale in primis quella di Vattel, il quale, come si è visto nel II capitolo aveva ampiamente sviluppato la problematica relativa all'interpretazione grammaticale e letterale e la volontà delle parti. Vattel affermava che "puisque l'interprétation légitime d'un acte ne doit tendre qu'à découvrir la pensée de l'Auteur, ou des Auteurs de cet Acte; dès qu'on y rencontre quel qu'obscurité, il faut chercher quelle a été vraisemblablement la pensée de ceux qui l'ont dressé, et interpréter en conséquence ». ${ }^{\mathbf{1 3 2}}$

Egli come si è visto nel precedente capitolo, ha inteso porre come compito principale dell'interprete la ricerca della volontà comune dei contraenti solo quando quest'ultima non sia afferrabile nella chiarezza logico-letterale del testo, e debba ricercarsi in tutti gli elementi ad essa relativi ed estrinseci al contenuto oggettivo dell'accordo.

La varietà di questioni giuridiche in cui Vattel era richiamato, mentre la comunità internazionale e ogni singola entità nazionale evolvevano con caratteristiche proprie, e la modalità con cui il trattato veniva citato tanto nelle controversie interne quanto internazionali, si collocano come passaggi importanti per soffermarsi sulla circolazione dell'opera, attraverso le edizioni del trattato pubblicate nel corso dell'Ottocento e sui relativi commenti. La presunta e controversa autorità di Vattel vacillava tra un concetto di moda $\mathrm{e}$ di modernità, a seconda delle necessità contingenti e anche quando vi erano forti critiche, proprio tali posizioni permettevano e garantivano all'opera una sorta di continuità nello spazio e nel tempo.

131 Etats Unis - Grande Bretagne, 22 avril 1822, in: Lapradelle / Politis (1905) 333 ss.

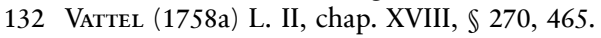





\section{Commentare e annotare il Droit des gens nel XIX secolo: esperienze giuridiche a confronto}

\section{Commentare il diritto e commentare il diritto internazionale}

Tutto il percorso della ricerca si snoda nell'investigare il fenomeno giuridico con una apertura alla interdisciplinarietà, intesa possibile strumento per comprendere i processi di appropriazione e di riproduzione, in relazione ai diversi contesti storico-geografici. L'obiettivo principale del capitolo è quello di valutare, prendendo come esempio Emer de Vattel, con quali modalità il diritto internazionale dell'Ottocento abbia preso forma anche attraverso l'opera di commento e di traduzione di trattati pubblicati (per la prima volta) tra il Sette e l'Ottocento.

Questo tipo di analisi permette di esaminare una serie di parametri che ricorrono sia nella storia del diritto internazionale sia nella storia del diritto. Il quadro d'insieme risulterà tanto più approfondito e preciso, quanto più si riuscirà, sebbene con le necessarie limitazioni nel tempo e nello spazio, a collegare il corso degli eventi che hanno condotto alla pubblicazione di testi del diritto delle genti commentati, con le condizioni dell'elaborazione scientifica di specifici ordinamenti e contesti normativi. Ciò comporta la necessità di affrontare il quesito storiografico in stretta correlazione con la prerogativa di tutto l'Ottocento, che ha portato ad un altissimo numero di traduzioni ed edizioni commentate.

E' stato scelto il metodo della comparazione sincronica perché, nel momento in cui si realizzano imprese editoriali di questo " tipo ", quasi ad un secolo dopo la pubblicazione del trattato di Vattel, rimane un punto di osservazione privilegiato: si possono infatti cogliere tutti gli intrecci, gli snodi di percorsi culturali e giuridici che non furono mai univoci e diretti; scoprire i motivi per cui gli internazionalisti sentivano l'esigenza di commentare testi sul diritto internazionale, cogliendo le opinioni della dottrina corrente, il linguaggio di una società internazionale in continua evoluzione; e interrogarsi sulle specifiche e apposite procedure che regolavano la disciplina. 
Come le glosse sono espressione del pensiero giuridico medievale, scriveva Paradisi dando l'omonimo titolo al suo saggio, è possibile sostenere che i commenti ai trattati di diritto delle genti e poi diritto internazionale, a loro volta, possano essere espressione del diritto internazionale dell'Ottocento. ${ }^{1}$

L'atteggiamento che si riscontra ha tutte le caratteristiche di un modo di «fare » e di «creare » il diritto internazionale e vuole essere un status omogeneo di comunicarlo a tutti i livelli: accademico, dottrinale, pratico, politico e diplomatico.

Prima però di analizzare questo singolare aspetto occorre necessariamente riscontrare che si tratta di un preciso approccio dell'epoca, presente in altre discipline del diritto. E' innegabile che nel XIX secolo si ravvisi nella scienza giuridica una tendenza marcatissima al lavoro di traduzione e di commento ai testi giuridici ed in particolare a quelli inerenti alla codificazione. $^{2}$

Non si è ritenuto opportuno ripercorrere sentieri già autorevolmente indagati, e per i quali rinomati studiosi hanno dato il loro significativo contributo, ma soltanto di mettere in luce un dato oggettivo che, con le dovute precisazioni, coinvolge anche il diritto internazionale.

Il caso giuridico delle traduzioni e della circolazione di opere commentate, come ben sottolineato da Ranieri, «si presenta nella letteratura giuridica del secolo scorso come un vero e proprio autonomo filone giuridicoletterario ", 3 ed è proprio attraverso questo approccio che si assiste nel corso del XIX secolo ad una facilitazione nella diffusione delle dottrine giuridiche.

Erano i primi dell'Ottocento quando un gruppo di giuristi di provenienza francese e belga, poi identificati come «scuola dell'esegesi » insegnavano il Codice Napoleonico con dovizia, articolo per articolo, facendo ricorso alla massima aderenza possibile al dettato legislativo, con l'aggiunta di annotazioni storiche e comparative e in qualche caso dei lavori preparatori. ${ }^{4} \mathrm{Ci}$

1 Paradisi (1987) 711-773.

2 Cfr. Viora (1967); Cavanna (1996) 659-760; Cappellini/Sordi (cura di) (2002); Ferrante (2015); Ferrante (2002); Ferrante (2011); anche Ferrante (2009) 223-237; Solimano (2009) 191-221.

3 RANIERI (2007) 51-66, qui 51.

4 Ferrante (2011) passim. 
furono moltissimi altri esempi nel corso dell'Ottocento, come la diffusione dei commenti all'Codice civile generale austriaco $^{5}$ o la circolazione della scuola storica e della pandettistica. ${ }^{6}$

Molto interessanti sono stati altresì gli studi che hanno indagato sui processi della cosiddetta "armonizzazione del diritto». Le esperienze di armonizzazione, che possono essere rintracciate nell'Europa dell'Ottocento, sono volte "all'insegna della costruzione concreta di ordinamenti in cui il diritto assumeva una natura positiva e prendeva la forma di un codice e si circoscriveva entro la cornice di sovranità statali distinte; una storia, dunque, di diritti non integrati a livello europeo, di diritti nazionali, per la promozione dei quali si attivavano dinamiche di uniformazione del diritto ${ }^{7}$

Codificazione e commento devono essere letti congiuntamente, così come si potrebbe affermare anche per il diritto internazionale del XIX secolo, che i commenti ai testi, in assenza di una " codificazione » tra Stati, abbiano contribuito incisivamente all'emergere della disciplina.

Nell'esprimersi sull'intima e necessaria relazione tra traduzione e commenti, Walter Benjamin argomentava che essi «stanno al testo come lo stile e la mimesi alla natura: lo stesso fenomeno sotto diversi aspetti. Sotto l'albero del testo sacro l'uno e l'altra sono solo le foglie che eternamente stormiscono: sull'albero del testo profano i frutti che cadono al momento giusto ${ }^{\mathbf{8}}$

I commenti, attraverso cui i giuristi continuamente realizzavano una comparazione, sono volti a dimostrare l'identità comune nella storia giuridica; al tempo stesso il lavoro realizzato da chi annota o commenta è sempre legato ad un procedimento logico fermamente condizionato dalla realtà e dal contesto di diffusione e dalle necessità a cui deve rispondere un determinato testo giuridico.

C'è sicuramente una coincidenza spazio-temporale che non può essere sottovalutata e riguarda la circolazione dei testi sul diritto delle genti: tra il

5 Si richiama in questa sede, solo a titolo meramente esemplificativo, per quanto riguarda il contesto italiano, il volume edito in occasione dei 200 anni dall'entrata in vigore dell'ABGB: Caroni/Ferrante (a cura di) (2015). Cfr. anche: Caroni/Dezza (a cura di) (2006).

6 Cfr. per quanto concerne il contesto italiano a: Furfaro (2011) 417-442; Furfaro (2012) 55-71.

7 Meccarelli (2004) 118.

8 Benjamin (1983) 12. 
1820 e il 1870 si assiste, quasi in contemporanea nei diversi Stati europei e non europei, alla pubblicazione e traduzione di opere sul diritto delle genti, quali in primis i testi di Grozio, Martens e Vattel, tutti commentati. Il compito del traduttore e dell'annotatore è stato svolto da giuristi, esperti di diritto internazionale e costituzionale, che nella loro veste di professori, diplomatici e avvocati, hanno aggiornato i più celebri testi della tradizione giuridica della scuola del diritto naturale. ${ }^{9}$

\section{Il Droit des gens nel XIX secolo in Francia tra diritto naturale e diritto delle genti}

Gérard de Rayneval, in Institutions du droit de la nature et des gens affermava che:

La distribution de mon Ouvrage n'est pas nouvelle, c'est à-peu-près celle de Vattel, qui lui-même l'a puisée dans le traité du célèbre Wolff: je l'ai choisie, parce que c'est elle qui m'a paru la plus naturelle, la plus simple et la plus claire; d'ailleurs je n'ai pas eu la petite prétention de perfectionner un ordre reconnu bon dans des auteurs estimés. ${ }^{10}$

La pubblicazione di testi di diritto delle genti in francese si concentrò per di più nella seconda metà del XIX secolo; essi erano per la maggior parte destinati ad un uso didattico, come nel caso del più celebre commentatore del Droit des gens, Pradier-Fodéré, che come si avrà modo di illustrare, scrisse in otto volumi tra il 1885 e il 1906 il Traité de droit international public européen et américan suivant les progrès de la science et de la pratique contemporaines. Nel 1894 Robert Piédelièvre scrisse Précis de Droit International Public ou Droit des gens, ${ }^{\mathbf{1 1}}$ precisando nell'Avant-Propos, che la scienza del diritto, applicata alle relazioni internazionali, era una scienza ancora giovane e ancora non sufficientemente completa. ${ }^{\mathbf{1 2}}$ Il trattato di Piédelièvre, però, aveva

9 Fiocchi Malaspina (2013) 303-319.

10 Rayneval (1803) V. Egli continuava: «mon unique but a été de mettre dans une étude abstraite, compliquée et d'une étendue presque sans bornes, autant de clarté, de précision et de briéveté qu'il a dépendu de moi, afin que le lecteur ne soit ni rebuté par une méthode fatigante, pénible et incohérente, ni égaré par des discussions et de longs raisonnements; ils n'auraient servi qu'à lui faire perdre le fil des premières idées, qui doivent être la base de son instruction »: Ivi, V-VI.

11 Piédeliévre (1894).

12 Ivi, Avant-Propos, III. 
come punto di partenza il lavoro di Vattel, che, a suo dire, riscuoteva ancora alla sua epoca moltissimo successo. ${ }^{13}$

Nello stesso anno Alfred Chrétien, titolare dal 1894 al 1924 a Nancy della cattedra di diritto internazionale pubblico e privato, dava alle stampe Principes de droit international public, specificando che tale opera era " accompagné d'indications bibliographiques, ansi que de notes intercalées dans le texte et suivi d'un résumé des principaux traités signés par la France depuis 1648 jusqu'à nos jours », ${ }^{14}$ e una delle fonti principali, più citate e più importanti è proprio Vattel.

Chrétien, tuttavia, pur considerando attuale il pensiero vatteliano, allo stesso tempo riteneva anche che

en France Vattel a trouvé le trop mince éloge de ceux qui systématiquement n'entendent voir en lui que le vulgarisateur de Wolff, auteur plus cité, mais moins digne de l'être, parce que moins puissant, que Grotius. ${ }^{15}$

Si riesce a comprendere pienamente il ruolo di Vattel, con le parole di Lapradelle, il quale riferendosi alla Francia del XIX secolo scrisse che Vattel fu uno degli autori che più influenzò lo studio del diritto delle genti. ${ }^{\mathbf{1 6}}$

Fino al 1884 nelle università francesi si insegnava diritto delle genti e soltanto successivamente la cattedra prese il nome di diritto internazionale pubblico, mentre per quanto riguarda il diritto internazionale privato si insegnava già da qualche anno prima, nel $1880 .{ }^{17}$

La scienza sino alla seconda metà inoltrata del XIX secolo era più incline all'espressione diritto delle genti piuttosto che diritto internazionale, nonostante l'ingresso di tale terminologia ad opera di Jeremy Bentham. Nella pratica e nella dottrina, pertanto, non è casuale che nel corso del secolo ancora non venisse utilizzato in modo uniforme e omogeneo il termine «diritto internazionale» ma si parlasse di diritto delle genti. Questo sem-

13 Ivi, 57.

14 Chretien (1893).

15 Ivi, 59. Cfr. anche: Pradier-Fodéré (1886) 282 ss.; Pillet (1893) 14.

16 Lapradelle, Introduction, in: Vattel (1916) XLI.

17 Sul punto si rimanda all'utilissima banca dati on line Siprojuris che permette una visualizzazione completa dell'andamento della cattedra di di diritto internazionale pubblico e privato nel corso dell'Otto e Novecento: http://siprojuris.symogih.org/siprojuris/enseigne ments?concat_standard_name=droit+international\&begin_year $\% 5 \mathrm{Bmin} \% 5 \mathrm{D}=\&$ begin_year $\% 5 \mathrm{Bmax} \% 5 \mathrm{D}=$, consultato il 25 giugno 2016. 
brerebbe in parte spiegare il motivo per cui diversi internazionalisti intitolino il loro trattato diritto delle genti e non diritto internazionale. In una opera divulgativa come il Grand dictionnaire universel du XIXe siècle, alla voce "droit des gens/droit international », dopo un breve excursus storico nel quale viene citato anche Vattel, viene data la seguente definizione:

Les principes du droit des gens sont devenus à la base des rapports des gouvernements entre eux, et l'ont pénétré profondément dans la conscience des peuples modernes. De nos jours l'idée que les peuples de l'Europe doivent former une vaste association fondée sur les principes de la justice est entrée profondément dans les esprits. Elle a deja produit d'hereux résultats en apportant des perfectionnements véritable au droit des gens. ${ }^{18}$

Théophile Funck-Brentano e Albert Sorel nel loro Précis du Droit des gens argomentavano che il diritto delle genti alla loro epoca non aveva ancora un significato fisso e determinato e si distingueva tra diritto delle genti «teorico » e diritto delle genti « réel ». ${ }^{19}$

Lo sviluppo del diritto delle genti può essere letto attraverso le diverse tipologie di edizioni del Droit des gens che si susseguirono a partire dal

18 Larousse (réd. par) (1870), Droit des gens ou Droit international, 1248-1249.

19 «L'un des plus renommés parmi les théoriciens du droit des gens, Vattel a intitulé son ouvrage: Le Droit des gens ou principes de la loi naturelle, appliqués à la conduite et aux affaires des Nations et des Souverains" e terminavano argomentando che i progressi dei teorici sul diritto delle genti sono una delle condizioni del progresso del diritto delle genti « réel », il quale invece consiste nella condotta vera e propria delle singole nazioni le une verso le altre. "Leur relations résultent de certains causes et produisent certains effets parfaitement déterminés; en un mot, elles ont leurs règles, qu'une observation attentive des faits permet peu à peu de dégager »: Funck-Brentano/Sorel (1877) 1-2. Cfr. Arthur De la Guéronnière, mentre passa in rassegna i maggiori esponenti postgroziani affermava «Ces auteurs auront bientôt pour continuateur un écrivant éminent, un maître dans la science du droit lui-même, Vattel, dont le nom ne peut être prononcé qu'avec respect par tous ceux qui traitent cette matière. Vattel, s'inspirant de tout le grand mouvement philosophique et intellectuel que nous allons dépeindre, eut l'avantage de poser des principes plus élevés, des régles plus positives; il élargit, en la continuant, l'œuvre de ses devanciers, et on reconnaît dans ses travaux une parenté de doctrine avec Montesquieu qu'il a connu dans son enfance, et Jean-Jacques Rousseau qui est son contemporain. Dans le traité de Vattel, les principes du Droit des gens s'élèvent à la hauteur des vérités du droit public »: De LA Guéronnière (1876) 186. Anche JANET (1887) 261-262: "Vattel supérieur à Burlamaqui pour l'éntendue et la force de l'esprit est devenue une véritable autorité pour le Droit des gens: son livre [...] est aussi souvent cité que celui de Grotius ». 
$1820 .^{20}$ Nel 1830 Paul Royer-Collard, ${ }^{21}$ titolare dal 1829 al 1865 della cattedra di diritto delle genti presso la facoltà di legge di Parigi, tradusse dall'inglese il Discourse on the Study of the Law of Nature and Nations di James Mackintosh e lo incluse alla nuova edizione del trattato di Vattel da lui curata (ed edita da Aillaud), redigendo anche un'appendice ai due volumi. ${ }^{22}$ Tale appendice è composta da una bibliografia, in cui sono stati scelti i maggiori testi di diritto della natura e delle genti. ${ }^{23}$

Cinque anni dopo Royer-Collard curò una nuova edizione del Droit des gens, servendosi della versione di Amsterdam $1775,{ }^{24}$ ma con l'aggiunta, questa volta, di un'ulteriore bibliografia redatta da M. de Hoffmanns. ${ }^{25}$

20 Ad esempio in questo anno veniva data alle stampe una nuova versione del trattato di Vattel. Nell'Avertissement si precisava che: «Vattel aura toujours le mérite d'avoir mis à la portée du plus grand nombre des lecteurs les travaux des grands hommes qui ont ouvert cette importante carrière. Son ouvrage du Droit des gens est devenu le manuel des diplomates; on l'appelle dans toute l'Europe le Code des ambassadeurs »: Avertissement in: VATTEL (1820a) II-III. Questa edizione, in due volumi in ottavo, è la ristampa dell'edizione di Amsterdam del 1775 e quindi correlata da tutte le note che Dumas aveva posto, come si è visto, al testo di Vattel.

21 Paul Royer-Collard (1797-1865) fu anche avvocato alla Corte Reale di Parigi. «Il a été, de 1845 à 1847, doyen de la Faculté de droit. A cette époque, il fut chargé, par le ministre des affaires etrangères, d'une mission en Sardaigne, qui lui valut le titre de chevalier de l'ordre des SS. Maurice et Lazare »: VAPEREAu (1870) 1591. Cfr. L'enseignement du droit international public en France (1955) 816-818, in particolare 816. Paul Royer-Collard è ricordato anche per l'apparato di note a: Bravard-VeYrières (1861).

22 Vattel (1830). Cfr. anche per i repertori francesi: Bragt (1995) 285-286.

23 Il titolo esatto è Bibliothèque choisie des livres qu'il est le plus utile d'acquérir et de connaître; pour l'étude du droit de la nature et des gens; extraite des bibliographies spéciales de Camus, Kluber, etc., in: VATTEL (1830) 435-448. Tale bibliografia contiene al suo interno un'analitica esposizione di testi riguardanti il diritto della natura e delle genti, con particolare riguardo ai suoi più importanti autori. Dopo una breve sezione dedita alla storia del diritto delle genti, si trovano elencati i Traités sur le Droit de la nature et des gens, in cui compaiono le opere di Grozio: Ivi, 437-448.

24 Cfr. Marvin (1847) 705.

25 VAttel (1835). Una tabella permette al lettore di orientarsi nella scelta dei testi indicati da Hoffmanns; vi è così una prima sezione in cui sono indicati i maggiori testi sulla storia del diritto della natura e sul diritto delle genti. Le successive sezioni sono dedicate alle opere didattiche e sistematiche per lo studio del diritto della natura e delle genti (e del diritto pubblico), al diritto marittimo (viene elencata l'opera di Domenico Azuni, tradotta in francese, Origine et progrès du Droit et de la législation maritime, avec des observations sur le consulat de la mer edita a Parigi nel 1810), a quelli sulla libertà del mare, sulla neutralità marittima e sulle leggi marittime (ad esempio il testo di Pardessus intitolato Collection des 
Nell'Avertissement venne esplicitato il desiderio di perfezionare il più possibile l'opera, togliendo tutto ciò che rendeva faticosa la lettura del testo ed eliminando le note ritenute superflue. ${ }^{\mathbf{2 6}}$

Sin dalle prime righe si tentò, inoltre, di prendere posizione contro coloro che « ingiustamente » avevano criticato il pensiero di Vattel. ${ }^{27}$ Per il curatore Vattel commise, nel redigere il trattato e nell'esporre il suo pensiero, senz'altro degli errori, ma questi sono stati fatti in buona fede, perché, a suo dire, scriveva «sull'influenza della sua coscienza $»^{28}$

Tuttavia questi errori non sono stati analizzati, il curatore sembra accennare al problema, evitando volutamente di entrare nel dettaglio, in riflessioni più argomentate e approfondite, per lasciare spazio alla affermata autorità di Vattel e al suo elogio. ${ }^{29}$

lois maritimes antérieures au XVIII siècle edito a Parigi tra il 1828 e il 1831 e quello di Martens, Lois et ordonnances des diverses puissances européennes concernant le commerce, la navigation et les assurances, depuis le milieu du XVII siècle, pubblicato a Gottinga nel 1802). Le sezioni successive sono costituite dall'elencazione di alcune opere di lessicografia, una particolare sezione (la nona) è dedicata alle opere relative alla storia e all'interpretazione dei trattati pubblici, un'altra alle memorie storiche in ambito di negoziazione, mentre le ultime sezioni sono dedicate ai trattati sulla storia politica, ai giornali e ai periodici dell'epoca. Apparentemente può sembrare superfluo aver riportato la descrizione dell'appendice, ma ciò offre un'attenta e precisa visione di quella che all'epoca era la diffusione dei testi relativi al diritto delle genti.

26 Così affermava Hoffmanns, Avertissement, in: Vattel (1835) V: « Nous avions reconnu par nos études antérieures sur Vattel, dans les éditions les plus répandues de son ouvrage, des intercorrections, et des transpositions dans le texte qui nuisaient sensiblement à sa clarté; il nous a été facile de les faire disparaître dans la nôtre, en conférant soigneusement les meilleures éditions avec les textes originaux que nous possédons".

27 Ivi, VI-VII: «Vattel, ou moins l'ouvrage qui nous occupe, a été, de la part de quelques écrivans qui doivent l'avoir mal lu, pour en parler comme ils l'ont fait, l'objet de quelques critiques aussi peu juste qu'imméritées".

$28 \mathrm{Ivi}$, VII.

29 Vattel (1835) 66: "par tous les États, lui [Vattel] acquit la réputation la mieux méritée, et lui concilia les suffrages des hommes d'Etat autant que l'estime des amis de l'humanité. On peut dire, en effet, qu'Emer de Vattel a déployé dans cette production toute l'étendue de son génie et la solidité de son esprit, à mesure qu'on y trouve l'empreinte des vertus qui formaient l'essence de son caractère. Tout y est clair, judicieux, systématique, et les préceptes sont appuyés par des exemples bien choisis. Tout y annonce le citoyen vertueux, l'ami des hommes, de la liberté, de la vraie gloire. Le sentiment vif et profond dont l'auteur était pénétré donne à son style une chaleur, une énergie qui ne se trouve pas dans les ouvrages purement didactiques; en un mot, Le Droit des gens d'Emer de Vattel sera toujours regardé comme un ouvrage d'une mérite incontestable, destiné à éclairer les nations sur leurs intérêts les plus essentiels ». 
Le critiche furono aggiunte all'interno di uno specifico volume, edito nel 1838 e contenente esclusivamente le note del giurista portoghese Pinheiro Ferreira.

Sempre nel 1838 fu pubblicata in due volumi, una ulteriore edizione di Vattel, précédée d'un essai de l'auteur sur le droit naturel pour servir d'introduction. ${ }^{30}$ In essa sono stati inseriti, per la prima volta, oltre al saggio di Vattel intitolato Essai sur le fondement du droit naturel, ${ }^{31}$ punto di partenza per la redazione del Droit des gens, sia le riflessioni contenute nelle Questions di Chambrier d'Oleyres, che invece criticavano in toto il trattato, sia le posizioni di Sulzer. L'edizione francese utilizzata come riferimento è sempre quella di Amsterdam (del 1775); mentre dal punto di vista formale è presente un'appendice bibliografica che venne redatta dal politico e diplomatico PierreLouis-Auguste-Bruno Blanc de La Nautte comte d'Hauterive. ${ }^{32}$

2.1. Il Droit des gens riletto dal portoghese Silvestre Pinheiro Ferreira: le Notes del 1838

L'editore Aillaud nel 1838 pubblicò una particolare edizione del Droit des gens in cui sono raccolte le notes di Pinheiro-Ferreira: ${ }^{33}$ si tratta di un commento analitico e critico alla maggior parte delle sezioni del trattato di Vattel, con un accentuato approccio più propriamente filosofico alla materia.

Silvestre Pinheiro Ferreira nacque a Lisbona nel 1769 e vi morì nel 1846. Ebbe una vita lavorativa movimentata: da giovane, per pochi anni, fu docente di filosofia all'Università di Coimbra, dove insegnò il pensiero di Locke e di Condillac. Costretto all'esilio, viaggiò moltissimo prima in Inghilterra, poi in Olanda e dal 1797 si stabilì a Parigi come «secrétaire de délegation du Ministre de Portugal ». ${ }^{34}$

30 Sulla pubblicazione di questa edizione cfr. anche: Веuснот (1839) 135, n. 1420.

31 Ci si riferisce al saggio di Vattel intitolato Essai sur le fondement du droit naturel, pubblicato a Parigi nel 1746. Questo saggio insieme Dissertation sur cette question: La loi naturelle peut-elle porter la société à la perfection sans le secours des lois politiques? costituiscono gli antecedenti del Droit des gens di Vattel e verranno inclusi in diverse edizioni francesi del XIX secolo.

32 Annuaire diplomatique de la Republique Française pour 1871-1872 (1872) 179.

33 Per le edizioni del Droit des gens di Vattel, in cui si parla in particolare proprio di quella curata da Pinheiro cfr. Bonfils (1904) 66-67.

34 Larousse (réd. par) (1874), Pinheiro Ferreira, Silvestre, 1036. 
Nel 1807 Napoleone obbligò Pinheiro a lasciare la Francia e a rientrare in patria, poichè aveva informato la monarchia portoghese sulle mire egemoniche francesi verso il Portogallo. Raggiunta Lisbona, Pinheiro salpò con la famiglia reale verso il Brasile, dove divenne consigliere di Giovanni VI, membro della direzione del commercio e successivamente segretario di Stato del regno portoghese.

Le sue abilità politiche emersero in questo contesto travagliato tanto in Europa quanto in Sud America. Egli suggerì al Sovrano di concedere la costituzione e la contestuale creazione di una monarchia rappresentativa, al fine di evitare la separazione tra Portogallo e il Brasile. Dopo la rivoluzione di Oporto del 1821 fu nominato ministro degli affari esteri e della guerra; successivamente si dimise per protesta al regime assolutistico ristabilito da Michele I e ritornò quindi a Parigi. ${ }^{35}$ Terminato il breve regno di Michele I, Pinheiro non rimpatriò sino al 1842 e fu eletto deputato « a distanza » per la provincia di Miho nel 1826 e $1837 .^{36}$

Su Pinheiro è stato scritto che

de todos los portugueses que se ocuparon de filosofia en los comienzos del siglo XIX, el más conocido es Silvestre Pinheiro Ferreira. [...] En derecho internacional adquirió también un nombre illustre. ${ }^{37}$

Numerosissime sono le opere scritte dal giurista-filosofo portoghese che, dalla vastità e complessità delle materie affrontate, risulta un vero e proprio eclettico. Pubblicò trattati di filosofia come la Preleções philosophicas sobre a theórica do discurso e da linguagem, a esthetica, a diceósyna e a cosmologia (Rio de Janeiro 1813), ma il settore in cui più si specializzò fu più propriamente il diritto costituzionale, basti solo citare il Projecto de Código Geral de leis fundamentais e constitutivas de uma Monarquia representativa (Parigi 1834), mentre nel 1836 elaborò una Declaração dos direitos e deveres do homem e do cidadão. $^{38}$

35 Vergé (1856) 51-70. A seguito, infatti, della morte di Giovanni VI, Pietro I (già imperatore del Brasile) diventò re del Portogallo, ma abdicò a favore della figlia Maria II, affidando la reggenza al fratello Michele, ma questi si proclamò re con l'intenzione di creare una monarchia assolutistica, ma Pietro intervenne rimettendo sul trono Maria II.

36 Su Pinheiro Ferreira: Silva (2012) 221-241. Per maggiori dettagli sulla biografia dell'autore si rimanda a: Louzada de Magalhães (1881); Silva (1975); Pereira (1974). Cfr. BARRETO (1975) 470-478.

37 Pinheiro-Ferreira Silvestre (1991) 913-914.

38 Dello stesso autore si ricordano anche: Observações sobre a Constituição do Império do Brazil, e sobre a carta constitutional do Reino de Portugal, segunda edição augmentada com 
Nel 1838 (anno in cui pubblicò il commento al Droit des gens) fu eletto corrispondente straniero presso l'Accademia delle Scienze morali e politiche (sezione di legislazione) dell'Istituto francese, in cui facevano parte anche grandi nomi del pensiero giuridico francese come André-Marie-Jean Dupin (1783-1865) e Philippe-Antoine Merlin (1754-1838); fu inoltre membro dell'Istituto Storico-Geografico del Brasile e, rientrato in Portogallo, divenne Presidente dell'Accademia di Scienze e Lettere (1843-1846), nonché socio onorario della Reale Accademia di Scienze di Lisbona. ${ }^{39}$

Pinheiro, oltre ad aver approfondito gli studi sul diritto costituzionale, ampliò anche alcune tematiche legate al diritto internazionale; infatti non si limitò ad aggiungere note solo al testo di Vattel ma «commentò » anche il testo di Martens Précis du Droit des gens. ${ }^{\mathbf{4 0}}$

Nell'introdurre il commento al Droit des gens, affermava che il trattato di Vattel era per quell'epoca « l'ouvrage classique de la science. Ce seul fait suffit pour en constater le mérite ${ }^{41}$ La società, continuava Pinheiro, non era più quella dei tempi nei quali Vattel scriveva, il principio costitutivo dei governi era stato cambiato o modificato. I rapporti commerciali tra le nazioni non erano più gli stessi, ${ }^{\mathbf{4 2}}$ ma soprattutto "Puis grand nombre de doctrines contenues dans ce livre, d'ailleurs très-remarquable, inexactes autrefois aux yeux des hommes de la science, sont aujourd'hui reconnues fausses toutes les lumières $»{ }^{43}$ Insomma il Droit des gens necessitava di una revisione e a tal fine sembrava utile per le giovani generazioni, di pubblicare un volume di sole note esplicative, relative ai passaggi del trattato del giurista di Neuchâtel. ${ }^{\mathbf{4 4}}$

as observações do mesmo autor sobre a lei das reformas do imperio do Brazil (Paris 1835); Observations sur la Charte constitutionelle de la France, extraites du cours de droit public (Paris 1833); Manual do cidadão em um governo representativo, ou principios de direito constitucional, administrativo e das gentes (Paris 1834); Projecto de ordenações para o Reino de Portugal (Paris 1831-1834).

39 Silva (2012) 229.

40 Martens (1831).

41 Pinheiro Ferreira (1838) Avertissement, V.

42 Ibidem.

43 Ibidem.

$44 I v i, \mathrm{VI}$ : «Nous avons donc cru rendre quelque service à la jeunesse en publiant ce volume de notes sur les passages du Traité de Vattel qui nous ont paru contenir le plus de principes généraux du droit international, tel que la raison le conçoit d'après le principe du juste, seule règle de toute morale et de toute politique ». 
Pinheiro sosteneva il commento al testo di Vattel più che mai necessario, ${ }^{45}$ ed aggiungeva che, per far fronte ai progressi del diritto delle genti, fosse opportuno prendere in considerazione anche le teorie contenute nelle opere di ulteriori giuristi quali ad esempio Droit des gens moderne de l'Europe di Johann Ludwig Klüber, il già citato Précis du Droit des gens moderne de l'Europe di Martens, mentre per l'aspetto più squisitamente pratico Le guide diplomatique, précis des droits et des fonctions des agents diplomatiques et consulaires, suivi d'un traité des actes et offices divers di Charles de Martens pubblicato nel 1822 a Parigi e a Lipsia.

L'intento del diplomatico portoghese fu subito apprezzato, anzi, in una recensione presente negli Archives du droit et de législation, fu scritto che Pinheiro aveva reso un gradissimo servizio alla scienza del diritto grazie all'aggiunta delle sue note e alla revisione di gran parte delle teorie sul diritto delle genti. ${ }^{46}$

Per meglio comprendere il lavoro fatto dall'infatigabile Pinheiro è utile accennare alla sua metodologia: egli rilegge il Droit des gens con la lente della sua epoca e dei fatti che tra il 1758 e i primi trent'anni dell'Ottocento si sono progressivamente succeduti. Ciò significa che vengono presi in considerazione, sebbene la prospettiva dell'argomentazione sia più squisitamente filosofica e dottrinale piuttosto che storica, i fallimenti e i successi statali tanto a livello nazionale quanto a livello internazionale. Sotto questo duplice profilo inaugura le sue Notes, partendo in primis dagli elementi essenziali di una

45 Ibidem: «nous semblait d'autant plus nécessaire que le droit des gens positif, tel qu'il est au moment même où écrivons, ne saurait être avoué dans toutes ses dispositions par aucun publiciste sincèrement constitutionnel. Nous ne parlons pas de ceux qui défendent encore les gouvernements absolus; car, admettant pour base de leurs doctrines la volonté du maître, l'arbitraire tempéré par l'instinct individuel de l'équité, ils rejettent par cela même tout principe partant de plus haut, c'est-à-dire de cette de tous les monarques et de tous les législateurs ".

46 Belpaire et al. (pub. par) (1840) 219-220: "a rendu un véritable service à la science en entreprenant ce travail. Il a publié sur le passage du traité de Vattel contenant le plus de principes généraux du droit international, des notes qui forment le 3 me volume de la nouvelle édition que nous annonçons. Dans ce travail, qui ne peut qu'ajouter à la haute réputation du savant et infatigable auteur [...], Pinheiro Ferreira commence par rectifier un grand nombre de definitions données par son devancier, et modifiés par le temps: il introduit ainsi dans l'étude du droit des gens toute la clarté et la précision dont elle est susceptible. Il établit ensuite les principes du droit international, tels que les conçoit la raison, d'après le principe du juste, seule règle de toute morale et de toute politique ». 
Nazione. Il giurista portoghese alla definizione di Vattel di Nazione o Stato, contenuta nei Préliminaires:

«Les Nations ou États sont des Corps Politiques, des Sociétés d'hommes unis ensemble pour procurer leur salut et leur avantage, à forces réünies, ${ }^{47}$

controribatteva

Cette définition est trop générale, car elle convient à toute société, quelconque, tandis qu'il s'agissait de définir ce que c'est qu'une nation ou un État. Vattel aurait dû dire que c'est une association contractée dans le but d'assurer mutuellement, envers et contre tous, la jouissance des droits naturels de sûreté, de liberté et de propriété. $^{48}$

Tale posizione veniva nuovamente ripresa quando Vattel argomentava al libro I, capitolo, I, $\mathbb{S}$ 4, che cosa fosse uno Stato sovrano: cioè ogni Nazione indipendente e che si governa da se medesima, sotto qualsivoglia forma, ed emanando le sue proprie leggi; Pinheiro Ferreira la riteneva assolutamente inesatta, perché sembrava racchiudere al suo interno una comunità di persone che non potessero essere identificate nel concetto di nazione: «Le nombre des individus n'entrant pas en considération, on pourrait conclure quel es flibustiers et pareilles masses de pirates ou forment une nation $[\ldots]$ ", per il giurista portoghese la condizione per l'esistenza di una nazione intesa come indipendente era «qu'il respecte lui-même celles des autres nations en général et de chacun de leurs membres en particulier ${ }^{\mathbf{4 9}}$

Vattel, dopo aver scritto sulla "théorie de la sociabilité », estendeva il discorso anche alle nazioni, poiché la società universale del genere umano è essa stessa una istituzione della natura, cioè una conseguenza necessaria della natura dell'uomo. ${ }^{50}$

Tutti gli uomini sono

obligés de la cultiver et d'en remplir les devoirs [...] Toute la différence consiste en ce qu'étant convenus d'agir en commun, et aïant remis leurs droits et soumis leur volonté au Corps de la Société, en tout ce qui intéresse le bien commun, c'est désormais à ce Corps, à l'État et à ses Conducteurs, de remplir les devoirs de l'humanité envers les Etrangers, dans tout ce qui ne dépend plus de la liberté des particuliers, et c'est à l'État particulièrement de les observer avec les autres États. ${ }^{\mathbf{5 1}}$

47 Vattel (1758a) L. I, Préliminaires, $\mathbb{S} 1,1$.

48 Pinheiro Ferreira, Notes, in: Vattel (1838) Préliminaires, $\mathbb{S} 1,1$.

49 Ivi, $₫ 4,29-30$.

50 Vattel (1758a) L. I, Préliminaires, $\$ 10,5-7$.

51 Ivi, $\mathbb{1 1}, 7$. 
Per Pinheiro in questa deduzione si insinuava un equivoco:

On voit qu'il n’y pas de cohérence dans ce raisonnement, car la réciprocité des services que les nations se doivent n'a rien de commun avec les rapports créés entre les citoyens par leur réunion en corps de nation. Il n'y a donc rien à conclure des devoirs de ceux-ci pour ce que se doivent les nations les unes envers les autres. S'il y a quelque rapport à établir, ce n'est pas entre les devoirs des nations entre elles et les devoirs des citoyens les uns envers les autres. ${ }^{52}$

Per il giurista portoghese, inoltre, non vi era alcuna differenza tra il diritto delle genti convenzionale e il diritto delle genti volontario, come invece sosteneva Vattel, o meglio per essere più precisi, si poteva riservare l'utilizzo del termine solo per designare le due specie di diritto delle genti: il diritto delle genti volontario le cui fonti erano i trattati e il diritto delle genti consuetudinario che si basava sugli usi consolidati nel tempo. Entrambi erano da intendersi come due modi attraverso cui i governanti e i popoli facevano conoscere le loro volontà. ${ }^{53}$

Serrate critiche sono successivamente rivolte alla sezione dedicata alla costituzione, che il giurista portoghese contestava in maniera capillare. La definizione data da Vattel sulla costituzione come regolamento fondamentale, contenuta, come si è visto nel libro I, al capitolo III, sembrava non essere esaustiva né esplicita per i tempi in cui Pinheiro Ferreira viveva. ${ }^{54}$ Vengono così illustrate tre tipologie diverse di leggi: fondamentali, costitutive e organiche ognuna delle quali riveste un preciso ruolo all'interno della Nazione. Questa tripartizione mirava a provare, secondo Pinheiro Ferreira, quanto la dicitura « regolamento fondamentale» non fosse calzante con il concetto vero e proprio di costituzione moderna. Le prime s'identificavano ne «la déclaration des droit naturels et invariables", in quanto «si tout le monde était d'accord sur les principes qui doivent être compris dans la déclaration des droits dont nous venons de parler, cette déclaration pourrait

52 Pinheiro Ferreira, Notes, in: Vattel (1838) Préliminaires, $\mathbb{1} 11,13$. Continuava il giurista portoghese « Mais il n'était ancore qu'à la moitié de son raisonnement, lorsqu'il s'aperçut que les nations ne pouvaient pas former entre elles des sociétés civiles, sans cesser d'être des nations. N'importe, s'est-il dit, ce sera là la différence entre les nations et les individus; ceux-ci formeront des sociétés, mais les nations n'en formeront pas moins une société », e ancora « Il y a donc abus de mot à donner le nom de société à cet ensemble de droits et de devoirs qui existent entre tous les membres de l'espèce humaine »: Ivi, $\mathbb{1} 11,13-14$.

$53 \mathrm{Ivi}, \mathbb{S} 24,22-23$.

54 Ivi, L. I, $\mathbb{S} 27,34-35$. 
être omise mais, dans l'état actuel de la société il est essentiel de poser nettement ces principes qui sont la loi des lois parce qu'il n'est jamais permis aux législateurs eux-mêmes de s'en écarter $» .{ }^{55}$ Mentre le leggi costitutive avevano per scopo la determinazione delle attribuzioni di chi esercitava il potere politico; quelle organiche, invece regolavano l'esercizio di tali attribuzioni. ${ }^{56}$ Il fine della costituzione è individuato da Vattel nella felicità della Nazione, ma per Pinheiro Ferreira tale caratteristica non era affatto sufficiente, perchè la costituzione doveva essere integrata con la promulgazione delle leggi cosiddette organiche, oltre all'esigenza dell'ordine e della stabilità del governo. Secondo Pinheiro la breve durata di molte costituzioni sia in America che in Europa nel corso della fine del Settecento e nei primi anni dell'Ottocento è stata causata dall'assenza di leggi organiche "sans lesquelles ou ne saurait même concevoir qu'une constitution puisse être autre chose que lettre morte $»:{ }^{57}$ rimanendo quindi senza esecuzione, non potevano essere oggetto di denunce contro il governo, che, solo per questo, veniva collocato "dans une fausse position ${ }^{58}$

Dalla lettura dei vari commenti apportati da Pinheiro Ferreira in diversi e molteplici passaggi delle Notes, emerge forte criticità nei confronti del pensiero vatteliano; Pinheiro non vuole "aggiornare ", il suo scopo ultimo è spiegare o meglio ancora riformulare le teorie di Vattel. È interessante rilevare che in moltissime occasioni, Pinheiro utilizza espressioni alquanto negative riguardo al trattato e in particolare, come è stato rilevato da Chetail definisce la dottrina di Vattel come "generica ", "vaga », mentre si rivolge alle sue teorie come colme di «equivoci " e contraddizioni. ${ }^{59} \mathrm{Ma}$ al tempo stesso in alcune occasioni sembra però giustificare le manchevolezze del giurista Neuchâtel affermando che "notre auteur [est] guidé par l'instinct de son bon sens, plutôt que par l'exactitude de ses principes ", ${ }^{\mathbf{6 0}}$ quasi a volerlo difendere dalle accuse che lui stesso aveva rivolto nelle sue Notes.

55 Ivi, 35 .

56 Ivi, 35.

57 Ivi, 36.

58 Ibidem.

59 Chetail (2011) 424.

60 Pinheiro Ferreira, Notes, in: Vattel (1838) L. I, $\int S 88-89$, 99. Pinheiro in una altra nota così argomentava: "Toujours la saine raison de Vattel, l'emportant sur le prejudice, finit par le forcer à sortir de la thèse dont il s'agit et dont il sent l'absurdité, pour se jeter dans une autre dont il n'est nullement question ». Ivi, L. I, $\mathbb{S} 132,120$. Alla stessa conclusione si 


\subsection{Un altro Droit des gens nelle note di Paul Pradier-Fodéré (1863)}

Nel 1863 fu pubblicata una nuova edizione del testo di Vattel ricca di allegati e con maggiori note da parte del curatore Paul Pradier-Fodéré ed intitolata: Le Droit des gens ou Principes de la loi naturelle appliqués à la conduite et aux affaires des nations et des souverains par Vattel, nouvelle edition précédé d'un essay et d'une dissertation (de l'auteur) accompagnée des notes de Pinheiro Ferreira et du Baron de Chambrier d'Oleires augmentée du discours sur l'étude du droit de la nature et des gens, par sir James Mackintosh (traduction nouvelle) mise au courant des progrès du droit public moderne et suivie d'une table analytique des matières. ${ }^{\mathbf{6 1}}$

Essa è considerata la migliore edizione dell'opera di Vattel nella Francia del XIX secolo. ${ }^{62}$ L'editore parigino Guillaumin così annunciò l'uscita:

Bien que Vattel ait écrit dans le siècle dernier, sa réputation est loin d'être épuissé, et son livre est demeuré le Traité du Droit des gens le plus généralement répandu. Le nombre des éditions que l'on a faites de cet ouvrage en toutes les langues et dans tous le pays s'est accru au point que leur énumération raisonnée pourrait former un travail volumineux. ${ }^{63}$

Sono riportate le note del giurista portoghese Pinheiro Ferreira, le riflessioni di Chambrier d'Oleyres, già raccolte nell'edizione del 1838 curata d'Hauterive, ma Pradier-Fodéré ne aggiunse delle altre per far fronte ai cambiamenti ottocenteschi tanto a livello dottrinale quanto politico. ${ }^{64}$

giunse ancora durante i primi anni del Novecento. Van der Vlugt scrisse: «malgré tout, ce dernier des internationalistes d'un grand renom a été un homme aux vues étroites, dénué de sens logique, se pendant à tout moment dans les contradictions qu'il se flatte de résoudre simplement par juxtaposition des termes »: VAN DER Vlugt (1925) 467. Mallarmé sul punto rileva che il Droit des gens: "constituent une vaste encylopédie qui s'étend à une foule de questions et qui fournit, pour chacune, une solution généralement nette mais qui n'est pas bâtie sur un système solide pouvant servir de trame à des développements; de sorte que les différents paragraphes se présentent comme une série de tiroirs, ayant chacun son objet propre et ne se reliant pas, le plus souvent, au precedent »: Mallarmé (1904) 492. Cfr. anche Chetail (2011) 424.

61 Esattamente come per l'edizione precendente edita con il volume delle note di Pinheiro anche in questa sono state inseriti i due saggi antecedenti il Droit des gens e cioè Essai sur le fondament du droit naturel et sur le premier principe de l'obligation où se trouvent les hommes d'en observer les lois, e la Dissertation sur cette Question: la loi naturelle peut-elle porter la société à la perfection, sans le secours de Lois politiques.

62 Bonfils (1904) 66-67: «Eine vorzügliche Ausgabe mit Anmerkungen ist 1863 von Pradier-Fodéré besorgt worden »; Calvo (1885) 297; Rivier (1896) 405.

63 Bulletin de la librairie Guillaumin et C. Suite au catalogue général (1863) 7-8.

64 Pradier-Fodéré, Avant propos, in: Vattel (1863) V. 
Sotto il profilo biografico Paul Luis Ernest Pradier-Fodéré (1827-1904), iniziò la sua carriera prima come avvocato poi si dedicò allo studio del diritto commerciale, amministrativo e a Parigi insegnò, presso il Collegio Armeno Moorat, diritto pubblico. Soggiornò per molto tempo in Perù, dove a Lima fondò la facoltà di scienze politiche e amministrative; è dipinto come uno dei giuristi più importanti e più rinomati nella Francia dell'Ottocento per il diritto internazionale.

La sua produzione scientifica si caratterizza soprattutto nel diritto delle genti e nel diritto commerciale, è sufficiente solo citare il Précis de droit administrativ del 1853 o il Précis de droit commercial del 1854; i nove volumi del Traité de droit international public européen et américain (1885-1906), l'edizione in lingua francese del trattato di Pasquale Fiore intitolato Droit international privé del 1875 ed infine la nuova edizione del Droit des gens di Vattel con l'aggiunta di importanti note. ${ }^{65}$

Nell'introdurre l'opera vatteliana Pradier-Fodéré, esattamente come aveva fatto Pinheiro, dedicava ampio spazio ai mutamenti susseguitesi nell'Ottocento: «le plus magnifique essor aux arts de la paix; il a consolidé l'édifice des libertés politiques ", nel cuale si è consacrato il dogma della sovranitá del popolo. ${ }^{66}$ Per il giurista francese era stato proprio il suo secolo ad aver messo "alla prova dei fatti» tutte le questioni che il precedente aveva lasciato irrisolte: ci furono tentativi temerari di monarchia universale e di creazione di piccoli Stati, di reminiscenze assolutistiche e le più alte aspirazioni di libertà.

Tuttavia, secondo Pradier-Fodéré, l'Ottocentro era stato un'epoca di azione ed eclettica:

Son rôle n'aura point été d'avoir donné la prééminence à telle ou telle idée: plus large, plus tolérant, il a tout compris, il s'est tout approprié. En politique, il a cherché à combiner les formes désormais impossibles de la monarchie absolue, avec les conditions praticables du governement républicain; en matière religieuse, il a voulu réunir la raison et la foi; dans les arts libéraux, il a poursuivi le mélange du beau réel avec le beau idéal. ${ }^{67}$

Per il commentatore, poichè il diritto naturale era ancora uno degli argomenti più dibattuti, bisognava che fossero sufficientemente approfonditi $\mathrm{i}$

65 Su Pradier-Fodéré per maggiori dettagli biografici: Saint-Bonnet (2007) 641; Elliot (1908) 245-254.

66 Pradier-Fodéré, Avant propos, in: Vattel (1863) V.

67 Ivi, VI-VII. 
principi sui quali si snodava. ${ }^{68}$ Per ovviare a tale mancanza Pradier-Fodéré introduceva l'opera di Vattel in quanto essa

ne sera jamais déplacé, même dans les temps où l'esprit général sera plus détourné des études spéculatives. Quelles que soient les tendances d'un siècle, il arrivera toujours un moment où les hommes d'État éprouveront le besoin de relier les faits à des principes certains; et peut-être même sera-t-il quelquefois utile de rappeler aux générations trompées par le succés éphémère de la force, l'éternelle vérité du droit ${ }^{69}$

sottolineando, inoltre, che il Droit des gens ebbe un successo immediato perché elegante e semplice. Wolff non aveva mai lavorato se non per la scienza: «Jamais il n'avait quitté ces hauteurs où son esprit philosophique se complaisait dans l'étude abstraite de la théorie du droit ${ }^{\mathbf{7 0}}$

Vattel, al contrario, aveva trasportato le astrazioni al servizio della pratica, « mettendo il diritto delle genti alla portata del mondo », applicando cioè i principi del diritto naturale alla condotta delle nazioni e dei governanti, sostituendo alla lingua latina la lingua che nel frattempo era divenuta d'uso nella diplomazia: il francese. ${ }^{71}$

Dunque il Droit dens gens è una vera e propria enciclopedia della scienza politica, dove sono state raccolte riflessioni sul diritto costituzionale, penale, sull'economia, sul diritto civile. Pradier-Fodéré nello specifico raccolse tutto il lavoro di annotazioni svolto dai diversi curatori delle precedenti edizioni francesi e pose a fondo pagina i suoi commenti, che sono facilmente riconoscibili delle iniziali del suo cognome: si tratta di annotazioni e riflessioni per lo più di carattere dottrinale e comparativo tra le teorie che nel merito erano state avanzate da diversi giuristi esperti di diritto internazionale del XIX secolo; in particolare tali note sono arricchite con molta bibliografia che facilita il reperimento delle fonti da cui Pradier-Fodéré ha attinto.

Se, infatti, Pinheiro aveva cercato di colmare le contraddizioni, le "vaghezze », di Vattel, Pradier-Fodéré, fece un passo in avanti, in quanto cercò di completare il Droit des gens non solo con le osservazioni di Pinheiro, che

68 Ivi, VII: «Vainqueurs et vaincus, oppresseurs et victimes, despotes et peuples, l'ont invoqué tour à tour pour legitimer leur domination, ou pour justifier leurs vengeances. Il serait grand temps, cependant, de s'entendre sur les principes fondamentaux de cette loi primordiale, dont on peut méconnaître les règles, qu'on peut même traiter de chimère, mais qui est immutable et commune à toutes les époques et à toutes les nations. »

69 Ivi, VII-VIII.

70 Ivi, VIII.

71 Ibidem. 
riportò in molti casi in toto, ma aggiungendo le teorie della dottrina internazionalistica, civilista e costituzionalista dell'epoca. Ad esempio per quanto riguarda la definizione di Stato o Nazione contenuta nel Droit des gens, dopo aver riportato interamente il testo del giurista portoghese, Pradier-Fodéré la completava il tema riassumendo la posizione di Henry Wheaton, il quale specificava che nella massima vatteliana non rientravano né le corporazioni create da uno Stato né quelle come l'antica Compagnia delle Indie, perchè esisteva solo in forza di una autorizzazione statale; tantomeno non si chiamavano Stati le associazioni volontarie, anche se gli individui si erano riuniti per assicurarsi dei vantaggi singoli e reciproci. Inoltre Wheaton asseriva la necessità di distinguere uno Stato da una comunità nomade, che ancora non poteva essere considerata una società civile; infine differenziava uno Stato da una nazione, in certi casi, quando vi erano più popoli sottomessi ad un'unica autorità suprema come l'impero d'Austria o quello di Prussia. ${ }^{72}$

Pradier-Fodéré continuava la sua riflessione parafrasando anche le teorie di Heffter, secondo il quale, invece gli Stati erano delle riunioni permanenti di uomini create per loro volontà e per soddisfare i propri bisogni morali e fisici e per lo sviluppo razionale dell'essere umano e della sua libertà. L'esistenza di uno Stato era però subordinata a tre condizioni: formare una comunità in grado di mantenersi con le sue forze; avere una volontà organica esclusiva per dirigere questa riunione di persone; possedere un territorio sufficiente a contenere la comunità. ${ }^{73}$

In questa articolata ed erudita digressione, citava anche Martens e i significati che "Stato, Popolo, Nazione " potevano avere, per poi soffermarsi sulla teoria di Klüber: egli, come Vattel, indistintamente attribuiva il termine Stato e Nazione all'unione di un gruppo di uomini che avevano fissato la loro dimora e si sottomettevano ad un sovrano con l'intenzione di vivere insieme per la sicurezza di tutti. ${ }^{74}$

L'osservazione di Pradier-Fodéré anche per quanto riguarda la costituzione risulta interessante: dopo aver fornito in modo molto didascalico, la differenza etimologica dal punto di vista sociale, politico e giuridico, richiamava la posizione di Pinheiro Ferreira e la sua tripartizione in leggi fonda-

72 Ivi, Préliminaires, $\mathbb{} 1,72$, nota 1 .

73 Ibidem.

74 Ibidem. 
mentali, costitutive e organiche. Secondo Pradier-Fodéré, Pinheiro Ferreira aveva confuso la legge fondamentale, che era la costituzione di uno Stato, con le dichiarazioni dei diritti dell'uomo, dove venivano riassunti i principi del diritto naturale, e che sono state inaugurate dalle colonie americane e poi portate nel contesto francese. ${ }^{75}$ Per chiarire l'utilità di tali dichiarazioni citava autori come Jeremy Bentham e Pierre Lanfrey:

«Elles ont, dit-on, l'avantage d'indiquer aux législateurs l'esprit dans lequel ils doivent composer leur lois; mais on objecte que, réduites à quelques maximes dénuées de developpements, leur brièveté engendre de graves inconvénientes. Si on laisse, en effet, au pouvoir législatif, la faculté de décréter lex exceptions jugées nécessaires, la déclaration deviant illusoire; si elle énonce les droits d'une manière absolue, elle deviant pour les législateurs une entrave perpétuelle ${ }^{76}$

Si soffermava sulla differenza ontologica tra la dichiarazione americana da quella francese, ribadendo che la prima ineriva all'indipendenza dalla madrepatria e la contestuale nascita di una nuova nazione, mentre quella francese era caratterizzata dalla condanna e rottura con l'Ancien régime, per tali argomentazioni Pradier-Fodéré attingeva dal pensiero del giurista e politico francese Anselme Batbie e dalla sua imponente opera in sette volumi (edita tra il 1861 e il 1868), intitolata Traité théorique et pratique de Droit public et administratif. ${ }^{77}$

Il lavoro di Pradier-Fodéré è minuzioso ed è testimoniato dalla vastità delle fonti consultate e dalla varietà delle note che si alternano tra quelle più squisitamente dottrinali a quelle storiche: è sufficiente solo citare l'approfondita nota al libro I, capitolo XVIII, $\mathbb{2} 210$, relativo allo stanziamento delle colonie e della loro naturale appartenenza alla madrepatria. Vattel, come aveva notato Pinheiro, sul delicato tema delle colonie si era limitato a poche righe, mentre Pradier-Fodéré descriveva l'impero coloniale francese degli inizi dell'Ottocento, illustrando come si fosse "ridotto » alle isole di Martinica e Guadalupe e all'arcipelago di Saint-Pierre e Miquelon (a sud di Terranova), a pochi scali in Senegal e a cinque porti commerciali in India e alla Réunion; nel 1817 si aggiunse il territorio della Guyana francese; nel 1830 la Francia ottenne l'Algeria e sotto Napoleone III promosse i propri interessi economici in Senegal, in Cina, in Indocina e in Nuova Caledonia, e,

75 Ivi, L. I, chap. III, 154, nota 1.

76 Ivi, 155.

77 Ivi, 156. 
senza successo, in Siria e in Messico. ${ }^{78}$ Invece per quanto concerne la stessa legittimità della colonizzazione argomentata nel paragrafo precedente da Vattel $(\$ 209)$, nella quale come si è visto vengono elogiate le modalità di conquista dei Quaccheri, Pradier-Fodéré, citando Raynal e la sua Histoire, riscontrava come in nome della civilizzazione, la gran parte delle nazioni europee si erano impadronite di territori non di loro appartenenza. ${ }^{79}$

Osservando nel complesso e dal punto di vista metodologico l'opera di Pradiér-Fodéré, si constata che vengono apposte note ad ogni sezione di cui sono composti i quattro libri di Vattel. Egli espone la dottrina di Vattel, elabora i concetti, li fa propri e li pone a confronto con le teorie dei più autorevoli giuristi dell'epoca. Questa sua « nuova » edizione pare destinata ad un pubblico di studiosi della disciplina, i quali, senza cercare in altre fonti, potevano avere, attraverso le note e il "testo base » di Vattel, una visione completa dal punto di vista dottrinale e dal punto di vista storico dell'evoluzione del diritto delle genti, del diritto pubblico e del diritto internazionale.

Come spiegare nell'Ottocento le due edizioni di Vattel contenenti le note di Pinheiro e di Pradier-Fodéré? Una risposta si potrebbe ricavare dalle parole di Lapradelle, il quale sottolinea come il testo di Vattel fosse l'unico a tenere insieme la scienza francese del diritto internazionale che ancora non aveva le caratteristiche dell'autonomia e della specificità, ma guardava ai modelli passati per costruire il suo presente. ${ }^{\mathbf{8 0}}$

Un'altra risposta potrebbe ricavarsi dalle opere stesse. Pradier-Fodéré, nel 1863, riprese in toto le note di Pinheiro Ferreira aggiungendo ad esse un profilo storico e dottrinale alla luce delle vicende internazionali succedutesi dal 1758, della trasformazione della comunità internazionale e dell'allora più recenti teorie sul diritto delle genti o diritto internazionale, contenute nei lavori di internazionalisti quali Wheaton, Heffter e Martens.

Dal lavoro di Pradier-Fodéré risaltano sia l'intento didascalico-correttivo sia la necessità di non mettere da parte il Droit des gens anzi, di aggiornarlo e di illustrarne gli sviluppi, senza quindi apparentemente porre in discussione la portata delle sue teorie. Ma occorre segnalare che la metodologia adottata dal portoghese Pinheiro Ferreira e da Pradier-Fodéré, non riguardava esclu-

78 Ivi, L. I, chap. XVIII, 496-498, nota 1.

79 Ivi, 496, nota 1.

80 LApradelle, Introduction, in: VATtel (1916) XLI. 
sivamente il trattato di Vattel: entrambi i giuristi hanno commentato anche altri trattati di diritto internazionale. Pinheiro Ferreira nel 1831 pubblicava una nuova edizione del Précis du Droit des gens di Martens; Pradiér-Fodéré, nel 1867, tradusse il De iure belli ac pacis di Grozio: esse furono sempre annotate e in particolare nella nuova versione di Grozio, il professore di diritto internazionale, mise ancora più in evidenza le osservazioni che Jean Barbeyrac aveva posto al testo del giurista olandese, auspicando ad un ritorno al testo originale con la traduzione letterale. ${ }^{\mathbf{8 1}}$

L'intento quindi di ripubblicare il Droit des gens di Vattel, con il complesso groviglio di varianti proposte da Pinheiro Ferreira e da Pradier-Fodéré, rientra e trova la sua giustificazione in una particolare tendenza dell'epoca, volta a costruire la dottrina del diritto internazionale basandosi sull'autorevolezza del pensiero dei grandi nomi del passato.

\section{Diritto costituzionale nelle note di Manuel Pascual Hernández} al Derecho de Gentes (1820 e 1834)

In Spagna il contesto storico è chiaramente differente da quello francese, ma il Droit des gens è ugualmente considerato come uno dei testi più importanti per il diritto internazionale.

Luis Arias, nelle note che appose al trattato sulla storia del diritto internazionale di Nussbaum, precisò che nel corso dell'Ottocento « las traducciones castellanas del Droit de gens de E. de Vattel servirán acaso de única guía a los estudiosos españoles del Derecho de Gentes $\gg .^{\mathbf{8 2}}$

Nonostante nella seconda metà del XVIII secolo il Droit des gens fosse stato messo all'indice, circolava lo stesso sia nelle biblioteche private sia indirettamente, con le opportune correzioni o censure e attraverso le opere dei docenti, nelle università, prima fra tutte quella di Salamanca, vera e propria fucina di idee liberali. ${ }^{\mathbf{8 3}}$

Nel 1798 il trattato vatteliano veniva già segnalato come manuale per tutti gli studenti e non solo per coloro che intraprendevano gli studi giuridici; addirittura nel Plan de Educacion de la nobleza. Trabajado de Orden del Rey si trova riportato che:

81 Grotius (1867).

82 Arias/Nussbaum (1949) 495.

83 Addy (1966); Suárez (2002) 121 ss.; anche Robledo (2003) 49-80; Fernández Rozas / André Sáenz de Sante María (2010) 495-570. 
El derecho de gentes [...] se dará por Wattel, corrigiendo los capítulos que justamente han movido su prohibición: pues una vez purgado de ellos, aunque sin algunos tratados importantes, como éstos, se han proporcionado a los seminaristas en fuentes más puras, quedará por su concisión, claridad y especialísimo método geométrico,

ma soprattutto il Droit des gens era ritenuto il testo più opportuno per fornire ai giovani idee precise e universali sul diritto delle genti e adatto alla formazione di qualsiasi tipo di carriera. ${ }^{\mathbf{8 4}}$

Vattel definito «larva retracta» nel XVIII secolo, ${ }^{85}$ raggiunse nel XIX secolo il massimo successo, soprattutto nella fase precedente e contemporanea all'emanazione della costituzione spagnola. Recenti saggi, pubblicati in occasione del bicentenario della costituzione di Cadice, offrono molteplici riflessioni sul ruolo del giusnaturalismo e in particolare di Vattel per la sua redazione. ${ }^{86}$ Essa, come è noto, restò in vigore per brevi e non continuativi periodi, dal 1812 al 1814, poi dal 1820 al 1823 durante il cosiddetto Triennio Liberale, fu successivamente abrogata e restaurata e di nuovo tra il 1836 e il 1837, dopo lo Statuto Reale della sovrana Maria Cristina di Borbone. Quando il 19 marzo 1812 si dette così vita alla creazione della costituzione spagnola fondata sui valori liberali il Droit de gens era citato come « el manual de referencia ${ }^{8}{ }^{87}$

In effetti il pensiero di Vattel in merito alla costituzione aveva particolarmente affascinato anche gli spagnoli. Per usare le parole di Tomas y Valiente, con Vattel si è passati dalla legge del patto come legge fondamentale astratta alla costituzione dello Stato come legge positiva, come norma fondamentale: "alcuni articoli della Costituzione di Cadice, per esempio il terzo, sembrano essere scritti con il libro di Vattel davanti ${ }^{\mathbf{8 8}}$

84 «Y aunque los destinados a la toga tendrán que ampliarle mucho, los que abracen la de la Espada, la del Comercio u otras, cuyo fin primario no sea la Jurisprudencia, tendrán este preliminar preciso en toda educación completa, si ha de ser con adecuada extensión »: Plan de Educacion de la nobleza. Trabajado de Orden del Rey en 1798. Precedido de un estudio preliminar por Miguel Adellac Gonzáles de Agüero catedrático y director del Instituto de Jovellanos de Gijón (1915) 227.

85 Gutierrez Vega (2010) 119-125; Gutierrez Vega (2004) 537-568.

86 Tra le molte pubblicazioni si segnalano: Martínez Martínez (2013) 39 ss.; González Diez (2013) 139. Anche per le fonti dottrinali della costituzione: Fernández García (2010) 46 ss.

87 Portillo Valdés (2007) 19-35, in particolare 20-21.

88 Tomás y Valiente (2003) 34-35. 
Il Droit des gens tuttavia non venne subito tradotto nella lingua nazionale e si dovettero aspettare altri otto anni per la pubblicazione della prima versione spagnola, nonostante già con Cadice fosse stata ufficialmente garantita la libertà di stampa. ${ }^{89} \mathrm{Nel} 1820$, durante il Trienio Liberal, la Spagna visse in un clima di profondo cambiamento socio-politico e si assistette progressivamente ad un altissimo numero di pubblicazioni di opere, anche di contenuto giuspubblicistico, che prima erano state messe all'indice. ${ }^{\mathbf{9 0}}$

Tra il 1820 e il 1846 ci furono otto edizioni del Derecho de Gentes e il giurista di Neuchâtel fu considerato il più importante e autorevole autore del diritto delle genti. ${ }^{91}$ A Madrid nel 1820, fu pubblicato per la prima volta in lingua spagnola El derecho de gentes, ó Principios de la ley natural, aplicados á la conducta, y á los negocios de las naciones y de los soberanos tradotto dall'avvocato Manuel Maria Pascual Hernández ed edito successivamente nel 1834 con diverse modifiche. ${ }^{92}$

Due anni dopo sempre nella capitale fu data alle stampe un'altra versione « en Castellano por D. Lucas Miguel Otarena, de la última edición francesa

89 Gutierrez Vega (2010) 122.

90 In realtà la pubblicazione delle opere su temi politico-costituzionali ebbe inizio già precedentemente quando, ad esempio, un anonimo diede alle stampe nel 1812 il testo di Gabriel Bonnot de Mably intitolato: Derechos y Deberes del Ciudadano che venne di nuovo ritradotto nel 1820 da Hernández, il quale sempre in quell'anno curò e pubblicò, come si vedrà, la prima edizione spagnola di Vattel; cfr. Astigarraga (2005) 238-264.

91 Herrero Rubio (1954) 132-133; Ruddy (1972) 178. La traduzione in lingua spagnola di diverse opere giuridiche, tra cui anche Vattel, si riassume in questa riflessione: "Diríamos así que en el siglo XIX las gentes de habla castellana se han quedado sin tradición jusinternacional y que buscan en los autores extranjeros los pivotes para construir una ciencia jurídica internacional hispánica. Ello explica, [...], el contenido positivista que en los primeros tiempos influye fuertemente en la doctrina hispánica, reflejo de la ciencia continental europea y anglosajona, si bien hay que señalar desde un comienzo el carácter jusnaturalista, propio de la doctrina española »: Arias/Nussbaum (1949) 495. Anche: PANTín Fernández (2001) 18-26, in particolare 20.

92 Compare come Hernández e come Fernandez, fu avvocato presso la corte di Madrid e membro dell'Accademia Greco-Latina: Novales (dir.) (1991) 509; Martínez (1992) 103; Álvarez LondoÑo (2000) 91-93. Così veniva introdotta la prima traduzione spagnola: «El deseo de propagar en España el importante estudio del derecho natural y de gentes, limitado hasta ahora á un corto número de literatos que se dedicaban á cultivarle privadamente, nos hizo concebir la idea de publicar traducida en castellano la obra de Mr. Vattel, que goza de una reputacion general, y es acaso la mas completa y apreciable en su género »: Advertencia de los editores in: VaTtel (1820b) XXXVIII. 
publicada en Paris, corregida y aumentata con notas del autor y de los editores ", ${ }^{93}$ che vide poi una ulteriore ripubblicazione a Burdeos, sempre nel 1822, e a un'altra a Parigi nel 1824.

L'edizione francese del Droit des gens utilizzata come originale per le traduzioni in spagnolo è quella francese pubblicata a Parigi nel 1820, la quale a sua volta si riferiva alla precedente del 1775 , curata da Dumas. Nel 1836 , invece, fu tradotta in spagnolo quella del 1835, con al suo interno il discorso di Mackintosh e l'inserzione di una biblioteca selecta de la mejores obras sobre la materia; infine nel 1840 e nel 1846 a Madrid vennero pubblicate due ulteriori versioni del Derecho de Gentes, senza però che vi fossero apportati commenti o saggi introduttivi.

La versione del 1834 è senza dubbio la più interessante: Hernández, come curatore e traduttore, aggiunse alcune note che rispecchiano l'ultimo trentennio costituzionale della Spagna dell'Ottocento.

Sin nella Advertencia affermava: "Sale de nuevo á luz el Derecho de Gentes de Vattel, impreso todo por una mano diestra é inteligente », chiarendo però che «El testo está corregido en muchos lugares, como lo convencerá el simple contejo de una impresion con otra; y á las antiguas notas se han aumentado otras, previa censura ${ }^{94}$

Soprattutto Hernández sottolineva che le sue note avrebbero contenuto « unas sacadas de l'Historia de España y de nuestra legislacion, con aplicacion de ellas á la doctrina del autor $» .{ }^{95}$ È molto importante la precisazione che effettua, perchè consultando questa edizione si resta sorpresi dalla quantità di dettagli storici e legislativi contenuti. Essi si riferiscono a precise situazioni della Spagna sia dell'epoca che precedenti, anche di carattere legislativo, in cui emerge l'applicazione pratica dei diversi principi vatteliani al caso concreto spagnolo.

L'attualità dell'opera di Vattel nel 1834 era tale da essere considerata non solo un testo di studio ma, secondo Hernández, un testo da cui la legislazione spagnola doveva trarne insegnamento e quasi si può dire fondamento. Una delle parti più commentate dal traduttore riguarda proprio l'assetto interno di uno Stato e in particolare la redazione di una carta

93 Bibliographie de la France, ou journal genéral de l'imprimerie et de la librairie (1824) 673.

94 Hernandez, Advertencia, in: Vattel (1834a) 7.

95 Ibidem. 
costituzionale. Hernández scriveva in un momento decisivo per la storia costituzionale spagnola. Nello stesso anno in cui ripubblicava il suo $D e-$ recho de Gentes, la sovrana Maria Cristina, vedova di Ferdinando VII, promulgò l'Estatuto Real, che rimase in vigore per pochissimi anni. Il commento di Hernández, alle sezioni che Vattel dedica alla costituzione, ha proprio ad oggetto l'Estatuto Real, promulgato nell'aprile 1834 ed è di questo tenore:

El Estatuto Real, si en el dia no puede rivalizar con una de las constituciones mas liberales de Europa; es sin duda la ley fundamental que por ahora conviene en général á la nacion española y al espíritu del siglo: está sostenida, en derecho, por dos cosas las mas esenciales á todo gobierno representativo: la libertad moderada de la imprenta, y la independecia del poder judicial, al paso que no excluye la administracion en comun de los intereses de los pueblos por medios que se han elegido. ${ }^{96}$

Le note si concentrano soprattutto nel primo libro del giurista di Neuchâtel, pochissimi sono i commenti agli altri libri di ambito più internazionalistico ed è interessante osservare che l'autorità di Vattel in principio nacque soprattutto per le sue teorie sullo Stato e solo successivamente si diffuse anche al il diritto internazionale. Il trattato di Vattel «tuvo una gran difusión en Europa, así concebida como el "reglamento fundamental" de la "autoridad pública" que eran tres: la abundancia (comercio, adunadas, moneda y cambio); la felicitad (instrucción, educación, ciencias, artes, premios públicos), y la protección o la defensa (poder nacional, valor, virtudes militares, riquezas e impuestos y rentas del Estado) . $^{\text {97 }}$

Per quanto concerne le pubblicazioni di testi di diritto internazionale spagnoli, possono essere divise in due grandi filoni: nella prima metà dell'Ottocento si riscontrano per lo più testi che raccolgono trattati internazionali e negoziati spagnoli come il Codigo de derecho internacional, o sea colección metódica de los tratados de paz, amistad y comercio entre España y las demas naciones di Ferrater, ${ }^{\mathbf{9}}$ oppure gli Elementos de derecho público internacional:

96 Vattel (1834a) L. I, cap. III, $\mathbb{S} 31,35$, nota 1 . Precisava inoltre che «Esta ley no es una coleccion de principios sujetos ya á consulta no un hacinamiento de máximas sin aplicacion necesaria bien al contrario, define nuestros derechos; declara y limita yes secundarias que la contradicen es superior á las demas ». Ivi, 35-36, nota 1.

97 Astigarraga (2008) 150, nota 64.

98 Ferrater (1846-1847). 
con explicación de todas las reglas que, según los tratados, estipulaciones, leyes vigentes y costumbres, costituyen el derecho internacional español di Riquelme. ${ }^{99}$

Nella seconda metà dell'Ottocento accanto ai trattati simili al genere sopraesposto, ${ }^{\mathbf{1 0 0}}$ vi sono anche opere di carattere più sistematico, come quella del marchese de Olivart che nel 1887 diede alle stampe il suo Tratado y notas de derecho internacional público ed annovera tra le fonti principali anche il Droit des gens. ${ }^{101}$

\section{Il Droit des gens manuale didattico-politico in America Latina}

La fama del Droit des gens oltrepassò l'oceano fino a giungere in Sud America, al punto tale che venne considerato una vera e propria guida ideologica, con precise finalità didattiche e politiche; ${ }^{\mathbf{1 0 2}}$ Vattel è stato un giurista particolarmente citato non solo in Messico ma in tutta l'America Latina nei diversi ferventi rivoluzionari della prima metà dell'Ottocento, quando si realizzò progressivamente il processo di decolonizzazione, la nascita di nuovi Stati all'interno del panorama internazionale e la conseguente promulgazione delle rispettive carte costituzionali.

Come è avvenuto per i paesi anglo-americani, anche nei territori dell'America meridionale l'autorità vatteliana compariva sui giornali dell'epoca e si riferiva alle particolari situazioni statali tanto interne quanto internazionali: così il richiamo a Vattel è molto ampio quando si parla di trattati stipulati con Stati limitrofi e in moltissime altre questioni internazionali, quali ad esempio il problema della naturalizzazione degli stranieri, come attestato nel

99 Riquelme (1849).

100 Mozo (1898).

101 Olivart (1887) 25. Sul diritto internazionale nella Spagna dell'Ottocento: De La Rasilla Del Moral (2013) 48-65.

102 Più in generale si rimanda a: MücKe (2008) 134 ss.; VAN Young (2004) 127-172. LyNCH (1994); RodRíGuEz (1998). Vattel era inoltre utilizzato come testo di diritto naturale presso il collegio universitario di San Ildefonso a Città del Messico: "los autores usados en derechos canonico, natural y publico fueron Cavallario y Vattel »: Mayagoitia STone (2005) 322; cfr. anche Clavero (2002). Anche in altri collegi era usato come testo di studio nell'edizione spagnola del 1820: Collegio di Purísima, Conception, Guanajuato, Collegio di San Juan de Letrán, México, Collegio San Gregorio, México: Mijangos y Gonzalez (2015) 59. Per la formazione universitaria in Colombia e Vattel: BoHorquez (2002). 
Peruano $^{103}$ e nei dibattiti costituzionali messicani degli stessi anni editi nel $E l$ Siglo Diez y Nueve. ${ }^{104}$

Un esempio dell'influenza vatteliana e del suo utilizzo pratico si verifica nel territorio venezuelano dove a Caracas vennero pubblicati alcuni importanti saggi nei primi trenta anni dell'Ottocento ad opera di giuristi-politici con il preciso scopo di introdurre le teorie del diritto delle genti, attraverso traduzioni (in senso lato) e compendi del Droit des gens di Vattel. Tre in particolare sono le figure che si inseriscono in questa dinamica: Francisco Javier Yanes, Tomás Lander e Andrés Bello.

Francisco Javier Yanes è stato un illustre avvocato, giornalista e politico, partecipando attivamente al processo di emancipazione del Venezuela: fu tra i firmatari dell'Atto d'Indipendenza e della carta costituzionale insieme a personaggi del calibro di Cristóbal de Mendoza, Miguel Peñalver, Miguel José Sanz e Francisco Javier Ustáriz. Si trasferì a Caracas nel 1802, dopo la laurea in diritto civile, iniziò a lavorare come avvocato e nel 1810 partecipò attivamente agli eventi del 19 aprile, con cui iniziarono le fasi dell'indipendenza venezuelana. Venne nominato dalla Giunta di Caracas commissiario per la città di Araure, entrò nella Società Patriottica e nel Congresso Costituente, firmando rispettivamente il 5 luglio e il 21 dicembre 1811, la Dichiarazione d'Indipendenza (con la proclamazione della Repubblica del Venezuela) e la prima costituzione. Nel mese di agosto dello stesso anno divenne presidente del Congresso e della commissione della legislazione di Caracas. ${ }^{105}$

Come parlamentare e giurista, Yanes apportò contributi di grande rilevanza, seguendo sempre lo spirito liberale del tempo, al primo codice civile e

103 Sui giornali peruviani: Peruano (28 Octubre 1840) 3: in un articolo in cui si tratta della naturalizzazione degli stranieri sul territorio peruviano, viene citato in toto il passaggio del Droit des gens, contenuto al Libro II, Capitolo VIII, $\mathbb{S} 114$; in tema di poteri dei consoli, ad esempio si fa riferimento a Vattel nel: Peruano (6 Noviembre 1844) 3; sul prestito tra governi, Peruano (11 Noviembre 1845) 2; in tema di commercio e di trattati: Peruano (22 Abril 1846) 2.

104 El Siglo Diez y Nueve (18 Mayo 1842) 2; El Siglo Diez y Nueve (6 Octubre 1842) 2; El Siglo Diez y Nueve (30 Octubre 1842) 3; El Siglo Diez y Nueve (31 Octubre 1842) 3; El Siglo Diez y Nueve (6 Noviembre 1842) 3; El Siglo Diez y Nueve (13 Noviembre 1842) 3; El Siglo Diez y Nueve (31 Deciembre 1842) 5; El Siglo Diez y Nueve (10 Abril 1843) 2.

105 Rodríguez (2010) 32-59. Per riferimenti biografici su Yanes: CañIzales Verde (1997); Pino Iturrieta (1971); Pino Iturrieta (1987). Sulla storia della Prima Repubblica venezuelana si rimanda sinteticamente a: LóPEz BoHórQuez (2008) 553-566; ThIBAUd (2007) $185-222$. 
criminale per il Venezuela, alla legge sul matrimonio, all'abolizione della tortura e dei tribunali inquisitoriali; mentre in ambito più squisitamente costituzionale prese parte alla redazione della Declaración de los Derechos y Deberes del Pueblo e alla prima legge sulla libertà di stampa. ${ }^{106}$

Tuttavia nel 1812, soltanto dopo un anno dalla proclamazione della Repubblica, la fazione realista ebbe la meglio; furono necessari quasi dieci anni di lotta senza quartiere perché i creoli venezuelani guidati da Simon Bolívar, magistralmente immortalato nel celebre romanzo storico di Gabriel García Márquez, riuscissero infine ad affrancarsi dal dominio spagnolo. ${ }^{107}$ Il 12 dicembre 1816 Yanes era nell'esercito capeggiato dal generale Páez per la batalla de El Yagual. ${ }^{108}$ Nel 1819 il congresso di Angostura (ora Ciudad Bolívar) lo nominò membro della Corte Suprema di Giustizia del Venezuela e nel 1820 presidente della Corte del Almirantazgo. ${ }^{109}$ Morto nel 1842, la salma venne trasferita nel 1876 presso il Panteón Nacional di Caracas.

Yanes si prodigò moltissimo anche come pubblicista per diffondere il pensiero rivoluzionario e lo spirito moderno e liberale: tra il 1824 e il 1825 fece circolare a Caracas, con l'aiuto di Cristóbal de Mendoza, il periodico intitolato El Observador Caraqueño. ${ }^{\mathbf{1 1 0}} \mathrm{Fu}$ inoltre autore di numerose opere di taglio storico-politico. ${ }^{\mathbf{1 1 1}}$

Il periodo legato all'indipendenza nazionale venezuelana fu un processo molto complesso e gravoso, ma permise una riforma significativa per l'istruzione universitaria: si assistette, infatti, ad una drastica riduzione nell'insegnamento del diritto romano e di quello canonico per lasciare spazio al diritto delle genti, al diritto politico, al diritto patrio, ai principi di legislazione universale e l'economia politica. ${ }^{112}$

106 Rodríguez (2010) 34. Nel momento in cui veniva approvata la Declaración de los Derechos $y$ Deberes del Pueblo, Yanes celebrava l'evento in questi termini: "Caracas ha comenzado a dar los pardos lo que les corresponde de justicia y sus resoluciones liberales le harán siempre un alto honor entre las naciones que conocen los derechos de la humanidad »: così citato in: Guerrero/Leal/ Plaza (2012) 469-470.

107 Si rimanda a: SAMudio (2009) 195-216.

108 Ibidem.

109 Ibidem.

110 Rodríguez (2010) 35.

111 Nel 1821 una Relación documentada de los principales sucesos ocurridos en Venezuela: desde que se declaró estade independiente hasta el año de 1821; nel 1840 un Compendio de la historia de Venezuela desde su descubrimiento hasta que se declaró Estado independiente: RAYNERo (2001) 18-19.

112 Pérez Perdomo (1987) 450. Anche Falcón (2009) 543. 
Gli effetti di questo cambiamento influirono anche sulla produzione di opere legate alla massima divulgazione delle teorie del diritto delle genti ed in questo contesto che si inserisce nel 1824 la pubblicazione da parte di Yanes dell'Idea general o principios elementales de derecho de gentes, extractos de Vattel y otros autores. ${ }^{113}$ Il testo è introdotto da un ringraziamento, datato 8 dicembre 1824 da parte di Felipe Fermín de Paul, uno dei firmatari dell'atto di indipendenza, all'epoca rettore dell'Universidad Central del Venezuela, fondata nel 1721. ${ }^{114}$ Questa opera di raccolta in forma semplificata delle dottrine del diritto delle genti era pertanto indirizzata alle giovani generazioni che si affacciavano allo studio del diritto pubblico e necessitavano di una solida base di conoscenza del diritto naturale nei suoi rapporti con il diritto pubblico. Proprio nel 1824 si decise l'introduzione dello studio del diritto pubblico, poi stabilito nel 1827 nei programmi di studio. ${ }^{\mathbf{1 1 5}}$

Cinque sono i capitoli del saggio di Yanes, in cui si snodano i capisaldi della dottrina vatteliana. Il primo è dedicato alle nociones generales acerca de la ley de las naciones, y necesidad de su estudio, in cui riprende in toto la Préface di Vattel, ripercorrendo il pensiero di Grozio, Pufendorf, Barbeyrac, Wolff, sino ad arrivare al "sabio " Vattel; ${ }^{\mathbf{1 1 6}}$ nel secondo è spiegato che cosa sia una nazione e a quale norma sia soggetta, richiamando le idee vatteliane per cui « las naciones o estado son unos cuerpos político o sociedades de hombres, que reuniendo sus fuerzas físicas y morales procuran su conservación y utilidad» e ancora "el derecho de gentes es la ciencia de las leyes que se observan entre las naciones o estados, y de las obligaciones que les corresponden ». ${ }^{\mathbf{1 1 7}}$ La differenza tra diritto naturale e diritto delle genti è l'oggetto del terzo capitolo: Yanes presentava un excursus molto succinto del tema, illustrando in pochissime righe le posizioni di Cicerone, Seneca, Grozio, Hobbes, Pufendorf, Burlamaqui e Vattel. Il quarto capitolo vede al centro i diritti e i doveri delle nazioni, sulla base del primo e del secondo libro del Droit des gens, differenziando tra quelli interni, nei confronti dei cittadini e quelli esterni verso le altre nazioni: la pace, per Yanes, è il maggior bene; la

113 Il testo si trova interamente riprodotto in YANES (1959).

114 Per approfondimenti sul testo: Pérez Perdomo (2011) 35-69; Pérez Perdomo (2013) 51 ss.

115 Ivi, 43.

116 YANes (1959) 199.

117 YANES (1959) 203.

196 Capitolo IV 
prima obbligazione generale di una nazione è la contribuzione alla felicità e alla perfezione delle altre nel rispetto dell'indipendenza e della libertà di ciascuna di esse, da cui si ricava la seconda seconda obbligazione generale: «a cada nación debe dejarse la posesión pacífica de aquella libertad que le concedió la naturaleza ». I successivi principi concernono la definizione di diritto interno ed esterno, perfetto e imperfetto, il fondamento del diritto delle genti volontario e il rispetto del diritto delle genti da parte di tutte le nazioni. ${ }^{118}$ L'ultimo capitolo è dedicato ai trattati ed è impostato come il Droit des gens: dopo una analisi delle diverse forme di diritto delle genti vengono così illustrate i tipi di trattati uguali, inuguali, reali, personali, e le modalità con cui un trattato possa cessare di esistere tra le nazioni che lo avevano stipulato. ${ }^{119}$

Tuttavia la produzione di Yanes oltre ai soli fini didattici comprende anche aspetti più politici, che ben si trovano palesati nel Manual Político del Venezolano, pubblicato nel 1839 con lo pseudonimo " un Venezolano ». In esso, avvalendosi di illustri illuministi quali Montesquieu, Rousseau, Beccaria, Bentham e Constant, spiegava i principi costitutivi di uno Stato, dividendo la trattazione in sei capitoli. I primi due sono rispettivamente dedicati al governo rappresentativo e al governo rappresentativo federale, sono densi di citazioni a Montesquieu e riferimenti storici alla rivoluzione nordamericana e alla costituzione del 1787. Yanes argomentava la diversità di origine delle colonie nordamericane, " por las transacciones del pacífico Penn », l'eco che ebbe l'illuminismo sulle teorie legislative, « en un país donde no tenían que luchar y vencer un poder militar, ni limitar una autoritad absoluta, ni tampoco despojar á un clero dominante de su poder á una nobleza de sus derechos privilegiados, á una multidud de familias de sus fortunas, ni de construir su nuevo edificio sobre despojos cimentados en la sangre $[\ldots] \gg .{ }^{\mathbf{1 2 0}}$ Per questi motivi il popolo nordamericano riuscì a fondare le sue istituzioni sui principi della ragione, della libertà, dell'uguaglianza politica e civile e della giustizia universale. "Un solo esfuerzo, una sola guerra para sacudir el yugo de la madre patria fué bastante para libetarse de toda carga, y sua leyes hechas únicamente con el objeto del interés general ». ${ }^{\mathbf{1 2 1}}$

118 Ivi, 221-229.

119 Ivi, 231-246.

120 YANES (1839) 102-103.

121 Ivi, 103. 
Partendo proprio dai principi costitutivi nordamericani e facendo riferimento anche ad altri eventi storici significativi, i successivi capitoli hanno ad oggetto la libertà e le distinzioni tra la libertà civile, individuale e politica, che devono « concederse ó dispensare á los ciutadadanos en la mayor porción possible ${ }^{\mathbf{1 2 2}}$ e ricordava: la storia inglese e i rappresentanti di Cromwell; l'uguaglianza sull'idea sancita durante la rivoluzione francese; la proprietà con riferimenti a Raynal e alla sua Histoire, soprattutto in merito all'importanza della libertà intellettuale; ${ }^{123}$ infine la certezza della legge, «la seguriad », cuore di tutta la società. Yanes prendeva come esempio alcuni articoli contenuti nella neo carta costituzionale venezuelana promulgata nel 1830, che garantivano fondamentali diritti in materia penale, così all'articolo 199 veniva stabilito che nessuno potesse essere arrestato senza che ci fossero fondati indizi e che si fosse provveduto ad informare la persona che la condotta sarebbe stata punibile con la restrizione della libertà personale; all'articolo 200 secondo cui l'arresto e la detenzione dovevano essere autorizzate esclusivamente dall'organo competente; all'articolo 195 nel quale si statuiva il principio fondamentale del giudice naturale stabilito dalla legge, proibendo l'istaurazione di commissioni speciali e tribunali straordinari. ${ }^{\mathbf{1 2 4}}$

Sostiene Cañizales Verde che: «El conceptuoso libro del prócer Francisco Javier Yanes [...] son elocuente testimonio del pensamiento y el ideal de una generación de pensadores, ubicados en la misma trinchera ideológica de la soberanía popular, en irrenunciable defensa de los fueros de la emancipación nacional », ${ }^{\mathbf{1 2 5}}$ ed è soprattutto una valida testimonianza dell'utilizzo di Vattel con uno specifico fine didattico-giuridico e politico.

122 Ivi, 117.

123 «El pensamiento del hombre, verdadero ó erróneo, es su propriedad la más sagrada, y bien sea un error, ó bien una verdad, los tiranos son igualmente culpables cuando intentan atacarlo. La propriedad de nuestros pensamientos es una importantísima parte de la propriedad personal [...] Así que ninguna autoridad tiene derecho de turbarnos en el goce y ejercicio de una propriedad tan natural y legítima »: Ivi, 201-202. Il passaggio di Raynal a cui si fa riferimento è il seguente: «En todas partes se ve ahogada esta libertad con supersticiones extravagantes, estilos bárbaros, y leyes anticuadas: ella sin embargo renacerá de sus propias cenizas algún día, y recuperará el hombre sus derechos, a proporción que hagan progresos la morale y la política ». Esso è stato estrapolato da Yanes che sicuramente aveva tra le sue mani uno dei compendi dell'Histoire di Raynal, che contenevano i passaggi più salienti e che molto circolavano all'epoca: Cfr. RaYnAL (1823) 60.

124 Yanes (1839) 250.

125 Cañizales Verde (1997) 37. 
Il Manual e l'Idea di Yanes devono essere letti congiuntamente come prodotti di una specifica inclinazione alla diffusione didattica e politica delle teorie del diritto naturale, delle genti e della maggior parte della cultura giuridica europea, con particolare attenzione all'ambito del diritto costituzione e penale.

Ma non furono casi isolati in Venezuela perché negli stessi anni in cui Yanes pubblicava l'Idea general sempre a Caracas vedeva la luce il Manual del colombiano ó explicación de la ley natural van añadidos los deberes y derechos de la nación y del ciudadano (1825), che è uno scritto parallelo a quello di Yanes, ma ancora pù divulgativo. Esso è stato attribuito al politico liberale Tomás Lander (1792-1845), anche se alcuni storici hanno sollevato dei dubbi. ${ }^{\mathbf{1 2 6}} \mathrm{Il}$ Manual si pone lo scopo di offrire una panoramica sulle leggi naturali e sui diritti e doveri del cittadino che sono alla base di tutta la società, di tutti i governi giusti e liberali e devono essere conosciuti soprattutto dai giovani. ${ }^{\mathbf{1 2 7}}$ Il testo, infatti, è articolato in una serie di domande e risposte che spaziano appunto dal concetto di legge naturale e ai suoi caratteri, all'elencazione e analisi delle cosidette virtù sociali, in cui ci sono diretti ed indiretti rimandi alle teorie del diritto naturale e delle genti settecentesche, tra cui anche quelle vatteliane.

Contemporaneamente si affermava in Venezuela la complessa figura di Andrés Bello. Nato a Caracas nel 1781 e morto a Santiago del Cile nel 1865 è considerato uno dei più importanti giuristi del Sud America, non solo per il suo fondamentale contributo alla scienza del diritto internazionale sudamericano, ma anche per il suo impulso sia alla formazione universitaria, con la creazione dell'Università del Cile nel 1842 sia alla redazione del Codice civile cileno (tra il 1840 e il 1855), considerato una delle opere più originali della legislazione sudamericana. ${ }^{\mathbf{2 8}}$

Il soggiorno di Bello a Londra, avvenuto tra il 1810 e il 1829, per una delicata missione diplomatica, come rappresentante della nascente Repub-

126 Secondo Pérez Perdomo «Lander era un acerrimo avversario dell'Unione colombiana e quindi il titolo non sarebbe giustificato ": Pérez Perdomo (1987) 454.

127 LANDER (1983) 54.

128 Su Bello cfr. Krebs (1969) 152-174; Lynch (ed.) (1987); JaKsić (2001); si segnala anche la tesi di dottorato di Keller-Kemmerer (2016) svolta presso il Max-Planck-Institut für europäische Rechtsgeschichte di Francoforte sul Meno. Sul contributo di Bello alla codificazione civile cilena si rimanda, tra i molti contributi, alla raccolta pubblicata in occasione dell'anniversario della sua promulgazione: Martinic/ TAPIA (dir.) (2004). 
blica del Venezuela, fu determinante per la sua specializzazione nel diritto internazionale e soprattutto per la pubblicazione nel 1832 dei Principios de derecho de gentes, un trattato che ebbe moltissimo successo in sud America e fu destinato soprattutto ai fini didattici.

Sin dal prologo dei Principios si legge che uno dei personaggi cardine del suo pensiero è stato proprio Vattel e questo si riflette nella prima edizione del trattato. ${ }^{129}$ Per Bello il giurista di Neuchâtel è in assoluto lo scrittore più elegante e più conosciuto della scienza del diritto internazionale:

Su obra ha sido citada con respeto en los juzgados de almirantazgo, donde se ventilan de ordinario causas que conciernen á esta clase de jurisprudencia, en los debates de las asambleas legislativas y en las negociaciones diplomáticas. ${ }^{130}$

L'uso delle teorie vatteliane da parte di Bello è un perfetto esempio di come si possano innescare i meccanismi di appropriazione delle teorie del diritto delle genti, con il preciso scopo di ricrearle in forma nuova al fine di rispondere alle esigenze storiche, sociali e politiche dell'America del Sud. ${ }^{\mathbf{1 3 1}}$

L'originalità del lavoro emerge con molta evidenza, nella teoria del nonintervento, di cui Vattel e Bello si fecero portavoci, ma con sviluppi diversi. In molti passaggi del Droit des gens Vattel si esprimeva sul non intervento collegando tale principio alla sua centrale idea di Stato. ${ }^{\mathbf{1 3 2}}$ Egli affermava esplicitamente che la giurisdizione statale fosse inviolabile, pur ammettendo

129 «Incorporando lo que he tomado de estas fuentes con la doctrina de Vattel, ajustada á los límites de unos elementos que pudieran servir á la instruccion de los alumnos de jurisprudencia »: Bello (1832) VI. Il testo fu poi pubblicato a Caracas nel 1837, a Bogotà nel 1839 e a Madrid nel 1843.

130 Ivi, 14-15.

131 Afferma Liliana Obregón Tarazona: «Bello informa a sus lectores que su obra no es original, sino un repertorio bien organizado de las obras de muchos autores. Se presenta como un editor ilustrado y selectivo que, según su propia descripción, adopta, revisa, discute, cita, prueba, presenta, contradice y reescribe las obras de los autores que ha consultado. Podríamos decir que Bello conversa con estos autores a lo largo del libro y mediante la edición cuidadosa, la homogenización del lenguaje y estilo, y la adición de múltiples pies de página, nos proporciona, de hecho, una voz autorizada sobre sus ideas. Incluso, la selección de textos que resume o reescribe (a menudo sin citarlos) hace difícil distinguir si se encuentra editando más de lo que reconoce en su prólogo o si realmente lo que está escribiendo es un texto original »: Obregón Tarazona (2010) 70.

132 Vattel (1758a) L. II, chap. IV, \54, 297. Cfr. Chetail (2014) 291-292; Fiocchi Malaspina/Keller (2014) 214-227. Su Vattel e la disciplina dell'intervento: Zurbuchen (2010) 69-84; Distefano (2011) 226; Beaulac (2004) 150-155; Glanville (2013) 56-59; PITTS (2013) 132-153. 
alcuni casi di intervento: per «buoni uffici, qualora non fosse richiesto di farlo o indotti da particolari motivi ", ${ }^{\mathbf{1 3 3}}$ nel caso di autoconservazione, ${ }^{134}$ per una obbligazione morale, per fermare una condotta non conforme ai principi del diritto delle genti, ${ }^{\mathbf{1 3 5}}$ e per liberare un popolo oppresso dal suo tiranno. ${ }^{136}$

Bello, dal canto suo, sulla scia di Vattel formulava il non intervento sul presupposto della sovranità statale e al tempo stesso, precisando maggiormente le sue teorie: «De la independencia y soberanía de las naciones sigue que a ninguna de ellas es permitido dictar a otra la forma de gobierno, la relijion [sic], o la administracion que esta deba adoptar ».137

Riteneva Bello solo in un caso giustificabile l'intervento per ragioni di autoconservazione: " No hay duda que cada nacion tiene derecho para proveer a su propria conservacion y tomar medidas de seguridad contra cualquier peligro ». ${ }^{138}$ Rispetto a Vattel limitava il più possibile questa eccezione solo quando il pericolo fosse stato "significativo, evidente ed imminente", non condividendo quindi totalmente la condotta degli Stati europei e le loro dinamiche di intervento. ${ }^{139}$ L'appropriazione delle teorie vatteliane da parte di Bello è molto interessante. Come Koskenniemi ha scritto «Latin American international law textbooks have adapted the universal vocabulary of European writings into a 'professional style uniquely Latin American', thus supporting not the passive assimilation of the region to Europe, but its asserted distinctiveness from it ${ }^{\mathbf{1 4 0}}$

Il trattato di Bello, nonostante abbia avuto tra le sue fonti il testo di Vattel, è stato redatto in un particolare momento storico e usato non solo per spiegare i principi che regolavano le relazioni in Europa, ma anche per essere applicato nelle dinamiche latino-americane. Bello, soprattutto nelle successive edizioni del suo trattato, rimodella le teorie del diritto internazionale per instaurare un dialogo con l'Europa servendosi dell'autorità di Vattel e di

133 Vattel (1758a) L. I, chap. III, $\mathbb{S} 37,38$.

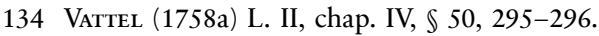

135 Vattel (1758a) L. I, Preliminaries, $\mathbb{\$} 22,12-13$.

136 Cfr. ReCH (2013) 218-224.

137 BeLlo (1832) 15.

138 BeLlo (1832) 16.

139 Ibidem. Cfr. Fiocchi Malaspina/Keller (2014) 222-223.

140 Koskenniemi (2011b) 173. Cfr. anche: Obregón Tarazona (2009a) 189-218; Obregón Tarazona (2009b) 154-164; Obregón Tarazona (2008) 111-128. 
un discorso giuridico conosciuto come era il Droit des gens ${ }^{\mathbf{1 4 1}}$ In questo caso, in modo evidente, si è in presenza di un uso più ampio del termine traduzione intesa come metafora al trasferimento di idee e concetti in contesti geo-temporali diversi. Come è stato argomentato: « The concept and practice of appropriation may thus reconfigure the status of translation as the production of texts that are not simply consumed by the target language and culture but which, in turn, become creative and productive, stimulating reflections, theorizations, and representation within the target of cultural context ${ }^{\mathbf{1 4 2}}$

I Principios di Bello, l'Idea di Yanes e il Manual del Colombiano di Lander furono stampati a Caracas, riscuotendo, soprattutto nel caso di Bello un grande favore del pubblico. Sottolinea Rogelio Pérez-Perdomo, quanto all'epoca e in un simile contesto, la vendita già di mille esemplari sia da ritenersi un successo editoriale. I testi "giuridici avevano un pubblico maggiore che non quello formato da soli studenti e laureati in diritto "; i manuali politici erano essenziali per il buon andamento della società e per la massima divulgazione della cultura, affinchè i paesi sudamericani potessero partecipare alla comunità mondiale delle nazioni. ${ }^{143}$ Essi non pretendono erudizione e rigore poiché i fini sono squisitamente pragmatici, con un impatto politico: ${ }^{\mathbf{1 4 4}}$ "Scopo degli autori è la produzione scientifica rivolta alla divulgazione di una scienza estremamente importante per il destino della patria ». ${ }^{\mathbf{4 5}}$

Yanes sosteneva che si trattava di diffondere « cognizioni, sane ed utili [...] convinti che molti dei mali che ci affliggono sono causati dall'ignoranza o dall'errata comprensione dei principi ». ${ }^{\mathbf{1 4 6}}$

In merito alla originalità dei lavori occorre tuttavia precisare che il primo Bello era fortemente ancorato alle teorie di Vattel e di Burlamaqui, come lui stesso nella prefazione aveva anticipato, ma nelle successive edizioni del trattato tale riferimento alla fonte non c'è più. Questo è un forte e significativo segno di come con il tempo le teorie siano diventate autonome, indipendenti e abbiano preso la loro forma «latina», come si è visto nel

141 Arias Ospina (2013) 189-204.

142 Saglia (2002) 96. Anche Bastin / Echeverri / Campo (2010) 62-63.

143 Pérez Perdomo (1987) 455-456.

144 Pérez Perdomo (2008) 168-183, qui 180.

145 Pérez Perdomo (1987) 454.

146 Yanes (1839) 35. 
caso ad esempio del principio del non intervento. Il "primo» Bello (del 1832), esattamente come Yanes e Lander, erano il frutto della concezione culturale tipica della loro epoca: all'inizio del XIX era loro chiesto di tradurre, riportare in frammenti e rendere accessibile le teorie europee. «In Vattel è espressa la ragione - scrive Rogelio Pérez Perdomo - ovvero la Verità. Questa Verità doveva essere diffusa nell'America Spagnola, sopprimendo qualsiasi deviazione eterodossa (ossia che si scontrasse con altre verità) ». ${ }^{\mathbf{1 4 7}}$

Il Droit des gens ripreso da rivoluzionari nordamericani, da quelli francesi non poteva non essere un punto di riferimento per i fervori liberali dell'America Latina e anche in Argentina, come argomentato da José Carlos Chiaramonte, l'utilizzo del trattato è politico-costituzionale. ${ }^{\mathbf{1 4 8}}$

Nel contesto brasiliano il Droit des gens, durante la prima metà dell'Ottocento, è un testo di riferimento per la guerra dei Farrapos o rivoluzione di Farroupilha, che vide schierati in prima linea Giuseppe Garibaldi e altri italiani legati alla Giovine Italia tra cui l'esule ligure Giuseppe Stefano Grondona. Bento Gonçalvez, uno dei principali leader rivoluzionari, nonchè primo e ultimo presidente della Repubblica Riograndense, in una lettera del 1844 in cui riferiva delle sue trattative di pace con l'impero scriveva che il diritto delle genti «deriva e nasce dal Droit des gens di Vattel ». ${ }^{\mathbf{4 9}}$

147 Pérez Perdomo (1987) 457.

148 «[..] Vattel no era solamente una autoridad para la enseñanza del derecho natural y de gentes. Era también leído por los políticos de la época. [...] Comprobamos que en la sesión segreta de la Junta de Representantes de Buenos Aires, del 24 de enero de 1831, destinada a discutir los artículos del tratado de la Liga del Litoral - tratado que luego se convertiría en el Pacto Federal, al ser suscripto por el resto de las provincias argentinas, e inauguraría la débil confederación vigente hasta caída de Juan Manuel de Rosas en 1852 se destaca explícitamente a Vattel como autoridad en lo relativo a la entrega de delincuentes entre los estados participantes: "algunos tratadistas notables, y entre ellos Vattel, aplaudía este medio de reprimir los delitos, que según se expresaba, hacía que los pueblos tomasen el aspecto de una República”. Y en la siguiente sesión, del 24 de enero, se apela al derecho de gentes para legitimar el artículo en discusión y se vuelve a destacar el nombre de Vattel entre sus exponentes. El articulo «lejos de estar en oposición con los principios generales del derecho público de las naciones, era conforme a éste, y a las doctrinas de los tratadistas más clásicos entre los que se citó a Batel »: Chiaramonte (2002) 116.

149 «Donde veio este Direito das Gentes? Responderei que de Vattel. É ele quem diz que o uso dá o nome de guerra civil a toda a guerra que se faz entre os membros de una mesma Sociedade Política: se estãode um lado os cidadãos e de outros o Soberano com aqueles que Ihe obedecem, basta que os descontentes tenham alguma razão de tornar as armas, 
Sotto l'aspetto del diritto internazionale, oltre a Bello, tra i giuristi latino americani interessante è, senza dubbio, prendere in considerazione Carlos Calvo, il quale insieme agli italiani Mancini e Pierantoni fu tra i fondatori, nel 1873, dell'Institut de Droit International di Gand. ${ }^{\mathbf{1 5 0}}$

Calvo nato a Montevideo nel 1824, dopo gli studi a Buenos Aires, trascorse gran parte della sua vita in Europa, in particolare a Parigi (dove vi morì nel 1906), lavorando come diplomatico per conto dell'Argentina e scrivendo numerosi testi di diritto internazionale scegliendo la lingua diplomatica francese. ${ }^{151}$

Nel 1868, dopo la prima edizione spagnola, pubblicò Le droit international théorique et pratique précédé d'un exposé historique des progrés de la science du Droit des gens; come altri, anche Calvo, sostenne che Vattel fosse il semplice divulgatore delle idee di Wolff, alle quali riuscì a dare più accessibilità alla loro lettura, e per questo il suo trattato era molto più citato di quello di Grozio, anche se «son mérite relatif soit beaucoup moindre ». ${ }^{\mathbf{1 5 2}}$

Benché Calvo sembri non dare molto peso al Droit des gens, non appena procedeva con la trattazione specifica dei concetti di diritto internazionale quali la sovranità e l'indipendenza degli Stati, oppure la materia dei trattati, il punto di riferimento e di partenza dei suoi ragionamenti rimaneva la posizione di Vattel. Così anche nell'illustrare i principi generali e le fonti del diritto internazionale, Calvo si ispirò al sistema vatteliano, riconoscendogli il merito di aver sistematizzato il diritto delle genti: « le grand résultat des travaux de Vattel a été de prouver que la base du droit des gens réside dans le droit naturel, quelles que soient d'ailleurs les modifications que ce dernier ait eu à subir dans son application aux relations internationales ». ${ }^{\mathbf{1 5 3}}$

para que se chame a esta desordem guerra civil e não rebelião ": così riportato in CHIAramonte (2004) 34. Cfr. anche Chiaramonte (2010) 15-31; Parada (1998) 131-136. Per la rivoluzione di Farroupilha: Medianeira Padoin (2000); Medianeira Padoin (2001).

150 Per una ricostruzione sui giuristi esperti di diritto internazionale nel contesto dell'America del Sud: Alvarez (1910); Jacobini (1954) Obregón Tarazona (2009a) 189-218; Becker Lorca (2014).

151 Grafton Wilson (1907) 137-138. Per la dottrina di Calvo si rimanda: Tamburini (2002) 81-104.

152 Calvo (1868) 45.

153 Ivi, 127. 


\section{L'utilizzo casistico del Law of Nations: le note di Joseph Chitty e di Edward Ingraham alle edizioni inglesi e nord americane (1834 e 1852)}

Il successo di Vattel iniziato nel XVIII secolo raggiunse il suo culmine nei paesi di common law anche durante tutto il corso dell'Ottocento, dove si contano numerose edizioni, aggiornate e annotate, al fine di « modernizzare il diritto internazionale con le vicende della prassi contemporanea ». ${ }^{\mathbf{1 5 4}}$

La più famosa edizione, oggetto di numerose ristampe, fu curata da Chitty e pubblicata nel 1834 . Joseph Chitty (1775-1841) era un avvocato inglese e autore di molti testi di diritto, con spiccati risvolti nella pratica giuridica: come il Treatise on Commercial Law del 1818 e il Treatise on the Law of the Legal Prerogatives of the Crown del $1820 .{ }^{155}$

Nel 1833, ritiratosi a vita provita si prodigò moltissimo per il Law of Nations, basandosi sull'edizione inglese del 1797 , a cui vi appose ulteriori note, spiegando la necessità di una revisione nella celebre prefazione scritta presso la Chancery Lane. ${ }^{156}$

Chitty, sin dalle prime righe fece trasparire la sua devozione per il Droit des gens di Vattel, sottolineando i meriti e la crescente utilità di questo ammirabile trattato che, all'epoca in cui scriveva, " addirittura non era sufficientemente conosciuto o giustamente apprezzato». Egli era fermamente convinto che il Law of Nations potesse servire non soltanto agli uomini di governo o politici, ma «a tutti». Chiunque avesso letto questo trattato avrebbe dovuto ammettere che: "he has acquired knowledge of superior sentiment, and more important information than he ever derived form any other work ». ${ }^{157}$

154 Ziegler (2007) 339-340; cfr. anche ReEves (1909) 547-561.

155 Hunter (2010) 22. Riferimenti biografici: Montmorency (1909) 17 nota 1, 19, 20 nota 1. 156 «Many years have elapsed since the original work was published, long before the invaluable decisions of sir W. Scott, sir C. Robinson, and sir J. Nichol, and other eminent Judges in the Courts of Admiralty, Prize and other Courts; and the last edition upon which any care was bestowed, was published in AD 1797; since which time, and especially during the last general war, many of most important rules respecting the Law of Nations were established. The object of the present Editor has, therefore, been to collect and condense, in numerous notes, the modern rules and decisions, and to fortify the positions in the text by reference to other author of eminence, and by which he hopes that this edition will be found of more practical utility, without interfering with the text, or materially increasing its size »: Chitty, Preface to the present edition, in: Vattel (1834b) III-IV.

157 Ivi, IV. 
La versione commentata da Chitty, rispetto alle edizioni francesi, ha uno spiccato risvolto pratico, vi sono infatti numerosissime citazioni di casi riguardanti il diritto del mare, emersi soprattutto durante l'epoca napoleonica e dopo il Congresso di Vienna, come afferma Ian Hunter "Chitty's edition adapted Vattel's jus gentium to British constitutional thought and English common-law Culture ». ${ }^{\mathbf{1 5 8}}$ La quantità di riferimenti a casi pratici e giurisprudenziali tipici del sistema di common law è altissima e fa comprendere quanto ancora Vattel fosse tenuto in considerazione e applicato a fattispecie concrete. In particolare la definizione che Vattel dava al diritto delle genti ovvero « The law of nations is the science which teaches the right subsisting between nations or states, and the obligations correspondent to those rights », permetteva a Chitty di avanzare alcune osservazioni che trascendono dalla dottrina o dalla teoria internazionalistica, ma trovano la ragione d'essere solo nella sfera pratica. ${ }^{159}$

L'avvocato inglese evidenziava le difficoltà del diritto internazionale del suo tempo, l'assenza di un "general moral international code ", illustrava altresì le fonti di tale diritto e si soffermava in particolare sulle violazioni del diritto delle genti durante la guerra. ${ }^{\mathbf{1 6 0}}$

Per Chitty l'esigenza di avere regole e principi da utilizzare nella pratica internazionale rivestiva così un'assoluta importanza e pertanto all'epoca in cui scriveva l'unica fonte autorevole era il Law of Nations, che doveva quindi essere utilizzato come se fosse un codice di comportamento tra le nazioni, soprattutto in caso di conflitto. ${ }^{\mathbf{1 6 1}}$

Negli Stati Uniti, nel 1852, l'avvocato americano Edward Duncan Ingraham curò una nuova edizione del Law of Nations, avendo come punto

158 Hunter (2010) 22.

159 Così Chitty in: VAttel (1834b) Preliminaires, $\mathbb{3}$ 3, LV, nota 1.

$160 \mathrm{Ivi}, \mathrm{LV}$ : "Unhappily, especially in modern times, we found that the law of nations has sometimes been set at naught by over-powerful states, adhering [...] rather to Common Law than stopping to enquire whether the law of nature and of justice had not become and been declared in that instance, part of the law of nations. It may therefore be asked, of what utility is the law of nations, since it is of such imperfect and inefficient obligation? The answer is, that all nations, although for a time astounded and surprised by the unexpected aggression of an oppressive and ambitious conqueror, will yet ultimately feel, and endeavour to give effect to, the true law of nations, lest, by suffering its continued violations, they may individually be sacrificed; and consequently, as in the instance alluded to, they will ultimately coalesce and associate in one common cause, to humiliate and overcome the proud invader of all just right and principle».

161 Ibidem. 
partenza proprio quella curata da Chitty una ventina di anni prima. Ingraham sottolineava nella Preface di avere eseguito un lavoro di comparazione con tutte le edizioni a lui conosciute, a partire dalla prima (Londres 1758) da quella di Amsterdam del 1775 curata da Dumas, le migliori, a suo parere conosciute e servendosi di quella di Hoffmanns del 1835, « the last and best edition ", a cui come si è detto, Pinheiro-Ferreira aggiunse il terzo volume, contenente le sue note. ${ }^{\mathbf{1 6 2}}$

Successivamente Ingraham tenne in considerazione tutte le British decisions riportate da Chitty; ma, nello studiare attentamente questa edizione, scoprì le inesattezze delle citazioni inerenti alle cause in cui era stato riportato il pensiero di Vattel a sostegno della decisione, perciò oltre effettuare le dovute correzioni, chiarì e ampliò, a suo dire, alcuni punti dottrinali in modo più argomentato e più intelleggibile, inserendoli all'interno di virgolette per non offuscare il testo originale. ${ }^{163}$

Il curatore precisò altresì che, vista l'entità dell'opera, decise di discostarsi dall'aggiungere la bibliografia sistematica di diritto della natura e delle genti, presente nell'edizione francese di Hoffmanns e ritenuta « an excellent guide in the choice of Works upon a subject much less attended to than is demanded by its importance ». ${ }^{\mathbf{1 6 4}}$ Quella di Ingraham fu riedita sempre a Philadelphia diverse volte tra il 1853 e il $1883 .{ }^{165}$

In tutte queste versioni anglo-americane si riscontra una costante: in esse, a differenza di quelle francesi, non sono mai stati inclusi il Discourse di Mackintosh; i saggi di Vattel precedenti la stesura del Droit des gens; le riflessioni di Chambrier d'Oleyres e tantomeno un'appendice bibliografica dei testi di diritto delle genti. Questa scelta editoriale era certamente frutto dell'utilizzo squisitamente pratico e politico del trattato, che poco si conciliava con riflessioni di stampo più dottrinale, ma privilegiava l'attenzione alla realtà storica del tempo.

Negli Stati Uniti il Law of Nations era il testo per eccellenza: il presidente Millard Fillmore nell'organizzare, tra il 1850 e il 1853, la biblioteca presidenziale volle il trattato tra i libri; ${ }^{\mathbf{1 6 6}}$ Esattamente come era accaduto anche

162 Ingraham, Preface to the present edition, in: VATTEL (1852) III.

163 Ibidem.

164 Ibidem.

165 LAPRADELLE, Introduction, VATTEL (1916) LIX.

166 «The Executive Mansion Purchased two copies of this book, one in December of 1850 and a second in October of 1852 . The first copy was probably the 1839 edition that Little 
in passato, durante le sessioni della United States House of Representatives frequentissimi erano altresì i richiami all'opera per tutte le questioni che attenevano al diritto internazionale, come ad esempio nella circostanza della disastrosa guerra messicano statunitense, combattuta tra il 1846 e il $1848 .{ }^{167}$

Il conflitto esplose a seguito di questioni tra il Messico e la Repubblica del Texas, una repubblica indipendente che i coloni americani avevano creato su territori messicani, mai riconosciuta dal Messico, in quanto la considerava una semplice provincia. Ma nel momento in cui il Texas chiese di entrare a far parte degli Stati Uniti, e fu accolto, il Messico rivendicava la lesione della sua sovranità territoriale.

Tuttavia furono gli Stati Uniti a dichiarare guerra al Messico, poichè il Presidente americano James Knox Polk ambiva alla conquista di un'altra provincia messicana: la California e il pretesto per il casus belli fu l'uccisione di alcuni soldati americani che erano schierati al confine con il Messico. In questo contesto Polk giustificava il necessario inizio della guerra prendendo spunto dall'opera di Vattel e allo stesso tempo la maggior parte dei rappresentanti della Camera degli Stati Uniti nei loro discorsi sostennero tale posizione riportando interi passaggi del Droit des gens. ${ }^{168}$

Il conflitto messicano statunitense è solo un esempio dell'influenza del trattato Vattel in ambito internazionalistico, perchè negli Stati Uniti durante il XIX secolo in tutta la pratica internazionalistica e all'interno della giurisprudenza ebbe un ruolo fondamentale e venne citato e definito come la massima autorità. ${ }^{169}$

and Brown listed for sale in its 1850 catalogue of law books: Although there were later editions available, it is reasonable to assume that Little and Brown would sell what they had in stock. The second copy would have been the 1852 edition that appears in the Hayes catalogue, which is based on the 1797 London edition but contains notes added by $\mathrm{E}$. Ingraham along with his preface and an index »: PARISIAN (2010) 330.

167 Johannsen (1985) 288 ss.; Gilmore (1891) 51 ss.; Gardner (1860) 45.

168 Sims (1847) 4-5. Cfr. per la sempre più frequente tendenza a citare nei propri discorsi l'autorità di Vattel: MoOre (1857) 102-107.

169 Cfr. Garrison (2009) 60 ss.; Dodge (2010) 544 ss.; Sloss / Ramsey/Dodge (2011) 8 ss.; Chetail (2014) 262 ss. Anche durante la seconda metà dell'Ottocento sono molti i proceedings e le decisioni giurisprudenziali che si appellano all'autorità di Vattel: Statement of the Claim of the State of Alabama against the United States; with argument in support thereof, and numerous precedents from usage of the Government, and Cases in point, now before congress (1850) 23: «It only remains to be seen what the law of nations, and the policy of our own laws suggest as applicable to the case. But two authorities will be cited. Let them speak for themselves "; Russell (1851), Argument for the Defendants, in 
Anche in questo secolo il nome del diplomatico di Neuchâtel ritorna sui giornali e riviste americane sia per "pubblicizzare» le nuove edizioni di Vattel, sia, come accadeva tra il XVIII e i primi anni dell'Ottocento, per riportare interi passaggi del Droit des gens e poi commentarli con le più importanti vicende diplomatico-internazionali nelle quali erano coinvolti gli Stati Uniti. ${ }^{170}$

Il testo di Vattel è il vero e proprio manuale d'eccellenza per l'insegnamento del diritto costituzionale e delle genti o diritto internazionale, anzi viene definito la prima guida per gli studenti (suggerendo lo studio sulla versione Chitty): ${ }^{171}$ punto di riferimento nel manuale di Edward Miner Gallaudet, ${ }^{172}$ in quello di Theodore Dwight Woolsey, ${ }^{173}$ di John Norton Pomeroy, ${ }^{174}$ di James Gilmore ${ }^{175}$ ed è in assoluto il più citato nel 1809 da

the Case of the State of Pennsylvania, vs. the Wheeling \& Belmont Bridge Co. and others, in the Supreme Court of the United States, 32, 50; Howard (1857), A Report of the decision of the Supreme Court of the United States, and the Opinions of the Judges thereof, in the Case of Dred Scott versus John F. A. Sandford, 89, 95-96; Report of the Lemmon Slave Case: Containing Points and Arguments of Counsel of both Sides, and Opinions of all the Judges (1860) 6-9, 10, 25, 67, 93; Murphy (1861), Full Report of the Trial of William Smith for Piracy, as one of the Crew of the Confederate Privateer, the Jeff Davis. Before Judges Grier and Cadwalader, in the Circuit Court of the United States, for the Eastern District of Pennsylvania, Held at Philadelphia, in October, 54 ss.

170 A titolo esemplificativo compare nei seguenti articoli e lettere all'editore sulle più importanti tematiche di diritto internazionale e diritto pubblico: « Privateering » in: Daily National Intelligencer, issue 2055 (Friday, 13 August 1819); «Important Slave Case » in: Fayetteville Observer, issue 44 (Thursday, 18 November 1852): «Right of Transit by Owners of Slaves» in: Daily National Intelligencer, issue 12448 (Tuesday, 18 January 1853); «Central American Affairs » in: North American and United States Gazette, issue 18693 (Thursday, 17 February 1853); «The United States and Austria» in: The Weekly Raleigh Register, issue 52 (Wednesday, 12 October 1853); « The British Recruiting Case at Cincinnati » in: Daily National Intelligencer, issue 13226 (Wednesday, 18 July 1855); The Charleston Mercury, issue 9558 (March, 21 Friday 1856): «Vattel the most generally accepted authority on international law ».

171 Cushing (1843) 251-252.

172 Gallaudet (1879) 14; cfr. anche Wharton (1872). Al college William and Mary della Virginia il giudice e professore Nathaniel Beverley Tucker, a partire dal 1833, per il suo corso di Political Law and Government aveva adottato come libro di testo Vattel; egli fu anche autore di A Running Commentary on Vattel's Law of Nations: Hunter (2013a) 480, nota 15.

173 Woolsey (1860).

174 Pomeroy (1886).

175 Gilmore (1891). 
William John Duane nel curiosissimo e divulgativo testo di diritto delle genti rivolto ai «farmers of the United States ». ${ }^{176}$

Allo stesso modo l'influenza di Vattel emerge marcatamente dalle parole del giudice inglese William Scott, Lord Stowell, il quale, per il caso Maria, relativo al commercio degli Stati neutrali, scrisse ormai all'alba dell'Ottocento (precisamente nel 1799), che sarebbe stato solo necessario riferirsi a Vattel per dirimere la questione in oggetto, in quanto non era un semplice giurista " but as a witness asserting the fact - the fact that such is the existing practice of modern Europe ». ${ }^{177}$

Come avvenuto nel secolo precedente i giornali inglesi continuavano a citare Vattel; Inghilterra e America quindi conservano anche in questo aspetto un identico approccio al Law of Nations. È ancora il Times durante il corso XIX secolo a riportare interi passaggi del trattato, a consigliare la sua lettura e il suo studio. ${ }^{178}$

176 Duane (1809).

177 Così riportato in Fenwick (1914) 406-407, nota 421.

178 Tra i numerosi contributi si segnalano: The Times, issue 10949 (Wednesday, 10 November 1820) 4, che annunciava la pubblicazione dell'edizione francese; The Times, issue 12132 (Thursday, 11 March 1824) 2, dove si trova scritto che "Vattel who is among the first authority on the law of nations "; The Times, issue 16809 (Thursday, 16 August 1838) 2, in cui veniva edita una lettera all'editore intitolata " Para Claims »; The Times, issue 16833 (Thursday, 13 September 1838) 2, intervento sul giornale intitolato "Loans to Greece », si riferisce alla situazione finanziaria greca; a seguito della pubblicazione del libro di Henry Parish intitolato Diplomatic and Financial History of Greece, viene argomentato che «Vattel $[\ldots]$ is not generally admitted to be the highest authority, not merely on points of international law, but on the obligations by which sovereigns and their subjects are reciprocally bound, it is the duty of the supreme power in the state to protect the interests of private individuals, as a portion of the property of the nation "; The Times, issue 16923 (Thursday, 27 December 1838) 5, viene pubblicata una lettera all'editore del giornale sulla questione della Florida in cui compare una diretta citazione del Droit des gens al libro II, capitolo VI, $\mathbb{7 1}$ ss., relativa alla nazione e alla possibilità di intervenire nelle azioni dei suoi cittadini; The Times, issue 17158 (Friday, 27 September 1839) 6, sul commercio dell'oppio in Cina; The Times, issue, 18805 (Friday, 27 December 1844) 3, lettera all'editore per gli effetti della stipula da parte degli inglesi e dei Maori del trattato di Waitangi del 6 febbraio 1840; The Times, issue 20567 (Friday, 28 June 1850) 8, si tratta di un intervento molto interessante perchè riguarda la lettera di un lettore, intitolata "Greece. - Vattel versus Palmerston", l'autore si firmò come "An Ionian ", esplicitando la sue criticità nei confronti di Henri John Palmerston, Ministro degli esteri inglese, quando nel 1850 decise di ripristinare l'influenza britannica in Grecia con un colpo di forza. Prendendo a pretesto le richieste insoddisfatte di indennizzo da parte di un ebreo considerato suddito inglese, David Pacifico, le rivendicazioni dei marinai delle isole Ionie, e la 
Il successo del Droit des gens nella sfera pratica non coincide però parimenti con le posizione della dottrina internazionalistica inglese e americana, che sembra invece prenderne le distanze, o meglio ancora elaborare diversi e più aggiornati orientamenti alla luce anche del progressivo evolversi della disciplina.

Ampi riferimenti al testo vatteliano si trovano nell'imponente opera del chancellor James Kent, che tra il 1826 e il 1830 pubblicò i Commentaires on American Law. Kent non esitava più volte a ribadire la popolarità di Vattel per il diritto delle genti e a richiamarlo all'interno della sua opera, ma al tempo stesso lo riteneva purtroppo ancora troppo impreciso nelle definizioni filosofiche: "his topic are loosely and often tediously and diffusively discussed, and he is not sufficiently supported by the authority of precedents, which constitute the foundation of the positive law of nation ». ${ }^{179}$

Per comprendere la portata di Vattel e l'influenza esercitata nella dottrina internazionalistica del XIX secolo in America e in Inghilterra è necessario prendere come esempi il concetto di trattato e in particolare la sua interpretazione. Nei paesi di common law ampio era il dibattito sull'elaborazione di regole che vincolassero l'interprete nei trattati internazionali. Nella dottrina inglese, Theodore Sedgwick scrisse che la ricerca di regole vincolanti per l'interprete appare « as vain [...] as to endeavor, in the same way, to define the mode by which the mind shall draw conclusions from testimony $\gg .{ }^{\mathbf{1 8 0}}$ Henry Halleck, pur riconoscendo l'importanza dell'opera di Vattel, le cui regole di interpretazione riassumeva in dodici punti, sottolineava l'esigenza di evitare « all metaphysical distinction, minute subdivision of terms and the

questione degli isolotti dei Cervi e Sapienza (reclamati dalla Gran Bretagna) il governo inglese ordinò il blocco della Grecia. Ancora sulla Grecia: The Times, issue 20568 (Thursday 15 August 1850) 3; The Times, issue 21539 (Wednesday, 21 September 1853) 6, sulla politica del Pacifico da parte dell'Inghilterra. Ancora: The Times, issue 24823, (Tuesday, 11 June 1867) 9, relativo " on the Luxemburg guarantee "; The Times, issue 26951 (Wednesday, 4 January 1871) 4, in merito ai rapporti tra Stati Uniti e Canada; The Times, issue 29973 (Monday, 30 August 1880) 8, è presente un report sulla Conferenza di Berna sul diritto internazionale; The Times, issue 34498 (Tuesday, 12 February 1895) 13, sull'« Indian Standard the gold finance». I riferimenti a Vattel continuano ancora, anche se in forma decisamente ridotta, durante tutto il XX secolo: The Times, issue 36027 (Monday, 1 January 1900) 8, lettera all'editore che ha come tema il contrabbando nei territori africani; The Times, issue 37362 (Thursday, 7 April 1904) 8, lettera all'editore intitolata "The Hague tribunal and the war "; The Times, issue 37401 (Monday, 23 May 1904) 8, lettera all'editore sulle acque internazionali.

179 KeNT (1826) 18 ss.

180 SedGWICK (1857) 228. 
use of arbitrary formulae ». ${ }^{\mathbf{1 8 1}}$ William Hall considerava la maggior parte delle regole suddette "either unsafe in their application or of doubtful applicability", tuttavia ne fissò alcune "against which no objections can be urged, and which probably are sufficient for all purposes ». ${ }^{\mathbf{1 8 2}}$

In nordamerica Henry Wheaton, celebre giurista e diplomatico, nei suoi Elements of International Law, opera che riscosse molto successo a cui seguirono diverse edizioni americane durante il corso del XIX secolo, procedeva ad uno studio sistematico del pensiero dei maggiori autori del diritto delle genti e dopo aver passato in rassegna Grozio e Wolff, descriveva i capisaldi contenuti nel Droit des gens di Vattel, soffermandosi in particolare sull'analisi della Préface. Il trattato vatteliano anche in questo caso risulta la fonte principale per Wheaton; egli però esprimeva una sfiducia analoga a quella degli internazionalisti inglesi circa l'utilità ed il valore pratico dei principi interpretativi applicabili ai trattati internazionali elaborati da Vattel. ${ }^{183}$ Thomas Lawrence, pur elogiando Vattel per il suo imponente lavoro, fu molto critico e drastico nelle parti più filosofiche del Droit des gens come le regole interpretative. Egli riferendosi a Vattel scriveva: «a vast amount of misplaced ingenuity has been expended on this subject » e osservava come sarebbe illusorio credere nell'efficacia prescrittiva di simili regole nella società internazionale "since States have no common superior to adjust their difference and declare with authority the real meaning and force of their international documents ». ${ }^{\mathbf{1 8 4}}$ John Westlake, in merito a quale tipo di interpretazione privilegiare, interveniva nel dibattito sostenendo che: «the important point is to get at the real intention of the parties, and that inquiry is not to be shackled by any rule of interpretation which may exist in a particular national jurisprudence, but is not generally accepted in the civilized world ». ${ }^{\mathbf{1 8 5}}$

Nella pratica viceversa le teorie di Vattel erano sempre molto influenti sui giuristi esperti di diritto internazionale. Robert Phillimore celebre politico e giurista inglese, nominato avvocato generale della regina, in una lettera inviata a quest'ultima datata 19 giugno 1863, all'interno di una complicata vicenda con il Brasile, per la distruzione e il saccheggio della nave britannica Prince of Wales, inviava l'intero estratto di Vattel dedicato alla rappresaglia,

181 Halleck (1861) 905.

182 HaLl (1880) 281.

183 Wheaton (1836) 204. Sulle regole interpretative cfr. anche: Chetail (2014) 291 ss.

184 Lawrence (1898) 286.

185 Westlake (1910) 293-294. 
alle sue ipotesi di legittimità e i suoi limiti. ${ }^{\mathbf{1 8 6}}$ Le rappresaglie infatti «were taken under the advise of Sir Robert Phillimore only " ${ }^{\mathbf{1 8 7}}$ e anche nel contestuale dibattito parlamentare l'autorità di Vattel e la successiva presa di posizione di Phillimore erano le uniche ad essere rilevanti. ${ }^{\mathbf{1 8 8}}$

186 La lettera conservata presso: The National Archives (United Kingdom), Kew, Richmond, con questi riferimenti: Folios 379-381: Sir Robert Phillimore, MP, Queen's advocate, 1862-1867: Sending extract from Vattel on reprisals (Book 2 cap $18 \mathrm{sec} 342$ ). Scriveva Vattel sulle rappressaglie che: « Reprisals are used between nation and nation in order to do themselves justice when they cannot otherwise obtain it. If a nation has taken possession of what belongs to another, - if she refuses to pay a debt, to repair an injury, or to give adequate satisfaction for it, - the latter may seize something belonging to the former, and apply it to her own advantage till she obtains payment of what is due to her, together with interest and damages, or keep it as a pledge till she has received ample satisfaction. In the latter case, it is rather a stoppage or a seizure, than reprisals: but they are frequently confounded in common language. The effects thus seized on are preserved while there is any hope of obtaining satisfaction or justice. As soon as that hope disappears, they are confiscated, and then the reprisals are accomplished. If the two nations, upon this ground of quarrel, come to an open rupture, satisfaction is considered as refused from the moment that war is declared or hostilities commenced; and then also the effects seized may be confiscated".

187 Fitzmaurice (2014) 230.

188 Affermava, infatti, l'Earl of Malmesbury il 19 giugno 1863, in occasione della lettera che Phillimore inviava alla regina con le teorie vatteliane sulle rappresaglie, quanto fosse determinante la posizione autorevole di Vattel e di Phillimore: "I think that is a proposition that cannot be disputed, and I am supported in that opinion by the authority of Vattel and of Dr. Phillimore. Vattel says - International law does not permit reprisals, except for a cause evidently just, for a debt clear and undoubted; for he who puts forward a doubtful pretension can only ask at first an equitable inquiry into his right. In the second place, before a recourse to reprisals, one must have uselessly demanded justice at least, have good reason to believe that it would be demanded in vain; then only may one obtain justice by one's own bands. Reprisals ought to be grounded on a denial of justice, either an actual denial or one which there is good reason to apprehend. In all cases susceptible of doubt, a Sovereign ought not to listen to the complaints of his subjects against a foreign tribunal, nor to attempt to screen them from the effects of a sentence passed in doe form, for that would be the means of exciting continual troubles. The law of nations directs that States should reciprocally pay that kind of deference to each other's jurisdiction, for the reason that definitive sentence shall be esteemed just. Dr. Phillimore lays down the same doctrine. He says - Letters of reprisal are not to be granted without a full knowledge of the causes that justify them. Moreover, it must be res minime dubia, in which justice has been denied, and it must have been absolutely denied by all the tribunals of the country before which the cause could be brought. An erroneous sentence conscientiously given by free judges, unbiassed and unintmidated by any judicial authority, affords no just ground for reprisals »: l'intero testo è tratto dalle edizioni digi- 
Nella varietà di posizioni sul pensiero vatteliano si rileva che i giuristi anglo-americani tendevano a circoscrivere o, meglio ancora, modellare tutti quei passaggi del Droit des gens più filosofici che poco si addicevano alla pratica internazionale, esattamente come Chitty e Ingraham si erano rapportati all'opera di Vattel, inserendo nel testo in nota i casi giudiziari: infine se la dottrina giuridica nella teoria vacillava è la stessa dottrina nella pratica a sostenere le proprie argomentazioni citando Vattel, ${ }^{189} \mathrm{e}$ Phillimore è stato un esempio.

\section{L'autonomia del diritto internazionale e il Droit des gens nei paesi di lingua tedesca}

I paesi di lingua tedesca conservano una loro peculiare caratteristica: dopo la traduzione in tedesco del Droit des gens ad opera di Schulin del 1760, nel XIX secolo non vi furono altre edizioni, ristampe e compendi che in qualche modo potessero richiamare alla memoria il pensiero del giurista di Neuchâtel.

In effetti nessun altra realtà europea ebbe una così vasta produzione di opere sul diritto delle genti sia dal punto di vista dottrinale che pratico, ma soprattutto emerge con forza come il diritto internazionale fosse concepito in modo del tutto diverso rispetto agli altri Stati. ${ }^{\mathbf{1 9 0}}$

La così profonda e diversa impostazione alla materia si discosta dalla visione degli altri Stati continentali e americani che invece presero come modello Vattel e alcuni giuristi sulla falsariga del Droit des gens elaborarono i loro trattati. Le ragioni di una tale differenza sono riconducibili alla signi-

tali della Commons and Lords Hansard si trova on line al sito: http://hansard.millbank systems.com/lords/1863/jun/19/brazil-papers-moved-for, consultato il 29 giugno 2016.

189 Con enfasi Lapradelle affermava che: "Quel que soit le jugement que portent sur les mérites de son œuvre, Mackintosh, Ward, Manning, Phillimore, Hall, Travers Twiss, Westlake, Lawrence, Oppenheim, aucun des maîtres anglais du droit des gens, qui se sont succédés, du XIXe Siècle au commencement du XXe, n'a jamais mis en doute la valeur de son autorité, comparable pour la formation du droit international anglais à celle de Pothier pour l'interprétation au XIXe siècle du Code Civil français »: Lapradelle, Introduction, in: VATTEL (1916) XXXIV.

190 Cfr. per una completa disamina dei rapporti tra giusnaturalismo, diritto pubblico e diritto delle genti nel corso dell'Ottocento si rimanda alle importanti riflessioni di STOLLEIS (2014). Per l'evoluzione della disciplina del diritto internazionale in Germania nella seconda parte XIX secolo: KosKenNiemi (2004b) 179-264. 
ficativa attenzione al diritto naturale e al diritto delle genti che ha da sempre caratterizzato questi territori; al diverso contesto storico-politico che si realizza nel corso dell'Ottocento in questa area geografica, ma principalmente all'influenza che anche sul diritto internazionale ebbe la scuola storica tedesca, la quale, come è ben noto, non solo metteva in discussione le codificazioni illuministiche della Prussia, della Francia e dell'Austria ma anche le tesi giusnaturalistiche, comprese quelle «di confine» come la teoria vatteliana.

Karl Friedrich von Savigny ridimensionò in qualche modo la tendenza giusnaturalistica a concepire la ricezione del diritto romano nell'Europa moderna in modo astratto. Egli osservava che il diritto è espressione della realtà storica o dello spirito di ciascun popolo e che, pertanto, la nascita di una comunità dotata di una certa comunione di idee precede, o almeno, determina tutte le vicende, dalla nascita alla estinzione, proprie di ciascun ordinamento giuridico. Come ha rilevato Nuzzo, le teorie di Savigny che individuavano nella coscienza del popolo l'origine del diritto positivo non potevano non condizionare anche il concetto di diritto internazionale: ${ }^{\mathbf{1 9 1}}$ "Con Savigny il diritto internazionale si afferma come il prodotto della coscienza giuridica europea, il frutto di un processo storico che permetteva agli Stati europei di affidare le loro relazioni giuridiche ad una serie regole condivise, $[\ldots]$ dopo Savigny nessuna rappresentazione giuridica avrebbe potuto qualificarsi scientifica se non fosse stata storica e soprattutto sistematica $»{ }^{\mathbf{1 9 2}}$

Le opere di diritto internazionale di provenienza tedesca, sembrano così tradurre il tradizionale frasario della dottrina giusnaturalistica in un nuovo vocabolario più sensibile alle vicende del corso storico di cui gli Stati sono protagonisti (comunità giuridica del genere umano, comunità dei popoli, comunità delle nazioni, consenso comune delle genti). ${ }^{193}$ In un simile contesto le idee vatteliane passarono quasi in secondo piano rispetto al pensiero forte e ben elaborato e strutturato dei giuristi tedeschi del XIX secolo.

La circolazione del Droit des gens di Vattel e la sua recezione in questo ambiente si articola attraverso due modalità: da una parte vi furono giuristi che criticarono il suo pensiero, dall'altro, invece, sembra quasi che la figura e il trattato di Vattel fossero completamente superati e collocati nella storia ma, questa linea di confine non risulta nella realtà così netta, poichè vi

191 Nuzzo (2009b) 79; Nuzzo (2012b) 25-50.

192 Nuzzo (2009b) 81-82.

193 Panebianco (1974) 167-168. 
furono invece alcuni giuristi che utilizzarono Vattel, anche se non come fonte principale. ${ }^{194}$

Alla fine del XVIII secolo George Frederich von Martens, nonostante avesse profondamente attinto dal pensiero vatteliano, in parte oscurò la fama di Vattel. Fu proprio con il Précis du Droit des gens di Martens (1789), che iniziò a svilupparsi e a prendere forma la concezione più marcatamente positivistica del diritto internazionale, a rivestire un ruolo essenziale sia per la dottrina sia per la prassi internazionale tedesca. ${ }^{195} \mathrm{Il}$ trattato di Martens, incentrato esclusivamente sulle relazioni internazionali in tempo di pace e di guerra, senza però far cenno allo Stato e al suo diritto interno, vide un successo molto simile a quello di Vattel; ebbe numerose edizioni, traduzioni e diverse note, commenti ed allegati che furono inseriti come appendici insieme al testo principale. Nel 1831, dopo quasi dieci anni dalla morte di Martens, Pinheiro Ferreira, come si è visto, prima ancora della nuova versione vatteliana, annotò il trattato del giurista tedesco, divenendo il punto di riferimento per le successive pubblicazioni, tra cui del 1858, dove Charles Vergé curò una Bibliographie raisonnée du Droit des gens. ${ }^{196}$

L'opera di Martens fu il punto di partenza per la redazione della maggior parte dei testi tedeschi sul diritto delle genti e fu anche la fonte principale da cui attinsero autori come August Wilhelm Heffter, Johann Ludwig Klüber o Johann Caspar Bluntschli. Esattamente come Martens, anche Heffter si confrontò con l'opera di Vattel, e prese ampiamente spunto nel Das europäische Völkerrecht der Gegenwart. ${ }^{197}$ Klüber, per la stesura del Droit des gens moderne de l'Europe, richiamava «tra le dottrine autorevoli » Burlamaqui, Martens e soprattutto Vattel. ${ }^{198}$

194 Hueck (2004) 41-55, in particolare 48.

195 Koskenniemi (2008) 189-207; Rauschning (1987) 123-145. Anche: Figge (1914); HabeNICHT (1934).

196 Basti pensare al Prècis du Droit des gens moderne de l'Europe. Nouvelle edition revisée, accompagnée des notes de Pinheiro-Ferreira, precedée d'une introduction et completée par l'exposition des doctrines des publicistes contemporains et suivie d'une Bibliographie raisonnée du Droit des gens, par Ch. Vergé edito a Parigi nel 1858. Vi fu anche un'altra edizione del trattato di Martens nel 1864 sempre a Parigi ed intitolata Précis du Droit des gens moderne de l'Europe, fondé sur les traités et l'usage; pour servir d'introduction à un cours politique et diplomatique. Augmentée des notes de Silvestre Pinheiro-Ferreira, 2. éd. enrichie de nouvelles notes et mise au courant des événements contemporains par Ch. Vergé.

197 Heffter (1844). Su Heffter: Hueck (2004) 41-55.

198 KlüBER (1819) V. Cfr. sulla sua opera: Stolleis (2014) 52-55. 
Tuttavia questi giuristi rispecchiavano un atteggiamento nei confronti del Droit des gens che era già iniziato ad intravedersi alla fine del Settecento e ridimensionava storicamente il successo del trattato: nel 1797, Karl Friedrich Häberlin scriveva che il trattato Vattel (insieme alle precedenti opere di Grozio e Pufendorf) veniva considerato prima come un Coder des Völkerrecht, in diverse parti del mondo ed era giunto ormai il momento di superarlo. ${ }^{199}$ Nel 1808 Johann Christian Meister all'interno del Lehrbuch des Natur-Rechtes citava Vattel solo una volta precisando che il suo diritto delle genti non era modellato sulle idee di Wolff, sia in contenuti che in struttura. ${ }^{200}$

Heinrich Escher rilevava ancora l'autorità di Vattel, ma solo nel contesto della prassi diplomatica affermando che: «Beliebt bei den Diplomaten des gewöhnlichen Schlages, weil mäßig ausführlich und durchsichtig (seicht) ist immer noch Vattel ». ${ }^{201}$

Nell'opera di Bluntschli, un vero e proprio lavoro di codificazione del diritto internazionale pubblico, all'interno del quale vi sono note esplicative scritte allo scopo di indurre gli ambienti ufficiali ad avviare il perfezionamento redazionale del diritto delle genti, i riferimenti a Vattel diventano sempre più sporadici. ${ }^{202}$

Lo stesso distacco si rileva per Franz Liszt autore del celebre Das Völkerrecht systematisch dargestellt, dove si trova un breve riferimento a Vattel soltanto nell'introduzione, in cui chiarisce che il Droit des gens non è stata la sua fonte principale, attribuendo a Wolff il merito della separazione tra diritto delle genti naturale e diritto delle genti positivo. ${ }^{203}$

Senza dubbio celebri sono le posizioni di Robert von Mohl (1799-1875), professore di scienze politiche prima a Tubinga poi ad Heidelberg, fu ministro della giustizia nel governo provvisorio nato dopo la riunione del Parla-

199 Häberlin (1797) 12-13 nota *\%. Su Häberlin cfr. Stolleis (2014) 24-25.

200 Meister (1808) 546.

201 ESCHER (1864) 624.

202 Bluntschli (1868). Cfr. anche: Baker (2007) 342-358; Schindler (2007) 437-454; MetzNer (2009); Stolleis (2014) 643-647.

203 "Christian Wolf [...], der gegenüber der rein idealistischen Richtung der Pufendorfschen Schüler wieder die Scheidung des „natürlichen“ und des „positiven“ Völkerrechts durchzuführen sich bemühte. Als Wolfs Schüler hat der Schweizer Vattel [...] durch sein 1758 erschienenes Droit des gens den meisten Einfluß auf die Gelehrten wie auf die Staatsmänner der folgenden Jahrzehnte gewonnen »: Liszt (1898) 11. Su Liszt: HerrmanN (2001). 
mento di Francoforte e poi nel 1874 membro del Reichstag come deputato liberale. Egli è considerato uno dei fondatori del moderno giuspubblicismo. ${ }^{204}$

Mohl pubblicò nel 1855 Die Geschichte und Literatur der Staatswissenschaften, all'interno del quale scrisse che Vattel era considerato nella prima metà del XIX secolo da parte dei diplomatici come un oracolo incofutabile e infallibile:

«Es gilt bei Diplomaten und namentlich bei Consuln als eine Art von Orakel, wird nicht selten sogar in Staatsschriften als ein unbeantwortbarer Beweis angeführt, und bietet somit das eigenthümliche Schauspiel eines fast zum positiven Gewohnheitsrechte gewordenen Lehrgebäudes dar ». ${ }^{\mathbf{2 0 5}}$

Pur rilevandone l'autorità soprattutto in altri contesti europei, lo definiva un semplice traduttore di Wolff. ${ }^{206}$

Significativo che si associ al trattato il termine « oracolo » perché oltre alla sacralità insita in esso, necessitava anche un ulteriore successivo passaggio di traduzione del messaggio stesso: la risposta era di conseguenza soggetta ad interpretazione a seconda delle diverse esigenze e del contesto in cui il Droit des gens doveva essere applicato e attualizzato.

A partire dalla seconda metà dell'Ottocento è interessante rilevare l'alternanza della presenza di Vattel all'interno delle opere enciclopediche sia per quanto riguarda la sua trattazione separata, come giurista, sia nelle voci dedicate al diritto delle genti e alla sua evoluzione; ${ }^{207}$ inoltre nel XIX secolo

204 Padoa Schioppa (2007) 513.

205 Mohl (1855) 386. Su Mohl cfr. Stolleis (2014) 223-230.

206 Mohl (1855) 386: «Vattel ist bekanntlich eine grosse Auctorität. Schon längst hat in den ausserdeutschen Ländern keine Bearbeitung des philosophischen Völkerrechtes eine so allgemeine Billigung und Benützung gefunden, als eben sein Handbuch. Alle Auseinandersetzungen, dass dasselbe denn doch nur eine lesbarere, zum Theile sogar verschlimmbesserte Uebersetzung von Wolf's Werk sei, dass dasselbe sich keineswegs durch eine tiefe Auffassung und kernhafte Beweisführung auszeichne dass es um Jahrhunderte hinter dem jetzigen Stande der Philosophie zurück sei, haben lediglich nichts verfangen ». Ullmann, riferendosi a Vattel, disse: «Die Hauptvertreter dieser Richtung in jener Epoche sind Christian Wolff [...] und Emerich von Vattel [...], Le Droit des gens [...], seither in zahlreichen neuen Ausgaben verbreitet; englische Übersetzung von Chitty 1834. Vattel besaß namentlich in diplomatischen Kreisen bis in die neuere Zeit ein unbestrittenes Ansehen »: UllmanN (1908) 32.

207 Nello Staats-Lexikon di Karl Baumbach, alla voce «Völkerrecht», viene citato Hobbes, Pufendorf, Klüber senza accenno al pensiero o all'opera vatteliana: BAUмвасн (1882) 604-606. Nel celebre Staats-Lexikon edito da Rotteck e Welcker nel 1843 non c'è la voce 
i dottorandi tedeschi che si occuparono della storia del diritto delle genti o di tematiche di diritto internazionale, non annoverano il Droit des gens tra le fonti principali nelle loro ricerche. ${ }^{208}$

Dunque nella maggior parte dei trattati tedeschi i riferimenti alla dottrina vatteliana sono contrastanti: ${ }^{209}$ come si evince nell'opera di Theodor Schmalz, ${ }^{210}$ di Julius Schmelzing che sporadicamente citò il giurista di Neuchâtel $^{\mathbf{2 1 1}} \mathrm{ma}$ anche successivamente in August Quaritsch ${ }^{\mathbf{2 1 2}} \mathrm{e}$ in August Bulmerincq. ${ }^{213}$

Vi furono altresì esempi di giuristi tedeschi che non esitarono ad esprimersi anche con metafore ironiche sul Droit des gens, come Karl Baron Kaltenborn von Stachau (1817-1866). Egli fu Privatdozent ad Halle poi professore di diritto pubblico all'università di Köningsberg, nel 1864 nominato consigliere presso il ministero degli affari esteri a Kassel. ${ }^{214}$ È ricordato per

«Vattel », né alla sintetica voce «Völkerrecht, natürliches europäisches » si parla della sua opera: Welcker (1843) 731-735; lo stesso anche nello Staats-Lexikon del 1848, Welcker (1848) 789-792; nello Staats-Lexikon del 1866 è invece presente la voce sul giurista di Neuchâtel, Held (1866) 326-327; nello Staats- und Gesellschafts-Lexikon di Wagener compare la voce su Vattel e ampio spazio è dedicato alle diverse traduzioni del Droit des gens dell'Ottocento, Wagener (1866) 185-186.

208 In particolare: ZALESKI (1874) 69-76, dove Vattel viene citato solo nell'ultimo capoverso della tesi, come chiusa finale, ma non è la fonte principale dell'elaborato. Cfr. anche dello stesso autore Zaleski (1866) 96-101. Nel 1888 venne pubblicato il lavoro di: Gareis (1888), dove a Vattel nel capitolo Geschichtliche Entwicklung des Völkerrechts dedica solo qualche riga: l'autore si limita ad un semplice elenco nel momento in cui parla dei successori di Grozio, passa in rassegna sia Pufendorf, che Barbeyrac e alla fine Wolff e Vattel, mentre ampio spazio è dato ad internazionalisti come Bluntschli, Heffter, Martens.

209 Anche il tema della neutralità esposto da Vattel, che, come si è visto nel precedente capitolo ottenne critiche e al tempo stesso consensi in diversi Stati europei alla fine del XVIII secolo, non viene mai affrontato con alcun riferimento al diplomatico di Neuchâtel da parte degli internazionalisti tedeschi. Cfr. a titolo esemplificativo: Gessner (1855). Assenza di citazioni, riferimenti o teorie vatteliane anche nel precedente trattato di SAALFELD (1809) dove la fonte principale è ancora una volta il testo di Martens. Anche in: SAALFELD (1833) 11, passa in rassegna i giuristi che si sono maggiormente dedicati al diritto delle genti e cita anche Vattel. Cfr. Miruss (1847) 228.

210 Schmalz (1817). L'opera, composta da otto libri, fu tradotta anche in italiano anche in italiano da Giovanni Fontana e pubblicata a Pavia nel 1821, Del diritto delle genti europee in otto libri.

211 Schmelzing (1818-1820).

212 QUARITSCH (1875).

213 BulmerincQ (1889) 182.

214 Koskenniemi (2004b) 24; Hueck (2004) 48; Teichmann (1882) 43-45. Sulla produzione scientifica per quanto riguarda il diritto pubblico tedesco: Stolleis (2014) 475-476. 
essere un profondo conoscitore del diritto delle genti, scrisse nel 1847 Kritik des Völkerrechts, e dopo essersi soffermato in modo molto approfondito sull'opera e sul pensiero di Wolff, del Droit de gens di Vattel disse:

Es sind allerdings in beiden Schriften die Völkerrechtsverhältnisse in ihrem positiven Wesen nicht ganz verläugnet; nur sind alle darin abgehandelten positiven Materien nicht in ihrer lebendigen Fülle und Frische, nicht in ihrer positiven Unmittelbarkeit dargelegt, sondern unter dem verzehrenden Hohlspiegel der Wolff'schen Methode zu dürren Gerippen zusammengeschrumptt oder unter dem Filtrirbeutel der Vattel'schen Art aus krä̈tigem Mineralwasser zu distillirtem, allerdings klaren aber abschmeckenden Wasser verwandelt worden. ${ }^{215}$

Le argomentazioni avanzate portano concretamente a ritenere che nei paesi di lingua tedesca il diritto internazionale nell'Ottocento sia stato contraddistinto dalla presenza di forti modelli dottrinali, quali, tra i molti, ad esempio, le opere di Martens, Heffter e Bluntschli. Tale caratteristica trae fondamento dalla storica e radicale attenzione ai temi del diritto delle genti e del diritto naturale che da secoli ha delineato queste terre e ha permesso di conseguenza uno sviluppo della disciplina più svincolato da modelli giuridici come il Droit des gens.

Vattel acquistò molta importanza in contesti in cui non vi furono modelli forti e alternativi; mentre nei paesi tedeschi dove la tradizione del diritto delle genti o internazionale si era formata, esportando essa stessa modelli, questo non accadde, rimanendo particolarmente radicata. L'opera di Martens, i primi germogli del positivismo giuridico in ambito internazionalistico, i lavori dei suoi successori ed inoltre la diffusione della scuola storica tedesca in qualche modo non contribuirono al ritorno a trattati di per sè considerati già superati, come il Droit des gens, che rispecchiavano e rivisitavano teorie, argomenti e metodologia di illustri giusnaturalisti, quale in primis Christian Wolff.

\section{7. «La relazione diretta con i bisogni e gli affari presenti della nostra patria »: il Droit des gens in Grecia (1825 e 1831)}

La conferma, ancora una volta, della strategia traduttiva come strumento fondamentale per la veicolazione delle idee e la maggiore assimilazione di esse è la scelta di tradurre il Droit des gens per una finalità politico costitu- 
zionale in occasione dell'indipendenza greca. A partire dal 1821 la Grecia combatté la sua guerra di liberazione contro l'occupazione ottomana, iniziata quattro secoli prima nel 1453 con la caduta di Costantinopoli, a cui seguì la presa di Atene nel 1456 e nel 1460 l'estensione del dominio su tutta la Grecia continentale.

Come ha scritto Richard Clogg qualcosa, però, tra Sette e Ottocento cambiò radicalmente nel popolo greco, ponendo le basi politiche e sociali per l'indipendenza. Sporadiche erano state le insurrezioni che si erano realizzate nei secoli precedenti e per lo più erano sprovviste di pianificazione: «solo ora alcuni greci cominciavano a lavorare in direzione di una rivoluzione coordinata come conseguenza logica degli sforzi dell'intellighenzia di installare una coscienza nazionale nei connazionali ${ }^{216}$

Questa intellighenzia era stata affascinata e influenzata dagli sconvolgimenti della Rivoluzione francese e aveva permesso che nel popolo greco si iniziasse a sentire forte la coscienza nazionale, l'identità culturale, di tradizioni e politica. ${ }^{217}$ Il kapetànios dei klèftes, uno dei simboli della guerra di indipendenza greca, Theòdoros Kolokotrònis, aveva scritto che « la rivoluzione francese e le gesta di Napoleone hanno aperto gli occhi al mondo ». ${ }^{\mathbf{2 1 8}}$

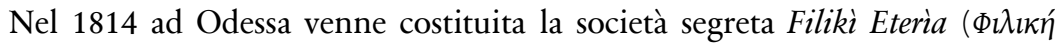

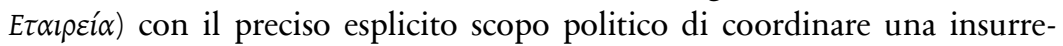
zione contro il governo ottomano e di conseguenza di liberare la madrepatria dall'oppressore.

Si trattava di un momento significativo perché le precedenti società come

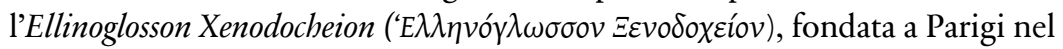

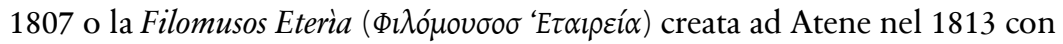
sede anche a Vienna, accanto ad obiettivi squisitamente culturali perseguivano solo implicitamente quelli politici. ${ }^{219}$

Alcuni storici, con approfonditi studi anche statistici, hanno messo in rilievo che la composizione della Filiki Eteria fosse per lo più rappresentata dalla borghesia commerciale, attenta e interessata ad investire nel movimento nazionale con tutto il peso e rilievo economico che avrebbe potuto otte-

216 Clogg (1996) 49. La bibliografia sulla vicenda greca è molto ricca, per la presente trattazione si rimanda a: Finlay (1971) 109 ss.; Pizanias (2011).

217 SFOINI (2015) 47.

218 Frammento citato in Clogg (1996) 49.

219 Ivi, 53. 
nere. ${ }^{220}$ Tra i membri della Società ci furono diverse tensioni interne generatesi riguardo alla possibilità dell'appoggio russo per la lotta all'indipendenza; in più di una occasione si era, infatti, cercato di coinvolgere Ioànnis Capodistrias, il conte corfiota futuro fautore della Grecia indipendente, e che, nel 1816 era ministro degli esteri aggiunto presso la corte russa dello zar Alessandro I. Tuttavia Capodistrias, né nel 1817 né nel 1820, diede alcun contributo e incoraggiamento ai piani rivoluzionari, ma con certezza informò lo zar di quanto si volesse realizzare; egli, senza mai sconfessare l'Eteria, consigliò i connazionali, prevedendo a breve una nuova guerra russo-turca, a seguito della quale i greci avrebbero potuto sperare per una nuova fase della propria storia. ${ }^{221}$

L'insurrezione si concretizzò nel 1821: Alèxandros Ypsilàntis, di origine fanariota, che in passato aveva servito nell'esercito dello zar, era stato scelto come leader e presidente dell'Eteria, la quale nel frattempo si era assicurata l'alleanza con Alī Tepedelenlī, pascià di Giannina, l'ex collaboratore del sultano e di origini albanesi con mire indipendentiste verso la Sublime Porta. ${ }^{222}$ Il 7 marzo Ypsilàntis entrò in Moldavia dove tentò, senza risultato, di sollevare la popolazione, occupando Bucarest, ma fu costretto a fuggire in Ungheria. Mentre il fratello Dimìtrios fu a capo della rivolta del Peloponneso, che venne portata a termine; il 1 gennaio 1822 fu proclamata a Epidauro l'indipendenza e venne costituito un governo nazionale. ${ }^{223}$ La mappa geopolitica, cos̀̀ come disegnata durante il Congresso di Vienna, veniva modificata. La guerra proseguì con fasi alterne e in favore della Grecia si mobilitò l'opinione pubblica liberale di tutta Europa. Come è noto il filoellenismo, che si era sviluppato e prendeva forza in diversi contesti socioculturali, vedeva schierati non solo l'inglese Lord Byron, il capitano Frank Abney Hastings o il generale sir Richard Church, ma soprattutto gli italiani, come Santorre di Santarosa, Vincenzo Gallina e Pietro Gamba, che si sentivano legati alle sorti della Grecia, condividendo con quel popolo il forte sentimento d'indipendenza. ${ }^{224}$

220 Ivi, 54; Frangos (1973) 87 ss.

221 Clogg (1996) 55. Il trattato di Bucarest del 1812 aveva concluso la guerra russo-turca combattuta tra il 1807 e il 1812, ma aveva lasciato aperte molte questioni che si sarebbero protratte sino ai primi anni del Novecento.

222 Fleming (1999) 32 ss.; Arafat (1987) 172-182.

223 Clogg (1996) 55-60; Frary (2015) 29 ss.

224 Era proprio Santorre di Santarosa, patriota piemontese che morì durante la battaglia di Sfactèria, a scrivere una lettera al filosofo Victor Cousin in cui affermava: «Amico mio, 
La rivoluzione politica e sociale della Grecia prendeva il suo corso accompagnata da una vera e propria "rivoluzione morale", che si realizzò nello strumento della traduzione dei testi europei dell'illuminismo nella lingua greca. ${ }^{225}$ Si tratta di un transfer che permette una maggiore assimilazione delle idee illuministiche di matrice europea, le quali prendono forza attraverso l'opera traduttiva da parte di greci formatisi nei territori francesi o italiani.

Già alla fine del Settecento, in coincidenza con la Rivoluzione francese, la Grecia assistette ad un incremento dei testi tradotti e all'impegno per la formazione ideologica dei compatrioti, basti pensare ad Adamantios Koraï, il quale si espresse a favore dei fermenti francesi nel suo Canto, pubblicato nel 1800 incitando i Greci a combattere per la libertà insieme ai francesi che avrebbero potuto costituire una unione «francogreca ». ${ }^{26} \mathrm{Fu}$ proprio Korais a tradurre in greco e ad arricchire di note l'opera di Cesare Beccaria. ${ }^{227}$ Molto successo riscuotevano anche trattati che riguardavano il rapporto tra la politica e la morale: nel 1813 Ioannis Kaskabas dava alle stampe a San Pietroburgo la traduzione del celebre testo di Mably Entretiens de Phocion sur le rapport de la morale avec la politique (1763), poiché in esso erano contenute delle « convinzioni elevate che dovevano essere lette e comprese dai greci,

amo la Grecia, patria di Socrate, di un amore che ha in sé qualcosa di sacro. Il popolo greco, valoroso, buono, che è sopravvissuto a secoli di schiavitù, è fratello del popolo mio. Comuni le sorti d'Italia e di Grecia, e dato che non posso far nulla per la mia patria ho il dovere di dedicare i pochi anni di vigoria che ancora mi restano a questa nobile causa »: così riportato in ClogG (1996) 60. Sulle ripercussioni degli eventi risorgimentali greci in Italia: Noто (2015) $47 \mathrm{ss.}$

225 Per l'utilizzo di «rivoluzione morale »: SForni (2015) 53; anche SForni (2014) 127-138. Sulla Rivoluzione greca e illuminismo: KiтromiLides (2015) 21 ss.

226 Sfoini (2015) 53. Koraïs (1800). Su Koraï: Frangiskos (2010); Kitromilides (ed.) (2010). In un altro scritto Korais afferma energicamente che: «L'admiration des prodiges opérés par les armées de la République se répandit de proche en proche, et rappela le souvenir de ceux qu'avoient opérés autrefois les armées Grecques. Dès ce moment la vanité nationale fit place à la contenance d'un peuple qui se prépare à devenir nation. L'enthousiasme de n'être gouverné que par des lois fut si général, qu'on ne parlait que des Français [...] Si parmi les causes de la révolution morale qui s'opère dans ce moment chez les Grecs, j'ai assigné la dernière place a la Révolution française, c'est qu'en effet, elle est la dernière dans l'ordre du temps, quoiqu'elle soit celle qui a le plus puissamment contribué à consolider dans les esprits des Grecs l'idée salutaire qu'ils avoient déjà conçue de la nécessité de s'éclairer ": Konaïs (Paris 1803) 43-44, 52-53.

227 L'opera fu pubblicata a Parigi nel 1802. Sulla traduzione di Cesare Beccaria: SForni (2015) 55; anche: Camariano-Cioran (1967) 193-202; Di Salvo (1982). 
affinché si potessero ricordare della loro antica patria e dei valenti uomini ", a cui seguì una seconda traduzione di Aikatérini Soutzou. ${ }^{228}$

Le opere tradotte prima della Rivoluzione greca avevano come fine la massima divulgazione delle idee illuministiche, mentre un significato diverso è dato da quelle tradotte durante: è pur vero che si riducono le traduzioni rispetto alla fase precedente, ma si specializzano nelle tematiche giuridicopolitiche per essere più consoni alle nuove esigenze. Si assiste così ad una nuova edizione del trattato di Beccaria nel $1823 ;^{229}$ ad una versione del contratto sociale di Rousseau grazie a Grigorios Zalykoglou, pubblicata a Parigi nel $1829 .^{230}$

Come giustamente affermato da Alexandra Sfoini: «Parmi les nombreuses brochures qui circulèrent au cours de la période 1821-1833 dans toutes les langues européennes (français, anglais, allemand, italien, russe, langues suisses et scandinaves), certaines furent traduites aussi en grec. Les philhellènes étaient principalement des libéraux, mais il y avait aussi des royalistes ou bien des partisans de la monarchie constitutionnelle, ce qui faisait du mouvement philhellène le lieu idéalise de dépassement des différences politiques ${ }^{231}$

L'attenzione politica si concretizza su più livelli attraverso le traduzioni delle costituzioni più moderne, al fine di essere utili modelli alla formazione del sistema greco, ${ }^{232}$ le versioni in lingua greca di opere dedicate alle armi e alle strategie belliche, soprattutto navali, ${ }^{233}$ infine la revisione trattati sul diritto delle genti.

Una parte del Droit des gens venne tradotta da Spyridon Skouphos, che

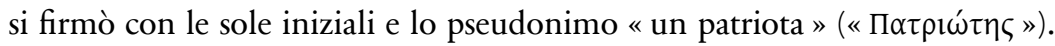

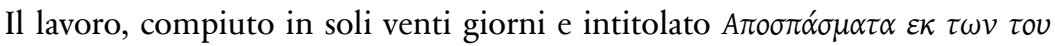

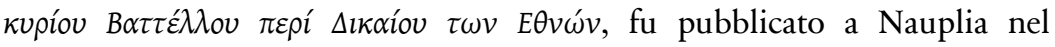
$1825 .{ }^{234}$ La scelta delle parti del Droit des gens selezionate si presenta molto

228 Così in SFoINI (2015) 58.

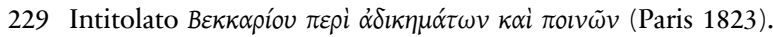

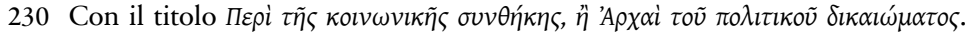

231 SFoINI (2015) 63.

232 Ivi, 68.

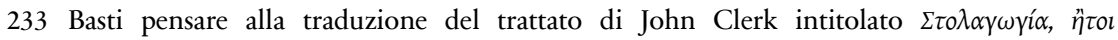
$\theta \alpha \lambda \alpha \sigma \sigma \imath v \dot{\eta} \tau \alpha \kappa \tau \imath \kappa \dot{~(M o s c a ~ 1823) ~ t r a d o t t o ~ d a ~ Z a c h a r i a s ~ A n d r e ́ a d i s: ~ i b i d e m . ~}$

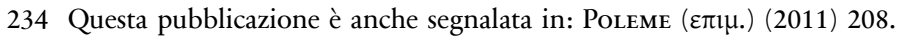


interessante: Skouphos, per rispondere alle esigenze rivoluzionarie, seleziona alcuni capitoli e paragrafi del trattato. ${ }^{235}$ Egli nella premessa dedicata al popolo ellenico non esitò ad affermare l'importanza dell'opera e il valore della traduzione, sostenendo che i passaggi scelti avevano una relazione

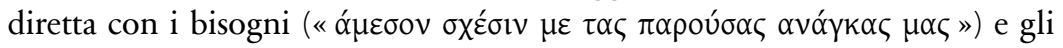

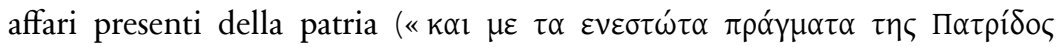
$\mu \alpha \varsigma ») .{ }^{236}$

Skouphos non indica né in nota né nel testo esattamente a quali capitoli e sezioni del Droit des gens si riferisce, ma leggendo i titoli si risale agevolmente alla fonte: tradusse gran parte del capitolo VII del libro III dedicato alla neutralità. In particolare scelse i paragrafi dal 103 al 118, relativi al passaggio delle truppe in uno Stato neutro, con riguardo alla condotta che deve essere osservata da quest'ultimo ( $(104)$; al commercio con i belligeranti ( $\$ 111$ ss.); ai beni oggetto di contrabbando $(\mathbb{S 1 1 2}$ ); ai vascelli neutrali al loro commercio e ai beni appartenenti al nemico a bordo di essi ( $\$ 115)$; ai doveri imparziali dei popoli neutrali $(\mathbb{S 1 1 8}$ ). Con un breve salto nella traduzione delle successive parti, Skouphos la riprese dal $\ 132$, riguardante il divieto di esercizio di qualunque ostilità in un paese neutrale, continuando e concludendo la selezione dei brani sulla neutralità con il $\$ 133$, secondo cui un paese neutro non può prestarsi a ricevere truppe di uno Stato belligerante al fine di attaccare il loro nemico. ${ }^{237} \mathrm{La}$ seconda parte scelta da Skouphos riguarda alcuni paragrafi del capitolo successivo, l'VIII, dedicato al delicatissimo tema del trattamento del nemico in guerra e di conseguenza di cosa sia lecito e giusto fare. Il traduttore greco si concentrava: sul $₫ 138$, riguardante il diritto di debilitare il nemico in tutti i modi leciti; sul diritto sulla persona del nemico ( $(139)$ e sui suoi limiti, e sul divieto di uccisione quando cessa di resistere $(\mathbb{1 4 0})$; su di un caso in cui fosse possibile ucciderlo $(\mathbb{1} 141)$; sulle rappresaglie $(\$ 143)$, e sulla possibilità che il nemico potesse uccidere il comandante di piazza resistente nella difesa. Le successive parti, analizzate da Vattel e riprese da Skouphos sono rivolte a determinate categorie di soggetti e al loro trattamento in caso di guerra: il $\$ 144$ ha ad oggetto rifugiati e disertori; il $\ 145$ donne, fanciulli, anziani ed infermi; il $₫ 146$

235 Sulla figura di Skouphos cfr. ANDriotis (1996) 181-190, qui 181-182.

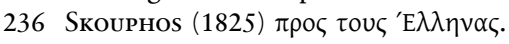

237 Ккоирнол (1825) 1-35. 
ministri di culto e sui letterati; il $₫ 147$ agricoltori e in generale tutto il popolo senza armi. Il $\$ 148$ è inerente al diritto di fare prigionieri di guerra, da cui discendono una serie di altri principi tra i quali: il divieto della loro uccisione $(\mathbb{1 4 9 9 )}$, il loro trattamento $(\mathbb{1 5 0})$; la liceità di uccisione quando non si possano custodire o alimentare $(\mathbb{1 5 1})$; la possibilità di renderli schiavi $(\$ 152)$; il loro riscatto $(\$ 153)$; l'obbligo dello Stato di liberarli $(\mathbb{1 5 4})$, per poi concludere con il $\$ 158$, in cui sono dettate disposizioni per relazionarsi al nemico. È molto significativo che Skouphos termini con tale passaggio: era infatti Vattel in questo paragrafo a ricordare che i nemici sono pur sempre uomini e "ridotti alla dolorosa necessità di rivendicare il nostro diritto con l'uso della forza e delle armi » non è possibile spogliarsi della carità che lega tutto il genere umano; " con coraggio pertanto saranno difesi i diritti della patria senza però che vengano offesi quelli della umanità ». ${ }^{238}$

L'iniziativa intrapresa da Skouphos, nei primi anni della rivoluzione gre$\mathrm{ca}$, non fu un singolo episodio anzi, ebbe uno sviluppo maggiore proprio

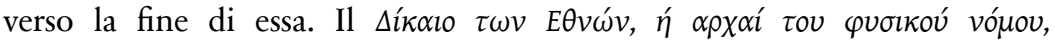

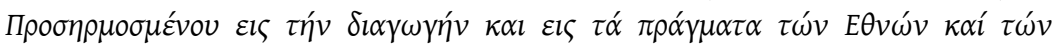

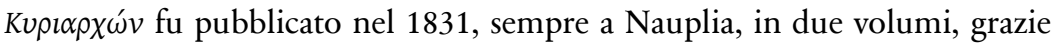
alla traduzione di Georgios Rallis (1804-1883), noto avvocato e politico greco. Dal punto di vista biografico a seguito dell'uccisione del padre Alexandros, diplomatico dell'impero ottomano che fu impiccato dai turchi dopo lo scoppio della rivoluzione nel 1821, Georgios lasciò la Grecia e studiò legge sia a Parigi che a Vienna. Ritornato in patria nel 1829 proseguì la sua carriera prima nella magistratura, dove divenne presidente della Corte Suprema (1849-1861), poi in accademia. Nel 1837 fu nominato professore di diritto commerciale presso la facoltà giuridica di Atene, dove fu rettore per due mandati (dal 1838 al 1841 e dal 1867 al 1869). ${ }^{239}$ Oltre a dedicarsi al diritto commerciale fu particolarmente attento al diritto fallimentare e al diritto delle genti, divenendo una figura centrale per la teoria giuridica e istituzionale greca. ${ }^{\mathbf{2 4 0}}$

Storia, contesto e idee politiche sono determinanti per comprendere l'impresa vatteliana condotta da Rallis. Egli infatti, dedicava il lavoro di

238 Vattel (1758a) L. III, chap. VIII, $\mathbb{1} 158,131-132$.

239 Petmezas (2009) 126; anche Sotiropoulos (2015) 277.

240 Sotiropoulos (2015) 41.

226 Capitolo IV 
traduzione a Ioannis Capodistrias, apponendovi come data il 20 aprile 1831, esattamente sette mesi prima della sua cruenta uccisione, che avverrà a Nauplia il 9 ottobre. Sotto il profilo dell'iter rivoluzionario nel 1827, Inghilterra, Francia e Russia decisero nel trattato di Londra, di imporre ai turchi un armistizio e il riconoscimento dell'autonomia ellenica. Avendo il sultano rifiutato, ne scaturì una guerra che vide impegnate le tre potenze contro i turchi e si concluse con la pace di Adrianopoli (1829). Nel frattempo Capodistrias, nell'aprile del 1827 era stato nominato dall'assemblea nazionale di Trezene alla presidenza del potere esecutivo per un mandato settennale e, date le dimissioni al governo russo, nel febbraio del 1828 assunse a Nauplia tale carica di governo. ${ }^{241}$ La Conferenza di Londra, il 3 febbraio 1830, confermando la sovranità della Grecia, stabilì che la corona ellenica venisse affidata al principe Leopoldo di Sassonia-Coburgo, il quale il 2 maggio dello stesso anno rinunciò formalmente ad essa. Capodistrias allora fu sospettato di ambire al trono ellenico e accusato di aver favorito la rinuncia del Principe: improvvisamente esplosero i dissensi che portarono progressivamente alla guerra civile e all'assassinio di Capodistrias il 9 ottobre $1831 .^{\mathbf{2 4 2}}$

La strategia della traduzione di Rallis è molto eloquente perché, nel 1831, ormai verso la fine della Rivoluzione, non era sufficiente tradurre solo alcuni frammenti dedicati alla guerra, come era successo nel 1825, si sentiva invece il bisogno di tradurre interamente il primo libro e il secondo libro, dedicati rispettivamente alla nazione e alle relazioni internazionali, in quanto la Grecia, stava ora iniziando la sua fase di (ri)nascita. ${ }^{243}$ Era necessario che nella nuova nazione tutto fosse disciplinato, soprattutto dal punto di vista

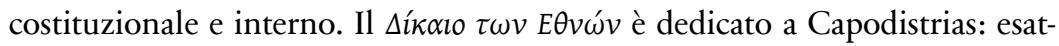
tamente come Vattel indirizzava la sua opera a coloro che governano, così Rallis è della stessa idea.

Nei Prolegomena, dopo aver illustrato molto brevemente la vita di Vattel, Rallis precisa che l'idea di una versione greca era nata durante i suoi studi a Parigi, ${ }^{244}$ confermando così, ancora una volta, quanto il trattato circolasse in ambiente accademico; prende in considerazione il lavoro svolto nell'edizione «parziale » di Skouphos, e ribadisce che questo tipo di iniziative traduttive

241 Clogg (1996) 68 ss.

242 Ibidem.

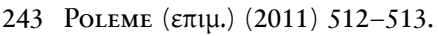

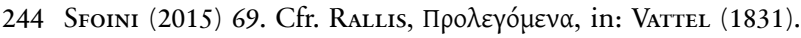


servono per favorire e diffondere il linguaggio giuridico e le teorie sul diritto delle genti. ${ }^{245}$

Come ha scritto Michail Sotiropoulos: "The reception and dissemination of Vattel's translation was the first among many to show that Greece was touched in these years by emerging global currents of liberal thought ${ }^{246}$ Queste correnti globali del pensiero liberale hanno avuto risvolti, ad esempio, sulle vicende italo-greche. Un possibile anello di collegamento per leggere le rivoluzioni greca e italiana è proprio il Droit des gens, che fu anche il punto di riferimento per gli esuli italiani e in particolare per il conte Alerino Palma, coinvolto politicamente e intellettualmente negli sconvolgimenti greci.

L'intreccio tra le vicende rivoluzionarie e l'utilizzo del Droit des gens rappresenta una costante ottocentesca: la politica espressa nel trattato, la possibilità che il traduttore possa fare una selezione delle parti interessanti al momento storico contingente e i contenuti che ben rispondono ad una rosa eterogenea e multiforme di situazioni politiche e diplomatiche, garantiscono al trattato una sorta di «astoricità » e «atemporalità ».

\section{Diritto pubblico e diritto costituzionale: il Droit des gens nell'Italia risorgimentale}

Come sostenuto da Eugenio di Rienzo, tra gli anni 1820 e 1830 si assistette ad una "Vattel Reinassance " non solo nel contesto europeo, ma anche in quello italiano. Le teorie vatteliane influenzarono in modo significativo il pensiero di alcuni giuristi e patrioti italiani. ${ }^{247}$

Vattel fu tra i più citati sia durante le grandi rivoluzioni europee e americane, sia nella creazione di costituzioni come quella di Cadice del 1812, ed ebbe anche un ruolo di rilievo nell'epoca risorgimentale italiana.

Lo scontento dell'ordine restaurato dal Congresso di Vienna si manifestò nella forma delle società segrete che si diffusero in tutta Europa, in particolare basti pensare alla Carboneria, la quale, nata nell'Italia meridionale, fece numerosi adepti in Spagna, nel Regno sabaudo, nel Lombardo-Veneto e a Londra, dove Luigi Angeloni e Giovanni Battista Marochetti fondarono la

245 Ivi.

246 Sotiropoulos (2015) 41.

247 di Rienzo (2002) 449. 
cosiddetta «Vendita carbonara degli italiani rifugiati al Tamigi ». ${ }^{\mathbf{2 4 8}}$ Tale società fu subito messa sotto stretta sorveglianza da parte delle autorità inglesi e ciò costrinse gli aderenti a pubblicare, il 17 giugno 1823, una dichiarazione finalizzata a smentire le voci diffuse sugli uomini che ne facevano parte: nel documento programmatico si scorgevano le idee e il pensiero politico di Angeloni e di Marochetti in merito alla questione nazionale, entrambi convinti sostenitori della creazione di uno Stato italiano a struttura federale. ${ }^{249}$

Angeloni (1758-1842) fu una delle maggiori figure dell'esulato italiano, di cui Mazzini sottolineò «la fede nella vita, nelle capacità e della forza della propria nazione $»{ }^{250}$ Prima dell'esperienza londinese nel 1814 ebbe insieme a Buonarroti svolse una funzione primaria: la sua intransigenza repubblicana, i suoi legami con tutti i maggiori esponenti liberali europei, fecero di lui il centro di riferimento e la guida morale a Parigi di tutto il movimento liberale italiano. ${ }^{251}$ E fu proprio a Parigi che scrisse e pubblicò Dell'Italia, uscente il settembre 1818, in cui denunciava apertamente il tradimento delle promesse fatte agli italiani da parte degli alleati e della Santa Alleanza e rivendicava il diritto dell'Italia all'indipendenza e alla libertà, basandosi sui due esempi di Stati: gli Stati Uniti, di cui si dichiarò sempre grande ammiratore e la Svizzera. ${ }^{252}$ Angeloni, infatti, riprendeva le riflessioni esposte nel suo precedente saggio $^{253}$ e teorizzava con più forza e maggiori argomentazioni la sua tesi confederale, non escludendo che la confederazione italiana potesse essere di carattere misto e comprendere Stati monarchici e Stati repubblicani.

Nella Dedicatoria agl'Italiani proponeva il rafforzamento territoriale del Regno di Sardegna, la salvaguardia delle esigenze del papato, esortando gli italiani ad attivarsi per avere la loro indipendenza. ${ }^{254}$

248 Sulla carboneria si rimanda a titolo non esaustivo a: Pieri (1931); Leti (1966); Dito (1978); Chiarle (1999); De Mattei (2001) 127-154; Conti (2003) Berti/Della Perruta (a cura di) 2004; Isabella (2006) 493-520; Isabella (2008) 37-58. Per una disamina sotto il profilo storico-giuridico: LATINI (2009) 553-577.

249 Morelli (1979) 3-13.

250 Mazzini (1842) 40-41.

251 Di Sabantonio (1977) 3 ss.

252 De Felice (1961) 242-249. Sulla figura di Angeloni si rimanda a: Vannucci (1887) 289, e 558 ss.; Di Sabantonio (1977); Ricciotti (2009).

253 Sopra l'ordinamento italiano che dover avrebbero i governi d'Italia (Parigi 1814).

254 L'invito veniva pronunciato con forti parole: « Non già particolarmente all'une che all'altre italiche genti io di favellare intesi in questi miei Ragionamenti, ma comprensivamente, e senza distinzione alcuna a tutti voi, Uomini d'Italia, che quantunque separati per più 
E poi aggiungeva, per rivendicare tale indipendenza, che Vattel nella sua bellissima opera con molta sagacia poneva "innanzi tratto ch'essi hanno dalla natura una libertà ed una indipendenza che essi non possono perdere [...] e poscia che le nazioni sono così tra loro naturalmente eguali, come sono gli uomini gli uni verso gli altri ». ${ }^{255} \mathrm{Si}$ soffermava sulla teoria dello Stato, sulla sovranità come principio cardine di una nazione, riportando, a supporto delle sue argomentazioni, interi paragrafi del Droit des gens. ${ }^{256}$

Ma la posizione di Angeloni non fu un caso isolato, i riferimenti e le citazione al testo di Vattel nel pensiero degli esuli si fanno ancora più frequenti proprio in quegli anni. Maurizio Isabella sottolinea sul punto che questi patrioti si riferivano al giurista di Neuchâtel come fosse « an advocate of a liberal international order $»{ }^{257}$

Marochetti o Marocchetti (1772-1851) vicinissimo ad Angeloni, biellese di origine, fine giurista e avvocato, partecipò attivamente all'insurrezione giacobina in Val d'Ossola; dopo il moto scoppiato a Torino il 12 marzo 1821, quando alcuni reparti militari conquistarono la cittadella e chiesero al re di concedere la Costituzione, Marochetti venne nominato dalla Giunta di governo capo politico di Biella. ${ }^{258}$

Organizzò la rivolta sulla convinzione che essa fosse sostenuta da larghi strati della popolazione: sostituì i consiglieri comunali realisti con altri liberali, epurò gli impiegati dell'amministrazione statale contrari alla nuova

maniere di governi, meritissimamente pur vi tenete, e tener dovetevi per i cittadini di una stessa patria, siccome quelli che, oltre ad una meravigliosa conformità di indole, d'ingegno e di costumi, avete in comunità una medesima religione, una medesima lingua, una medesima storia politica, scientifica e letteraria ed un paese il quale così è ben segreto da tutti gli altri, e pe' mari che l'accerchiano e per gli altissimi monti che fascianlo, che sole le isole sono meglio di quello della terra ferma dispartite »: Angeloni (1818) vol. I, Dedicatoria, I-II.

255 Ivi, 90.

256 Angeloni (1818) vol. II, Ragionamento III, 95: «Or così intorno a ciò ragiona il Vattel, la cui autorità di quanto peso sia in così fatte cose sel sanno troppo bene tutti gli statisti europei. E, per rispetto all'Italia, quanto queste vere e ragionate dottrine s'avvalorino, nelle stranezze che miseramente or la deturpano; tutti quegli uomini bene li conoscono pe' quali, ciò che fruttuosamente fondamenta il viver civile, è siccome essere dee, il supremo de' beni, tra i tanti mali di questa nostra misera terra ».

257 Isabella (2009) 99-100.

258 Novarino (2008) 637-640. Anche: Torrione/ Tamaroglio (a cura di) (1960) 17-19, 21, 33; Torrione (1968) 21-28; Marengo/Parlato (1982) 97; Della Perruta (1989) 328339; Presa (2002) 12-22. 
politica. Ma, dopo la sconfitta degli insorti a Novara, la maggior parte dei costituzionalisti piemontesi partirono per l'esilio: Marochetti con un passaporto falso scappò in Svizzera e da lì raggiunse Angeloni a Londra.

Nel frattempo però, con una sentenza del 3 settembre 1821, veniva pubblicamente condannato a morte in contumacia "per essere stato uno dei principali cospiratori, autori e complici della rivolta seguita nella città di Biella, col premeditato oggetto di sconvolgervi il legittimo governo di Sua Maestà ».259

L'attivismo politico si riflette anche nella sua produzione saggistica infatti, ${ }^{260}$ come Angeloni, sosteneva la struttura federativa per l'Italia e in Le congrès bienfaisant (Parigi 1826), ripubblicato qualche anno più tardi nel 1830 con il titolo Indepéndance de l'Italie, moyen de établir dans l'intérêt général de l'Europe, considéré spécialement sous le point de vue de l'équilibre politique, ou Partage de la Turquie, affrontava il problema italiano nell'ottica di un vero e proprio nuovo equilibrio europeo: egli proponeva l'abbandono del Lombardo-Veneto da parte dell'Austria, destinata a spostare gli interessi nell'area danubiano-balcanica, sfruttando la crisi dell'Impero ottomano. ${ }^{\mathbf{2 6 1}}$

Questo in sintesi il progetto dell'avvocato biellese: il Lombardo-Veneto e i Ducati di Parma e Modena venivano annessi al Regno sabaudo, che, a sua volta, avrebbe ceduto sia la Sardegna alla Francia e sia la Savoia alla Confederazione elvetica, ottenendo in cambio il Canton Ticino. ${ }^{262}$ Dal crollo dell'Impero ottomano e conseguentemente dal nuovo assetto europeo si sarebbero previsti: l'assegnazione all'Austria di Bulgaria, Serbia, Bosnia, Croazia e Albania; il riconoscimento della piena sovranità della Russia sulla Moldavia e sulla Valacchia, e infine la creazione di un Regno del Bosforo per il duca di Modena. ${ }^{263}$

Per penisola italiana Marochetti era propenso ad una soluzione unitaria, in linea con Angeloni, al fine di evitare resistenze internazionali, e proponeva un sistema federativo strutturato in tre Stati: il Regno dell'Alta Italia, il Regno delle Due Sicilie e lo Stato pontificio, con una forte egemonia della

259 Torrione (1968) 26. Cfr. Segre (1927) 238-302.

260 Le idee del Marochetti sarebbero poi state riprese da Cesare Balbo nella seconda edizione del suo famoso scritto Le speranze d'Italia del 1844.

261 Novarino (2008) 637 ss.

262 Novarino (2008) 637-640.

263 Ibidem. Anche Marochetti (1830) Avant-Propos, V ss. 
dinastia sabauda, mentre il Papato sarebbe stato ridotto a esercitare il potere temporale soltanto su Roma. Tale disegno geopolitico poteva prendere consistenza solo se la Francia si fosse fatta promotrice di un programma di rinnovamento europeo. ${ }^{264}$

L'avvocato biellese constatava che in Europa ci fu una vera e propria distruzione del principio dell'equilibrio di potere, iniziata con Napoleone e continuata con i risultati ottenuti con la Santa Alleanza. Quest'ultima venne da lui raffigurata come un insieme di Stati dominatori, all'interno dei quali non facevano parte gli Stati più piccoli, definiti di secondo e terzo ordine, come il Piemonte, la Baviera e il regno di Napoli. ${ }^{265}$

Secondo Marochetti non potevano esistere queste forti diversità tra gli Stati né alcuni di essi dovevano subire le decisioni di altri, perché si contravveniva al fondamentale principio argomentato da Vattel su cui poggia l'intero diritto internazionale: tutti gli Stati sono uguali, senza alcuni tipo di distinzione e hanno gli stessi diritti all'interno della comunità internazionale. ${ }^{\mathbf{2 6 6}}$

Inoltre nel 1829 Alerino Palma conte di Cesnòla pubblicava la sua Difesa dei Piemontesi inquisiti a causa degli avvenimenti del $1821{ }^{267}$ Palma (17761851), precedentemente commissario straordinario a Vercelli sotto Napoleone, fu magistrato a Ivrea e un fervido rivoluzionario, tanto da sostenere i moti del 1821, e al fallimento di essi scappò prima in Spagna, poi a Londra e infine in Grecia. ${ }^{268}$

264 Novarino (2008) 637 ss.

265 Marochetti (1830) 108: "Qui en effet a autorisé quatre ou cinq puissances à s'arroger exclusivement la dictature du reste de l'Europe? Depuis quand et pourquoi des états comptant plusieurs millions de population, l'Espagne, par example, Naples, la Bavière, le Piémont, la Suède, etc., et aussi anciens, aussi respectables, au moins, que la Russie et la Prussie, ne seront-ils plus consultés et appelés dans l'aréopage des souverains, et ne seront destinés qu'à obéir comme des ilotes? Y aurait-il en Europe deux espèces de souverains, comme de sujets, ainsi que dans l'ancien système féodal, les uns plus nobles, plus relevés, les autres moins; les uns sujets directs et immédiats du maître, les autres médiats comme servi servorum?".

266 Ibidem.

267 Occorre precisare che le opere sui moti del 1821 pubblicate in quello stesso anno o successivamente furono moltissime, si ricordano: Baggiolini (1821); Arborio GatTinara Di Breme (1822). Più in generale sulla situazione politica e sugli eventi si rimanda a: Beolchi (1864) 425-464; Braggio (1892) 183-225; Bobbio (1922) 3-44; Rinieri (1927) 543-649; Ricuperati (a cura di) 1968; Carpanetto (1980) 598-614; Colombo (2002) 147-176.

268 Damilano (2014) 586-588. 
Infatti dal 1826, dopo "aver colà onorato la patria colla dottrina e colle nobili virtù del suo animo ", fino alla morte visse in Grecia, dove fu coinvolto politicamente e attivamente nella rivoluzione in corso; successivamente ebbe dal governo greco l'incarico di ordinare i tribunali, svolgendo l'attività di magistrato e rifiutandosi di chiedere a Carlo Alberto la domanda di grazia per ritornare in patria. ${ }^{269}$ La Difesa dei Piemontesi fu voluto da un personaggio di grandissimo rilievo internazionale: lo statista e primo ministro inglese George Canning (1770-1827), il quale si oppose alla politica austriaca, appoggiò le istanze costituzionali e indipendentiste che da molte parti dell'Europa e del Sud America si levavano, favorendo, di conseguenza, moltissimi movimenti liberali e nazionali. ${ }^{270}$

Il saggio di Palma è diviso in tre parti, nella prima l'autore intendeva dimostrare come l'imputazione di lesa maestà a coloro che presero parte agli avvenimenti del marzo 1821 fosse illegittima dal punto del diritto civile e politico, e " presso il foro dell'onestà e buon senso e appo quello della legge scritta $»{ }^{271}$ Nella seconda parte, argomentava come le condanne contro tali uomini fossero non solo ingiuste " ma viziate eziandio di nullità radicale a forza delle legge medesime del Piemonte ${ }^{272}$ Infine nella terza sezione illustrava che «i riffugiti Piemontesi han diritto a richiedere onesta accoglienza e soccorso in ogni contrada governata con ordini costituzionali, e come giusta sia in essi l'aspettazione, che ivi la causa propria della loro patria ad un tempo, sia per avere protezione ${ }^{273}$

Palma introduceva quest'ultima parte con parole di solidarietà e vicinanza verso coloro che avevano ricevuto la condanna a morte. ${ }^{274} \mathrm{Il}$ magistrato eporediese appoggiava i moti torinesi che avevano come obiettivo il ribaltamento della monarchia e l'adozione di una carta costituzionale ispirata a quella di Cadice, nella quale venissero previsti maggiori diritti per il popolo

269 Pinoli Maritano (1975) 80 ss.; cfr. anche Cantù / Barbati (1859) 519.

270 Si rimanda a: Hinde (1973); Rolo (1965).

271 Palma (1829) 7.

272 Ivi, 8.

273 Ibidem.

274 Ivi, 70: « La simpatia inverso ogni uomo travolto nella sciagura, non per sua colpa, ma per cagione della più nequitosa ingiustizia, è propria di tutti gli uomini, che non vanno privi del sentimento d'umanità. Da questa simpatia tanto connaturale all'uomo non corrotto, dalla inclinazione che lo spinge a confortare, e a proteggere l'infelice, ed alleviare le pene, ella, è derivata quella legge naturale, la quale ci comanda di fare ad altri quello che in simile congiuntura giustamente vorremmo che a nostro pro si facesse ». 
piemontese e una riduzione del potere del sovrano. Palma, all'atteggiamento avuto dal Re sabaudo nei confronti di queste richieste costituzionali, così si espresse: «se un popolo, che indarno ha lunga pezza aspettato l'adempimento delle promesse del suo re bandisce egli stesso alla per fine una costituzione che il re non può schermirsi dal non accettare, e dal darvi la sua sanzione con giuramento, eccoti tosto accampato il diritto d'intervenzione contro il popolo, col pretesto che l'onore reale sia perciò offeso, ed avvilito; e si fa scrupolo d'esercitar questo diritto a fine che i principi cessino dall'avvilire essi medesimi il proprio onore col frodare le loro promesse a prò de' popoli! ». ${ }^{275}$ È proprio in questo contesto che Palma cita con veemenza Vattel riportando interamente tradotto il passaggio contenuto nel Droit des gens: « una nazione può con tutta giustizia ribellarsi ed ogni potenza straniera ha diritto di soccorso quando questa chieda aiuto: tutto ciò è mera giustizia il sovvenire ad un popolo prode che difende la propria libertà $» .{ }^{\mathbf{2 7 6}}$

Il costante richiamo alle teorie vatteliane da parte dello scrittore Angeloni, dell'avvocato Marochetti e del giudice Palma permette innanzitutto la comprensione delle dinamiche che regolavano la circolazione del trattato in questa delicata fase storica risorgimentale dell'Italia; fornisce inoltre un preciso quadro della tipologia di riflessioni fatte da alcuni patrioti italiani con l'ausilio delle fonti vatteliane; infine pone le premesse per affrontare la ricezione nel sud dell'Italia, nel Regno delle Due Sicilie, che vedrà come protagonista la traduzione di Terenzio Sacchi del Droit des gens.

\subsection{Nel Regno delle Due Sicilie: Terenzio Sacchi rilegge} il Diritto delle genti (1854)

In Italia il Droit des gens "piaceva » non solo ai patrioti e filocostituzionali, come Angeloni, Marochetti e Palma, ma anche ai conservatori come Terenzio Sacchi, al punto tale che lo tradusse in italiano.

Esattamente come il XVIII secolo ebbe una sola edizione pubblicata del trattato vatteliano, curata dal modenese Loschi, anche nel XIX secolo vi fu una sola versione con moltissime particolarità: Il Diritto delle genti di E. de Vattel applicato allo stato attuale delle nazioni venne dato alle stampe nel 1854 dal napoletano Terenzio Sacchi (1809-1865), membro dell'Accademia Pon- 
taniana, «valoroso scrittore e stimato cittadino » e ufficiale del Ministero degli affari interni nel Regno delle Due Sicilie. ${ }^{277}$

Nel 1845 Sacchi partecipò anche all'Adunanza degli Scienziati Italiani che si tenne a Napoli, venendo indicato come "segretario in capo della Sezione delle due Sicilie, della Società generale de' naufraghi ». ${ }^{278}$

Prima ancora della pubblicazione del Diritto delle genti, nel 1846, aveva scritto Prospectus d'un essai des institutions qui régissent le Royaume des deux Siciles, che fu inviato al Congresso dei germanisti tenutosi a Francoforte dal 24 al 26 settembre 1846 a cui partecipò anche Karl Joseph Mittermaier. ${ }^{279}$ Il Prospectus era stato redatto a seguito della pubblicazione nel 1830 de l'Histoire de la civilisation en France di François Guizot, che per Sacchi era una opera di « ingegno e vasta dottrina » e intendeva estenderla anche al Regno delle Due Sicilie perché «le leggi di questo Reame sono per avventura una espressione integra di questa civiltà ». ${ }^{280}$

Il giurista napoletano focalizzò la sua attenzione sulle istituzioni che governarono il Regno delle Due Sicilie, con particolare riguardo alle rela-

277 Atti dell'accademia pontaniana (1851) XIX; Cotugno (1923) 96; Annali civili del regno delle Due Sicilie (1839) 69.

278 Atti della settima adunanza degli scienziati italiani tenuta a Napoli dal 20 di settembre al 5 di ottobre del 1845 (1846) parte seconda, 35.

279 Renscher (1846) 494-527. Sulla partecipazione di Mittermaier a tale congresso: BaLeSTRERI (1983) 97-140, in particolare 101. Il testo di Sacchi presentato, come si è detto, in quel contesto fu molto recensito anche in Germania. Si segnala in particolare la recensione di Schmidt che avanza alcune critiche al Prospectus: "Einer Auseinandersetzung derselben müssen wir uns enthalten, weil ein Excerpt uns zu weit führen würde, der Gegenstand auch mehr in die Wissenschaft des Rechts als der Geschichte einschlägt und die Titelangabe genügt um dem lüsternen Fachgenossen zur Erreichung der Schrift zu verhelfen. Ebensowenig können wir aber auch andererseits dem Wunsche des Vf. entsprechen, ihn auf etwa anderwärts befolgte Methoden in der Bearbeitung der Staatswissenschaften als eines Ganzen oder einer Einheit zu verweisen, weil solcher Versuche denn doch schon zu viele wenn auch mangelhafte gemacht sind, als dass wir nicht befürchten müssten den deutschen Leser durch die bibliographischen Anführungen zu belästigen und weil es dem Vf. unmöglich schwer werden kann, auf anderem Wege zur Kenntniss dessen zu gelangen, was er in der Form einer Anfrage an die Germanistenversammlung zu wissen begehrt. Im Uebrigen freuen wir uns aufrichtig über die Achtung, die der italienische Vf. der deutschen Wissenschaft, und über die Theilnahme die er der Germanistenversammlung wenn auch in etwas befremdender Weise bezeugt hat. Denn was sollte aus dieser werden, wenn jeder Schriftsteller verlangen wollte, von ihr Recensionen seiner Werke zu empfangen! »: Sснміdт (1847) 276.

280 Riferimenti al Prospectus riportati in: Sacchi, Prefazione, in: Vattel (1854) 16. 
zioni internazionali mantenute con gli altri Stati nella forma di trattati di pace e di commercio, per poi concentrarsi sull'assetto interno dello Stato, nella suddivisione tra diritti individuali (uguaglianza civile, libertà civile e proprietà) e interesse pubblico (religione, istruzione, industria, agricoltura e commercio). ${ }^{281}$

Sacchi, infatti, acceso sostenitore nonché servitore della monarchia borbonica, visse il periodo della rivoluzione indipendentista siciliana del 1848 quando il re, Ferdinando II, cercò di arginare le richieste liberali concedendo la costituzione con il regio decreto del 29 gennaio 1848.

Fu a seguito di detta rivolta e delle successive, che scrisse Delle istituzioni di governo del reame delle Due Sicilie, ragionamenti (Napoli 1849), in cui contestò il diritto alla rivoluzione, sostenendo energicamente il sovrano e le sue direttive. $^{282}$

Per Sacchi l'onda rivoluzionaria che si «abbatteva » su gran parte degli Stati europei era frutto di « un prestigio di idea e non un'idea vera» e si sentiva sempre più urgente la necessità che "venisse neutralizzata l'azione di questo prestigio, sì che non torni più a turbare le condizioni e la pace de' popoli, e di fare una prova generosa dei fatti che ha prodotto ${ }^{283}$ Delle istituzioni di governo del reame delle Due Sicilie fu altresì redatta per criticare l'opera del letterato e concittadino Michitelli Francesco (1797-1863) intitolata Storia degli ultimi fatti di Napoli fino a tutto il 15 maggio 1848, che venne stampata nel 1848 in mille esemplari dalla tipografia Barone di Napoli e

281 Il saggio si concludeva con queste parole: "J'espère avant tout que le Congrès composé des hommes les plus illustres de la Savante Allemagne voudra bien donner le programme d'un ouvrage dont la méthode pût être semblable pour tous les États. La Création n'a qu'un principe unique. Les éléments de la vie sont les mêmes pour tous les hommes, sauf les conditions des différents endroits de la terre. Les lumières de l'intelligence sont presque également répandues partout. La loi d'amour est imprimée dans tous les cœurs. Le commerce rapproche les hommes des régions les plus éloignées, et les unit par leurs sentiments et leurs intérêts. Et pourquoi les institutions ne pourraient-elles pas être, si non les mêmes, du moins semblables? »: SACCHI (1846) 55-56.

282 I Ragionamenti contenuti nell'opera di Sacchi sono due ed erano stati pubblicati separatamente, come afferma lo stesso autore, solo qualche mese prima dal periodico L'Araldo giornale militare, politico, scientifico letterario (ai numeri 86-89), che all'epoca era diretto da Michele Rota, ufficiale dell'esercito Reale: SACCHI (1849) 3. Su questo periodico e sulla sua diffusione nel Regno delle Due Sicilie si rimanda a: Ottonello (1977) 34; Di Stasio (1995) 33 ss.

283 SACCHI (1849) 61.

236 Capitolo IV 
ancora nel 1849 senza indicazioni tipografiche; ${ }^{284}$ essa è divisa in tre parti, dedicate rispettivamente ad una introduzione storica, alla ricostruzione dei fatti rivoluzionari ed infine all'illustrazione di documenti, cronache giornalistiche e informazioni anche riservate. Michitelli divenne per le sue posizioni un fervente antiborbonico e fu subito segnalato al governo. Il saggio di Sacchi invece sosteneva i Borboni, opponendosi alle tesi del Michitelli, il quale subì pesanti persecuzioni e nel 1853 fu prima rinchiuso in carcere e poi allontanato da Napoli. ${ }^{285}$

La posizione di Sacchi è espressamente filomonarchica: a Ferdinando II dovevano essere attribuiti due titoli di gloria, il primo «a' miglioramenti che negli anni decorsi del suo regnare hanno ricevuto le istituzioni medesime, e più ancora le condizioni del Reame; il secondo veniva attribuito " alla saviezza e alla forza con che ha salvato le nostre istituzioni e le nostre condizioni dai violamenti e dalle ruine che han sofferto specialmente gli altri Stati d'Italia e che ha mostrato fra le relazioni e le complicazioni della politica estera ${ }^{286}$

Il Sovrano aveva concesso la costituzione, ma era «stato il primo ad avvedersi degli eccessi a' quali il predominio della demagogia trasportava i popoli, ed a reprimerne gli attentati ", ${ }^{\mathbf{2 8 7}}$ vagliando « i principi della ragione de' popoli quelli che sono stati sostenuti dalle armi del Re ${ }^{288}$

Le insurrezioni del Regno delle Due Sicilie diventano cos̀̀ spunto per una riflessione sul concetto di sovranità, sul principio di nazionalità e sull'equilibrio politico degli Stati, attraverso un excursus storico e una comparazione tra i diversi Stati europei.

Egli sosteneva che:

Gli uomini della rivoluzione del 1848 han dato da intendere due errori, le cui tristi conseguenze è pur toccato ai popoli di sperimentare: 1 . che i trattati stabiliti nel Congresso di Vienna del 1815 e posteriori avessero violato o distrutto il principio della nazionalità, e che eglino volessero ricomporlo; 2 . che il principio del non intervento avesse rivocato quello determinati e guarentiti dalle Potenze di Europa co' i trattati medesimi, e che sorreggesse non meno le ribellioni in ciascuno Stato, ma con una aperta contraddizione di principii, anche la guerra generale in Europa, ch'eglino moveano con prestigio di ricomporre le nazionalità », ${ }^{289}$

284 Ciferri (2010) 339-341.

285 Ibidem.

286 SACCHI (1849) 4-5.

287 Ivi, 8.

288 Ivi, 11.

289 SACCHI (1849) 46-47. 
concludendo con una forte e drastica posizione contro i rivoluzionari e la loro «ideologia ». 290

Un ulteriore episodio merita di essere richiamato e contribuisce a delineare ancora di più la complessa e attiva figura di Sacchi: è lo scontro diplomatico-politico con lo statista inglese William Ewart Gladstone, il quale nel 1851, di ritorno da un soggiorno a Napoli, aveva denunciato aspramente il regime poliziesco borbonico attraverso due lettere indirizzate a George Hamilton Gordon, conte di Aberdeen, suo compagno del partito Tory, già in passato Segretario agli Affari Esteri e futuro Primo Ministro. Le due missive furono poi raccolte nel volume intitolato Two letters to the Earl of Aberdeen, on the state prosecutions of the Neapolitan government (London, Murray). ${ }^{291}$ Sacchi prontamente nell'ottobre 1851 diede alle stampe le sue

290 Ivi, 52: "Se gli uomini della rivoluzione del 1848 fossero stati di buona fede nel voler costituire il governo monarchico temprato dalle leggi, noi li chiameremmo con ugual buona fede a disaminare (esame che eglino han voluto evitare perché non avevano la coscienza e forse né anche la capacità di sostenere) le leggi e i principi co' i quali è ordinata l'azione dei governi delle Monarchie costituite in Europa [...], ma il disegno che guidava la rivoluzione e che successivamente è stato svelato era quello di aggiungere alla repubblica, in cui la setta con la sua fazione si fosse costituita a potere, non che sovrano costituente, minaccioso di spargere a mezzo dei popoli il terrore del sangue e degli orrori del comunismo ». Sacchi è anche autore di diversi trattati di argomento per lo più filo-monarchico: Della quistione italiana per quanto potesse riguardare il reame delle Due Sicilie (1859a), e Intorno al richiamo delle legazioni francese ed inglese da Napoli (1859b). Inoltre tra il 1836 e il 1848 scrisse Osservazioni sul la giustizia convenienza e possibilità della conversione delle rendite pubbliche (Napoli 1836); Discorso di Terenzio Sacchi fra' $i$ suoi colleghi nel real ministero degli affari interni (Napoli 1840).

291 Cfr. Gajo (1973) 31-47. Gladstone nella sua prima lettera per giustificare la necessità di scrivere a Gordon nel manifestargli le sue impressioni a seguito del viaggio a Napoli argomentava che: "Without entering at length into the reasons which have led me thus to trouble you, I shall state these three only: first, that the present practices of the Government of Naples, in reference to real or supposed political offenders, are an outrage upon religion, upon civilisation, upon humanity, and upon decency. Secondly, that these practices are certainly, and even rapidly, doing the work of republicanism in that country: a political creed, which has little natural or habitual root in the character of the people. Thirdly, that as a member of the Conservative party in one of the great family of European nations, I am compelled to remember, that that party stands in virtual and real, though perhaps unconscious, alliance with all the established Governments of Europe as such; or and less that, of according moral detriment to the measure from its of reverses, its influence, and they drive suffer strength more, and encouragement from its successes. This principle, which applies with very limited force to the powerful States, whose Governments are strong, not only in military organization, but in the habits and affections of the 
Brevi risposte in cui, scrivendo " come napoletano ", puntualmente contestò le accuse del Ministro inglese, rivendicando quanto il sovrano si fosse prodigato per il suo popolo e ribadendo la sua posizione filoborbonica. ${ }^{292}$

Dopo questo saggio maturò l'idea di dedicarsi alle teorie sulla sovranità e sulla nazionalità, iniziando la stesura del Diritto delle genti di E. de Vattel applicato allo stato attuale delle nazioni che fu pubblicata nel 1854 e fu recensita anche all'estero. ${ }^{293}$ Tale opera consiste solo nella parziale traduzione del primo libro di Vattel, quello relativo ai principi che regolano una nazione.

Come acceso sostenitore della monarchia borbonica dedicò il suo lavoro a Ferdinando II, il quale ufficialmente accettò l'omaggio di Sacchi all'interno

people, is a principle of great practical importance in reference to the Government of Naples, which, from whatever cause, appears to view its own social, like its physical, position, as one under the shadow of a volcano, and which is doing everything in its power from day to day to give reality to its own dangers, and fresh intensity, together with fresh cause, to its fears »: GLadstone (1851) 6.

292 Sacchi così rispondeva alle invettive dell'illustre politico inglese: «Noi, nel volere ancora rispondere con animo napoletano alle lettere del signor Gladstone, muoveremo da altro principio, il quale non ci potrà essere contraddetto. Lo Stato, che nella presente civiltà di Europa è la espressione politica delle nazioni, comprende insieme il popolo con le sue condizioni e il governo con la sua azione. Il perché la civiltà di questo Reame, di per sè ed anche nella relazione delle nazioni incivilite, formata da questi due elementi con le memorie che vi si congiungono, non è patrimonio ed interesse del solo governo; ma ogni nazionale che ne è a parte à essenzialmente il debito di sostenerla, non solo ne' conflitti armati che attentassero alla sicurezza dello Stato, ma anche nel contrasto d'idee e di fatti che si cercasse far valere per vulnerarne la opinione. Chè se pure in tal maniera di differenze altri potesse mai prevalere o per maggiori mezzi personali, o per forza influenza dominio più esteso dello Stato a cui appartenga; la ragione e il diritto sono sempre uguali indistintamente per tutti, che possano anche soggiacere ad una condizione di fatto ". SACCHI (1851) 8-9.

293 Questa edizione parziale non compare nel repertorio di NAPOLI (1986). Si segnala in modo particolare la recensione di Derôme pubblicata sulla Revue critique de législation: " aujourd'hui même une importante publication excite l'intérêt de tout ce que le royaume des Deux-Siciles renferme d'esprits élevés, et nous croyons devoir appeller l'attention des jurisconsultes français sur un ouvrage qui promet au droit international un docte et profond interprète. L'auteur, M. Terenzio Sacchi, a rempli d'éminentes fonctions dans l'administration de son pays. [...] Ainsi, d'une part, sa position nous garantit que, mêlé aux affaires publiques, il ne saurait être confondu avec ces écrivains que l'inexpérience de la vie pratique expose aux aberrations des théories inapplicables; d'un autre cotê, une sorte d'adoption royale imprime à son ouvrage un caractère semI-officiel qui a bien son intérêt, puisqu'il nous autorise en quelque sorte à voir dans les doctrines d'un simple particulier les principes reconnus et avoués d'un gouvernement »: Derôme (1856) 455-469, qui 457. 
del Consiglio ordinario di Stato del 14 ottobre 1853. Al termine della dedica, Sacchi illustrava immediatamente il metodo e l'approccio utilizzato per la traduzione dell'opera di Vattel al fine di «rettificare coteste teoriche, con riportare i principî a' loro elementi ed applicarli allo stato attuale delle nazioni, ovvero al grado di civiltà a cui sono pervenute, è opera degna dell'età nostra ». ${ }^{294}$

Il suo scopo si concretizzava nel voler applicare, ma al tempo stesso a correggere, il Diritto delle genti di Vattel in base allo stato attuale delle nazio$\mathrm{ni}$, ripercorrendo le varie fasi del trattato e delle annotazioni che furono apportate da alcuni giuristi soprattutto durante il XIX secolo. Proprio su questo aspetto si discostava dal commento di Pinheiro Ferreira, pubblicato come terzo volume al Droit des gens nel 1838.

Non solo si oppose al metodo di lavoro utilizzato da Pinheiro, in particolare al suo intento di voler completare l'opera, ma sentì l'esigenza di modificare l'ordine con cui Vattel aveva scritto. Per il giurista napoletano la divisione in quattro libri e l'inserimento dei Preliminari non era corretta e necessitava di una profonda revisione nella struttura e nell'importanza delle materie da trattare. Analizzava altresì scrupolosamente il pensiero del giurista di Neuchâtel articolando le sue critiche in tre punti precisi.

Il primo concerneva la somiglianza del Droit des gens all'opera di Wolff, « essendo il suo trattato in certa guisa un compendio del diritto di natura e delle genti di questo capo-scuola; la quale scuola cadeva nell'errore di considerare gli uomini nel così detto stato di natura, ch'è una ipotesi ideologica cui contrastano insieme la natura della vita umana e gli elementi delle relazioni degli uomini e delle nazioni ». ${ }^{295}$

Nel secondo punto contestava a Vattel l'aver consacrato il "possibile morale » come principio del diritto mancando «di forza di fede più che di dottrine, per seguitare non che i principî propri della moderna civiltà da' i quali era discostato dal sistema d'idee cui ha seguito, ma gli stessi pretesi principî della sua scuola, [...] chiama eccessi dello spirito democratico, che già cominciavano a mostrarsi nelle opere di taluni scrittori ${ }^{296}$

294 SACCHI, Alla sacra real Maestà di Ferdinando II. Re del Regno delle Due Sicilie, in: Vattel (1854).

295 Sacchi, Prefazione, in: Vattel (1854) 18.

296 Ibidem. 
Nel terzo passaggio, viceversa, si scagliava contro quanto sostenuto dal pubblicista portoghese (cioè Pinheiro Ferreira) per la sua giustificazione alle teorie di Vattel quando scrisse che:

cependant nous devons dire, à l'honneur de Vattel, que le style embarrassé dans lequel sont exposées les doctrines révoltantes que nous venons de lire, surtout si on les compare avec la lucidité des principes de haute morale publique que respire d'un bout à l'autre son bel ouvrage, nous conduit à penser que son intention était moins de faire l'apothéose des Tibères ou des Héliogabales, que mettre un frein au débordement de l'esprit démocratique, qui, de son temps, commençait déjà à se faire jour dans les ouvrages de quelques écrivains célèbres. ${ }^{297}$

L'osservazione di Pinheiro fu il pretesto per Sacchi di ampliare la sua critica contro Vattel, le cui teorie erano prive "de' maggiori lumi, di che la scienza, se pure ad una scienza unica possano essere ridotti i principî di cui trattiamo e le loro applicazioni, è stata cresciuta nel volgere dell'ultimo secolo ». ${ }^{298}$ Tuttavia si discostava anche dalle pretese di completezza di Pinheiro Ferreira il quale per "giustificare " le teorie di Vattel, che lui stesso definiva generiche, aveva fatto ricorso alle posizioni di alcuni giuristi e soprattutto alla scuola di Bentham. Per Sacchi il cosiddetto "principio utilitario ",

demoralizzava la stessa idea del giusto assunta come principio del diritto: ovvero prentendeva stabilire, giovandosi di ciò che era già fatto, una specie di sistema di principi, che annodando i passati a successivi rivolgimenti, avesse potuto poi reggere un sistema di cose, i cui elementi fossero improntati alle idee di questa scuola. ${ }^{299}$

Analizzò anche l'Avertissement di Pinherio Ferreira al Droit des gens di Vattel: era concorde nel ritenere tale testo un'opera classica. Essa « è ancora citata come testo di dottrine, specialmente nel diffinire i diritti delle nazioni ed i mezzi per sperimentarli »; sosteneva Sacchi che, ovviamente i rapporti politici e commerciali tra le nazioni fossero cambiati rispetto al secolo precedente quando Vattel dava alle stampe il suo trattato, ma questo cambiamento, a differenza di Pinheiro, non influiva sugli elementi dello stato politico delle nazioni, sul principio costituivo dei governi che, a pare suo, rimaneva invariato. $^{300}$

Il giurista napoletano dunque non solo prese le mosse dalle teorie di Pinherio, ma addirittura «le diffidava e cominciava a disdergnarsene ». ${ }^{\mathbf{3 0 1}}$

297 Pinheiro Ferreira, in: Vattel (1838) L. I, $\mathbb{S}$ 49-50, 55.

298 Sacchi, Prefazione, in: Vattel (1854) 20.

299 Ibidem.

300 Ivi, 21.

301 Ibidem. 
Già all'interno di questa lunga e interessante Prefazione, avvisava il lettore che le fonti principali da lui utilizzate per commentare il Droit des gens furono gli scritti di François Guizot, celebre politico e storico francese, deputato conservatore, che difese la monarchia liberale di Luigi Filippo e si oppose al costituzionalismo orleanista nella sua accezione democratica. ${ }^{302}$

Sacchi si riferiva soprattutto alle dottrine di Guizot contenute nelle quattordici lezioni sulla storia generale della civilizzazione in Europa, all'interno delle quali concepiva la civiltà come un destino inarrestabile, che dal crollo dell'Impero romano reggeva la storia del mondo senza tenere conto degli eventi transitori e vedeva la civiltà comporsi di due sviluppi, lo «sviluppo sociale » e lo «sviluppo morale», proclamandone l'intima e necessaria connessione. ${ }^{303}$

Guizot, prendendo spunto dalla sua epoca, sosteneva, nelle relazioni tra i fatti e le idee, che occorresse far prevalere di più i fatti sull'ordine intellettuale e all'interno dei fatti la ragione: Sacchi citava proprio l'intero passaggio dello storico francese:

depuis quelque temps se manifeste parmi nous un goût déclaré, je dirai même une sorte de prédilection pour les faits, pour le point de vue pratique, pour le côté positif des choses humaines $[\ldots]$ nous sommes appelés à considérer, à faire marcher ensemble, la science et la réalité, la théorie, et la pratique, le droit et le fait [...] c'est un progrès nouveau, un grand pas dans la connaissance et vers l'empire de le vérité [...] nous n'éviterons nullement les questions générales et philosophiques; nous n'irons pas les chercher, mais quand les faits nous y amèneront, nous les aborderons sans hésitation sans embarras. ${ }^{304}$

A queste riflessioni il giurista napoletano ne aggiunse delle altre che si rendevano necessarie, a seguito degli ultimi fatti storici (accaduti anche nel Regno delle Due Sicilie), per chiarire e giustificare l'elaborazione della sua nuova versione del Droit des gens di Vattel.

Sacchi sottolineava che nel momento in cui Guizot nel 1843 pubblicava la sua opera l'Europa non aveva ancora attraversato le grandi prove e i grandi sconvolgimenti interni, che sarebbero arrivati pochi anni dopo. Infatti la stessa Francia nel febbraio 1848 fu travolta dall'insurrezione che destituì la monarchia, mandando in esilio Guizot e proclamando la Repubblica con la

302 Su Guizot cfr. Chamboredon (actes réunis par) (2010); Clerici (2012) 47-69; Theis (2014). Anche i contributi di: Ihl (2004) 125 ss.; Pozzi (2010) 1-8; Pozzi (2014) 73-85.

303 Pozzi (2010) 1-8.

304 Guizot (1863) 95-97. 
contestuale formazione di un governo provvisorio formato da repubblicani, democratici e socialisti; le insurrezioni durante il regno di Ferdinando II, che Sacchi visse in prima persona; il 1848 nell'Italia centro-settentrionale; la rivoluzione nell'Impero asburgico e nella Confederazione germanica.

"L'età nostra, diceva Sacchi, è ingiustamente sopraccaricata di accuse, come se fossero veramente sue colpe i disordini di cui è stata tratta, ed i mali cagionati ». ${ }^{305}$ Egli, riprendendo le parole di Guizot, sostenne fermamente che scienza e realtà, teoria e pratica, diritto e fatto dovessero essere considerate come un tutt'uno, evitando di cadere in questioni generali e filosofiche perché un principio "non può essere mai razionale se non è basato con elementi del vero in cui gli uomini abbiano fede ", e qualora venisse abbattuto ed distrutto dalla forza che domina tutti gli avvenimenti storici, di certo «non può mancare a se stesso ed alla propria azione ». ${ }^{306}$

Per applicare il trattato di Vattel « allo stato attuale delle Nazioni », Sacchi effettuò qualche modifica nell'ordine dei capitoli e dei paragrafi del Droit des gens "secondochè sarà necessario » e ponendo le osservazioni, "nel fine di ridurre le une e le altre teoriche agli elementi del vero, pe' quali unicamente possono essere certi i principi della scienza ». ${ }^{\mathbf{3 0 7}}$

Egli precisò il contesto storico (riferendosi al Regno delle Due Sicilie), l'anno e il luogo di pubblicazione. La sua opera era stata redatta nel 1854 dopo «le prove che gli Stati della più parte di Europa han fatto tanto dei principi che saranno fermati ed applicati allo stato attuale delle nazioni, quanto dei pretesi principi che da più o meno remota stagione si è cercato di sostituirvi ». ${ }^{308}$

Sacchi, Prefazione, in: Vattel (1854) 26.

306 Ivi, 25. Ecco così spiegato l'intento di Sacchi che attraverso le teorie di Vattel e quelle che sono state aggiunte da Pinheiro, ha voluto illustrare: «i principi con i quali, nella maggiore civiltà delle genti e tra le prove che hanno subito nel trionfare degli avvenimenti, sono costituite le Sovranità degli Stati, e sono ordinati lo stato e le relazioni delle persone e delle nazioni. Nè qui vogliamo tacere delle polemiche, che dagli ultimi anni si sono levate a propugnare i principii medesimi a fronte dei pretesi principii che si è cercato di sostituirvi: ammiriamo in esse più elevate e secure dottrine; ma in verità non siamo gran fatto persuasi del metodo che, rendendo tali dottrine troppo staccate e speciali, potesse mai sviare i principii medesimi dalla unità e dalla misura che debbono serbare nelle loro applicazioni, ed anche estendere l'azione di un principio a danno dell'altro »: Ivi, 26.

307 Ivi, 28.

308 Ibidem. 
Inoltre la scelta di Napoli, come luogo di pubblicazione, per Sacchi significava che «in questo Reame le genti vivono e s'inspirano nella fede della Chiesa cattolico-apostolica-Romana, ch'è per noi inviolabile; sì che escluderemo dalle teoriche di Vattel e del giurista portoghese, quelle che mai attentassero o contrastassero alla inviolabilità della Religione che professiamo ${ }^{309}$

La modifica dei Preliminari dimostra quanto Sacchi sia intervenuto sull'ordine strutturale e formale stabilito da Vattel. Mentre il giurista di Neuchâtel introduceva la sua opera con la definizione di Stato, Sacchi partiva dalla definizione di diritto delle genti, aggiungeva la posizione di Pinheiro, che accusava Vattel di essere stato semplicistico e squisitamente filosofico, per poi concludere con le sue riflessioni. ${ }^{310}$ Sacchi si chiedeva se il trattato stabilisse solidamente gli obblighi ed i diritti delle nazioni, oppure facesse conoscere come gli Stati avrebbero dovuto regolare le loro azioni e altresì se il diritto delle genti fosse una scienza del diritto o disciplinasse solo i principi della legge naturale per la condotta dei Sovrani.

In una lunga dissertazione, mentre affermava che la teoria vatteliana era «luminosa» e «raggiunge nel «possibile morale» il principio del diritto così per gli uomini come per le nazioni "; ${ }^{311}$ si discostava dalla posizione assunta da Pinheiro, poiché aveva sostenuto, riprendendo le teorie di Bentham, che il principio di ogni diritto e di ogni dovere non fosse altra cosa che il principio del giusto e "non vi è giusto che se non quello che produce la maggior somma di bene a tutti».

Sacchi constatava però che il principio del giusto nel pensiero di Pinheiro e Bentham restringeva il concetto del « possibile morale » limitando la teoria di Vattel:

imperocchè la moralità comune degli uomini e delle nazioni impone due doveri, che sono ovvi nella ragion comune: l'uno quasi negativo di non fare agli altri ciò che non si vorrebbe fatto a se stesso; l'altro positivo, di fare agli altri quello che si vorrebbe fatto per se. Il principio del giusto quasi si restringe al primo dettato: il possibile morale li comprende entrambi, ${ }^{312}$

tale idea per Sacchi non solo era falsa ma anche impossibile poiché:

309 Ibidem.

310 Ivi, 35-36.

311 Ibidem.

312 Ivi, 37. 
la maggior somma di bene a tutti in generale ed a ciascuno in particolare è conseguenza e non principio: l'elemento del possibile morale, anzi che nel bene e nell'utile che deve derivarne, sta nel vero che si contiene negli esseri della natura che sono gli elementi della vita, nelle leggi eterne della creazione di Dio che soli avvanzano le misure del tempo e dello spazio. [...] gli uomini e le nazioni stanno più securi su la via per la quale possono giungere alla conoscenza ed alla fede del vero, che su quella in cui fossero già sotto l'azione ed il dominio dell'errore. ${ }^{313}$

Anche negli ulteriori commenti Sacchi non si sottrae a lunghi ragionamenti filosofici. Ad esempio nell'annotare la definizione di Vattel sul diritto della natura e delle genti il giurista napoletano utilizza come fonte il Discourse on the study of the law of nature di James Mackintosh di cui traduce interi passaggi e ne commenta il pensiero e, a suo dire, lo completa con osservazioni di stampo per lo più cattolico che trovano la loro origine nella rivelazione divina e nel rapporto tra la ragione, come mezzo per l'uomo di arrivare alla conoscenza, e il vero, cioè l'insieme delle leggi eterne della creazione. ${ }^{314}$

Dalle considerazioni e dai commenti di Sacchi si possono trarre le seguenti osservazioni. In primo luogo il giurista napoletano, esattamente come aveva anticipato nella sua Prefazione, non esita a rivestire la dottrina vatteliana di un velo più marcatamente cattolico, dalla quale si possono ricavare $\mathrm{i}$ principi non solo per quanto riguarda le relazioni umane, ma anche per quelle internazionali. In secondo luogo si delinea un approccio volto ad applicare il trattato allo « stato attuale delle Nazioni » nella tensione di voler conciliare « la scienza e la realtà » e « la pratica e la teoria ».

Si assiste così ad una sorta di "snaturalizzazione » formale e sostanziale del testo di Vattel. Formale poiché vengono cambiati gli ordini dei paragrafi, le note prima poste a piè pagina vengono ora tradotte e collocate a livello del testo principale e le Notes di Pinheiro vengono altresì intercalate con il testo del giurista di Neuchâtel. Sostanziale poiché Sacchi sembra quasi forzare, in virtù di una inclinazione cattolica e della "ricerca degli elementi del vero ", una lettura al testo di Vattel in chiave per così dire moderna, capace quindi di poter essere ben accettata dalle istituzioni e dal governo del Regno delle Due Sicilie.

Non si conoscono i motivi dell'interruzione della traduzione di Terenzio Sacchi, ma, evitando una lettura forzata, molto probabilmente Sacchi aveva intuito che il suo tentativo di attualizzare, soprattutto dal punto di vista

313 Ivi, 38.

314 Ivi, 59-60. 
costituzionale, il Droit des gens mal si conciliava con le esigenze e i fatti storici del periodo, e alle istanze di unificazione italiana che si levavano sempre più forti su tutta la penisola.

Accanto all'unica edizione italiana del Droit des gens, alle posizioni dei giuristi patrioti italiani nel corso del periodo risorgimentale è opportuno evidenziare un terzo livello di ricezione del pensiero vatteliano che si manifesta nel giudizio espresso da parte della dottrina italiana di diritto internazionale. Nicola Rocco, sostituto procuratore del Tribunale civile di Palermo poi procuratore della Gran Corte civile napoletana, fu un illustre professore di diritto commerciale e internazionale nell'Università di Napoli, dando un significativo impulso all'esposizione sistematica del diritto internazionale privato. ${ }^{315}$ In Dell'uso ed autorità delle Leggi nel Regno delle due Sicilie considerate nelle relazioni con le persone e col territorio degli stranieri pubblicato da Rocco a Napoli nel 1837, Vattel veniva definito come un autore di estrema lucidità nell'esporre in particolare le questioni che attengono alla disciplina dell'immunità dei ministri pubblici presso gli Stati esteri; alle regole da osservarsi verso gli stranieri che sono di passaggio o residenti in un altro paese, soffermandosi sulle controversie che possono sorgere tra uno straniero e un cittadino e la scelta del giudice competente. ${ }^{316}$ Per Rocco Vattel era «l'ultimo più grande giuspubblicista ». ${ }^{\mathbf{3 1 7}}$

Fu proprio tale opera a fare ottenere a Rocco fama a livello internazionale: Joseph-Marie Portalis nel 1842 in una relazione tenuta presso l'Accademia delle Scienze morali e politiche francese, notò l'alto valore scientifico che acquistò sin da subito autorità dottrinale nelle scuole e nel foro; ${ }^{318}$ è inoltre Mancini nell'Esame di un'opera di dritto internazionale a tesserne gli elogi attraverso un bilanciamento tra le argomentazioni di Rocco e i più spinosi casi di diritto internazionale privato, senza omettere le critiche sull'elaborazione, ad esempio, dei criteri per la risoluzione dei conflitti di legge nello spazio e alla esigenza che vi fosse un «principio supremo unico » che

315 Sulla figura di Rocco cfr. Zaniboni (2013) 1709-1710. Per le argomentazioni di Rocco sul diritto internazionale privato e sulla condizione giuridica dello straniero: STORTI (1990) 212 ss.

316 Rocco (1837) XVII, 88, 109, 142, 151-157, 166-167, 177-178, 196, 256, 273-275.

317 Ivi, 109.

318 Per Portalis il trattato di Rocco «è un eccellente manuale di diritto civile internazionale ", meritevole di traduzione. Il Rapport di Joseph-Marie Portalis pubblicato sul Moniteur Universel del luglio 1842 ed è interamente trascritto in: Rocco (1859) III-XXVIII. 
regolasse tanto la pratica quanto le questioni generali. ${ }^{319} \mathrm{Ma}$ in questo saggio Mancini non esita anche ad offrire un excursus storico sulla disciplina, mettendo in luce il merito di Rocco che va oltre il lavoro compiuto dai precedenti giusnaturalisti e non esitando a dipingere Vattel come un giurista superato, collocato insieme a Grozio e a Pufendorf nella storia, le cui teorie erano definite come «secche » e non più utilizzabili in comunità internazionale in continua evoluzione. ${ }^{\mathbf{3 2 0}}$

Nel lavoro di Rocco frequenti sono i riferimenti all'opera di Vattel: le sue teorie sono il punto di partenza e di confronto per gli sviluppi e le argomentazioni successive, oscillando tra l'appartenenza di Vattel al pensiero giusnaturalista e le esigenze, a distanza di quasi un secolo, di specializzazione e scientificità nel diritto internazionale privato. Emerge nell'analisi della dottrina internazionale italiana ottocentesca una doppia posizione nei confronti di Vattel che è frutto della realtà contingente: riconoscevano durante la loro epoca che egli godeva ancora di una notevole autorità soprattutto in ambito diplomatico e politico, ma si assiste progressivamente alla collocazione del suo pensiero nella storia del diritto delle genti, prediligendo quindi un sviluppo del diritto internazionale autonomo nel contesto in cui è stato creato.

Massimo Panebianco argomenta acutamente che in Italia le opere di diritto internazionale erano più sensibili alle vicende del corso storico di cui gli Stati erano protagonisti: ${ }^{321}$ le Lezioni del diritto internazionale di Ludovico Casanova pongono "la storia come fonte del diritto internazionale» per giustificare una tradizione che appunto sembra in qualche modo caratterizzare la scienza italiana, senza essere abbandonata sino al secolo XX,

319 Mancini (1844) 16; anche Storti (2013) 1247. Augusto Pierantoni formula un giudizio favorevole sull'opera di Rocco: nel 1869 nella sua Storia degli studi del diritto internazionale in Italia così si espresse: "guardando l'epoca di questa pubblicazione [1837] si ravvisa immantinente che un tal libro provveda all'avanzata necessità del tempo, nel quale si erano strette relazioni e create novelle attinenze tra $\mathrm{i}$ popoli risguardati quali individui di una sola universale famiglia " e poi continuava "Rocco indovinò la materia e servì nobilmente la scienza »: Pierantoni (1869) 105-106.

320 Scriveva Mancini sulla necessità di indagare sulle dinamiche che regolavano i principi del diritto internazionale privato: «si è a poco a poco avvertito il bisogno di studiare gli effetti giuridici derivanti dalle indicate relazioni, e così si è venuta formando una massa considerevole di dottrine che costituiscono oggi la scienza del dritto civile internazionale. Ma questa scienza può dirsi ancora nascente »: MancinI (1844) 12-13.

321 Panebianco (1974) 167-168. 
con diversi indirizzi, basti solo pensare agli scritti di Luigi Olivi e di Giovanni Lomonaco. ${ }^{322}$

È dunque in tale panorama che si riescono a ben comprendere le criticità di molti giuristi italiani nei confronti dell'opera di Vattel. Pasquale Stanislao Mancini, infatti, nella sua celebre prolusione Delle nazionalità come fondamento del diritto delle genti, tenuta presso l'Università di Torino nel 1851, riferendosi a Vattel scrisse che era considerato una sorta di oracolo valido esclusivamente per gli uomini di governo, e nonostante giuristi come Heffter e Wheaton si fossero ispirati al suo sistema, nella dottrina non veniva più preso in considerazione:

La impopolare aridità di questo metodo indusse in pochi anni appresso il Vattel a rendere francese la dottrina Wolfiana, e ad offrirne una specie di compendio, il quale cadde nel vizio opposto per una troppo superficiale leggerezza scientifica e per la frequente oscillazione ed incertezza nell'applicazione de' principii. Ciò non ostante il Vattel continua ad essere anche el presente l'oracolo degli uomini di governo, perché sebbene in esse non manchino talvolta soluzioni liberali e generose, pur tuttavia fra le eccezioni e le distinzioni di cui soprammodo abbonda è ancora facile assai spesso attingerne le soluzioni più opposte; e ad eccezione de' progressi posteriormente avvenuti in alcuni argomenti parziali della disciplina, può dirsi che il sistema di questo scrittore abbia inspirato tutti gli altri più recenti fino all'Heffter ed al Wheaton recentissimi. ${ }^{323}$

Augusto Pierantoni è ancora più incisivo e pungente nei confronti di Vattel, di Pufendorf e Wolff, ma in linea con il pensiero di Mancini: ${ }^{324}$

il Vattel non ammetteva una società naturale tra i popoli, e negava che le società civili fossero una creazione della natura. Strana contraddizione davvero in uno scrittore, che volendo formolare le leggi naturali regolatrici degli stati esordiva col negar loro una esistenza naturale, la quale si spiega soltanto per l'avvertenza che nessun uomo giunge a isolarsi pienamente dai pensieri del suo tempo, ${ }^{325}$

322 Ibidem. Cfr. anche: Storti (2012) 51-146; Fugazza (2014). Su Casanova: Piergiovanni (2001) 193-216; Storti (2001) 53-94; Braccia (2013) 474-475. Per Olivi Luigi: Passero (2013a) 1455; Su Lomonaco Giovanni: VANO (2013) 1192-1193.

323 Mancini (1851) 19. Solo sinteticamente si rimanda per Mancini a: Storti (2013) 1244-1248; Mongiano (2013) 85-97; Nuzzo (2012c) 307-311; Mancini Pasquale Stanislao (2007) 537-547; Mele (2002) 9 ss.; Pene Vidari (2002) 274 ss.; Nishitani (2001) 23 ss.; Torre (1995) 404-405; Jayme (1987); Morelli (1955) 49 ss.; Droetto (1954) 29 ss.

324 Cfr. su Pierantoni: Passero (2013b) 1576; Fiocchi Malaspina (2015b) 890-891; Sereni (1943) 178-180.

325 Pierantoni (1874) 16. 
e continuando la sua posizione così scriveva: «Tralasciando di ripetere le censure, che meritarono le opere di questi scrittori, i quali lungi dal prendere ispirazione dalla realtà della storia e del progresso, si raccolsero in nude astrazioni e sconobbero quasi sempre la efficacia di un diritto delle genti necessario, avvolgendolo delle collisioni e negli attriti di un diritto volontario ${ }^{326}$

Egli affermava e confermava che in Italia «Scarsa fu la influenza delle loro opere dettate men per il popolo che per essere i manuali degli uomini di gabinetto $»{ }^{327}$

A queste efferate critiche si accostavano quelle che vedevano Vattel come per Giuseppe Carnazza Amari, l'imitatore di Wolff, ${ }^{328}$ ma anche posizioni decisamente più miti che appoggiavano l'incidenza del Droit des gens sull'elaborazione e sugli sviluppi della scienza giuridica internazionale: Pasquale Fiore all'interno delle sue opere richiamava il giurista di Neuchâtel, e sebbene sviluppò un suo originale pensiero, si sentiva influenzato per formazione giuridica e culturale alla corrente giusnaturalistica, ${ }^{329}$ per poi intrecciarsi inevitabilmente con « criteri di positività e di equilibrato realismo ». ${ }^{330}$

Dionisio Anzilotti nei primi anni del Novecento sottolinea l'autorità di Vattel, ancora attuale, alla sua epoca e nei diversi contesti europei, tanto nella scienza quanto nella pratica. ${ }^{331}$

In Italia lo sviluppo del diritto internazionale coincise con la presa di coscienza dell'identità e dell'indipendenza nazionale; Vattel era ormai un autore troppo lontano per le aspettative italiane che necessitavano invece

326 Ibidem.

327 Ibidem. In un'altra occasione Pierantoni afferma che l'opera di Vattel « con eccessiva leggerezza scientifica compendiò e rese francese la dottrina che il Wolff aveva esposto in forme geometriche »:Pierantoni (1866) 116. Anche nella celebre ricostruzione che effettua sulla storia degli studi di diritto internazionale in Italia in moltissime occasione richiama Vattel per essere utilizzato molto nella pratica diplomatica, ma superato in dottrina: Pierantoni (1869) 55, 70-71, 106, 208, 224, 229, 245-246.

328 Carnazza Amari (1865) 15. Cfr. Brugnatelli (2013) 455.

329 Scriveva Fiore nel Nuovo diritto internazionale pubblico nel riferirsi alle « diverse scuole di diritto delle genti » che: «nell'esporre in poche parole quale sia il sistema che secondo noi si avvicina di più ai veri principii, a noi sembra doverci accostare alla opinione del Vattel »: Fiore (1865) 29. Sul punto anche: Marzano (1923) 44. Su Fiore cfr. Conetti (2013) 874-876; Augusti (2015) 37-61; Fiocchi Malaspina (2015a) 488-489.

330 Conetti (2013) 875.

331 Anzilotti (1928) 11. Si rimanda per Anzilotti a: Passero (2010); Salerno (2013c) 84-87. 
di un diritto internazionale più vicino alle esigenze del periodo e che prenderà forma da giuristi, ad esempio, come Rocco, Mancini, Pierantoni, ma al tempo stesso non venne messo da parte e fu il punto di partenza per una autonoma, peculiare e originale trattazione del diritto internazionale sia privato che pubblico.

Nel corso dell'Ottocento il diritto delle genti subì un profondo periodo di innovazione dopo le tensioni e i cambiamenti non solo storici ma giuridici ottocenteschi e la scuola italiana del diritto internazionale ben rappresentata da Mancini e dal suo principio della nazionalità fu poi proseguita da Pasquale Fiore, che propugnò energicamente una organizzazione giuridica della comunità internazionale, auspicandone la sua codificazione nei diversi rami del diritto internazionale pubblico e privato per il raggiungimento della pace. ${ }^{332}$ Il Droit des gens non forniva un valido sostegno " politico-giuridico » alle esigenze del diritto internazionale italiano ma, rientrando in quei classici del diritto delle genti, sui quali formarono gli stessi giuristi, non poteva essere ignorato e, pur nelle controverse posizioni, il suo pensiero veniva riportato, adattato, criticato senza però perdere l'aura di autorità che lo ha sempre caratterizzato.

In questo complesso scenario si delinea la figura di Vattel nelle sue molteplici sfumature: i tre livelli, giuridico, dottrinale e politico che si intrecciano permettono di cogliere differenti aspetti della circolazione del trattato. Il richiamo al pensiero vatteliano da parte dei patrioti e giuristi italiani legati al periodo risorgimentale fa risaltare il suo contributo alla teoria della sovranità statale; le posizioni di Sacchi all'interno della traduzione italiana sono propense per una lettura costituzionale con ripercussioni nella costruzione dell'ordine internazionale, nella convinzione che il Diritto delle genti dovesse essere applicato allo stato «attuale » delle nazioni, tentando quindi di unire «il diritto al fatto ", "la realtà alla scienza »; i giuristi internazionalisti italiani lo tengono in considerazione, ne rilevano l'importanza soprattutto in ambito diplomatico, senza mai concretamente mettere in discussione la loro formazione classica e l'essersi forgiati su teorie di matrice giusnaturalistica che, sebbene superate, incidevano sulle loro elaborazioni giuridiche. 


\section{Epilogo}

1. Il fenomeno del Droit des gens in prospettiva globale: una goccia nell'oceano?

L'attenzione in questa ricerca si è focalizzata su alcune realtà dell'Europa occidentale, del Nord e del Sud America, incentrando la disamina su uno studio comparativo sincronico e diacronico e dimostrando che, a più livelli, emergevano quasi simultaneamente strategie di assimilazione, riproduzione, adattamento giuridico, a seconda di precise circostanze spazio-temporali. Il fenomeno assume una rilevanza globale, come constatato dagli studi sulla storia intellettuale globale e transnazionale. ${ }^{1}$ Infatti nei paesi orientali è accaduto qualcosa di simile: ad esempio, in Cina si è assistito ad una selezione dei passaggi del trattato vatteliano, quando, alla vigilia della guerra dell'oppio, Lin Zexu, consigliere dell'imperatore Qing, entrò in contatto con il Droit des gens grazie alla traduzione di Yuan Dehui e Peter Parker. Il fine era l'instaurazione di un dialogo con gli inglesi sulle tematiche di diritto internazionale che regolavano il sistema europeo, rafforzando anche in questo caso la profonda relazione tra il linguaggio e la traduzione, e trasponendo nella scrittura le rappresentazioni di concetti distanti dalla visione orientale. ${ }^{2}$

Alla luce di quanto argomentato quale ruolo hanno avuto le traduzioni del Droit des gens e, più in generale, le opere di diritto internazionale e i relativi commenti durante il XIX secolo nel contesto globale?

Arthur Lovejoy, in una celebre frase, affermava l'intrinseco dinamismo delle idee e la loro straordinaria capacità migratoria. ${ }^{3}$ Durante il corso del XIX secolo i principi legati e vincolati all'Europa avevano una fertilissima diffusione globale, proprio attraverso il numero di traduzioni, edizioni, rie-

1 Armitage (2013) 17-32; Armitage (2004) 108-09; Moyn/Sartori (eds.) (2013). Anche: IrIYE (2013); IRIYe/ SAUNIER (eds.) (2009).

2 TANG (2012) 704-705; Svarverud (2007) 77 ss.

3 Lovejoy (1904) 4. 
dizioni, di trattati, di compendi e di manuali. Questo processo di divulgazione ha avuto una notevole rilevanza sul piano giuridico, perché ha creato la percezione che le categorie europee fossero sempre e ovunque valide:

As concepts moved - come ha scritto Christopher Hill - around the world, they experience multiple mediations, including translation into other languages and mass reproduction in the form of textbooks and publication for popular audiences. The resulting process of abstraction attenuated the concepts' connection to their originators and to the European examples from which they were derived. Mediation and abstraction allowed the 'universalization' of concepts in a specific sense. The use of a concept as if it were valid in all places at all times. ${ }^{4}$

Se è vero che "mediazione » e "astrazione " permettono la "universalizzazione » nel senso specifico del termine, allora si deve constatare che le teorie del diritto internazionale necessariamente sono incluse all'interno di questo fenomeno. Il diritto internazionale, infatti, per il suo intrinseco e peculiare fine di regolare le relazioni tra gli Stati, di creare un ordine internazionale, garante della pace, della sicurezza e del rispetto dei trattati internazionali, era sicuramente uno dei «campi » prediletti e ideali per la proliferazione di idee di matrice europea con caratteristiche universali. ${ }^{5}$

Il compito europeo per la massima fruibilità del diritto internazionale veniva svolto concretamente dagli esperti di diritto internazionale, che furono coinvolti in una sorta di lavoro di mediazione: c'erano le istanze dello Stato in relazione agli altri Stati, ma c'erano anche le istanze di tutti gli Stati all'interno della comunità internazionale, duplice obiettivo in cui intervenivano per dare risposte giuridiche. ${ }^{6}$

Un così frastagliato e disomogeneo panorama politico, sociale e culturale ha permesso ai giuristi di ritagliarsi un ruolo che servisse da ponte tra l'Europa e il resto del mondo. Molti sono i casi di cariche accademiche e governative attribuite a diversi e famosi esperti di diritto internazionale. Come Gustave Rolin Jaequemyns, uno dei fondatori dell'Institut de Droit

4 Hill (2013) 135.

5 Becker Lorca (2010) 475-552, qui 476; Becker Lorca (2014). Cfr. anche: Fiocchi Malaspina/Keller-Kemmerer (2014) 214-227. Per il contesto orientale e il processo di universalizzazione del diritto internazionale europeo Keun-Gwan Lee argomenta che esso «was an unprecedented event of world historical significance. One may describe this historical process as the replacement of one (Sino-centric) universality by another (Eurocentric) universality »: LEE (2011) 419.

6 Nuzzo/Vec (2012) XII. 
international, che venne nominato consigliere del re del Siam tra il 1892 e il 1902; ${ }^{7}$ oppure Paul Luis Ernest Pradier-Fodéré, commentatore delle edizioni del Droit des gens di Vattel e del De iure belli ac pacis di Grozio, fondatore e direttore a Lima della facoltà di scienze politiche e amministrative, ${ }^{\mathbf{8}}$ dove insegnò diritto internazionale, divenendo anche consulente legale presso il governo peruviano. ${ }^{9}$ L'apporto di Pradier-Fodéré nelle questioni e nelle dinamiche diplomatiche del Perù è stato significativo, ed è grazie al suo duplice impegno che si riesce a cogliere la mediazione tra la figura del professore di diritto internazionale e l'applicazione pratica degli stessi principi nella seconda metà dell'Ottocento. ${ }^{\mathbf{1 0}}$ Gli anni in cui visse in Perù coincisero con lo scoppio della guerra tra le forze alleate del Perù e della Bolivia contro il Cile, più nota come guerra del Pacifico (1879-1884), all'interno della quale Pradier-Fodéré seppe distinguersi tanto come giurista quanto come uomo diplomatico. ${ }^{\mathbf{1 1}}$

7 Becker Lorca (2010) 484, nota 15. "According to his biographer, Rolin Jaequemyns played a vital role in Siam with his daily advice on foreign affairs and domestic judicial reforms »: Loos (2006) 55.

8 LACHS (1987) 77.

9 BeCKer LoRCa (2010) 484, nota 15.

10 Elliot (1908) 246 ss.

11 Tra i molti esempi del suo contributo diplomatico si ricorda, in particolare, quello relativo ad una controversia sorta per il trasporto di armi da parte della nave Luxor, che navigava in acque peruviane. Nel 1879 infatti il Perù sequestrò presso il porto di Callao, il vascello della compagnia tedesca Kosmos, Luxor, con l'accusa di avere portato materiale di guerra da Montevideo a Valparaiso, dove il Luxor ancorò il 4 maggio. Appena questa notizia fu nota al governo tedesco venne inviato a Lima l'ordine telegrafico di stendere una relazione sull'incidente e di reclamare dal governo peruviano il rilascio del Luxor. La risoluzione della controversia fu decisa dal Tribunale delle prede il quale ritenne, nell'ottobre 1879 , che il sequestro della nave fosse legittimo, innescando una vera e propria querelle giuridica tanto pratica quanto dottrinale. In merito alla questione della Luxor si distinse il professore francese inviando un preciso memoriale al governo peruviano in cui "showed a very enlightened spirit on some of the most difficult questions of international law; his ideas respecting the security of private property upon the high seas were most liberal and he advocated with vigor the rights of neutral commerce. He laid particular emphasis upon the fact that contraband trade was not in itself an illegal act nor an act of hostility, but that the basis of the right of seizure was the right of self defense on the part of the belligerent making the seizure; that the seizure of contraband goods was an exception to the rule of the freedom of neutral commerce and did not necessarily extend to the ship, which was liable to confiscation only when the owner authorized or tolerated the acts of the captain. Regarding the question of capture after the contraband articles have been deposited, the question, that is, of the "return voyage", he held that after the vessel 
È altresì eloquente che da un lato, mentre si spostavano i giuristi europei verso territori latino-americani e asiatici, dall'altro $i$ «nuovi » esperti di diritto internazionale provenienti da quelle terre sentivano l'esigenza di formarsi nelle accademie e nelle sedi diplomatiche europee. Andrés Bello lavorò a Londra a partire dal 1810, per una ventina di anni, come diplomatico al servizio del governo venezuelano: questa fu l'occasione per entrare in contatto con le teorie del diritto delle genti e con i testi che all'epoca circolavano, tra cui in primis quelli Martens e Vattel. ${ }^{\mathbf{1 2}}$ Anche il nordamericano Henry Wheaton fu nominato chargé d'affaires in Danimarca e, tra il 1837 e il 1846, assunse l'incarico di ministro degli Stati Uniti presso la Corte di Berlino; ${ }^{13}$ allo stesso modo l'argentino Carlos Calvo si formò in Europa e scrisse le sue opere sia in spagnolo sia nella lingua diplomatica francese così «that he would reach a broader European audience $",{ }^{\mathbf{1 4}}$ mentre tradusse in spagnolo la celebre Histoire des Progrès du Droit des gens en Europe et en Amérique (Lipsia, 1846), sentendosi parte dell'universo europeo. Pertanto si può considerare che Bello, Wheaton e Calvo "promoted the European legal doctrine. Thus they took an active part in the globalization and globalization of international law ${ }^{15}$

Questo dinamismo è la manifestazione del potere intrinseco esercitato dallo scambio di idee che avveniva reciprocamente e congiuntamente tra $\mathrm{i}$ giuristi di diversa provenienza, rientrando perfettamente nel grande e complesso tema della circolazione dei testi di diritto internazionale, nelle sue più ampie varianti, di manuali, compendi, trattati di diritto delle genti (e anche di storia) e di diritto internazionale, che dalla seconda metà del Settecento venivano prodotti, editi, riediti, tradotti in diverse parti del globo. ${ }^{\mathbf{1 6}}$

has deposited the contraband, she then begins a new voyage and is not liable to capture; that the offense is deposited with the contraband-a position now all but universally recognized »: Elliot (1908) 246 ss.

12 JaKsIC (2001) 8.

13 LiU (2012) 1132-1136, qui 1133.

14 Obregón (2009) 158.

15 VeC (2012) 674.

16 Becker Lorca (2010) 484, nota 15. È pertanto rilevante quanto Peter Macalister-Smith e Joachim Schwietzke osservano: «A greatly increased market demand for the works on international law that were printed in the $19^{\text {th }}$ century is evident not only from the large quantity of very similar titles of publications in this period, but also from the number of editions and translations which were produced »: Macalister-SMith / SchwiETzKe (2001) 78. 
L'autorità che ebbero i testi di diritto internazionale e l'influenza sono state decisive; addirittura ancora nei primi anni del Novecento, durante la sessione annuale dell'Institut de Droit International, veniva constatato che

The relations of Sovereigns States, which acknowledge no common legislator, can be regulated, in the absence of treaty, only by usage formed by opinion; and although international opinion is largely shaped by trains of circumstances, it looks also for articulate and reasoned guidance, and finds what it looks for mainly in the teachings of international jurists. Such writers as Grotius and Vattel, Martens and Hefter have done more than sum up existing rules, or throw out suggestion for their improvement. So far as their conclusions has been adopted by the diplomacy of States, they have given a new scope to the law itself by expressing it in literary form and deducing the conclusions which were logically involved in its premises. ${ }^{17}$

La traduzione, intesa nel suo significato più ampio, non solo di formulare in una altra lingua il testo originale, ma anche di « portare al di là » (« trans »«oltre», e «ducere» - «portare»), ha senz'altro giocato un importante ruolo. Tradizionalmente rilegata ad essere semplicemente oggetto di prefazioni, introduzioni, note del traduttore, è invece stata l'anello di congiungimento nel processo di professionalizzazione del diritto internazionale. I traduttori, esattamente come i giuristi, possono essere dipinti come mediatori e diplomatici perché erano chiamati in prima persona a gestire problemi di enorme distanza culturale, linguistica, di tempo, di spazio ed erano gli agenti centrali delle idee e dei valori europei. ${ }^{\mathbf{1 8}}$

Nell'Ottocento i traduttori dei trattati erano per la maggior parte avvocati, profondi conoscitori del diritto internazionale e commerciale sotto il profilo sia pratico che dottrinale: è sufficiente ricordare che tutte le traduzioni del Droit des gens di Vattel, sono state realizzate da giuristi qualificati come Paul Pradier-Fodéré, Silvestre Pinheiro Ferreira, Joseph Chitty, Manuel Hernández, Georgios Rallis, Terenzio Sacchi, i quali con le loro differenti modalità recepivano e trasmettevano il significato del trattato di Vattel, alla luce dei progressi del diritto delle genti, delle realtà nazionali giuridiche e diplomatiche. Questi fenomeni « can be just as illuminating as the original work in registering the process whereby, in this case, international law has been globalized and universalized $[\ldots] \gg .{ }^{19}$

17 Queste le parole espresse nell'articolo «The Institute of International Law » scritto dal corrispondente del Times inviato alla sessione annuale dell'Institut del 22 settembre 1904 a Edimburgo: The Times, issue 37507 (Friday, 23 September 1904) 5.

18 Liv (1999) 128.

19 Ibidem. 
Come ha precisato Immanuel Maurice Wallerstein, l'universalismo ha visto la sua massima espressione nella metà dell'Ottocento in quanto esso coesisteva paradossalmente con un universalismo settoriale e «the core of each variants was a pair of premises that the path of knowledge begins with the particular and ends with the abstract-<universalizing thought » ${ }^{\mathbf{2 0}}$

In realtà si percepisce come tale universalismo fosse soggetto ai meccanismi di appropriazione e di assimilazione, che si innescavano inevitabilmente nel momento in cui un trattato di diritto internazionale veniva tradotto, commentato o annotato. Il diritto internazionale rientra, infatti, in un continuo dinamismo di traduzione culturale e si spinge oltre il contesto linguistico e letterario nel quale si utilizza, diventando un vero e proprio sinonimo di transculturazione, di negoziazione e di selezione interculturale.

Calzante è richiamare, come similitudine, il celebre episodio dell'Iliade, quando Glauco e Diomede, in procinto di combattersi, scoprono che i loro

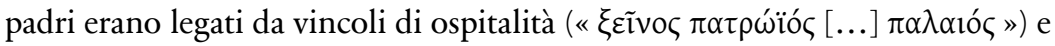
decisero di scambiarsi le armi nonostante fosse uno scambio non paritario, oro per il bronzo. ${ }^{21}$ Allo stesso modo questa è la traduzione che coinvolge anche il fenomeno giuridico, il cui risultato è qualcosa di nuovo che racchiude in sé le caratteristiche di entrambe le parti, senza venga posta attenzione sulla possibile disuguaglianza originaria.

Così la costruzione identitaria degli stati europei e non europei genera una esigenza di traduzione come forma di appropriazione, ma anche di identità, bilanciata con quelle che sono le interazioni derivanti dalle diversità culturali e sociali. La figura del mediatore (giuridico) venne svolta concretamente da coloro che si occupavano di diritto internazionale. I giuristi presero attivamente parte al processo di costruzione del diritto (internazionale) e ne favorirono l'espansione. Il risultato fu sostanzialmente qualcosa di profondamente diverso dalla classica e statica rappresentazione del diritto internazionale come sapere a dimensione universale, ma al contrario provvide a esaltarne la specificità, svelando, attraverso le sovrapposizioni tra piano statale e interstatale e il dinamico intreccio tra culture, società, diritti, e linguaggi, il suo carattere ibrido. Questo emerge dai molteplici contesti presi in considerazione in cui il Droit des gens di Vattel era utilizzato, rivisitato e aggiornato alla luce delle contingenti situazioni politiche, giuridiche, interne e internazionali dell'Ottocento.

20 Wallerstein (2001) 191.

21 Omero (2014) VI, 119-236, 114 ss. 


\section{Il Droit des gens nella sua polifonia}

Vattel è colui che ha vissuto un momento di transizione, ha saputo cogliere dal passato la tradizione giusnaturalistica di cui è figlio, per trasformarla nel linguaggio statale e politico in continuo divenire. Ha elaborato il Droit des gens nella consapevolezza che ci fosse una tensione tra diritto interno e diritto internazionale, focalizzando l'attenzione sul concetto di Nazione e delineandone tutti i requisiti per il raggiungimento della sua conservazione, perfezione e felicità, attraverso la promulgazione della costituzione. Il dialogo tra le nazioni si realizza all'interno di una comunità di stati indipendenti e sovrani, intesi come soggetti del diritto delle genti. Il risultato che emerge nel corso del Sette e Ottocento è stata una rilettura del trattato che oscilla tra il diritto costituzionale e il diritto internazionale. Così in Spagna si pubblicò per la prima volta il Derecho de Gentes nel 1820, in coincidenza con il cosiddetto " Triennio Liberale », grazie al giurista Hernández, il quale nella successiva edizione del 1834, con le sue significative note, approfondì le teorie di Vattel per quanto riguarda il concetto di costituzione e integrando il testo con precisi esempi sulla storia costituzionale spagnola. Determinante è stata la circolazione anche nei territori latino-americani attraverso i diversi manuali didattici, quali in primis quello di Francisco Javier Yanes o i trattati di diritto delle genti come la prima edizione del Derecho de gentes di Andrés Bello, fortemente ancorata all'opera vatteliana.

Durante la rivoluzione greca un primo approccio al trattato si ebbe attraverso la traduzione parziale di Skouphos del 1825, incentrata sulle tematiche della neutralità e del trattamento del nemico, che agevolò l'utilizzo per $\mathrm{i}$ «bisogni e gli affari » greci, a cui seguì la traduzione di Rallis del Droit des gens nel 1831, dopo la nomina di Capodistrias quale leader della Grecia indipendente.

Nel 1834, in Inghilterra, è invece l'avvocato Chitty, a consigliare l'opera specialmente dal punto di vista pratico, annotando il Law of Nations di circoscritti esempi giurisprudenziali e di " prassi internazionale ", seguito poi dall'americano Ingraham, il quale, qualche anno più tardi, nel 1852, completò le note di Chitty alla luce dei successivi casi decisi negli Stati Uniti, incarnando, in quest'ultimo contesto, la tensione politico-giuridica del diritto internazionale. ${ }^{22}$

Una lettura al testo di Vattel più marcatamente giuridico-filosofica la si riscontra in ambiente francese e precisamente nelle note del portoghese 
Pinheiro Ferreira, il quale nel 1838 aggiunse un terzo volume al trattato del giurista di Neuchâtel, commentandone all'incirca ogni paragrafo. Egli pur riconoscendo la portata del Droit des gens, pur constatandone i pregi, si dimostrò molto critico, definendo le teorie " ambigue ", "vaghe » ed « equivoche». Il pensiero di Pinheiro Ferreira fu ripreso da Pradier-Fodéré, che pubblicò nel 1863 una nuova edizione del trattato di Vattel aggiungendovi numerosissime note di carattere storico e dottrinale ed evidenziando così le vicende storiche succedutesi dopo 1758, con particolare riguardo alle teorie sul diritto delle genti o diritto internazionale contenute nei lavori di illustri internazionalisti quali Wheaton, Heffter e Martens.

Dal lavoro di Pradier-Fodére risaltano l'intento didascalico e la necessità soprattutto di non mettere da parte l'opera di Vattel, anzi di aggiornarla e di illustrarne gli sviluppi del diritto delle genti. Infatti il metodo esegetico di Pinheiro Ferreira e di Pradier-Fodéré, non riguardava esclusivamente il Droit des gens, perchè entrambi i giuristi hanno commentato anche altri trattati di diritto internazionale, quali rispettivamente una nuova edizione del Précis $d u$ droit des gens di Martens e una nuova traduzione del De iure belli ac pacis di Grozio.

Se per il diritto internazionale nei territori tedeschi il Droit des gens era ormai classificato nella storia del diritto delle genti, nella pratica diplomatica continuava a rivestire una influenza quasi sacrale, paragonabile a quella di un « oracolo » con ripercussioni duplici: da un lato si era in presenza di una autorità infallibile, ma dall'altro anche di una risposta che doveva essere «tradotta » e applicata al caso concreto, dimostrando la necessità di leggere il trattato con gli occhi dell'attualità e del preciso contesto storico-politico. Nell'Italia dell'Ottocento ritorna energicamente il binomio costituzionale / internazionale: si assistette ad una oscillazione tra l'utilizzo da parte di molti patrioti e giuristi sostenitori dell'indipendenza nazionale e l'intento conservatore e poco incline allo spirito rivoluzionario, incarnati nella traduzione del napoletano Terenzio Sacchi del 1854.

Centrale, altresì, è la definizione di diritto delle genti, che, come si è visto, è « est une science particulière, la quelle consiste dans une application juste et raisonnée de la loi naturelle aux affaires et à la conduite des Nations ou des Souverains $»{ }^{23}$ Il diritto delle genti, benché ancora leggato al diritto naturale, è la scienza da applicarsi esclusivamente alle relazioni tra le Nazioni. È 
con questa affermazione che Vattel indirettamente ammette, come per tutte le scienze, una serie di caratteristiche: la sua dinamicità con la possibilità di apportare " progressi » e la sua conformazione alla contingente realtà storica, prevedendo ovviamente anche il suo superamento.

Tali progressi sono ben delineati dall'esigenza dei giuristi del XIX secolo di commentare l'opera, contribuendo a dipingere la poliedricità del Droit des gens. Essa è stata analizzata mediante le molteplici edizioni e traduzioni che sono state pubblicate tra Sette e Ottocento e attraverso l'impatto che il pensiero vatteliano ha avuto a più livelli nella scienza giuridica. Il continuo richiamo al trattato si percepisce tanto nei più diversi contesti quanto nelle numerose fonti giuridiche e diplomatiche. La coincidenza delle edizioni e traduzioni (in senso più ampio) in occasione delle rivoluzioni europee $\mathrm{e}$ americane testimonia l'influenza giuridico-politica e la straordinaria capacità di adattamento alle più svariate realtà. La divulgazione realizzatasi nella Scuola Romanda del diritto naturale è stata trasferita da Vattel al piano delle scelte giuridiche statali con un marcato approccio pratico e risvolti politici, contribuendo all'emergere della regolamentazione giuridica delle forme statali e alla loro "governabilità " anche nelle relazioni internazionali. Queste ripercussioni sono state "concretizzate» nella connessione tra moda e modernità insita nel Droit des gens, che ha permesso di selezionare e definire la sua dinamicità all'interno di intrecci storici, giuridici e diplomatici settecenteschi per poi realizzarsi nel corso dell'Ottocento.

Dalle ricerche compiute sulla circolazione del Droit des gens si delinea un movimento polifonico: Vattel, con lo sguardo rivolto verso il passato ma proiettato al futuro, realizza un percorso che dialoga con la cultura giuridica nel tempo e nello spazio, imprimendo e garantendo così al trattato il suo eterno ritorno. 



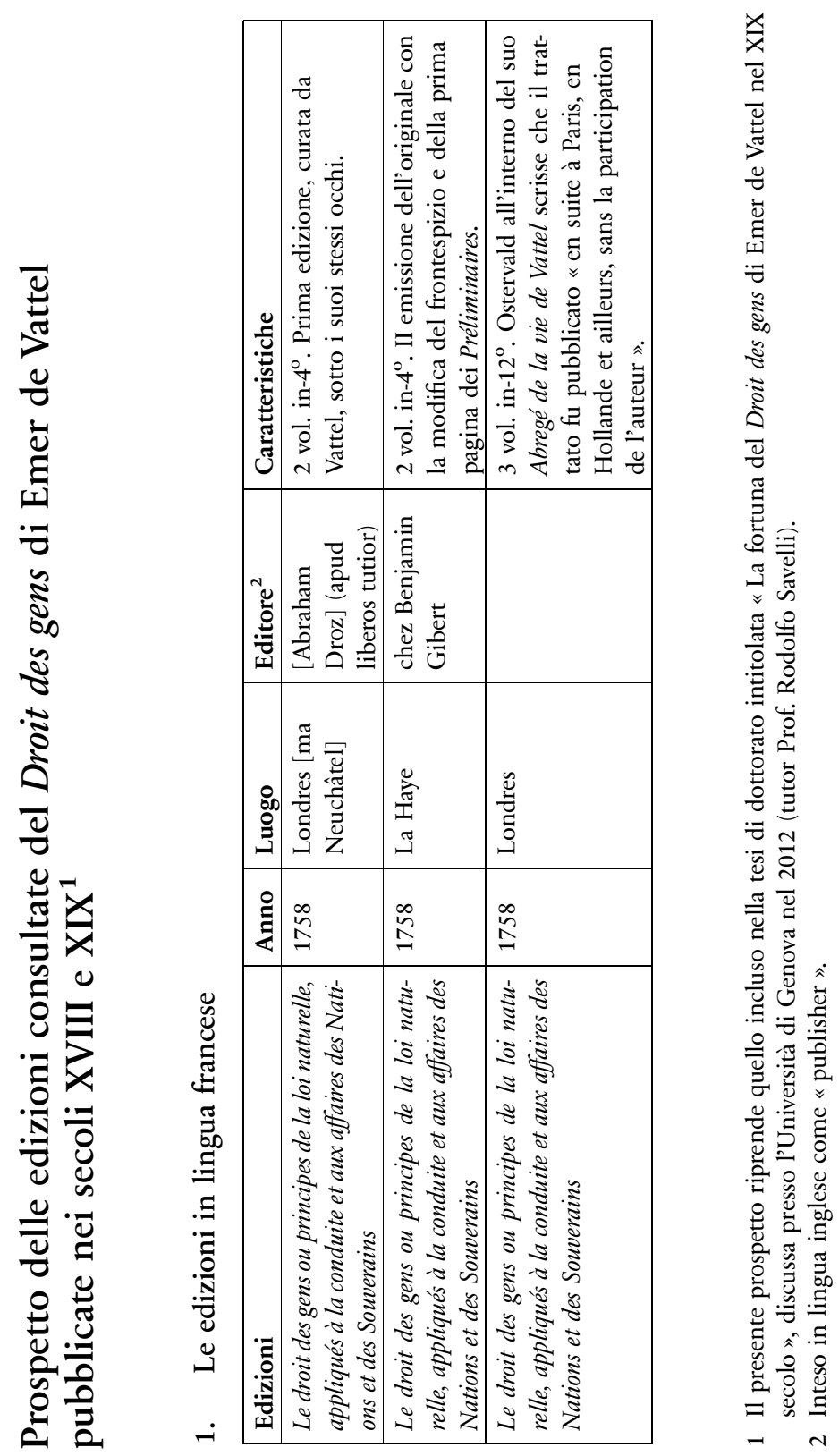

Prospetto 


\begin{tabular}{|c|c|c|c|c|c|}
\hline & 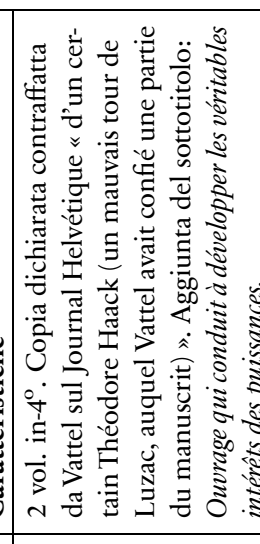 & 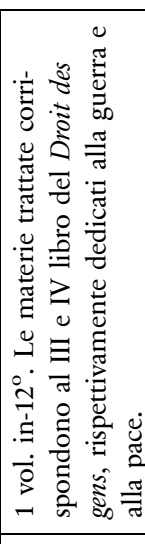 & 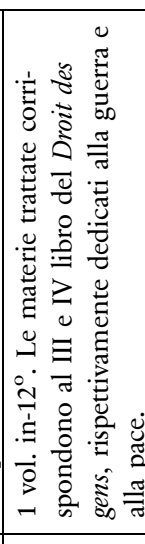 & 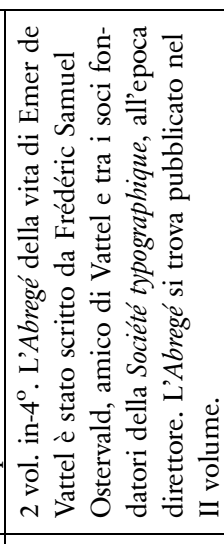 & $\begin{array}{l}\stackrel{\dot{I}}{\grave{I}} \\
\stackrel{.}{\Xi} \\
\dot{0} \\
\dot{m}\end{array}$ \\
\hline \multicolumn{2}{|r|}{ 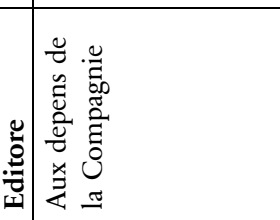 } & 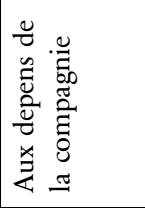 & 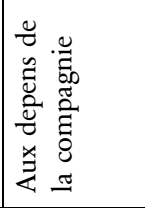 & 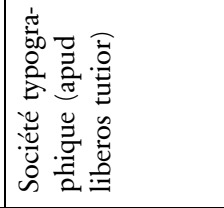 & 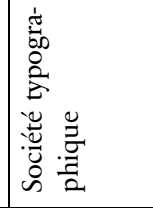 \\
\hline \multicolumn{2}{|c|}{$\begin{array}{ll} & 5 \\
50 \\
3\end{array}$} & 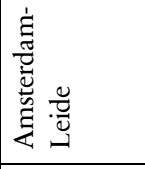 & 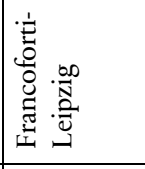 & 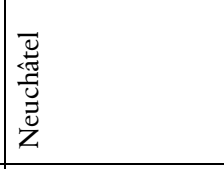 & 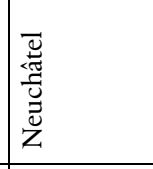 \\
\hline 娄 & $\stackrel{\infty}{\stackrel{2}{\Lambda}}$ & {$\left[\begin{array}{l}\infty \\
\stackrel{1}{1}\end{array}\right.$} & 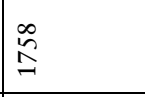 & $\stackrel{R}{\hat{E}}$ & $\underset{\stackrel{t}{I}}{\stackrel{t}{\prime}}$ \\
\hline (:) & 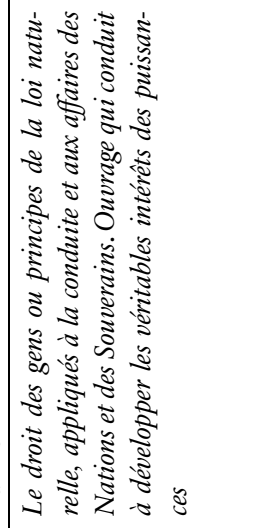 & 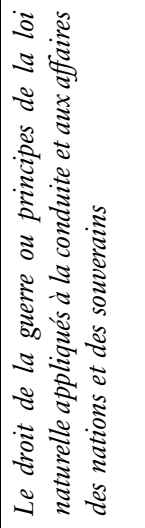 & 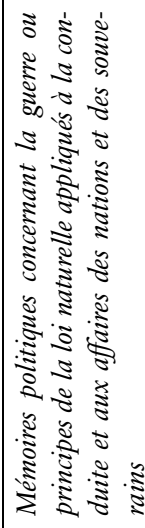 & 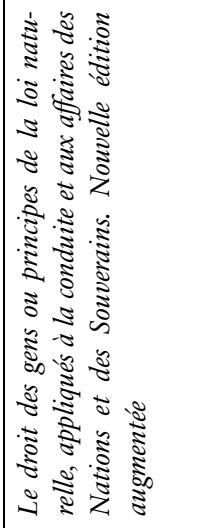 & 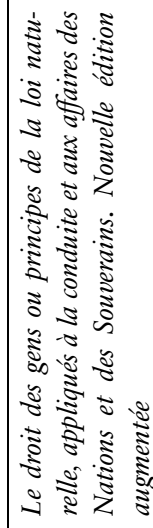 \\
\hline
\end{tabular}




\begin{tabular}{|c|c|c|c|c|c|c|}
\hline 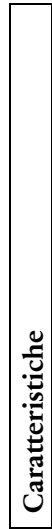 & 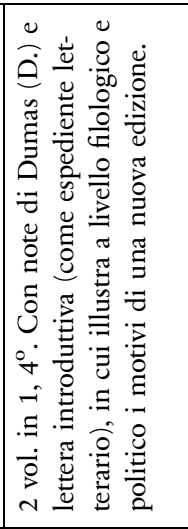 & $\begin{array}{l}0 \\
\stackrel{7}{1} \\
\dot{\Xi} \\
\dot{0} \\
\dot{0} \\
\dot{m}\end{array}$ & 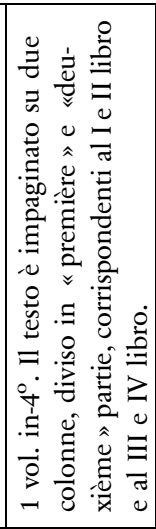 & 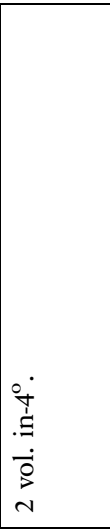 & $\begin{array}{l}0 \\
\stackrel{1}{1} \\
. j \\
\dot{1} \\
\dot{0} \\
\dot{m} \\
m\end{array}$ & 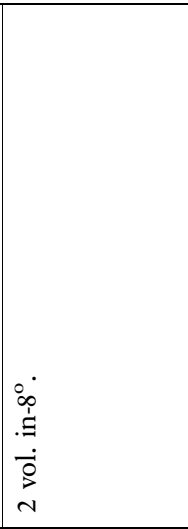 \\
\hline & 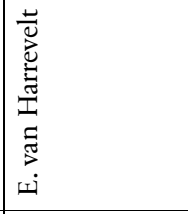 & 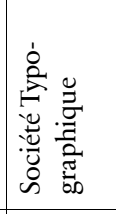 & 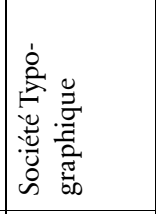 & 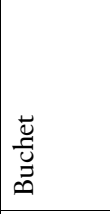 & 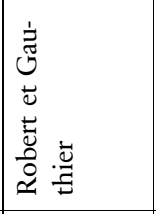 & 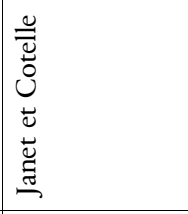 \\
\hline & 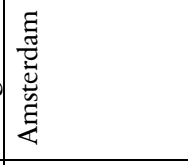 & 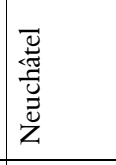 & 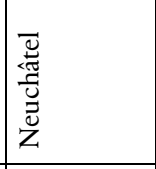 & 节 & ఏ્ટ & 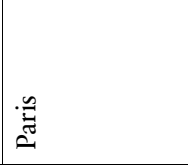 \\
\hline$\stackrel{8}{\sharp}$ & $\stackrel{n}{\hat{\imath}}$ & I & $\stackrel{ }{\stackrel{ }{A}}$ & $\stackrel{\infty}{\stackrel{\infty}{\simeq}}$ & $\underset{\infty}{\infty}$ & $\begin{array}{l}\stackrel{1}{1} \\
\underset{-\infty}{-1}\end{array}$ \\
\hline : & 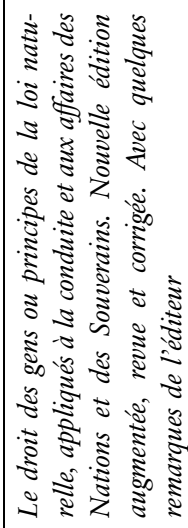 & 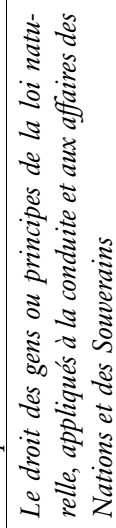 & 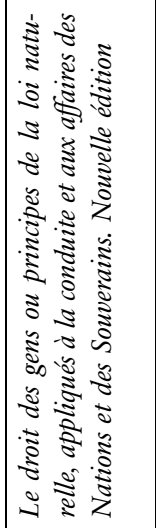 & 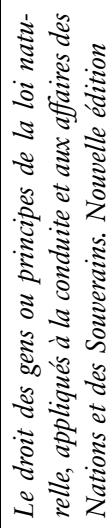 & 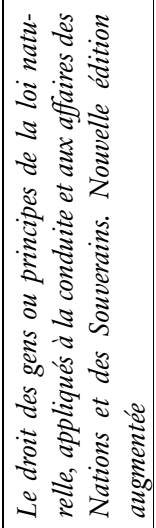 & 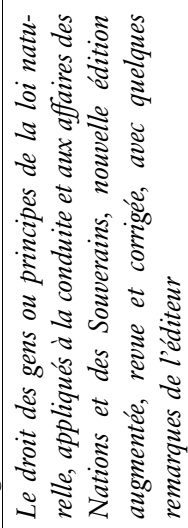 \\
\hline
\end{tabular}




\begin{tabular}{|c|c|c|c|}
\hline 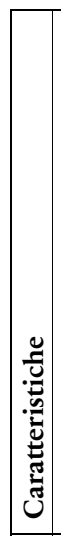 & & 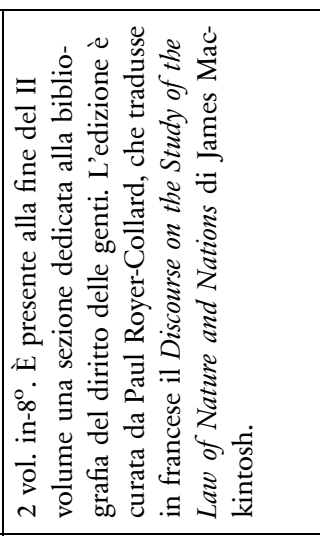 & 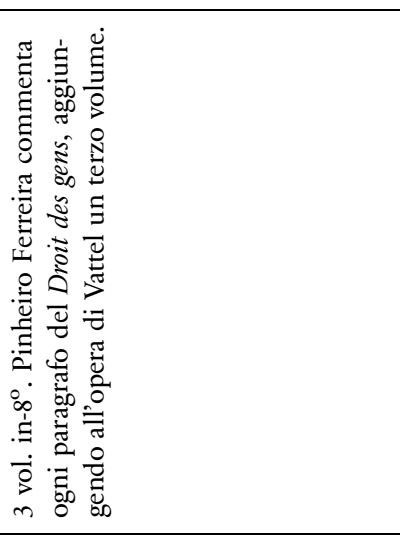 \\
\hline & 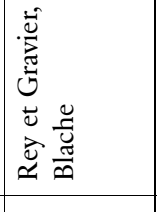 & 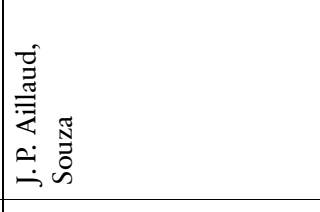 & 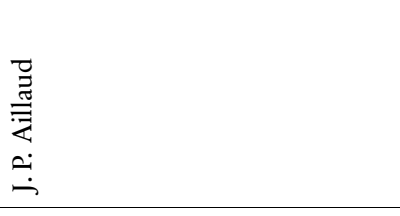 \\
\hline & & 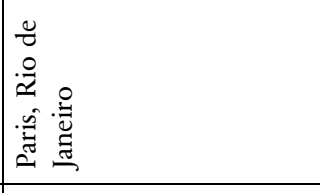 & 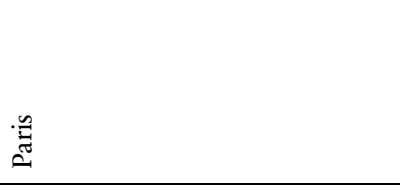 \\
\hline 妾 & 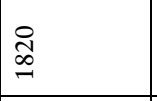 & $\underset{\infty}{\infty}$ & 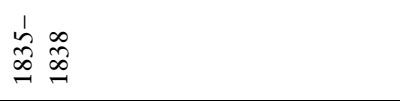 \\
\hline & 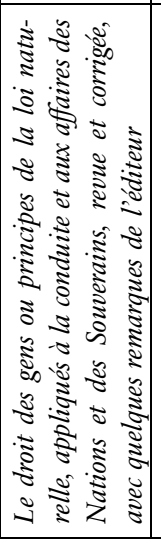 & 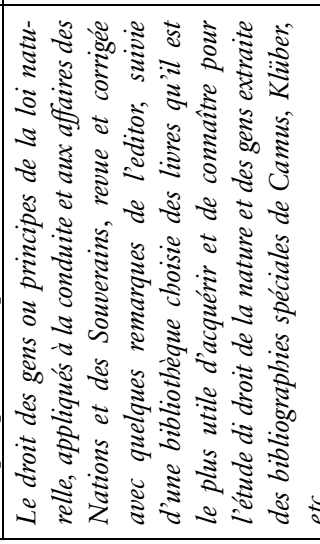 & 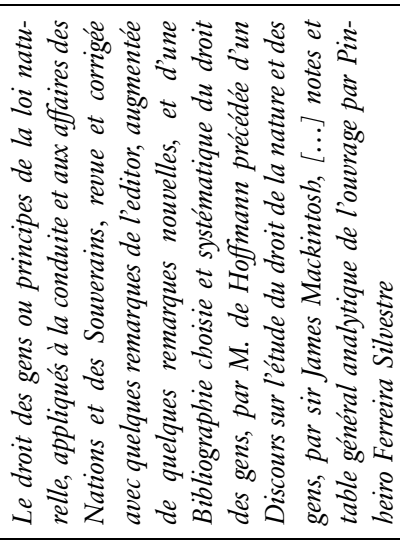 \\
\hline
\end{tabular}




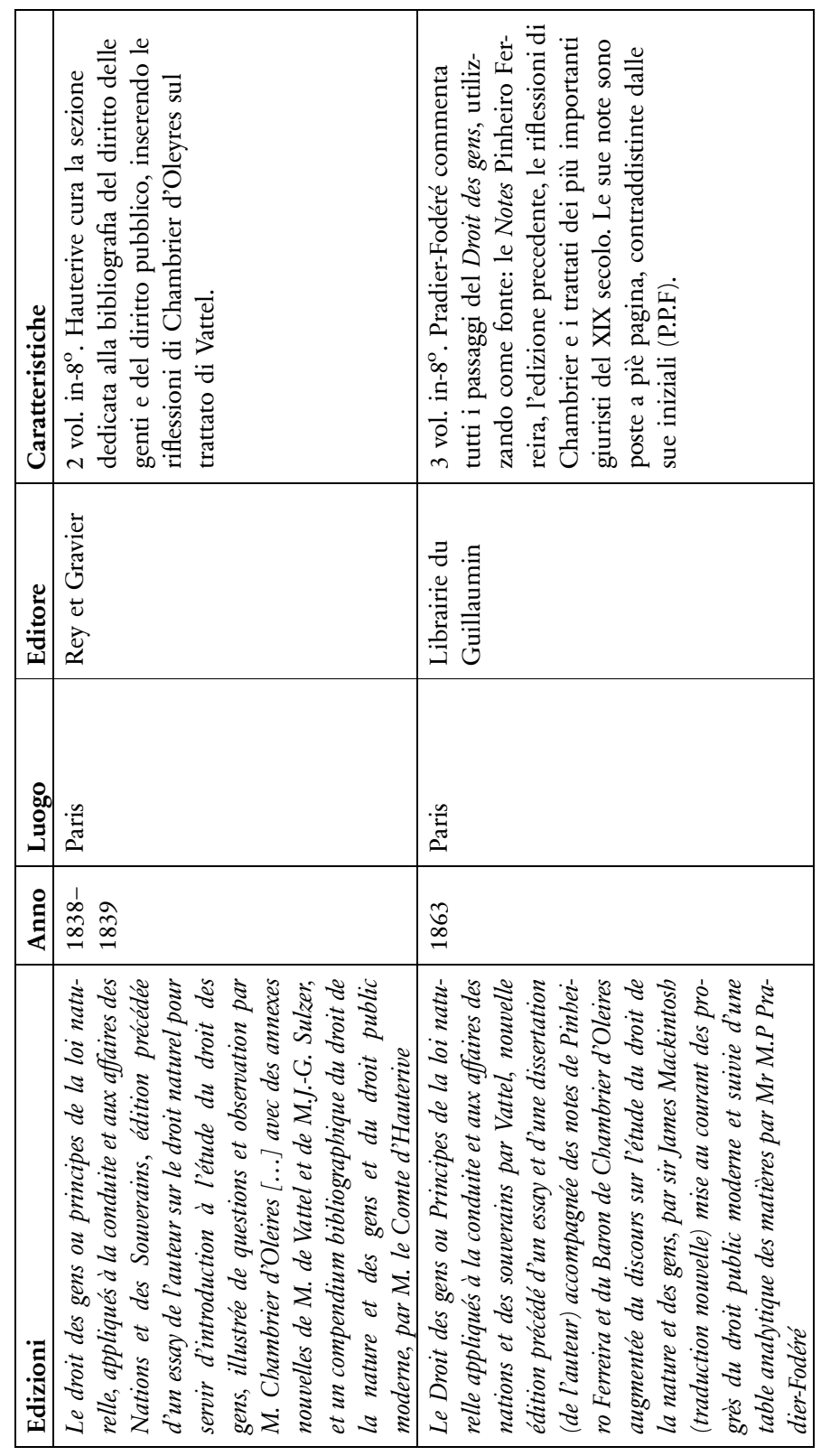




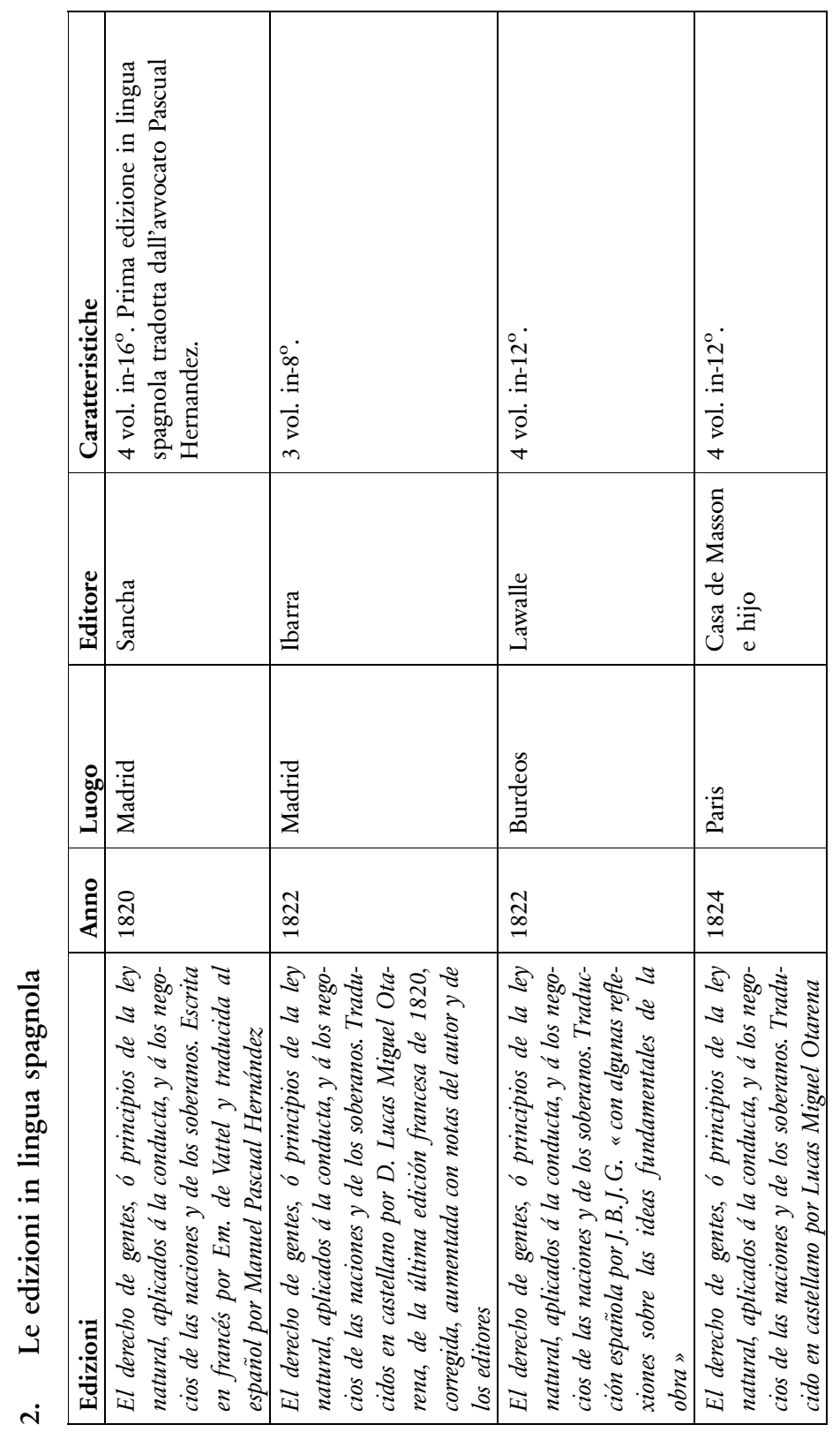




\begin{tabular}{|c|c|c|c|c|}
\hline & 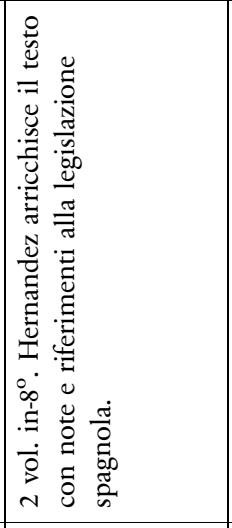 & 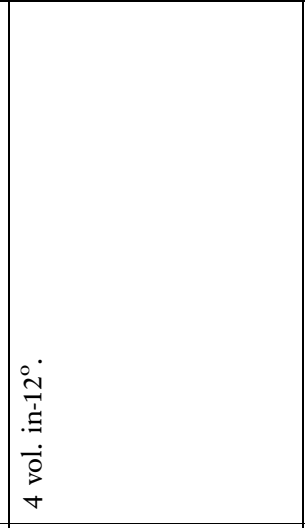 & 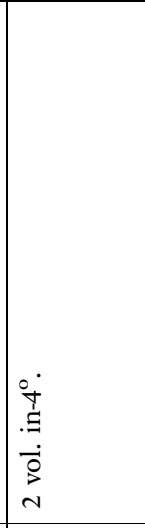 & 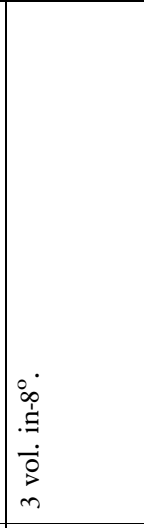 \\
\hline 悹 & 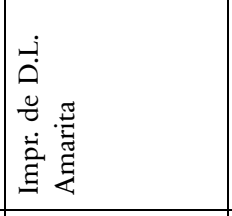 & 节 & 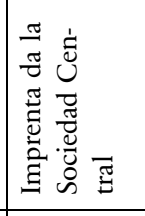 & \\
\hline & & 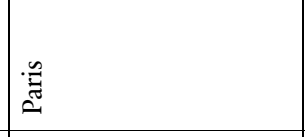 & $\mid \begin{array}{l}\frac{D}{\bar{E}} \\
\frac{\pi}{\Sigma} \\
\frac{\pi}{2}\end{array}$ & \\
\hline & $\underset{\infty}{\stackrel{\infty}{\infty}}$ & 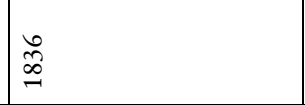 & 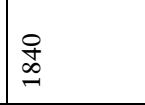 & \begin{tabular}{l}
0 \\
\multirow{2}{0}{} \\
-1
\end{tabular} \\
\hline & 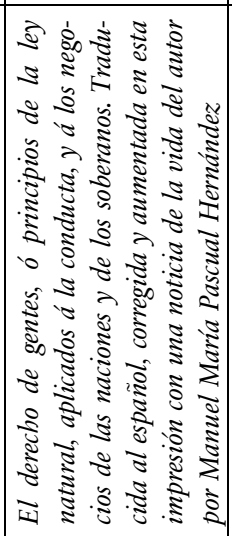 & 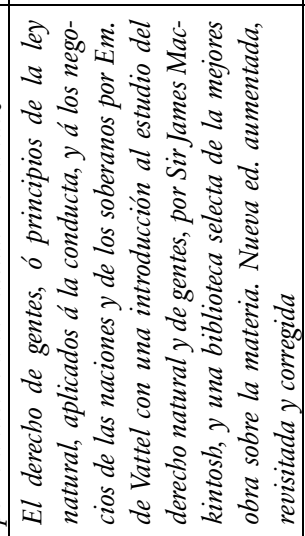 & 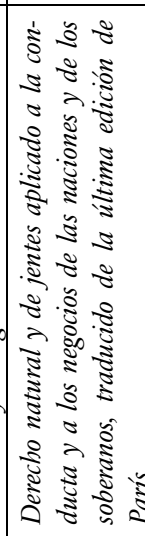 & 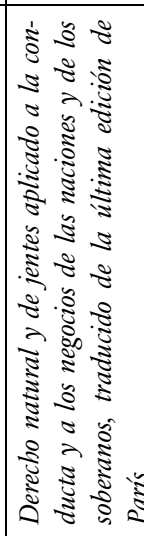 \\
\hline
\end{tabular}




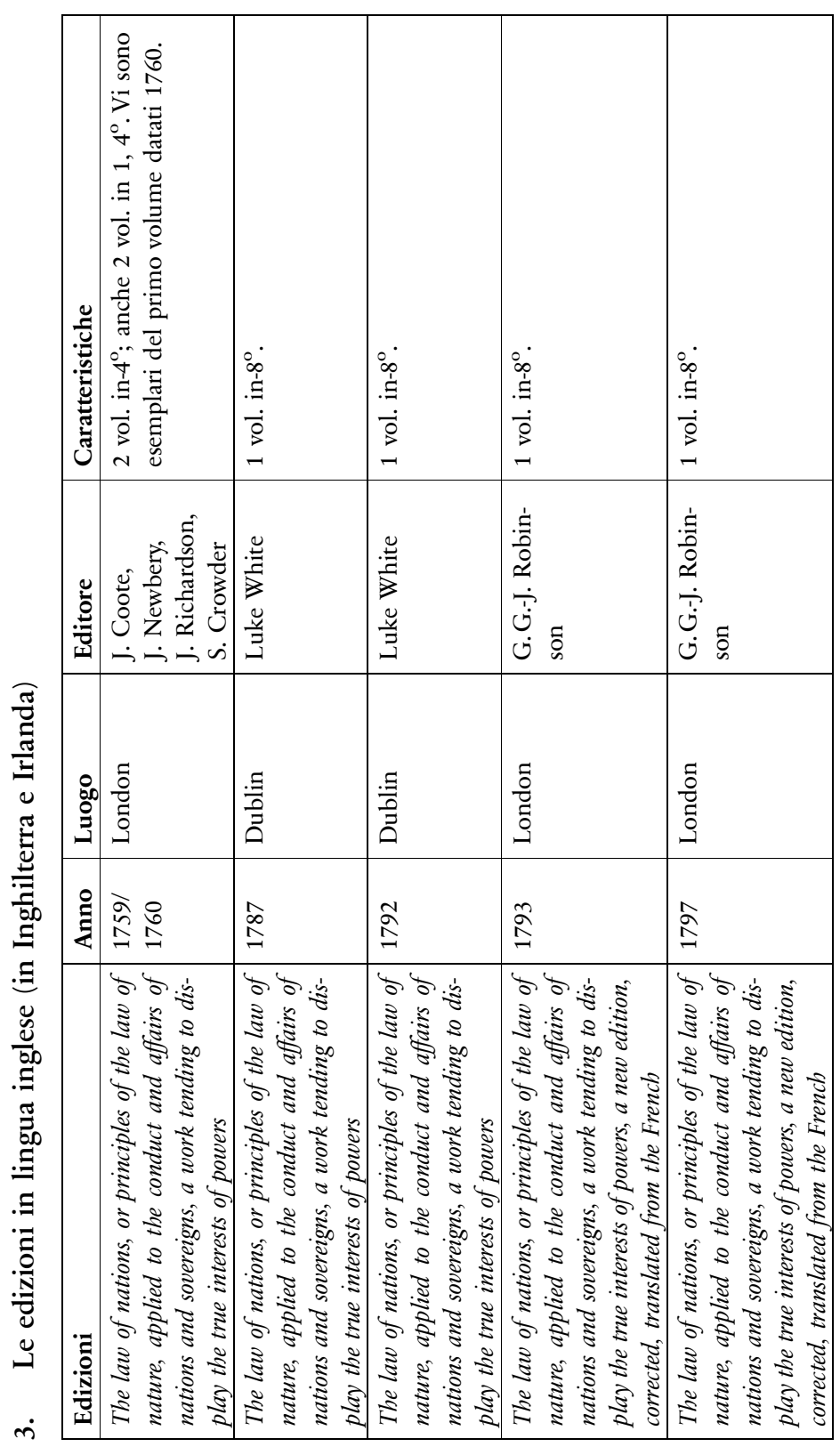



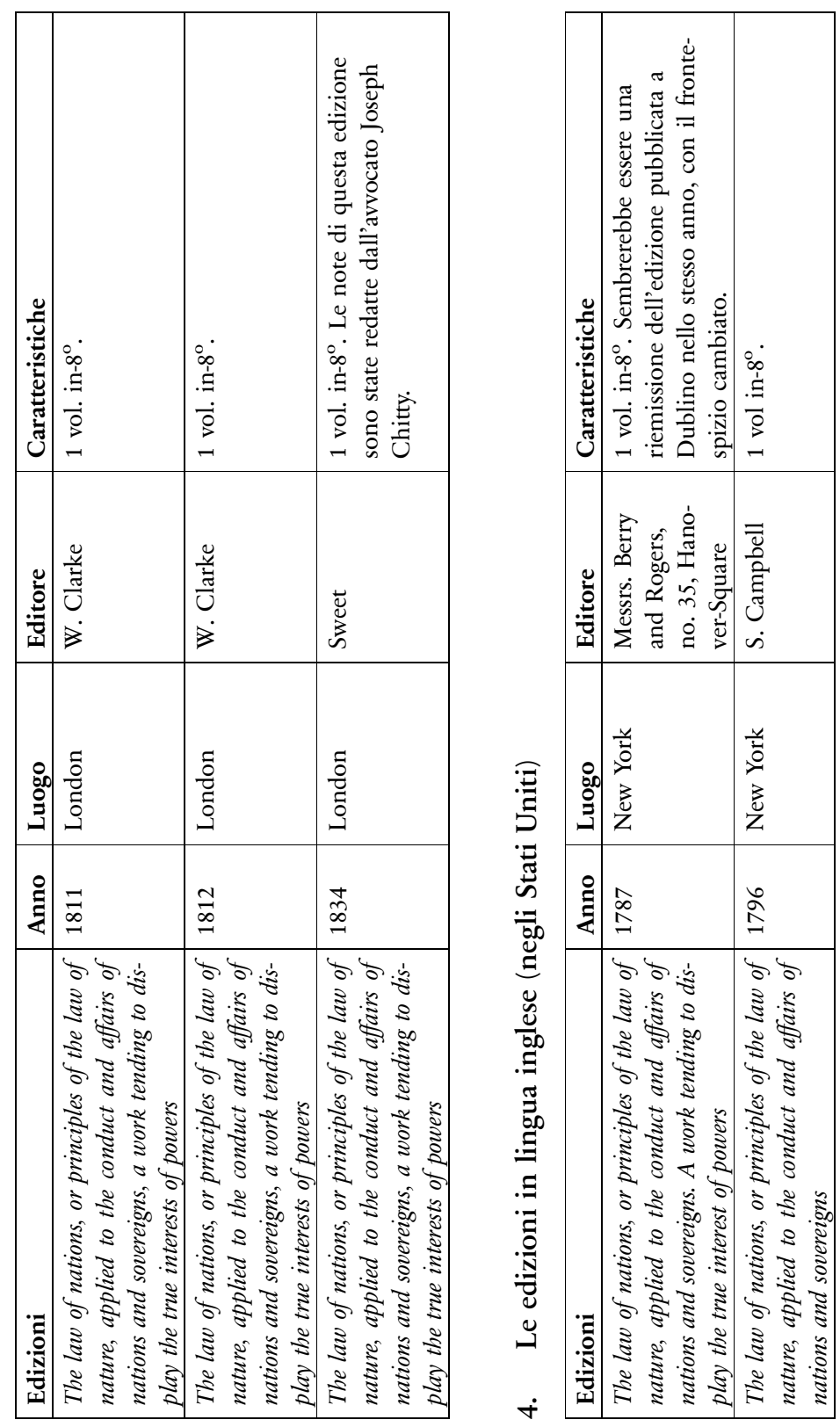

Prospetto 


\begin{tabular}{|c|c|c|c|c|c|c|c|c|}
\hline 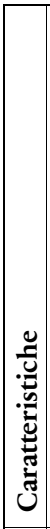 & 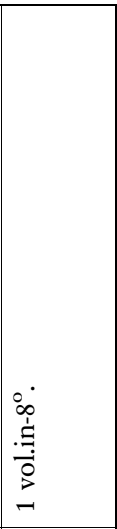 & 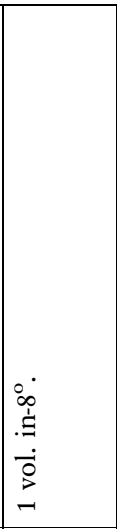 & 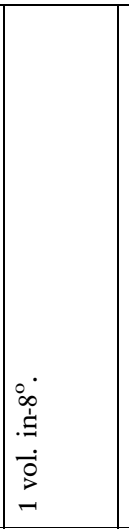 & 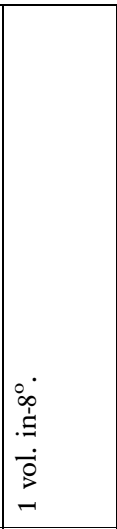 & 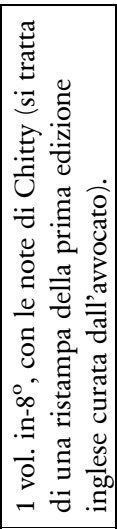 & 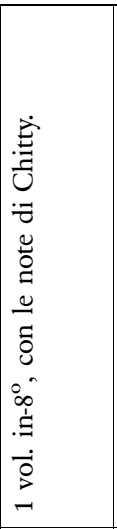 & 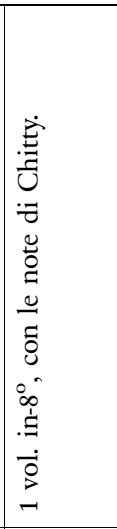 & 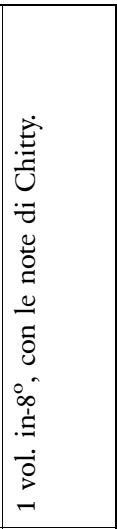 \\
\hline 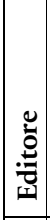 & 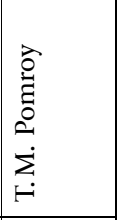 & $\begin{array}{l}\overline{\widetilde{\widetilde{J}}} \\
\text { के } \\
\dot{<}\end{array}$ & $\begin{array}{l}\overrightarrow{\vec{u}} \\
\vec{\Xi} \\
\dot{\omega}\end{array}$ & 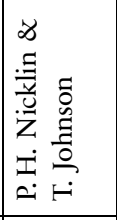 & 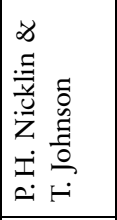 & 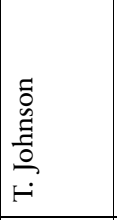 & $\begin{array}{l}\stackrel{\tilde{D}}{0} \\
\stackrel{0}{0} \\
\stackrel{0}{0} \\
\stackrel{0}{H}\end{array}$ & $\begin{array}{l}\stackrel{\Xi}{0} \\
0 \\
\stackrel{0}{0} \\
\stackrel{0}{0} \\
\stackrel{H}{H}\end{array}$ \\
\hline $\begin{array}{l} \\
\stackrel{8}{8} \\
\stackrel{8}{8} \\
\stackrel{8}{3} \\
3\end{array}$ & 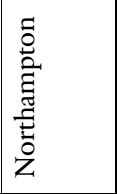 & 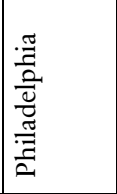 & 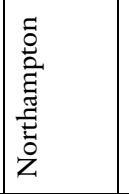 & 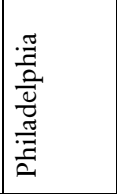 & 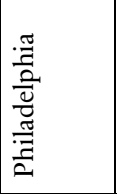 & 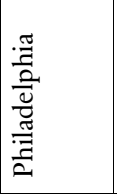 & 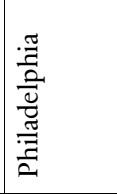 & 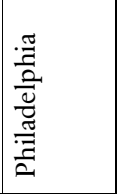 \\
\hline$\stackrel{8}{\sharp}$ & $\begin{array}{l}2 \hat{\infty} \\
\infty \\
-\end{array}$ & $\stackrel{\wedge}{\stackrel{\infty}{\rightarrow}}$ & 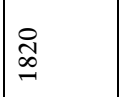 & 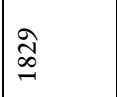 & $\stackrel{\infty}{\infty}$ & $\stackrel{\infty}{\infty}$ & 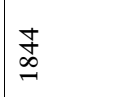 & $\underset{\infty}{\stackrel{े}{\sigma}}$ \\
\hline 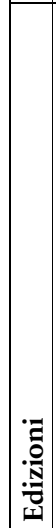 & 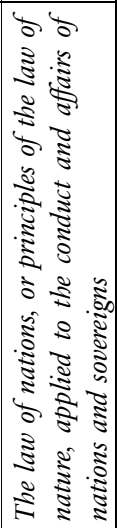 & 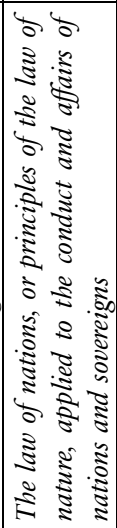 & 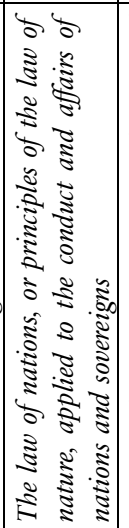 & 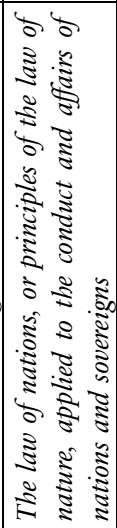 & 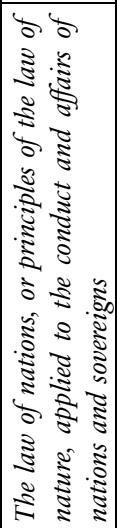 & 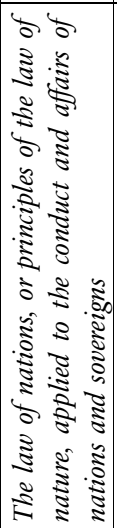 & 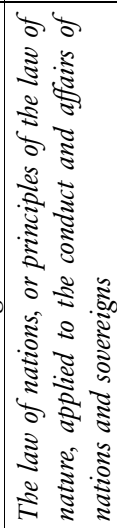 & 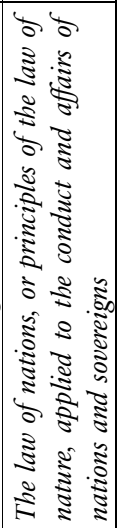 \\
\hline
\end{tabular}




\begin{tabular}{|c|c|c|c|}
\hline 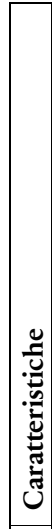 & 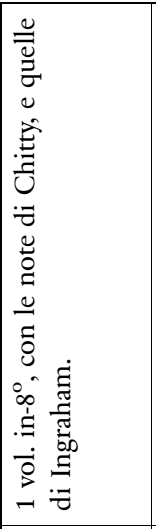 & 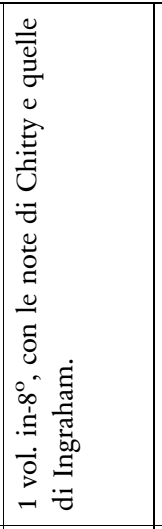 & 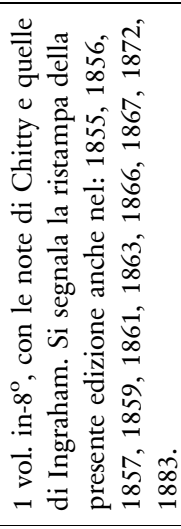 \\
\hline : & 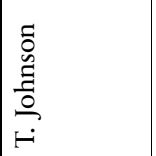 & 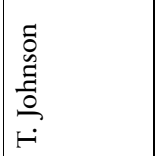 & $\begin{array}{l}\underset{0}{0} \\
\stackrel{0}{E} \\
\stackrel{\Xi}{0} \\
\stackrel{\circ}{\circ}\end{array}$ \\
\hline & \begin{tabular}{|l}
$\frac{\pi}{3}$ \\
$\frac{2}{2}$ \\
$\frac{\pi}{2}$ \\
$\frac{\pi}{\pi}$ \\
$\frac{\pi}{2}$ \\
$\frac{\pi}{2}$
\end{tabular} & 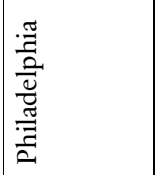 & 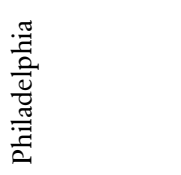 \\
\hline$\stackrel{\Xi}{\sharp}$ & $\begin{array}{l}1 \\
\sigma \\
\infty \\
\sim\end{array}$ & $\stackrel{\infty}{\infty}$ & $\underset{\infty}{\stackrel{\Delta}{\infty}}$ \\
\hline 㱐 & 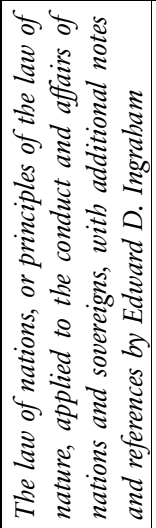 & 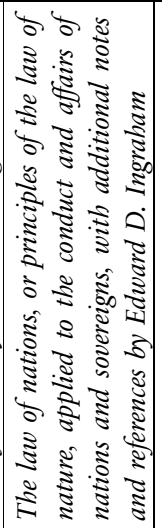 & 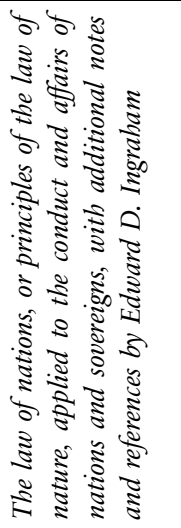 \\
\hline
\end{tabular}

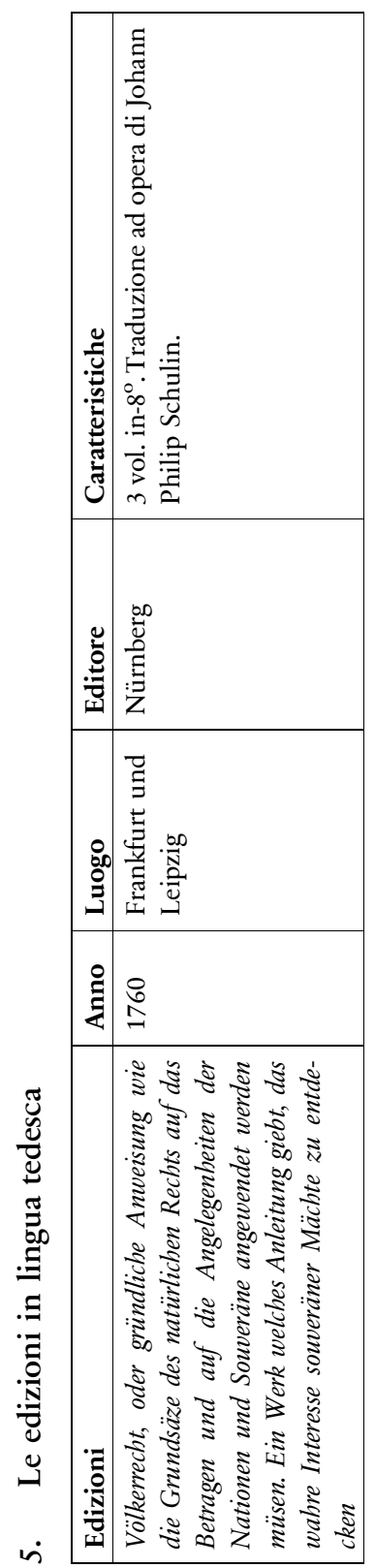

Prospetto 

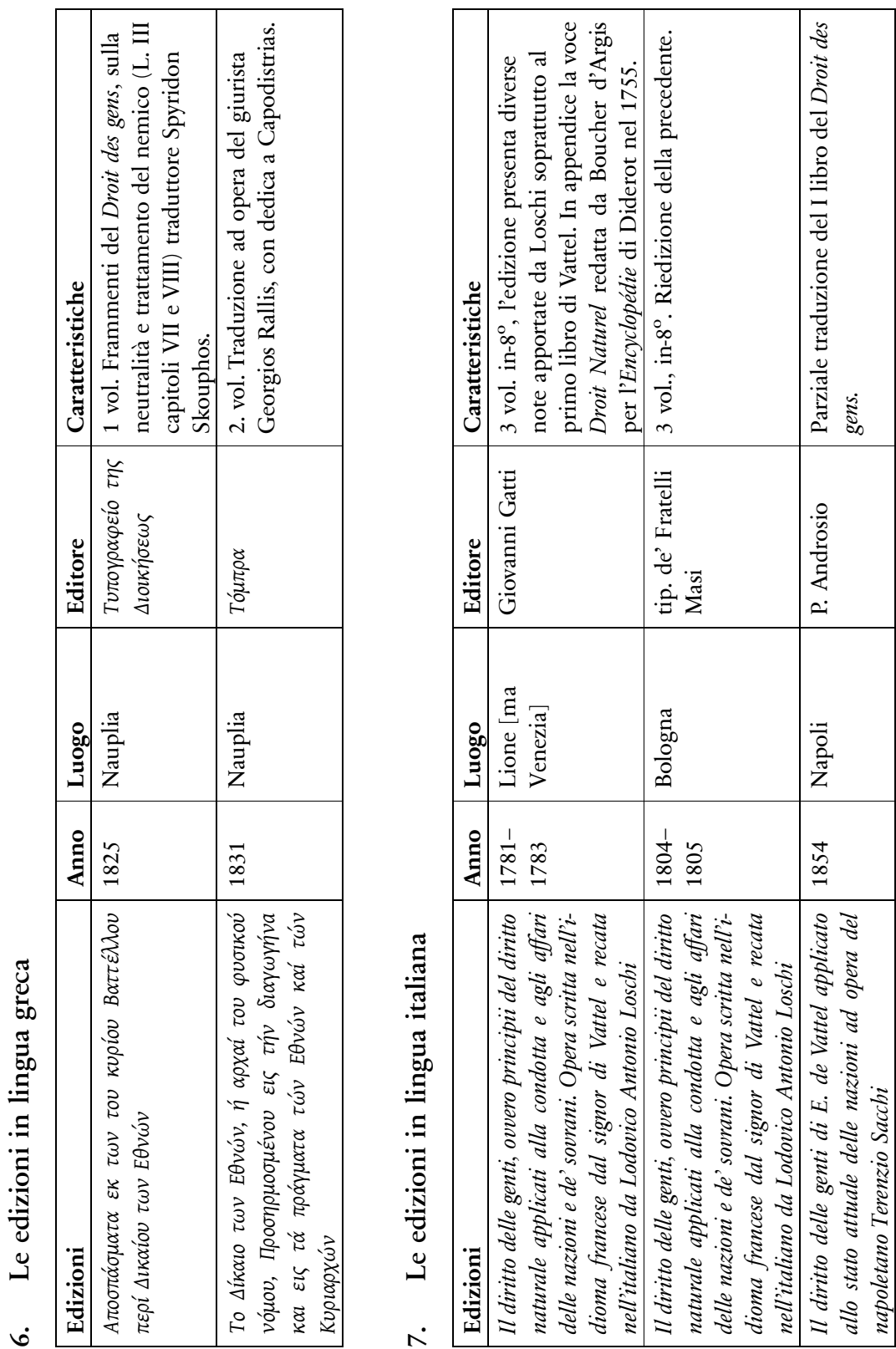

272 Prospetto 


\section{Bibliografia}

a. Articoli su riviste e quotidiani del XXI secolo

Goldsmith, Belinda (Thursday, May 20, 2010), George Washington's library book returned 221 yrs late http://www.reuters.com/article/us-library-washingtonidUSTRE64J4EG20100520, consultato il 26 maggio 2016

Senger, Harro von (22. April 2014), Schweizer Gesetzestexte für China, in: Panorama, Neue Zürcher Zeitung, 92, Seite 22, Teil 01

Senger, Harro von (Vendredi 25 avril 2014), Un Neuchâtelois adopté par l'Empire du Milieu, in: Temps, Dèbats, 12

b. Riviste, periodici e cataloghi bibliografici del Settecento

Bibliographie de la France, ou journal général de l'imprimerie et de la librairie, 35 (Samedì 28 août 1824)

Bibliothèque Impartiale (Janvier et Fevrier 1758), t. XVII, première partie, Göttingue, Leide

Bibliothèque Impartiale, pour le moins de janvier et février 1758 (1758), première partie, Göttingue, Leide

Journal Encyclopédique ou universel (15 juin 1763), t. IV, troisième partie, Bouillon Journal Encyclopédique ou universel (15 juin 1758), t. IV, troisième partie, Bouillon Journal Encyclopédique ou universel (Ir mars 1768), t. II, deuxième partie, Bouillon Journal Helvétique ou Recueil de pièces fugitives de littérature choisie (août 1755), Neuchâtel

Journal Helvétique ou Recueil de pièces fugitives de littérature choisie (avril 1757), Neuchâtel

Journal Helvétique ou Recueil de pièces fugitives de littérature choisie (septembre 1757), Neuchâtel

Journal Helvétique ou Recueil de pièces fugitives de littérature choisie (avril 1758), Neuchâtel

Mercure Suisse, ou recueil de nouvelles historiques, politiques, littéraires et curieuses (septembre 1757) Neuchâtel 
c. Riviste, cataloghi, annali e annuari dell'Ottocento

A catalogue of the Library of the London Institution (1813), London

A catalogue of the printed books and manuscripts in the library of the Inner Temple (1833), London

Annali civili del regno delle Due Sicilie (1839), fascicolo 37, Napoli

Annuaire diplomatique de la République Française pour 1871-1872 (1872) Paris

Atti dell'accademia pontaniana (1851), Napoli, vol. IV

Atti della settima adunanza degli scienziati italiani tenuta a Napoli dal 20 di settembre al 5 di ottobre del 1845 (1846), parte seconda, Napoli

Bibliothèque de M. Quatremère de Quincy collection d'ouvrages relatifs aux beaux arts et à l'archéologie (1850), Paris

Bulletin de la librairie Guillaumin et C. Suite au catalogue général (5 Mars 1863), Paris

Catalogue of the library or congress (December 1830), Washington

Clarke's Bibliotheca legum. Complete catalogue of the common and statute law-books of the United Kingdom with an account of their dates and prices, arranged in a new manner, interspersed with observations, from the Authorities, on the principal works (1819), London

The Edinburgh Annual register for 1811, Edinburgh vol. IV, part. II

The European Magazine and London Review (1804), vol. 45

The London and Edinburgh philosophical Magazine and Journal of Science (1833), Brewster, David, Richard Taylor, Richard Philipps (conducted by), London, vol. II, January-June

\section{d. Giornali inglesi del Settecento: "17th-18th Century Burney Collection Newspapers » 1}

Lloyd's Evening Post, issue 1248 (July 8, 1765-July 10, 1765)

Public Advertiser, issue 7509 (Wednesday, 22 November 1758)

London Evening Post, issue 5091 (19 July 1760)

London Chronicle, issue 548 (June 28, 1760-July 1, 1760)

Public Ledger or The Daily Register of Commerce and Intelligence, issue 162 (Friday, 18 July 1760)

St. James Chronicle or British Evening Post, issue 334 (April 26, 1763-April 28 1763)

Morning Chronicle and London Advertiser, issue 4258 (Thursday, January 9, 1783)

London Chronicle, issue 5673 (Thursday, 27 December 1792)

Morning Post, issue 6142 (Saturday, 29 December 1792)

Star, issue 1456 (Tuesday, 8 January, 1793)

1 Consultazione del catalogo del Gale Group grazie all'accesso fornito dalla Max Planck Society in data 27 maggio 2016. 
St. James's Chronicle or the British Evening Post, issue 6536 (November 7, 1799 November 9, 1799)

e. Giornali inglesi: «The Time Digital Archive 1785-1985 » ${ }^{2}$

The Times, issue 2796 (Thursday, 3 October 1793)

The Times, issue 6159 (Tuesday, 23 October 1804)

The Times, issue 8507 (Wednesday, 12 August 1812)

The Times, issue 9086 (Wednesday, 8 December 1813)

The Times, issue 10636 (Thursday, 1 April 1819)

The Times, issue 10949 (Wednesday, 10 November 1820)

The Times, issue 12132 (Thursday, 11 March 1824)

The Times, issue 16809 (Thursday, 16 August 1838)

The Times, issue 16833 (Thursday, 13 September 1838)

The Times, issue 16923 (Thursday, 27 December 1838)

The Times, issue 17158 (Friday, 27 September 1839)

The Times, issue, 18805 (Friday, 27 December 1844)

The Times, issue 20567 (Friday, 28 June 1850)

The Times, issue 20568 (Thursday, 15 august 1850)

The Times, issue 21539 (Wednesday, 21 September 1853)

The Times, issue 24823, (Tuesday, 11 June 1867)

The Times, issue 26951 (Wednesday, 4 January 1871)

The Times, issue 29973 (Monday, 30 August 1880)

The Times, issue 34498 (Tuesday, 12 February 1895)

The Times, issue 36027 (Monday, 1 January 1900)

The Times, issue 37362 (Thursday, 7 April 1904)

The Times, issue 37401 (Monday, 23 May 1904)

The Times, issue 37507 (Friday, 23 September 1904)

f. Giornali Nord americani dell'Ottocento: « Nineteenth Century U.S. Newspapers ${ }^{3}$

The National Intelligencer and Washington Advertiser, issue 1105 (Friday, 13 November 1807)

The National Intelligencer and Washington Advertiser, issue 1421 (Friday, 17 November 1809).

2 Consultazione del catalogo del Gale Group grazie all'accesso fornito dalla Max Planck Society in data 27 maggio 2016.

3 Consultazione del catalogo del Gale Group grazie all'accesso fornito in data 27 maggio 2016 dalla Max Planck Society. 
National Intelligencer, issue 1663 (Saturday, 8 June 1811)

Daily National Intelligencer, issue 116 (Saturday, 15 May 1813)

The Mississipi State Gazette, issue 14 (Saturday, 4 April 1818)

The Mississipi State Gazette, issue 15 (Saturday, 11 April 1818)

The Mississipi State Gazette, issue 17 (Saturday, 25 April 1818)

Daily National Intelligencer, issue 2055 (Friday, 13 August 1819)

Daily National Intelligencer, issue 2240 (Friday, 17 March 1820)

Daily National Intelligencer, issue 44 (Thursday, 18 November 1852)

Daily National Intelligencer, issue 12448 (Tuesday, 18 January 1853)

Daily National Intelligencer, issue 18693 (Thursday, 17 February 1853)

The Weekly Raleigh Register, issue 52 (Wednesday, 12 October 1853)

Daily National Intelligencer, issue 13226 (Wednesday, 18 July 1855)

g. Giornali latino americani: «Latin American Newspapers (1805-1922)» 4

Peruano (28 Octubre 1840)

El Siglo Diez y Nueve (18 Mayo 1842)

El Siglo Diez y Nueve (6 Octubre 1842)

El Siglo Diez y Nueve (30 Octubre 1842)

El Siglo Diez y Nueve (31 Octubre 1842)

El Siglo Diez y Nueve (6 Noviembre 1842)

El Siglo Diez y Nueve (13 Noviembre 1842)

El Siglo Diez y Nueve (31 Deciembre 1842)

El Siglo Diez y Nueve (10 Abril 1843)

Peruano (6 Noviembre 1844)

Peruano (11 Noviembre 1845)

Peruano (22 Abril 1846)

h. American Proceedings: "The Making of Modern Law.

Trials $1600-1926 »^{5}$

Report of a Case, Decided on Saturday, the 16th of November, 1793, in The General Court of Virginia (1794), Philadelphia

4 Consultazione del catalogo della Readex (Latin American Newspaper [1805-1922]) grazie all'accesso fornito in data 20 marzo 2016 dalla Bodleian Library di Oxford.

5 Consultazione del catalogo del Gale Group grazie all'accesso fornito in data 27 maggio 2016 dalla Max Planck Society.

276 Bibliografia 
Proceedings on the Impeachment of William Blount, a Senator of the United States From the State of Tennessee, for High Crimes and Misdemeanors (1799), Philadelphia

The Claim and Answer with the Subsequent Proceedings, in the Case of the Right Reverend Charles Inglis, against the United States (1799), Philadelphia

Bache, Richard (reported by) (1813), The Case of Alien Enemies, considered and decided upon a writ of Habeas Corpus, allowed on the petition Charles Lockington, an Alien Enemy, Philadelphia

Opinions of the Judges of the Supreme Court of the United States, in the Case of the Proceedings of Charles River Bridge vs. the Proprietors of Warren Bridge and Others. Delivered at the January Term of the Court, at Washington (1837), Boston

Statement of the Claim of the State of Alabama against the United States; with Argument in Support thereof, and Numerous Precedents from Usage of the Government, and Cases in Point, now Before Congress (1850), Washington

Russell, Charles W. (1851), Argument for the Defendants, in the Case of the State of Pennsylvania, vs. The Wheeling \& Belmont Bridge Co. and Others, in the Supreme Court of the United States, Philadelphia

Howard, Benjamin C. (1857), A Report of the Decision of the Supreme Court of the United States, and the Opinions of the Judges thereof, in the Case of Dred Scott versus John F. A. Sandford, New York

Report of the Lemmon Slave Case: Containing Points and Arguments of Counsel of both Sides, and Opinions of all the Judges (1860), New York

Murphy, D. F. (1861), Full Report of the Trial of William Smith for Piracy, as one of the Crew of the Confederate Privateer, the Jeff Davis. Before Judges Grier and Cadwalader, in the Circuit Court of the United States, for the Eastern District of Pennsylvania, Held at Philadelphia, in October, Philadelphia

i. Fonti $^{6}$

Авbattista, Guido (2008), Edmund Burke, la guerra e i " poveri ebrei di St. Eustatius ». L'impero atlantico e il diritto delle genti, in: Cromohs, 13, 1-33, http:// www.fupress.net/index.php/cromohs/article/view/15494/14728

Авватtista, Guido (2011), Una mappa per una svolta transnazionale, in: Contemporanea, $4,773-780$

6 Nel ricostruire la presente bibliografia si è ritenuto opportuno riportare sotto il nome del traduttore e/o curatore tutte le indicazioni bibliografiche delle prefazioni, introduzioni, avvertimenti, redatti alle opere di Grozio, Pufendorf, Burlamaqui, e Vattel citate all'interno dei capitoli, nonché sotto il nome degli autori le edizioni stesse, anche quelle più recenti. 
Abreu y Bertodano, José Antonio de (1740-1751), Coleccion de los tratados de paz, alianza, neutralidad, garantia, proteccion, tregua, mediacion, accesion, reglamento de limites, comercio, navegacion, \&c., hechos por los pueblos, reyes, y principes de España con los pueblos, reyes, principes, republicas, y demàs potencias de Europa, y otras partes del mundo: Desde antes del establecimiento de la monarchia gothica, hasta el feliz reynado del rey N.S.D. [Fernando VI], 12 vol., Madrid, Peralta

Abreu y Bertodano, José Antonio de (1746), Tratado jurídico-político sobre pressas de mar y calidades que deben concurrir para hacerse legítimamente el Corso, Cadiz, Impr. Real de Marina

Addy, George M. (1966), The Enlightenment in the University of Salamanca, Durham

Aguilar Piñal, Francisco (1969), La Universidad de Sevilla en el siglo XVIII. Estudio sobre la primera reforma universitaria moderna, Sevilla

Almici, Giovambattista (1757), Prefazione, in: Pufendorf, Samuel, Il diritto della natura e delle genti o sia sistema generale de' principii li più importanti di morale, giurisprudenza e politica, rettificato, accresciuto e illustrato, Venezia, Pietro Valvasense, t. I, I-XII

Álvarez, Alejandro (1910), Le droit international américain. Son fondement - sa nature d'après l'histoire diplomatique des Etats du Nouveau Monde et leur Vie Politique et Economique, Paris

Álvarez de Morales, Antonio (1988), La Ilustración y la Reforma de la Universidad en la España del siglo XVIII, Madrid

Álvarez de Morales, Antonio (1998), La difusión del derecho natural y de gentes europeo en la universidad española de los siglos XVIII y XIX, in: Doctores y escolares. II congreso internacional de Historia de las Universidades Hispánicas (Valencia 1995), Valencia, vol. I, 49-60

Álvarez de Morales, Antonio (2003), Los origines del derecho natural y de gentes en España, in: Peset, Mariano (coord.), Aulas y Saberes. VI Congreso Internacional de Historia de las Universidades Hispánicas, Valencia, 141-158

Álvarez de Morales, Antonio (2008), El salmantino condado y la difusión del Derecho Natural y de Gentes, in: Rodríguez-San Pedro Bezares, Luis E., Juan Luis Polo Rodríguez (eds.), Universidades Hispánicas: Modelos territoriales en la Edad Moderna (II): Valencia, Valladolid, Oñate, Oviedo y Granada. Miscelánea Alfonso IX, Salamanca, 271-281

Álvarez Londoño, Luis Fernando (2006), Vattel (Emerico de) 1714-1767, in: Álvarez Londoño, Luis Fernando, La historia del derecho internacional público, Bogotà, 91-93

Andreu, Antonio Jara (1977), Derecho natural y conflictos ideológicos en la Universidad española (1750-1850). Estudios de historia de la administracion, Madrid

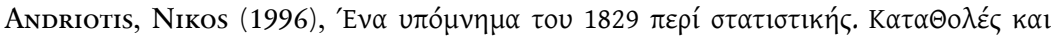
$\varepsilon \pi 1 \delta \rho \alpha ́ \sigma \varepsilon 1 \varsigma$, in: Mvฑ́ $\mu \omega v, 18,181-190$ 
Angeloni, Luigi (1818), Dell'Italia, uscente il settembre 1818, ragionamenti IV, di Luigi Angeloni Frusinate, dedicati all'Italica Nazione, Parigi, vol. I-II

Anghie, Antony (2011), Vattel and Colonialism: Some Preliminary Observations, in: Chetail, Vincent, Peter Haggenmacher (eds.), Vattel's International Law in a XXIst Century Perspective, Le droit international de Vattel vu du XXIe siècle, Leiden, Boston, 237-253, https://doi.org/10.1163/ej.9789004194632.i-443.62

Anghie, Antony (2014), Vattel, Internal Colonialism, and the Rights of Indigenous People, in: Nichols, Robert, Jakeet Singh (eds.), Freedom and Democracy in an Imperial Context, Abingdon, London, 81-99

Anzilotti, Dionisio (1928), Corso di diritto internazionale, Roma

Arafat, Karim (1987), A Legacy of Islam in Greece: 'Ali Pasha and Ioannina, in: British Society for Middle Eastern Studies. Bulletin, 14, 2, 172-182

Arborio Gattinara di Breme, Ludovico Pietro (1822), Brevi osservazioni di un piemontese intorno ad alcune inesattezze di quattro racconti venuti in luce sopra la tentata rivoluzione del Piemonte nel 1821, Parma

Arias Ospina, Felipe (2013), Dinámica del derecho a la no-intervención en América del siglo XIX, in: Pensamiento Jurídico, 36, enero-abril, 189-204, http://www. revistas.unal.edu.co/index.php/peju/article/viewFile/40322/pdf_501

Arias, Garcia Luis, Arthur Nussbaum (1949), Historia del derecho internacional. Adiciones sobre la historia de la doctrina hispanica de derecho internacional, Madrid

Armando, David (2014), Pacca Bartolomeo, in: Dizionario Biografico degli Italiani, Roma, 80, 39-45

Armani, Giuseppe (1979), Aspetti della diffusione delle idee illuministiche nei territori estensi, in: Berengo, Marino, Sergio Romagnoli (a cura di), Reggio e $\mathrm{i}$ territori estensi dall'Antico regime all'età napoleonica. Atti del convegno di studi 18-20 Marzo 1977, Reggio Emilia, Parma, vol. II, 345-371

Armitage, David (2000), Edmund Burke and Reason of State, in: Journal of the History of Ideas, 61, 4, 2000, 617-634, https:/doi.org/10.1353/jhi.2000.0033

Armitage, David (2002), The declaration of Independence and International law, in: The William and Mary Quarterly, 59, 1, 39-64, https://doi.org/10.2307/ 3491637

Armitage, David (2004), The Fifty Years' Rift: Intellectual History and International Relations, in: Modern Intellectual History, 1, 97-109, https:/doi.org/10.1017/ S1479244303000027

Armitage, David (2013), Foundations of the Modern International Thought, Cambridge

Astigarraga, Jesús (2005), I traduttori spagnoli di Filangeri e il risveglio del dibattito costituzionale (1780-1839), in: Trampus, Antonio (a cura di), Diritto e costituzione. L'opera di Gaetano Filangeri e la sua fortuna europea, Bologna, 238-264

Astigarraga, Jesús (2008), Iusnaturalismo moderno de la mano de la economía política: las «Apuntaciones al Genovesi » de Ramón de Salas, in: Historia Constitucional (revista electrónica), 9, 135-161, http://www.historiaconstitu 
cional.com/index.php/historiaconstitucional/article/view/147/131 consultato il 24 maggio 2016

Augusti, Eliana (2013), Questioni d'Oriente: Europa e Impero Ottomano nel diritto internazionale dell'Ottocento, Napoli, Roma

Augusti, Eliana (2015), Peace by Code. Draft Solution for a Codification of International Law, in: Hippler, Thomas, Miloš Vec (eds.), Paradoxes of Peace in 19th Century Europe, Oxford, 37-61, https:/doi.org/10.1093/acprof:oso/ 9780198727996.003.0003

Ayling, Stanley (1988), Edmund Burke: his Life and Opinions, New York

Azcárate, Gumersindo de (2002), Ensayo sobre el derecho de gentes, Madrid

Azouvi, François, Dominique Burel (1991), De Königsberg à Paris, la réception de Kant en France (1788-1804), Paris

Bachmann, Adrian (1993), Die preussische Sukzession in Neuchâtel: ein ständisches Verfahren um die Landesherrschaft im Spannungsfeld zwischen Recht und Utilitarismus (1694-1715), Zürich

Bachmann, Hanns-Martin (1977), Die naturrechtliche Staatslehre Christian Wolffs, Berlin

Bachmann-Medick, Doris (2006), Cultural Turns, Neuorientierungen in den Kulturwissenschaften, Reinbek bei Hamburg

BAggiolini, Cristoforo (1821), Relazioni di un carbonaro piemontese ad un carbonaro spagnolo sugli affari presenti a Torino, Torino

Baker, Betsy (2007), The "Civilised Nation" in the work of Johann Caspar Bluntschli, in: Kremer, Markus, Hans-Richard Reuter (Hrsg.), Macht und Moral - Politisches Denken im 17. und 18. Jahrhundert, Stuttgart, 342-358

Baker, Keith Michael (1990), Inventing the French Revolution: Essays on French Political Culture in the Eighteenth Century, Cambridge

Baker, Keith Michael (1994), The Idea of Declaration of Rights, in: Van Kley, Dale (ed.), The French Idea of Freedom. The Old Regime and the Declaration of Rights of 1789, Stanford, 154-198

Balbo, Cesare (1844), Le speranze d'Italia, Capolago

Balch, Thомas W. (1915), The United States and the Expansion of the Law between Nations, in: University of Pennylvania Law Review and American Law Register, 64, 2, 113-140, https://doi.org/10.2307/3313623

Balcou, Jean, Sophie, Barthélémy, André Cariou (dir.) (2001), Élie Fréron, polémiste et critique d'art, Rennes

Baldwin, Geoffrey P. (2007), The translation of political theory in early modern Europe, in: Burke, Peter, Ronnie Po-chia Hsia (eds.), Cultural Translation in Early Modern Europe, Cambridge, 101-124, https:/doi.org/10.1017/ CBO9780511497193.007

Balestreri, Paola (1983), Mittermaier e l'Italia. Orientamenti politici e dottrine processualistiche in un carteggio di metà Ottocento, in: Ius Commune, 10, $97-140$

Bancarel, Gilles (2004), Raynal ou le devoir de vérité, Paris 
Bancarel, Gilles (2006), Préface, in: Raynal, Guillaume-Thomas François, Histoire philosophique et politique des établissements et du commerce des Européens dans les deux Indes, Paris, 9-38

Bancarel, Gilles (2011), L’Histoire des deux Indes ou la découverte de la mondialisation, in: Internationale Zeitschrift für Humboldt-Studien, 22, 23-34

Bandeira Galindo, George Rodrigo (2005), Martti Koskenniemi and the Historiographical Turn in International Law, in: European Journal of International Law, 16, 539-559, ejil.org/pdfs/16/3/308.pdf, https://doi.org/10.1093/ ejil/chi130

Bandelier, André (1996), De Berlin à Neuchâtel: la genèse du Droit des gens d'Emer de Vattel, in: Fontius, Martinus, Helmut Holzhey (Hrsg.), Schweizer im Berlin des 18. Jahrhunderts, Berlin, 45-56, https://doi.org/10.1515/ 9783050072296-004

Bandelier, André (2012), Emer de Vattel à Jean Henry Samuel Formey. Correspondance autour du Droit des gens, Paris

Baratta, Giorgio (2007), Antonio Gramsci in contrappunto: dialoghi con il presente, Roma

Barbeyrac, Jean (1714), Discours sur l'utilité des lettres et des sciences par rapport au bien de l'Etat, Genève, Fabri \& Barrillot

Barbeyrac, Jean (1724), Préface du traducteur in: Grotius, Hugo, Le droit de la guerre et de la paix. Nouvelle traduction par Jean Barbeyrac. Avec les notes de l'Auteur même, qui n'avoient point encore paru en françois; et de nouvelles notes du traducteur, Amsterdam, P. de Coup, I-XLIII

Barreto, Vicente (1975), Uma introdução ao pensamento político de Silvestre Pinheiro Ferreira, in: Revista Brasileira de Filosofia, 25, 100, 470-478

Barton Korty, Margaret (1965), Benjamin Franklin and Eighteenth-Century American Libraries, in: Transactions of the American Philosophical Society, New Series, 55, 9, 1-83

Bassi, Shaul, Andrea Sirotti (a cura di) (2010), Gli studi postcoloniali: un'introduzione, Firenze

Bassnett, Susan (1980), Translation Studies, 1st ed., London

Bassnett, Susan (2014), Translation Studies, 4th ed., New York

Bassnett, Susan, André Lefevere (1990), Translation, History and Culture, London

Bassnett, Susan, André Lefevere (1998), Constructing Culture, Essay on Literary Translation, Clevedon, Philadelphia

Bassnett, Susan, Harish Trivedi (1999), Introduction, in: Bassnett, Susan, Harish Trivedi (eds.), Post-Colonial Translation. Theory and Practice, London, 1-18

Basso, Alberto (2004), La musica nella corrispondenza di Isabelle de Charrière con Jean-Pierre Chambrier d'Oleyres, in: Antolini, Bianca Maria, Teresa Maria Gialdroni, Annunziato Pugliese (a cura di), "Et facciam dolçi canti ». Studi in onore di Agostino Ziino in occasione del suo $65^{\circ}$ compleanno, Lucca, 943-962

Basso, Alberto (coord.) (1995), Contrappunto, in: Enciclopedia della musica, Novara, 244-245 
Bastin, Georges, Álvaro Echeverri, Ángela Campo (2010), Translation and the Emancipation of Latin America, in: Tymoczko, Maria (ed.), Translation, Resistance, Activism, Amherst, 42-64

Baumbach, Karl (1882), Völkerrecht, in: Staats-Lexikon. Handbuch für jeden Staatsbürger zur Kenntnis des öffentlichen Rechts und des Staatslebens aller Länder, insbesondere des Deutschen Reichs, Leipzig, 604-606

Bazzoli, Maurizio (1979), Giambattista Almici e la diffusione di Pufendorf nel Settecento Italiano, in: Critica Storica, 16, 3-100

Bazzoli, Maurizio (1986), Il pensiero politico dell'assolutismo illuminato, Firenze

Bazzoli, Maurizio (2001), L'ordine internazionale secondo Mably: dal Droit public de l'Europe ai Principes des négociations, in: Mazzanti Pepe, Fernanda (a cura di), Costituzione e diritti fondamentali in Mably. Atti della Giornata di Studio di Genova 25 novembre 1998, Genova, 43-58

Bazzoli, Maurizio (2005), Aspetti della recezione di Pufendorf nel Settecento italiano, in: Ferronato, Marta (a cura di), Dal «De Jure Naturae et gentium » di Samuel Pufendorf alla codificazione prussiana del 1794. Atti del convegno internazionale, Padova, 25-26 ottobre 2001, Padova, 41-60

Beaulac, Stéphane (2003), Emer de Vattel and the Externalization of Sovereignty, in: Journal of the History of International Law, 5, 237-292, https:/doi.org/ $10.1163 / 157180503770735849$

Beaulac, Stéphane (2004), The Power of Language in the Making of International Law: The Word Sovereignty in Bodin and Vattel and the Myth of Westphalia, Leiden, Boston

Beaumont, Christophe (2012), Mandement de Mgr l'Archevêque de Paris portant condamnation d'un livre qui a pour titre «Émile ou l'éducation " par JeanJacques Rousseau citoyen de Genève, in: Trousson, Raymond, Frédéric S. Eigeldinger (sous la direction de), Jean Jacques Rousseau, Cuvres Complètes, Genève, Paris, vol. VIII, 1128-1141

Beccaria, Cesare (1984), Dei delitti e delle pene, Francioni, Gianni (a cura di), con Le edizioni italiane del «Dei delitti e delle pene» di Firpo, Luigi, Milano

Becedas González, Margarita (2004), Los libros de Ius hispanum o Derecho patrio en Salamanca entre los siglos XVIII y XIX: del plan de 1771 a la ley moyano de 1857, in: Salustiano de Dios, Yavier Infante, Eugenia Torijano (coords.), El Derecho y los Juristas en Salamanca (siglos XVI a XX), en memoria de Francisco Tomás y Valiente, Salamanca, 311-336

Becedas González, Margárita (2005), La renovación de las lecturas en la Universidad de Salamanca (1625-1771) y su reflejo en la Biblioteca Universitaria, in: Rodríguez-San Pedro Bézares, Luis Enrique, Juan Luis Polo Rodríguez (coords.), Saberes y disciplinas en las universidades hispánicas, Salamanca, 181-207

Becedas González, Margárita, Oscar Lilao Franca (2006), La Biblioteca General Universitaria: evolución histórica y fondos, in: Saberes y confluencias, in: Rodríguez-San Pedro Bezares, Luis Enrique (coord.), Historia de la Universidad de Salamanca, III.2, Salamanca, 880-953 
Becker Lorca, Arnulf (2010), Universal International Law: Nineteenth Century Histories of Imposition and Appropriation, in: Harvard International Law Journal, 51, 2, 475-552

Becker Lorca, Arnulf (2012), Eurocentrism in the History of International Law, in: Fassbender, Bardo, Anne Peters (eds.), The Oxford Handbook of the History of International Law, Oxford, 1034-1057, https:/doi.org/10.1093/ law/9780199599752.003.0044

Becker Lorca, Arnulf (2014), Mestizo International Law: a Global Intellectual History (1842-1933), Cambridge

BÉguelin, Edouard (1929), En souvenir de Vattel (1714-1767), in: Recueil de travaux offert par la Faculté de Droit de l'Université de Neuchâtel à la Société Suisse de Juristes à l'occasion de sa reunion à Neuchâtel, 15-17 Septembre 1929, Neuchâtel, 35-176

Belissa, Marc (1998), Fraternité Universelle et Intérêt National (1713-1795). Les cosmopolitiques du droit des gens, Paris

Belissa, Marc (2010), La déclaration du droit des gens de l'abbé Grégoire (juin 1793, 4 floréal an III). Etudes, in: Révolution Française.net, http://revolutionfrancaise.net/2010/10/06/399-declaration-droit-des-gens-abbe-gregoire-juin-1793, consultato il 21 maggio 2016

Belissa, Marc (2013), La place du droit naturel chez Mably. Eléments de débat, in: Corpus, 64, 111-128

Belissa, Marc (2015), De la critique de «l'art de négocier " à l'apprentissage de la «politique »: mort du «bon ambassadeur » et apparition du «diplomate » (c. 1750 - c. 1830 ), in: Andretta, Stefano, Stéphane Péquignot, Jean-Claude WAQUET (ét. réunis par), Les écrits relatifs à l'ambassadeur et à l'art de négocier du Moyen Âge au début du XIXe siècle, Rome, 523-558

Bellia, Anthony J., Clark R. Bradford (2011), The Alien Tort Statute and the Law of Nations, in: The University of Chicago Law Review, 78, 2, 445-552

Bello, Andrés (1832), Principios de Derecho de Gentes, Santiago de Chile

Belpaire, Antoine et al. (pub. par) (1840), Bibliographie. Le droit des gens ou principes de la loi naturelle, appliqués à la conduite et aux affaires des Nations et des Souverains, revue et corrigée avec quelques remarques de l'editor, augmentée de quelques remarques nouvelles, et d'une bibliographie choisie et systématique du droit de la nature et des gens, par M. de Hoffmann, note et table général par Pinheiro Ferreira, in: Archives de droit et de législation, Bruxelles, t. III, 219-220

Benjamin, Walter (1961), Die Aufgabe des Übersetzers, in: Benjamin, Walter, Illuminationen. Ausgewählte Schriften, Frankfurt am Main, 56-70

Benjamin, Walter (1976), Il compito del traduttore, in: Benjamin, Walter, Angelus Novus. Saggi e frammenti, trad. it. Solmi, Renato, 37-50

Benjamin, Walter (1983), Strada a senso unico. Scritti 1926-1927. Edizione italiana (a cura di) Agamben, Giongio, Torino 
Benjamin, Walter (2006), Parco centrale, in: Opere Complete di Walter Benjamin, Tiedemann Rolf, Hermann Schweppenhäuser (a cura di), edizione italiana di Ganni Enrico, Torino, vol. VII, 179-209

Benot, Yves (2000), À propos de la quatrième édition de l'Histoire des deux Indes, in: Bancarel, Gilles, Gianluigi Goggi (Textes réunis et présentés par), Raynal de la polémique à l'Histoire, Oxford, 165-171

Bentham, Jeremy (1789), An introduction to the principles of morals and legislation, London

Benton, Lauren (2002), Law and Colonial Cultures. Legal Regimes in World History, $1400-1900$, Cambridge

Benton, Lauren, Lisa Ford (2016), Rage for Order: The British Empire and the Origins of International Law, 1800-1850, Cambridge

Beolchi, Carlo (1865), Il Piemonte nel 1821, in: Rivista Contemporanea, 38, 425-464, Torino

Bernardi, Bruno, Florent Guérard, Gabriella Silvestrini (sous la direction de) (2005), La religion, la liberté, la justice. Un commentaire des Lettres écrites de la montagne de Jean-Jacques Rousseau, Paris

Berti, Francesco (2010), Illuminismo, rivoluzione, modernità: lo spirito del secolo in alcuni significativi scritti dei gesuiti espulsi, in: Baldini, Ugo, Gian Paolo Brizzi (a cura di), La presenza in Italia dei gesuiti iberici espulsi. Aspetti religiosi, politici, culturali, Bologna, 321-336

Berti, Giampietro, Franco Della Perruta (a cura di) (2004), La nascita della nazione, la carboneria: intrecci veneti, nazionali e internazionali. Atti del 26 convegno di studi storici, Rovigo, Crespino, Fratta Polesine, 8, 9, 10 novembre 2002, Rovigo

Beuchot, Adrien Jean Quentin (1839), Bibliographie de la France ou Journal Bibliographie de la France ou Journal général de l'Imprimerie et de la Librairie, et des cartes géographiques, gravures, lithographies et œuvres de musique, XXVIII année, Paris

Внавна, Номі K. (2001), I luoghi della cultura, Roma

Birocchi, Italo (2002), Alla ricerca dell'ordine. Fonti e cultura giuridica nell'età moderna, Torino

Birocchi, Italo (2013), De Simoni Alberto, in: Birocchi, Italo, Ennio Cortese, Antonello Mattone, Marco Nicola Miletti (diretto da), Dizionario Biografico dei Giuristi Italiani (XII-XX secolo), Bologna, vol. I, 718-772

Biucchi, Basilio M. (1983), Profilo di storia economica e sociale della Svizzera, Locarno

Blanning, Timothy Charles William (1986), The Origins of the French Revolutionary Wars, London, New York

Blanning, Timothy Charles William (1996), The French Revolutionary Wars (1789-1802), London, New York

Bluntschli, Johann Caspar (1868), Das moderne Völkerrecht der civilisirten Staten als Rechtsbuch dargestellt, Nördlingen 
Bobbio, Giovanni (1922), Appunti e note critiche sulla Rivoluzione piemontese del 1821, in: Rivista di Storia, Arte, Archeologia della Provincia di Alessandria, 4, $3-44$

Bobbio, Norberto (2009), Il Giusnaturalismo moderno, Greco, Tommaso (a cura di), Torino

Bodin, Jean (1583), Les six livres de la République, Paris, Puys

Bohorquez, Julio Gaitán (2002), Huestes de estado: la formacion universitaria de los juristas en los comienzos del estado colombiano, Bogotà

Bonfils, Henry (1904), Lehrbuch des Völkerrechts für Studium und Praxis, Berlin

Borgeaud, Charles (1938), La publication des "Principes du droit politique » de Burlamaqui, in: Recueil des travaux publiés par la Faculté de droit de l'Université de Genève, Genève, 73-82

Bork, Kennard B. (1974), The geological insights of Louis Bourguet (1678-1742); in: Journal of the scientific laboratories, 55, 49-77

Bosica, Ludovica (2015), Droit et politique au XVIIIe siècle: considérations à propos du droit public européen, relazione presentata in occasione del convegno intitolato «L'Histoire du droit entre science et politique. Colloque international jeunes chercheurs » (Bordeaux, 15-16 ottobre 2015), i cui atti sono in corso di stampa

Bots, Hans, Johan Nicolas Joseph Schillings (2001), Lettres d'Élie Luzac à Jean Henri Samuel Formey: regard sur les coulisses de la librairie hollandaise du XVIIIe siècle, Paris

Bourguignon, Henri J. (1977), Incorporation of the Law of Nations during the American Revolution, The Case of the San Antonio, in: The American Journal of International Law, 71, 2, 270-295, https:/doi.org/10.2307/2199529

Boye, Thorvald (1938), Quelques aspects du développement des règles de la neutralité, in: Recueil des cours de l'Académie de droit international de la Haye, 64, II, 157-231, https://doi.org/10.1163/ej.9789028610125.157-231

Braccia, Roberta (2013), Casanova, Luigi (Ludovico), in: Birocchi, Italo, Ennio Cortese, Antonello Mattone, Marco Nicola Miletti (diretto da), Dizionario Biografico dei Giuristi Italiani (XII-XX secolo), Bologna, vol. I, 474-475

Braggio, Carlo (1892), La rivoluzione piemontese del 1821, in: Giornale ligustico, $19,183-225$

Bragt, Katrine van (1995), Bibliographie des traductions françaises (1810-1840). Répertoire par disciplines, Louvain

Brandli, Fabrice (2006), Une résidence en République: le résident de France à Genève et son rôle face aux troubles politiques de 1734 à 1768, Genève

Brandli, Fabrice (2012a), Le nain et le géant. La République de Genève et la France au XVIIIe siècle, cultures politiques et diplomatie, Rennes

Brandli, Fabrice (2012b), Pierre-Michel Hennin, la République de Genève et le républicanisme, in: Musicorum, 13, 15-26

Brandli, Fabrice (2014), La République de Genève et la France au XVIIIe siècle: diplomatie asymétrique et culture politique, in: Revue d'histoire moderne et contemporaine, 61/4bis, 4, 65-93, https://doi.org/10.3917/rhmc.614.0065 
Brandt, Reinhard (2007), Hobbes, Locke, Wolff, in: Ecole, Jean, Jürgen Stolzenberg, Oliver Pierre Rudolph (Hrsg.), Wolffiana II.1 - Christian Wolff und die europäische Aufklärung (Akten des 1. Internationalen Christian Wolff-Kongresses, Halle (Saale), 4.-8. April 2004, Teil 1), Christian Wolff, Gesammelte Werke, Materialien und Dokumente, Bd. 101, Hildesheim, Zürich, New York, 113-132

Bravard-Veyrières, Pierre Claude Jean Baptiste (1861), Des Prises Maritimes, d'après l'ancien et le nouveau droit tel qu'il résulte du traité de Paris et de la déclaration du 16 avril 1856, avec des notes par Paul Royer-Collard, Paris

Bravetti, Patrizia, Orfea Granzotto (a cura di) (2008), False date. Repertorio delle licenze di stampa veneziana con falso luogo di edizione, Firenze

Briasson, Antoine-Claude, Nicolas-Charles-Joseph Trublet (1996), Correspondance passive de Formey. Antoine-Claude Briasson et Nicolas Charles Joseph Trublet. Lettres adressées à Jean-Henri-Samuel Formey (1739-1770), Fontius, Martin, Rolf Geissler, Jens Häseler (textes édites par), Paris, Genève

Brierly, James Leslie (1967), The Law of Nations, 6th edition, Oxford

Brooke Clarke, Thomas (1803), An historical and political view of the disorganisation of Europe wherein the Laws and characters of Nations, and the Maritime and Commercial system of Great Britain and other States, are vindicated against the imputations and revolutionary proposals of M. Talleyrand and M. Hauterive, Secretaries of State to the French Republic, London

Broussois, Lisa (2015), Burlamaqui and Rousseau on the Law of War and the Law of Nations, relazione presentata durante la conferenza «The Law of Nations and Natural Law 1625-1850» (Losanna, 5-6 novembre 2015), i cui atti sono in corso di stampa

Brownlie, Ian (1984), The Expansion of International Society: the Consequences for the Law of Nations, in: Bull, Hedley, Adam Watson (eds.), The Expansion of International Society, Oxford, 357-369

Brugnatelli, Stefano (2013), Carnazza Amari Giuseppe, in: Birocchi, Italo, Ennio Cortese, Antonello Mattone, Marco Nicola Miletti (diretto da), Dizionario Biografico dei Giuristi Italiani (XII-XX secolo), Bologna, vol. I, 455 Buddeus, Johann Franz (1697), Elementa philosophiae practicae, Halle, Zeidler BulmerincQ, August von (1889), Völkerrecht oder das internationale Recht. Systematisch dargestellt, mit einem Nachtrag vermehrte Ausgabe, Freiburg

Buonafede, Appiano (1792), Della restaurazione di ogni filosofia pe' secoli XVI, XVII, XVIII, Milano, Venezia, Pasquali, vol. III

Burke, Edmund (1797), Three Memorials on French Affairs, Written in the Years 1791, 1792 and 1793, London, Rivington

Burke, Edmund (1816), Speech on the Seizure and Confiscation of Private Property in St. Eustatius, in: The Speeches of the Right Honourable Edmund Burke, in the House of Commons and in Westminster-Hall, London, vol. II, 244-269

Burlamaqui, Jean Jacques (1751), Principes du droit politique, Amsterdam, Zacharias Chatelain, t. II 
Burlamaqui, Jean Jacques (1768), Les Principes du droit de la nature et des gens de J.J. Burlamaqui avec la Suite du Droit de la nature qui n'avait point encore paru, le tout considérablement augmenté par Mr le professeur De Felice, Yverdon, t. VII

Burlamaqui, Jean Jacques (1780), Principii del diritto naturale di G.G. Burlamachi consigliere di Stato, già professore di diritto naturale e civile in Ginevra. Traduzione dal francese del C.B.C., 2 vol., Venezia, Giovanni Gatti

Burlamaqui, Jean Jacques (1780-1782), Principi del diritto della natura e delle genti. Colla continuazione del diritto della natura aggiunta nell'ultima edizione d'Yverdon. Tutto notabilmente accresciuto da de Félice. Traduzione dal francese, 8 vol., Siena, Bindi

Cadalso, José (1772), Los eruditos a la violeta ó courso completo de todas las ciencias dividido en siete lecciones para los siete dias de la semana con el Suplemento de este compuesto por don Joseph Vazquez, quien lo publica en obsequio de los que pretenden saber mucho, estudiando poco, Madrid, Antonio de Sancha

Cagnoli, Luigi (1837), Lodovico Antonio Loschi, in: Notizie biografiche in continuazione della biblioteca modenese del cavaliere e abate Girolamo Tiraboschi, Reggio, t. V, 333-341

Calamo Specchia, Marina (2015), Burke e la Rivoluzione in Francia, ovvero la sublimazione del panta rei/Burke and the Revolution in France, that is the sublimation of the panta rei, in: Giornale di Storia Costituzionale, Il pensiero di Edmund Burke. Costituzionalismo ereditato e costituzioni rivoluzionarie/ The thought of Edmund Burke. Inherited constitutionalism and revolutionary constitutions, 29, 1, 71-88

Calvo, Carlos (1885), Vattel Emer de, in: Calvo, Carlos, Dictionnaire de droit international public et privé, Paris, Berlin, t. II, 296-297

Camariano-Cioran, Ariadna (1967), «L'œuvre de Beccaria 'Dei delitti e delle pene' et ses traductions en langue grecque et roumaine ", in: Revue des etudes sudest europeennes, 5, 193-202

Candaux, Jean-Daniel (1973), Les gazettes helvétiques: inventaire provisoire des périodiques littéraires et scientifiques de langue française publiés en Suisse de 1693 à 1795, in: Couperus, Marianne (dir.), L'étude des périodiques anciens. Colloque d'Utrecht (9-10 janvier 1970), 126-171

Candaux, Jean-Daniel (1988), Le Mercure suisse dans son premier lustre (17321737): un périodique à la recherche de son public, in: Bots, Hans (éd.), La diffusion et la lecture des journaux de langue française sous l'Ancien Régime. Actes du colloque international de Nimègue (3-5 juin 1987), Amsterdam, $49-57$

Candaux, Jean-Daniel (1991a), Mercure Suisse I (1732-1737), in: Sgard, Jean (sous la direction de), Dictionnaire de la presse 1600-1789, I. Dictionnaire de journaux, Paris, vol. II, j-v, n. 950, 884-885 
Candaux, Jean-Daniel (1991b), Journal Helvétique (1738-1769), in: Sgard, Jean (sous la direction de), Dictionnaire de la presse 1600-1789, I. Dictionnaire de journaux, Paris, vol. II, n. 743, 682-683

Cañizales Verde, Francisco (1997), Francisco Javier Yanes: Teniente Justicia Mayor (Diputado al Congreso de 1811 por la Villa de Araure), Barquisimeto

Cantù, Cesare (1864), Il tempo dei Francesi (1796-1815), brani di storia d'Italia, Napoli

Cantù, Cesare, Gaetano Barbati (1859), Storia degli italiani, Napoli, vol. VI

Cappellini, Paolo, Bernardo Sordi (a cura di) (2002), Codici. Una riflessione di fine millennio. Atti dell'incontro di studio, Firenze 26-28 ottobre 2000, Milano

Carafa, Giuseppe Maria (1769), Difesa del vescovo di Mileto e del Collegio dei Greci in Roma contro un'istanza fiscale ed una scrittura stampata col titolo di Dimostrazione del padronato della Real Corona, sulla chiesa e badia della Trinità di Mileto, Napoli

Carnazza Amari, Giuseppe (1865), Prelezione al corso di diritto internazionale letta nella Regia Università degli studi di Catania, Catania

Caroni, Pio, Ettore Dezza (a cura di) (2006), L’ABGB e la codificazione asburgica in Italia e in Europa, Atti del Convegno Internazionale, Pavia, 11-12 ottobre 2002, Padova

Caroni, Pio, Riccardo Ferrante (2015), La codificazione del diritto tra il Danubio e l'Adriatico. Per i duecento anni dall'entrata in vigore dell'ABGB. Atti del convegno internazionale. Trieste, 25-27 ottobre 2012, Torino

Carozzi, Marguerite (1986), From the concept of salient and reentrant angles by Louis Bourguet to Nicolas Desmarest's description of meandering rivers, in: Archives des sciences, 39, 25-51

Carpanetto, Dino (1980), Moti del 1820-1821, in: Bongiovanni, Bruno, Gian Carlo Jocteau, Nicola Tranfaglia (a cura di), Il mondo contemporaneo. Storia d'Europa, Firenze, vol. II, 598-614

Carrera, Alberto (2012), Il diritto di resistenza nella dottrina giuridica di Emer de Vattel, in: Sciumè, Alberto (a cura di), Il diritto come forza, la forza del diritto. Le fonti in azione nel diritto europeo tra Medioevo ed età contemporanea, Torino, 81-109

Carrera, Alberto (2015), Pietro Tamburini «Giurista ». Per una storia della cultura giuridica giansenista italiana, tesi di dottorato discussa presso l'Università degli studi di Milano, tutor prof.ssa Maria Gigliola di Renzo Villata

Cassi, Gellio (1931), Il cardinal Consalvi ed i primi anni della restaurazione pontificia (1815-1819), Firenze

Castiglione, Tommaso R. (1965), Fortunato Bartolomeo De Felice tra Voltaire e Rousseau, in: Studi di letteratura, storia e filosofia in onore di Bruno Revel, Firenze, $155-178$

Cavallar, Georg (2008), Vitoria, Grotius, Pufendorf, Wolff and Vattel: Accomplices of European Colonialism and Exploitation or True Cosmopolitans?, in: Jour- 
nal of the History of International Law, 10, 2, 181-209, https:/doi.org/ $10.1163 / 157180508 X 359828$

Cavanna, Adriano (1996), Codificazione del diritto italiano e imperialismo giuridico francese nella Milano Napoleonica. Giuseppe Luosi e il diritto, in: Ius Mediolani. Studi di storia del diritto milanese offerti degli allievi a Giulio Vismara, Milano, 659-760

Cavelti, Ulrich A. (1979), Einflüsse der Aufklärung auf die Grundlagen des Schweizerischen Staatskirchenrechts: mit besonderer Berücksichtigung des Schrifttums Emer de Vattels und Josef Anton Felix Balthasars, Freiburg, Schweiz

Cernuschi, Alain (1993), Lectures de l'Encyclopédie d'Yverdon. Images d'une œuvre et réflexions méthodologique à partir des comptes rendus du « Journal helvétique », in: Annales Benjamin Constant, 14, 85-109

Cernuschi, Alain (2005), L'Encyclopédisme de Lumières et ses modèles, in: Candaux, Jean Daniel et al. (éds.), L'Encyclopédie d'Yverdon et sa résonance européenne, Genève, 470-476

Chamboredon, Robert (actes réunis par), François Guizot, 1787-1874: passé, présent, Paris

Chambrier, Frédéric de (1840), Histoire de Neuchâtel et Valangin jusqu'à l'avènement de la Maison de Prusse, Neuchâtel

Chambrier d'Oleyres, Jean-Pierre (1789), Notices Préliminaires sur des recherches historiques relatives à l'Etat de Neuchâtel et Vallengin

Chambrier d'Oleyres, Jean-Pierre (1793), Questions de Droit des Gens et Observations sur le Traité du Droit des Gens de M. de Vattel, in: Mémoires de l'Académie Royale des Sciences et Belles Lettres, 1788-1789, Berlin, 436459, anche online in: http://bibliothek.bbaw.de consultato il 24 maggio 2016

Chambrier d'Oleyres, Jean-Pierre (1795), Essay sur le Droit des Gens, Parma

Chambrier d'Oleyres, Jean-Pierre (1796), Questions de Droit des Gens et Observations sur le Traité du Droit des Gens de M. de Vattel: suite, in: Mémoires de l'Académie Royale des Sciences et Belles Lettres, 1790-1791, Berlin, 419-430, anche online, in: http://bibliothek.bbaw.de, consultato il 24 maggio 2016

Checchini, Aldo (1950), Vecchi e nuovi metodi della storiografia giuridica, in: Scritti Giuridici in onore a Francesco Carnelutti, Padova, vol. I, 389-415

Chetail, Vincent (2011), Vattel et la sémantique du droit des gens: une tentative de reconstruction critique, in: Chetail, Vincent, Peter Haggenmacher (eds.), Vattel's International Law in a XXIst Century Perspective - Le droit international de Vattel vu du XXIe siècle, Leiden, Boston, 387-434, https://doi.org/ 10.1163/ej.9789004194632.i-443.103

Chetail, Vincent (2014), Vattel and the American Dream: An Inquiry into the Reception of the Law of Nations in the United States, in: Dupuy, PierreMarie, Vincent Chetail (eds.), The Roots of International Law. Les fondements du droit international: Liber Amicorum Peter Haggenmacher, Leiden, $251-300$ 
Chetail, Vincent, Peter Haggenmacher (eds.) (2011), Vattel's International Law in a XXIst Century Perspective - Le droit international de Vattel vu du XXIe siècle, Leiden, Boston, https://doi.org/10.1163/ej.9789004194632.i-443.103

Chevalley, Lucie (1912), La déclaration du droit des gens de l'Abbé Grégoire (1793-1795). Étude sur le droit international public intermédiaire, Paris, Le Caire

Chiaramonte, José Carlos (2002), Fundamentos iusnaturalista de los movimientos de independencia, in: Terán, Marta, José Antonio Serrano Ortega (eds.), Las guerras de independencia en la América española, Zamora, 99-122

Chiaramonte, José Carlos (2004), Nación y Estado en Iberoamérica. El lenguaje político en tiempos de la independencia, Buenos Aires

Chiaramonte, José Carlos (2008), Conceptos y lenguajes político en el mundo iberoamericano, 1750-1850, in: Revista de Estudios políticos, 140, 11-31

Chiaramonte, José Carlos (2010), Fundamentos intelectuales y políticos de las independencias. Notas para una nueva historia intelectual de Iberoamérica, Buenos Aires

Chiarle, Aldo (1999), Carboneria: storia-documenti 1809-1931, Firenze

Chitty, Joseph (1834), Preface to the present edition, in: Vattel, Emer de, The law of nations, or principles of the law of nature, applied to the conduct and affairs of nations and sovereigns, a work tending to display the true interests of powers, London, III-IV

Chretien, Alfred (1893), Principes de droit international public, accompagné d'indications bibliographiques ainsi que de notes intercalées dans le texte et suivi d'un résumé des principaux traités signés par la France depuis 1648 jusqu'à nos jours, Paris

Christov, Theodore (2005), Liberal Internationalism Revisited: Grotius, Vattel and the International Order of States, in: The European Legacy, 10, 6, 561-584, https://doi.org/10.1080/10848770500254076

Christov, Theodore (2013), Vattel's Rousseau: Ius gentium and the Natural Liberty of States, in: Skinner, Quentin, Martin van Gelderen (eds.), Freedom and the Construction of Europe: Free Persons and Free States, Cambridge, vol. II, 167-187, https://doi.org/10.1017/CBO9781139519298.012

Ciferri, Elvio (2010), Michitelli Francesco, in: Dizionario Biografico degli Italiani, Roma, 74, 339-341

Clavero, Bartolomé (2002), Ama llunku, abya yala: Constituyencia Indígena y Código Ladino por America, Madrid 2002

Clerici, Alberto (2012), Contro l'uguaglianza, contro il privilegio. Il giovane Guizot e i suoi critici (1820-1821), in: Ruocco, Giovanni, Luca Scuccimarra (a cura di), Il governo del popolo. 2. Dalla Restaurazione alla guerra francoprussiana, Roma, 47-69

Clogg, Richard (1996), Storia della Grecia moderna dalla caduta dell'impero bizantino a oggi, Milano

Colombo, Paolo (2002), I presupposti dello Statuto albertino. Dai moti del 1821 alle riforme del 1847, Historia Constitucional (revista electrónica), 3, 147-170, 
http://www.historiaconstitucional.com/index.php/historiaconstitucional/ article/view/174/155

Colombo, Paolo (2014), Emmanuel Sieyès, la storia costituzionale e il sistema istituzionale rivoluzionario, in: Aimo, Piero, Elisabetta Colombo, Fabio Rugge (a cura di), Autonomia, forme di governo e democrazia nell'età moderna e contemporanea. Scritti in onore di Ettore Rotelli, Pavia, 117-128

Comanducci, Paolo (1981), Il Settecento conservatore: Lampredi e il diritto naturale, Milano

Comanducci, Paolo (2013), Lampredi, Maria Giovanni, in: Birocchi, Italo, Ennio Cortese, Antonello Mattone, Marco Nicola Miletti (diretto da), Dizionario Biografico dei Giuristi Italiani (XII-XX secolo), Bologna, vol. I, 1138-1141

Comini, Mariano (1992), Pietro Tamburini, 1737-1827: un giansenista lombardo tra riforma e rivoluzione, Brescia

Comparato, Vittor Ivo (2014), Il diritto di natura a Perugia tra la Repubblica romana e l'Unità, in: Annali di storia delle università italiane, 18, 221-242

Conetti, Giorgio (2013), Fiore Pasquale, in: Birocchi, Italo, Ennio Cortese, Antonello Mattone, Marco Nicola Miletti (diretto da), Dizionario Biografico dei Giuristi Italiani (XII-XX secolo), Bologna, vol. I, 874-876

Consalvi, Ercole (1834), Nota su gli affari temporali della S. Sede con cui fu accompagnata la protesta latina indirizzata agli otto principali ministri della Alte Potenze che sottoscrissero il trattato di Parigi del 30 maggio dell'anno 1814 compito col trattato di Vienna del 9 giugno 1815, in: Fea, Carlo, Il diritto sovrano della Santa Sede sopra le valli di Comacchio e sopra la Repubblica di San Marino, Roma, 36-40

Contr, Fulvio (2003), Storia della massoneria italiana. Dal Risorgimento al fascismo, Bologna

Conti, Vittorio (a cura di) (2002), La recezione di Grozio a Napoli nel Settecento, Firenze

Corio, Alessandro (2007), Elogio dell'ibridità, esotismo postcoloniale e mercato globale: per una genealogia critica del discorso della contaminazione, in: Experience, 10, "Contaminazioni», 1-14, http://www2.lingue.unibo.it/franco fone/corio_contaminazione.pdf, consultato il 4 novembre 2016

Coronas GonzÂles, Santos M. (1998), Jovellanos, ante el plan de estudios ovetense de 1774, in: Doctores y escolares. El congreso internacional de Historia de las universidades Hispánicas (Valencia 1995), Valencia, vol. I, 93-100

Coronas Gonzâles, Santos M. (2008), Jovellanos y la Universidad, Gijón

Coronas Gonzales, Santos M. (2009), Marín y Mendoza, Joachín, in: Diccionario Biográfico Español, Madrid, vol. XXXII, 581

Cotugno, Raffaele (1923), Tra reazioni e rivoluzioni. Contributo alla storia dei Borboni, Lucera

Courtin, Antoine de (1687), Avertissement du Traducteur, in: Grotius, Hugo, Le droit de la guerre et de la paix, divisé in trois livres où il explique le droit de la 
nature, le droit des gens et les principaux Points du droit public, ou qui concerne le gouvernement public d'un Etat, Paris, Arnold Seneuze, t. I, I-X

Courtney, Cecile, Jenny Mander (eds.) (2015), Raynal's Histoire des deux Indes. Colonialism, Networks and Global Exchange, Oxford

Craven, Matthew (2016), Theorising the Turn to History in International Law, in: Orford, Anne, Florian Hoffmann, The Oxford Handbook of the Theory of International Law, in corso di stampa, online http://eprints.soas.ac.uk/21492/, consultato il 24 maggio 2016

Crispi, Benedetto Conte (1780), Il traduttore a chi legge, in: Burlamaqui, Jean Jacques, Principii del diritto naturale di G. G. Burlamachi consigliere di Stato, già professore di diritto naturale e civile in Ginevra, Venezia, Venezia, Giovanni Gatti, vol. I, III-VIII

Crucitti, Ullrich, Francesca Bianca (1974), La «Bibliothèque italique ». Cultura « italianisante » e giornalismo letterario, Milano

Crucitti, Ulrichi, Francesca Bianca (1991), Bibliothèque Italique 1728-1734, in: Sgard, Jean (sous la direction de), Dictionnaire de la presse 1600-1789, I. Dictionnaire de journaux, Paris, vol. I, a-i, n. 165, 191-192

Cruise O'Brien, Conor (1992), The Great Melody: a Thematic Biography and Anthology of Edmund Burke, London

Cunningham, George Godfrey (1839), Sir James Mackintosh, in: The English Nation; or a History of England in the lives of Englishmen, London, vol. V, 208-220

Curzi, Valter (2010), Il patrimonio artistico e monumentale nello Stato pontificio negli anni dell'editto Pacca, in: La Monica, Denise, Francesca Nanni (a cura di), Municipalia. Storia della tutela, Pisa, 207-215

Cushing, Luther (1843), American Jurist and Law Magazine, from April 1838 to January 1843, Boston, vol. X

D’Aguesseau, Henri François (1819), Première Instruction, contenant un Plan général d'Etudes, et en particulier celle de la Religion et celle du Droit, in: CEuvres complètes du Chancelier D’Aguessau. Nouvelle édition, augmentée de pièces échappées aux premiers éditeurs, et d'un discours préliminaire par M. Pardessuss, Paris, t. XV, 1-29

D'Aspremont, Jean (2011), Formalism and the Sources of International Law. A Theory of the Ascertainment of Legal Rules, Oxford

Damilano, Roberto (2014), Palma di Cesnola Alerino, in: Dizionario Biografico degli Italiani, Roma, 80, 586-88

Darnton, Robert (1982), L'aventure de l'Encyclopédie 1775-1800, Paris

Darnton, Robert (1984a), Le livre prohibé aux frontières: Neuchâtel, in: Martin, Henri-Jean, Roger Chartier, Jean-Pierre Vivet (dir.), Histoire de l'édition française: le livre triomphant (1660-1830), Paris, vol. II, 342-359

Darnton, Robert (1984b), Sounding the Literary Market in Prerevolutionary France, in: Eighteenth-Century Studies, 17, 4, 477-492, https:/doi.org/ $10.2307 / 2738131$ 
Darnton, Robert (2008), La science de la contrefaçon, in: Tilkin, Françoise (éd.), L'Encyclopédisme au XVIIIe siècle, Actes du Colloque organisé par le Groupe d'étude du XVIIIe siècle de l'Université de Liège (Liège 30-31 octobre 2006), Genève, 277-292

Darnton, Robert (2009), The Business of Enlightenment. A Publishing History of the Encyclopédie 1775-1800, Cambridge, London

Darnton, Robert, Michel Schlup (éd.) (2005), La Société typographique de Neuchâtel (1769-1789). Actes du colloque organisé par la Bibliothèque publique et universitaire de Neuchâtel et la Faculté des lettres de l'Université de Neuchâtel: Neuchâtel 31 octobre-2 novembre 2002, Neuchâtel

De Felice, Renzo (1961), Angeloni Luigi, in: Dizionario Biografico degli Italiani, Roma, 3, 242-249

De la Guéronnièr Arthur (1876), Le droit publique et l'Europe moderne, Paris, t. I

De la Muela, Miaja (1945), Una sátira española contra los internacionalistas del siglo XVIII: Lección quarta de "Los eruditos a la violeta », in: Colección de estudios en homenaje al profesor Camilo Barcia Trelles en sus bodas de plata de catedrático de derecho internacional, Santiago, 103-131

De la Rasilla del Moral, Ignacio (2013), El estudio del Derecho internacional en el corto siglo XIX español, in: Rechtsgeschichte - Legal History, 21, 48-65, data.rg.mpg.de/rechtsgeschichte/rg21_048rasilla.pdf

De Majo, Silvio (1998), Galiani Ferdinando, in: Dizionario Biografico degli Italiani, Roma, 51, 456-465

De Mattei, Roberto (2001), Le società segrete nelle rivoluzioni italiane, in: Viglione, Massimo (a cura di), La rivoluzione italiana. Storia critica del Risorgimento, Roma, 127-154

De Simoni, Alberto (1776), Del furto e sua pena, Lugano, Agnelli

Defourneaux, Marcelin (1973), Inquisición y censura de libros en la España del siglo XVIII, version española de Tellechea Idigoras, José Ignacio, Madrid

Degan, Vladimir-Djuro (1989), L'affirmation des principes du Droit naturel par la Révolution française. Le project de Déclaration du Droit des Gens de l'abbé Grégoire, in: Annuaire français de droit international, 35, 1, 99-116

Del Vecchio, Giorgio (1964), Le droit international et le problème de la paix, Paris

Deleplace, Marc (2002), L'anarchie de Mably à Proudhon, 1750-1850. Histoire d'une appropriation polémique, Paris

Della Perruta, Franco (1989), Conservatori, liberali e democratici del Risorgimento, Milano

Deloche, Régis (1988), En marge d'un bicentenaire: Galiani et l'apologue, in: Revue économique, 39, 6, 1143-1157

Derôme, Théodore (1856), Bibliographie. Le Droit des gens d'Emmerich de Vattel, in: Revue critique de législation et de jurisprudence, 6, 455-470

Detti, Tommaso (2005), Per una prospettiva di storia globale, relazione presentata al Convegno: L'insegnamento della storia nei licei: riflessioni della comunità degli storici sui nuovi programmi ministeriali, promosso da Reti Medievali; 
SISEM - Società Italiana degli Storici dell'età moderna; SIS - Società Italiana delle Storiche; SISSCO - Società Italiana per lo Studio della Storia Contemporanea, Bologna, 24 giugno 2005, http://www.sissco.it/articoli/linsegnamen to-della-storia-nei-licei-565/, consultato il 24 maggio 2016

Devetak, Richard (2011), Law of Nations as Reason of State: Diplomacy and the Balance of Power in Vattel's Law of Nations, in: Parergon, 28, 2, 105-128, https://doi.org/10.1353/pgn.2011.0080

Dhondt, Frederik (2015a), Historical Exempla in Legal Doctrine, Vattel and Réal de Curban on the War of the Spanish Succession, in: De Ruysscher, Dave, KaAt Cappelle et al. (red.), Rechtsgeschiedenis op nieuwe wegen. Legal History, moving in new directions, Antwerpen, 367-394

Dhondt, Frederik (2015b), Balance of Power and Norm Hierarchy. Franco-British Diplomacy after the Peace of Utrecht, Leiden, Boston

Dhondt, Frederik (2016a), Vattel's Le droit des gens, in: Dauchy, Serge, Georges Martyn et al. (eds.), The Books that made Law in the Western World (13th century-1940), Heidelberg, 247-250

Dhondt, Frederik (2016b), Recent Research in the History of International Law, in: Tijdschrift voor Rechtsgeschiedenis/Revue d'Histoire du Droit/The Legal History Review, 84, 1-2, 313-334, https://doi.org/10.1163/15718190-08412p10

Dhondt, Frederik (2016c), The Law of Nations and Declarations of War after the Peace of Utrecht, in: History of European Ideas, 42, 3, 329-349, https:// doi.org/10.1080/01916599.2015.1118333

Dhondt, Frederik (2016d), Equilibre et hiérarchie: l'argumente juridique dans la diplomatie française et anglaise après la Paix d'Utrecht, in: Drocourt, Nicolas, Eric Schnakenbourg (dir.), Thémis en diplomatie: l'argument juridique dans les relations internationales de l'antiquité tardive à la fin du XVIIIe siècle, Rennes, 67-83

Di Donato, Francesco (2007), La contribution de Jean Barbeyrac au renouvellement du droit européen, in: Poncelet, Christian, Rolf Büttiker, Giovanni Busıno (éd.), Genève et la Suisse dans la pensée politique. Actes du Colloque de Genève (Septembre 2006), Aix-en-Provence, 409-418

Di Piazza, Elio (2004), Studi (post) coloniali, in: Cometa, Michele, Roberta Coglitore, Federica Mazzara (a cura di), Dizionario degli studi culturali, Roma, 417-424

Di Renzo Villata, Maria Gigliola (2004), Introduzione. La formazione del giurista in Italia e l'influenza culturale europea tra Sette e Ottocento: il caso della Lombardia, in: Di Renzo Villata, Maria Gigliola (a cura di), Formare il giurista. Esperienze nell'area lombarda tra Sette e Ottocento, Milano, 1-106

Di Renzo Villata, Maria Gigliola (2008), Le droit public en Lombardie au XVIIIe siècle et l'Europe, in: Krynen, Jacques, Michael Stolleis (dir.), Science politique et droit public dans les facultés de droit européennes (XIIIe-XVIIIe siècle), Frankfurt am Main, 583-612 
Di Rienzo, Eugenio (2002), Decadenza e caduta del cosmopolitismo: Francia/Europa, 1792-1848. Note per una ricerca, in: Bianchi, Lonenzo (a cura di), L'idea di cosmopolitismo: circolazione e metamorfosi, Napoli, 419-458

Di Sabantonio, Bruno (1977), Luigi Angeloni tra liberalismo e democrazia, in: Rassegna Storica del Risorgimento, 64, 3-21

Di Salvo, Ines (1982), Beccaria nelle cultura neogreca antecedente a Koraís, Palermo

Di Simone, Maria Rosa (2005), L'influenza di Christian Wolff sul giusnaturalismo dell'area asburgica e italiana, in: Ferronato, Marta (a cura di), Dal « De jure naturae et gentium»di Samuel Pufendorf alla codificazione prussiana del 1794. Atti del Convegno Internazionale, Padova, 25-26 ottobre 2001, Padova, 221-268

Di Simone, Maria Rosa (2006), Stato e diritto nel pensiero di Gian Rinaldo Carli, in: Di Simone, Maria Rosa, Percorsi del diritto tra Austria e Italia (secoli XVII-XX), Milano, 135-158

Di Stasio, Raffaele (1995), La stampa militare dei Borboni a Napoli: 1848-1849, Napoli

Diaz, Furio (1968), L'abate Galiani consigliere di commercio estero del Regno di Napoli, in: Rivista storica italiana, 80, 854-909

Dickinson, Edwin (1932), Changing Concepts and the Doctrine of Incorporation, in: American Journal of International Law, 26, 2, 239-260, https://doi.org/ $10.2307 / 2189346$

Dimitru, Rodica (2006), The Cultural Turn in Translation Studies, Iaşi

Distefano, Giovanni (2011), Les compétences territoriales, in: Chetail, Vincent, Peter Haggenmacher (eds.), Vattel's International Law in a XXIst Century Perspective, Leiden, Boston, 211-236, https://doi.org/10.1163/ej.9789004 194632.i-443.57

Dito, Oreste (1978), Massoneria, Carboneria ed altre società segrete nella storia del Risorgimento italiano, Bologna

Djeffal, Christian (2016), Static and Evolutive Treaty Interpretation. A Functional Reconstruction, Cambridge

Dodge, William S. (2010), Customary, International Law, Congress and the Courts: Origins of the Later-in-Time Rule, in: Bekker, Pieter H. F., Rudolf Dolzer, Michael Waibel (eds.), Making Transnational Law Work in the Global Economy. Essays in Honour of D. Vagts, Cambridge, New York, 531-559, https:// doi.org/10.1017/CBO9780511675881.028

Domingo, Rafael (2011), Gaius, Vattel, and the New Global Law Paradigm, in: European Journal of International Law, 22, 3, 627-647, https:/doi.org/ 10.1093/ejil/chr050

Donato, Clorinda (1992a), The Letters of Fortunato Bartolomeo De Felice to Pietro Verri, in: Modern Language Notes, 107, 74-111, https://doi.org/10.2307/ 2904677

Donato, Clorinda (1992b), An Intellectual Exile in the 18th Century: Fortunato Bartolomeo De Felice in Switzerland, in: Romance Languages Annual, 243-247 
Donato, Clorinda (1993), L'Encyclopédie d'Yverdon et l'Encyclopédie di Diderot et d'Alembert, in: Annales Benjamin Constant, 14, 53-86

Donato, Clorinda (1996), Jean Henry Samuel Formey's Contribution to the Encyclopédie d'Yverdon, in: Fontius, Martinus, Helmut Holzhey (Hrsg.), Schweizer im Berlin des 18. Jahrhunderts, Berlin, 87-98, https://doi.org/10.1515/ 9783050072296-007

Donato, Clorinda (1997), Fortunato Bartolomeo De Felice e l'edizione di Yverdon dell'Encyclopédie, in: Studi settecenteschi, 16, 374-396

Donato, Clorinda (2002), Rewriting Heresy in the Encyclopédie d'Yverdon 1770-1780, in: Cromohs, 7, 1-26, http:/www.fupress.net/index.php/cromohs /article/view/15718/14607, consultato il 22 maggio 2016

Dörr, Oliver (2012), Interpretation of Treaties, in: Dörr, Oliver, Kirsten Schmalenbach (eds.), Vienna Convention on the Law of Treaties. A Commentary, Berlin, Heidelberg, 477-604, https://doi.org/10.1007/978-3-64219291-3_31

Douglass, Robin (2011), Rousseau's Debt to Burlamaqui: The Ideal of Nature and the Nature of Things, in: Journal of the History of Ideas, 72, 2, 209-230, https://doi.org/10.1353/jhi.2011.0014

Douki, Caroline, Philippe Minard (2007), Histoire globale, histoires connectées: un changement d'échelle historiographique?, in: Revue d'histoire moderne et contemporaine, 54, 7-21, https://doi.org/10.3917/rhmc.545.0007

Dreyer, Frederick (1979), Burke's Politics. A Study in Whig Orthodoxy, Ontario

Droetto, Antonio (1954), Pasquale Stanislao Mancini e la scuola italiana di diritto internazionale del secolo XIX, Milano

Duane, William John (1809), The law of nations investigated in a popular manner addresses to the Farmers of the United States, Philadelphia

Dufour, Alfred (1976), Le Mariage dans l'École allemande du droit naturel moderne au XVIIIe siècle, Paris

Dufour, Alfred (1979), Die Ecole romande du droit naturel - ihre deutschen Wurzeln, in: Thieme, Hans (Hrsg.), Humanismus und Naturrecht in Brandenburg-Preußen, Berlin, 133-143

Dufour, Alfred (1999), L'ambivalence politique de la figure du contrat social chez Pufendorf e chez les Fondateurs de l'Ecole romande du droit naturel au XVIIIe siècle, in: Kervégan, Jean-François, Heinz Mohnhaupt (Hrsg.), Gesellschaftliche Freiheit und vertragliche Bindung in Rechtsgeschichte und Philosophie. Liberté sociale et lien contractuel dans l'histoire du droit et la philosophie. Zweites deutsch-französisches Symposion vom 12. bis 15. März 1997 in der Herzog August Bibliothek Wolfenbüttel, Frankfurt am Main, $35-74$

Dumas, Charles-Guillaume Frédéric (1775), Lettre de l'éditeur à Monsieur ***; in: Vattel, Emer de, Le droit des gens ou Principes de la loi naturelle, appliqués à la conduite et aux affaires des nations et des souverains. Nouvelle édition augmentée, revue, corrigée, avec quelques remarques de l'Editeur, Amsterdam, van Harrevelt, I-X 
Dupuy, Pierre-Marie (2011), Vattel et le droit des traités, in: Chetail, Vincent, Peter Haggenmacher (eds.), Vattel's International Law in a XXIst Century Perspective, Le droit international de Vattel vu du XXIe siècle, Leiden, Boston, 151-166, https://doi.org/10.1163/ej.9789004194632.i-443.42

Duve, Thомas (2012), Von der Europäischen Rechtsgeschichte zu einer Rechtsgeschichte Europas in globalhistorischer Perspektive, in: Rechtsgeschichte Legal History, 20, 18-71, https://doi.org/10.12946/rg20/018-071

Duve, Thomas (2013), European Legal History - Global Perspectives. Working Paper for the Colloquium European Normativity - Global Historical Perspectives' (Max Planck-Institute for European Legal History, September, 2nd-4th 2013), in: Max Planck for European Legal History Research Paper Series, 2013-06, http://ssrn.com/abstract=2292666

Duve, Thomas (2014a), European Legal History - Concepts, Methods, Challenges, in: Duve, Thomas (ed.), Entanglements in Legal History: Conceptual Approaches, Global Perspectives on Legal History 1, Max Planck Institute for European Legal History, Open Access Publication, Frankfurt am Main, 29-66, https://doi.org/10.12946/gplh1

Duve, Thomas (2014b), La historia del derecho y la necesidad de reflexionar sobre la formación de espacios jurídicos, in: Albani Benedetta, Samuel Barbosa, Thomas Duve, La formación de espacios jurídicos iberoamericanos (s. XVIXIX): Actores, artefactos e ideas. Comentarios introductorios, Max Planck for European Legal History Research Paper Series, No. 2014-07, http://ssrn.com/ abstract $=2532868$

Duve, Thомаs (2014c), German Legal History: National Traditions and Transnational Perspectives, in: Rechtsgeschichte - Legal History, 22, 2014, 16-48, https:// doi.org/10.12946/rg22/016-048

Duve, Thомаs (2016), Global Legal History - A Methodological Approach, in: Max Planck for European Legal History Research Paper Series, 2016-04, http:// ssrn.com/abstract $=2781104$

Edelstein, Dan (2008), War and Terror: the Law of Nations from Grotius to the French Revolution, in: French Historical Studies, 31, 229-262, https:/doi.org/ 10.1215/00161071-2007-021

Edelstein, Dan (2009), The Terror of Natural Right: Republicanism, the Cult of nature, and the French Revolution, Chicago

Ehrlich, Ludwik (1928), L'interprétation des traités, in: Recueil des Cours de l'Académie de Droit International de La Haye, 24, IV, 5-145, https:/doi.org/ 10.1163/ej.9789028606128.001-145

EISen, Cliff (1991), New Mozart Documents: A Supplement to O. E. Deutsch's Documentary Biography, Stanford

Elliot, Edward (1908), M. Pradier-Fodéré, in: American Political Science Review, 2, 2, 245-254, https://doi.org/10.2307/1944776

Enciso Recio, Luis Miguel (1985), La reforma de la Universidad española en la época de Carlos III, in: Pinto, Mario (a cura di), Borbone di Napoli e i Borbone di Spagna. Un bilancio storiografico, Napoli, vol. II, 191-239 
Escher, Heinrich (1864), Handbuch der praktischen Politik, Leipzig, Bd. II

Falcón, Fernando (2009), Federación/Federalismo. Venezuela, in: Sebastián, Javier Fernández (dir.), Diccionario político y social del mundo iberoamericano. La era de las revoluciones, 1750-1850. [Iberconceptos-I], Madrid, $535-547$

Fassbender, Bardo, Anne Peters (eds.) (2012), The Oxford Handbook of the History of International Law, Oxford, https://doi.org/10.1093/law/9780199599752.001.001

Felice, Bartolomeo Fortunato de (1772), Constitution d'État, in: Encyclopédie ou dictionnaire universel raisonné des connoissances humaines, Yverdon, vol. XI, cong - cot, 189-191

Fenwick, Charles G. (1913), The Authority of Vattel, in: The American Political Science Review, 7, 3, 395-410, https://doi.org/10.2307/1944965

Fenwick, Charles G. (1914), The Authority of Vattel II, in: The American Political Science Review, 8, 3, 375-392, https://doi.org/10.2307/1946172

Fernández García, Antonio (2010), Las Cortes y la Constitución de Cádiz, Madrid Fernández Rozas, José, Paz André Sáenz de Sante María (2010), La aportación de la Facultad de Derecho de la Universidad de Oviedo al progreso del Derecho internacional, in: Coronas Gonzáles, Santos M. (coord.), Historia de la Facultad de Derecho (1608-2008), 495-570

Ferrante, Riccardo (2002), Dans l'ordre établi par le code civil. La scienza del diritto al tramonto dell'illuminismo giuridico, Milano

Ferrante, Riccardo (2009), Traduzione del codice e tradizione scientifica: la cultura giuridica italiana davanti al Codice Napoleone, in: Tavilla, Elıo (a cura di) (2009), Giuseppe Luosi, giurista italiano ed europeo. Traduzioni, tradizioni e tradimenti della codificazione. A 200 anni dalla traduzione in italiano del Code Napoléon (1806-2006). Quaderni dell'archivio storico XXV, Atti del convegno Internazionale di Studi, Mirandola 19-20 ottobre 2006, Modena, $223-237$

Ferrante, Riccardo (2011), Codificazione e cultura giuridica, Torino

Ferrante, Riccardo (2015), Un secolo sì legislativo. La genesi del modello ottonovecentesco di codificazione e la cultura giuridica, Torino

Ferrari, Stefano (2008), Il rifugiato e l'antiquario. Fortunato Bartolomeo De Felice e il transfert italo-elvetico di Winckelmann nel secondo Settecento, Rovereto

Ferrari, Stefano (2010), La conversione «filosofica» di Fortunato Bartolomeo De Felice, in: Cantarutti, Giulia, Stefano Ferrari (a cura di), Illuminismo e protestantesimo, Milano, 87-106

Ferrario, Giulio (1833), Il costume antico e moderno di tutti i popoli d'Europa, ovvero Storia del governo, della milizia, delle religione, delle arti, scienze ed usanze di tutti i popoli antichi e moderni, provata coi monumenti dell'antichità e rappresentata con analoghi disegni, Torino, vol. X

Ferrater, Esteban (1846-1847), Codigo de derecho internacional, o sea colección metódica de los tratados de paz, amistad y comercio entre España y las demas naciones, 2 vol., Barcelona 
Ferreira Silvestre Pinheiro (1991), in: Enciclopedia universal ilustrada europeo americana, Madrid 23, 913-914

Figge, Robert (1914), Georg Friedrich von Martens: Sein Leben und seine Werke. Ein Beitrag zur Geschichte der Völkerrechtswissenschaft, Gleiwitz

Finlay, George (1971), History of the Greek Revolution and the Reign of King Otho, London

Fiocchi Malaspina, Elisabetta (2012), La fortuna del Droit des gens di Emer de Vattel nel XIX secolo, tesi di dottorato, Università degli Studi di Genova, tutor prof. Rodolfo Savelli

Fiocchi Malaspina, Elisabetta (2013), La ricezione e la circolazione de Le droit des gens di Emer de Vattel nel XIX secolo in: Materiali per una storia della cultura giuridica, 43, 2, 303-319

Fiocchi Malaspina, Elisabetta (2014), Le droit des gens di Emer de Vattel: le vicende editoriali di un successo destinato a durare per secoli, in: Nuova Rivista Storica, 98, 2, 733-754

Fiocchi Malaspina, Elisabetta (2015a), Fiore Pasquale, in: Dizionario del Liberalismo Italiano, t. II, 488-489

Fiocchi Malaspina, Elisabetta (2015b), Pierantoni Augusto, in: Dizionario del Liberalismo Italiano, Soveria Mannelli, t. II, 890-891

Fiocchi Malaspina, Elisabetta, Nina Keller-Kemmerer (2014), International Law and Translation in the 19th century, in: Rechtsgeschichte - Legal History, 22, 214-227, http://dx.doi.org/10.12946/rg22/214-226

Fioravanti, Marco (2012), Il pregiudizio del colore. Diritto e giustizia nelle Antille francesi, Roma

Fiore, Pasquale (1865), Nuovo diritto internazionale pubblico internazionale secondo i bisogni della civiltà, Milano

Fitzmaurice, Andrew (2014), Sovereignty, Property and Empire, 1500-2000, Cambridge

Fleming, Katherine Elizabeth (1999), The Muslim Bonaparte: Diplomacy and Orientalism in Ali Pasha's Greece, Princeton

Focarelli, Carlo (2013a), La persona umana nel diritto internazionale pubblico, Bologna

Focarelli, Carlo (2013b), Il diritto internazionale umanitario e la Croce Rossa dal 1859 al 1914, in: Cipolla Costantino, Paolo Vanni (a cura di), Storia della Croce Rossa Italiana dalla nascita al 1914. I. Saggi, Milano, 103-128

Foljanty, Lena (2015), Legal Transfer as Processes of Cultural Translation: on the Consequences of a Metaphor, Max Planck Institute for European Research Paper series 2015-09, http://ssrn.com/abstract=2682465

Formey, Samuel Henri (1758), Principes du droit de la nature et des gens, Extrait du grand ouvrage latin de Mr de Wolff, Amsterdam, Marc Michel Rey

Forsyth, William (1853), History of the Captivity of Napoleon at St. Helena; from the letters and journals of the late Lieut.-Gen. Sir Hudson Lowe and official documents not before made public, New York, vol. I 
Foucault, Michel (2005a), Nascita della biopolitica. Corso al Collège de France, 1978-1979. Edizione stabilita sotto la direzione di Françors Ewald e Alessandro Fontana da Michel Senellart, trad. it. Bertani, Mauro, Valeria Zini, Milano

Foucault, Michel (2005b), Sicurezza, territorio, popolazione. Corso al Collège de France (1977-1978), traduzione italiana Napoli, Paolo, Milano

Foucault, Michel (2012), Kant, Che cos'è l'illuminismo, Udine, Milano

Francioni, Gianni (1984), Nota al testo, in: Beccaria, Cesare, Dei delitti e delle pene, Francioni, Gianni (a cura di), con Le edizioni italiane del « Dei delitti e delle pene » di Firpo, Luigi, Milano, 215-335

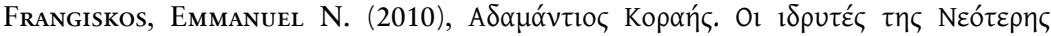

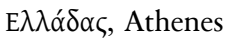

Frangos, George (1973), The Philiki Etairia: a Premature National Coalition, in: Clogg, Richard (ed.), The Struggle for Greek Independence, London, $87-103$

Frary, Lucien J. (2015), Russia and the Making of Modern Greek Identity, 18211844 , Oxford

Fremont-Barnes, Gregory (2001), The French Revolutionary Wars, Oxford

Fréron, Elie (1759), Année Littéraire, Paris, vol. VIII, 48-63

Friedemann, Peter (2014), Die Politische Philosophie des Gabriel Bonnot de Mably (1709-1785). Eine Studie zur Geschichte des republikanischen und des sozialen Freiheitsbegriffs, Münster

Fugazza, Emanuela (2014), Ercole Vidari internazionalista. Dal magistero pavese alla collaborazione con l'Institut de droit international, Historia et Ius. Rivista di storia giuridica dell'età medievale e moderna, 5 , paper $8,1-31$, www.histori aetius.eu/uploads/5/9/4/8/5948821/fugazza_5.pdf

Funck-Brentano, Théophile, Albert Sorel (1877), Précis du Droit des gens, Paris

Furfaro, Federica (2011), «Il più minuto, il più completo e il più pratico di tutti $\mathrm{i}$ libri giuridici italiani ». La versione italiana del Commentario alle Pandette di Christian Friedrich von Glück, in: Rivista di storia del diritto italiano, 84, $417-442$

Furfaro, Federica (2012), The connections between German Pandectist School and the Italian legal culture at the end of XIX century, in: Sources of Law and Legal Protection, Triestine Lectures 1, Trieste, 55-71

Gagnebin, Bernard (1944), Burlamaqui et le droit naturel, Genève

Gajo, Maria Gaia (1973), Le lettere di Gladstone ad Aberdeen, in: Rassegna Storica del Risorgimento, 60, 4, 31-47

Galiani, Ferdinando (1782), De' doveri e de' principi neutrali verso i guerreggianti e di questi verso i principi neutrali, Napoli

Gallaudet, Edward Miner (1879), A Manual of International Law, New York

Gardner, Daniel (1860), Institutes of International Law, Public or Private, as Settled by the Supreme Court of the United States, and by our Republic. With Reference to Giudicial Decision, New York 
GareIs, KarL (1888), Institutionen des Völkerrechts, ein kurzgefasstes Lehrbuch des positiven Völkerrechts in seiner geschichtlichen Entwicklung und heutigen Gestaltung, Giessen

Garrison, Tim Alan (2009), The Legal Ideology of Removal; the Southern Judiciary and the Sovereignty of Native American Nations, Athens, London

Gatti, Roberto, Giulio Maria Chiodi (a cura di) (2014), La filosofia politica di Rousseau, Milano

Gatti, Roberto (2005), Rousseau: la politica come libertà, in: Rousseau, Jean Jacques, Il contratto sociale, Gatti, Roberto (a cura di), Milano, 5-40

Gaven, Jean-Christophe (2007), Boucher d'Argis, Antoine-Gaspard, in: Arabeyre, Patrick, Jean-Louis Halpérin, Jacques Krynen (sous la dir. de), Dictionnaire historique des juristes français (XIIe-XXe siècle), Paris, 113-115

Gembicki, Dieter (1979), Histoire et politique à la fin de l'Ancien Régime. Jacob Nicolas Moreau (1717-1803), Paris

Gemma, Scipione (1902), Introduzione allo studio del diritto pubblico internazionale considerato nel suo svolgimento scientifico, Fascicolo II. La scuola del diritto naturale, Bologna

Genta, Enrico (2004), Principi e regole internazionali tra forza e costume. Le relazioni anglo-sabaude nella prima metà del Settecento, Napoli

GÉrAud-LlorCa, Edith (1993), L'actualité du Droit des gens à la veille de la Révolution; le traité anonyme de 1786, in: Revue d'Histoire de droit français et étranger, 71, 211-225

Gessner, Ludwig (1855), Das Recht des neutralen Seehandels und eine Revision der darüber geltenden Grundsätze des Völkerrechts, Bremen

Gilmore, James (1891), Notes of a Course of Lectures on Vattel's Law of Nation, Charlottesville

Gladstone, William Ewart (1851), Two letters to the Earl of Aberdeen, on the state prosecutions of the Neapolitan government, London

Glanville, Luke (2013), Sovereignty and the Responsibility to Protect. A New History, Chicago

Godet, Philippe (1893), Emer de Vattel, in: Musée Neuchâtelois. Recueil d'histoire nationale et d'archéologie, 30, 221-222

Godwin, Parke (1866), Mackintosh James, in: The Cyclopaedia of Biography: a Record of the Lives of Eminent Person. New Edition. With a Supplement, brought down to the present time by George Sheppard, New York, 605-606

Goldworth, John Alger (1904), Napoleon's British Visitors and Captives 1801-1815, London

Golove, David, Daniel J. Hulsebosch (2010), A Civilized Nation: the Early American Constitution, the Law of Nations, and the Pursuit of International Recognition, in: New York University Law Review, 85, 4, 932-1066

Gómez, Edgardo Rodríguez (2007/2008), El jusnaturalismo y la guerra en el pensamiento de Jean-Jacques Burlamaqui y Emer de Vattel en el siglo XVIII, in: Revista Telemática de Filosofia del Derecho, 11, 41-56, www.rtfd.es/ numero11/3-11.pdf 
González Diez, Emiliano (2013), La monarquía constitucionalisada por la Nación, in: Palacios Bañuelos, Luis, Ignacio Ruiz Rodríguez (dir.), Cádiz 1812. Origen del constitucionalismo español, Madrid, 117-166

Good, Christoph (2011), Emer de Vattel (1714-1767) - Naturrechtliche Ansätze einer Menschenrechtsidee und des humanitären Völkerrechts im Zeitalter der Aufklärung, Zürich

Good, Christoph (2012), Menschenrechte ohne Deklaration - Die naturrechtliche Begründung von Freiheitsrechten in der «école romande du droit naturel», in: Arlettaz, Silvia et al. (Hrsg.), Menschenrechte und moderne Verfassung. Die Schweiz im Übergang vom 18. zum 19. Jahrhundert/Droits de l'homme et constitution moderne. La Suisse au tournant des XVIIIe et XIXe siècles (= Travaux sur la Suisse des Lumières, 14), Genf, 15-33

Good, Christoph (2013), Emer de Vattel: Völkerrecht - Droit des Gens - Law of Nations, in: Zelle, Carsten, Heidi Eisenhut, Anett Lütteken (Hrsg.), Europa in der Schweiz - Grenzüberschreitender Kulturaustausch im 18. Jahrhundert. 3. Trogener Bibliotheksgespräch, Trogen, 10.-13. Juni 2009, Göttingen, 117-128

Gordley, James (2013), The Jurists. A Critical History, Oxford

Gouhier, Henri (1983), Rousseau et Voltaire: portraits dans deux miroirs, Paris

Goyard-Fabre, Simone (1996), Barbeyrac et la thèorie du droit naturel, in: GoyardFabre, Simone (prés. par), Thesaurus de philosophie du droit, Paris, 1-74

Gozzi, Gustavo (2010), Diritti e civiltà. Storia e filosofia del diritto internazionale, Bologna

Grafton Wilson, George (1907), Carlo Calvo, in: American Journal of International Law, 1, 1, 137-138

Grandi, Michele (1761), Avvertimento sopra questa traduzione italiana, in: Pufendorf, Samuel, I doveri dell'uomo e del cittadino: tali che a lui dalla legge naturale sono prescritti, dalla versione francese di Giovanni Barbeyrac tradotti, e con molte aggiunte corretti ed illustrati da Michele Grandi accademico di Udine, t. I, IX-XII

Grandpierre, Louis (1889), Histoire du Canton de Neuchâtel sous le Roi de Prusse, 1707-1848, Leipzig

Grewe, Wilhelm G. (2000), The Epochs of International law, translated and revised by Byers, Michael, Berlin, New York

Grimm, Dieter (2002), B. Verfassung II. Konstitution, Grundgesetz(e) von der Aufklärung bis zur Gegenwart, in: Mohnhaupt, Heinz, Dieter Grimm, Verfassung. Zur Geschichte des Begriffs von der Antike bis zur Gegenwart, zwei Studien, Berlin, 100-141

Grotius, Hugo (1625), De iure belli ac pacis libri tres, in quibus ius naturae et gentium: item iuris publici praecipua explicantur, Pariis, Nicolaum Buon

Grotius, Hugo (1724), Le droit de la guerre et de la paix. Nouvelle traduction par Jean Barbeyrac. Avec les notes de l'Auteur même, qui n'avoient point encore paru en françois; et de nouvelles notes du traducteur, Amsterdam, P. de Coup 
Grotius, Hugo (1867), Le droit de la guerre et de la paix, divisé en trois livres ou sont expliqués le droit de la nature et des gens et les principaux points du droit public. Nouvelle traduction, précédé d'un Essai biographique et historique sur Grotius et son temps, accompagnée d'un choix de notes de Gronovius, Barbeyrac, etc. complétée par des notes nouvelles mise au courant des progrès du Droit publique moderne, et suivie d'une table analytique des matières par M. P. Pradier-Fodéré, Paris

Guasti, Niccolò (2006a), L'esilio italiano dei gesuiti spagnoli. Identità, controllo sociale e pratiche culturali (1767-1798), Roma

Guasti, Niccolò (2006b), Gesuiti spagnoli espulsi (1767-1815): politica, economia, cultura, in: Bianchini Paolo (a cura di), Morte e resurrezione di un Ordine religioso, Milano, 15-52

Guerrero, Carolina, Carole Leal, Elena Plaza (2012), Amigos de la libertad: presencias liberales en Venezuela (1750-1850), in: SEbastián, Javier FERnÁndez (coord.), La aurora de la libertad. Los primeros liberalismos en el mundo iberoamericano, Madrid, 459-492

Guggenheim, Paul (1956), Emer de Vattel et l'étude des relations internationales en Suisse, Genève

Guggenheim, Paul (1959), Einleitung, Emer de Vattel und das Völkerrecht, in: Vattel Emer DE, Das Völkerrecht oder Grundsätze des Naturrechts, angewandt auf das Verhalten und die Angelegenheiten der Staaten und Staatsoberhäupter, Dt. Übers. von Euler, WILHELM, Tübingen, XV-XXXII

Guizot, François (1863), Histoire de la civilisation en Europe depuis la chute de l'Empire Romain jusqu'à la Révolution Française, VIII éd., Paris

Gumbrecht, Hans Ulrich (1978), Modern/Modernität/Moderne, in: Brunner, Otto, Werner Conze, Reinhart Koselleck (Hrsg.), Geschichtliche Grundbegriffe: Historisches Lexikon zur politisch-sozialen Sprache in Deutschland IV, Stuttgart, 93-131

Gumbrecht, Hans Ulrich (1997), La trasformazione del concetto di moda nella letteratura e nell'arte, in: Koselleck, Reinhart (a cura di), Gli inizi del mondo moderno, Milano, 499-508

Günther, Gоттlob (1787), Europäisches Völkerrecht in Friedenszeiten nach Vernunft, Verträgen und Herkommen, mit Anwendung auf die teutschen Reichsstände, Altenburg, Richter

Gutierrez Vega, Pablo (2004), Vattel larva detracta: reflexiones sobre la recepción del Ius Publicum Europaeum en la Universidad preliberal española, in: Bermejo Castrillo, Manuel Ángel (ed.), Manuales y textos de enseñanza en la universidad liberal / VII Congreso Internacional sobre la Historia de las Universidades Hispánicas, Madrid, 537-568

Gutierrez Vega, Pablo (2010), Vattel, larva retracta (reception in Spain), in: Sandoz, Yves (éd.), Réflexions sur l'impact, le rayonnement et l'actualité du 'Droit des gens' d'Emer de Vattel - Reflections on the Impact, Influence and Continuing Relevance of the 'Law of Nations' by Emer de Vattel, Bruxelles, 119-125 
HAAG, Eugène (1847), La France protestante ou vies des protestants français que se sont fait un nom dans l'histoire, depuis les premiers temps de la réformation jusqu’à la reconnaissance du principe de la liberté des cultes par l'Assemblée nationale, Genève

Habenicht, Walter (1934), Georg Friedrich von Martens: Eine biographische und völkerrechtliche Studie, Göttingen

Häberlin, Karl Friedrich (1797), Handbuch des Teutschen Staatsrechts, nach dem System des Herrn Geheimen Justizrath Pütter zum gemeinnützigen Gebrauch der gebildeten Stände in Teutschland mit Rücksicht auf die neuesten merkwürdigsten Ereignisse bearbeitet, Berlin, Bd. III

Habscheid, Walther J. (1967), Jean-Jacques Burlamaqui (1694-1748) und seine Principes du Droit Naturel, in: Mayer, Franz (Hrsg.), Staat und Gesellschaft: Festgabe für Günther Küchenhoff zum 60. Geburtstag am 21. August 1967, Göttingen, 5-24

Hall, William E. (1880), A Treatise on International Law, Oxford

Halleck, Henry W. (1861), International Law, or Rules Regulating the Intercourse of States in Peace and War, London

Hampsher-Monk, Iain (2005), Edmund Burke's Changing Justification for Intervention, in: The Historical Journal, 48, 1, 65-100, https://doi.org/10.1017/ S0018246X04004224

Harvey, Ray Forrest (1937), Jean Jacques Burlamaqui: A Liberal Tradition in American Constitutionalism, Chapel Hill

Häseler, Jens (2005), L'encyclopédisme protestant de Formey à la lumière de sa correspondance avec De Felice, in: Candaux, Jean Daniel et al. (éd.), L'Encyclopédie d'Yverdon et sa résonance européenne, Genève, 121-140

Haupt, Heinz-Gerhard, Jürgen Kocka (Hrsg.) (1996), Geschichte und Vergleich. Ansätze und Ergebnisse international vergleichender Geschichtsschreibung, Frankfurt am Main

Haupt, Heinz-Gerhard, Jürgen Kocka (2009), Comparison and Beyond: Traditions, Scope and Perspective of Comparative History, in: Haupt, HeinzGerhard, Jürgen Kocka (eds.), Comparative and Transnational History: Central European Approaches and New Perspectives, New York, Oxford, 1-30

Hébié, Mamadou (2015), Souveraineté territoriale par traité: Une étude des accords entre puissances coloniales et entités politiques locales, Paris

Heffter, August Wilhelm (1844), Das europäische Völkerrecht der Gegenwart, Berlin

Held, Joseph (1866), Vattel (Emrich von), in: Rotteck, Carl von, Carl Welcker (Hrsg.), Das Staats-Lexikon. Encyklopädie der sämmtlichen Staatswissenschaften für alle Stände. In Verbindung mit vielen der angesehensten Publicisten Deutschlands, Leipzig, Bd. XIV, 326-327

Helfman, Tara (2005), Neutrality, the Law of Nations, and the Natural Law Tradition: A Study of the Seven Years' War, in: Yale Journal of International Law, 30, 549-586

Hermon-Belot, Rita (2000), L’Abbé Grégoire, la politique et la vérité, Paris 
Herrero Rubio, Alejandro (1947), Internacionalistas Españoles del siglo XVIII, Don Joseph de Olmeda y Leon (1740-1805), Valladolid

Herrero Rubio, Alejandro (1952), Le droit des gens dans l'Espagne du XVIIIe siècle, in: Recueil des cours de l'Académie de Droit international de La Haye, 81, II, 309-450

Herrero Rubio, Alejandro (1953), Internacionalistas Españoles del siglo XVIII. Pedro Josef Pérez Valiente (1713-1789), Valladolid

Herrero Rubio, Alejandro (1954), Nociones de Historia del Derecho de Gentes y de las relaciones internacionales, Valladolid

Herrmann, Florian (2001), Das Standardwerk. Franz von Liszt und das Völkerrecht, Baden-Baden

Hervouet, Blandine (2009), Jacob-Nicolas Moreau, le dernier des légistes, une défense de la Constitution monarchique au siècle des Lumières, Paris

Hill, Christopher L. (2013), Conceptual Universalization in the Transnational Nineteenth Century, in: Moyn, Samuel, Andrew Sartori (eds.), Global Intellectual History, New York, 134-158, https://doi.org/10.7312/moyn16048-006

Hinde, Wendy (1973), George Canning, London

Hinsley, Francis Harry (1963), Power and the Pursuit of Peace, Cambridge

Hippler, Thomas, Miloš Vec (eds.) (2015), Paradoxes of Peace in 19th Century Europe, Oxford

Новвеs, Тномаs (1760), Elementa Philosophica. De Cive, Lausanne, Franciscum Grasset

Hochstrasser, Timothy (1993), Conscience and Reason: the Natural Law Theory of Jean Barbeyrac, in: The Historical Journal, 36, 2, 289-308, https:/doi.org/ 10.1017/S0018246X00019245

Hochstrasser, Timothy J., (2000), Natural Law Theories in the Early Enlightenment, Cambridge, https://doi.org/10.1017/CBO9780511490552

Hoffmanns, M. de (1835), Avertissement, in: Vattel, Emer de, Le droit des gens ou principes de la loi naturelle, appliqués à la conduite et aux affaires des Nations et des Souverains, revue et corrigée avec quelques remarques de l'editeur, augmentée de quelques remarques nouvelles, et d'une bibliographie choisie et systématique du droit de la nature et des gens, par M. de Hoffmann, Paris, t. I, V-VII

Hofmann, Hasso (2005), Riflessioni sull'origine, lo sviluppo e la crisi del concetto di Costituzione, in: Chignola, Sandro, Giuseppe Duso (a cura di), Sui concetti giuridici e politici della costituzione dell'Europa, Milano, 227-238

Hofmann, Hasso (2009a), Nascita sviluppo e crisi del concetto di Costituzione, in: Carrino, Agostino (a cura di), La libertà dello stato moderno. Saggi di dottrina della Costituzione, Napoli, 7-24

Hofmann, Hasso (2009b), Sull'idea di legge fondamentale dello Stato, in: Carrino, Agostino (a cura di), La libertà dello stato moderno. Saggi di dottrina della Costituzione, Napoli, 95-138 
Holland, Ben (2011), The Moral Person of the State: Emer de Vattel and the Foundations of International Legal Order, in: History of European Ideas, 37, 4, 438-445

Höpfner, Ludwig Julius Friedrich (1780), Naturrecht der einzelnen Menschen, der Gesellschaften und der Völker, Giessen, Krieger

Howard, George VI Earl of Carlisle (17 February 1783), Debate in the Lords on the Preliminary Articles of Peace, Fifteenth Parliament of Great Britain: third session (5 December 1782-16 July 1783), in: Parliamentary Register 1780-1796, vol. XI, 30-93

Hübner, Martin (1759), De la saisie des bâtiments neutres, ou Du Droit qu'ont les Nations Belligérantes d'arrêter les Navires des Peuples Amis, 2 vol., La Haye

Hueck, Ingo (2001), The Discipline of the History of International Law - New Trends and Methods on the History of International Law, in: Journal of the History of International Law, 3, 194-217, https:/doi.org/10.1163/ 15718050120956956

Hueck, Ingo J. (2004), Pragmatism, Positivism and Hegelianism in the Ninetheenth Century. August Wilhelm Heffter's Notion of Public International Law, in: Stolleis, Michael, Masaharu Yanagihara (Hrsg.), East Asian and European perspectives on international law, Baden-Baden, 41-55

Huguenin, SÉverine (2012), Sociétés littéraires et «Journal helvétique» (1732-1782): un échange de bons procédés, in: Revue historique vaudoise, 120, 315-327

Huguenin, Séverine, Timothée Léchot (2012), Les noyés du « Mercure suisse »: production et diffusion d'un savoir scientifique dans la première moitié du XVIIIe siècle, in: Léchot, Pierre-Olivier, Virginie Pasche (dir.), Neuchâtel dans le concert des Lumières européennes. Acteurs locaux et cultures transnationales/Neuchâtel im Konzert der Europäischen Aufklärung. Lokale Akteure und transnationalen Kulturen, in: xviii.ch. Annales de la Société suisse pour l'étude du XVIIIe siècle, vol. III, 55-71

Huguenin, Séverine, Timothée Léchot (dir.) (2016), Lectures du Journal helvétique 1732-1782. Actes du colloque de Neuchâtel 6-8 mars 2014, Genève, in corso di stampa

Hunter, Ian (1982), The Concept of Context and the Problem of Reading, in: Southern Review, 15, 1, 80-91

Hunter, Ian (2004), Conflicting Obligations: Pufendorf, Leibniz and Barbeyrac on Civil Authority, in: History of Political Thought, 25, 4, 670-699

Hunter, IAN (2007), Natural law, Historiography, and Aboriginal Sovereignty, in: Legal History, 11, 137-168

Hunter, IAN (2010a), Vattel's Law of Nations: Diplomatic Casuistry for the Protestant Nation, in: Grotiana 31, 108-140

Hunter, Ian (2010b), Global Justice and Regional Metaphysics: On the Critical History of the Law of Nature and Nations, in: Dorsett, Shaunnagh, Ian Hunter (eds.), Law and Politics in British Colonial Thought: Transpositions of Empire, New York, 11-29, https://doi.org/10.1057/9780230114388_2 
Hunter, Ian (2011), Law, War, and Casuistry in Vattel's Jus gentium, in: Parergon, 28, 2, 87-104, https://doi.org/10.1353/pgn.2011.0070

Hunter, Ian (2012), 'A Jus gentium for America': The Rules of War and the Rule of Law in the Revolutionary United States, in: Journal of the History of International Law, 14, 173-206

Hunter, IAn (2013a), Kant and Vattel in Context: Cosmopolitan Philosophy and Diplomatic Casuistry, in: History of European Ideas, 39, 4, 477-502, https:// doi.org/10.1080/01916599.2012.727145

Hunter, IAn (2013b), The Figure of Man and the Territorialisation of Justice in 'Enlightenment' Natural Law: Pufendorf and Vattel, in: Intellectual History Review, 23, 3, 289-307

Hunter, Ian (2013c), Vattel in Revolutionary America: From the Rules of War to the Rule of Law, in: Ford, LisA, Tim Rowse (eds.), Between Indigenous and Settler Governance, New York, 12-22

Hunter, Ian (2016), About the dialectical historiography of international law, in: Global Intellectual History, 1-32, http://dx.doi.org/10.1080/23801883.2016. 1155863, consultato il 15 giugno 2016

Hurd, John Codman (1858), The law of freedom bondage in the United States, Boston, vol. I

Ibbetson, David (2012), Comparative Legal History: A Methodology, in: Musson, Anthony, Chantal Stebbings (eds.), Making Legal History: Approaches and Methodologies, Cambridge, 131-145

Ibbetson, David (2013), The Challenges of Comparative Legal History, in: Comparative Legal History, 1, 1, 1-11, https://doi.org/10.5235/2049677x.1.1.1

Iggers, Georg, Edward Wang, Supriya Mukherjee (2008), A Global History of Modern Historiography, London, New York

Ihl, Olivier (2004), Conspirations et science du pouvoir chez François Guizot, in: Revue Française d'Histoire des Idées Politiques, 19, 1, 125-150

Indice ultimo de los libros prohibidos y mandados a expurgar: para todo los reynos y señorios del catolico rey de las Españas el Señor don Carlos IV (1790), Madrid

Ingraham, Edward D. (1852), Preface to this edition, in: Vattel, Emer De, The law of nations, or principles of the law of nature, applied to the conduct and affairs of nations and sovereigns, with additional notes and references by Edward D. Ingraham, Philadelphia, III

Iriye, Akira (2013), Global and Transnational History, the Past, the Present, and the Future, Basingstoke

Iriye, Akira, Paul Yves Saunier (eds.) (2009), The Palgrave Dictionary of Transnational History, Basingstoke

Isabella, Maurizio (2006), Exile and Nationalism: the Case of the Risorgimento, in: European History Quarterly, 36, 493-520, https:/doi.org/10.1177/026569 1406068126

Isabella, Maurizio (2008), Mazzini's Internationalism in Context: From the Cosmopolitan Patriotism of the Italian Carbonari to Mazzini's Europe of the Nations, in: Bayly, Christopher, Eugenio Biagini (eds.), Giuseppe Mazzini 
and the Globalisation of Democratic Nationalism 1820-1920, Oxford, 37-58, https://doi.org/10.5871/bacad/9780197264317.003.0003

Isabella, Maurizio (2009). Risorgimento in Exile. Italian Émigrés and Liberal International in the Post-Napoleonic Era, Oxford, https://doi.org/10.1093/acprof: oso/9780199570676.001.0001

Jacobini, H. B. (1954), A Study of the Philosophy on International Law as Seen in Works of Latin American Writers, The Hague, https://doi.org/10.1007/978-94011-8798-5

Jacobs, Beatrix Christina, Raymond Kubben, Randall Lesaffer (eds.) (2008), In the Embrace of France: the Law of Nations and Constitutional Law in the French Satellite States of the Revolutionary and Napoleonic Age (1789-1815). Acts of the International Conference held in Tilburg University on 27 and 28 April 2006, Baden-Baden

Jaksic, Ivan (2001), Andrés Bello: Scholarship and Nation-Building in NineteenthCentury Latin America, Cambridge

Janet, Paul (1887), Histoire de la science politique dans ses rapports avec la morale, Paris, t. II

Janis, Marc Weston (2004), The American Tradition of International Law: Great expectation, 1789-1914, Oxford

Janis, Marc Weston (2010), America and the Law of Nations 1776-1939, Oxford

Jayme, Erik (1987), Pasquale Stanislao Mancini (1817-1888): l'attualità del suo pensiero, Trento

Jeanneret, Frédéric, James Henri Bonhôte (1863a), Bourguet Louis, in: Biographie neuchâteloise, Locle, vol. I, 59-79

Jeanneret, Frédéric, James Henri Bonhôte (1863b), Ostervald Samuel Frédéric, in Biographie Neuchâteloise, Locle, vol. II, 145-148

Jelmini, Jean-Pierre (2010), Neuchâtel 1011-2011, mille ans - mille questions mille et une réponses, Hauterive

Johannsen, Robert W. (1985), To the Halls of the Montezumas: The Mexican War in the American Imagination, New York

Johnston, Raúl Pérez (2005), Jean Jacques Burlamaqui and the Theory of Social Contract, in: Revista Electrónica de Historia Constitucional, 6, 331-374, http://www.historiaconstitucional.com/index.php/historiaconstitucional/article/ download/83/69, consultato il 22 maggio 2016

Jouannet, Emmanuelle (1998), Emer de Vattel et l'émergence doctrinale du droit international classique, Paris

Jouannet, Emmanuelle (2000), Vattel et la sujétion directe de l'état au droit international, in: Goyard-Fabre, Simone (ét. réunies par), L'État moderne 1715-1848, Paris, 153-179

Jouannet, Emmanuelle (2006), What is the Use of International Law?, in: Ruiz Fabri, Hélène, Emmanuelle Jouannet, Vincent Tomkiewicz (eds.), Select Proceedings of the European Society of International Law, Oxford, Portland, Oregon, 1, 51-96 
Jouannet, Emmanuelle (2011), Les dualismes du Droit des gens, in: Chetail, Vincent, Peter Haggenmacher (eds.), Vattel's International Law in a XXIst Century Perspective, Le droit international de Vattel vu du XXIe siècle, Leiden, Boston, 133-150, https://doi.org/10.1163/ej.9789004194632.i-443.37

Jouannet, Emmanuelle (2012a), The Liberal-Welfarist Law of Nations. A History of International Law, translated by Sutcliffe Christopher, Cambridge

Jouannet, Emmanuelle (2012b), Emer de Vattel (1714-1767), in: Fassbender, Bardo, Anne Peters (eds.), The Oxford Handbook of the History of International Law, Oxford, 1118-1121, https://doi.org/10.1093/law/9780199599752. 003.0054

Kaelble, Hartmut, Jürgen Schriewer (Hrsg.) (2003), Vergleich und Transfer: Komparatistik in den Sozial-, Geschichts- und Kulturwissenschaften, Frankfurt am Main

Kaltenborn, Carl Baron von Stachau (1847), Kritik des Völkerrechts nach dem jetzigen Standpunkte der Wissenschaft, Leipzig

Kämmerer, Jürgen (1991), Nouvelle Bibliothèque Germanique, in: Sgard, Jean (sous la direction de), Dictionnaire de la presse 1600-1789, I. Dictionnaire de journaux, Paris, vol. I, a-i, n. 163, 188-189

Kant, Immanuel (1795), Zum ewigen Frieden, ein philosophischer Entwurf, Frankfurt, Leipzig

Kant, Immanuel (2004), Per la pace perpetua. La pace come destinazione etica e politica nella storia dell'umanità, Pancaldi, Maurizio (a cura di), Roma

Kapossy, Béla, Richard Whatmore (2008a), Introduction, in: Vattel, Emer de, The Law of Nations Applied to the Conduct and Affairs of Nations and Sovereigns, Indianapolis, IX-XX

Kapossy, Béla, Richard Whatmore (2008b), Emer de Vattel's Mélanges de littérature, de morale et de politique (1760), in: History of European Ideas, 34, 1, 77-103, https://doi.org/10.1016/j.histeuroideas.2007.12.002

Kapossy, BÉLA (2010), Rival Histories of Emer de Vattel's Law of Nations, in: Grotiana 31, 5-21, https://doi.org/10.1163/187607510x543481

Kates, Gary (1998), The French Revolution. Recent Debates and New Controversies, New York

Keitner, Chimène I. (2007), The Paradoxes of Nationalism. The French Revolution and Its Meaning for Contemporary Nation Building, New York

Keller, Alexis (2006), Emer de Vattel: la tradition du droit des gens et la question des peuples autochtones, in: Revue suisse d'histoire, 56, 387-409

Keller, Helen (2003), Rezeption des Völkerrechts, Berlin, New York

Keller-Kemmerer, Nina (2016), Die Mimikry des Völkerrechts: Andrés Bellos "Principios de Derecho Internacional ", tesi di dottorato svolta presso il MaxPlanck-Institut für europäische Rechtsgeschichte di Francoforte sul Meno, tutor prof. Dr. Miloš Vec

Kemmerer, Alexandra (2008), The Turning Aside. On International Law and Its History, in: Miller, Russell A., Rebecca M. Bratspies (eds.), Progress in International Law, Leiden, 71-94 
Kemmerer, Alexandra (2015), "We do not need to always look to Westphalia ...» A Conversation with Martti Koskenniemi and Anne Orford, in: Journal of the History of International Law, 17, 1-14, https://doi.org/10.1163/ 15718050-12340032

Kent, James (1826), Commentaries on American law, New York, vol. I

Kitromilides, Paschalis M. (ed.) (2010), Adamantios Korais and the European Enlightenment, Oxford

KLüber, Johann Ludwig (1819), Droit des gens moderne de l'Europe, Stuttgart

Kocka, Jürgen (2003), Comparison and Beyond, in: History and Theory, 42, 1, 39-44, https://doi.org/10.1111/1468-2303.00228

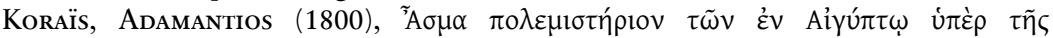

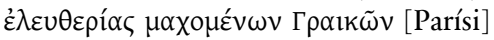

Koraïs, Adamantios (1803), Mémoire sur l'état actuel de la civilisation dans la Grèce, lu à la Société des Observateurs de l'Homme. Le 16 Nivôse, an XI (6 Janvier 1803), Paris

Korkman, Petter (2002), Civil Sovereign and the King of Kings: Barbeyrac and the Creator's Right to Rule, in: Hunter, Ian, David Saunders (eds.), Natural Law and Civil Sovereignty: Moral Right and State Authority in Early Modern Political Thought, New York, 109-122, https://doi.org/10.1057/9781403919 $533 \_8$

Korkman, Petter (2003), Voluntarism and Moral Obligation: Barbeyrac's Defence of Pufendorf Revisted, in: Hochstrasser, Timothy, Peter Schröder (eds.), Early Modern Natural Law Theories: Context and Strategies in the Early Enlightenment, London, 195-225, https://doi.org/10.1007/978-94-017-03918_8

Korkman, Petter (2006), Life, Liberty, and the Pursuit of Happiness. Human Rights in Barbeyrac and Burlamaqui, in: Mäkinen, Virpi, Petter Korkman (eds.), Transformations in Medieval and Early Modern Rights Discourse, Dordrecht, 257-282, https://doi.org/10.1007/1-4020-4212-4_10

Koselleck, Reinhart (1972), Critica illuminista e crisi della società Borghese, traduzione italiana Panzieri, Giuseppina, Bologna

Koskenniemi, Martti (2004a), Why History of International Law Today?, in: Rechtsgeschichte - Legal History, 4, 61-66, http://dx.doi.org/10.12946/rg04/ 061-066

Koskenniemi, Martti (2004b), The Gentle Civilizer of Nations. The Rise and Fall of International Law, Cambridge

Koskenniemi, Martti (2005), From Apology to Utopia: The Structure of International Legal Argument, Cambridge

Koskenniemi, Martti (2008), Into Positivism, Georg Friedrich Martens (1756-1821) and Modern International Law, in: Constellations, 15, 2, 189-207, https:// doi.org/10.1111/j.1467-8675.2008.00484.x

Koskenniemi, Martti (2009), Miserable Comforters: International Relations as New Natural Law, in: European Journal of International Relations, 15, 3, 395-422, https://doi.org/10.1177/1354066109338229 
Koskenniemi, Martti (2010), International Law and raison d'état: Rethinking the Prehistory of International Law, in: Kingsbury, Benedict, Benjamin Straumann (eds.), The Roman Foundations of the Law of Nations: Alberico Gentili and the Justice of Empire, Oxford, 297-339, https://doi.org/10.1093/ acprof:oso/9780199599875.003.0015

Koskenniemi, Martti (2011a), 'International Community' from Dante to Vattel, in: Chetail, Vincent, Peter Haggenmacher (eds.), Vattel's International Law in a XXIst Century Perspective, Le droit international de Vattel vu du XXIe siècle, Leiden, Boston, 51-75, https://doi.org/10.1163/ej.9789004194632.i-443.17

Koskenniemi, Martti (2011b), Dealing with Eurocentrism, in: Rechtsgeschichte Legal History, 19, 152-176, https://doi.org/10.12946/rg19/152-176

Koskenniemi, Martti (2012a), A History of International Law Histories, in: Fassbender, Bardo, Anne Peters (eds.), The Oxford Handbook of the History of International Law, Oxford, 943-971, https://doi.org/10.1093/law/ 9780199599752.003.0040

Koskenniemi, Martti (2012b), The Public Law of Europe. Reflections on a French 18th century, Debate, in: Lindemann, Helena et al. (Hrsg.), Erzählungen vom Konstitutionalismus, Baden-Baden, 43-73

Koskenniemi, Martti (2012c), Il mite civilizzatore delle nazioni. Ascesa e caduta del diritto internazionale 1870-1960, Gozzi, Gustavo, Lorenzo Gradoni, Paolo Turrini (a cura di), Roma, Bari

Koskenniemi, Martti (2013), Histories of International Law: Significance Problems for a Critical View, in: Temple International and Comparative Law Journal, $27,2,215-240$

Koskenniemi, Martti (2014), Vitoria and Us. Thoughts on Critical Histories of International Law, in: Rechtsgeschichte - Legal History, 22, 119-138, http:// dx.doi.org/10.12946/rg22/119-138

Koskenniemi, Martti (2016), Expanding Histories of International Law, in: American Journal of Legal History, 56, 1, 2016, 104-112, http://ajlh.oxfordjournals. org/content/ajlh/56/1/104.full.pdf

Kossmann, Ernest Heinrich (1966), Verlicht conservatisme: over Elie Luzac, Groningen

Krauss, Werner (1963), La correspondance de Formey, in: Revue d'Histoire littéraire de la France, 63, 2, 207-216

Krebs, Ricardo (1969), La idea de nación en el pensamento di Andrés Bello, in: Revista de Historia de América, 67/68, enero-diciembre, 152-174

Kroll, Stefan (2012), Normgenese durch Re-Interpretation - China und das europäische Völkerrecht im 19. und 20. Jahrhundert, Baden-Baden, https://doi.org/ $10.5771 / 9783845236506$

Kubben, Raymond (2011), Regeneration and Hegemony. Franco-Batavian Relations in the Revolutionary Era, 1795-1803, Leiden, https://doi.org/10.1163/ 9789004189515

L'enseignement du droit international public en France (1955), in: Annuaire Français du droit international, 1, 816-818 
Labriola, Giulia Maria (2003), Barbeyrac interprete di Pufendorf e Grozio. Dalla costruzione della sovranità alla teoria della resistenza, Napoli

Lachs, Manfred (1987), The Teacher in International Law: Teachings and Teaching, Dordrecht

Lampredi, Maria Giovanni (1788), Lampredi, Giovanni Maria: Del commercio dei popoli neutrali in tempo di guerra, Firenze

LANDer, Tomás (1983), Manual del colombiano ó Explicación de la ley natural. Van añadidos los deberes y derechos de la nacion y del ciudadano, in: La doctrina liberal. Tomás Lander, Caracas, vol. IV, 53-99

LANDi, SANDro (2000), Il governo delle opinioni. Censura e formazione del consenso nella Toscana del Settecento, Bologna

Langhorne, Richard (1996), Arbitration: the first phase, 1870-1914, in: Dockrill, Michel, Brian McKercher (eds.), Diplomacy and World Power: Studies in British Foreign Policy, 1890-1950, Cambridge, 43-55, https://doi.org/10.1017/ CBO9780511560934.004

Lapradelle, Albert de (1916), Introduction, in: Vattel, Emer de, Le droit des gens ou Principes de la loi naturelle, appliqués à la conduite et aux affaires des nations et des souverains, Washington, vol. I, I-LV

Lapradelle, Albert de, Nicolas Politis (1905), Recueil des arbitrages internationaux 1798-1855, Paris, t. I

Lapradelle, Albert de, Nicolas Politis (1923), Recueil des arbitrages internationaux 1856-1872, Paris, t. II

Lapradelle, Albert de, Nicolas Politis (1954), Recueil des arbitrages internationaux 1872-1875, Paris, t. III

Larousse, Pierre (réd. par) (1870), Droit des gens ou Droit international, in: Grand Dictionnaire universel du XIXe siècle, français, historique, géographique, mythologique, bibliographique, littéraire, artistique, scientifique, Paris, t. VI, 1248-1249

Larousse, Pierre (réd. par) (1874), Pinheiro-Ferreira Silvestre, in: Grand Dictionnaire universel du XIXe siècle, français, historique, géographique, mythologique, bibliographique, littéraire, artistique, scientifique, Paris, t. XII, 1036

Latini, Carlotta (2009), Processare il nemico. Carboneria, dissenso politico e penale speciale nell'Ottocento in: Quaderni fiorentini per la storia del pensiero giuridico, 38, t. I, 553-577

LAUterpacht, Hersch (1934), Les travaux préparatoires et l'interprétation des traités, in: Recueil des cours de l'Académie de droit international de la Haye, 48, II, 709-816, https://doi.org/10.1163/ej.9789028608528.709-846

Lawrence, Thomas (1898), The Principles of International Law, London

Lazzarich, Diego (2012), Stato moderno e diritto delle genti. Vattel tra guerra e politica. Benevento

Lee, Keun-Gwan (2011), The « Reception » of European International Law in China, Japan and Korea: A Comparative and Critical Perspective, in: Maraunn, Thilo, Heinard Steiger (eds.), Universality and Continuity in International Law, The Hague, 419-445

312 Bibliografia 
Le Fur, Louis, George Chrlaver (éd.) (1934), Recueil de textes de droit international public, deuxième édition, Paris

Lefevere, André (1978), Translation Studies. The Goal of the Discipline, in: Holmes, James J., José Lambert, Raymond Van den Broeck (eds.), Literature and Translation. New Perspective in Literary Studies with Basic Bibliography of books on Translation Studies, Leuven, 234-235

Lefevere, André (1992a), Translating Literature: Practice and Theory in a Comparative Literature, New York

Lefevere, André (1992b), Translation/History/Culture. A Sourcebook, London

Legarre, Santiago (2007), The Historical Background of the Police Power, in: University of Pennsylvania Journal of Constitutional Law, 9, 3, 745-796

Legarre, Santiago (2012), Police and Police Power: Domestic and International Law at the Crossroads, in: Nuzzo, Luigi, Miloš Vec (eds.), Constructing International Law. The Birth of a Discipline, Frankfurt am Main, 431-445

Lengel, James H. (1999), The Role of International Law in the Development of Constitutional Jurisprudence in Supreme Court: The Marshall Court and American Indians, in: The American Journal of Legal History, 43, 2, 117132, https://doi.org/10.2307/846282

LesAfFer, RANDALl (2007), International Law and its History: The Story of an Unrequited Love, in: Craven, Matthew, Malgosia Fitzmaurice, Maria Vogiatzi (eds.), Time, History and International Law, Leiden, 27-41

Lesaffer, Randall (2011a), A Schoolmaster Abolishing Homework? Vattel on Peacemaking and Peace Treaties, in: Chetail, Vincent, Peter HaggenMACHer (eds.), Vattel's International Law in a XXIst Century Perspective, Le droit international de Vattel vu du XXIe siècle, Leiden, Boston, 353-384, https://doi.org/10.1163/ej.9789004194632.i-443.98

Lesaffer, Randall (2011b), Roman Law and the Early Historiography of International Law: Ward, Wheaton, Hosack and Walker, in: Marauhn, Thilo, Heinhard Steiger (eds.), Universality and Continuity in International Law, The Hague, 149-184

Lesaffer, Randall (2012), Peace Treaties and the Formation of International Law, in: Fassbender, Bardo, Anne Peters (eds.), The Oxford Handbook of the History of International Law, Oxford, 71-94, https://doi.org/10.1093/law/ 9780199599752.003.0004

Leti, Giuseppe (1966), Carboneria e massoneria nel Risorgimento italiano, Bologna

LetzTer, Jacqueline (1998), Intellectual Tacking: Questions of Education in the Works of Isabelle de Charrière, Amsterdam, Atlanta

Lianeri, Alexandra (2002), Translation and Establishment of Liberal Democracy in Nineteenth-Century England: Constructing the Political as an Interpretative Act, in: Tymoczko, Maria, Edwin Gentzler (eds.), Translation and power, Amherst, Boston, 1-24

Lilao Franca, Óscar, Carmen Castrillo González (1997), Catálogo de manuscritos de la Biblioteca Universitaria de Salamanca, I, Manuscritos 1-1679bis, Salamanca 
Linklater, Andrew (1982), Men and Citizens in the Theory of International Relations, New York, https://doi.org/10.1007/978-1-349-16692-3

Liszt, Franz von (1896), Das Völkerrecht systematisch dargestellt, Berlin

Liu, Lydia H. (1999), Legislating the Universal: the Circulation of International Law in the Nineteenth Century, in: Liu, Lydia H. (ed.), Tokens of Exchange. The Problem of Translation in Global Circulation, Durham, 127-164, https:// doi.org/10.1215/9780822381129-005

Liu, Lydia H. (2004), The Clash of Empires. The invention of China in Modern World Making, Cambridge, London

Liu, Lydia H. (2012), Wheaton Henry, in: Fassbender, Bardo, Anne Peters (eds.), The Oxford Handbook of the History of International Law, Oxford, 1132-1136, https://doi.org/10.1093/law/9780199599752.003.0057

Lombardi-Diop, Cristina, Caterina Romeo (eds.) (2012), Postcolonial Italy: Challenging National Homogeneity. The Colonial Past in Contemporary Culture, New York

Losada, Ángel (1989), El Abate Grégoire, lector de los humanistas y juristas españoles de los siglos XV a XVII fuentes ideológicas españolas de la revolución francesa, in: Historia Mexicana, 39, 1, 71-88

Loschi, Lodovico Antonio (1781), Avvertimento del volgarizzatore, in: Vattel, EMER DE, Il diritto delle genti, ovvero principii del diritto naturale applicati alla condotta e agli affari delle nazioni e de' sovrani, Lione, vol. I, VI

Louzada de Magalhães, João José (1881), Silvestre Pinheiro Ferreira, sein Leben und seine Philosophie mit einer Einleitung über die wichtigsten portugiesischen Philosophen vor ihm, Bonn

Lovejoy, Arthur O. (1904), Reflections on the History of Ideas, in: Journal of the History, 1, 3-23

Lovrić-Pernak, Kristina (2013), Morale internationale und humanité im Völkerrecht des späten 19. Jahrhunderts. Bedeutung und Funktion in Staatenpraxis und Wissenschaft, Baden-Baden, https://doi.org/10.5771/9783845250724

Lovrić-Pernak, Kristina (2015), Aim: Peace - Sanction: War. International Arbitration and the Problem of Enforcement, in: Hippler, Thomas, Miloš Vec (eds.), Paradoxes of Peace in 19th Century Europe, Oxford, 62-74, https:// doi.org/10.1093/acprof:oso/9780198727996.003.0004

Luporini, Luigi (1987), Per una lettura della voce « Droit naturel» nell'Encyclopédie, in: Studi Storici, 28, 3, 679-697

Lüsebrink, Hans-Jürgen (1997), Civilizzazione, in: Ferrone, Vincenzo, Daniel Roche (a cura di), L'Illuminismo. Dizionario Storico, Roma, Bari, 168-178

Lynch, John (ed.) (1987), Andrés Bello. The London Years, Richmond

Lynch, John (1994), Latin America Revolutions 1808-1826, Old and New World Origins, Norman

López Bohórquez, Alí Enrique (2008), La Real Audiencia de Caracas y la independencia de Venezuela, in: Puente Brunke, José de la, Jorge Armando Guevara Gill (eds.), Derecho, instituciones y procesos históricos, Lima, $553-566$ 
Macalister-Smith, Peter, Joachim Schwietke (2001), Bibliography of the Textbooks and Comprehensive Treaties on Positive International Law of the 19th Century, in: Journal of the History of International law, 3, 75-142, https:// doi.org/10.1163/15718050120956901

Maccabez, Eugène (1903), Fortunato Bartolomeo De Felice (1723-1789) et son Encyclopédie, Yverdon 1770-1780, Bâle

Machet, Anne (1986), Clients italiens de la Société typographique de Neuchâtel, in: Richner, Jacques, Michel Schlup (études réunis par), Aspect du livre neuchâtelois, Neuchâtel, 158-185

Mackintosh, James (21 February 1815), Transfer of Genoa, Commons Sitting of Tuesday, in: House of Commons Hansard, George III year 55, vol. XXIX, 920-955

Mackintosh, James (1846), Discourse on the study on the law of nature and Nations, in: Mackintosh, James, The miscellaneous works of the Right Honourable Sir James Mackintosh, Philadelphia, vol. I, 27-43

Mackintosh, Robert James (1855), The life of the right honourable Sir James Mackintosh, 2 vol., London

Madonia, Claudio (2013), Fra l'orso russo e l'aquila prussiana. La Polonia dalla Repubblica Nobiliare alla IV Repubblica (1506-2006), Bologna

Mallarmé, André (1904), Emer de Vattel, in: Pillet, Antoine (éd.), Les fondateurs du droit international, Leurs œuvres - leurs doctrines, Paris, 595-596

Mancini, Pasquale Stanislao (1844), Esame di un'opera di dritto internazionale pubblicata da Nicola Rocco, e del rapporto fatto dal Portalis sulla stessa all'Accademia delle scienze morali e politiche di Francia, in: Le ore solitarie. Biblioteca di scienze morali legislative ed economiche accresciuta di conoscenze universali e de' memoriali accademici italiani. Continuazione, 1, 10-30

Mancini, Pasquale Stanislao (1851), Della nazionalità come fondamento del diritto delle genti. Prelezione al corso di diritto internazionale e marittimo pronunziata nella R. Università di Torino nel dì 22 gennaio 1851, Torino

Mancini, Pasquale Stanislao (2007), in: Dizionario biografico degli italiani, Roma, $68,537-547$

Mancuso, Francesco (2002), Diritto, Stato, Sovranità. Il pensiero politico-giuridico di Emer de Vattel tra assolutisimo e rivoluzione, Napoli

Mancuso, Francesco (2008), Introduçao, in: Vattel, Emer de, O direito das gentes ou Príncipios da lei natural aplicados à condução e aos negócios das nações e dos governantes, Florianopolis, 17-67

Mancuso, Francesco (2009), Le Droit des gens come apice dello jus publicum europaeum? Nemico, guerra, legittimità nel pensiero di Emer de Vattel, in: Quaderni Fiorentini per la storia del pensiero giuridico moderno, 38, t. II, 1277-1310

Mancuso, Francesco (2015a), L'altro, il selvaggio, il pirata: Emer de Vattel e l'eccezione del «politico », in: Iura and Legal Systems, 2, 65-81 
Mancuso, Francesco (2015b), Vattel: l'altro, il selvaggio, il pirata, il nemico, la costituzione, in: Foscari, Giuseppe (a cura di), L'Europa e la scoperta dell'Altro, S. Maria Capua Vetere, 29-51

Mannoni, Stefano (2003), Stato nazionale di diritto e diritto internazionale, in: Costa, Pietro, Danilo Zolo (a cura di), Lo stato di diritto: storia, teoria, critica, Milano, 485-505

Mannoni, Stefano (2014), Da Vienna a Monaco (1814-1938). Ordine europeo e diritto internazionale, Torino

Mantese, Giovanni (1942), Pietro Tamburini e il giansenismo lombardo, Brescia

Manz, Johannes Jacobus (1971), Emer de Vattel Versuch einer Würdigung unter besonderer Berücksichtigung seiner Auffassung von der individuellen Freiheit und der souveränen Gleichheit, Zürich

Marchetti, Maria Cristina (2011), La moda oltre le mode: lineamenti di sociologia della moda, Roma

Marcu, Eva (1953), Un encyclopédiste oublié: Formey, in: Revue d'Histoire littéraire de la France, 53, 296-305

Marengo, Giorgio, Giuseppe Parlato (1982), Marochetti Giovanni Battista, in: Marengo, Giorgio, Giuseppe Parlato (a cura di), Dizionario dei Piemontesi compromessi nei moti del 1821, Torino, vol. II, 97

Marín y Mendoza, Joaquín (1776), Historia del derecho natural y de gentes, Madrid, D. Manuel Martín

Marini, Giuliano (2000), Dal diritto naturale alla filosofia del diritto, in: Storia dell'Università di Pisa, Pisa, vol. II, 635-661

Marochetti, Giovanni Battista (1830), Indépendance de l'Italie, moyen de établir dans l'intérêt général de l'Europe, considéré spécialement sous le point de vue de l'équilibre politique, ou Partage de la Turquie, Paris

Martens, Georg Friedrich von (1788), Précis du Droit des gens moderne de l'Europe fondé sur les traités et l'usage, Gottingue

Martens, Georg Friedrich von (1831), Précis du Droit des gens moderne de l'Europe, fondé sur les traités et l'usage; pour servir d'introduction à un cours politique et diplomatique. Nouvelle édition avec des notes de M.S. PinheiroFerreira, Paris

Martens, Georg Friedrich von (1858), Précis du Droit des gens moderne de l'Europe. Nouvelle edition revisée, accompagnée des notes de Pinheiro-Ferreira, precedée d'une introduction et completée par l'exposition des doctrines des publicistes contemporains et suivie d'une Bibliographie raisonnée du Droit des gens, par Ch. Vergé, Paris

Martens, Georg Friedrich von (1864), Précis du Droit des gens moderne de l'Europe, fondé sur les traités et l'usage; pour servir d'introduction à un cours politique et diplomatique. Augmentée des notes de Silvestre Pinheiro-Ferreira, II édition. enrichie de nouvelles notes et mise au courant des événements contemporains as Ch. Vergé, Paris

Martínez Martínez, Faustino (2013), Repensar la Constitución de 1812: Cádiz o el imposible constituyente, in: Historia et Ius. Rivista di storia giuridica dell'età 
medievale e moderna, 3, paper 6, 1-118, http://www.historiaetius.eu/uploads/ 5/9/4/8/5948821/martinez.pdf

Martínez, Jesus A. (1992), La lectura y lectores en el Madrid del siglo XIX, Madrid Martinic, María Doria G., Mauricio R. Tapia (dir.) (2005), Sesquicentenario del Código civil de Andrés Bello: pasado, presente y futuro de la codificación, 2 t., Santiago de Chile

Marvin, John Gage (1847), Legal Bibliography, or a Thesaurus of American, English, Irish and Scotch law books. Together with some continental treaties. Interspersed with critical observations upon their various editions and authority to which is prefixed a copious list of abbreviations, Philadelphia

Marx, Jacques (1968), Un grand imprimeur du XVIIIe siècle: Élie Luzac Fils (1723-1726), in: Revue belge de philologie et d'histoire, 46, 3, 779-786

Marx, Jacques (1991), Bibliothèque Impartiale, in: SGARD, JeAn (sous la direction de), Dictionnaire de la presse 1600-1789, I. Dictionnaire de journaux, vol. I, a-i, Paris, n. 164, 189-191

Marzano, Umberto Renato (1923), Pasquale Fiore. L'uomo e il giurista, Bari

Mayagoitia Stone, Alejandro (2005), Los abogados y el Estado Mexicano: desde la independencia hasta las grandes codificaciones, in: Historia de la justicia en Mexico, siglos XIX y XX, Ciudad de México, t. I, 263-406

Mazzanti Pepe, Fernanda (2010), Mably. Principi, regole e istituzioni per una democrazia a misura d'uomo, Roma

Mazzetti, Roberto (1948), Pietro Tamburini: la mente del giansenismo italiano, Messina

Mazzini, Giuseppe (1842), Luigi Angeloni, in: Apostolato popolare, 1, 40-41

Meccarelli, Massimo (2004), Corti supreme e armonizzazione del diritto privato nella percezione della scienza giuridica tra otto e novecento, in: Troiano, Onofrio, Giunio Rizzelli, Marco Nicola Miletti (a cura di), Harmonisation involves history? Il diritto privato europeo al vaglio della comparazione e della storia. Atti del Convegno di Foggia del 20 e 21 giugno 2003, Milano, 117-170

Meccarelli, Massimo, María Julia Solla Sastre (eds.) (2016), Spatial and Temporal Dimensions for Legal History. Research Experiences and Itineraries, Global Perspectives on Legal History 6, Max Planck Institute for European Legal History, Open Access Publication, Frankfurt am Main, http://dx.doi.org/ 10.12946/gplh6

Meccarelli, Massimo, Stefano Solimano (20. März 2007), A colloquio con Paolo Grossi in: Forum Historiae Iuris, http://www.forhistiur.de/2007-03-meccarellisolimano/, consultato il 24 maggio 2016

Medianeira Padoin, Maria (2001), Federalismo gaúcho. Fronteira platina, direito e revolução, São Paulo

Meister, Johann Christian Friedrich (1808), Lehrbuch des Naturrechtes, Freystadt

Mele, Franca (2002), Un codice unico per un'Italia nuova. Il progetto di codice penale di Pasquale Stanislao Mancini, Roma 
Meriggi, Marco (2015), Intervento sulla World History, in: Giornale di storia, 17, $1-10$

Mestad, Ola (2015), The Most Awkward and Embarrassing Question: British Treaty Obligations, the Role of the Law of Nations and the Establishment of the Swedish Norwegian Union in 1814, in: Scandinavica, 54, 1, 48-64

Metzner Carolin (2009), Johann Caspar Bluntschli, Leben Zeitgeschehen und Kirchenpolitik 1808-1881, Frankfurt am Main

Meusel, Johann Georg (1815), Vattel de (Emerich), in: Lexikon der vom Jahr 1750 bis 1800 verstorbenen teutschen Schriftsteller, Leipzig, 431-432

Meylan, Philippe (1937), Jean Barbeyrac (1674-1744) et les débuts de l'enseignement du droit dans l'ancienne Académie de Lausanne. Contribution à l'histoire du droit naturel, Lausanne

Miglio, Gianfranco (2009), La controversia sui limiti del commercio neutrale fra Giovanni Maria Lampredi e Ferdinando Galiani, prefazione di Ornaghi, LoREnzo, Milano

Mijangos y Gonzalez, Pablo (2015), The Lawyer of the Church. Bishop Clemente de Jesús Munguía and the Clerical Response to the Mexican Liberal Reforma, Lincoln

Millán, Carmen, Francesca Bartrina (eds.) (2013), The Routledge Handbook of Translation Studies, London

Miruss, Alexander (1847), Das Europäische Gesandschaftsrecht, nebst einem Anhange von dem Gesandschaftsrechte des Deutschen Bundes, einer Bücherkunde des Gesandschaftsrecht und erläuternden Beilagen, Leipzig

Mohl, Robert von (1855), Die Geschichte und Literatur der Staatswissenschaften, in Monographieen dargestellt, Erlangen, Bd. I

Mohnhaupt, Heinz (1990), Verfassung (I.) Konstitution, Status, Lex fundamentalis, in: Brunner, Otto, Werner Conze, Reinhart Koselleck (Hrsg.), Geschichtliche Grundbegriffe, Stuttgart, vol. VI, 858-870

Mohnhaupt, Heinz (2003), Verfassung, in: Cancik, Hubert, Helmut Schneider, (Hrsg.), Der Neue Pauly. Enzyklopädie der Antike, Stuttgart, B. Suppl. IV, 969-982

Mohnhaupt, Heinz (2008), Costituzione: storia di un concetto dall'antichità a oggi, edizione italiana (a cura di) Ascheri, Mario, Simona Rossi, Roma

Mongiano, Elisa (2013), Il principio di nazionalità nella formazione dello stato unitario italiano: il contributo di Pasquale Stanislao Mancini, in: Revista europea de historia de las ideas políticas y de las instituciones públicas, 6, 85-97, http://www.eumed.net/rev/rehipip/06/em.pdf

Montmorency, James E. G. de (1909), The Great Jurist of the World: Emmerich de Vattel, in: Journal of Society of Comparative Legislation, 10, 17-39

Moore, Frank (1857), William Pinkney's speech, in: American eloquence: a collection of speeches and addresses, by the eminent orators of America; with biographical sketches and illustrative notes, New York, vol. II, 102-107 
Moreau, Jacob Nicholas (1757), Mémoires pour servir à l'Histoire de notre temps, par l'Observateur hollandois, rédigez et augmentez par M.D.V., Frankfort, Leipzig, Aux dépens de la compagnie

Morelli di Schönfeld, Carlo (1855), Istoria della Contea di Gorizia, Gorizia, vol. III, Gorizia

Morelli, Emilia (1955), Tre profili. Benedetto XIV, Pasquale Stanislao Mancini, Pietro Roselli, Roma

Morelli, Emilia (1979), Gli esuli italiani e la società inglese nella prima metà dell'Ottocento, in: Rassegna Storica del Risorgimento, 66, 3-13

Morice, Joseph R. (1961), The Contributions of Charles W. F. Dumas to the Cause of American Independence, in: Duquesne Review, 7, 17-28

Morley, Michael T. (2002), The Law of Nations and the Offenses Clause of the Constitution: A Defense of Federalism, in: The Yale Law Journal, 112, 1, 109-143, https://doi.org/10.2307/1562235

Moser, Johann Jасов (1750), Grundsätze des jetzt üblichen europäischen Völkerrechts in Friedenszeiten, Hanau

Moser, Johann Jасов (1752), Grundsätze des jetzt üblichen europäischen Völkerrechts in Kriegszeiten, Tübingen

Moser, Johann Jaсов (1777-1780), Versuch des neuesten Europäischen VölkerRechts in Friedens- und Kriegszeiten, 10 Bde., Frankfurt am Main, Varrentrapp und Wenner

Moyn, Samuel, Andrew Sartori (eds.) (2013), Global Intellectual History, New York

Moynihan, Daniel Patrick (1990), On the Law of Nations, Cambridge

Mozo, Manuel J. (1898), Tratado elemental de derecho de gentes y marítimo internacional: con varios apéndices que contienen documentos nacionales y extranjeros referentes al asunto, Madrid

MücKe, Ulrich (2008), Gegen Aufklärung und Revolution. Die Enstehung konservativen Denkens in der iberischen Welt (1770-1840), Köln, Weimar, Wien

Munday, Jeremy (2013), Introducing Translation Studies: Theories and Applications, London

Murphy, Cornelius F. (1985), The Search for World Order: a Study of Thought and Action, Dordrecht

Naguib Leerberg, Nora (2015), The Legal Politics of Neutrality in the Age of Privateering: Martin Hübner's Law of Neutrality and Prize, Oslo

Nakhimovsky, IsaAc (2007), Vattel's theory of the International Order: Commerce and the Balance of Power in the Law of Nations, in: History of European Ideas, 33, 2, 157-173, https://doi.org/10.1016/j.histeuroideas.2006.11.006

Naкнiмovsкy, IsaAc (2010), Carl Schmitt's Vattel and the «Law of Nations» between Enlightenment and Revolution, in: Grotiana, 31, 141-164

Naкнiмоvsкy, Isaac (2011a), The Closed Commercial State: Perpetual Peace and Commercial Society from Rousseau to Fichte, Princeton

Naкhimovsky, Isaac (2011b), The 'Ignominious Fall of the European Commonwealth': Gentz, Hauterive and the Armed Neutrality of 1800, in: STAPELBRoek, 
Koen (ed.), COLLeGium, Trade and War: The Neutrality of Commerce in the Inter-State System. Studies across Disciplines in the Humanities and Social Sciences 10, Helsinki, 212-228, https://helda.helsinki.fi/bitstream/handle/ 10138/25843/010_11_Nakhimovsky_2011.pdf\%20?sequence=01, consultato il 27 maggio 2016

Napoli, Maria Teresa (1986-1987), La cultura giuridica europea in Italia. Repertorio delle opere tradotte nel secolo XIX, t. I-III, Napoli

Nardi, Carla (2005), Napoleone a Roma. Dalla Consulta Romana al ritorno di Pio VII (1811-1814), Roma

Nardin, Terry (2014), Historian or Philosopher? Ian Hunter on Kant and Vattel, in: History of European Ideas, 40, 1, 122-134, https://doi.org/10.1080/01916599. 2012.725669

Neff, Stephen C. (2005), War and the Law of Nations: A General History, Cambridge, https://doi.org/10.1017/CBO9780511494253

Neri, Sergio (1958), Sull'interpretazione dei trattati nel diritto internazionale, Milano

Nippold, Otfried (1934), Einleitung, in: Wolff, Christian, Jus gentium methodo scientifica pertractatum, Oxford, London, XIII-LVI

Nishitani, Yuko (2001), Mancini e l'autonomia della volontà nel diritto internazionale privato, in: Rivista di diritto internazionale privato e processuale, 37, 22-44

Noto, Giovanni Andrea (2015), La ricezione del Risorgimento greco in Italia (1770-1844). Tra idealità filelleniche, stereotipi e Realpolitik, Roma

Novales, Alberto Gil (dir.) (1991), Pascual Hernández, Manuel Maria, in: Diccionario Biografico Triennio Liberal, Madrid, 509

Novarino, Marco (2008), Marochetti Giovanni Battista, in: Dizionario Biografico degli Italiani, Roma, 70, 637-640

Nussbaum, Arthur (1954), A Concise History of the Law of Nations, New York

Nuzzo, Luigi (2009a), Un mondo senza nemici. La costruzione del diritto internazionale e il controllo delle differenze, in: Quaderni fiorentini per la storia del pensiero giuridico, 38 , t. II, 1311-1381

Nuzzo, Luigi (2009b), Alberico Gentili «internazionalista » tra storia e storiografia, in: Lacchè, Luigi (a cura di), «Ius Gentium ius communicationis ius belli» Alberico Gentili e gli orizzonti della modernità. Atti del convegno di Macerata in occasione delle celebrazioni del quarto anniversario della morte di Alberico Gentili, Macerata, 6-7 dicembre 2007, Milano, 75-99

Nuzzo, Luigi (2012a), Origini di una Scienza. Diritto internazionale e colonialismo nel XIX secolo, Frankfurt am Main

Nuzzo, Luigi (2012b), History, Science and Christianity. International law and Savigny Paradigm, in: Nuzzo, Luigi, Miloš Vec (eds.), Constructing International Law. The Birth of a Discipline, Frankfurt am Main, 25-50

Nuzzo, Luigr (2012c), Pasquale Stanislao Mancini, in: Enciclopedia Treccani, Il contributo italiano alla storia del pensiero - Diritto, Roma, 307-311 
Nuzzo, Luigi (2013), La storia del diritto internazionale e le sfide del presente (a proposito di Martti Koskenniemi, Il mite civilizzatore delle Nazioni. Ascesa e caduta del diritto internazionale 1870-1960, Laterza, 2012), in: Quaderni fiorentini per la storia del pensiero giuridico, 42, 683-701

Nuzzo, Luigi, Miloš Vec (2012), The Birth of International Law as a Legal Discipline, in: Nuzzo, Luigi, Miloš Vec (eds.), Construction International Law. The Birth of a Discipline, Frankfurt am Main, IX-XVI

Nys, Ernest (1896), La Révolution française et le droit international, in: Nys, Ernest (éd.), Etudes du droit international et de droit politique, 318-406

O’Connell, Mary Ellen (2013), Historical Development and Legal Basis, in: Fleck, Dieter (ed.), The Handbook of International Humanitarian Law, 3th edition, Oxford, 101-151

Obregón Tarazona, Liliana (2008), Between Civilization and Barbarism: Creole Interventions in International Law, in: FALK, Richard, BaLAKrishnaN Rajagopal, Jacqueline Stevens (eds.), International Law and the Third World: Reshaping Justice, London, 111-128

Obregón Tarazona, Liliana (2009a), Construyendo la región americana: Andrés Bello y el Derecho Internacional, in: González, Stephana Beatriz, Juan Poblete (eds.), Andrés Bello y los Estudios Latinoamericanos. Serie Críticas, Pittsburgh, 189-218

Obregón Tarazona, Liliana (2009b), Latin American International Law, in: Amstrong, David (ed.), Routledge Handbook of International Law, New York, 154-164

Obregón Tarazona, Liliana (2010), Construyendo la región americana: Andrés Bello y el derecho internacional, in: Gamorra Chopo, Yolanda (coord.), La idea en el pensamiento ius internacionlista del siglo XXI: Estudios a propósito de la commemoración de los bicentenarios de las independencias de las repúblicas latinoamericanas, Zaragoza, 65-86, http://ifc.dpz.es/recursos/publica ciones/30/12/06obregon.pdf

Occhipinti, Egidia (2011), Teopompo e la politica macedone, in: Klio, 93, 2, 291-307

Oeter, Stefan (1988), Ursprünge der Neutralität. Die Herausbildung des Instituts der Neutralität im Völkerrecht der frühen Neuzeit, in: Zeitschrift für ausländisches öffentliches Recht und Völkerrecht, 48, 447-488

Oeter, Stefan (2011), Neutrality and Alliances, in: Chetail, Vincent, Peter Haggenmacher (eds.), Vattel's International Law in a XXIst Century Perspective, Le droit international de Vattel vu du XXIe siècle, Leiden, Boston, 335-352, https://doi.org/10.1163/ej.9789004194632.i-443.91

O’Leary, Patrick (1989), Sir James Mackintosh: the Whig Cicero, Aberdeen

Olivart, Ramón María de Dalmau Marqués de (1887), Tratado y notas de derecho internacional público, Madrid

Olivart, Ramón María de Dalmau Marqués de (1891), Tratado de derecho internacional público, Madrid, vol. I 
Olivart, Ramón María de Dalmau Marqués de (1905), Bibliographie du droit international, Paris

Olmeda y Leon José (1771), Elementos del derecho público de la paz y de la guerra, ilustrados con noticias históricas, leyes y doctrinas del derecho español, 2 vol., Madrid, Manuel Fernandez

Omero (2014), Iliade, Ciani, Maia Grazia (a cura di), Venezia

Ompteda, Dietrich Heinrich Ludwig von (1785), Literatur des gesamten sowohl natürlichen als positiven Völkerrechts, Regensburg, J. L. Montags, Bd. I

Onuf, Nicholas Greenwood (1994), Civitas Maxima: Wolff, Vattel and the Fate of Republicanism, in: The American Journal of International Law, 88, 2, 280-303, https://doi.org/10.2307/2204100

Onuf, Nicholas Greenwood (1998), The Republican Legacy in International Thought, Cambridge

Oosterveld, Willem Theo (2015), The Law of Nations in Early American Foreign Policy: Theory and Practice from the Revolution to Monroe Doctrine, Leiden, Boston

Orakhelashvili, Alexander (2011), The Origins of Consensual Positivism - Pufendorf, Wolff and Vattel, in: Orakhelashvili, Alexander (ed.), Research Handbook on the Theory and History of International Law, Cheltenham, Northampton, 93-110, https://doi.org/10.4337/9780857933089.00011

Orestano, Riccardo (1982), 'Institution' Barbeyrac e l'anagrafe di un significato, in: Itinerari moderni della persona giuridica, Quaderni fiorentini per la storia del pensiero giuridico, 11/12, t. I, Milano, 169-178

Orford, Anne (2013a), The Past as Law or History? The Relevance of Imperialism for Modern International Law, in: Toufayan, Mark, Emmanuelle TourmeJouannet, Hélène Ruiz-Fabri (eds.), Droit international et nouvelles approches sur le tiers-monde: entre répétition et renouveau, Paris, 97-118

Orford, Anne (2013b), On International Legal Method, in: London Review of International Law, 1, 1, 166-197, https://doi.org/10.1093/lril/lrt005

Orford, Anne (2014), Scientific Reason and the Discipline of International Law, in: European Journal of International Law, 25, 2, 369-385, https:/doi.org/ 10.1093/ejil/chu030

Ortega y Cotes, Ignacio José (1747), Questiones del derecho público en interpretación de los tratados de paces, Madrid, Peralta

Ortiz de la Torre, José Antonio Tomas (1970), L'établissement de l'enseignement officiel en Espagne du droit international, in: Yearbook of the Association of Attenders and Alumni of the Hague Academy of International law, 40, $122-132$

Ossipow, William, Dominik Gerber (2010), La réception du «Droit des gens » (1758) d'Emer de Vattel aux États-Unis: résultat d'une recherche et quelques expériences méthodologiques avec le concept d'autorité textuelle, in: SANDoz, Yves (éd.), Réflexions sur l'impact, le rayonnement et l'actualité du 'Droit des gens' d'Emer de Vattel - Reflections on the Impact, Influence and Continuing Relevance of the 'Law of Nations' by Emer de Vattel, Bruxelles, 79-118 
Ostervald, Samuel Frédéric (1773), Abrégé de la vie de M. de Vattel, in: Vattel, Emer de, Le droit des gens ou Principes de la loi naturelle, appliqués à la conduite et aux affaires des nations et des souverains. Nouvelle édition augmentée, Neuchâtel, Société typographique de Neuchâtel, I-VI

Ottonello, Franco (1977), Cultura filosofica nella stampa periodica dell'Italia meridionale, Milano, vol. I

Pacca, Bartolomeo (1843), Memorie storiche del ministero, de' due viaggi in Francia, e della prigionia nel forte di S. Carlo in Fenestrelle, Orvieto

Padoa Schioppa, Antonio (2007), Storia del diritto in Europa. Dal medioevo all'età contemporanea, Bologna

Pärvärinne, Meri (2012), Translating Grotius's De jure belli ac pacis: Courtin vs Barbeyrac, in: Translation Studies, 1, 33-47, https://doi.org/10.1080/ 14781700.2012.628811

Palladini, Fiammetta (2011), Die Berliner Hugenotten und der Fall Barbeyrac: Orthodoxe und «Sozinianer » im Refuge (1685-1720), Leiden, Boston

Palma, Alerino (1829), Difesa dei piemontesi inquisiti causa degli avvenimenti del 1821 con un'invocazione ai ministri costituzionali, Bruxelles

Panebianco, Massimo (1974), Ugo Grozio e la tradizione storica del diritto internazionale, Napoli

Panizza, Diego (1969), La traduzione italiana del « De iure naturae» di Pufendorf: giusnaturalismo moderno e cultura cattolica nel Settecento, in: Studi Veneziani, 11, 483-528

Panizza, Diego (1997), Genesi di una ideologia. Il conservatorismo moderno in Robert Ward, Padova

Pantín Fernández, Francisco José (2001), La «Bibliografia Nacional y Estranjera » de Antonio Miyar, un instrumento bibliográfico al servicio del progreso de España, in: Boletín de la Asociación Asturiana de Bibliotecarios, Archiveros, Documetalistas y Museólogos, 12, 2, 18-26

Papa, Emilio (1993), Storia della Svizzera, dall'antichità ad oggi. Il mito del federalismo, Milano

Parada, Alejandro (1998), El mundo del libro y de la lectura durante la época Rivadavia. Una aproximación a través de los avisos de La Gaceta Mercantil (1823-1828), Buenos Aires

Paradisi, Bruno (1973), Indirizzi e problemi della più recente storiografia giuridica italiana, in: PAradisı, Bruno, Apologia della storia giuridica, Bologna, $173-258$

Paradisi, Bruno (1987), Le glosse come espressione del pensiero giuridico medievale, in: Paradisi, Bruno, Studi sul medioevo giuridico, Roma, vol. II, 711773

Parisian, Catherine M. (2010), Catalogue of the First White House Library, in: Parisian, Catherine M. (ed.), The First White House Library: a History and Annotated Catalogue, Pennsylvania, 97-356

Passero, Laura (2009), Dalle Convenzioni di Ginevra alla 'bancarotta' del diritto internazionale. Il rapporto tra il 'nuovo' jus in bello otto-novecentesco e la 
catastrofe della prima guerra civile europea, in: Quaderni fiorentini per la storia del pensiero giuridico, 38, t. II, 1479-1504

Passero, Laura (2010), Dionisio Anzilotti e la dottrina internazionalistica tra Otto e Novecento, Milano

Passero, Laura (2013a), Olivi Luigi, in: Birocchi, Italo, Ennio Cortese, Antonello Mattone, Marco Nicola Miletti (diretto da), Dizionario Biografico dei Giuristi Italiani (XII-XX secolo), Bologna, vol. II, 1455

Passero, Laura (2013b), Pierantoni, Augusto, in: Birocchi, Italo, Ennio Cortese, Antonello Mattone, Marco Nicola Miletti (diretto da), Dizionario Biografico dei Giuristi Italiani (XII-XX secolo), Bologna, vol. II, 1576

Pasta, Renato (1990a), Prima della Rivoluzione: il mercato librario italiano nelle carte della Société typographique de Neuchâtel (1769-1789), in: Mélanges de l'École française de Rome et Méditerranée, 102, 2, 281-320

Pasta, Renato (1990b), Beccaria tra giuristi e filosofi: aspetti della sua fortuna in Toscana e nell'Italia centrosettentrionale, in: Romagnoli, Sergio, Gian Domenico Pisapia (a cura di), Cesare Beccaria tra Milano e l'Europa, Milano, Roma, 512-533

Pasta, Renato (1997a), Editoria e cultura nel Settecento, Firenze

Pasta, Renato (1997b), Dei delitti e delle pene et sa fortune italienne: milieu juridiques et lecture "philosophique", in: Porret, Michel (éd.), Beccaria et la culture juridique des Lumières, (Actes du colloque européenne de Genève 25-26 Novembre 1995), Genève, 119-148

Patmore, Peter George (1854), My Friends and Acquaintances: Memorials of Deceased Celebrities of the Nineteenth Century, London, vol. II

Pecquet, Antoine (1741), Arte de negociar con los soberanos, Madrid, Peralta

Pedroni, Marco (2013), La moda. Dinamiche di innovazione e spinte conservatrici nel campo, in: Balbi, Gabriele, Cecilia Winterhalter (a cura di), Antiche novità. Una guida transdisciplinare per interpretare il vecchio e il nuovo, Napoli, Salerno, 99-115

Pejrone, Giulietta (1987), De Felice, Francesco Placido Bartolomeo (in religione Fortunato Bartolomeo), in: Dizionario Biografico degli Italiani, Roma, 33, 682-686

Pene Vidari, Gian Savino (2002), Un secolo e mezzo fa (22 gennaio 1851): la lezione torinese di Pasquale Stanislao Mancini sulla nazionalità, in: Studi Piemontesi, 31, 2, 273-285

Pereira, José Esteves (1974), Silvestre Pinheiro Ferreira, o seu pensamento politíco, Coimbra

Pérez Perdomo, Rogelio (1987), Giuristi e cultura giuridica nel Venezuela all'epoca di Bolívar, in: Rivista trimestrale di diritto e procedura civile, 41, 2, 447-459

Pérez Perdomo, Rogelio (2008), Los juristas como intelectuales y el nacimiento de los estados naciones en América Latina, in: Alatamirano, Carlo (dir.), Jorge Myers (ed.), Historia de los intelectuales en América Latina. I. La ciudad letrada, de la conquista al modernismo, Buenos Aires, 168-183 
Pérez Perdomo, Rogelio (2011), Los juristas académicos en la independencia de Venezuela, in: Revista Mexicana de Historia del Derecho, 23, 35-69

Pérez Perdomo, Rogelio (2013), Los juristas académico de Venezuela. Historia Institucional y biografía colectiva, Caracas

Perkins, Bradford (1970), Prologue to War. England and the United States 1805-1812, Berkeley

Perret, Jean-PIerre (1945), Les imprimeries d'Yverdon au XVIIe et au XVIIIe siècle, Lausanne

Perrochon, Henri (1951), Un homme du XVIIIe siècle: Louis Bourguet, Lausanne

Peset, Mariano (1974), La Universidad española (siglos XVIII y XIX). Despotismo ilustrado y Revolución liberal, Madrid

Petmezas, Socrates (2009), From Privileged Outcasts to Power Players: the «Romantic » redefinition of the Hellenic Nation in the mid-nineteenth Century, in: Beaton, Roderick, David Ricks (eds.), The Making of Modern Greece, Nationalism, Romanticism and the Uses of the Past (1797-1896), London, 126-139

Petronio, Giuseppe (1981), L'autore e il pubblico, Pordenone

Petronio, Giuseppe (1986), Metodo e polemica, Palermo

Phipps, Edmund (1850), Memories of the Political and Literary Life of Robert Plumer Ward, with Selections from his Correspondence, Diaries and Unpublished Literary Remains, 2 vol., London

Piédeliévre, Robert (1894), Précis de Droit International Public ou Droit des gens, Paris

Pierantoni, Augusto (1866), Il progresso del diritto pubblico e delle genti. Introduzione allo studio del diritto costituzionale ed internazionale, Modena

Pierantoni, Augusto (1869), Storia degli studi del diritto internazionale in Italia, Modena

Pierantoni, Augusto (1874), La riforma del diritto delle genti e l'istituto di diritto internazionale di Gand, in: Field, Dudley Davide, Prime linee di un codice internazionale del giurista americano, trad. it. Pierantoni, Augusto, Napoli, $1-86$

Piergiovanni, Vito (2001), Lo Statuto albertino in Liguria: le lezioni di diritto costituzionale di Lodovico Casanova, in: Atti della Società Ligure di Storia Patria, 41, 2, 193-216

Pieri, Piero (1931), Le società segrete e i moti degli anni 1820-1821 e 1830-1831, Milano

Pietropaoli, Stefano (2009), Jus ad bellum e ius in bello. La vicenda teorica di una " grande dicotomia» del diritto internazionale, in: Quaderni fiorentini per la storia del pensiero giuridico, 38, t. II, 1169-1213

Pillet, Antoine (1893), Droit de la guerre, Paris, vol. I

Pinheiro Ferreira, Silvestre (1813), Preleções philosophicas sobre a theórica do discurso e da linguagem, a esthética, a diceosyna e a cosmologia, Rio de Janeiro 
Pinheiro Ferreira, Silvestre (1831-1834), Projecto de ordenações para o Reino de Portugal, t. I-III, Paris

Pinheiro Ferreira, Silvestre (1833), Observations sur la Charte constitutionelle de la France, extraites du cours de droit public, Paris

Pinheiro Ferreira, Silvestre (1834a), Manual do cidadão em um governo representativo ou principios de direito constitucional administrativo e das gentes, t. I-III, Paris

Pinheiro Ferreira, Silvestre (1834b), Projecto de Código Geral de leis fundamentais e constitutivas de uma monarquia representativa, Paris

Pinheiro Ferreira, Silvestre (1835), Observações sobre a Constituição do Império do Brazil, e sobre a Carta Constitucional do Reino de Portugal, segunda edição augmentada com as observações do mesmo autor sobre a lei das reformas do imperio do Brazil, Paris

Pinheiro Ferreira, Silvestre (1836), Declaração dos direitos e deveres do homen e do cidadão, Paris

Pinheiro Ferreira, Silvestre (1838), Avertissement, in: Vattel, Emer de, Le droit des gens ou principes de la loi naturelle, appliqués à la conduite et aux affaires des Nations et des Souverains, revue et corrigée avec quelques remarques de l'editeur, augmentée de quelques remarques nouvelles, et d'une bibliographie choisie et systématique du droit de la nature et des gens, par M. de Hoffmann, Paris, t. III, V-VII

Pino Iturrieta, Elías (1987), Las ideas de los primeros venezolanos, Caracas

Pino Iturrieta, Elías, (1971), La mentalidad venezolana de la emancipación (1810-1812), Caracas

Pinoli Maritano, Elena (1975), Ivrea e il Canavese nella Rivoluzione Piemontese del 1821, Ivrea

Piro, Francesco (1999), Leibniz tra i due Thomasius. Identificare o differenziare «Honestum » e "Justum »?, in: Cacciatore, Giuseppe et al. (a cura di), La filosofia pratica tra metafisica e antropologia nell'età di Wolff e Vico, Napoli, 409-443

Piro, Francesco (2000). Il corpo politico dell'Europa: da Leibniz a Vattel, in: Bianchi, Lorenzo, Alberto Postigliola (a cura di), Un «progetto filosofico » della modernità. "Per la pace perpetua » di Immanuel Kant, Napoli, 65-94

Pisanó, Attilio (2002), Il diritto dei popoli nella Rivoluzione Francese, Milano

Pitts, Jennifer (2013), Intervention and Sovereign Equality: Legacies of Vattel, in: Recchia, Stefano, Jennifer M. Welsch (eds.), Just and Unjust Military Intervention: European Thinkers from Vitoria to Mill, Cambridge, 132-153, https://doi.org/10.1017/CBO9781107323681.007

Pizanias, Petros (2011), The Greek Revolution of 1821: a European event, Istanbul Plan de Educacion de la nobleza. Trabajado de Orden del Rey en 1798. Precedido de un estudio preliminar por Miguel Adellac Gonzáles de Agüero catedrático y director del Instituto de Jovellanos de Gijón (1915), Gijón

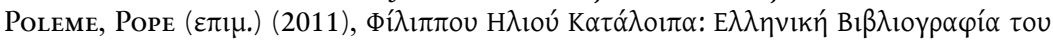

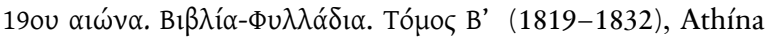


Pomeroy, John Norton (1886), Lectures in time of peace, Boston, New York

Popkin, Jeremy, Richard Popkin (eds.) (2000), The Abbé Grégoire and his World, Dordrecht

Porras, Ileana (2014), Appropriating Nature: Commerce, Property, and the Commodification of Nature in the Law of Nations, in: Leiden Journal of International Law, 27, 3, 641-660, https://doi.org/10.1017/S0922156514000247

Portillo Valdés, José M. (2007), Crisis e independencias: España y su monarchía. Multiple crises and independences: Spain and its Monarchy in Cuadernos Dieciochistas, 8, 19-35

Pott, SANdra (2002), Reformierte Morallehren und deutsche Literatur von Jean Barbeyrac bis Christoph Martin Wieland, Tübingen, https://doi.org/10.1515/ 9783110940558

Poudret, Jean-François (2006), La leçon inaugurale de Jean Barbeyrac à l'Académie de Lausanne, in: Pena, Marc (éd.), Les grands juristes. Actes des journées internationales de la Société d'Histoire du Droit. Aix-en-Provence, 22-25 Mai 2003, Aix-en-Provence, 105-110

Pozzi, Regina (2010), Guizot, o dell'Europa una e molteplice, in: Cromohs, 15, 1-8, http://www.fupress.net/index.php/cromohs/article/view/15470, consultato il 27 giugno 2016

Pozzi, Regina (2014), Guizot et l'Europe des nations: une âme aux différents caractères, in: The Tocqueville Review/La revue Tocqueville, 35, 1, 73-85, https:// doi.org/10.1353/toc.2014.0007

Pradier-Fodéré, Paul (1863), Avant propos, in: Vattel, Emer de, Le droit des gens ou principes de la loi naturelle, appliqués à la conduite et aux affaires des Nations et des Souverains, complétée par l'exposition des doctrines des publicistes contemporains mis au courant des progrès du droit public moderne, Paris, t. I, III-XI

Pradier-Fodéré, Paul (1886), Traité de droit international public européen et américain suivant les progrès de la science et de la pratique contemporaines, Paris, vol. II

Pradier-Fodéré, Paul Louis (1867), Avant-Propos, in: Grotius, Hugo (1867), Le droit de la guerre et de la paix, divisé en trois livres ou sont expliqués le droit de la nature et des gens et les principaux points du droit public. Nouvelle traduction, précédé d'un Essai biographique et historique sur Grotius et son temps, accompagnée d'un choix de notes de Gronovius, Barbeyrac, etc. completée par des notes nouvelles mise au courant des progrès du Droit publique moderne, et suivie d'une table analytique des matières par M. P. PradierFodéré, Paris, III-XII

Prendergast, Harris (1855), The Law Relating to Officers in the Army, London

Presa, Diego (2002), Protagonista della rivoluzione, Giovanni Battista Marochetti, in: Rivista Biellese, 6, 3, 12-22

Pufendorf, Samuel (1672a), De iure naturae et gentium libri octo, Londini scanorum, Junghans 
Pufendorf, Samuel (1672b), Elementorum iurisprudentiae universalis libri duo. Unà cum appendice de sphaera morali et indicibus, Cantabrigiae, J. Hayes et J. Creed

Pufendorf, Samuel (1706), Le droit de la nature et des gens, ou système général des principes les plus importans de la morale, de la jurisprudence et de la politique. Traduit du latin de feu Mr le baron de Pufendorf, par Jean Barbeyrac, 2 vol., Amsterdam, Henri Schelte

Pufendorf, Samuel (1712), Le Droit de la nature et des gens ou système général des principes les plus importans de la morale, de la jurisprudence, et de la politique. Traduit du Latin de feu Mr le Baron de Pufendorf par Jean Barbeyrac, professeur en Droit, Seconde Edition, revûë \& augmentée considerablement, Amsterdam, P. de Coup

Pufendorf, Samuel (1757-1759), Il diritto della natura e delle genti o sia sistema generale de' principii li più importanti di morale, giurisprudenza e politica, rettificato, accresciuto e illustrato da Giovambattista Almici, Venezia, Pietro Valvasense, vol. I-IV

Pufendorf, Samuel (1761-1767), I doveri dell'uomo e del cittadino: tali che a lui dalla legge naturale sono prescritti, dalla versione francese di Giovanni Barbeyrac tradotti, e con molte aggiunte corretti ed illustrati da Michele Grandi accademico di Udine, 3 t., Venezia, Pitteri

Quaglioni, Diego (1999), Pufendorf in Italia. Appunti e notizie della prima diffusione della traduzione italiana del De iure naturae et gentium, in: Il Pensiero Politico, 32, 23-250

Quaritsch, August (1875), Compendium des europäischen Völkerrechts: Lehrbuch und Repertorium, Berlin

Quartier-la-Tente, Edouard (1903), Les familles bourgeoises de Neuchâtel. Essais généalogiques, Neuchâtel

Quérard, Joseph-Marie (1834), Moreau Jacob-Nicolas, in: La France littéraire, ou dictionnaire bibliographique des savants, historiens et gens de lettres de la France, ainsi que des littérateurs étrangers qui ont écrit en français, plus particulièrement pendant les XVIIIe et XIXe siècles, Paris, tome sixième, 288-290

Rabkin, Jeremy (1997), Grotius, Vattel and Locke: an Older View of Liberalism and Nationality, in: The Review of Politics, 59, 2, 293-322, https://doi.org/10.1017/ S0034670500026644

Raine, Jonathan (12 February 1805), Commons Sitting of Tuesday, House of Commons Hansard, in: House of Commons Parliamentary Papers, George III year 45 , vol. III, 212-250

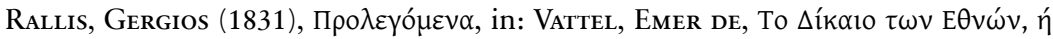

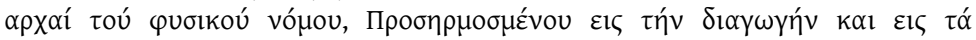

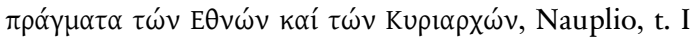

Randeria, Schalini (1999), Geteilte Geschichte und verwobene Moderne, in Rüsen, Jörn, Hanna Leitgeb, Norbert Jegelka (Hrsg.), Zukunftsentwürfe: Ideen für eine Kultur der Veränderung, Frankfurt am Main, 87-96 
RANieri, Filippo (2007), Le traduzioni e le annotazioni di opere giuridiche straniere nel XIX secolo come mezzo di penetrazione e di influenza delle dottrine, in: Ranieri, Filippo, Das Europäische Privatrecht des 19. und 20. Jahrhunderts. Studien zur Rechtsgeschichte und Rechtsvergleichung, Berlin, 51-66

Rao, Anna Maria (1990), La stampa francese a Napoli negli anni della Rivoluzione, in: Mélanges de l’École française de Rome. Italie et Méditerranée, 102, 2, 469-520, https://doi.org/10.3406/mefr.1990.4120

Rauschning, Dietrich (1987), Georg Friedrich von Martens (1756-1821) Lehrer des praktischen Europäischen Völkerrechts und der Diplomatie zu Göttingen, in: Loos, Fritz (Hrsg.), Rechtwissenschaft in Göttingen. Göttinger Juristen aus 250 Jahren, Göttingen, 123-145

Raynal, Guillaume-Thomas François (1781), Histoire philosophique et politique des établissements et du commerce des Européens dans les deux Indes, Genève, chez Jean-Leonard Pellet, t. IX

Raynal, Guillaume-Thomas François abbé (1823), De los pueblos y gobiernos: colleción de pensamientos, extraidos de la Historia filosófica de las dos Indias. Traducido al castellano por S. D.V., Londres

Raynero, Lucía (2001), La noción de libertad en los políticos venezolanos del siglo XIX, 1830-1848, Caracas

Rayneval, Joseph Matthias Gérard de (1803), Institutions du droit de la nature et des gens, II ed., Paris

Rech, Walter (2013), Enemies of Mankind. Vattel's Theory of Collective Security, Leiden, Boston

Reeves, Jesse S. (1909), The Influence of the Law of Nature Upon International Law in the United States, in: American Journal of International Law, 3, 3, 547-561, https://doi.org/10.2307/2186682

Reeves, Jesse S. (1924), La communauté internationale, in: Recueil des cours de l'Académie de droit international de la Haye, 3, 1924, 1-94, https://doi.org/ 10.1163/ej.9789028604025.001-094

Reeves, Jeesse (1937), The First American Treatise on International Law, in: The American Journal of International Law, 31, 4, 697-702

Regoli, Roberto (2006), Ercole Consalvi: le scelte per la Chiesa, Roma

Reibstein, Ernst (1958), Die Dialektik der souveränen Gleichheit bei Vattel, in: Zeitschrift für ausländisches öffentliches Recht und Völkerrecht, 19, 607-636

Renault, Louis (1905), Préface, in: Lapradelle, Albert de, Nicolas Politis (1905), Recueil des arbitrages internationaux t. I, 1798-1855, Paris, III-XV

Renscher, August Ludwig (1846), Bericht über die Germanisten-Versammlung zu Frankfurt a. M., am 24., 25., 26. September 1846, in: Zeitschrift für deutsches Recht und deutsche Rechtswissenschaft, 10, 494-527

Report of Decisions in the Supreme Court of the United States (1855), vol. III, Boston

Représentations des citoyens et bourgeois de Genève au Premier Syndic de cette République avec les réponses du Conseil à ces représentations (1763), Genève 
Ricciotti, Domenico (2009), In ricordo di Luigi Angeloni: scrittori e pensatori politici dell'Ottocento frusinate, Frosinone

Richardson, Brian (2012), The Use of Vattel in the American Law of Nations, in: The American Journal of International Law, 106, 3, 547-571, https://doi.org/ 10.5305/amerjintelaw.106.3.0547

Ricuperati, Giuseppe (a cura di) (1968), I moti piemontesi del 1821, Firenze

Rieppel, Olivier (1987), "Organization » in the Lettres philosophiques of Louis Bourguet compared to the writings of Charles Bonnet, in: Gesnerus, 44, 125-132

Rinieri, Ilario (1927), La rivoluzione in Piemonte, le società segrete, l'Austria e il principe di Carignano, in: Rossi, Teofilo, Carlo Pio Demagistris (a cura di), La rivoluzione piemontese del 1821. Studi e documenti, Torino, vol. I, 543649

Riquelme, Antonio (1849), Elementos de derecho público internacional: con explicación de todas las reglas que, según los tratados, estipulaciones, leyes vigentes y costumbres, costituyen el derecho interncional español, Madrid

Rivier, Alphonse (1896), Principes du Droit des gens, Paris, t. I

Robinson, John Martin (1987), Cardinal Consalvi 1757-1824, London

Robledo, Ricardo (2003a), Tradición e ilustración en la Universidad de Salamanca: sobre los orígenes intelectuales de los primeros liberales, in: Robledo, Ricardo, Irene Castelis María Cruz Romeo (eds.), Orígenes del liberalismo. Universidad, política, economía, Salamanca, 49-80

Robledo, Ricardo (2003b), Tradición en la Universidad de Salamanca, in: Robledo, Ricardo, Irene Catells, María Cruz Romeo (eds.), Origínes del Liberalismo. Universidad, politíca, economía, Salamanca, 49-80

Rocco, Niccola (1837), Dell'uso e autorità delle leggi del Regno delle Due Sicilie considerate nelle relazioni con le persone e col territorio degli stranieri, Napoli

Rocco, Niccola (1859), Trattato di diritto civile internazionale ossia dell'uso e autorità delle leggi considerate nelle relazioni con le persone e col territorio degli stranieri, Livorno, parte prima

Rodríguez, Frank (2010), La noción de representación política en Francisco Javier Yanes, in: Conhisremi, 6, 1, 32-59, conhisremi.iuttol.edu.ve/pdf/ARTI0000 86.pdf

Rodríguez, Jaime E. (1998), The Independence of Spanish America, Cambridge, https://doi.org/10.1017/CBO9780511721137

Roggerone, Giuseppe A. (1991), L'abbé de Mably: politico della teologia illuminata, Firenze

Rolo, Paul J.V. (1965), George Canning, three biographical studies, London

Rosset, François (1996), La vie littéraire et intellectuelle en pays romand au XVIIIe siècle, in: Francillon, Roger (dir.), Histoire de la littérature en Suisse romande, Lausanne, t. I, 193-223

Rossi, Pietro (1990), La storia comparata. Approcci e prospettive, Milano 
Roulet, Louis-Edouard (1987), Du début du XVIIIe siècle à 1848, in: Scheurer, Rémy, Louis-Edouard Roulet, Jean Courvoisier (éd.), Histoire du Conseil d'Etat neuchâtelois: des origines à 1945, Neuchâtel, 99-151

Rousseau, Jean Jacques (2012a), Lettre de Jean-Jacques Rousseau à Mgr Christophe de Beaumont, in: Trousson, Raymond, Frédéric S. Eigeldinger (sous la direction de), Jean Jacques Rousseau, CEuvres Complètes, Genève, Paris, vol. VIII, 1169-1292

Rousseau, Jean Jacques, (2012b), Lettres écrites de la montagne, Dufour, Alfred, Gabriella Silvestrini (éd. par), in: Trousson, Raymond, Frédéric S. Eigeldinger (sous la direction de), CEuvres complètes de J.-J. Rousseau, Paris, Genève, vol. VI, 159-540

Roveri, Alessandro, Michele Fatica, Francesca Cantù (a cura di) (1973), La missione Consalvi e il Congresso di Vienna, I serie: 1814-1830, volume terzo, 1 febbraio 1815 - 23 giugno 1815, Roma

Royen, Willem Paul Johannes Adriaen van (1938), Analyse du problème de la neutralité au cours de l'évolution du droit des gens, La Haye

Ruck, Erwin (1917), Die römische Kurie und die deutsche Kirchenfrage auf dem Wiener Kongreß, Basel

Ruddy, Francis Stephen (1972), The Acceptance of Vattel, in: Grotian Society Papers, 177-196

Ruddy, Francis Stephen (1975), International Law in the Enlightenment. The Background of Emmerich de Vattel's 'Le Droit des Gens', New York

Ruiz, Alain (1980), A l'aube de kantisme en France: Sieyès, Karl Friederich Reinhard et le traité «Vers la paix perpetuelle»(1795-1796), in: Cahiers d'études germaniques, 4, 147-193

Rus Rufino, Salvador (1998), Joaquín Marín Mendoza: El Derecho natural académico en el siglo XVIII, in: Rus Rufino, Salvador, Francisco de Paula Puy MuÑoz, La historia de la filosofía jurídica española, Santiago de Compostela, 163-198

Rus Rufino, Salvador (2000), Una versión del « estado de naturaleza » en la España del siglo XVIII el texto de Joaquín Marín y Mendoza, in: Cuadernos Dieciochistas, 1, 257-282

Ruyssen, Thèodore (1961), Les sources doctrinales de l'internationalisme, Paris, vol. III

SAALFeld, Friedrich (1809), Grundriss eines Systems des europäischen Völkerrechts, Göttingen

SaAlfeld, Friedrich (1833), Handbuch des positiven Völkerrechts, Tübingen

SAcchi, Terenzio (1836), Osservazioni sulla giustizia convenienza e possibilità della conversione delle rendite pubbliche, Napoli

Sacchi, Terenzio (1840), Discorso di Terenzio Sacchi fra' i suoi colleghi nel real ministero degli affari interni, Napoli

Sacchi, Terenzio (1846), Prospectus d'un essai des institutions qui régissent le royaume des Deux Siciles, Naples 
Sacchi, Terenzio (1849), Delle istituzioni di governo del reame delle Due Sicilie, ragionamenti, Napoli

Sacchi, Terenzio (1851), Brevi risposte alle lettere di Sir W. E. Gladstone indiritte al Conte Aberdeen intorno al governo e al popolo napoletano, Napoli

Sacchi, Terenzio (1854), Prefazione, in: Vattel, Emer de, Il diritto delle genti di E. de Vattel applicato allo stato attuale delle nazioni, Napoli, 15-29

SAcchi, Terenzio (1859a), Della quistione italiana per quanto potesse riguardare il reame delle Due Sicilie, Napoli

Sacchi, Terenzio (1859b), Intorno al richiamo delle legazioni francese ed inglese da Napoli, Napoli

SAdosky, Leonard J. (2009), Revolutionary Negotiations: Indians, Empires and Diplomats in the Founding of America, Charlottesville

SAglia, Diego (2002), Translation and Cultural Appropriation: Dante, Paolo and Francesca in British Romanticism, in: Quaderns. Revista de traducció, 7, 95-119, http://www.raco.cat/index.php/quadernstraduccio/article/viewFile/ 25310/25144, consultato il 10 dicembre 2016

SAID, EdwARD W. (1998), Cultura e imperialismo. Letteratura e consenso nel progetto coloniale dell'Occidente, Roma

SAID, Edward W. (2002), Orientalismo. L'immagine europea dell'Oriente, Milano

Saint-Bonnet, François (2007), Pradier-Fodéré, Paul Louis Ernest, in: Arabeyre Patrick, Jean-Louis Halpérin, Jacques Krynen (sous la dir. de), Dictionnaire historique des juristes français (XIIe-XXe siècle), Paris, 641

Salerno, Francesco (2013), Anzilotti Dionisio, in: Birocchi, Italo, Ennio Cortese, Antonello Mattone, Marco Nicola Miletti (diretto da), Dizionario Biografico dei Giuristi Italiani (XII-XX secolo), Bologna, vol. I, 84-87

Samudio, Edda (2009), El proceso emancipador de Venezuela, in: Frasquet, Ivana, Andréa Slemian (eds.), De las independencias iberoamericanas a los estados nacionales (1810-1850), 200 años de historia, Madrid, Frankfurt, 195-216

SANdoz, Yves (éd.) (2010), Réflexions sur l'impact, le rayonnement et l'actualité du 'Droit des gens' d'Emer de Vattel - Reflections on the Impact, Influence and Continuing Relevance of the 'Law of Nations' by Emer de Vattel, Bruxelles

SAunders, David (2003), The Natural Jurisprudence of Jean Barbeyrac: Translation as an Art of Political Adjustment, in: Eighteenth-Century Studies, 36, 4, 473-490, https://doi.org/10.1353/ecs.2003.0053

Schaeper, Thomas J. (1995), France and America in the Revolutionary Era. The Life of Jacques-Donatien Leray de Chaumont, 1725-1803, New York, Oxford

Schaer, Jean Paul (1996), Louis Bourguet, philosophe et naturaliste (1678-1742), in: Schlup, Michel (dir.), Biographies neuchâteloises. Des lumières à la révolution, t. I: De saint Guillaume à la fin des Lumières, Hauterive, 16-22

Scheidemantel, Heinrich Gottfried (1770-1773), Das Staatsrecht nach der Vernunft und den Sitten der vornehmsten Völker betrachtet, 3 Bde., Jena, Cröckers

Scheidemantel, Heinrich Gottfried (1775), Das allgemeine Staatsrecht überhaupt und nach der Regierungsform, Jena, Cröckers 
Scheidemantel, Heinrich Gottfried (1778), Leges naturales systematice pertractatae, Jena, Cröckers

Scheurer, Remy (1987), Des origines au début de XVIIIe siècle, in: Scheurer, Remy, Louis Eduard Roulet, Jean Courvoisier (éd.), Histoire du conseil d'Etat neuchâtelois, Neuchâtel, 7-84

Schindler, Dietrich (2007), J. C. Bluntschli's contribution to the law of war, in: Kohen, Marcelo Gustavo (ed.), Promoting justice, human rights and conflict resolution through international law - La promotion de la justice, des droits de l'homme et du règlement des conflict par le droit international, Leiden, 437-454

Schlup, Michel (1988), Diffusion et lecture du "Journal helvétique » au temps de la Société typographique de Neuchâtel, 1769-1782, in: Bots, Hans (éd.), La diffusion et la lecture des journaux de langue française sous l'Ancien Régime. Actes du colloque international de Nimègue (3-5 juin 1987), Amsterdam, 59-70

Schlup, Michel (dir.) (2002), L'édition neuchâteloise au siècle des Lumières. La Société typographique de Neuchâtel (1769-1789), Neuchâtel

Schlup, Michel, Pierre-Yves Tissot, Fernand Douzé et al. (éds.) (1983), Le livre neuchâtelois 1533-1983, Neuchâtel

Schmalz, Theodor A. H. (1817), Das europäische Völker-Recht, Berlin

Schmelzing, Julius (1818-1820), Systematischer Grundriß des praktischen Europäischen Völkerrechts, Rudolstadt

Schmidt, Wilhelm Adolf (1847), Angelegenheiten der historischen Vereine. Die Germanistenversammlung, in: Allgemeine Zeitschrift für Geschichte, Berlin, B. VIII, 275-276

Schmitt, Carl (1991), Il nomos della terra nel diritto pubblico internazionale dello "ius publicum europaeum ", trad. it. Castrucci, Emanuele, Milano

SCHNAKEnBourg, Éric (2011), From a right of war to a right of peace: Martin Hübner's contribution to the reflection on neutrality in the eighteenth century, in: Alimento, Antonella (ed.), War, Trade and Neutrality. Europe and the Mediterranean in seventeenth and eighteenth centuries, Milano, 203-216

Schnakenbourg, Éric (2013), Entre la guerre et la paix. Neutralité et relations internationales, XVIIe-XVIIIe siècles, Rennes

Schnoebelen, Anne (1979), Padre Martini's Collection of Letters in the Civico Museo Bibliografico Musicale in Bologna. An Annotated Index, New York

Schott, Clausdieter (2001), "Interpretatio cessat in claris ». Auslegungsfähigkeit und Auslegungsbedürftigkeit in der juristischen Hermeneutik, in: SCHRöDER, Jan (Hrsg.), Theorie der Interpretation von Humanimus bis zur Romantik. Rechtswissenschaft, Philosophie, Theologie, Stuttgart, 155-190

Schulze, Thies (2013), Grenzüberschreitende Religion. Vergleichs- und Kulturtransferstudien zur neuzeitlichen Geschichte, Göttingen, https://doi.org/10.13109/ 9783666310218

Scovazzi, Tullio (2012), The «First Time Instance» as Regards Restitution of Removed Cultural Properties, in: Agenda Internacional, 30, 9-19 
Sears, Louis Martin (1919), Jefferson and the Law of Nations, in: The American Political Science Review, 13, 3, 379-399, https://doi.org/10.2307/1945957

SEaward, Louise (2014), The Société Typographique de Neuchâtel (STN) and the Politics of the Book Trade in Late Eighteenth-Century Europe, 1769-1789, in: European History Quarterly, 44, 3, 439-457, https://doi.org/10.1177/ 0265691414535025

Sedgwick, Theodore (1857), A Treatise on the Rules which govern the interpretation and application of statutory and constitutional law, New York

Segre, Arturo (1927), Note e documenti sui casi e sui profughi piemontesi del 1821, in: Rossi, Teofilo, Carlo Pio Demagistris (a cura di), La rivoluzione piemontese del 1821. Studi e documenti, Torino, vol. I, 238-302

Senellart, Michel (2004), La qualification de l'ennemi chez Emer de Vattel, in: Astérion, 2, http://asterion.revues.org/82, consultato il 24 maggio 2016

Sepinwall, Alyssa G. (2005), The Abbé Grégoire and the French Revolution: the Making of Modern Universalism, Berkeley, https://doi.org/10.1525/california/ 9780520241800.001.0001

Sereni, Angelo Piero (1943), The Italian Conception of International Law, New York

Sfoini, Alexandra (2014), Loyaume and Nomarchie: Keywords of the French revolution in the Greek vocabulary, in: The Historical Review/La Revue Historique, 11, 127-138, https://doi.org/10.12681/hr.319

Sfoini, Alexandra (2015), Transfert des idées par la voie de la traduction pendant l'ère révolutionnaire grecque (1797-1832), in: The Historical Review/La Revue Historique, 12, 47-74, https://doi.org/10.12681/hr.8801

Shuttleworth, Mark, Moira Cowie (1997), Dictionary on Translation Studies, Manchester

Silva, Ana Cristina Nogueira da (2012), Libertad, derechos naturales y "multiculturalismo » en el pensamiento de Silvestre Pinheiro Ferreira (1769-1846), in: Naranjo, Estaban Conde (ed.), Vidas por el derecho, Madrid, 221-242

Silva, Maria Beatriz Nizza (1975), Silvestre Pinheiro Ferreira: ideologia e teoria, Lisboa

Silvestrini, Gabriella (2007), Guerre juste et droit de résistance dans la tradition protestante du droit naturel, in: Poncelet, Christian, Rolf Büttiker, Giovanni Busino (éd.), Genève et la Suisse dans la pensée politique. Actes du Colloque de Genève (Septembre 2006), Aix-en-Provence, 103-116

Silvestrini, Gabriella (2008), Giustizia della guerra e disuguaglianza: Vattel, l'aggressore ingiusto e il nemico del genere umano, in: Filosofia politica, 22, 3, 381-400

Silvestrini, Gabriella (2010a), Justice, War and Inequality. The Unjust Aggressor and the Enemy of Human Race in Vattel's Theory of the Law of Nations, in: Grotiana, 31, 44-68

Silvestrini, Gabriella (2010b), Rousseau e la tradizione repubblicana: repubblica, democrazia ed elezioni, in: Lenci Mauro, Carmelo Calabrò (a cura di), 
Viaggio nella democrazia. Il cammino dell'idea democratica nella storia del pensiero politico, Pisa, 69-87

Silvestrini, Gabriella (2010c), Diritto naturale e volontà generale. Il contrattualismo repubblicano di Jean-Jacques Rousseau, Torino

Silvestrini, Gabriella (2011), Vattel, Rousseau et la question de la 'justice' de la guerre, in: Chetail, Vincent, Peter Haggenmacher (eds.), Vattel's International Law in a XXIst Century Perspective, Le droit international de Vattel vu du XXIe siècle, Leiden, Boston, 101-129, https://doi.org/10.1093/ 9780199599752.003.0028

Simmel, Georg (1998), La moda, trad. it. Perucchi, Lucio, Milano

Simonin, Jérémy (2007), L'homme et le Citoyen dans la science du droit naturel de J. J. Burlamaqui, in: Poncelet, Christian, Rolf Büttiker, Giovanni Busino (éd.), Genève et la Suisse dans la pensée politique. Actes du Colloque de Genève (Septembre 2006), Aix-en-Provence, 89-101

Sims, Alexander D. (1847), Speech of Mr A. D. Sims, of South Carolina, on the Mexican war. Delivered in the House of Representatives, Thursday, January 28, 1847, Washington

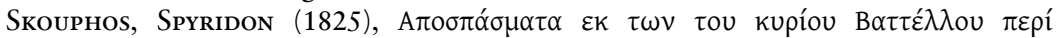

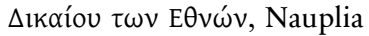

Skouteris, Thomas (2012), Engaging History in International Law, in: Beneyto, José Maria, David Kennedy (eds.), Approaches to International Law, The Hague, 99-122, https://doi.org/10.1007/978-90-6704-879-8_4

Sloan, Philipp R. (2006), Natural History, in: Haakonssen, Knud (ed.), The Cambridge History of Eighteenth Century Philosophy, Cambridge, vol. II, 903-938

Sloss, David, Michael D. Ramsey, William S. Dodge (2011), International Law in the Supreme Court to 1860, in: Sloss, David, Michael D. Ramsey, William S. Dodge (eds.), International Law in the U.S. Supreme Court, Cambridge, 7-54, https://doi.org/10.1017/CBO9780511977121.002

Smith, Paul H. (1976), Letters of Delegates to Congress, volume: 3 January 1, 1776 May 15, 1776, Washington

Snell-Hornby, Mary (2006), The Turns of Translation Studies: New Paradigms or Shifting Viewpoints, Amsterdam, Philadelphia

Solimano, Stefano (2001), Paolo Risi e il processo penale (1766), in: Studi di storia del diritto, Milano, vol. III, 419-519

Solimano, Stefano (2009), Le sacre du printemps, L'entrata in vigore del codice civile del Regno italico, in: Tavilla, Elıo (a cura di), Giuseppe Luosi, giurista italiano ed europeo. Traduzioni, tradizioni e tradimenti della codificazione. A 200 anni dalla traduzione in italiano del Code Napoléon (1806-2006), in: Quaderni dell'archivio storico XXV, Atti del convegno Internazionale di Studi, Mirandola 19-20 ottobre 2006, Modena, 191-221

Solimano, Stefano (2013), Risi Paolo, in: Birocchi, Italo Ennio Cortese, Antonello Mattone, Marco Nicola Miletti (diretto da), Dizionario Biografico dei Giuristi Italiani (XII-XX secolo), Bologna, vol. II, 1694-1696 
Sotiropoulos, Michail (2015), European jurisprudence and the intellectual origins of the Greek state: the Greek jurists and liberal reforms (ca 1830-1880), PhD in Philosophy, Queen Mary, University of London, supervised by VarouXaKIs, Georgios, Maurizio Isabella http:/qmro.qmul.ac.uk/jspui/handle/ 123456789/9111, consultato il 17 giugno 2016

Spagnesi, Enrico (2011), The trade of " neutral nations " as viewed by the publicist Lampredi, in: Alimento, Antonella (ed.), War, Trade and Neutrality. Europe and the Mediterranean in seventeenth and eighteenth centuries, Milano, 233-246

Stagg, John Charles Anderson (1983), Mr Madison's War: Politics, Diplomacy and Warfare in the Early America Republic 1783-1830, Princeton

Stanlis, Peter J. (1953), Edmund Burke and the Law of Nations, in: The American Journal of International Law, 47, 3, 397-413, https://doi.org/10.2307/2194680

Stapelbroek, Koen (2001), Galiani's concept of commerce in On Money and the eighteenth-century Neapolitan languages of commerce and liberty, in: History of Economic Ideas, 9, 3, 137-169

Stapelbroek, Koen (2006a), Commerce and Morality in Eighteenth Century Italy, in: History of European Ideas, 32, 361-367, https://doi.org/10.1016/j.histeu roideas.2006.08.004

Stapelbroek, Koen (2006b), Preserving the Neapolitan state: Antonio Genovesi and Ferdinando Galiani on Commercial Society and Planning Economic Growth, in: History of European Ideas, 32, 406-429, https://doi.org/10.1016/j.histeu roideas.2006.08.008

Stapelbroek, Koen (2008a), Universal Society, Commerce and the rights of Neutral Trade: Martin Hübner, Emer de Vattel and Ferdinando Galiani, in: Korkmann, Petter, Virpi Mäkinen (eds.), Universalism in International Law and Political Philosophy, COLleGIUM, Studies across Disciplines in the Humanities and Social Sciences 4, Helsinki, 63-89, https://helda.helsinki.fi/ bitstream/handle/10138/25780/04_stapelbroek_2008_4.pdf?sequence=1, consultato il 27 maggio 2016

Stapelbroek, Koen (2008b), Love, Self-Deceit and Money: Commerce and Morality in the Early Neapolitan Enlightenment, Toronto

Stapelbroek, Koen (2011), I significati della neutralità: la storia del commercio mediterraneo in Dei Doveri dei Principi Neutrali, in: Trampus, Antonio (a cura di), Il linguaggio del tardo illuminismo. Politica, diritto e società civile, Roma, 53-79

Stapelbroek, Koen, Antonio Trampus (2014), Vattels «Droit des gens » und die europaeischen Handelsrepubliken im 18. Jahrhundert, in: Asbach, Olaf (Hrsg.), Der moderne Staat und 'le doux commerce'. Politik, Ökonomie und internationale Beziehungen im politischen Denken der Aufklärung, BadenBaden, 181-206

Starke, James G. (1972), The Influence of Grotius upon the Development of International Law in the Eighteenth Century, in: Alexandrowicz, Charles Henri 
(ed.), Studies on the History of the Law of Nations, Grotian Society Papers, The Hague, 162-176

Steiger, Heinhard (2004), Peace treaties from Paris to Versailles, in: Lesaffer, Randall (ed.), Peace Treaties and International Law in European History: From the Late Middle Ages to World War One, Cambridge, 59-99, https:// doi.org/10.1017/CBO9780511494239.004

Steiger, Heinhard (2009), Peace treaties from Paris to Versailles, in: Steiger, Heinhard, Von der Staatengesellschaft zur Weltrepublik? Aufsätze zur Geschichte des Völkerrechts aus vierzig Jahren, Baden-Baden, 513-555

Stella, Pietro (1993), Pietro Tamburini nel quadro del giansenismo italiano, in: Corsini, Paolo, Daniele Montanari (a cura di), Atti del Convegno internazionale in occasione del $250^{\circ}$ della nascita (Brescia, 25-26 maggio 1989), Brescia, 151-204

Stoffella, Stefania (2000), Assolutismo e diritto naturale in Italia nel Settecento, in: Annali dell'Istituto storico italo-germanico, 26, 137-175

Stoffella, Stefania (2001), Il diritto di resistenza nel Settecento Italiano. Documenti per la storia della traduzione del De iure naturae et gentium di Pufendorf, in: Magistrature et politique, 2, 173-199, online http://laboratoireitalien.re vues.org/261, consultato il 23 maggio 2016, https://doi.org/10.4000/laboratoire italien.261

Stoffella, Stefania (2013), Almici Giovambattista, in: Birocchi, Italo, Ennio Cortese, Antonello Mattone, Marco Nicola Miletti (diretto da), Dizionario Biografico dei Giuristi Italiani (XII-XX secolo), Bologna, vol. I, 45

Stolleis, Michael (2008), Storia del diritto pubblico in Germania. I. Pubblicistica dell'Impero e scienza di polizia. 1600-1800, Milano

Stolleis, Michael (2014), Storia del diritto pubblico in Germania. II. Dottrina del diritto pubblico e scienza dell'amministrazione 1800-1914, Milano

Stolleis, Michael, Masaharu Yanagihara (Hrsg.) (2004), East Asian and European Perspectives on International Law, Baden-Baden

Storti Storchi, Claudia (1990), Ricerche sulla condizione giuridica dello straniero in Italia. Dal tardo diritto comune all'età unitaria. Aspetti civilistici, Milano

Storti, Claudia (2001), Ludovico Casanova e le sue Lezioni di diritto internazionale in: Varnier, Giovanni Battista (a cura di), Giuristi Liguri dell'Ottocento. Atti del Convegno Genova 8 aprile 2000, Genova, Accademia Ligure di Scienze e Lettere, 53-94

Storti, Claudia (2012), Empirismo e scienza: il crocevia del diritto internazionale, nella prima metà dell'Ottocento, in: Nuzzo, Luigi, Miloš Vec (eds.), Constructing International Law. The Birth of a Discipline, Frankfurt am Main, $51-145$

Storti Claudia (2013), Mancini Pasquale Stanislao, in: Birocchi, Italo, Ennio Cortese, Antonello Mattone, Marco Nicola Miletti (diretto da), Dizionario Biografico dei Giuristi Italiani (XII-XX secolo), Bologna, vol. II, 1244-1248 
Stribrny, Wolfgang (1998), Die Könige von Preußen als Fürsten von NeuenburgNeuchâtel (1707-1848). Geschichte einer Personalunion, Berlin

Suárez, Federico (2002), Las Cortes de Cádiz, Madrid

Svarverud, Rune (2001), The Notions of 'Power' and 'Rights', in Chinese Political Discourse, in: Lackner, Michael, Iwo Amelung, Joachim Kurtz (eds.), New Terms for New Ideas: Western Knowledge and Lexical Change in Late Imperial China, Leiden, Boston, Köln, 125-143

Svarverud, Rune (2007), International Law as World Order in Late Imperial China. Translation, Reception and Discourse 1847-1911, Leiden, Boston, https:// doi.org/10.1163/ej.9789004160194.i-322

Sylvester, Douglas J. (1999), International Law as Sword or Shield? Early American Foreign Policy and the Law of Nations, in: New York University Journal of International Law and Politics, 32, 1, 1-87

Tamburini, Francesco (2002), Historia y destino de la "doctrina Calvo »: ¿Actualidad u obsolescencia del pensamiento de Carlo Calvo?, in: Revista de Estudios Histórico-Juridicós [Sección Historia del Derecho International] 24, 81-104

Tang, Chi-Hua (2012), China - Europe, in: Fassbender, Bardo, Anne Peters (eds.), The Oxford Handbook of the History of International Law, Oxford, 701-723, https://doi.org/10.1093/law/9780199599752.003.0030

TAppy, Denis (2010), De Barbeyrac aux premiers Masters en droit de la Faculté de droit et des sciences criminelles de l'Unil: 300 ans d'enseignement du droit à Lausanne, in: Tappy, Dennis, Bettina Kahil-Wolff, Léonard Bruchez (textes réunis par), 300 ans d'enseignement du droit à Lausanne, Genève, Zurich, Bâle, 1-46

Tavilla, Elio (2009), Guerra, assoggettamento, consenso: temi settecenteschi di Ius publicum universale tra Italia e Germania, in: Cassi, Aldo Andrea (a cura di), Guerra e diritto. Il problema della guerra nell'esperienza giuridica occidentale tra medioevo ed età contemporanea, Soveria Mannelli, 85-108

Teichmann, Albert (1882), Kaltenborn von Stachau, Karl Baron, in: Allgemeine Deutsche Biographie, Leipzig, B. XV, 43-45

Thayer, James Bradley (1895), Cases on Constitutional law, Cambridge

The National Archives (United Kingdom), Kew, Richmond: Folios 379-381: Sir Robert Phillimore, MP, Queen's advocate, 1862-1867: Sending extract from Vattel on reprisals (Book 2 cap $18 \mathrm{sec} 342$ )

Theis, Laurent (2014), Guizot: la traversée d'un siècle, Paris

Thévenaz, Henri (1957), Vattel ou la destinée d'un livre, in: Schweizerisches Jahrbuch für Internationales Recht, 14, 9-16

Thévenaz, Henri (1958), Emer de Vattel: pour le deuxième centenaire du Droit des gens, Tirage à part, Extrait du Musée neuchâtelois, september - october, $129-135$

Thévenaz, Louis (1948), Histoire du pays de Neuchâtel, Neuchâtel

Thibaud, Clément (2007), Definiendo el sujeto de la soberanía: Repúblicas y guerra en la Nueva Granada y Venezuela 1808-1820, in: Chust, Manuel, Juan 
Marchena (eds.), Las armas de la Nación. Independencia y ciudadanía en Hispanoamérica (1750-1850), Madrid, Frankfurt am Main, 185-222

Tillet, Edouard (2007), La contribution de Jean Barbeyrac à la naissance d'un langage constitutionnel nouveau, in: Poncelet, Christian, Rolf Büttiker, Giovanni Busino (éd.), Genève et la Suisse dans la pensée politique. Actes du Colloque de Genève (Septembre 2006), Aix-en-Provence, 373-396

Tittel, Gotтloв August (1786), Natur- und Völkerrecht, Frankfurt am Main, Garbe Tomás y Valiente, Francisco (1964), Expedientes de censura de libros jurídicos por la Inquisición a finales del siglo XVIII y principio del XIX, in: Anuario de Historia del Derecho español, 34, 417-462

Tomás y Valiente, Francisco (2003), Genesi di un costituzionalismo euro-americano. Cadice 1812. Con un'autobiografia dell'autore, trad. ital. Cocchiara, Antonella M., Milano

Torre, Stefania (1995), Mancini Pasquale Stanislao, in: Stolleis, Michael (Hrsg.), Juristen. Ein biographisches Lexikon von der Antike bis zum 20. Jahrhundert, München, 404-405

Torrione, Pietro (1968), L'autobiografia di Giovanni Battista Marochetti il più grande rivoluzionario biellese, in: Rivista Biella, 4, 21-28

Torrione, Pietro, Pier Giorgio Tamaroglio (a cura di) (1960), Il Biellese nell'epopea del Risorgimento, Biella

Toyoda, Tetsuya (2001), Theory and Politics on the Law of Nations. Political Bias in International Law Discourse of Seven German Court Councilors in the Seventh and Eighteenth Centuries, Leiden, Boston

Toyoda, Tetsuya (2009), La doctrine vattelliene de l'égalité souveraine dans le contexte neuchâtelois, in: Journal of the History of International Law, 11, 103-124, https://doi.org/10.1163/157180509X421395

Toyoda, Tetsuya (2010), Le fondement universel du Droit des gens vattelien et l'entrée du Japon dans l'ordre juridique international, in: Sandoz, Yves (éd.), Réflexions sur l'impact, le rayonnement et l'actualité du 'Droit des gens' d'Emer de Vattel - Reflections on the Impact, Influence and Continuing Relevance of the 'Law of Nations' by Emer de Vattel, Bruxelles, 59-74

Trampus, Antonio (2002), La traduzione settecentesca di testi politici: il caso della Scienza della legislazione di Gaetano Filangeri, in: Rivista internazionale di tecnica della traduzione, 6, 19-44

Trampus, Antonio (2004), Filangieri in Austria: la traduzione perduta della "Scienza della Legislazione », in: Römische Historische Mitteilungen, 46, 283-304

Trampus, Antonio (2011a), Il ruolo del traduttore nel tardo illuminismo: Lodovico Antonio Loschi e la versione italiana del Droit de gens di Emer de Vattel, in: Trampus, Antonio (a cura di), Il linguaggio del tardo illuminismo. Politica, diritto e società civile, Roma, 81-108

Trampus, Antonio (2011b), The circulation of Vattel's Droit des gens in Italy: the doctrinal and practical model of government, in: Alimento, Antonella (ed.), War, Trade and Neutrality. Europe and the Mediterranean in seventeenth and eighteenth centuries, Milano, 217-232 
Trampus, Antonio (2013), La traduzione toscana del Droit des gens di Emer de Vattel (circa 1780): contesti politici, transferts culturali e scelte traduttive, in: Cantarutti, Giulia, Stefano, Ferrari, Traduzione e Transferts nel XVIII secolo tra Francia, Italia e Germania, Milano, 153-174

Trampus, Antonio (2015), Le costituzioni italiane prime di Cadice: Vattel e le radici democratiche italiane, in: García Sanz, Fernando, Vittorio Scotti Douglas et al. (a cura di), Cadice e oltre: costituzione, nazione e libertà. La carta gaditana nel bicentenario, Roma, 161-172

Trampus, Antonio (2016), Dalla libertà religiosa allo Stato nazione: Utrecht e le origini del sistema internazionale di Emer de Vattel, in: Ieva, FréDÉrIC (a cura di), I trattati di Utrecht: una pace di dimensione europea, Roma, 93-106

Tuccillo, Alessandro (2013), Il commercio infame. Antischiavismo e diritti dell'uomo nel Settecento italiano, Napoli

Ullmann, Emanuel von (1908), Völkerrecht, Tübinigen

Ulrych, Margherita (1997), Introduzione, in: Ulrych, Margherita (a cura di), Tradurre. Un Approccio Multidisciplinare, Torino, XI-XIII

Valeri, Stefania (2006), Libri nuovi scendono le Alpi: venti anni di relazioni franco-italiane negli archivi della Société typographique de Neuchâtel (1769-1789), Macerata

Valiente, Pedro José Pérez (1744), Dissertatio politico-juridica de maris imperio, Madrid, Peralta

Van der Vlugt, Willem (1925), L'œuvre de Grotius et son influence sur le développement du droit International, in: Recueil des cours de l'Académie de droit international de la Haye, 7, II, 399-507

Van Kley, Dale K. (2014), From the Catholic Enlightenment to the Risorgimento: the Exchange Between Nicola Spedalieri and Pietro Tamburini, 1791-1797, in: Past \& Present, 224, 1, 109-162, https://doi.org/10.1093/pastj/gtu010

VAN Young, ERIC (2004), « To throw off a tyrannical Government »: Atlantic Revolutionary Traditions, and Popular Insurgency in Mexico, 1800-1821, in: Morrison, Michael A., Melinda Zook (eds.), Revolutionary Current. Nation Building in the Transatlantic world, Lanham, 127-172

Vandiver Nicassio, Susan (2009), Imperial City: Rome under Napoleon, Chicago

VAnnini, Fabrizio (2004), Lampredi Giovanni Maria, in: Dizionario Biografico degli Italiani, Roma, 63, 259-262

Vannucci, Аtтo (1887), I martiri della libertà italiana dal 1794 al 1848, Milano

Vano, Cristina (2013), Lomonaco Giovanni, in: Birocchi, Italo, Ennio Cortese, Antonello Mattone, Marco Nicola Miletti (diretto da), Dizionario Biografico dei Giuristi Italiani, Bologna (XII-XX secolo), vol. II, 1192-1193

Vapereau, Gustave (1870), Royer-Collard Paul, in: Vapereau, Gustave (par), Dictionnaire universel des contemporains, contenant toutes les personnes notables de la France et des pays étrangers, quatrième édition, entièrement refondue et considérablement augmentée Paris, 1591

Vattel, Emer de (1747), Loisir philosophique, ou piéces diverses de Philosophie de Morale et d'Amusemens, Dresde, George Conrad Walther 
Vattel, Emer de (1757), Poliergie au mélange de littérature et de poesie, Amsterdam, Arkstée et Merkus

VATtel, Emer de (1758a), Le droit des gens ou principes de la loi naturelle, appliqués à la conduite et aux affaires des Nations et des Souverains, Londres [Neuchâtel, Abraham Droz]

VAttel, Emer de (1758b), Le droit des gens ou principes de la loi naturelle, appliqués à la conduite et aux affaires des Nations et des Souverains. Ouvrage qui conduit à développer les véritables intérêts des puissances, Leiden, Aux depens de la Compagnie

VAtTel, Emer de (1758c), Mémoires politiques concernant la guerre ou principes de la loi naturelle appliqués à la conduite et aux affaires des nations et des souverains, Frankfurt, Leipzig, Aux dépens de la compagnie

Vattel, Emer de (1760), Mélanges de littérature, de morale, et de politique, Neuchâtel

Vattel, Emer de (1762), Questions de droit naturel et observations sur le Traité du droit de la Nature de M. le Baron de Wolff, Berne

Vattel, Emer de (1765), Amusemens de littérature, de morale, et de politique, La Haye

VATtel, Emer de (1773), Le droit des gens ou Principes de la loi naturelle, appliqués à la conduite et aux affaires des nations et des souverains. Nouvelle édition augmentée, Neuchâtel, Société typographique de Neuchâtel, t. II

Vattel, Emer de (1775), Le droit des gens ou Principes de la loi naturelle, appliqués à la conduite et aux affaires des nations et des souverains. Nouvelle édition augmentée, revue, corrigée, avec quelques remarques de l'Editeur, Amsterdam, van Harrevelt, vol. I

VAttel, Emer de (1781), Il diritto delle genti, ovvero principii del diritto naturale applicati alla condotta e agli affari delle nazioni e de' sovrani, trad. it. Lodovico Antonio Loschi, Lione, vol. I

VAtTel, Emer de (1797), The Law of Nations or Principles of the Law of Nature Applied to the Conduct and Affairs of Nations and Sovereigns, London, Robinson, Paternoster-Row

VATtel, Emer DE (1820a), Le droit des gens ou principes de la loi naturelle, appliqués à la conduite et aux affaires des Nations et des Souverains, nouvelle édition augmentée, revue et corrigée avec quelques notes de l'auteur et des éditeurs, Paris, vol. I

Vattel, Emer de (1820b), El derecho de gentes, ó principios de la ley natural, aplicados á la conducta, y á los negocios de las naciones y de los soberanos. Escrita en francés por Em. de Vattel y traducida al español por Manuel Pascal Hernández, Madrid, t. I

VATtel, Emer de (1830), Le droit des gens ou principes de la loi naturelle, appliqués à la conduite et aux affaires des Nations et des Souverains, revue et corrigée avec quelques remarques de l'editeur, suivie d'une bibliothèque choisie des livres qu'il est le plus utile d'acquérir et de connaître; pour l'étude du droit 
de la nature et des gens; extraite des bibliographies spéciales de Camus, Klüber, etc., Paris

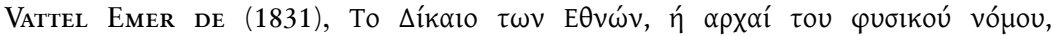

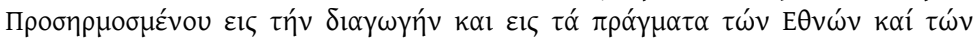

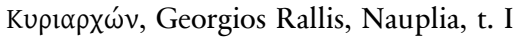

VAttel, Emer de (1834a), El derecho de gentes, ó principios de la ley natural, aplicados á la conducta, y á los negocios de las naciones y de los soberanos. Traducida al español, corregida y aumentada en esta impresión con una noticia de la vida del autor por Manuel María Pascual Hernández, Madrid, I vol.

VAtTel, Emer de (1834b), The law of nations, or principles of the law of nature, applied to the conduct and affairs of nations and sovereigns, a work tending to display the true interests of powers. A new edition by Joseph Chitty, London

VATtel, Emer de (1835), Le droit des gens ou principes de la loi naturelle, appliqués à la conduite et aux affaires des Nations et des Souverains, revue et corrigée avec quelques remarques de l'editeur, augmentée de quelques remarques nouvelles, et d'une bibliographie choisie et systématique du droit de la nature et des gens, par M. de Hoffmann, Paris, t. I

Vattel, Emer de (1838), Le droit des gens ou principes de la loi naturelle, appliqués à la conduite et aux affaires des Nations et des Souverains, revue et corrigée avec quelques remarques de l'editeur, augmentée de quelques remarques nouvelles, et d'une bibliographie choisie et systématique du droit de la nature et des gens, par M. de Hoffmann, notes et table général analytique de l'ouvrage par Pinheiro Ferreira Silvestre, Paris, t. III

Vattel, Emer de (1852), The law of nations, or principles of the law of nature, applied to the conduct and affairs of nations and sovereigns, with additional notes and references by Edward D. Ingraham, Philadelphia

VATtel, EMER DE (1867), Le droit des gens ou principes de la loi naturelle, appliqués à la conduite et aux affaires des Nations et des Souverains, complétée par l'exposition des doctrines des publicistes contemporains mis au courant des progrès du droit public moderne et suivi d'une table analytique des matières de Mr M. P. Pradier-Fodéré, t. I

Vattel, Emer de, (1916), Le droit des gens ou Principes de la loi naturelle, appliqués à la conduite et aux affaires des nations et des souverains, with an introduction by Albert de Lapradelle, Washington, vol. I

VAttel, Emer de (1959), Das Völkerrecht oder Grundsäze des Naturrechts, angewandt auf das Verhalten und die Angelegenheiten der Staaten und Staatsoberhäupter, Dt. Übers. von Euler, Wilhelm, Einl. von Guggenheim, Paul, Tübingen

VAttel, Emer de (2008a), The Law of Nations, Or, Principles of the Law of Nature, Applied to the Conduct and Affairs of Nations and Sovereigns, with Three Early Essays on the Origin and Nature of Natural Law and on Luxury, edited and with an introduction by Kapossy, Bèla, Richard Whatmore, Indianapolis 
Vattel, Emer de (2008b), O direito das gentes ou Príncipios da lei natural aplicados à condução e aos negócios das nações e dos governantes, introduçao de Mancuso, Francesco, Florianopolis

VAttel, Emer de (2011), The law of nations, or principles of the law of nature, applied to the conduct and affairs of nations and sovereigns, a work tending to display the true interests of powers, new edition by Joseph Chitty (1834), Cambridge

Vec, Miloš (2012), From the Congress of Vienna to the Paris Peace Treaties, in: Fassbender, Bardo, Anne Peters (eds.), The Oxford Handbook of the History of International Law, Oxford, 654-678

Vec, Miloš (2015), L'ambassade dans la science du droit des gens, in: Andretta, Stefano, Stéphane Péquignot, Jean-Claude Waquet (ét. réunis par), Les écrits relatifs à l'ambassadeur et à l'art de négocier du Moyen Âge au début du XIXe siècle, Rome, 487-522

Velema, Wyger Rykele Elzo (1985), Homo Mercator in Holland. Elie Luzac en het achttiende-eeuwse debat over de koophandel, in: Bijdragen en mededelingen betreffende de geschiedenis der Nederlanden, 100, 427-444, http://doi.org/ 10.18352/bmgn-lchr.2624, consultato il 24 maggio 2016

Velema, Wyger Rykele Elzo (1987), The Rise and Fall of Morality. Elie Luzac on the History of the Science des Mœurs, in: Theoretische geschiedenis, 14, 143-156

Velema, Wyger Rykele Elzo (1993), Enlightenment and conservatism in the Dutch Republic. The political thought of Elie Luzac (1721-1796), Assen, Maastricht

Venturi, Franco (1990), Settecento riformatore, V/2, L'Italia delle riforme. La Repubblica di Venezia (1761-1797), Torino

Venuti, Lawrence (2013), Translation Changes Everything. Theory and Practice, London

Vergé, Charles-Henri (1856), Diplomates et publicistes. - Maurice d'Hauterive. De Gentz. - Pinheiro-Ferreira. - Ancillon. - D’Entraigues. - Sièyes. - Chateaubriand. Mignet, Paris, 51-70

Vermeer, Hans Josef (1986), Übersetzen als kultureller Transfer, in: Snell-Hornby, Mary (Hrsg.), Übersetzungswissenschaft - eine Neuorientierung. Zur Integrierung von Theorie und Praxis, Tübingen, 30-53

Verri, Alessandro (1818), Discorso XI. Di alcuni sistemi del diritto pubblico, in: Discorsi vari del conte Alessandro Verri pubblicati nel giornale letterario intitolato Il Caffe, Milano, 349-375

Verzella, Emanuela (1998), Nella rivoluzione delle cose politiche e degli umani cervelli: il dibattito sulle Lettere teologico-politiche di Pietro Tamburini, Firenze

Via, Rachele (1995), Il libro e la storia delle idee. Le società tipografiche di Napoli e Neuchâtel alla fine del ' 700 , Soveria Mannelli

Vier, Jacques (1969), Élie Catherine Fréron, in: Annales de Bretagne, 76, 2, 475-483, https://doi.org/10.3406/abpo.1969.2525 
Villani, Pasquale (1962), Contributo alla storia dell'anticurialismo napoletano: l'opera di G.F. Conforti, in: Villani, Pasquale, Mezzogiorno tra riforme e rivoluzione, Bari, 187-264

Villari, Lucio (1996), La schiavitù dei moderni. Illuminismo e colonialismo: Raynal e Diderot, Roma

Viola, Francesco (2001), Apporti alla pratica interpretativa del diritto internazionale alla teoria generale dell'interpretazione giuridica, in: Ragion pratica, 17, $53-71$

Viora, Mario E. (1967), Consolidazioni e codificazioni. Contributo alla storia della codificazione, Torino

Vismara, Paola (2012), Pietro Tamburini e il "dispotismo pontificio ", in: Negruzzo, Simona (a cura di), Il giansenismo e l'Università di Pavia. Studi in ricordo di Pietro Stella, Milano, 95-114

VitANYI, Béla (1980), L'interprétation des traités dans la théorie du droit naturel, in: Revue générale droit international public, 84, 535-586

Vollenhoven, Cornelius van (1919), Les trois phases du droit des gens, La Haye

Voltaire (1974), The complete works of Voltaire. Correspondence and related documents. XXXI, October 1766-March 1767: letters D 13596-D 14077, Besterman, Theodore (ed.), Geneva, vol. 115

Voltaire (1994), Il secolo di Luigi XIV, trad. it. Morra, Umberto, Torino

Wagener, Herrmann (1866), Vattel (Emmerich de), in: Wagener, Herrmann (Hrsg.), Staats- und Gesellschafts-Lexikon, Berlin, Bd. XXI, Ungarische Revolution bis Weimar, 185-186

Wallerstein, Immanuel (2001), Unthinking Social Science: The Limits of Nineteenth-century Paradigms, Philadelphia

Ward, Robert Plumer (1795), An Enquiry into the Foundation and History of the Law of Nations in Europe from the Time of the Greeks and Romans to the Age of Grotius, London, Butterworth, vol. II

Weber, Nadir (2012), Zwei preussische Diplomaten aus Neuchâtel. Jean de Chambrier und Jean-Pierre Chambrier d'Oleyres zwischen Fürstendienst, Familieninteressen und Vaterlandsdiskursen, in: Léchot, Pierre-Olivier, Virginie PAsche (Hrsg.), Neuchâtel im Konzert der europäischen Aufklärung. Lokale Akteure und transnationale Kulturen (Jahrbuch der Schweizerischen Gesellschaft für die Erforschung des 18. Jahrhunderts, xviii.ch.:3), 142-157

Weidlich, Christof (1783), Schulin Johann Philip, in: Biographische Nachrichten von den jetztlebenden Rechts-Gelehrten in Deuchtsland, Halle, 290-291

Weifang, He (2010), Vattel's China, in: Sandoz, Yves (éd.), Réflexions sur l'impact, le rayonnement et l'actualité du 'Droit des gens' d'Emer de Vattel - Reflections on the Impact, Influence and Continuing Relevance of the 'Law of Nations' by Emer de Vattel, Bruxelles, 75-78

Welcker, Carl (1843), Völkerrecht, natürliches europäisches, in: Rotteck, Carl von, Carl Welcker (Hrsg.), Das Staats-Lexikon oder Encyklopädie der Staatswissenschaften in Verbindung mit vielen der angesehensten Publicisten Deutschlands, Altona, Bd. XV, 731-735 
Welcker, CARl (1848), Völkerrecht; natürliches; europäisches, in: RotTeck, Carl von, Carl Welcker (Hrsg.), Encyklopädie der sämmtlichen Staatswissenschaften für alle Stände. In Verbindung mit vielen der angesehensten Publicisten Deutschlands, Altona, Bd. XII, 789-792

Welsh, Jennifer M. (1995), Edmund Burke and International Relations. The Commonwealth of Europe and the Crusade against the French Revolution, Oxford

Werner, Michael, Bénédicte Zimmermann (2002), Vergleich, Transfer, Verflechtung. Der Ansatz der Histoire croisée und die Herausforderung des Transnationalen, in: Geschichte und Gesellschaft, 28, 607-636

Werner, Michael, Bénédicte Zimmermann (2003), Penser l'histoire croisée: entre empirie et réflexivité, in: Annales. Histoire, Sciences Sociales, 58, 1, 7-36

Westlake, John (1910), International Law, Cambridge, vol. I

Wharton, Francis (1872), A treatise on the conflicts of law, or private international law, including a comparative view of Anglo-American, Roman, German and French jurisprudence, Philadelphia

Wharton, Francis (ed.) (1889), The Revolutionary Diplomatic Correspondence of the United States, vol. II. September 1775 to December 1775, Washington, 64-66

Whatmore, Richard (2006), Rousseau and the Representants: the Politics of the Lettres Ecrites de la Montagne, in: Modern Intellectual History, 3, 3, 385-413, https://doi.org/10.1017/S1479244306000850

Whatmore, Richard (2010), Vattel, Britain and Peace in Europe, in: Grotiana, 31, $85-107$

Whatmore, Richard (2015), What is Intellectual History?, Chichester

Whatmore, Richard, Brian Young (eds.) (2016), A Companion to Intellectual History, Chichester

Wheaton, Henry (1836), Elements of International Law, vol. I, London,

Wheaton, Henry (1845), History of the Law of Nations in Europe and America: From the Earliest Times to the Treaty of Washington, 1842, New York

Whelan, Frederick G. (1999), Vattel's Doctrine of the State, in: Haakonssen, Knud (ed.), Grotius, Pufendorf and Modern Natural Law, Aldershot, 403-434

Widmer, Paul (1988), Der Einfluß der Schweiz auf die amerikanische Verfassung von 1787, in: Schweizerische Zeitschrift für Geschichte, 38, 359-389

Willcox, William B. (ed.) (1982), The Papers of Benjamin Franklin, vol. 22, March 23, 1775, through October, 1776, New Haven, London, 1982

Wilson, George Grafton (1936), The United States and International law, in: Proceedings of the American Society of International Law, 30, 14-25

Winterhoff, Christian (2007), Verfassung - Verfassunggebung - Verfassungsänderung. Zur Theorie der Verfassung und der Verfassungsrechtserzeugung, Tübingen

Wolff, Christian (1750), Institutiones iuris naturae et gentium in quibus ex ipsa hominis natura continuo nexu omnes obligationes et iura omnia deducuntur, Halae, Magdeburgicae, officina Rengeriana 
Wolff, Christian (1764), Ius gentium methodo scientifica pertractatum in quo ius gentium naturale $\mathrm{ab}$ eo quod voluntarii pactitii et consuetudinarii est, accurate distinguitur, Francofurti, Lipsiae, Societatis Venetae

Wolff, Christian (1764-1766), Jus naturae methodo scientifica pertractatum, pars prima [-octava], et nunc primum cum viri cl. de Vattel animadversionibus, editio novissima emendatior, et auctior, Francofurti, Lipsiae

Woolsey, Theodore Dwight (1860), Introduction to the study of international law, designed as an aid in teaching, and historical studies, Boston, Cambridge

Wright, Benjamin F. Jr. (1926), American Interpretations of Natural Law, in: The American Political Science Review, 20, 3, 524-547, https://doi.org/10.2307/ 1945286

Wright, Johnson (1997), A Classical Republican in Eighteenth-Century France: The Political Thought of Mably, Stanford

Yanes, Francisco Javier (1839), Manual político del venezolano ó breve exposición de los principios y doctrinas de la ciencia social que deben ser conocidos por la generalidad de los venezolanos, Caracas

Yanes, Francisco Javier (1959), Manual político del venezolano. Idea general, o principales del derecho de gentes; estracto de Vattel y otros autores. Estudio preliminar por SAlOM, Ramón Escovar, Caracas

Zaleski, Witold (1866), Zur Geschichte und Lehre der internationalen Gemeinschaft. [...] zur Erlangung der Würde eines Magisters der diplomatischen Wissenschaften mit Genehmigung einer Hochverordneten Juristen-Facultät der Kaiserlichen Universität zu Dorpat, Dorpat

Zaleski, Witold (1874), Die völkerrechtliche Bedeutung der Kongresse. [...] behufs Erlangung der Würde eines Doctors des Staats- und Völkerrechts [...] mit Genehmigung einer Hochverordneten Juristenfacultät der Kaiserlichen, Dorpat

Zaniboni, Eugenio (2013), Rocco Nicola, in: Birocchi, Italo, Ennio Cortese, Antonello Mattone, Marco Nicola Miletti (diretto da), Dizionario Biografico dei Giuristi Italiani (XII-XX secolo), Bologna, vol. II, 1709-1710

Ziegler, Karl-Heinz (2007), Emer de Vattel und die Entwicklung des Volkerrechts im 18. Jahrhundert, in: Kremer, Markus, Hans-Richard Reuter (Hrsg.), Macht und Moral. Politisches Denken im 17. und 18. Jahrhundert, Stuttgart, $321-340$

Zordan, Giorgio (1999), L'insegnamento del diritto naturale nell'Ateneo Patavino e i suoi titolari (1764-1855), in: Rivista di Storia del Diritto Italiano, 72, 5-76

Zurbuchen, Simone (1998), Die schweizerische Debatte über die Leibniz-Wolffsche Philosophie und ihre Bedeutung für Emer von Vattels philosophischen Werdegang, in: Coleman, Patrick von, Anne Hofmann, Simone Zurbuchen (Hrsg.), The Swiss Enlightenment: Reconceptualizing Science, Nature, and Aesthetics, Genf, 91-113

Zurbuchen, Simone (2003), Patriotismus und Kosmopolitismus. Die Schweizer Aufklärung zwischen Tradition und Moderne, Zürich 
Zurbuchen, Simone (2004), Das Prinzip des Naturrechts in der école romande du droit naturel, in: Jahrbuch für Recht und Ethik, 12, 189-211

Zurbuchen, Simone (2009), Vattel's Law of Nations and Just War Theory, in: History of European Ideas, 35, 4, 408-417, https://doi.org/10.1016/j.histeuroideas. 2009.05.001

Zurbuchen, Simone (2010), Vattel's «Law of Nations " and the Principle of NonIntervention, in: Grotiana, 31, 69-84 



\section{About the Author}

Elisabetta Fiocchi Malaspina is Assistant Professor of Legal History at the Law Faculty of the University of Zurich (Switzerland). She received her MA in Law at the University of Milan and her PhD in Legal History at the University of Genoa (Italy). Moreover, she was awarded several research grants from the Max Planck Institute for European Legal History (Frankfurt am Main, Germany); was visiting research fellow at the Erik Castrén Institute of International Law and Human Rights at the Law Faculty of the University of Helsinki (Finland); and participated in the Law and Public Affairs Discussion Group at the Law Faculty of the University of Oxford (England) during a research stay. Her main research fields include history of international law, circulation and diffusion of natural law and law of nations theories between the 18th and 19th centuries, history of water law, history of land ownership and land registration (19th and 20th centuries). 



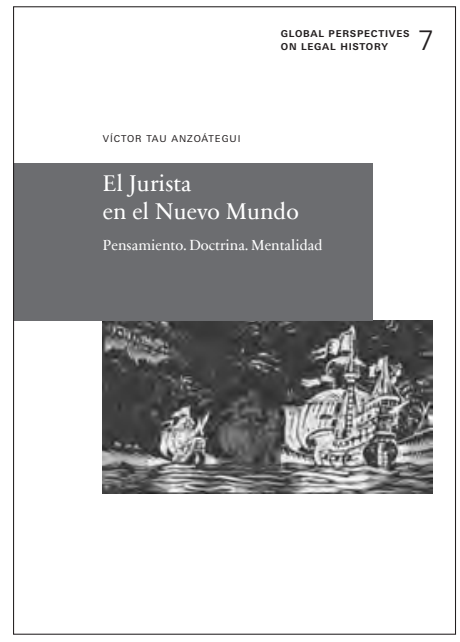

Víctor Tau Anzoátegui

El Jurista en el Nuevo Mundo

Pensamiento. Doctrina. Mentalidad

Global Perspectives on Legal History 7

Frankfurt am Main: Max Planck Institute for European Legal History 2016.

280 p., € 14,19 D

ISBN 978-3-944773-06-3

Open Access Online Edition:

http://dx.doi.org/10.12946/gplh7

This study of the history of Derecho Indiano proposes to analyze the role played by the Spanish-American jurist in the New World, as both elaborator as well as a practitioner of an art and a practice. While contributing to the development of public authority within a new society, the jurist faced episodes, realities, and circumstances of huge diversity. The studies collected in this volume appeared originally in journals and collected works in various countries. They are offered today in a revised edition. 
Global Perspectives on Legal History is a book series edited and published by the Max Planck Institute for European Legal History, Frankfurt am Main, Germany. As its title suggests, the series is designed to advance the scholarly research of legal historians worldwide who seek to transcend the established boundaries of national legal scholarship that typically sets the focus on a single, dominant modus of normativity and law.

The series aims to privilege studies dedicated to reconstructing the historical evolution of normativity from a global perspective.

It includes monographs, editions of sources, and collaborative works. All titles in the series are available both as premium print-on-demand and in the open-access format.

\section{Released volumes}

7 | Víctor Tau Anzoátegui

El Jurista en el Nuevo Mundo.

Pensamiento. Doctrina. Mentalidad

6 | Massimo Meccarelli, María Julia Solla Sastre (eds.)

Spatial and Temporal Dimensions for Legal History.

Research Experiences and Itineraries

4 | Osvaldo Rodolfo Moutin

Legislar en la América hispánica en la temprana edad moderna.

Procesos y características de la producción de los Decretos del Tercer Concilio Provincial Mexicano (1585)

3 | Thomas Duve, Heikki Pihlajamäki (eds.)

New Horizons in Spanish Colonial Law.

Contributions to Transnational Early Modern Legal History

2 | María Rosario Polotto, Thorsten Keiser, Thomas Duve (eds.)

Derecho privado y modernización.

América Latina y Europa en la primera mitad del siglo XX

1 | Thomas Duve (ed.)

Entanglements in Legal History: Conceptual Approaches

More information on the series and forthcoming volumes: http://global.rg.mpg.de 



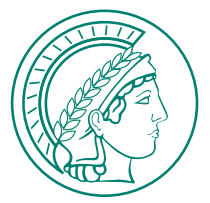

The numerous editions and early translations produced throughout the eighteenth century enabled the broad dissemination of Emer de Vattel's juridical-political work Droit des gens. This book investigates the global impact of the Droit des gens with regard to the different political realities, the historical and legal contexts as well as the attempts, mechanisms and strategies used to put these ideas into practice and establish new doctrine between the eighteenth and nineteenth centuries.

http://global.rg.mpg.de

ISBN 978-3-944773-07-0 